\title{
"Pathophysiological basis of hepatic Encephalopathy": a multi-organ perspective in patients with liver failure
}

Citation for published version (APA):

Olde Damink, S. W. (2005). "Pathophysiological basis of hepatic Encephalopathy": a multi-organ perspective in patients with liver failure. [Doctoral Thesis, Maastricht University]. https://doi.org/10.26481/dis.20050531so

Document status and date:

Published: 01/01/2005

DOI:

10.26481/dis.20050531so

Document Version:

Publisher's PDF, also known as Version of record

Please check the document version of this publication:

- A submitted manuscript is the version of the article upon submission and before peer-review. There can be important differences between the submitted version and the official published version of record. People interested in the research are advised to contact the author for the final version of the publication, or visit the DOI to the publisher's website.

- The final author version and the galley proof are versions of the publication after peer review.

- The final published version features the final layout of the paper including the volume, issue and page numbers.

Link to publication

\footnotetext{
General rights rights.

- You may freely distribute the URL identifying the publication in the public portal. please follow below link for the End User Agreement:

www.umlib.nl/taverne-license

Take down policy

If you believe that this document breaches copyright please contact us at:

repository@maastrichtuniversity.nl

providing details and we will investigate your claim.
}

Copyright and moral rights for the publications made accessible in the public portal are retained by the authors and/or other copyright owners and it is a condition of accessing publications that users recognise and abide by the legal requirements associated with these

- Users may download and print one copy of any publication from the public portal for the purpose of private study or research.

- You may not further distribute the material or use it for any profit-making activity or commercial gain

If the publication is distributed under the terms of Article $25 \mathrm{fa}$ of the Dutch Copyright Act, indicated by the "Taverne" license above, 


\title{
PATHOPHYSIOLOGICAL BASIS OF HEPATIC ENCEPHALOPATHY:
}

A multi-organ perspective in patients with liver failure

\author{
DISSERTATION \\ To obtain the degree of Doctor at \\ the Universiteit Maastricht,
} on the authority of the Rector Magnificus, Prof. dr. G.P.M.F. Mols in accordance with the decision of the Board of Deans, to be defended in public on

Tuesday 31 May 2005 at 14:00 hours

by

Stephanus Wilibrordus Maria Olde Damink

16 September 1968

Oldenzaal, The Netherlands

and

Rajiv Jalan

14 December 1962

Calcutta, India 



\author{
SUPERVISORS: \\ Prof. Dr. P.B. Soeters \\ Prof. Dr. P.C. Hayes, University of Edinburgh \\ CO-SUPERVISORS TO S.W.M. OLDE DAMINK: \\ Dr. N.E.P. Deutz \\ Dr. C.H.C. Dejong \\ CO-SUPERVISOR TO R. JALAN: \\ Dr. N.E.P. Deutz
}

AsSESSMENT COMMITTEe to S.W.M. OldDE DAMINK:

Prof. Dr. R.W. Stockbrügger, Chair

Prof. Dr. A.T. Blei, Northwestern University, Chicago

Prof. Dr. M.P. van Dieijen-Visser

Prof. Dr. T.M. van Gulik, Universiteit van Amsterdam

Prof. Dr. M.J.H.M. Jacobs

ASSESSMENT COMMITTEE TO R. JALAN:

Prof. Dr. M.J.H.M. van Dieijen-Visser, Chair

Prof. Dr. R.F. Butterworth, University of Montreal

Prof. Dr. M.J.H. Slooff, Rijksuniversiteit Groningen

Prof. Dr. C.D.A. Stehouwer

Prof. Dr. R.W. Stockbrügger 


\title{
CONTENTS
}

\author{
General introduction / 1
}

Aims of the thesis / 59

\section{SECTION 1}

\section{Interorgan ammonia and amino acid metabolism}

CHAPTER $1 / 63$

Interorgan ammonia and amino acid metabolism in metabolically stable patients with cirrhosis of the liver and a tipps

ChAPTER $2 / 83$

The kidney plays a major role in the hyperammonemia seen after simulated or actual GI bleeding in patients with cirrhosis

CHAPTER $3 / 103$

Can we influence renal ammoniagenesis or excretion

Chapter 3A / 103

The kidney plays a major role in ammonia homeostasis after portasystemic shunting in patients with cirrhosis

CHAPTER 3B / 121

Reversal of diuretic-induced hepatic encephalopathy with infusion of albumin but not colloid

ChAPTER 4 / 139

The use of the simulated bleed model to study hepatic encephalopathy

CHAPTER 4A/141

Oral amino acid load mimicking hemoglobin results in reduced regional cerebral perfusion and deterioration in memory test in patients with cirrhosis of the liver

CHAPTER 4B/161

Induced hyperammonemia alters neuropsychology, brain MR spectroscopy and magnetization transfer in cirrhosis

\section{SECTION 2 \\ Catabolic effects of upper GI bleeding and the role of isoleucine}

\section{CHAPTER $5 / 183$}

Medical hypothesis: upper gastrointestinal bleeding: an ammoniagenic and carabolic event due to the total absence of isoleucine in the haemoglobin molecule

CHAPTER $6 / 195$

Protein synthesis is severely diminished following a simulated upper GI bleed in parients with cirrhosis

Chapter $7 / 211$

Isoleucine infusion during simulated upper gastrointestinal bleeding improves liver and muscle protein synthesis in cirrhotics 



\section{SECTION 3}

Pathophysiological basis of hepatic encephalopathy in Liver Failure and role of hypothermia

CHAPTER $8 / 237$

Increased cerebral and peripheral vasodilation, and, whole body nitric oxide production after portacaval shunting in cirrhosis

CHAPTER $9 / 259$

Pathogenesis of intracranial hypertension in acute liver failure: inflammation, ammonia and cerebral blood flow

CHAPTER $10 / 281$

Treatment of uncontrolled intracranial hypertension in acute liwer failure with moderate hypothermia

ChAPTER 11/295

Restoration of cerebral blood flow autoregulation and reactivity to carbon dioxide in acute liver failure by moderate hypothermia

CHAPTER $12 / 311$

Moderate hypothermia prevents cerebral hyperemia and increase in intracranial pressure in patients undergoing liver transplantation for acute liver failure

CHAPTER $13 / 327$

Moderate hypothermia in patients with acute liver failure and uncontrolled intracranial hypertension

Samenvatting/Summary / 347

Perspectives for the future / 365

Acknowledgements / 371

Curricullum vitae / 374

List of publications / 376 


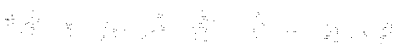

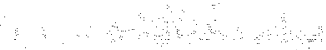

$+\quad \times \quad$ 
To the memory of my mentor the late Dr Robin Walker, who trusted in my ability and made me look beyond what I thought I was capable of doing.

Aan mijn ouders, ter nagedachtenis aan Sjan Buijs 


\title{
GENERAL INTRODUCTION
}

\author{
HEPATIC ENCEPHALOPATHY IN LIVER FAILURE, \\ THE ROLE OF AMMONIA AND OTHER FACTORS
}

This chapter is a condensation of reviews published in The Lancet 1997; 350: 1309-15, Neurochemistry International 2002; 41: 177-88, The International Journal of Biochemistry of Cell Biology 2003; 35: 1175-81 Seminars in Liver Disease 2003;23:271-82 


\section{OUTLINE}

1.0 Background

2.0 The definition of HE, type A, B and C

2.1 $\mathrm{HE}$ associated with cirrhosis, type $\mathrm{C}$

2.2 $\mathrm{HE}$ occurring in patients with acute liver failure, type $\mathrm{A}$

3.0 Pathogenesis of HE

3.1 Alterations in blood-brain barrier

3.2 Changes in energy metabolism

3.3 The role of gut-derived factors

3.4 Changes in cerebral neurotransmission

3.5 Changes in cerebral blood flow

3.6 Additional effects of inflammation

4.0 The role of ammonia in the pathogenesis of $\mathrm{HE}$

4.1 Arterial concentrations

4.2 Cerebral metabolism

4.2.1 Cerebral ammonia and glutamine metabolism during liver failure

4.3 Proposed mechanisms of how ammonia produces $\mathrm{HE}$

4.3.1 The ammonia-glutamine-brain oedema hypothesis

4.3.2 Direct ammonia related neurotoxicity

4.3.3 Ammonia and altered gene expression in liver failure

5.0 Interorgan ammonia metabolism

5.1 Intestinal metabolism

5.1.1 Ammonia liberating events

5.1.2 Intestinal ammonia and glutamine metabolism during liver failure

5.2 Liver metabolism

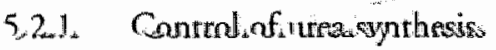

5.2.2 Hepatic ammonia and glutamine metabolism during liver failure

5.3 Muscle metabolism

5.3.1 Muscle ammonia and glutamine metabolism during liver failure

5.4 Renal metabolism

5.4.1 Renal ammonia and glutamine metabolism during liver failure

6.0 Treatment of $\mathrm{HE}$

6.1 Treatment of HE in cirrhosis, type $\mathrm{C}$

6.1.1 Treatment aimed at gut-derived factors, including ammonia and the excretion of nitrogenous waste

6.1.1.1 Diet

6.1.1.2 Non-absorbable disaccharides

6.1.1.3 Antibiotics

6.1.1.4 Sodium benzoate

6.1.1.5 L-Ornithine-L-Aspartate

6.1.2 Treatment aimed at changes in neurotransmission

6.1.2.1 Flumazenil. 
6.1.3 Treatment based on the GABAergic hypothesis

6.2 Treatment of HE in ALF, type A

6.2.1 General measures

6.2.2 Treatment aimed at gut-derived factors, including ammonia and the excretion of nitrogenous waste

6.2.2.1 Non-absorbable disaccharides

6.2.2.2 L-Ornithine-L-Aspartate

6.2.3 Treatment aimed at cerebral edema

6.2.3.1 Mannitol

6.2.3.2 Hyperventilation

6.2.3.3 Thiopental sodium

6.2.3.4 Indomethacin

6.2.3.5 Propofol

6.2.4 Treatment aimed at changes in neurotransmission

6.2.4.1 N-Methyl-D-Aspartate antagonism

6.2.4.2 Phenytoin

6.2.5 Treatment alimed at reducing additional effects of inflammation

6.2.5.1 N-Acetylcysteine

6.2.5.2 Dexamethasone

6.2.5.3 Antibiotics

6.2.5.4 Heparecromy

7.0 Summary 


\section{Table 1}

West Haven Criteria for grading mental state in $\mathrm{HE}$ (39)

Grade 0

No abnormality detected

Grade 1

Trivial lack of awareness, euphoria, and anxiety

Shortened attention span

Impaired performance in adding or substracting

Grade 2

Lethargy, apathy, disorientation for time and place

Obvious personality change

Inappropriate behaviour

Grade 3

Somnolence to semi-stupor, but responsive to stimuli

Confusion

Gross disorientation

Grade 4

Coma

Mental state not testable 


\subsection{BACKGROUND}

The word 'encephalopathy' originates from the Greek language and literally means 'inside head suffering'. In 1893 members of Pavlov's group in St. Petersburg described 'The Meat Intoxication Syndrome' (1). They described a causal relationship whereby the shunting of blood from the portal vein into the caval vein (bypassing the liver), resulted in the liver not being able to metabolise ammonia into urea leading to ammonia accumulation in the blood. In dogs, they observed a rise in arterial blood ammonia levels after a major protein meal that was associated with behavioural disturbances ( 2 , 3). Over fifty years later the same observations of elevated arterial ammonia levels and behavioural alterations were made in human patients with cirrhosis of the liver (4). Direct evidence linking ammonia to the pathogenesis of hepatic encephalopathy (HE) was produced using positron emission tomography with $13 \mathrm{~N}$ Ammonia as tracer. The rate of uptake of ammonia into the brain of patients with $\mathrm{HE}$ was shown to be significantly higher than in healthy volunteers (5). Recent studies have shown that arterial ammonia levels of $>150 \mathrm{mmol} / \mathrm{L}$ in patients with acute liver failure (ALF) predict a greater likelihood of dying from brain herniation (6). It has been estimated, from studies in experimental animals, that brain ammonia influx in ALF may be up to 45 -fold higher than normal (7).

The whole area of HE moved forward with a Consensus being reached on the definitions of this syndrome. HE is defined as a spectrum of neuropsychiatric abnormalities seen in patients with liver dysfunction after exclusion of other known brain disease (8). The multiaxial definition of HE is required such that both the type of hepatic abnormality and the duration/characteristics of neurologic manifestations in cirrhosis can be incorporated. In ALF, patients can succumb to a neurologic death, with brain edema and intracranial hypertension (9). In patients with cirrhosis, a wide spectrum of symptoms are summarised under the term HE. These neuropsychiatric abnormalities range from minor sleep disturbances, through discernable alterations in brain function, overt psychiatric/neurological symptoms to deep coma (see Table 1). However, recent studies have suggested that patients with cirrhosis can develop cerebral changes that are indistinct from that observed in ALF suggesting that the underlying pathogenic mechanisms in the development of this syndrome are likely to be similar $(10,11)$. Both in cirrhosis and in ALF, the neurones and blood brain-barrier are thought to be anatomically intact (12). HE in cirrhosis is characterised neuropathologically by Alzheimer Type II astrocytosis (Figure 1a) (13). Similarly, in ALF the astrocytes are swollen too (Figure 1b) (12). Astrocytes occupy about one third of the cortical volume and form a barrier segregating neurons from the external environment. Astrocytes are the site of ammonia detoxification in the brain and eliminate ammonia by the synthesis of gluta- 
mine through deamidation of glutamate by glutamine synthetase (14). The finding that similar changes can be induced experimentally in astrocytes in culture following exposure to ammonia suggests that $\mathrm{HE}$ is primarily due to dysfunction of these cells with neuronal dysfunction occurring secondarily (13-15). Astrocytes maintain and regulate the extracellular environment and influence neuronal excitability and neurotransmission.
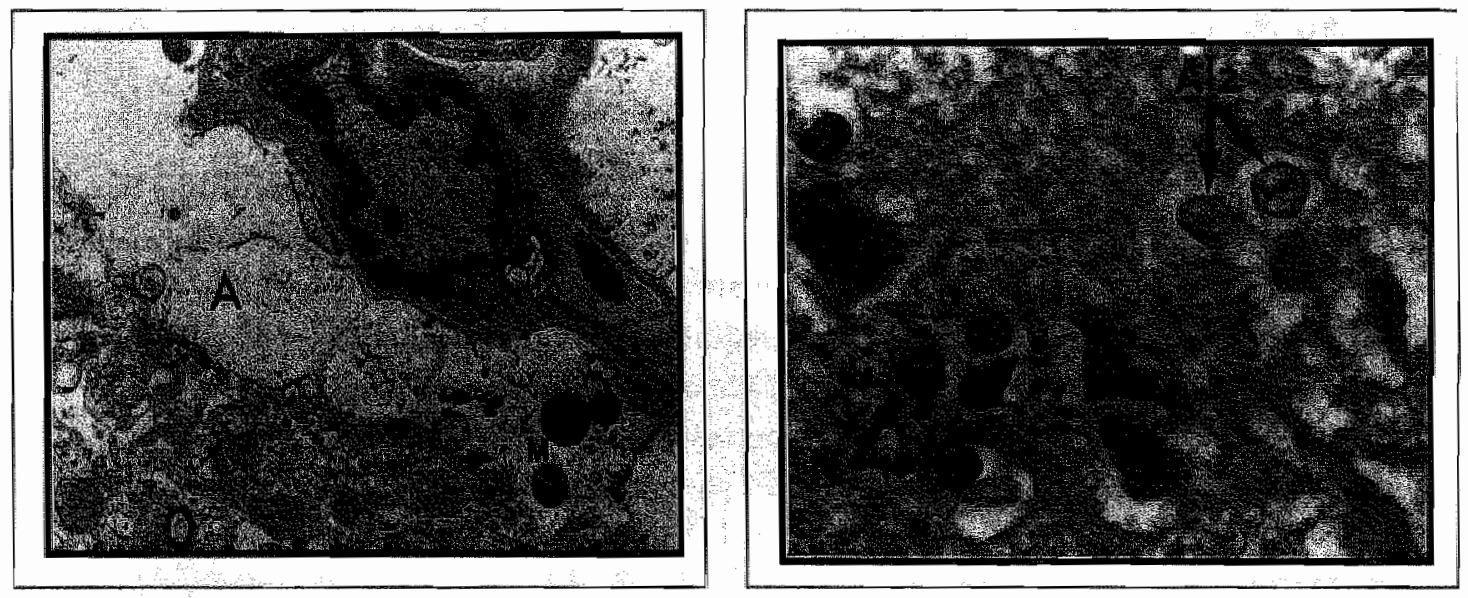

FIGURE 1A (LEFT). Electron micrograph of the brain from a patient dying from acute liver failure showing a swollen astrocyte $(A)(M$ : mitochondria).

FIGURE 1B (RIGHT). Histopathology of the brain from a patient dying from cirrhosis showing evidence of type II astrocytosis (Alz) and a normal neuron (n) (68). 


\subsection{THE DEFINITION OF HE: TYPE A, B AND C}

In a recent consensus meeting on $\mathrm{HE}(8)$, the following definition of $\mathrm{HE}$ was proposed. Type A HE is encephalopathy associated with acute liver failure. HE that is associated with portosystemic bypass, but without any evidence of underlying liver disease is referred to as Type $B$. This is a rare syndrome and very few data are available about the clinical consequences of this syndrome. Type C HE is encephalopathy associated with cirrhosis and portal hypertension or portal-systemic shunts. The following section focuses on Types $\mathrm{C}$ and $\mathrm{A}$, which are commonly seen in clinical practice.

\subsection{HE associated with Cirrhosis, Type C:}

The spectrum of $\mathrm{HE}$ in cirrhosis extends from minimal alterations in the neuropsychological function (minimal HE) to advanced stages where disturbances in consciousness are obvious (overt HE).

Minimal HE: Minimal $\mathrm{HE}$ is viewed as a mild neurocognitive disorder present in patients with cirrhosis (16). Individuals with this condition have cognitive deficits that affect mostly attention, speed of information processing and motor abilities, and a pattern that suggests 'subcortical' brain involvement. The level of cognitive impairment is so minimal that it is not recognized in a standard neurologic examination. The diagnosis requires corroboration of cognitive deficits using additional tests. Neurophysiologic tests (e.g., spectral electroencephalogram, evoked potentials) have been advocated as more sensitive than psychometric tests (17). However, these methods assess different aspects of the clinical syndrome of HE. The best characterization of minimal $\mathrm{HE}$ can be obtained through neuropsychologic measures, which also allow for a better estimation of the possible implications of such deficits on daily living $(18,19)$. It must be appreciated that assessment of the implications of minimal $\mathrm{HE}$ are confounded by many factors such as the effects of cirrhosis and alcohol consumption, both of which can result in impairment in cognitive defects. The neuropsychologic impairment of minimal HE is sufficiently mild so that effects on instrumental activities (e.g., shopping, answering the phone, taking public transportation) or basic activities of daily life (e.g., dressing, personal hygiene, eating) are not expected. However, the impairment of attention, executive function, and psychomotor skills may hamper the more complex activities such as appreciating the nuances of personal relationships, planning a trip, handling finances, gardening, performing a job, or driving a car.

There is some evidence to support the idea that this may be the case. In a study in 179 outpatients with cirrhosis, the Sickness Impact Profile questionnaire detected more frequent impairment in daily life activities related to alertness, social interactions, recreation, or work among those with minimal $\mathrm{HE}$, an effect that was maintained after 
adjusting for the severity of liver failure (20). Recent literature reports the results of a driving study in Germany where 48 subjects with cirrhosis were evaluated. Fourteen of these subject had minimal $\mathrm{HE}$, using a standardized driving test. Driving abilities were compared with a control group of 50 subjects at a stable phase of chronic gastro-intestinal disease to adjust for the possible effects of chronic illness. The main finding of the study were that ratings were worst in patients with minimal $\mathrm{HE}$, while subjects without cognitive impairment scored similar to controls (21).

'Overt HE: About $30-40 \%$ of patients presenting with cirrhosis will develop the first episode of $\mathrm{HE}$ within 1 year of diagnosis (Figure 2). The widespread use of the transjugular intrahepatic portosystemic stent-shunt (TIPSS) has brought back HE in the centre of interest of clinical practice in view of the fact that in $30-40 \%$ patients with this procedure HE occurred. Over a 7-year period, we followed 273 patients that had a TIPSS inserted (22). During follow-up, 94 episodes of HE were documented in 77 (28\%) patients, the first episode occurring at a median interval of 3 months after TIPSS. HE was present prior to TIPSS in $26 \%$ (79 patients); of these $25 / 79(32 \%)$ had HE after TIPSS. There were 46 episodes of de novo HE of which 30 were transient and 16 chronic. Thirty-eight episodes were induced by precipitating factors like bleeding $(n=26)$, sepsis $(n=6)$ and others $(n=6)$. Portal pressure gradients were lower in the chronic group ( $3 \mathrm{mmHg}$ ) compared to both the transient de novo $(9 \mathrm{mmHg})$ and precipitated groups (15 mmHg). Corresponding Child-Pugh scores were not different between the groups. Whilst most patients' episodes of HE were transient or responded to medical measures, 17 patients required narrowing or closure of the TIPSS as a result of intractable HE, Univariate analysis showed Child's class and prior HE to be significantly related to development of $\mathrm{HE}$, whereas multivariate analysis using these variables showed only prior HE to independently predict development of HE. Mortality was higher in patients that had HE before TIPPS, compared to those that developed HE, compared with those that did not (Figure 3) (25). Good data describing the short-term outcome of patients who have 'overt HE' has not been systematically studied, bur Gines et al. showed that the occurrence of the first episode of $\mathrm{HE}$ was associated with diminished survival. Approximately $50 \%$ of patients presenting with $\mathrm{HE}$ would be dead within 1 year of developing the complication (23) (Figure 4 and 5). 


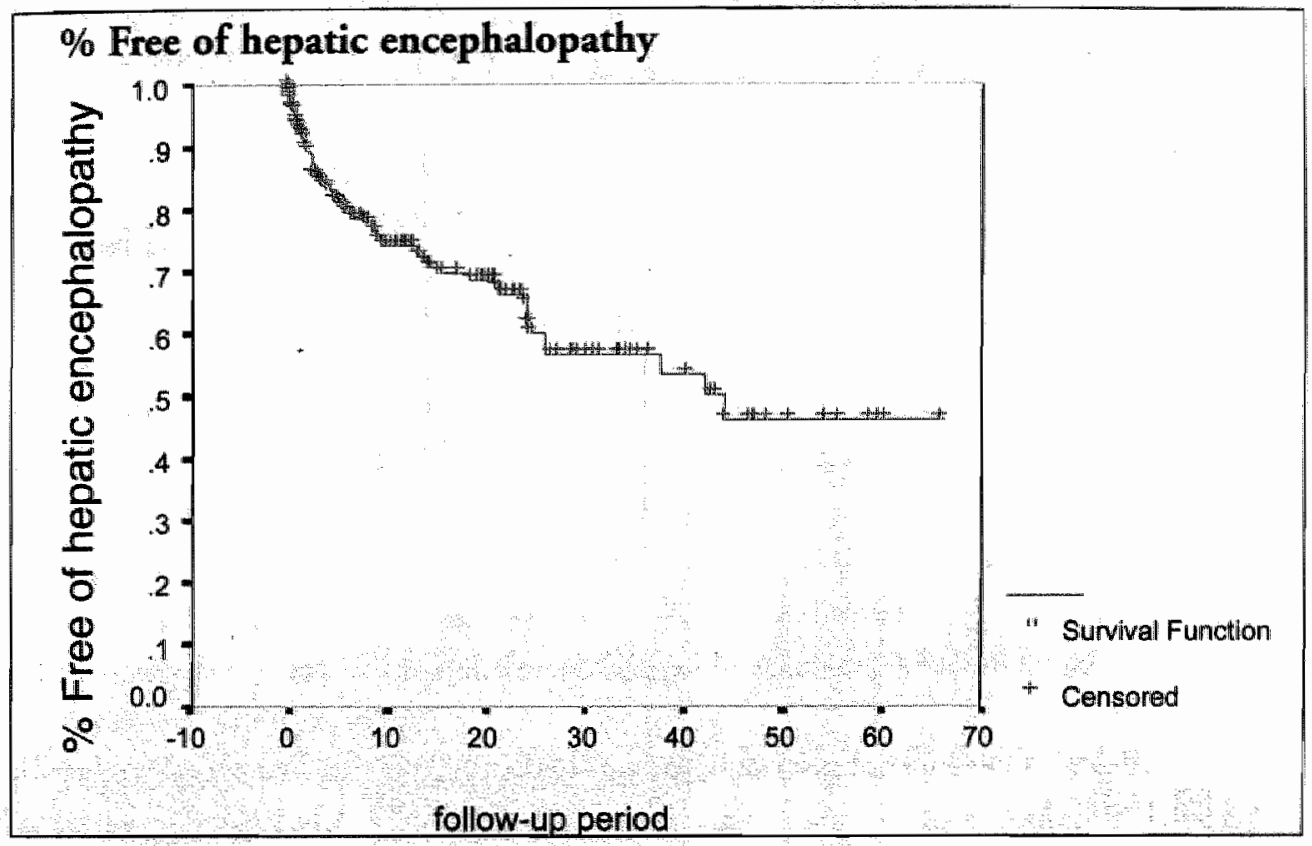

FIGURE 2 (TOP). Cumulative incidence of hepatic encephalopathy following insertion of transjugular intrahepatic portosystemic stent shunt (22). Follow-up period in months.

FigURE 3 (BELOW). Mortality of patients is significantly higher in patients that developed hepatic encephaloparthy (HE) following insertion of transjugular intrahepatic portosystemic stent shunt compared with those who did not develop HE (22). Follow-up period in months.

\section{$\%$ Mortality}

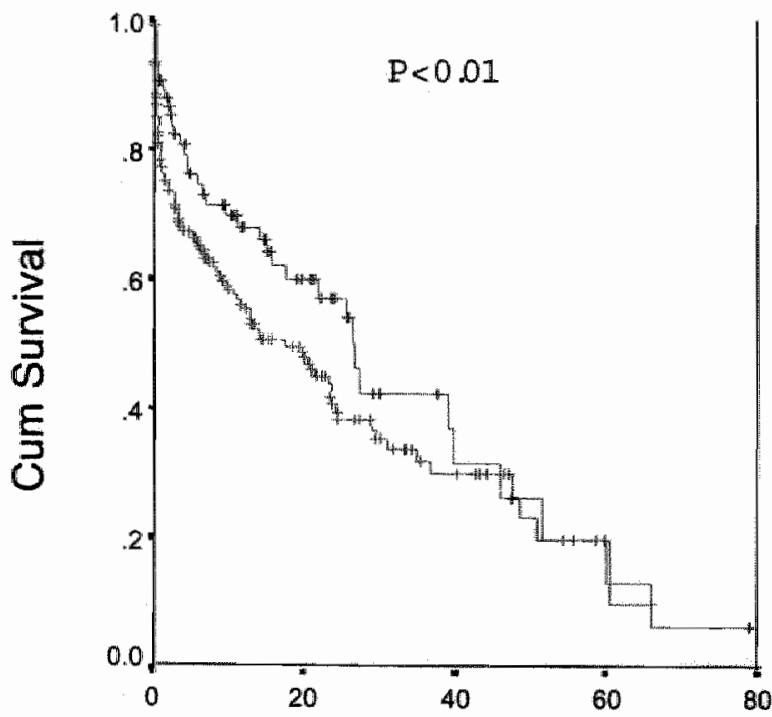

Without HE

With HE 

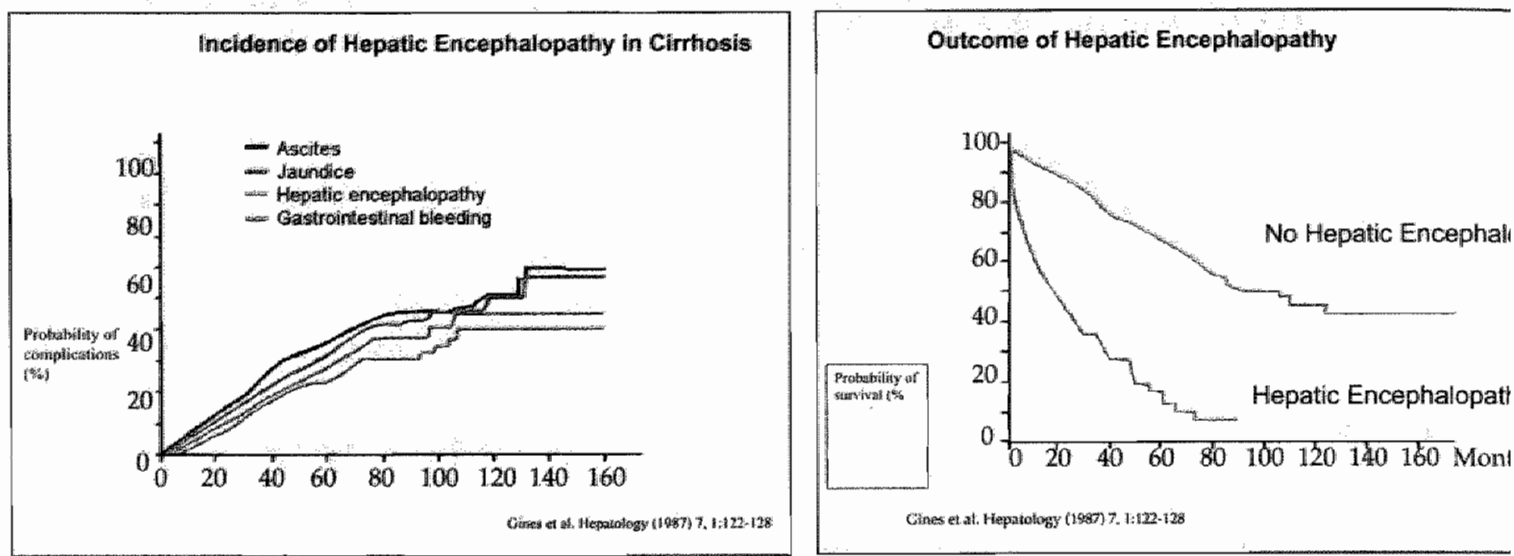

FIGURE 4 (LEFT). Cumulative incidence of hepatic encephalopathy in patients diagnosed with cirrhosis (23).

FIGURE 5 (RUGHT). Probability of survival in patients presenting with the first episode of hepatic encephaloparhy.

FiguRE 6 (BELOW). Outcome of parients referred to a single tertiary centre (Scottish Liver Transplantation Unit, Edinburgh) for management of severe liver dysfunction resulting from paracetamol overdose (OD) (data from reference 9). Criteria refer to the Kings College Criteria for poor prognosis; OLT: orthotopic liwer transplantation; MOFS: multiple organ failure syndrome CP. died of increased intracranial pressure.

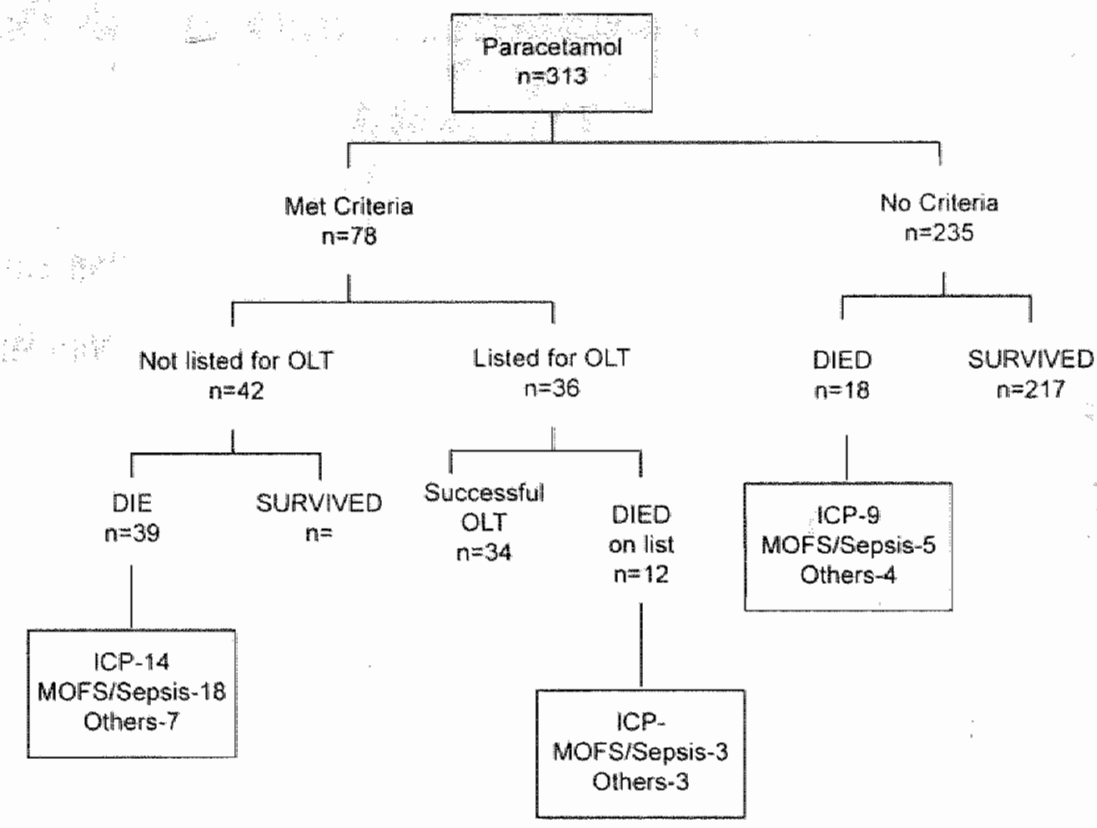




\subsection{HE occurring in patients with acute liver failure; Type A:}

The development of HE in patients with ALF is the key event that defines their prognosis (24-26). It is characterized by rapid deterioration in the level of consciousness, increased ICP and reduced cerebral perfusion pressure. Neuropathologically, the brain and astrocytes are oedematous (27). The exact pathophysiological mechanisms underlying the development of brain edema and increased ICP are not entirely clear but likely to be multifactor and will be discussed in detail later (28-30). The increase in ICP occurs in conjunction with dysfunction of multiple organ systems that occurs in patients with ALF. The circulatory disturbance in ALF is an early manifestation that tends to worsen during the course of the illness and is characterized by systemic vasodilatation which results in increased cardiac output and reduced systemic vascular resistance and mean arterial pressure. As a result, most patients in the advanced stage require intensive cardiovascular monitoring, and fluid and inotropic support. The increase in ICP occurs against this background and the strategies to manage increased ICP must be considered in this context.

Prognostic variables predicting the outcome of patients with ALF have been welldescribed and validared. The most comimonly used parameters to define the outcome of patients with ALF are the Kings College criteria, which have been used by us as criteria for poor prognosis. Data in relation to the impact of increased ICP on the outcome of patients with ALF are scanty, but over $90 \%$ mortality is expected in patients in whom ICP cannot be controlled with conventional measures. With OLT, survival rates of up to $80 \%$ can be achieved $(25,31)$. In ALF, $30-40 \%$ of parients die while waiting for a donor organ to become available, primarily due to the effects of increased ICP. Over a 7 -year period we followed 313 patients with acute liver injury due to paracetamol overdose (Figure 6) (9). Of the 78 patients that were in the group with a poor prognosis, 42 had other contraindications for orthotopic liver transplantation (OLT). Thirty-nine of these patients died and the immediate cause of death in these patients was brain herniation from elevated ICP in $14(35 \%)$. Of the 36 patients that were listed for OLT, 12 died after being removed from the waiting list (for uncontrolled increase in ICP, sepsis, bleeding or adult respiratory distress syndrome) while waiting for a donor organ to become available. Six of these deaths were from brain herniation (50\%). In those patients that underwent successful OLT, 2 patients died in the early post-transplant period from the effects of increased ICP. Of the 235 patients that were in the good prognosis group, 18 patients died. Death in 9 of these patients was the result of brain herniation (50\%). These data suggest that despite our best efforts in management, a significant proportion of patients continues to die from the effects of increased ICP (9). 

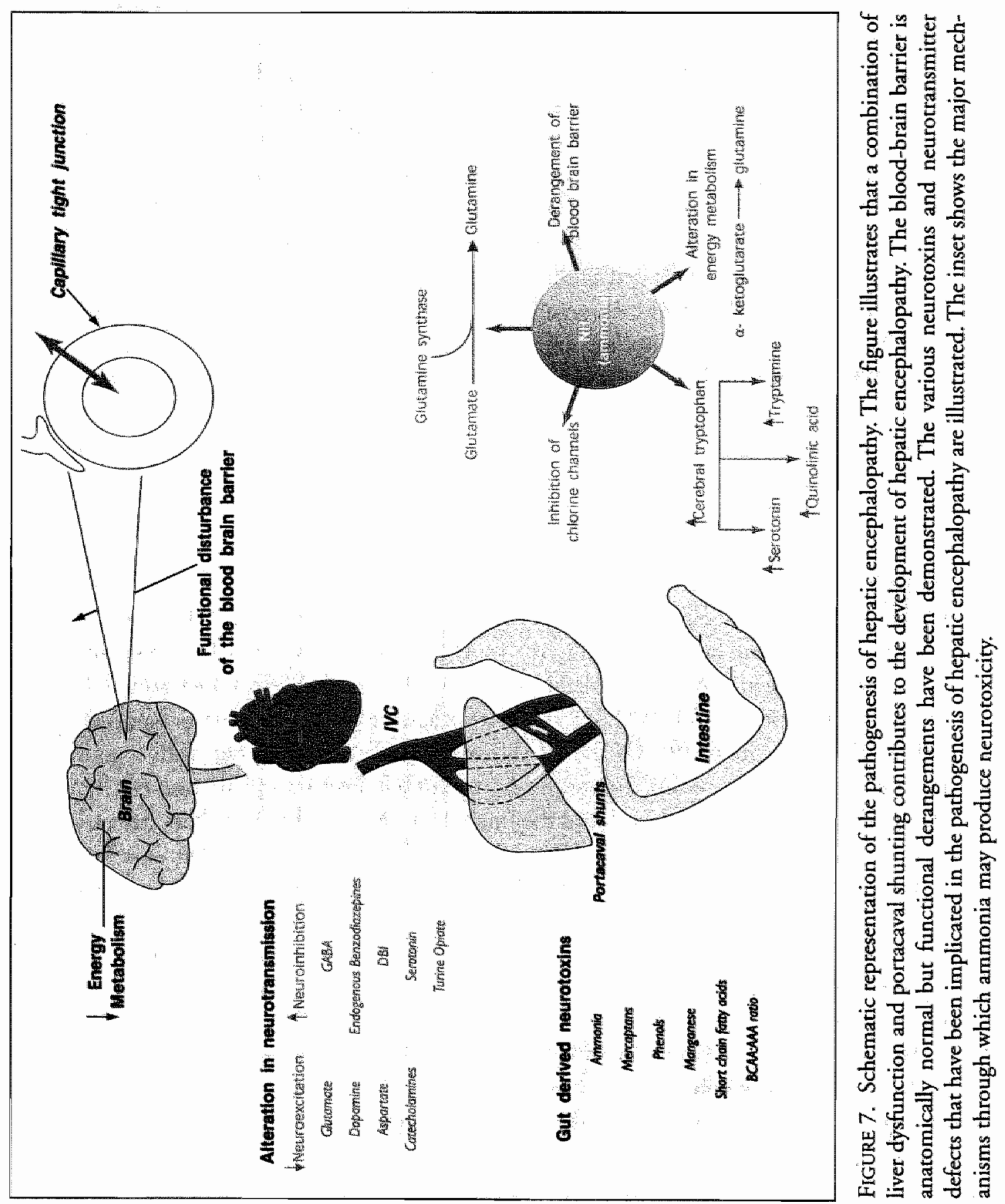


\subsection{Pathogenesis OF HE}

The pathogenesis of HE remains unclear and many mechanisms have been proposed at different times. The most important factors are summarized in Figure 7.

\subsection{Alterations in blood-brain barrier}

The blood-brain barrier ensures protection of the biochemical environment of the brain. Patients with HE have no anacomical defects of the blood-brain barrier (12) but functional derangements in the blood-brain barrier have been described resulting in increased transport of neutral amino acids and decreased transport of basic amino acids (32). The mechanism of this functional disturbance is not clear, although increased concentrations of ammonia and methanthiols have been implicated. This disturbance in the blood-brain barrier probably provides the mechanism by which other factors in HE may also operate (33).

\subsection{Changes in cerebral energy metabolism}

Patients with HE have decreased cerebral blood flow and decreased consumption of glucose and oxygen (34). Positron emission tomography scanning has shown correlations between alterations in cerebral blood flow and the severity of neuropsychological dysfunction (35). In animal models, abnormalities in cerebral energy metabolism occur after the onset of coma (36), but brain concentrations of high-energy phosphates (phosphocreatine, adenosine triphosphate) measured biochemically are not altered in experimental ALF (37). Extrapolation of this model to human beings is difficult, but changes in cerebral blood flow and glucose metabolism may be an epiphenomenon, secondary to a global depression in the function of the central nervous system, rarher than the cause of $\mathrm{HE}$. Longitudinal studies are required for further clarification.

\subsection{The role of gut-derived factors}

Ammonia has been judged the most important factor in the pathogenesis of $\mathrm{HE}$ (discussed in detail later). Concentrations of ammonia are raised in the systemic circulation and in the cerebrospinal fluid in patients with HE (38). Factors supporting the importance of ammonia include the production of a syndrome resembling $\mathrm{HE}$ by hyperammonemia, caused by factors unassociated with cirrhosis or portosystemic shunting (such as urea-cycle enzyme deficiencies); reduction of circulating ammonia concentrations by treatment with lactulose and antibiotics, which improves HE (39); precipitation of encephalopathy in patients with cirrhosis by administration of ammoniagenic substances (40-42), and the significant positive correlation that has been reported between arterial ammonia concentrations and the incidence of brain herniation due to raised ICP in patients with $\operatorname{ALF}(6)$. Observations against ammonia as the sole 
factor in the development of $\mathrm{HE}$ are the fact that HE occurs despite normal arterial concentrations of ammonia (43); and the seemingly contradictory effect of ammonia on the function of the central nervous system, with low ammonia concentrations producing excitation rather than neurodepression $(43,44)$. Studies have focused on the role of ammonia in the modulation of other neurotransmitter systems such as ?-aminobutyric acid (GABA), benzodiazepines, and serotonin (43).

Patients with cirrhosis have a characteristic abnormality on $T_{1}$-weighted magneticresonance imaging of the brain that is seen as hyperintensity of the globus pallidus (Figure 8) (45). $\mathrm{HE}$ and manganese toxic effects produce similar changes in magneticresonance images of the basal ganglia (45), and increased manganese concentrations have been found in the basal ganglia of patients with HE (46). Manganese concentrations may be raised in patients with cirrhosis because of impaired excretion in bile, but the clinical significance of these findings is uncertain (45).

\subsection{Changes in cerebral neurotransmission}

The normal state of consciousness is determined by a fine balance between the state of excitatory and inhibitory neurotransmission. Glutamate is the most important cerebral excitatory neurotransmitter in the brain (47) and its concentration at the synaptic level is dependent on the glutamate-glutamine cycle, in which glutamate is released by the presynaptic neurons; there, the glutamate reacts with the postsynaptic neurons and is inactivated to glutamine in the astrocyte (47). In patients with $\mathrm{HE}$, cerebral glutamate is decreased $(48,49)$, re-uptake mechanisms for glutamate are impaired, and down-regulation of glutamate-binding sites on the postsynapric neurons occurs (48), resulting in decreased neuroexcitation. GABA is an important inhibitory neurotransmitter, and although it does not cross the blood-brain barrier, GABA-ergic tone is increased in $\mathrm{HE}$ (43). Endogenous benzodiazepine concentrations are raised in plasma and cerebrospinal fluid of patients with HE (50), these endogenous benzodiazepines may be present in the diet or may be metabolic products of the microbial flora of the gut (51). Positron emission tomography suggests a diffuse increase in benzodiazepine receptor binding in the brain of patients with $\mathrm{HE}$ after administration of labelled flumazenil, a benzodiazepine receptor antagonist (9) (52). Diazepam-binding antagonists modulate the activity of GABA-ergic and glutamatergic tone by inducing the glial cells to produce neurosteroids, which have been implicated in the pathogenesis of HE (53). The significance of increased GABA-ergic tone is unclear, but it may contribute to the neurodepression seen in $\mathrm{HE}$.

Brain concentrations of catecholamines are decreased in animal models of HE but such a decrease has not been proven in human beings. Studies show that dopamine-2 receptors are downregulated in patients with severe $\mathrm{HE}$ and may contribute to the derangements in psychomotor function (54). Serotonin concentrations are increased in animal models of $\mathrm{HE}$, but the concentration of cerebral serotonin receptors is debated. 

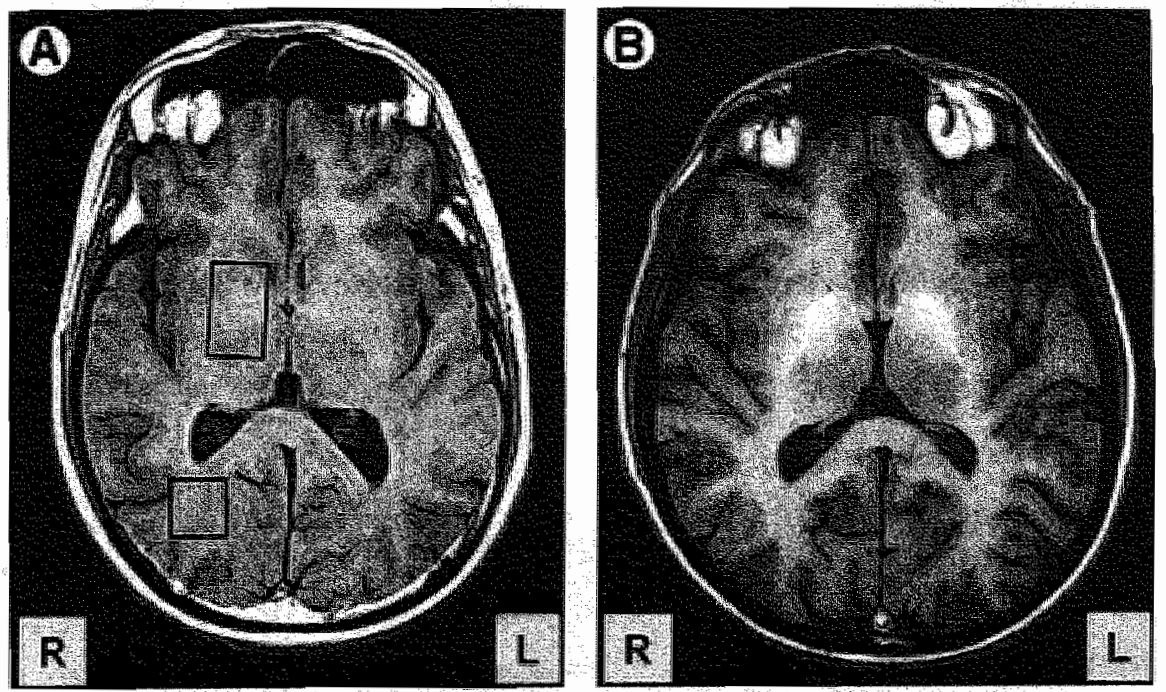

FIGURE 8 (ABOVE). Figure illustrating magnetic resonance inages of the brain from a healthy volunteer (A) and a patient with hepatic encephalopathy (B) showing brightness of the basal ganglia that is thought to result from manganese deposition (45).

FIGURE 9 (BELOW). These Positron Enission Tomographs images with $11 \mathrm{C}$ flumazenil show a significantly higher uptake of the ligand in all brain areas in a parient wirh grade I HE compared to a healthy volunteer $(p<0.0001)(68)$.

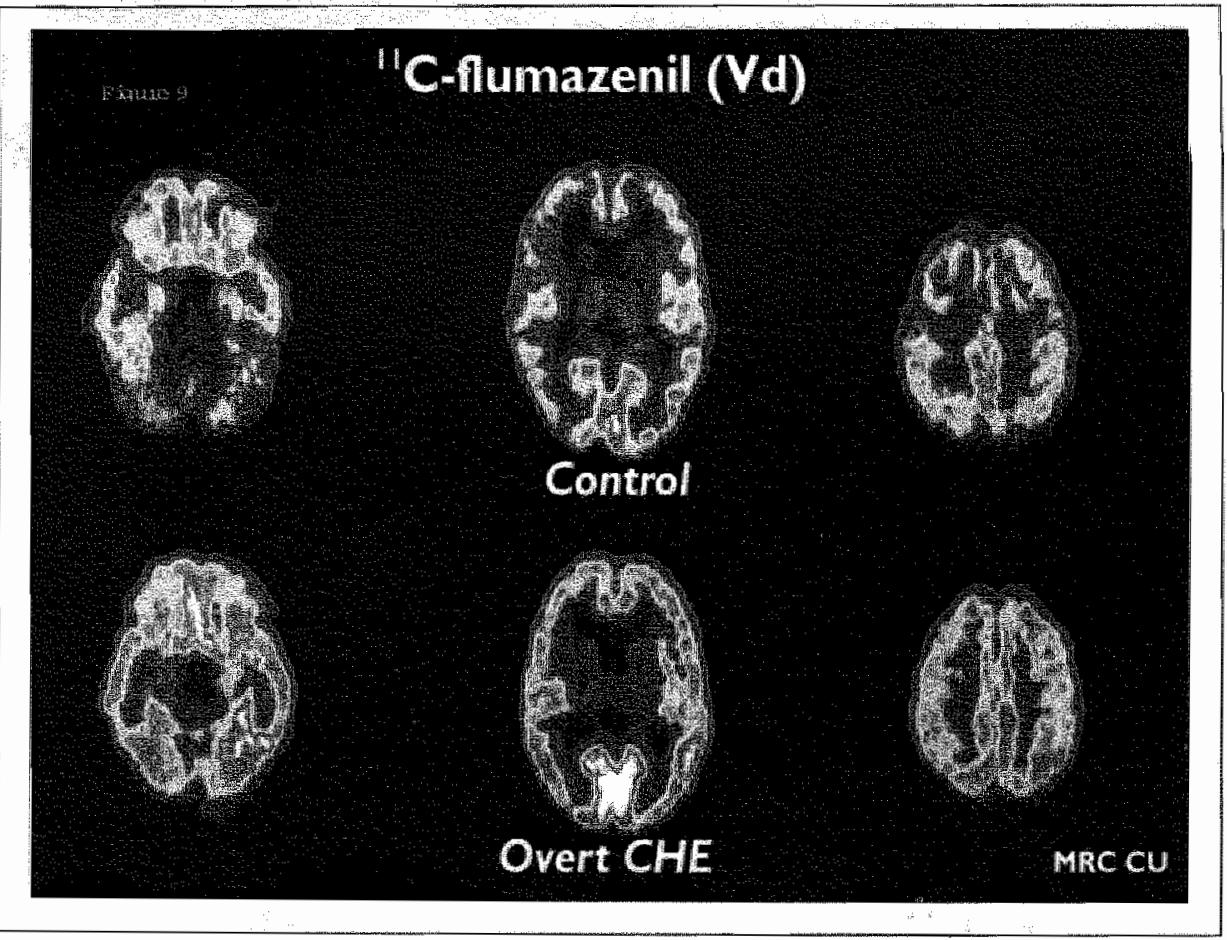


The increased cerebral concentrations of aromatic amino acids inhibit tyrosine hydroxylase, thereby generating false neurotransmitters, such as octopamine, tyramine, and phenylethanolamine, which are believed to compere with both catecholaminergic and dopaminergic neurotransmission. Although their concentrations are increased in $\mathrm{HE}$, direct injection of octopamine into the brains of animals did nor produce any substantial abnormality (55). Decreased aspartate (a neuroexcitator) has been found in comatose rats, concentrations of taurine (a neuroinhibitor) are increased in animal models, and the density of opiate receptors (a neuroinhibitor) is increased in dogs with portacaval shunts $(43,49)$.

\subsection{Changes in cerebral blood flow}

There is increasing evidence that increased cerebral blood flow may underlie the parhogenesis of elevated ICP in ALF, or type C HE $(56,57)$. The data from studies in animal models suggest that increases in cerebral blood flow are the important initiating mechanisms for increases in brain water, but studies in parients with ALF have shown wide variations in the measured values of cerebral blood flow (58-61). It is possible that these wide variations in cerebral blood flow reported in the literature represent different stages at which the measurements were made. Generally, the data suggest increased cerebral blood flow in those patients with high ICP with lower cerebral blood flow being reported in those that have minimal elevations in ICP. A part of the problem in assessing the role of altered cerebral blood flow in patients with ALF and how they relate to changes in ICP is the absence of longitudinal studies with serial measurements in the same patient and the effect of therapeutic intervention (such as Mannitol or Thiopentone) which may alter cerebral blood flow.

\subsection{Additional effects of inflammation}

Recent studies suggest that mediators of SIRS play a role in the development of intracranial hypertension. In a large series of patients with ALF, the presence of markers of inflammation or overt infection was associated with more severe encephalopathy and the likelihood of developing intracranial hypertension $(62,63)$. Moreover, studies in patients with head injury have suggested that increased brain cytokines may increase cerebral blood flow and ICP (64). Similarly, inflammatory conditions such as meningitis are associated with increased $\mathrm{CBF}$ and ICP (65). Reduction in the inflammatory response reduces $\mathrm{CBF}$ and ICP in head trauma (64-66).

The above studies support the notion that increases in CBF may be the critical event that produces a transition from minimally increased ICP to the advanced stages and mediators of inflammation may be the trigger that increases $\mathrm{CBF}$. Although we know that ammonia is critical to the pathogenesis of $\mathrm{HE}$, there is no direct correlation between the measured concentration of ammonia and the severity of $\mathrm{HE}$, which can occur with normal arterial concentrations of ammonia (43). Additional factors may therefore be important. Astrocytes belong to the macrophage lineage and therefore have 
the potential repertoire of cytokine responses. They contain most cytokines and have the ability to synthesise interleukin-1beta (IL-1?) in response to peripheral inflammation (67), which may induce soluble mediators such as nitric oxide, superoxide and prostaglandins, which make the brain more susceptible to the effects of hyperammonemia. We have recently confirmed that a reduction in circulating pro-inflammatory cytokines following hepatectomy is associated with a reduction in cerebral blood flow and ICP (68). We have also recently shown that the presence of markers of a systemic inflammatory response increases the deleterious neuropsychological effect of hyperammonemia (69). 


\subsection{THE ROLE OF AMMONIA IN THE PATHOGENESIS OF HE}

The liver plays a central role in whole body nitrogen metabolism. Liver failure and resulting hepatocellular dysfunction leads to disturbances in body nitrogen homeostasis. In liver failure; the diminished hepatic urea synthesis capacity (70) and the intraand/or extrahepatic shunting of portal blood into the systemic circulation are crucial (71). These characteristics lead to hyperammonemia resulting in an alteration in interorgan ammonia trafficking. Although ammonia is an important participant in several biochemical pathways, it is toxic at elevared levels $(47,72)$ and has been hypothesized to induce the syndrome of hepatic encephalopathy. Glutamine synthesis is the most important alternative pathway for ammonia detoxification. The cytosolic enzyme glutamine synthetase catalyses the synthesis (amidation) of glutamine from equimolar amounts of glutamate and ammonia (73). It is present in several organs in the body and its activity is increased by glucocortoid hormones, stress and glutamine depletion (74). Glutamine is a non-toxic, non-essential amino acid, consisting of a fivecarbon chain and two nitrogen residues. It is the most abundant free amino acid in the body with the highest plasma concentration and it constitutes approximately $50 \%$ of the whole body free amino acid pool (75).

Other organs can take up glutamine, where it is split by the intra-mitochondrial phosphate dependent enzyme glutaminase into glutamate and ammonia (76). Glutaminase in the liver is activated by ammonia, in contrast to other types of glutaminase, which are inhibited by ammonia (76). It can be deducted from the above that altered glutamine metabolism can counteract but also contribute to the development of hyperammonemia. Furthermore, systemic hyperammonemia has considerable impact on inter-organ glutamine metabolism. The existing data on interorgan trafficking of glutamine during liver failure will therefore also be discussed.

The disturbances in nitrogen homeostasis in patients and experimental animals with liver failure have been and still are subject to extensive research. In particular, the interplay between ammonia and glutamine metabolism during liver failure has received considerable attention, but was mostly studied in isolated organs or organ systems. The consequences of liver failure for inter-organ trafficking of ammonia have been studied sparsely, probably because these studies are difficult to perform, particularly in humans. However, in order to understand the aetiology and consequences of hyperammonemia and to be able to develop new therapies to lower arterial ammonia levels, knowledge of altered interorgan ammonia trafficking is essential.

More than a century ago Hahn and colleagues from Pavlov's group in Saint Petersburg $(1,2)$ were the first to systematically approach HE. They observed the devel- 
opment of stupor in dogs with an Eck fistula (a portacaval shunt), that were fed meat, and called it the meat intoxication syndrome. Behavioural derangement occurred only in dogs with patent shunts, and could be reversed if the dogs were switched to a milk and bread diet (1). In a follow-up study they determined ammonia concentrations in various organs and blood vessels of Eck fistula dogs (2). They observed a 3-fold higher ammonia concentration in the portal vein compared to arterial blood. More importantly, they reported increased blood and brain ammonia concentrations after the meat protein meal, and hypothesized that ammonia could be the cause of the cerebral disturbances. They concluded their series of experiments as follows: "Here we see the causal relationship: shunting of portal venous blood into the caval vein, by which the liver is not able to metabolise the ammonia of portal blood into urea, leading to ammonia accumulation in blood: intoxication" (2). Half a century later similar observations were made in humans: the 'meat-intoxication syndrome' was induced by administration of various nitrogenous substances in a patient with normal liver function, in whom an Eck-fistula had been construcred after portal vein resection for carcinoma (77). Behavioural alterations in this patient were associated with elevated ammonia levels. Similar observations were made in patients with cirrhosis of the liver (4).

\subsection{Arterial concentrations}

Ammonia in this thesis refers to the total ammonia concentration, i.e. $\mathrm{NH}_{3}$ $+\mathrm{NH}_{4}{ }^{+}$. Ammonium ion is formed from ammonia gas and vice versa in the equilibrium reaction $\mathrm{NH}_{3}+\mathrm{H}^{+}$? $\mathrm{NH}_{4}^{+}$. $(47,78)$. At physiological $\mathrm{pH}$ the overwhelming majority $(99 \%)$ is in the form of ammonium ion. The ammonia $\left(\mathrm{NH}_{3}\right)$ form is gaseous and lipophilic and can easily diffuse across cell membranes, whereas the ammonium ion is non-diffusible and can only be transported by carrier-mediated processes ( 47 , 78).

The arterial ammonia concentration in healthy volunteers has been reported to be about $45 \mathrm{mM}(79)$. In patients with chronic liver failure and proven cirrhosis the arterial ammonia level is elevated to $60 \mathrm{mM}(79,80)$ and slightly higher $(80 \mathrm{mM})$ in cirrhoric patients that are treated with a TIPSS (81). Higher arterial ammonia concentrations are documented for patients with acute-on-chronic liver disease ( 90 to $120 \mathrm{mM}$ ) and with ALF (150 to $180 \mathrm{mM})(6,79)$. The highest arterial ammonia concentrations were observed in patients with end stage ALF and elevated ICP that was unresponsive to conventional treatment $(340 \mathrm{mM})(82)$. An arterial concentration of ammonia of greater than $150 \mathrm{mM}$ was associated with brain hemiation in patients with ALF (6).

Arterial glutamine concentrations are of similar magnitude in healthy volunteers and patients with chronic and acute-an-chronic liver disease $(600 \mathrm{mM})$, but are extremely elevated during ALF to values of about $2500 \mathrm{mM}$ (79).

Similar observations were made in chronic portacaval shunted rats, who showed a 2-3 fold increase in arterial ammonia concentration with unchanged arterial glutamine 
concentration compared with normal control rats (83-86). During ALF in rats arterial ammonia levels are 15 to 20 times higher and glutamine concentrations are 5 times higher than in control animals (7).

\subsection{Cerebral metabolism (see figure 10)}

Because the brain does not express several of the enzymes of the urea cycle, ammonia removal by the brain relies almost exclusively on glutamine synthesis. Brain contains appreciable amounts of both glutamine synthetase $(14,47,87)$ and glutaminase activity $(47,88-90)$. These two enzymes are, however, to a considerable extent compartmentalized in brain. The astrocytes contain most of the total brain glutamine synthetase activity $(14,91)$, maximally $20 \%$ is localized in the neurons. Virtually all brain glutaminase activity is localized in the neurons (47).

Unidirectional brain ammonia uptake has been shown using positron-emitting isotope enriched ammonia $\left({ }^{13} \mathrm{~N}\right.$-ammonia). It was estimated that $47 \%$ of the arterial ammonia was extracted by the brain of healthy volunteers (92). Net ammonia uptake has been shown by virtue of the finding that a positive arterio-venous concentration difference exists in healthy human volunteers $(93,94)$. This could not be demonstrated in hospitalised patients without liver disease $(95,96)$.

FIGURE 10. Schematic representation of ammonia and glutamine trafficking in muscle and brain.

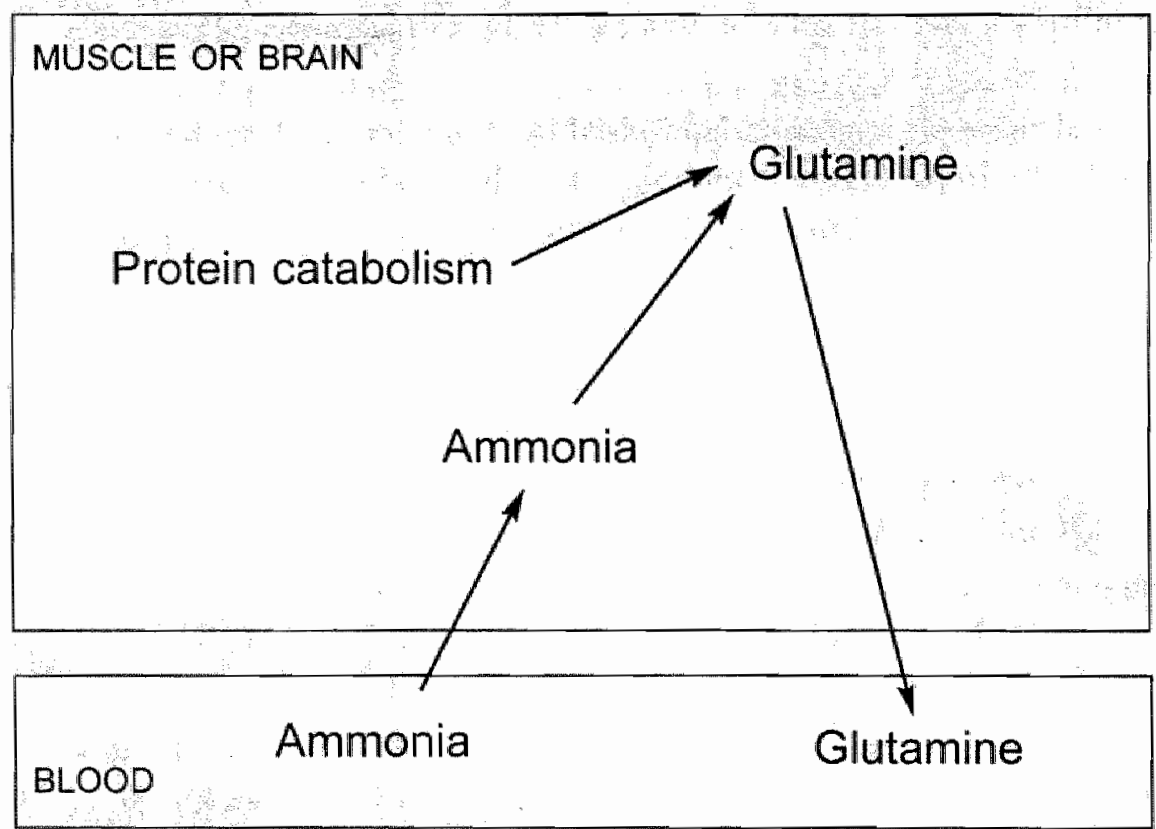




\subsubsection{Cerebral ammonia and glutamine metabolism during liver failure}

Hyperammonemia increases the delivery of ammonia to the brain when cerebral blood flow remains constant. Since the blood-brain barrier is freely permeable to ammonia, elevated arrerial ammonia levels would then lead to enhanced cerebral ammonia uptake, subsequent incorporation of ammonia into glutamine via the glutamine synthetase reaction and glutamine release into the blood $(47,97)$.

Unidirectional brain ammonia uptake has been repeatedly observed in various hyperammonemic states $(47,98)$. Studies of cerebral ammonia metabolism in non-comatose patients with liver disease showed a fractional extraction of ammonia ranging from $11 \%$ (99) to 20\% (100). Conflicting results were reported by Webster and Gabuzda, who could only show net cerebral ammonia uptake in comatose patients with liver disease and not in alert patients with liver disease (96). Bessman and Bessman (95) studied 13 patients who were (semi-) comatose due to acute on chronic liver disease and showed cerebral ammonia extraction (fractional extraction of $15 \%$ ) which was strongly correlated with arterial ammonia concentration $(r=0.82)$. Positive cerebral arteriovenous ammonia differences were also observed in patients with ALF $(6)$, and showed a strong correlation with arterial ammonia concentrations $(r=0.76)$, whereby arterial ammonia levels of $>150$ ?mol/1 predicted a greater likelihood of dying from brain herniation. To our knowledge net cerebral ammonia consumption or net glutamine release has not yet been described in patients with ALF.

Our group showed net consumption of ammonia and production of glutamine by rat cerebral cortex during chronic portacaval shunting and during acute liver ischaemia $(86,101)$. The consumption of ammonia by the brain was related to arterial ammonia levels. We calculated from our studies in rats with ALF that $66 \%$ of the ammonia that was taken up by the brain was metabolised and the remaining $33 \%$ was stored in the brain ammonia pool (7). Substantial ammonia storage in brain ammonia pools cannot take place in chronic liver failure, in which arterial ammonia concentrations reach steady state.

\subsection{Proposed mechanisms of how ammonia produces HE}

The exact mechanism of how ammonia produces HE is not entirely clear but the following section describes our thoughts on the role of ammonia in the pathogenesis of $\mathrm{HE}$.

\subsubsection{The 'ammonia-glutamine-brain oedema hypothesis'}

This hypothesis proposes that the accumulation of glutamine in the astrocytes induced by hyperammonemia produces osmotic stress and causes the astrocytes to swell. Infusion of ammonia into portacaval shunted rats produces an increase in brain glutamine and brain water, and the animals become comatose (see Figure 11) (36). This hypothesis has been explored almost in its entirety in humans. Haussinger and colleagues (102) demonstrated a disturbance in cell volume homeostasis and astrocyte swelling in 
patients with cirrhosis of the liver using proton magnetic resonance spectroscopy. They showed a depletion of myo-inositol, which correlated with an increase in the glutamine/ glutamate peak, a finding that has since been confirmed by a number of investigators. Cordoba and colleagues (103) performed proton magnetic resonance spectroscopy and determined the magnetisation transfer ratio, which is a measure of brain water, in 24 patients with liver disease. In a subgroup of 11 patients, the study was repeated after liver transplantation. In patients with liver disease the magnetisation transfer ratio was reduced and the glutamine/glutamate signal was increased, which correlated with deterioration in neuropsychological function, suggesting that hyperammonemia results in an increase in brain water, impairing neuropsychological function. These changes normalised after liver transplantation.

Astrocytes play a central role in the maintenance of central nervous system function by virtue of their interactions with other neural cells. The swelling of astrocytes activates extracellular regulated protein kinases, elevates intracellular calcium concentration, upregulates the expression of peripheral benzodiazepine receptors, affects multiple ion channels and amino acid transport, induces endosomal alkalinisation which affects receptor densities and neurotransmitter processing, induces deposition of glycogen and inhibition of glycogenolysis and increases the synthesis of neurosteroids which are potent modulators of neuronal ?-aminobutyric acid (GABA) receptor activity (see for review) (28). These mechanisms induce changes in multiple (neurotransmitter) systems that produce the neuropsychiatric disturbances that are observed in parients with $\mathrm{HE}$.

\subsubsection{Direct ammonia related neurotoxicity}

The ammonia concentrations that are reported in the brain in experimental liver failure impairs postsynaptic inhibition in cerebral cortex, brainstem, and spinal cord preparations by blocking chloride extrusion from the postsynaptic neuron thereby rendering the inbibitory neurotransmitter ineffective (47). Similar concentrations of ammonia also inhibit excitatory neurotransmission by a direct postsynaptic action (47). In millimolar concentrations, ammonia has the potential to cause cerebral energy failure through inhibition of ?-ketoglutarate dehydrogenase (104), a rate-limiting tricarboxylic acid cycle enzyme. In accordance with this finding liver failure has been reported to lead to increased brain concentrations of lactate. CSF lactate concentrations are increased in direct correlation with deterioration of neurological function $(32,37$, 105). Increased CSF lactate has also been reported in cirrhotic patients with $\mathrm{HE}$ (106). These findings suggest that brain metabolism changes to anaerobic glycolysis following ammonia-induced inhibition of ?-ketoglutarate dehydrogenase and operation of the
Krebs cycle.

\subsubsection{Ammonia and altered gene expression in liver failure}

During liver failure and consequent hyperammonemia, the brain responds rapidly by altering the expression of genes that code for various proteins whose role is critical to 
CNS function including the maintenance of cell volume and neurotransmission (see for review) (28). Brain extracts from animals with experimental ALF show that the expression of several genes was altered. Not surprisingly the genes that show alterations in expression in ALF are those that code for proteins involved in astrocytic function. These genes include the astrocytic glutamate transporter (GLT-1), the astrocytic structural protein, glial fibrillary acidic protein, the "peripheral-type" benzodiazepine receptor and aquaporin IV, a protein implicated in astrocytic water channels (107). The parthophysiologic consequences of this altered gene expression are unclear and need to be studied in suitable knockout models.

FIGURE 11: Illustrating the ammonia-glutamine-brain oedema hypothesis.

(a) This hypothesis suggests that the accumulation of glutamine in the astrocytes induced by hyperammonemia produces osmotic stress and causes the astrocytes to swell (GLU: glutamate; GLN: glutamine; GS: glutamine synthetase).

(b) A proton magnetic resonance spectrum illustrating the different peaks and in particular the Glx (Glutamine/Glutamate) peak, which is increased in the patients with 'overt hepatic encephalopathy' (NAA: $\mathrm{N}$-acetyl aspartate; Cho: choline; Cr: creatine)

\section{The ammonia-glutamine-brain swelling hypothesis}

a

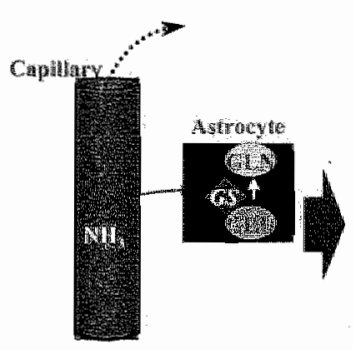

b

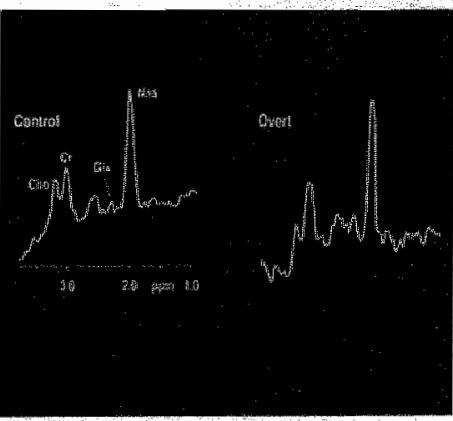




\subsection{INTERORGAN AMMONIA METABOLISM}

It is clear that multiple organs are involved in the homeostasis of ammonia and the arterial levels are critically determined by the production of ammonia and its metabolism. The following section describes the current knowledge about the interorgan metabolism of ammonia. We will use the terms uptake or release of ammonia if information is provided on the basis of arterio-venous concentration differences and consumption or production of ammonia if information is provided on ammonia flux across that organ: arterio-minus-venous concentration differences times organ blood flow.

\subsection{Intestinal metabolism (see Figure 12)}

Early clinical and experimental literature suggested the colonic bacterial flora to be the main source of ammonia in the body (108-110). Colonic bacteria produce ammonia by splitting urea and possibly amino acids $(111,112)$. However, this hypothesis does not explain hyperammonemia and $\mathrm{HE}$ in germ-free dogs with a portacaval shunt (1.13, 114) and the high portal-venous ammonia concentration in germ-free rats (1.15). The alternative and additional explanation is that hyperammonemia in germ-free animals is the result of intestinal breakdown of amino acids (111), especially glutamine (73, 116). The intestines have a high glutaminase activity, predominantly located in the enterocytes, and only little glutamine synthetase activity $(73,116)$, making it a major glutamine consuming organ. Glutamine utilization is essential for the maintenance of normal enterocyte function (117-121), which produces ammonia by converting blood borne as well as luminal glutamine to ammonia and glutamate $(73,111,112,122$, 123). Subsequently, glutamate is transaminated to alanine and to a lesser degree to other amino acids and organic acids $(73,116)$. The large bowel urilizes much less glutamine than the small intestine, but uses glucose, short chain fatty acids and ketone bodies as fuel (112).

In a study in post-absorptive healthy dogs it was estimated that intestinal glutamine consumption and subsequent metabolism accounted for half of the total ammonia produced from the portal drained viscera (112). The consumption of glutamine appeared to be stoichiometrically related with the production of ammonia into the mesenteric venous blood. The colon produced the other half. $42 \%$ of this colonic ammonia production was derived from uptake of urea from arterial blood, $9 \%$ from uptake of arterial glutamine and the remainder from the breakdown of nitrogen containing material within the colonic lumen (112).

Recently, arterio-venous concentration differences of ammonia and glutamine across the jejtunum, ileum and colon were documented in patients undergoing elective abdominal surgery (124). The fractional extraction of glutamine was the highest in the jejunum (24\%) compared with the ileum $(9 \%)$ and the colon $(8 \%)$. Glutamine extraction was associated with release of ammonia in the ileum and the jejunum. 


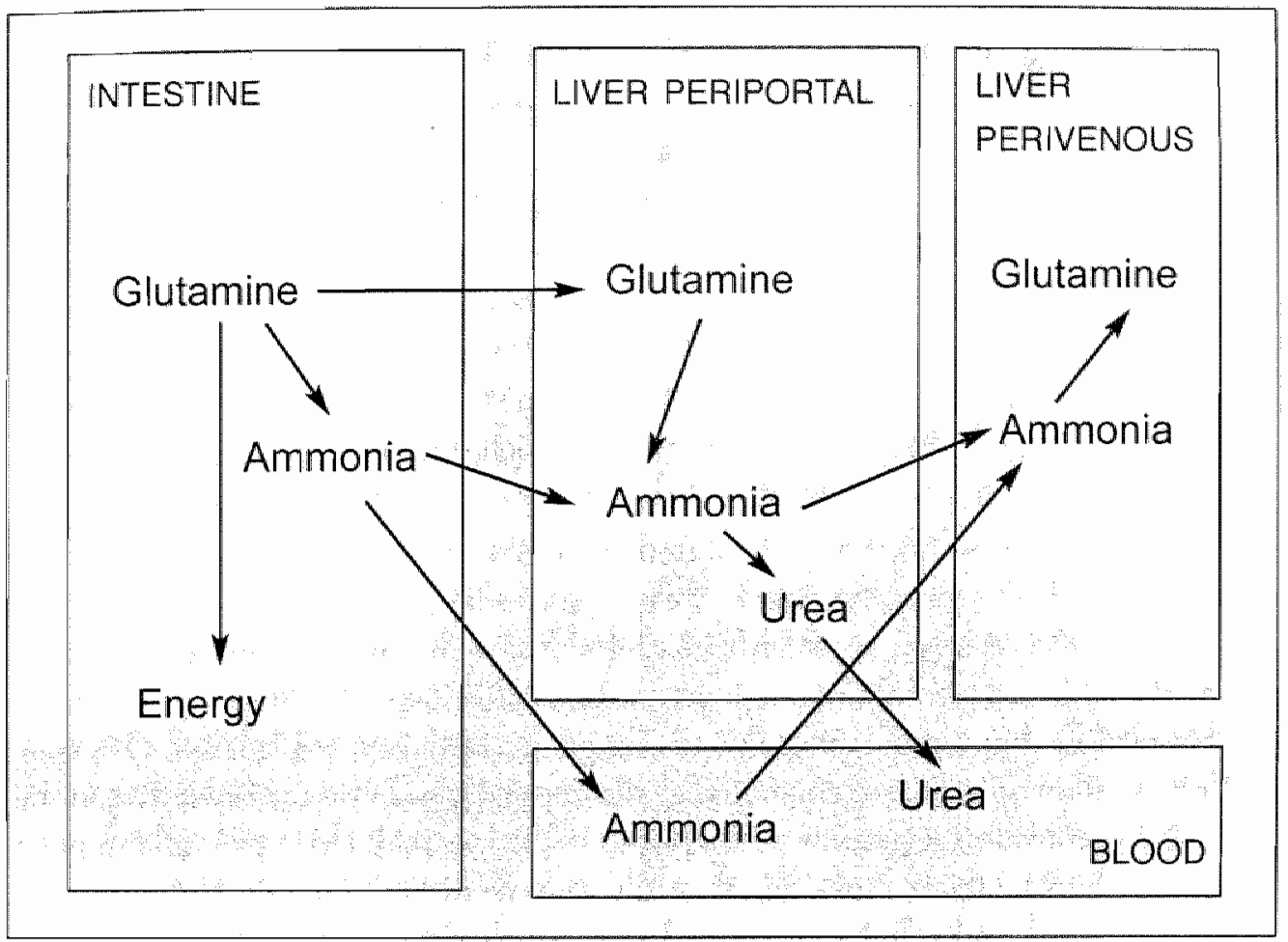

FIGUR: 12. Schematic representation of ammonia and glutamine trafficking between intestine and liver.

\subsubsection{Ammonia liberating events}

Hahn and colleagues were the first to describe post-feeding hyperammonemia (1). Hyperammonemia and $\mathrm{HE}$ are often precipitated by upper gastrointestinal bleeding or intake of nitrogenous substances $(4,77,96,125)$. The current generally accepted concept is still similar to the conclusions of the Saint Petersburg group that the post-feeding hyperammonemia is caused by enhanced intestinal ammonia liberation $(2,126)$. Furthermore, orally administered amino acids vary in their potential to produce ammonia (127), as do different protein sources, of which blood protein has the highest ammoniagenic potential $(128,129)$, probably related to the complete absence of the essential amino acid isoleucine in the haemoglobin molecule (129-132).

Meat is intermediate in elevating systemic ammonia concentration. Dairy proteins $(133,134)$ and vegetable proteins $(135,136)$ are thought to produce less ammonia than meat protein. These findings may be due to their amino acid composition and to accompanying carbohydrates in the meal $(137,138)$. Currently, standard clinical approaches focus on the removal of intestinal sources of ammonia by accelerating intestinal transit time by cathartic agents, reducing protein loads, acidification of intestinal contents with lactulose and reducing urease-containing bacterial flora with poorly resorbable antibiotics $(126,139)$. 


\subsubsection{Intestinal ammonia and glutamine metabolism during liver failure}

Intestinal ammonia production in portacaval shunted rats is similar to healthy postabsorptive rats ( $400 \mathrm{nmol} / 100 \mathrm{~g} \mathrm{bw} / \mathrm{min}$ ), and is stoichiometric to glutamine consumption (84). During acute liver ischaemia in rats there is an initial small increase of intestinal ammonia production and glutamine consumption. However, 4 hours after the induction of liver ischaemia, glutamine consumption ceased whereas ammonia production remained approximately $300 \mathrm{nmol} / 100 \mathrm{~g} \mathrm{bw} / \mathrm{min}$ despite an increase in arterial glutamine concentration from about $800 \mu \mathrm{M}$ to $2300 \mu \mathrm{M}$ (140). These observations are in agreement with the in vitro data which suggest that intestinal glutamine uptake increases linearly to a plateau at arterial concentrations of $600 \mu \mathrm{M}(73)$.

For obvious reasons, studies on intestinal ammonia and glutamine metabolism in patients with liver disease are sparse. Webster and colleagues (125) studied changes in ammonia concentrations in superficial abdominal veins, representing portal blood. They showed increased ammonia concentrations after oral administration of protein and glutamine, but did not relate this to changes in arterial ammonia levels. Owen and colleagues showed uptake of glutamine by the portal drained viscera via selective umbilicoportal cannulation in patients with cirrhosis of the liver with (141) and without portasystemic shunts (142). However, they did not measure ammonia concentrations. Recently, Plauth and colleagues reported negative arterio-venous concentration differences of ammonia across the superior mesenteric vein in overnight fasted patients with cirrhosis of the liver that where treated with a TIPSS (80). It is difficult to interpret their data because the patients they studied consisted of both stable post-absorptive cirrhotics with a TIPSS and patients who were undergoing portosystemic shunting for recurrent variceal bleeding. Moreover, the patients received an oral decontamination protocol to reduce ammonia production from intestinal bacteria. In their experiment they also showed increased arterio-venous ammonia differences across the superior mesenteric vein after an amino acid meal, but the absence of flow data precludes any conclusion about altered ammonia production by the gut (80).

In conclusion, the contribution of the intestine to hyperammonemia in acute and chronic liver failure is probably predominantly the result of existing intra-and/or extrahepatic portacaval shunts and not of altered intestinal ammonia production.

\subsection{Liver metabolism (see Figure 12)}

The pathways of urea and glutamine metabolism are compartmentalized in the liver acinus and interact in a sophisticated manner to maintain whole body ammonia homeostasis (see for review) (143). Ammonia detoxification takes place at two anatomically aligned sites. First, ammonia is converted to urea in the ornithine-cycle (= urea-cycle) in peri-portally located hepatocytes. Ornithine-cycle enzymes have a low affinity for ammonia but high capacity (144). Second, ammonia is scavenged by glutamine synthesis in peri-venous hepatocytes that are located around the terminal hepatic venules 
of the liver acini which represent only $7 \%$ of the total hepatocyte population (145). The enzyme glutamine synthetase has high-affinity but low capacity for ammonia, and is only present in the liver in peri-venous hepatocytes (145). Because of their location any ammonia escaping detoxification in the ornithine cycle can be scavenged by these hepatocytes and metabolised to glutamine.

Peri-portal hepatocytes contain glutaminase, carbamoyl-phosphate synthetase (the rate-controlling enzyme of the urea cycle) (146), alanine aminotransferase, aspartate aminotransferase and urea cycle enzymes (143). Liver glutaminase has the extraordinary characteristic of being activated by its product (ammonia) and has an important role in controlling ammonia inpur in carbamoyl-phosphate synthetase thereby regulating urea cycle flux (147). Therefore the ammonia that is produced by the breakdown of glutamine in the intestines and by peri-portal hepatocytes has a feed-forward effect on hepatic urea synthesis (147, 148). Periportal glutaminase and peri-venous glutamine synthetase operate simultaneously, resulting in the so-called intercellular glutamine cycle (147). In the physiological situation their activity is probably of equal magnitude and therefore the glutamine balance across the liver is close to zero. In view of the high hepatic capacities of glutaminase as well as glutamine synthetase $(87,148)$ and the way these enzymes are regulated (149), the liver can respond rapidly to changes in systemic ammonia levels by changing from glutamine uptake to release $(148,150)$.

\subsubsection{Control of urea synthesis.}

Urea synthesis is hypothesized to be partly controlled by bicarbonate production (for review see) (143), since urea synthesis is the only way by which bicarbonate can be removed (151). In vivo, portal ammonia accounts for $33 \%$ of total liver urea synthesis, portal vein glutamine for 6 to $13 \%$, mitochondrial glutamate via the glutamate dehydrogenase reaction for $20 \%$ and other sources including portal vein and hepatic artery alanine, hepatic artery ammonia and glutamine and the many liver enzymes that are capable of generating ammonia account for 33 to $40 \%$ (152). Increased delivery of amino acids to the liver increases the flux through carbamoyl-phosphate synthetase (144). The capacity for isolated hepatocytes to convert glutamine nitrogen to urea is high (153). Meijer (154) has suggested that ammonia derived from the glutaminase reaction is directly channelled to carbamoyl-phosphate synthetase. Alanine is an effective source of urea nitrogen (155). Peri-portal hepatocytes are especially rich in alanineand aspartate aminotransferases $(143,156)$, which facilitate transfer of nitrogen from alanine to urea.

To our knowledge, no information is available in the literature about ammonia and glutamine metabolism across the liver in healthy subjects. Data exist on ammonia efflux from the hepato-splanchnic region in post-absorptive healthy volunteers. The hepatic venous ammonia concentration was $20-40 \mathrm{mM}$ in these subjects and the net ammonia flux was not significantly different from zero $(6,79)$. This means that in 
healthy subjects, systemic ammonia concentrations are not determined by the splanchnic area but by other organs.

\subsubsection{Hepatic ammonia and glutamine metabolism during liver failure}

Patients with cirrhosis of the liver have intra- and/or extrahepatic portasystemic shunts that can account for up to $50 \%$ of the portal blood flow (157). This may be even higher in patients with iatrogenically created portosystemic shunts (93\%) (158). Furthermore, patients with cirrhosis have reduced ammonia detoxification capacity because of diminished urea and glutamine synthesis capacity $(70,149,159-161)$. In these patients the flux through glutaminase is 4 to 6 fold increased (149). This has been interpreted as a compensatory mechanism to maintain a normal flux through the urea cycle despite the decrease in urea cycle enzyme activities. However, peri-venous glutamine synthetase activity is not correspondingly increased and ammonia ions escape clearance by the liver and are released into the systemic circulation (143).

The above mentioned mechanism can explain the high hepatic venous ammonia concentrations observed in post-absorptive patients with chronic liver disease (40-60 $\mathrm{mM}$ ), acute-on-chronic liver disease (70-90 mM), and ALF (200-240 mM) (6, 79). There was a high correlation between arterial and hepatic venous ammonia concentrations $(r=0.85)(6)$. In a recent study, Clemmesen and colleagues showed that patients with ALF showed net ammonia release from the hepato-splanchnic region (110 $\mathrm{mmol} / \mathrm{min}$ ), suggesting defective ammonia scavenging by the liver. This was accompanied by net glutamine uptake $(240 \mathrm{mmol} / \mathrm{min})$. The observed hepato-splanchnic ammonia release does not exclude hepatic ammonia removal because there was no information about portal venous ammonia concentrations.

In conclusion, the contribution of the liver to hyperammonemia is the result of the existing intra- and/or extrahepatic portacaval shunts and of impaired capacity to produce urea in the periportal hepatocytes and glutamine in the perivenous hepatocytes.

\subsection{Muscle metabolism (see Figure 10)}

Glutamine synthetase activity in skeletal muscle is low (87), and it is mainly by virtue of its mass relative to other glutamine synthetase containing organs, that muscle is one of the principal glutamine synthesizing organs (162). Ammonia can be taken up by or released from the muscle. Skeletal muscle produces ammonia during exercise, probably related to purine nucleotide cycle activation (163). Unidirectional uptake of ammonia in healthy humans was shown in a tracer study using positron-emitting isotope enriched ammonia (13 $\mathrm{N}$-ammonia). It was estimated that $50 \%$ of the arterial ammonia was extracted by skeletal muscle (92). However, this measure of inward ammonia flux does not give any information on net ammonia consumption across skeletal muscle $(92,164)$. Furthermore, this estimate was based on measurements of an $8 \times 8 \mathrm{~cm}$ segment in the middle of the thigh, suggested to be $1 \mathrm{~kg}$ or $3,3 \%$ of total body skeletal 
muscle. Net ammonia uptake by skeletal muscle has been demonstrated by arteriovenous differences in fasted healthy human volunteers across the leg, although the arteriovenous differences were close to zero $(93,94,163,165)$. No ammonia uptake could be observed across the forearm $(95,96,162)$, invalidating the extrapolation of leg muscle metabolism to other skeletal muscles in the body. The considerable difference between the large unidirectional uptake of ammonia and the small arterio-venous differences for ammonia across muscle have been interpreted to signify that muscle must continuously use and produce ammonia (92). In conclusion, in healthy humans, ammonia uptake by skeletal muscle is close to zero.

\subsubsection{Muscle ammonia and glutamine metabolism during liver failure}

Unidirectional ammonia uptake by skeletal muscle has also been shown in patients with various degrees of liver failure (92). Data from arterio-venous catheterisation studies suggest that net ammonia uptake by muscle is probably related to (changes in) arterial ammonia levels. Net muscle ammonia uptake was shown in hyperammonemic patients with (decompensated) cirrhosis of the liver in arterio-venous difference measurements across the forearm $(95,96,100,162)$ and leg $(165,166)$. A good correlation between arterial ammonia concentrations and muscle ammonia uptake was observed in patients in hepatic coma with (96) or without (100) active upper gastrointestinal haemorrhage and in patients with decompensated cirrhosis (165) and gross muscle wasting (162). No significant correlation was found in stable patients with cirrhosis of the liver (96) with normal muscle composition (162). The fractional extraction of ammonia was reduced in patients with muscle wasting $(92,162)$, suggesting an important role for maintaining adequate muscle mass in these patients.

Arterio-venous differences do nor reflect the rate of production or consumption of ammonia since the latter also depends on organ flow. Organ flux measurements are necessary to enable quantification of metabolism and comparison of individual organs. Recently, net ammonia consumption by leg muscle has been quantified in patients with ALF (79) and was calculated to be $100 \mathrm{nmol} / 100 \mathrm{~g} / \mathrm{min}$. The authors did not document the correlation between arterial ammonia concentration and ammonia consumption by muscle, but leg ammonia consumption was correlated with glutamine production supporting the view that skeletal muscle participates in ammonia detoxification in ALF. Total amino acid production from muscle was 10 times higher than uptake of ammonia, indicating that production of glutamine was not only the result of ammonia consumption but also the result of net muscle catabolism during ALF (79). To our knowledge no data in the literature exist regarding the amount of ammonia that is removed by skeletal muscle in patients with chronic liver disease.

The fact that muscle ammonia uptake has only been shown in patients with decompensated chronic or ALF raised the question whether the observed ammonia uptake reflected net ammonia detoxification or changes in the muscle pool of ammonia or 
glutamine $(140,167,168)$. Net detoxification by muscle occurs if ammonia is taken up by muscle and glutamine is released. The discussion whether ammonia uptake by skeletal muscle will always lead to glutamine release and whether this glutamine release is stoichiometric to ammonia uptake is confounded by contrasting literature. Enhanced ammonia consumption by skeletal muscle was found in vivo after ammonia infusion in healthy rats (169-171), monkeys (172), dogs (172, 173) and pigs (174). This ammonia consumption enhanced glutamine production only in some of these studies (171, $172,174)$, whereas glutamine production remained unchanged in others $(169,173)$. Ammonia consumption either exceeded (174) or balanced the enhanced glutamine production (172). Experiments by our group in rats with chronic and ALF did not show a significant net uptake of ammonia across the hindquarter $(140,168)$. Muscle glutamine release was 2 to 4 fold higher in chronic portacaval shunted rats compared with healthy controls, whereas a corresponding net ammonia uptake was not observed. Enhanced muscle catabolism seemed to be the most likely cause of this enhanced glutamine production. An explanation might reside in the fact that most of these experiments do not take into account the changes in tissue concentrations. In ALF and acute-onchronic liver failure steady state is per definition lacking and part or all of the ammonia could be trapped in augmented tissue ammonia and amino acid (glutamine) pools. This need not necessarily induce a release of these amino acids. Therefore, net ammonia removal by muscle in chronic liver disease remains doubtful (7). Furthermore, the role of skeletal muscle in ammonia metabolism in patients with chronic liver disease has never been quantified.

Ammonia uptake by muscle, leading to glutamine synthesis and glutamine release from muscle does not necessarily indicate net ammonia detoxification. Glutamine released from skeletal muscle can be taken up in the splanchnic area or in the kidneys and can be metabolised to ammonia, abolishing the effect on whole body ammonia removal. Glutamine acts thereby as a non-toxic nitrogen carrier, without contributing to net nitrogen removal from the systemic circulation.

In conclusion, skeletal muscle metabolism probably plays an important role in ammonia metabolism in chronic and ALF. This is mainly because total body skeletal muscle mass probably constitutes the quantitatively most important localization of the enzyme glutamine synthetase.

\subsection{Renal metabolism (see Figure 13)}

The kidneys contain both glutaminase and glutamine synthetase (150) and are therefore capable of synthesizing and degrading glutamine in several species, including man. Glutamine is the main substrate for renal ammoniagenesis in the post-absorptive state (175-177). Glutamine is metabolised predominantly in the intramitochondrial phosphate-dependent glutaminase reaction situated within the proximal convoluted tubule (178). Kidney-type glutaminase is strongly inhibited by glutamate, and in contrast to 
liver-type glutaminase not activated by ammonia (76). It is not clear whether both nitrogen moieties of glutamine always serve renal ammoniagenesis (reviewed in) (78)). The amide moiety always serves ammoniagenesis in the glutaminase reaction, but subsequently three possible fates for the resulting glutamate remain. First, glutamate can be released into the renal vein. Second, glutamate can be used in transamination reactions, yielding predominantly alanine. Alanine can be released into the bloodstream, and transported to the liver where the carbon skeleton can be used for gluconeogenesis (179). Third, increased flux through glutaminase is accompanied by increased flux through mitochondrial glutamate dehydrogenase (180), yielding a-ketoglutarate and ammonia. a-Ketoglutarate is initially metabolised to phosphoenolpyruvate, which can be transformed to lactate and glucose (181) and released into the renal vein, or pyruvate may be oxidized to $\mathrm{CO}_{2}$ in the TCA cycle. The two decarboxylation reactions associated with the conversion of a-ketoglutarate to phosphoenolpyruvate result in the net synthesis of bicarbonate ions, which are released into the bloodstream (76).

McDermott and colleagues sampled renal vein and artery in 10 post-absorptive patients undergoing surgery and were the first to show that the kidneys release ammonia into the systemic circulation in men (182). This observation has been repeated in normal post-absorptive human volunteers $(93,94,176)$. Ammonia production into renal vein was quantified to be approximately $40 \mathrm{mmol} / \mathrm{min}$, of a similar magnitude as renal urinary ammonia excretion (176). During prolonged administration of ammonium chloride in these volunteers renal venous production of ammonia remained the same $(40 \mathrm{mmol} / \mathrm{min})$, but renal urinary ammonia excretion increased $(100 \mathrm{mmol} / \mathrm{min})(176)$. Similar observations were made in experimental animals. In the post-absorptive state in healthy dogs $(183,184)$, mini-pigs (185) and rats $(150,183)$, approximately $30 \%$ of rotal ammonia produced in the kidneys is released into the urine and the remaining $70 \%$ is released back into the renal vein. During chronic acidosis, however, the situation reverses because total ammoniagenesis is enhanced and $70 \%$ of produced ammonia is released into the urine $(150,183-185)$. Consequently, the kidney is an organ of net ammonia addition to the whole body in the normal physiologic state and an organ of net ammonia disposal during acidosis. This implies an important role for renal ammonia and glutamine metabolism in maintaining whole-body acid-base homeostasis, since glutamine breakdown and subsequent ammonia excretion has been suggested to be an efficient way of excreting protons and generating new bicarbonate (186).

\subsubsection{Renal ammonia and glutamine metabolism during liver failure}

The kidneys release ammonia in the circulation in post-absorptive patients with chronic liver disease $(165,166,187)$. Under normal post-absorptive conditions about $70 \%$ of total renal ammonia production is released into the renal vein and the remainder excreted in the urine. Induction of hyperammonemia in healthy volunteers turns the kidney into an organ of net ammonia uptake from the blood (188) and in cirrhotic 


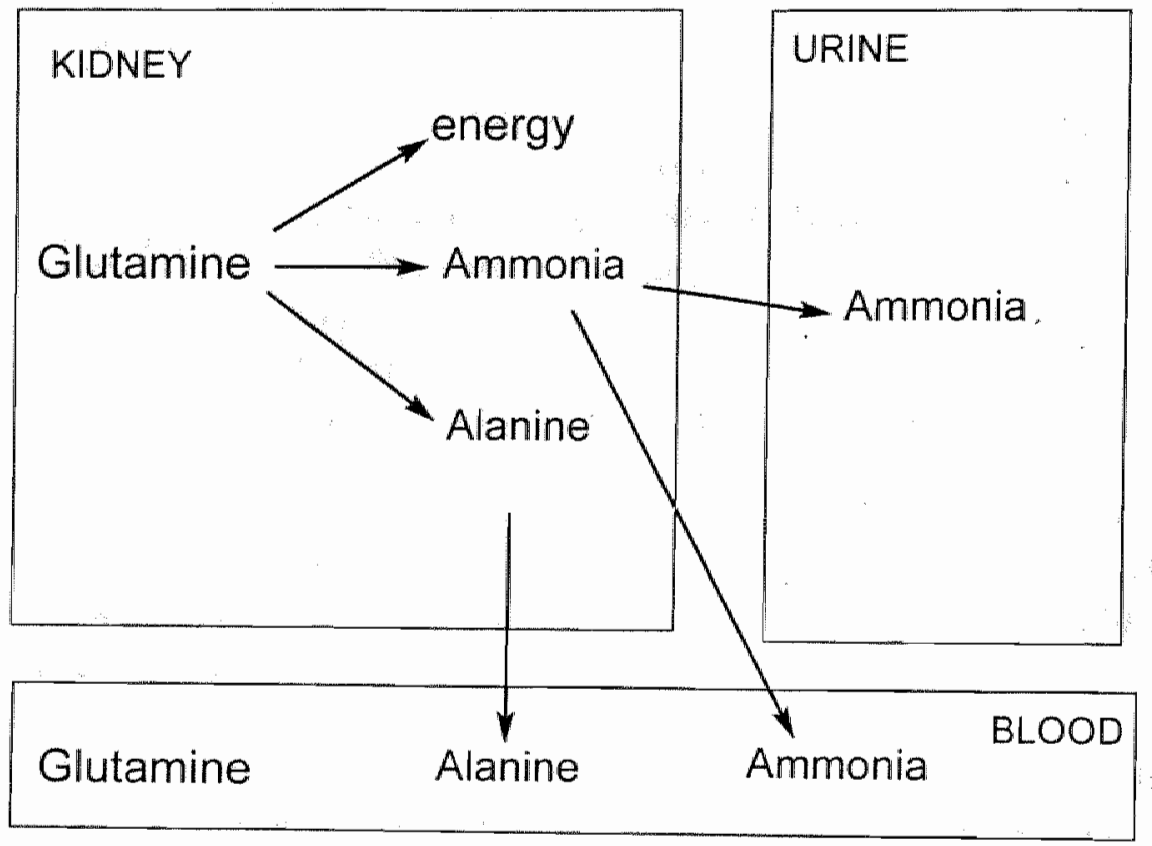

FicURE 13. Sichematic representation of ammonia and glutamine trafficking in kidney.

patients renal ammonia release into the circulation decreased at elevated ammonia concentrations (165). These early human studies do not provide data on the metabolism of glutamine or other amino acids. Recent literature concerning renal ammonia and amino acid metabolism during liver failure in humans is not available. Moreover, no information exists regarding renal ammonia metabolism durring ALF.

Recently, our group studied this subject in rats with acute and chronic liver failure $(83,85)$. As expected the kidneys of post-absorptive healthy control rats consumed glutamine and released ammonia back into the systemic circulation (83). Following creation of a portacaval shunt, renal ammonia production decreased drastically despite markedly elevated arterial ammonia concentrations $(83,85)$. Renal glutamine consumption was reduced compatible with the reduced ammonia production. Similar observations were made in rats with ALF. During acute and chronic liver failure $70 \%$ of total renal ammoniagenesis was excreted into urine and $30 \%$ released into the renal vein (83). This adaptive mechanism of the kidneys to reduce ammonia release in to the body ammonia pool can be interpreted as beneficial for the organism. From these experiments we concluded that during acute and chronic liver failure, the increase of arterial ammonia concentrations is mainly determined by portacaval shunting in combination with reduced hepatic urea synthesis capacity and not by increased renal ammonia efflux into the arterial pool (7). 
Recent studies by our group suggest that the kidneys have a crucial role in the occurrence of hyperammonemia following feeding in pigs (189). We observed increased ammonia release into the renal vein after an amino acid and a protein meal in normal pigs (189), with no change in ammonia release from the hepato-splanchnic region, suggesting that post-feeding hyperammonemia was mainly related to renal ammoniagenesis. This observation is in agreement with a previous study by Imler and colleagues (190): In this study, feeding a high protein diet to rats with a portal stricture and portasystemic shunting led to an increased ammonia release into the renal vein. Since renal plasma flow measurements were not performed, the authors were not able to quantify renal ammonia efflux.

In conclusion, the kidneys contribute to the hyperammonemic state in liver failure since they release ammonia into the renal vein. The kidneys have the ability to adapt to experimental hyperammonemia by increasing the percentage of total renal ammonia production that is excreted into the urine. 


\subsection{TREATMENT OF HEPATIC ENCEPHALOPATHY}

\subsection{Treatment of HE in cirrhosis, type $C$}

As described in previous sections, patients that develop type C HE largely fall into 2 groups. The first group consists of those parients who have episodes of encephalopathy and are well between attacks. Encephalopathy is often preceded by a precipitating event, such as: dietary-protein loading, constipation, gastrointestinal haemorrhage, sepsis, dehydration, hypokalaemia, hypoxia, systemic alkalosis, use of sedatives or exacerbation of the underlying liver disease. The second group is much smaller and consists of those patients that develop spontaneous encephalopathy. Management of type C HE therefore involves the detection and treatment of precipitating events, the encephalopathy itself, and the introduction of measures to prevent episodes of encephalopathy.

\subsubsection{Treatment aimed at gut-derived factors, including ammonia and the excre- tion of nitrogenous waste}

\subsubsection{Diet}

The major strategy for the therapy of $\mathrm{HE}$ is directed at ammonia reduction, which can be attained either by decreasing its absorption/production or increasing its removal. Traditionally, the gut has been thought to be the major site of ammonia production and current straregies are directed at methods of reducing ammonia absorption/production from the gut. Management of the precipitating event, restriction of dietary protein, avoidance of constipation, and manipulation of the bowel flora are the mainstays of therapy. Historically, patients presenting acurely with encephalopathy had dietary protein stopped or severely curtailed. However, the guidelines for nutrition in liver disease and transplantation of the European Society of Parenteral and Enteral Nutrition (191), recommend protein in amounts of $1.0-1.5 \mathrm{~g}$ per kilogram per day in patients with chronic liver disease, since many of these patients are anorectic and find restricted diets unpalatable and hence unacceptable $(192,193)$. In patients with a dietary protein tolerance of less than $1 \mathrm{~g}$ per kilogram, vegetable protein may improve nitrogen balance without precipitating or worsening encephalopathy $(136,194)$. Recent studies suggest the beneficial effects of a vegetable protein diet relates to the higher fibre content. Dietary fibre undergoes bacterial fermentation, lowering the $\mathrm{pH}$ of the colonic luminal contents $(195,196)$, which in turn reduces the formation of toxic fatty acids and ammonia from amino acids in the gut lumen. Clinical use of vegetable protein diets is, however, limited by poor patient compliance.

Hyperammonaemia is associated with zinc deficiency, which in turn decreases the activity of enzymes in the urea cycle responsible for metabolising ammonia to urea. 
Some studies support the supplementarion of zinc in cirrhotic patients with demonstrable deficiency, showing an objective improvement in $\operatorname{HE}(197,198)$.

Several studies have documented the effects of branched chain amino acid (BCAA) infusions in patients with acute and chronic $\mathrm{HE}$, showing normalisation of plasma amino acid concentration, but no benefit in terms of improved cerebral function or mortality data (199). Only studies using BCAA over greater than 3 months show a significant improvement in objective measures of disease severity, such as EEG and psychometric tests, suggesting that a subset of HE patients may benefit from long term use. The difficulty is identifying this cohort of patients who would benefit from this expensive treatment. However, BCAA do have a specific role in improving nitrogen balance in malnourished patients with cirrhosis who are intolerant of protein supplementation (200). This treatment needs to be revisited in future clinical trials.

\subsubsection{Non-absorbable disaccharides}

In addition to its osmotic action, the non-absorbable disaccharide lactulose stimulates the incorporation of ammonia into bacterial protein thereby reducing the amount available for absorption (201). Furthermore, bacterial fermentation lowers colonic $\mathrm{pH}$ and hence reduces the absorption of ammonia by non-ionic diffusion, whilst promoting the growth of non-urease producing lactobacilli and thus, reducing ammonia production (137). Recent studies also suggest that lactulose may inhibit the generation of ammonia in the gut mucosa by interfering with the uptake of glutamate and its subsequent metabolism (137). Despite these mechanisms, only four placebo-controlled studies have been reported on the use of lactulose in $\mathrm{HE}$. One of these reported no clinical benefit $(202,203)$, although these studies were small and not analysed on an 'intention to treat' basis. Moreover, a range of side effects, predominantly gastrointestinal, require discontinuation of treatment in at least $5 \%$ of cases.

The non-absorbable disaccharide lactitol has been shown to be as effective as lactulose in the management of $\mathrm{HE}(204,205)$ and a meta-analysis suggests that lactitol treated patients responded more quickly and with fewer side-effects (206). However, no published placebo controlled trials exist to appraise the efficacy of lactitol in HE.

\subsubsection{Antibiotics}

Neomycin inhibits growth of urease producing bacteria and early studies suggested and efficacy similar to that of lactulose in the treatment of $\mathrm{HE}(207,208)$. However, a placebo controlled study showed no clinical benefit from neomycin compared with placebo (209). In addition, up to $3 \%$ of neomycin is systemically absorbed, producing risks of nephrotoxicity and ototoxicity, and thus it should not be used routinely in clinical practice.

There are limited data to suggest that lactulose and neomycin may have an additive effect in reducing the intestinal production of ammonia in the majority of patients 
who have an inadequate response to lactulose alone (210). However, a placebo controlled trial of 80 patients with acute $\mathrm{HE}$ using a lactulose-neomycin combination, showed no benefit over placebo (211).

\subsubsection{Sodium benzoate}

Treatment with benzoate, which reacts with glycine to form hippurate, increases urinary nitrogen excretion by diverting nitrogen from the urea cycle. In a large prospective controlled trial in India, sodium benzoate was shown to be as effective as lactulose in producing objective improvement in encephalopathy and mortality in acute $\mathrm{HE}$ (212). The advantage of benzoate is that it is an inexpensive alternative to lactulose for use in developing countries and can be administered intravenously in un-cooperative patients.

\subsubsection{L-Ornithine-L-Asparate (LOLA)}

Ornithine and aspartate are important substrates in the metabolic conversion of ammonia to urea (urea cycle) and glutamine, respectively (213). In 126 patients enrolled in a randomised, double blind, placebo-controlled trial of intravenously administered LOLA, challenged with a protein meal, improvements were noted in postprandial venous ammonia concentrations as well as in performance times in number connection tests, compared with placebo (214). Similar studies have also shown the treatment to be well tolerated with a good compliance and no adverse events noted (215). These trials suggest that both the enteral and parenteral formulation of LOLA significantly reduce ammonia levels in cirrhotic patients, and may be at least as effective as lactulose when given to $\mathrm{HE}$ parients $(214,215)$.

More recently studies of LOLA infusions in rats with liver failure have shown a significant delay in onset of $\mathrm{HE}$ and brain oedema (38). The protective effects of LOLA are accompanied by an increased availlability of several amino acids including glutamate (a major alternative ammonia removal mechanism through glutamine synthesis), as well as branched-chain amino acids and GABA. Thus the protective effects of LOLA may be both peripherally mediated (increased urea and BCAA) and centrally mediated (increased glutamine synthesis). Further placebo-controlled trials to elucidate efficacy are clearly needed.

\subsubsection{Treatment aimed at changes in neurotransmission}

\subsubsection{Flumazenil}

Benzodiazepines exert their CNS depressant effects by interacting with high affinity binding sites on the GABA-Benzodiazepine receptor complex. Increased concentrations of benzodiazepine-like substances have been noted in the brains and CSF of

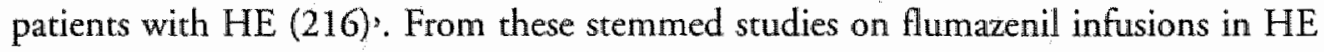
patients, which initially demonstrated a favourable response rate in an uncontrolled. clinical trial $(217,218)$. More recent studies, however, have failed to show any objective 
benefit in clinical outcome (219) and flumazenil should no longer be used routinely in the treatment of $\mathrm{HE}$.

\subsection{Treatment of HE in ALF, type A (see Figure 14)}

\subsubsection{General Measures}

It is important to note that patients with ALF can decompensate very rapidly often within a matter of hours and have a catastrophic event related to their airway and respiration, circulation or brain herniation. Close clinical monitoring is important during the observation period and particularly when moving a parient either between hospitals or between different wards. The blood pressure should be maintained within a narrow range to achieve a cerebral perfusion pressure (CPP, mean arterial pressure minus intracranial pressure (ICP)) of $>50 \mathrm{mmHg}$ but $<65 \mathrm{mmHg}$ to prevent cerebral hypoperfusion on the one hand and further cerebral hyperaemia on the other $(220,221)$. Hyperthermia should be prevented as it worsens intracranial hypertension (222). Glucose levels need to be maintained to prevent cerebrall and systemic effects of hypoglycaemia. Hyponatraemia can worsen brain edema and should be prevented or corrected (223). Hypercapnia should be avoided as it induces cerebral hyperaemia and increases ICP (224). Close attention to acid-base balance and correction of hyperlactaraemia is important as it can worsen cerebral hyperaemia $(225,226)$. Patients requiring renal support should have continuous veno-venous hemofiltration rather than haemodialysis to prevent rapid fluid shifts (227).

\subsubsection{Treatment aimed at gut-derived factors, including ammonia and the excre- tion of nitrogenous waste}

\subsubsection{Non-absorbable disaccharides}

There are no randomised controlled clinical trials of ammonia reducing strategies such as lactulose, branched amino acids or non-absorbable antibiotics in ALF patients. However, a recently reported study from the database of the United States ALF group compared the outcome of 70 patients with ALF who received lactulose with data from 47 parients who did not receive the drug. Despite the retrospective nature of the study, the parients were well matched for their demographics and coma score. "There was no significant difference berween the groups in the severity of $\mathrm{HE}$, stay in the intensive care unit, rate of infections, the percentage of patients that underwent OLT and the percentage of patients who died during follow up (228). The routine use of lactulose can therefore not be recommended.

\subsubsection{L-Ornithine-L-Asparate (LOLA)}

Although there are no data in humans with ALF, studies in experimental models of ALF suggests that the administration of this agent early in the course of illness may prevent the occurrence of brain edema (38). Because of the good safety profile of this drug, its further evaluation in ALF through a randomised controlled clinical trial would seem worthwhile. 


\subsubsection{Treatment aimed at reduction of cerebral edema}

\subsubsection{Mannitol}

The mainstay for the treatment of increased ICP is mannitol, which is administered intravenously as a bolus dose in a dose of $1-2 \mathrm{mg} / \mathrm{Kg}$ as a $20 \%$ solution. Its use is based upon the principle that mannitol administration results in an increase in the osmolality of the capillaries in the brain and this results in movement of water according to Starling's law. Canalise et al. studied 34 patients with ALF and showed that the episodes of cerebral edema resolved significantly more frequently in the 17 patients who received mannitol than in the 17 patients who did not (229). In those that received mannitol, the survival was significantly higher than those who did not receive it ( $47.1 \%$ and $5.9 \%$ respectively). There are however, problems with its usage particularly in those patients who have renal failure because repeated administration results in an increase in plasma osmolality and consequent loss of efficacy. Plasma osmolality needs to be measured if more than 2 doses are used to ensure it is less than $320 \mathrm{Osm} / \mathrm{L}$. In order to be able to use mannitol repeatedly, fluid can be taken off with hemofiltration, which, by itself reduces ICP. Although controlled data are lacking in the literature, reduction of blood volume (up to $500 \mathrm{ml}$ ) with hemofiltration is effective in reducing the ICP.

\subsubsection{Hyperventilation}

In patients with ALF, loss of CBF autoregulation contributes to cerebral vasodilatation (57). The induction of arterial hypocapnia by hyperventilation resulted in the restoration of cerebral vascular autoregulation (230). Ede et al. performed a controlled clinical evaluation of hyperventilation in ALF(231). Twenty patients were electively hyperventilated to maintain $\mathrm{PaCO}_{2}$ between 3.5 and $5 \mathrm{kPa}$. In the other 35 patients, mechanical ventilation was instituted only if severe hypoxia or hypercapnia occurred. Cerebral edema, diagnosed clinically or by a rise in ICP to greater than $30 \mathrm{~mm} \mathrm{Hg}$, occurred in $85 \%$ of hyperventilated patients and in $86 \%$ of controls. They observed no significant reduction in the number of episodes of cerebral edema in the hyperventilated patients ( 4.8 episodes $/ 24 \mathrm{~h}$ ) compared with the controls $(5.3$ episodes $/ 24 \mathrm{~h}$ ) but hyperventilation did appear to delay the onset of brain herniation. Their results suggest that hyperventilation may reduce ICP acutely but cannot be recommended for prolonged use.

\subsubsection{Thiopental Sodium.}

The use of thiopental sodium was prompted by the observation that its administration results in cerebral vasoconstriction possibly by inhibition of nitric oxide synthase, which is thought to be important in the pathogenesis of increased ICP in ALF. In 13 patients who had increased ICP that was unresponsive to standard medical therapy the dosage of thiopental was adjusted upwards until the ICP fell to within normal limits or adverse haemodynamic changes occurred (232). The ICP was reduced, in each case, by administration of 185 to $500 \mathrm{mg}$ thiopental over $15 \mathrm{~min}$. Five of the patients made 
complete recovery and there were only three deaths from intracranial hypertension. However, thiopentone sodium is not an easy drug to use and its administration to patients with ALF is associated with significant haemodynamic disturbances, which may require additional inotropes. Some of the benefits from reduction in ICP may be off set by a reduction in mean arterial pressure and thereby cerebral perfusion pressure. Its use should be limited to episodes of catastrophic increases in ICP particularly in relation to OLT.

\subsubsection{Indomethacin}

Indomethacin induces cerebral vasoconstriction through multiple mechanisms that include inhibition of the endothelial cyclooxygenase pathway, alterations in extracellu$\operatorname{lar} \mathrm{pH}$ and reduction in cerebral temperature (233). Its use has been investigated in a single patient with ALF in whom administration of $25 \mathrm{mg}$ of indomethacin resulted in normalization of ICP (234). Although the results are interesting and confirmed by data from studies in an animal model (235), indomethacin is toxic for the kidneys and the gur and cannot be recommended for use in patients with ALF. Studies using the cyclooxygenase- 2 inhibitors are awaited.

\subsubsection{Propofol}

Propofol is a highly lipophilic drug and rapidly distributes with the blood volume and brain and has a high metabolic clearance. In addition, propofol in a dose of $6 \mathrm{mg} / \mathrm{kg}$ per hour reduces $\mathrm{CBF}$ through metabolic suppression. Its use was investigated in 7 patients with ALF (236). The patients were managed with an infusion rate of $50 \mathrm{mg} / \mathrm{kg}$ per minute of propofol. The ICP at insertion was elevated in three of seven patients. The ICP remained within normal limits in six of seven patients. One of the patients died from increased ICP and one during OLT. Early data on the use of propofol in ALF are encouraging and support a fuller evaluation.

\subsubsection{Treatment aimed at changes in neurotransmission}

\subsubsection{N-methyl-D-aspartate antagonism}

Memantine is an inhibitor of the glutamate $\mathrm{N}$-methyl-D-aspartate receptor and has been used in studies in experimental animals with ALF. The results suggest that hyperammonemia results in an increase in extracellular glutamate concentrations which act upon the $\mathrm{N}$-methyl-D-aspartate receptors leading to an increase in sodium flux into the cell and brain swelling (48). Pre-treatment of rats with portacaval shunts resulted in a reduction in brain swelling and ICP (237), but there are no data of its use in patients with ALF.

\subsubsection{Phenytoin}

Phenytoin acts upon the $\mathrm{Na} / \mathrm{K}$ ATP-ase and has been investigated in a randomised clinical trial in 42 ALF patients admitted with Grade III-IV HE. In addition to ICP 
monitoring, the patients underwent continuous EEG monitoring. Although there were no significant differences in frequency of subclinical seizure in the 2 groups $(30 \%$ and $45 \%$ respectively) or the occurrence of increase in ICP ( $25 \%$ and $50 \%$ respectively), the autopsy examinations available in 19 patients showed signs of cerebral edema in only $22 \%$ of the phenytoin-treated patients compared with $70 \%$ of the controls ( $\mathrm{p}$ $<.03)(238)$. These data provide the rationale for the use of Phenytoin in ALF as prophylaxis in those that have reached Grade III-IV HE.

\subsubsection{Treatment aimed at reducing additional effects of inflammation}

As has been highlighted in the previous sections, there is increasing evidence that the systemic inflammatory response is important in the pathogenesis of increased ICP in ALF. At present, it is not clear what component of the observed inflammatory response is due to the release of humoral substances from the necrotic liver and what component is due to additional infection $(28,29,62,63)$.

\subsubsection{N-Acetylcysteine}

Although N-Acetylcysteine is used widely in the UK in the later stages of ALF (in contradistinction to its use as an antidote to paracetamol poisoning when administered within 24 hours), its use is difficult to justify on pathophysiologic grounds as its vascular effects are those of vasodilatation (239). A number of clinical studies support a role for this drug. Harrison et al. investigated the administration of $\mathrm{N}$-acetylcysteine in patients with paracetamol overdose who presented up to 36 hours afterwards and showed that the mortality was $37 \%$ in patients who received N-Acetylcysteine compared with $58 \%$ in the controls (240). In patients given $\mathrm{N}$-Acetylcysteine, progression to grade III-IV HE was lower $(51 \%$ vs. $75 \%$ ). Keays et al. performed a randomised controlled trial in paracetamol induced ALF and showed that survival was significantly higher in the N-Acetylcysteine treated group than in the controls ( $48 \%$ vs. $20 \%$; $\mathrm{p}<0.04)$ (241). The N-Acerylcysteine treated patients had a lower incidence of cerebral edema ( $40 \%$ vs. $68 \% ; \mathrm{p}<0.05$ ). In a subsequent study, they suggested that the improvement in survival was related to an increase in cardiac output, oxygen extraction ratio and oxygen consumption and it was proposed that the therapeutic benefit was caused by a reduction of tissue hypoxia (242). Walsh et al. studied the haemodynamic effects of $\mathrm{N}$-acetylcysteine during the first 5 hours of a standard infusion regime in 11 ALF patients and simultaneously measured oxygen consumption using a method based on respirarory gas analysis. They were unable to confirm that $\mathrm{N}$-Acetylcysteine infusion resulted in clinically relevant improvements in global oxygen consumption, or in clinical markers of tissue hypoxia (243). A multicenter trial regarding the value of $\mathrm{N}$ Acetylcysteine administration in the later stages of ALF is currently underway in the US. The drug should be discontinued in case of systemic hypotension or cerebral oedema. 


\subsubsection{Dexamethasone}

The use of steroids in ALF is based on the notion that inflammatory response is important in the progression of intracranial hypertension. A relatively old study addresses this question (229). Cerebral edema developed in 34 patients with similar frequency in those treated with and without dexamethasone, (16 of 21 and 18 of 23 respectively). Survival figures were also unaffected.

\subsubsection{Antibiotics}

There are no controlled data in the literature addressing the question whether early use of antibiotics is associated with a reduced incidence of $\mathrm{HE}$. As referred to earlier, Rolando et al. have suggested that systemic inflammatory response as a result of microbial infection is likely to be important in those who progress to advanced stages of $\mathrm{HE}$ (63). Whether such a progression is reduced by the use of antibiotics has not been demonstrated. As the prophylactic use of parenteral and enteral antibiotics are associated with lower rates of infection (244), these data suggest - in an indirect manner that prophylactic antibiotics may reduce the incidence of intracranial hypertension. The choice of the antibiotic should be decided on the basis of bacterial cultures and resident hospital flora.

\subsubsection{Hepatectomy}

The use of hepatectomy in patients awaiting OLT is a rather dramatic intervention but may be of value in desperate situations where all the available treatments have been applied and the patient continues to deteriorate. It is based upon the concept that the 'necrotic liver' is the source of unknown humoral substances that contribute to increased ICP. In patients with ALF who were likely to die while awaiting OLT, Ringe et al. performed hepatectomy with portacaval shunting in 32 patients (245). They observed stabilization of the cardiovascular and cerebrovascular state with 19 of 32 patients being successfully transplanted, 6-41 hours afterwards. In a single case, we have recently shown that the removal of the liver in an ALF patient resulted in improved ICP possibly through a reduction in $\mathrm{CBF}$, nitric oxide and liver derived proinflammatory cytokines (68). 


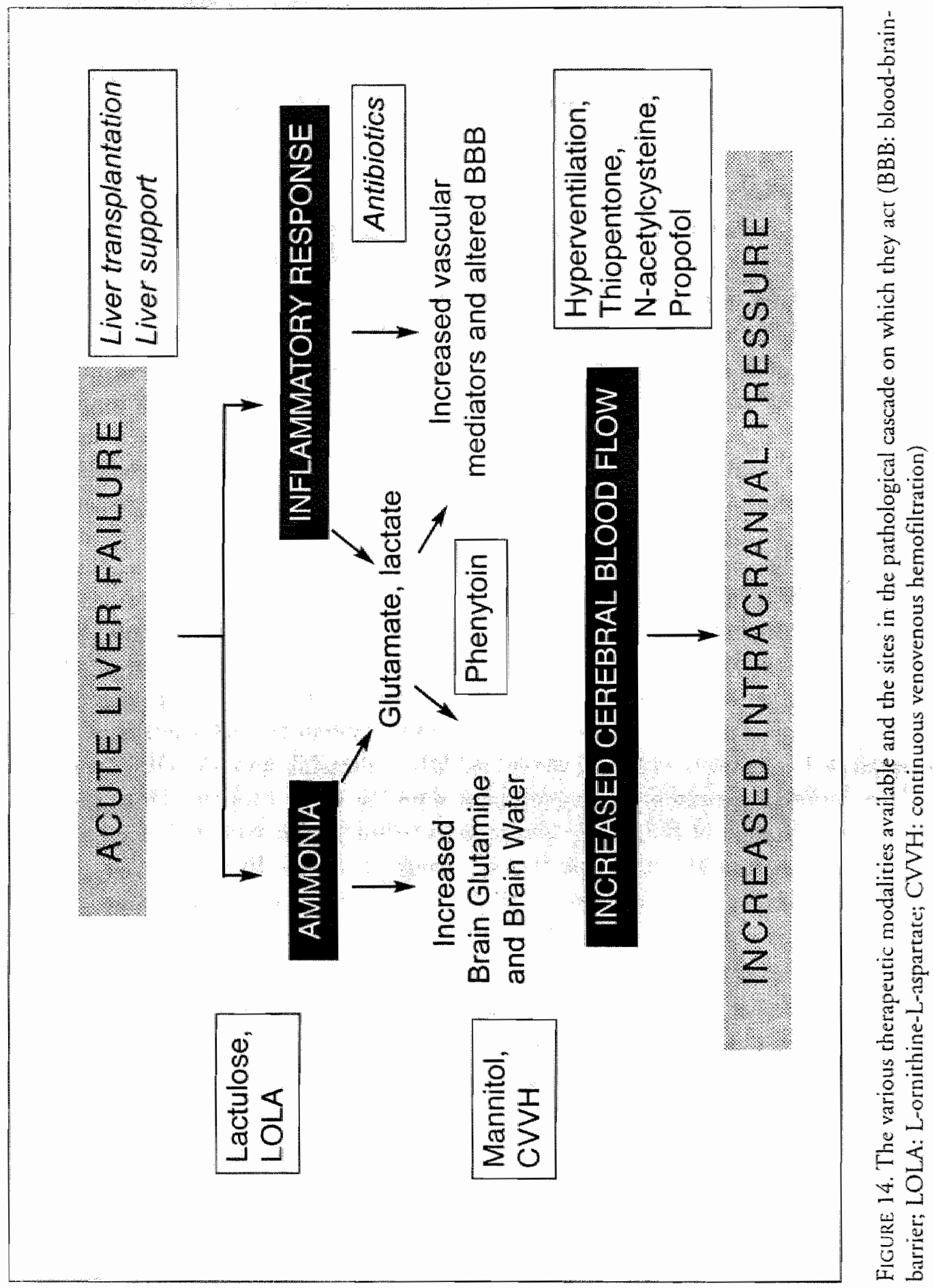




\subsection{SUMMARY}

HE incorporates a spectrum of neuropsychiatric abnormalities seen in patients with liver dysfunction that are potentially reversible. Distinct syndromes are identified in ALF and cirrosis. Rapid deterioration in consciousness level and increased ICP that may result in brain herniation and death are features of ALF whereas manifestations of $\mathrm{HE}$ in cirrhosis include psychomotor dysfunction, impaired memory, increased reaction time, sensory abnormalities, poor concentration and in severe forms, coma. No single mediator has been found to account for the syndrome, and HE may result from the effect of several interacting mediators. However, for over 100 years ammonia has been considered central to its pathogenesis.

The major ammonia producers in the body are the intestines and the kidneys. Ammonia is produced in equal amounts in the small and large bowel. Synthesis of ammonia in the small bowel is related to amino acid breakdown in the gut wall, whereas large bowel ammonia production is caused by bacterial breakdown of amino acids and urea. The amount of ammonia that is produced by the intestines does not change during experimental liver failure. The other ammonia producing organ, i.e. the kidney, has the interesting ability to adapt to experimental hyperammonemia by reducing ammonia release into the systemic circulation. The kidneys can switch from net ammonia production to net ammonia excretion, which could be beneficial for the hyperammonemic patient. Data in experimental animals suggest that the kidneys also have a major role in post-feeding hyperammonemia.

The liver contributes to the hyperammonemic state in patients with liver dysfunction due to the intra- and extra-hepatic portacaval shunts and because of reduced peri-portall urea synthesis capacity as well as a reduced peri-venous glutamine synthesis capacity. This results in a reduced hepatic capacity to detoxify ammonia in patients with chronic liver disease. Glutamine synthesis has been suggested to be an alternative route to detoxify ammonia and has been demonstrated in muscle and brain of experimental animals and in patients with liver disease. Concomitant release of glutamine has been demonstrated in experimental animals.

In the brain, the astrocyte is the main site for ammonia detoxification, during the conversion of glutamate to glutamine. An increased ammonia level raises the amount of glutamine within astrocytes, causing an osmotic imbalance resulting in cell swelling and ultimately brain edema. Multiple other factors interact with ammonia toxicity. Most recently the role of inflammation has arisen.

Management of HE involves the detection and treatment of precipitating events, the HE itself, and the underlying liver disease. The major strategy for the therapy of $\mathrm{HE}$ in cirrhosis is directed at ammonia reduction, which can be attained either by 
decreasing its absorption/production or increasing its removal. Traditionally, the gut has been thought to be the major site of ammonia production and current strategies are directed at methods of reducing ammonia absorption/production from the gut. The management of HE in ALF is much more unsatisfactory. Without liver transplantation, a mortality rate of $90 \%$ is expected in those patients with ALF who have increased ICP and fulfil criteria for poor prognosis. Effective therapy will emerge only once the pathogenesis of this syndrome is fully understood. 


\section{REFERENCES}

1. Hahn M, Massen O, Nencki M, Pawlow J. Die Eck'sche fistel zwischen der unteren hohlvene und der pfortader und ihre folgen für den organismus. Archiv fuer Experimentelle Pathologie und Pharmakologie 1893;32:161-210.

2. Nencki M, Pawlow JP, Zaleski J. Ueber den Ammoniakgehalt des Blutes und der Organe und die Harnstoffbildung bei den Saugethieren. Archiv fuer experimentelle Parhologie und Pharmakologie 1896;37:26-51.

3. Nencki $M$, Zalieski J. Ueber die Bestimmung des Ammoniaks in "Thierischen Fluessigkeiten und Geweben. Archiv fuer Experimentelle Pathologie und Pharmakologie 1895;36:385396.

4. Phillips GB, Schwarz R, Gabuzda GJ, Davidson CS. The syndrome of impending hepatic coma in patients with cirthosis of the liver given certain nitrogenous substances. $N$ Engl J Med 1952,247:239-246.

5. Lockwood AH, Tap EW, Rhoades HM, Wong WH. Altered cerebral blood flow and glucose metabolism in patients with liver disease and minimal encephalopathy. $J$ Cereb Blood Flow Metab 1991,11:331-6.

6. Clemmesen IO, Larsen FS, Kondrup J, Hansen BA, On P Cerebral herniation in patients with acute liver fallure is correlated whth arterial ammonia concentration. Hepatology $1999 ; 29: 648-53$.

7. Deutz NEP, Dejong $\mathrm{CHC}$, Soeters $\mathrm{PB}$. Interorgan ammonia and glutamine exchange during liver failure. In: Record CO, Mardini HA, editors. Advances in Hepatic Encephalopathy \& metabolism in liver disease. Newcastle upon Tyne: University of Newcastle upon Tyne; 1996. p. 87-99.

8. Ferenci P, Lockwood A, Mullen K, Tarter R, Weissenborn K, Blei AT. Hepatic encephalopathy-definition, nomenclature, diagnosis, and quancification: final report of the working party at the 11 th World Congress of Gastroenterology, Vienna, 1998. Hepatology 2002;35:716-21.

9. Jalan R. Intracranial hypertension in acute liver failure: pathophysiological basis of rational management. Semin Liver Dis 2003;23:271 82 .

10. Jalan R, Shawcross D, Davies $N$. The molecular pathogenesis of hepatic encephaloparhy. Int ] Biochem Cell Biol 2003;35:1175-81.

11. Donovan IP, Schafer DF, Shaw BW, Ji., Sorrell MF. Cerebrial ocdema and increased intracranial pressure in chronic liver disease. Lancer 1998;351:719-21.

12. Kato M, Hughes RD, Keays RT, Williams R. Electron microscopic study of brain capitlaries in cerebral edema from fulminant hepatic failure. Hepatology 1992;15:1060-6.

13. Norenberg MD. A light and electron microscopic study of experimental portal-systemic (ammonia) encephaloparhy. Progression and reversal of the disorder. Lab Invest 1977;36:618-27.

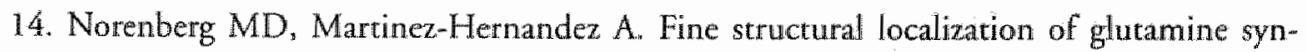
thetase in astrocytes of rat brain. Brain Res 1979;161:303-10.

15. Norenberg MD. Astrocytic-ammonia interactions in hepatic encephaloparhy. Semin Liver Dis $1996 ; 16: 245-53$. 
16. Krieger $S$, Jauss $M$, Jansen $O$, Theilmann L, Geissler M, Krieger D. Neuropsychiatric profle and hyperintense globus pallidus on $\mathrm{T} 1$ - weighted magnetic resonance images in liver cirthosis [see comments]. Gastroenterology 1996;111:147-55.

17. Morgan MY. Noninvasive neuroinvestigation in liwer disease. Semin Liver Dis $1996 ; 16: 293-314$

18. Weissenborn $K$, Ennen JC, Schomerus $H$, Ruckert $N$, Hecker H. Neuropsychological characterization of hepatic encephalopathy. I Hepatol 2001;34:768-73.

19. Kircheis $G$, Wettstein $M$, Timmermann L, Schnitzler A, Haussinger D. Critical flicker frequency for quantification of low-grade hepatic encephalopathy. Hepatology 2002;35:35766.

20. Groeneweg M, Quero JC, De Brujin I, Harmann IJ, Essink-bor ML, Hop WC, et al. Subdinical hepatic encephalopathy impairs daily functioning. Hepatology 1998;28:45-9.

21. Wein C, Koch H, Popp B, Oehler G, Schauder P. Minimal hepatic encephalopathy impairs firness to drive. Hepatology 2004,39:739-45.

22. Lui HF, Jalan R, Redhead DN, Hayes PC. Heparic Encephalopathy after transjugular intrahepatic stent-shunt (TIPSS): Analysis of prognostic variables. In: Yurdaydin $C_{\text {, }}$ Bozkaya $\mathrm{H}$, editors. Advances in hepatic encephalopathy and metabolism in liver disease; 1999. p. $4177-423$.

23. Gines P, Quintero E, Arroyo V, Teres J, Bruguera M, Rimola A, et al. Compensated cirthosis: natural history and prognostic factors. Hepatology 1987;7:122-8.

24. O'Grady JG, Alexander G], Hayllar KM, Williams R. Early indicators of prognosis in fulminant hepatic failure. Gastroenterology 1989;97:439-45.

25. Makin A], Wendon J, Williams R. A 7-year experience of severe acetaminophen-induced hepatotoxicity (1987-1993). Gastroenterology 1995; 109:1907-16.

26. Hoofnagle JH, Carithers RL, Jr., Shapiro C, Ascher N. Fulminant hepatic failure: summary of a workshop. Heparology 1995;21:240-52.

27. Norenberg MD. Astroglial dysfunction in hepatic encephalopathy. Metab Brain Dis $1998 ; 13: 319-35$.

28. Butterworth RF Molecular neurobiology of acute liver failure. Semin Liver Dis $2003 ; 23: 251-8$.

29. Vaquero J, Chung $\mathrm{C}$, Cahill ME, Blei AT. Pathogenesis of hepatic encephalopathy in acute liver failure. Semin Liver Dis 2003;23:259-69.

30. Larsen FS, Wendon J. Brain edema in liver failure: basic physiologic principles and management. Liver Transpl 2002;8:983-9.

31. Ascher NL, Lake JR, Emond JC, Roberts JP. Liver transplantarion for fulminant hepatic failure. Arch Surg 1993;128:677-82.

32. Mans AM, Biebuyck JF, Shelly K, Hawkins RA. Regional blood-brain barrier permeability to amino acids after portacaval anastomosis. J Neurochem 1982;38:705-17.

33. Kreis R, Farrow N, Ross BD. Localized 1H NMR spectroscopy in parients with chronic hepatic encephalopathy. Analysis of changes in cerebral glutamine, choline and inositols. NMR Biomed 1991;:109-16.

34. Lockwood AH, Yap EW, Wong WH. Cerebral ammonia metabolism in patients wich severe liver disease and minimal hepatic encephaloparhy. I Cereb Blood Flow Metab $1991 ; 11: 337-41$. 
35. O'Carroll RE, Hayes PC, Ebmeier KP, Dougall N, Murray C, Best J], et al. Regional cerebral blood flow and cognitive function in partients with chronic liver disease. Lancet 1991;337:1250-3.

36. Hindfelt B, Phum F, Duffy TE. Effect of acute ammonia intoxication on cerebral metabolism in rats with portacaval shunts. J Clin Invest 1977:59:386-96.

37. Mans AM, Dejoseph MR, Hawkins RA. Metabolic abnormalities and grade of encephallopathy in acute hepatic failure. J Neurochem 1994;63:1829-38.

38. Rose C, Michalak A, Rao KV, Quack G, Kircheis G, Butterworth RE L-ornithine-L-aspartate lowers plasma and cerebrospinal fluid ammonia and prevents brain edema in rats with acute liver failure. Hepatology 1999;30:636-40.

39. Conn HO, Leevy CM, Vlahcevic ZR, Rodgers JB, Maddrey WC, Seeff L, et all. Comparison of lactulose and neomycin in the treatment of chronic portal-systemic encephalopathy. A double blind controlled trial. Gastroenterology 1977;72:573-83.

40. Oppong KN, Al-Mardini H, Thick M, Record CO Oral glutamine challenge in cirrhotics pre- and post-liver transplantation: a psychometric and analyzed EEG study. Hepatology $1997: 26: 870-6$.

41 Balata 5 , Olde Damink SWM, Ferguson K, Marshall I, Hayes PC, Deutz NEP, et al. Induced hyperammonemia alters neuropsychology, brain MR spectroscopy and magnetization transfer in cirrhosis. Hepatology 2003,37:931-9.

42. Jalan $\mathbb{R}$, Olde Damink SWM, Lui HF, Glabus M, Deutz NEP, Hayes PC, et al. Oral amino acid load mimicking hemoglobin results in reduced regional cerebral perfusion and deterioration in memory tests in patients with cirrhosis of the liver. Metab Brain Dis 2003; 18:37-49.

43. Basile AS, Jones EA, Skolnick $\mathbb{P}$. The pathogenesis and treatment of hepatic encephalopathy: evidence for the involvement of benzodiazepine receptor ligands. Pharmacol Rev $1991 ; 43: 27-71$.

44. Stahl J. Studies of the blood ammonia in liver disease. Ann Int Med 1963;58:1-24.

45. Spahr L, Butterworth RF, Fontaine S, Bui L, Therrien G, Milette PC, et al. Increased blood manganese in cirthotic patients: relationship to pallidal magnetic resonance signal hyperintensity and neurological symptoms. Hepatology 1996;24:1116-20.

46. Pomier-Layrargues $G$, Spahr L, Butterworth RF. Increased manganese concentrations in pallidum of cirrhotic patients. Lancet 1995;345:735.

47. Cooper AJ, Plum F. Biochemistry and physiology of brain ammonia. Physiol Rev 1987;67:440-519.

48. Michalak A, Rose C, Butterworth J, Butterworth RF. Neuroactive amino acids and glutamate (NMDA) receptors in frontal cortex of rats with experimental acute liver failure. Hepatology $1996 ; 24: 908-13$.

49. Butterworth RF. Portal-systemic encephalopathy: a disorder of multiple neurotransmitter systems. Rev Gastroenterol Mex 1994;59:79-84.

50. Jones EA, Gammal SH, Martin P. Heparic encephalopathy: new light on an old problem. QJ Med $1988 ; 69: 851-67$.

51. Mullen KD, Jones EA. Natural benzodiazepines and hepatic encephalopathy. Semin Liver Dis $1996 ; 16: 255-64$.

52. Jalan R, Turjanski N, Taylor-Robinson SD, Koepp MJ, Richardson MP, Wilson JA, et al. 
Increased availability of central benzodiazepine receptors in patients with chronic hepatic encephalopathy and alcohol related cirrhosis. Gut 2000;46:546-52.

53. Itzhak Y, Roig-Cantisano A, Dombro RS, Norenberg MD. Acute liver failure and hyperammonemia increase peripheral-type benzodiazepine receptor binding and pregnenolone synthesils in mouse brain. Brain Res 1995;705:345-8.

54. Baraldi M, Zeneroli ML, Ricci P, Caselgrandi E, Ventura E. Down regulation of striatal dopamine receptors in experimental hepatic encephalopathy. Life Sci 1983;32:1417-25.

55. Fischer $J E$, Baldessarini RJ. False neurotransmitters and hepatic failure. Lancer 1971;2:7580.

56. Master $S$, Gottstein J, Blei AT. Cerebral blood flow and the development of ammoniainduced brain edema in rats after portacaval anastomosis. Hepatology 1999;30:876-80.

57. Larsen FS. Cerebral circulation in liver failure: Ohm's law in force. Semin Liver Dis 1996;16:281-92.

58. Ede RJ, Williams RW. Hepatic encephalopathy and cerebral edema. Semin Liver Dis $1986 ; 6: 107-18$.

59. Almdal T, Schroeder T, Ranek L. Cerebral blood flow and liver function in patients with encephalopathy due to acute and chronic liver diseases. Scand J Gastroenterol 1.989;24:299-303.

60. Aggarwal S, Kramer D, Yonas H, Obrist W, Kang Y, Martin M, et al. Cerebral hemodynamic and metabolic changes in fulminant hepatic failure: a retrospective study. Hepatology 1994;19:80-7.

61. Wendon JA, Harrison PM, Keays R, Williams R. Cerebral blood flow and merabolism in fulminant liver failure. Hepatology 1994;19:1407-13.

62. Vaquero J, Polson J, Chung C, Helenowski I, Schiodt FV, Reisch J, et al. Infection and the progression of hepatic encephalopathy in acute liver failure. Gastroenterology $2003 ; 125: 755-64$.

63. Rolando N, Wade J, Davalos M, Wendon J, Philpott-Howard J, Williams R. The systemic inflammatory response syndrome in acure liver failure. Hepatology 2000;32:734-9.

64. Marion DW, Penrod I.E, Kelsey SF, Obrist WD, Kochanek PM, Palmer AM, er al. Treatment of traumatic brain injury with moderate hypothermia. $N$ Engl J Med $1997 ; 336: 540-6$.

65. Angstwurm K, Reuss S, Freyer D, Arnold G, Dirnagl U, Schumann RR, et al. Induced hypothermia in experimental pneumococcal meningitis. J Cereb Blood Flow Metab 2000;20:834-8.

66. Muller M, Schwerdtfeger K, Maier B, Mautes A, Schiedat T, Bianchi O, et al. Cerebral blood flow velocity and inflammatory response after severe traumatic brain injury. Eur J Ultrasound 2001; 12:203-8.

67. Licinio J, Wong ML. Pathways and mechanisms for cytokine signaling of the central nervous system. J Clin Invest 1997;100:2941-7.

68. Jalan R, Pollok A, Shah SH, Madhavan K, Simpson KJ. Liver derived pro-inflammatory cytokines may be important in producing intracranial hypertension in acute liver failure. J Hepatol 2002;37:536-8.

69. Shawcross DL, Davies NA, Williams R, Jalan R. Systemic inflammatory response exacerbates the neuropsychological effects of induced hyperammonemia in cirrhosis. J Heparol 2004; $40: 247-54$. 
70. Rudman D, Difulco TI, Galambos JT, Smith RBd, Salam AA, Warren WD. Maximal rates of excretion and synthesis of urea in normal and cirrhotic subjects. J Clin Invest $1973,52: 2241-9$.

71. Zieve L. Pathogenesis of hepatic encephalopathy: Metab Brain Dis 1987:2:147-65.

72. Lin S, Raabe W. Ammonia intoxication: effects on cerebral cortex and spinal cord. J Neurochem 1985;44:1252-8.

73. Windmueller HG, Spaeth AE. Uptake and metabolism of plasma glutamine by the small intestine. J Biol Chem 1974;249:5070-9.

74. Labow BI, Souba WW, Abcouwer SF. Glutamine synthetase expression in muscle is regulated by transcriptional and posttranscriptional mechanisms. Am I Physiol 1999;276:E1136-45.

75. Lacey JM, Wilmore DW. Is glutamine a conditionally essential amino acid? Nutr Rev $1990 ; 48: 297-309$.

76. Curthoys NP, Watford M. Regulation of glutaminase activity and glutamine metabolism. Annu Rev Nutr 1995;15:133-59.

77. McDermote WV Adams RD. Episodic stupor associated with an Eck Fistula in the human with particular reference to the metabolism of ammonia. J Clin Invest. 1954;33:1-9.

78. Halperin ML, Kamel KS, Ethier JH, Stinebaugh BJ, Jungas RL. Biochemistry and Physiology of ammonium excretion. In. The Kidney: Physiology and pathophysiology. Ed: Seldin DW, Giebisch G, Raven Press, Ltd, New York 1992.

79. Clemmesen JO, Kondrup J. Ott P. Splanchmic and leg exchange of amino acids and ammonia in acute liver failure. Gastroenterology 2000;118:1131-9.

80. Plauth M, Roske AE, Romaniuk P, Roth E, Ziebig R, Lochs H. Post-feeding hyperammonaemia in patients with transjugular intrahepatic portosystemic shunt and liver cirrhosis: role of small intestinal ammonia release and route of nutrient administration. Gut $2000 ; 46: 849-55$.

81. Rees CJ, Oppong K, Al Mardini H, Hudson M, Record CO. Effect of L-ornithine-Laspartate on patients with and without TIPS undergoing glutamine challenge: a double blind, placebo controlled trial. Gut 2000;47:571-4.

82. Jalan R, Olde Damink SWM, Deutz NEP, Lee A, Hayes PC. Moderate hypothermia for uncontrolled intracranial hypertension in acute liver failure. Lancet 1999;354:1164×8.

83. Dejong CHC, Deutz NEP, Soeters PB. Renal ammonia and glutamine metabolism during liver insufficiency-induced hyperammonemia in the rat. J Clin Invest. 1993;92:28342840 .

84. Dejong CHC, Deutz NEP, Soeters PB. Intestinal glutamine and ammonia metabolism during chronic hyperammonaemia induced by liver insufficiency. Gut 1993;34:11121119 .

85. Dejong CHC, Detz NEP, Soeters PB. Metabolic adaptation of the kidney to hyperammonemia during chronic liver insufficiency in the rat. Hepatology 1993; 18:890-902.

86. Dejong CHC, Deutz NEP, Soeters PB. Cerebral cortex ammonia and glutamine metabolism in two rat models of chronic liver insufficiency-induced hyperammonemia: influence of pair-feeding. J Neurochem. 1993;60:1047-1057.

87. Lund P. A radiochemical assay for glutamine synthetase, and activity of the enzyme in rat tissues. Biochem J. 1970;1 18:35-39.

88. Bradford HF, Ward HK, Thomas AJ. Glutamine--a major substrate for nerve endings. J Neurochem 1978;30:1453-9. 
89. Benjamin AM. Control of glutaminase activity in rat brain cortex in vitro: influence of glutamate, phosphate, ammonium, calcium and hydrogen ions. Brain Res 1981;208:36377.

90. Kvamme $\mathrm{E}$, Lenda $\mathrm{K}$. Regulation of glutaminase by exogenous glutamate, ammonia and 2- oxoglutarate in synaptosomal enriched preparation from rat brain. Neurochem Res $1982 ; 7: 667-78$.

91. Martinez-Hernandez A, Bell KP, Norenberg MD. Glutamine synthetase: glial localization in brain. Science 1977;195:1356-8.

92. Lockwood AH, McDonald IM, Reiman RE, Gelbard AS, Laughlin JS, Duffy TE, et al. The dynamics of ammonia metabolism in man. Effects of liver disease and hyperammonemia. J Clin Invest 1979;63:449-60.

93. Warter JM, Brandt C, Marescaux C, Rumbach L, Micheletti G, Chabrier G, et al. The renal origin of sodium valproate-induced hyperammonemia in fasting humans. Neurology $1983 ; 33: 1136-1140$.

94. Warter JM, Marescaux C, Brandt C, Rumbach L, Micheletti G, Chabrier G, et al. Sodium valproate associated with phenobarbital: effects on ammonia metabolism in humans. Epillepsia 1983;24:628-633.

95. Bessman SP, Bessman AN. The cerebral and peripheral uptake of ammonia in liver disease with an hypothesis for the mechanism of hepatic coma. J Clin Invest 1955;34:622-628.

96. Webster LT, Gabuzda GJ. Ammonium uptake by the extremities and brain in hepatic coma. J Clin Invest 1958;37:414-424.

97. Gjedde A, Lockwood AH, Duffy TE, Plum F. Cerebral blood flow and metabolism in chronically hyperammonemic rats: effect of an acute ammonia challenge. Ann Neurol $1978 ; 3: 325-30$

98. Cooper AJ, Mora SN, Cruz NE, Gelbard AS. Cerebral ammonia metabolism in hyperammonemic rats. J Neurochem 1985;44:1716-23.

99. Fazekas JF, Ticktin WR, Ehrmantraut WR, Alman RW. Cerebral metabolism in hepatic insufficiency. Amer Med 1956,21:843-849.

100. Bessman SP Bradley JE. Uptake of ammonia by muscle. Its implications in ammoniagenic coma. N Engl] Med 1955;253:1143-1147.

101. Dejong CH, Kampman MT, Deutz NE, Soeters PB. Cerebral cortex ammonia and glutamine metabolism during liver insufficiency-induced hyperammonemia in the rat. J Neurochem 1992;59:1071-9.

102. Haussinger D, Laubenberger J, vom Dahl S, Ernst T, Bayer S, Langer M, et al. Proton magnetic resonance spectroscopy studies on human brain myo-inositol in hypo-osmolarity and hepatic encephalopathy. Gastroenterology 1994;107:1475-80.

103. Cordoba J, Alonso J, Rovira A, Jacas C, Sanpedro F, Castells L, et al. The development of low-grade cerebral edema in cirrhosis is supported by the evolution of $(1) \mathrm{H}$-magnetic resonance abnormaliries after liver transplantation. J Hepatol 2001;35:598-604.

104. Lai JC, Cooper AJ. Brain alpha-ketoglutarate dehydrogenase complex: kinetic properties, regional distribution, and effects of inhibitors. J Neurochem 1986;47:1376-86.

105. Peeling I, Shoemaker L, Gaurhier T, Benarroch A, Sutherland GR, Minuk GY. Cerebral metabolic and histological effects of thioacetamide-induced liver failure. Am J Physiol 1993;265:G572-8.

106. Yao H, Sadoshima S, Fujii $K$, Kusuda $K$, Ishirsuka T, Tamaki K, et al. Cerebrospinal fluid lactate in patients with hepatic encephalopathy. Eur Neurol 1987;27:182-7. 
107. Desjardins P, Belanger M, Butterworth RF. Alterations in expression of genes coding for key astrocytic proteins in acute liver failure. J Neurosci Res 2001;66:967-71.

108. Folin O, Denis W. Protein metabolism from the standpoint of blood and uissue analysis: the origin and significance of the ammonia in the portal blood. J Biol Chem 1912;1 1:161167.

109. Wolpert E, Phillips SF, Summerskill WH. Ammonia production in the human colon. Effects of cleansing, neomycin and acetohydroxamic acid. N Engl J Med 1970;283:15964.

110. Floch MH, Katz J, Conn HO. Qualitative and quantitative relationships of the fecal flora in cirrhotic patients with portal systemic encephalopathy and following portacaval anastomosis. Gastroenterology 1970:59:70-5.

111. Weber FL, Jr., Friedman DW, Fresard KM. Ammonia production from intraluminal amino acids in canine jejunum. Am J Physiol 1988;254:G264-8.

112. Weber $\mathrm{FL}, \mathrm{Jr}$, Veach $\mathrm{GL}$. The importance of the small intestine in gut ammonium production in the fasting dog. Gastroenterology 1979:77:235-40.

113. Nance FC, Kaufman HJ, Kline DG. Role of urea in the hyperammonemia of germ-free Eck fistala dogs. Gastroenterology 1974;66:108-12.

114. Nance FC, Kline DG. Edk's fistula encephalopathy in germfree dogs. Ann Surg $1971,174: 856-62$.

115. Schalm SW, van der Mey T. Hyperammonemic coma after hepatectomy in germ-free rats. Gastroenterology 1979;77:231-4.

116. Windmueller HG. Glutamine utilization by the small intestine. Adv.Enzyme Regul. 1982;53:210-237.

117. Windmueller HG, Spaeth AE. Identification of ketone bodies and glutamine as the major respiratory fuels in vivo for postabsorptive rat small intestine. J Biol Chem. 1978;253:69-76.

118. Hammarquist F, Wernerman J, Ali R, von der Decken A, Vinnars E. Addition of glutamine to total parenteral nutrition after elective abdominall surgery spares free glutamine in muscle, counteracts the fall in muscle protein syathesis, and improves nitrogen balance. Ann Surg 1989;209:455-61.

119. Wilmore DW, Smith RJ, O'Dwyer ST, Jacobs DO, Ziegler TR, Wang XD. The gut: A central organ after surgical stmess. Surgery 1988;104:917-923.

120. O'Dwyer ST, Smith RJ, Hwang TL, Wilmore DW. Maintenance of small bowel mucosa with glutamine-enriched parenteral nutrition. J Parenter Enteral Nutr 1989;13:579-585.

121. Jacobs DO, Evans DA, Mealy K, O'Dwyer ST, Smith RJ, Wilmore DW. Combined effects of glutamine and epidermal growth factor on the rat intestine. Surgery 1988; 104:358-364.

122. Windmueller $\mathrm{HG}$, Spaeth AE. Intestinal metabolism of glutamine and glutamate from the lumen as compared to glutamine from blood. Arch Biochem Biophys 1975;171:662672.

123. Chang TW, Goldberg AL. The metabolic fates of amino acids and the formation of glutamine in skeletal muscle. J Biol Chem 1978;253:3685-3695.

124. van der Hulst RR, von Meyenfeldr MF, Deurz NEP, Soeters PB: Glutamine extraction by the gut is reduced in depleted patients with gasturointestinal cancer. Ann Surg 1997;225:112-21. 
125. Webster LT, Davidson CS, Gabuzda GJ. Effect on portal blood ammonium of administering nitrogenous substances to patients with chronic hepatic disease. I Lab Clin Med. 1958:52:501-514.

126. Jalan R, Hayes PC. Heparic encephalopathy and ascites. Lancet 1997;350:1309-15.

127. Rudman D, Galambos JT, Smith RB, Salam AA, Warren WD. Comparison of the effect of various amino acids upon the blood ammonia concentration of patients with liver disease. An J Clin Nutr 1973;26:916-925.

128. Bessman AN, Hawkins R. The relative effecrs of enterically administered plasma and packed cells on circulating blood ammonia. Gastroenterology 1963;45:368-373.

129. van Berlo CL, van de Bogaard AE, van der Heijden MA, van Eijk HM, Janssen MA, Bost $\mathrm{MC}$, et al. Is increased ammonia liberation afrer bleeding in the digestive tract the consequence of complete absence of isoleucine in hemoglobin? A study in pigs. Hepatology 1989; 10:315-23.

130. Deutz NEP, Reijwen PL, Bost MC, van Berlo CL, Soeters PB. Modification of the effects of blood on amino acid metabolism by intravenous isoleucine. Gastroenterology 1991;101:1613-20.

131. Dejong CHC, Meijerink WJHJ, van Berlo CLH, Deurz NEP, Soeters PB. Decreased plasma isoleucine concentrations after upper gastrointestinal haemorrhage in humans. Gut 1996;39:13-17.

132. Olde Damink SWM, Dejong CHC, Deutz NEP, van Berlo CLH, Soeters PB. Upper gastrointestinal bleeding: an ammoniagenic and catabolic event due to the total absence of isoleucine in the haemoglobin molecule. Medical Hypotheses 1999;52:515-519.

133. Condon RE. Effect of dietary protein on symptoms and survival in dogs with an Eck fistula. Am J Surg 1971;121:107-114.

134. Fenton JC, Knight EJ, Humpherson PL. Milk-and-cheese diet in portal-systemic encephalopathy. Lancet 1966;1:164-6.

135. Greenberger NJ, Carley J, Schenker S, Bettinger I, Stamnes C, Beyer P. Effect of vegetable and animal protein diets in chronic hepatic encephalopathy. Am J Dig Dis $1977 ; 22: 845-55$.

136. Weber FL, Minco D, Fresard KM, Banwell JG. Effects of vegetable diets on nitrogen metabolism in cirrhotic subjects. Gastroenterology 1985;89:538-44.

137. Weber FL, Jr. Lacrulose and combination therapy of heparic encephalopathy: the role of the intestinal microflora. Dig Dis 1.996;14:53-63.

138. Deutz NEP, Ten Have GAM, Soeters PB, Moughan PJ. Increased intestinal amino-acid retention from addition of carbohydrates to a meal. Clin Nutrition 1995;14:354-364.

139. Jalan R, Seery JP, Taylor-Robinson SD. Review article: pathogenesis and treatment of chronic hepatic encephalopathy. Aliment Pharmacol Ther 1996;10:681-97.

140. Dejong CHC, Kampman MT, Deutz NEP, Soeters PB. Altered glutamine metabolism in rat portal drained viscera and hindquarter during hyperammonemia. Gastroenterology 1992;102:936-948.

141. Owen OE, Mozzoli MA, Reichle FA, Kreulen TH, Owen RS, Boden G, et al. Hepatic and renal metabolism before and after portasystemic shunts in parients with cirrhosis. J Clin lnvest 1985;76:1209-17.

142. Owen OE, Reichle FA, Mozzoli MA, Kreulen T, Patel MS, Elfenbein IB, et al. Hepatic, gut, and renal substrate flux rates in patients with hepatic cirrhosis. J Clin Invest $1981 ; 68: 240-52$. 
143. Haussinger D. Regularion of hepatic ammonia metabolism: the intercellular glutamine cycle. Adv Enzyme Regul 1986;25:159-80.

144. Meijer AI, Lamers WH, Chamuleau RA. Nirrogen merabolism and ornithine cycle funcrion. Physiol Rev 1990;70:701-48.

145. Gebhardr R, Mecke D. Heterogeneous distribution of glutamine synthetase among rat liver parenchymal cells in situ and in primary culture. Embo J 1983;2:567-70.

146. Gaasbeek Janzen JW, Lamers WH, Moorman AF, de Graaf A, Los JA, Charles R. Immunohistochemical localization of carbamoyl-phosphare synthetase (ammonia) in adult rat liver; evidence for a heterogeneous distribution. I Histochem Cytochem 1984;32:55764.

147. Haussinger D. Hepatocyte heterogeneity in glutamine and ammonia metabolism and the role of an intercellular glutamine cycle during ureogenesis in perfused rat liver. Eur J Biochem 1983;133:269-75.

148. Welbourne TC. Hepatic glutaminase flux regulation of glutamine homeostasis. Studies in vivo. Biol Chem 1986;367:301-5.

149. Kaiser S, Gerok W, Haussinger D. Ammonia and glutamine metabolism in human liver slices: new aspects on the pathogenesis of hyperammonaemia in chronic liver disease. Eur J Clin Invest $1988 ; 18: 535-42$.

150. Welboume TC, Childress D, Givens $G$. Renal regulation of interorgan glutamine flow in metabolic acidosis. Am J Physiol 1986;251:R858-R866.

151. Atkinson DE, Camien MN. The role or urea synthesis in the removal of metabolic bicarbonate and the regulation of blood pH. Curr 'Top Cell Regul 1982;21:261-302.

152. Cooper AJL, Nieves E, Fillc-DeRicco S, Gelbard AS. Short-term metabolic fate of [13N]ammonia, L-[13N]alanine, L-[13N]glutamate and L[amide-13N]g]utamine in normal rat liver in vivo. In: Soeters PB, Wilson JHP, Meijer AJ, Holm E, editors. Advances in ammonia metabolism and hepatic encephalopathy: Elsevier Science Publishers B.V; 1988. p. 11-25.

153. Ochs RS. Glutamine metabolism of isolated rat hepatocytes. Evidence for catecholamine activation of alpha-ketoglutarate delhydrogenase. J Biol Chem 1984;259:13004-10.

154. Meijer A]. Channeling of ammonia from glutaminase to carbomoyl-phosphate synthetase in liver mitochondria. FEBS. lett. 1985;191:249-251.

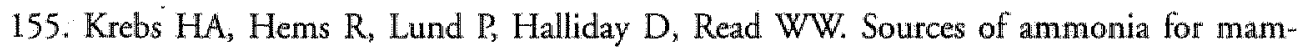
malian urea synthesis. Biochem J 1978;176:733-7.

156. Quistorff B. Gluconeogenesis in periportal and perivenous hepatocytes of rat liver, isolated by a new high-yield digitonin/collagenase perfusion technique. Biochem J $1985 ; 229: 221-6$.

157. Debatin JF, Zahner B, Meyenberger C, Romanowski B, Schopke W, Marincek B, et al. Azygos blood flow: phase contrast quantitation in volunteers and patients with portal hypertension pre- and postintrahepatic shunt placement. Hepatology 1996,24:1109-15.

158. Walser EM, Harris VM, Harman JT, Park HM, Siddiqui AR. Quantification of intrahepatic portosystemic shunting after placement of a transjugular intrahepatic portosystemic shunt. J Vasc Interv Radiol 1996;7:263-7.

159. Haussinger D. Glutamine metabolism in the liver: overview and current concepts. Metabolism 1989;38:14-7.

160. Fabbri A, Marchesini $G$, Bianchi $G$, Bugianesi $E$, Bortoluzzi L, Zoli $M$, et al. 
Unresponsiveness of hepatic nicrogen metabolism to glucagon infusion in patients with cirrhosis: dependence on liver cell failure. Hepatology 1993;18:28-35.

161. Shangraw RE, Jahoor F. Effect of liver disease and transplantation on urea synthesis in humans: relationship to acid-base status. Am J Physiol 1999;276:G1145-52.

162. Ganda OP, Ruderman NB. Muscle nitrogen metabolism in chronic hepatic insufficiency. Metabolism 1976;25:427-435.

163. Eriksson LS, Broberg S, Bjorkman O, Wahren J. Ammonia metabolism during exercise in man. Clin Physiol 1985;5:325-336.

164. Barrett Ef, Revkin JH, Young LH, Zaret BL, Jacob R, Gelfand RA. An isotopic method for measurement of muscle protein synthesis and degradacion in vivo. Biochem J $1987 ; 245: 223-228$.

165. Tyor MP, Owen EE, Berry JN, Flanagan JF. The relative role of extremity, liver and kidney as ammonia receivers and donors in patients with liver disease. Gastroenterology 1960;39:420-424.

166. Berry JN, Flanagan JF, Owen EE, Tyor MP. The kidney as a source of blood ammonia in resting and hyperventilated cirrhotics. Clin Res 1959;7:154-155.

167. Deutz NEP. Dejong CHC, Soeters PB. Ammonia and glutamine metabolism during liver insufficiency: the muscle- gut-liver axis. Ital J Gastroenterol 1993;25:79-86.

168. Dejong CHC, Deutz NEP, Soeters PB. Muscle ammonia and glutamine exchange during chronic liver insufficiency in the tat. J Hepatol 1994;21:299-307.

169. Chabrier G, Schlienger JL, Imler M. Etude du metabolisme musculaíre de l"ammoniaque sur le train posterieur du rat intact. CR Soc Biol 1982;176:716-722.

170. Rosado A, Flores G, Mora J, Soberon G. Distribution of an ammonia load in the normal rat. Am J Physiol 1962;203:37-42.

171. Ruderman NB, Lund P Amino acid metabolism in skeletal muscle. Regulation of glutamine and alanine release in the perfused rat hindquarter. Israel J Med Sci 1972;8:295-302.

172. Hills AG, Reid EL, Kerr WD. Circulatory transport of L-glutamine in fasted mammals: cellular sources of urine ammonia. Am J Physiol 1967;223,1470-1476.

173. Fine $A$. The effects of ammonia infusion on ammonia and glutamine metabolism by liver and muscle in the normal dog. Contrib Neplirol 1985;47:1-8.

174. Deutz NEP, Dejong CHC, Reijven PLM, Soeters PB. In vivo ammonia and glutamine flux measurements during hyperammonemia in rats and pigs. Prog Hepat Encephal Metabol Nitr Exch 1991:329-339.

175. Pltts RF, DeHlas J, Klein J. Relation of renal amino and amide nitrogen extraction to ammonia production. Am J Physiol 1963;204*187-191.

176. Owen EE, Robinson RR. Amino acid extraction and ammonia metabolism by the human kidney during prolonged administration of ammonium chloride. I Clin Invest $1963 ; 42: 263-276$.

177. Tannen RL. Ammonia metabolism. Am J Physiol 1978;235:F265-F277.

178. Good DW, Burg MB. Ammonia production by individual segments of the rat nephron. J Clin Invest 1984;73:602-10.

179. Stumwoll M, Meyer C, Perriello G, Kreider M, Welle S, Gerich J. Human kidney and liver gluconeogenesis: evidence for organ substrate selectivity. Am I Physiol 1998;274:E817-26. 
180. Schoolwerth AC, Nazar BL, LaNoue KF. Glutamate dehydrogenase activation and ammonia formation by rat kidney mitochondria. I Biol Chem 1978;253:6177-83.

181. Newsholme EA, Leech AR. Biochemistry for the Medical sciences. 1 ed. New York: John Wiley \& Sons; 1983.

182. McDermott WV, Adams RD, Riddell AG. Ammonia metabolism in man. Ann Surg 1954; 140:539-556.

183. Vinay $P$, Allignet $\mathbb{E}$, Pichette $C$, Watford M, Lemieux $G$, Gougoux A. Changes in renal metabolite profile and ammoniagenesis during acute and chronic metabolic acidosis in $\mathrm{dog}$ and rat. Kidney Int. 1980;17:312-325.

184. Vinay $P$, Lemieux $G$, Gougoux A, Halperin M. Regulation of glutamine metabolism in dog kidney in vivo. Kidney Int 1986;29:68-79.

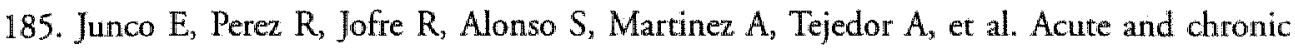
metabolic acidosis in the pig: renal metabolism and ammoniagenesis. in: Molecular aspects of ammoniagenesis. Contrib Nephrol. Ed: Endou H, Schoolwerth AC, Baverel G, Tizianello A. Karger, Basel, Switserland vol 1991;92:18-30.

186. Walser M. Roles of urea production, ammonium excretion, and amino acid oxidation in acid-base balance. Am I Physiol 1986;250:F181-F188.

187. Owen EE, Tyor MP, Flanagan JF, Berry JN. The kidney as a source of blood ammonia in patients with liver disease: the effect of acetazolamide. I Clin Invest 1960;39:288-294.

188. Owen EE, Johnson JH, Tyor MP. The effect of induced hyperammonemia on renal ammonia metabolism. J Clin Invest 1961;40:215-221.

189. Welters CE, Deutz NEP, Dejong CHC, Soeters PB. Enhanced renal vein ammonia efflux after a protein meal in the pig. J Hepatol 1999;31:489-96.

190. Imler M, Schlienger $J \mathrm{~L}$, Chabrier G, Comte F. Origine renale de l'hyperammoniemie provoquee par un regime hyperprotidique chez le rat normal ou porteur d'une stricture portale. Gastroenterol Clin Biol 1983;7:740-745.

191. Plauth M, Merli M, Weimann A, Ferenci P, Mueller MJ. ESPEN guidelines for nutrition in liver disease and transplantation. Clin Nutr 1997;16:43-55.

192. Kondrup J, Muller MJ. Energy and protein requirements of patients with chronic liver disease. J Hepatol 1997;27:239-47.

193. Soulsby CT, Morgan MY. Dietary management of hepatic encephalopathy in cirrhotic patients: survey of current practice in United Kingdom. BMJ1999;318:1391.

194. Bianchi GP, Marchesini G, Fabbri A, Rondelli A, Bugianesi E, Zoli M, et al. Vegetable versus animal protein diet in cirrhotic patients with chronic encephalopathy. A randomized cross-over comparison. J Intern Med 1993;233:385-92.

195. Uribe M, Marquez MA, Garcia Ramos $G$, Ramos-Uribe $M H$, Vargas $F$, Villalobos A, et al. Treatment of chronic portal--systemic encephalopathy with vegetable and animal prom tein diets. A controlled crossover study. Dig Dis Sci 1982;27:1109-16.

196. Uribe M, Dibildox M, Malpica S, Guillermo E, Villallobos A, Nieto L, et al. Beneficial effect of vegetable protein diet supplemented with psyllium plantago in patients with hepatic encephalopathy and diabetes mellitus. Gastroenterology 1985;88:901-7.

197. Reding P, Duchateau I, Bataille C. Oral zinc supplementation improwes hepatic encephalopathy. Results of a randomised controlled trial. Lancet 1984;2:493-5.

198. Van der Rijt CC, Schalm SW, Schat H, Foeken K, De Jong G. Overt hepatic encephalopathy precipitated by zinc deficiency. Gastroenterology 1991;100:1114-8. 
199. Wahren J, Denis J, Desurmont P, Eriksson LS, Escoffier JM, Gauthier AP, et al. Is intravenous administration of branched chain amino acids effective in the treatment of hepatic encephalopathy? A multicenter study. Heparology 1983;3:475-80.

200. Marchesini G, Bianchi G, Merli M, Amodio P, Panella C, Loguercio C, et al. Nutritional supplementation with branched-chain amino acids in advanced cirrhosis: a double-blind, randomized trial. Gastroenterology 2003;124:1792-801.

201. Vince A, Killingley M, Wrong OM. Effect of lactulose on ammonia production in a fecal incubation system. Gastroenterology 1978;74:544-9.

202. Simmons F, Goldstein H, Boyle JD. A controlled clinical trial of lactulose in hepatic encephalopathy. Gastroenterology 1970;59:827-32.

203. Elkington $S G$, Floch $M H$, Conn HO. Lactulose in the trearment of chronic portal-systemic encephalopathy. A double-blind clinical trial. N Engl J Med 1969;281:408-12.

204. Morgan MY, Hawley KE. Lactitol vs. lactulose in the treatment of acute hepatic encephaloparhy in cirrhotic patients: a double-blind, randomized trial. Hepatology 1987;7:1278-84.

205. Heredia D, Caballeria J, Arroyo V, Ravelli G, Rodes J. Lactitol versus lactulose in the treatment of acute portal systemic encephalopathy (PSE). A controlled trial. J Hepatology 1987;4:293-8.

206. Blanc P, Daures JP, Rouillon JM, Peray P, Pierrugues R, Larrey D, et al. Lactitol or lactulose in the treatment of chronic hepatic encephalopathy: results of a meta-analysis. Hepatology 1992;15:222-8.

207. Atterbury CE, Maddrey WC, Conn HO. Neomycin-sorbitol and lactulose in the treatment of acure portal-systemic encephalopathy. A controlled, double-blind clinical trial. Am J Dig Dis 1978;23:398-406.

208. Orlandi F, Freddara U, Candelaresi MT, Morettini A, Corazza GR, Di Simone A, et al. Comparison berween neomycin and lactulose in 173 parients with hepatic encephalopathy: a randomized clinical study. Dig Dis Sci 1981;26:498-506.

209. Strauss E, Tramote R, Silva EP, Caly WR, Honain NZ, Maffei RA, et al. Double-blind randomized clinical trial comparing neomycin and placebo in the treatment of exogenous hepatic encephalopathy. Hepatogastroenterology 1992;39:542-5.

210. Weber FL, Jr., Fresard KM, Lally BR. Effects of lactulose and neomycin on urea metabolism in cirrhotic subjects. Gastroenterology 1982;82:213-7.

211. Blanc P, Daures JP, Liautard J, Buttigieg R, Desprez D, Pageaux G, et al. Lactuloseneomycin combination versus placebo in the treatment of acute hepatic encephalopathy. Results of a randomized controlled trial. Gastroenterol Clin Biol 1994;18:1063-8.

212. Sushma S, Dasarathy S, Tandon RK, Jain S, Gupta S, Bhist MS. Sodium benzoate in the treatment of acure heparic encephalopathy: a double-blind randomized trial. Hepatology 1992;16:138-44.

213. Herlong HF, Maddrey WC, Walser $M$. The use of ornithine salts of branched-chain ketoacids in portal-systemic encephalopathy. Ann Intern Med 1980;93:545-50.

214. Kircheis G, Nilius R, Held C, Berndt H, Buchner M, Gortelmeyer R, et al. Therapeutic efficacy of L-ornithine-L-aspartate infusions in parients with cirrhosis and heparic encephalopathy: results of a placebo- controlled, double-blind study. Hepatology 1997;25:1351-60.

215. Stauch S, Kircheis G, Adler G, Beckh K, Ditschuneit H, Gortelmeyer R, et al. Oral L- 
ornithine-L-aspartate therapy of chronic hepatic encephalopathy: results of a placebo-controlled double-blind study. J Hepatol 1998;28:856-64.

216. Olasmaa $M$, Rothstein JD, Guidotti A, Weber RJ, Paul SM, Spector $S$, et al. Endogenous benzodiazepine receptor ligands in human and animal hepatic encephalopathy, I Neurochem 1990;55:2015-23.

217. Burke DA, Mitchell KW, al Mardini H, Recond CO. Rewersal of hepatic coma with fumazenil with improvement in visual evoked potentials. Lancet 1988;2:505-6.

218. Grimm G, Ferenci P, Katzenschlager R, Madl C, Schneeweiss B, Laggner AN, et al. Improvement of hepatic encephalopathy treated with flumazenil. Lancet 1988;2:1392-4.

219. Cadranel $J \mathrm{~F}$, el Younsi M, Pidoux B, Zylberberg $\mathrm{P}$, Benhamou Y, Valla D, et al. Flumazenil therapy for hepatic encephalopathy in cirthotic patients: a double-blind prag. matic randomized, placebo study. Eur J Gastroenterol Hepatol 1995;7:325-9.

220. McCashland TM, Shaw BW, Jr., Tape E. The American experience with transplantation for acute liver failure, Semin Liver Dis 1996;16:427-33.

221. Davies $\mathrm{MH}$, Mutimer D, Lowes J, Elias E, Neuberger J, Recovery despite impaired cerebral perfusion in fulminant hepatic failure Lancet 1994:343:1329-30.

222. Munoz SJ, Moritz MJ, Bell R, Northrup B, Martin B Radomski J. Factors associated with severe intracranial hypertension in candidates for emergency liver transplantation. Transplantation 199355:1071-4.

223. Cordoba J, Gottstein J, Blei AT. Chronic hyponatremia exacerbates ammonia-induced brain edema in rats after portacaval anastomosis. J Hepatol 1998;29:589-94.

224. van Hulst RA, Hasan D, Lachmann B. Intracranial pressure, brain PCO2, $\mathrm{PO}$, and pH during hypo- and hyperventilation at constant mean airway pressure in pigs. Intensive Care Med 2002;28:68-73.

225. Tofteng F, Jorgensen L, Hansen BA, Ot P, Kondrup J, Larsen FS. Cerebral microdialysis in patients with fulminant hepatic failure. Hepatology 2002;36:1333-40.

226. Zwingmann C, Chatauret N, Leibfritz D, Butterworth RF. Selective increase of brain lactate synthesis in experimental acute liver failure: results of a $[\mathrm{H}-\mathrm{C}]$ nudear magnetic resonance study. Hepatology 2003;37:420-8.

227. Winney RJ, Kean DM, Best J], Smith MA. Changes in brain water with haemodialysis. Lancet $1986 ; 2: 1107-8$.

228. Alba L, Hay JE, Angulo P, Lee WM. Lactulose therapy in acute liver failure. J Hepatol 2002;36:33

229. Canalese J, Gimson AE, Davis C, Mellon PJ, Davis M, Williams R. Controlled trial of dexamethasone and mannitol for the cerebral oedema of fulminant hepatic failure. Gut $1982 ; 23: 625-9$.

230. Strauss G, Hansen BA, Knudsen GM, Larsen FS. Hyperventilation restores cerebral blood flow autoregulation in patients with acute liver failure. J Hepatol 1998;28:199-203.

231. Ede RJ, Gimson AE, Bhari D, Williams R. Controlled hyperventilation in the prevention of cerebral oedema in fulminant hepatic failure. J Hepatol 1986;2:43-51.

232. Forbes A, Alexander GJ, O'Grady JG, Keays R, Gullan R, Dawling S, et al. "Thiopental infusion in the treatment of intracranial hypertension complicating fulminant hepatic failure. Hepatology 1989;10:306-10.

233. Jensen $\mathrm{K}_{\mathrm{s}}$ Ohrstrom J, Cold GE, Astrup J. Indomethacin (Confortid) in severe head injury and elevated intracranial pressure (ICP). Acta Neurochir Suppl 1992;55:47-8. 
234. Clemmesen JO, Hansen BA, Larsen FS. Indomethacin normalizes intracranial pressure in acute liver failure: a twenty-three-year-old woman treated with indomethacin. Hepatology 1997;26:1423-5.

235. Chung C, Gottstein J, Blei AT. Indomethacin prevents the development of experimental ammonia-induced brain edema in rats after portacaval anastomosis. Hepatology $2001 ; 34: 249-54$.

236. Wijdicks EF, Nyberg SL. Propofol to control intracranial pressure in fulminant hepatic failure. Transplant Proc 2002;34:1220-2.

237. Vogels BA, Maas MA, Daalhuisen J, Quack G, Chamuleau RA. Memantine, a noncompetitive NMDA receptor antagonist improves hyperammonemia-induced encephalopathy and acure heparic encephalopathy in rats. Hepatology 1997;25:820-7.

238. Ellis AJ, Wendon JA, Williams R. Subdinical seizure activity and prophylactic phenytoin infusion in acure liver failure: a controlled clinical trial. Hepatology 2000;32:536-41.

239. Jones AL, Bangash $I H$, Bouchier IA, Hayes PC. Portal and systemic haemodynamic action of N-acerylcysteine in patients with stable cirrhosis. Gut 1994;35:1290-3.

240. Harrison PM, Keays R, Bray GP, Alexander GJ, Williams R. Improved outcome of paracetamol-induced fulminant hepatic failure by late administration of acerylcysteine. Lancet $1990 ; 335: 1572-3$.

241. Keays R, Harrison PM, Wendon JA, Forbes A, Gove C, Alexander GJ, et al. Intravenous acetylcysteine in paracetamol induced fulminant hepatic failure: a prospective controlled trial. BMJ 1991;303:1026-9.

242. Harrison PM, Wendon JA, Gimson AE, Alexander GJ, Williams R. Improvement by acerylcysteine of hemodynamics and oxygen transport in fulminant hepatic failure. N Engl J Med 1991;324:1852-7.

243. Walsh TS, Hopton P, Phillips BJ, Mackenzie SJ, Lee A. The effect of $\mathrm{N}$-acetylcysteine on oxygen transport and uptake in patients with fulminant hepatic failure. Heparology $1998 ; 27: 1332-40$.

244. Rolando N, Gimson A, Wade J, Philpott-Howard J, Casewell M, Williams R. Prospective controlled trial of selecrive parenteral and enteral antimicrobial regimen in fulminant liver failure. Hepatology 1993;17:196-201.

245. Ringe B, Lubbe N, Kuse Ex Frei U, Pichlmayr R. Total hepatectomy and liver transplantation as two-stage procedure. Ann Surg 1993;218:3-9.

246. Plauth M, Raible A, Vieillard-Baron D, Bauder D, Kremer I, Hartmann F. Effect of glucose and ammonia on glutamine (GLN) metabolism in the isolated perfused rat small intestine. Gastroenterology 1991;100:A240. 


\section{AIMS OF THE THESIS}

The knowledge presented in the introduction formed the basis of the studies described in this thesis. From the overview of the literature it can be concluded that there are numerous possible research questions unanswered in $\mathrm{HE}$ associated with cirrhosis and acute liver failure. We decided to focus on three major topics during our collaborative research period: 1 . the interorgan metabolism of ammonia in patients with cirrhosis with specific attention on the effects of major ammonia liberating events and its consequences on brain ammonia handling; 2 . the specific ammoniagenic and catabolic consequences of the major HE-inducing event in patients with cirrhosis: an upper gastrointestinal bleed; and 3. the pathophysiological basis of HE in acute liver failure and the possible role of a new therapy: moderate hypothermia.

The three research topics will be described in separate sections in this thesis. The work presented is the resultant of a close collaboration between the two authors and their research groups and, apart from chapters $3 \mathrm{~b}$ and 5, both authors contributed to every chapter in this thesis. Chapters $1,2,3 a, 5,6$ and 7 were principally written by Steven WM Olde Damink and will be the topic of his part of the dissertation. The other chapters are principally written by Rajiv Jalan and will be the topic of his part of the dissertation. Both wrote the introduction, the summary and the perspectives for the future. 


\section{RESEARCH AIMS}

\section{SECTION 1: INTERORGAN AMMONIA AND AMINO ACID METABOLISM}

The aims of this part of our collaborative research were:

- To determine the contribution of the individual organs to whole body ammoniagenesis. We hypothesised that the gut would produce the most ammonia and the liver would be the major ammonia consumer

$o$ in the basal state after an overnight fast, and

o following upper gastrointestinal bleeding (simulated and actual)

- To determine whether renal ammonia handling in patients with cirrhosis could be influenced by

o insertion of a transjugular intrahepatic portosystemic stent-shunt

$o$ altering the volume status.

- To determine the effects of induced hyperammonemia on brain

o function (neuropsychology)

o glutamine metabolism

o hydration status

o blood flow

SECTION 2: CATABOLIC EFFECTS OF UPPER GI BLEEDING AND THE ROLE OF ISOLEUCINE The specific aims of this part of our research were:

- To determine whether a 'simulated bleed' results in reduced protein synthesis and branched chain amino-acid antagonism.

-To determine the effect of isoleucine administration following a 'simulated' bleed on

o gut, Liver, Muscle and Kidney ammonia and amino acid metabolism

o gut, Liver, Muscle and Kidney Protein synthesis

SECTION 3: PATHOPHYSIOLOGICAL BASIS OF HEPATIC ENCEPHALOPATHY IN LIVER FAILURE AND ROLE OF HYPOTHERMIA

Here, we wanted to:

- determine whether a insertion of a transjugular intrahepatic stent-shunt in patients with variceal bleeding results in alterations in

o systemic haemodynamics

o brain ammonia and amino acid metabolism

o cerebral blood flow

o whole body nitric oxide production 
- determine the pathophysiological basis of increased intracranial pressure in parients with acute liver failure and study the relative roles of

o brain ammonia metabolism

o cerebral blood flow

o systemic Inflammatory response

- determine the effect of moderate hypothermia on

o clinical outcome of patients with acute liver failure and uncontrolled intracranial hypertension

o intracranial Pressure and Cerebral Blood Flow

o systemic Haemodynamics

o brain Ammonia and Amino acid metabolism

o systemic Inflammatory response and its markers

- determine whether moderate hypothermia restores cerebral blood flow autoregulation - determine whether moderate hypothermia could prevent the occurrence of episodes of increases in intracranial pressure during liver transplantation in patients with acute liver failure 



\section{SECTION 1}

\section{CHAPTER 1}

INTERORGAN AMMONIA AND AMINO ACID METABOLISM IN METABOLICALLY STABLE PATIENTS WITH CIRRHOSIS OF THE LIVER AND A TIPPS

Steven WM Olde Damink ${ }^{1,3}$, Rajiv Jalan ${ }^{1,4}$, Doris $N$ Redhead ${ }^{2}$, Peter C Hayes ${ }^{1}$, Nicolaas EP Deutz ${ }^{3}$, Peter B Soeters ${ }^{3}$

${ }^{1}$ Liver Unit and 2Department of Radiology, Royal Infirmary of Edinburgh, Edinburgh, Scotland, UK, ${ }^{3}$ Department of Surgery, Maastricht University, Maastricht, The Netherlands and 4 Institute of Hepatology, Royal Free and University College London Medical School, University College London, London, UK.

Published in Hepatology 2002; 36:1163-1171 


\section{INTRODUCTION}

Ammonia plays a central role in the pathogenesis of hepatic encephalopathy (1). The prevalence of hepatic encephalopathy in patients with cirrhosis of the liver is high, and reaches about $30 \%$ if patients are treated with a transjugular intrahepatic portosystemic stent shunt (TIPSS) (2). Hyperammonemia in these patients has been suggested to result from diminished hepatic urea synthesis capacity, the main route of ammonia detoxification, and intra- and/or extra hepatic shunting of ammonia produced by the intestines (3).

Glutamine synthesis has been suggested to be the most important alternative route of ammonia detoxification (4). Glutamine acts as a non-toxic nitrogen carrier and can be taken up by various organs. The interorgan exchange of glutamine and ammonia is severely altered in experimental animals with liver failure $(5,6)$. Ammonia metabolism has been studied in individual organs of patients with cirrhosis, but quantification is sparse, as is its relation with amino acid metabolism. Furthermore, to our knowledge no quantitative data are available on interorgan ammonia and amino acid metabolism in patients with cirrhosis. This information is essential since it provides the rationale for (potential) clinical therapy. Currently, all clinical therapeutic strategies aim to lower systemic ammonia by reducing colonic ammonia production, but therapeutic success rate is low (1).

The aim of the present study was to determine the contribution of various organs to whole body ammoniagenesis. We hypothesized that the portal drained viscera (PDV) would produce the most ammonia and that the liver would be the major ammonia consumer. We used the fact that cirrhotic patients with a TIPSS need to undergo routine portography to check the TIPSS patency. 


\section{METHODS}

Patients. Twenty-four metabolically stable patients with biopsy proven cirrhosis of the liver who underwent routine portography to check TIPSS patency were studied (Table 1). Exclusion criteria were severe ascites, pitting peripheral oedema, hepatic encephalopathy, recent upper gastro-inrestinal bleed ( $<14$ days), diabetes, portacaval pressure gradient $>12 \mathrm{mmHg}$, clinically evident cardiovascular disease or renal dysfunction, active alcohol consumption, malignancy or pregnancy. Patients did not use lactulose, diuretics or antibiotics. The Lothian ethics commitree approved the study and written informed consent was obtained. Severity of liver disease was assessed by the Pugh-Child scoring system.

Study protocol. Studies were started between 6.00 and 9.00 A.M. after an overnight fast, three hours before the portography was planned. The protocol began after a catheter was placed in a dorsal hand vein for infusion of para-amminolaippuric acid (PAH, 20\% w/v solution, MSD, NL) to determine organ plasma flow (detailed later) and a mixture of stable isotopes to determine organ protein metabolism. Stable isotope data will be discussed elsewhere. Furthermore, patients drank a mixture of 20 gram deuterium oxide dilution (99.9 atom\%, D-4501, Sigma, USA) and $30 \mathrm{ml}$ of $150 \mathrm{mM}$ sodium bromide (Clinical pharmacy, AZM, NL) to determine total body water and extracellular water (see for details (7)). Portography was performed under analgesia (pethidine, $1 \mathrm{mg} / \mathrm{kg} \mathrm{bw}$ ) and sedation (midazolam, $0.1 \mathrm{mg} / \mathrm{kg} \mathrm{bw}$ ). If the shunt was shown to be patent (portal pressure gradient $<12 \mathrm{mmHg}$ ), the following catheters were inserted under direct $x$-ray screening. The right femoral artery was cannulated using an $18 \mathrm{G}$ needle (Vygon leader, France). A sheath with a sampling port (6F, Cordis, NL) was inserted through the right femoral vein. A $5 \mathrm{~F}$ catheter (Terumo, femoral-visceral angiographic catheter, Belgium) was introduced through the sheath and positioned in the right renal vein. A similar 5F sampling catheter was inserted through the $10 \mathrm{~F}$ sheath in the right internal jugular vein (used for TIPSS portography) and was used to selectively sample blood from the left gastric vein, the splenic vein, and the superior and inferior mesenteric vein. Furthermore, a sample was taken from the middle or right hepatic vein that did not contain the TIPSS to prevent mixing of hepatic and portal venous blood. Hereafter, a Swan Ganz catheter (Baxter, 131 HF7, USA) was modified such that the distance between the distal (infusion) and proximal (sampling) port was $10.5 \mathrm{~cm}$. This catheter was inserted through the jugular venous sheath and the proximal port was positioned in the trunk of the portal vein distal to the shunt.

Sampling and measurement of blood flow. Organ blood flow was determined using a slight modification of the protocol used by Owen et al (8) and ten Have (9). Kidney, 
leg and portal flow were determined using a primed $(0.56 \mathrm{ml} / \mathrm{kg} \mathrm{bw})$, continuous infusion $(50 \mathrm{ml} / \mathrm{h})$ of PAH and liver plasma flow was determined using a primed $(10 \mathrm{mg})$, continuous infusion $(0.2 \mathrm{mg} / \mathrm{min}$ ) of indocyanine green (ICG, Cardiogreen, BDMS, USA). Both solutions were infused into a dorsal hand vein. PAH infusion was started 180 minutes and ICG 60 minutes before the portography to enable steady state concentrations $(10,11)$. To measure portal flow, PAH infusion was switched from the dorsal hand wein to the distal port of the Swan-Ganz catheter. Sampling for PAH was performed through the proximal port of the Swan-Ganz catheter (for a detailed description see (12)). Renal blood flow was determined by measurement of PAH in the arterial and renal venous blood (13). Blood flow in a single leg was measured by switching PAH infusion from the Swan-Ganz carheter to the femoral artery, and after a stabilizing period of 5 minutes, femoral venous blood was sampled for PAH. Hepatic blood flow was measured by simultaneous sampling of arterial and hepatic venous blood (10). A blood sample for deuterium and bromide analysis was taken four hours after the ingestion. Blood samples were immediately put on ice.

Analyses. Blood gas and electrolyte concentrations were directly measured using a COoximeter (IL 282, Instrumentation Laboratories, USA). For PAH determinations, $300 \mathrm{~mL}$ heparinised blood was added to $600 \mathrm{~mL}$ of $12 \%$ trichloroacetic acid solution (w/v; Merck 807 , Germany), thoroughly mixed, centrifuged at $8,900 \mathrm{~g}$ at $4^{\circ} \mathrm{C}$ for 5 minutes, put into liquid nitrogen and stored at $-80^{\circ} \mathrm{C}$. Plasma for ammonia, urea and ICG was obtained by centrifugation of heparinised whole blood at $8,900 \mathrm{~g}$ at $4^{\circ} \mathrm{C}$ for 5 minutes. Plasma for ICG was put into liquid nitrogen and stored at $-80^{\circ} \mathrm{C}$. For ammonia, urea and amino acid determination, $500 \mathrm{~mL}$ of plasma was mixed with 50 $\mathrm{mL}$ of $50 \%$ trichloroacetic acid solution put into liquid nitrogen and stored at $-80^{\circ} \mathrm{C}$. For amino acid analysis $500 \mathrm{~mL}$ of plasma was deproteinised by mixing with $30 \mathrm{mg}$ dry sulfosalicylic acid put into liquid nitrogen and stored at $-80^{\circ} \mathrm{C}$.

Plasma ammonia, urea and PAH were determined spectrophotometrically on an automated analysis system Cobas Mira S (Roche Diagnostica, Basel, Switzerland) by standard enzymatic methods, using commercially available kits (14). Indocyanine green was determined spectrophotometrically (15). Amino acid and bromide concentrations were determined by a fully automated HPLC system $(7,16)$. Deuterium was analysed with an isotope ratio mass spectroscope (7).

Calculations. Plasma flow rate $(\mathrm{mL} / \mathrm{kg} \mathrm{bw} / \mathrm{min})$ of the portal vein, liver, kidneys and one leg were calculated using formulae based on the method of indicator dilution and Fick's principle $(8,13,14)$. PAH determined blood flow was converted to plasma flow using the haematocrit. Substrate fluxes ( $\mathrm{nmol} / \mathrm{kg} \mathrm{bw} / \mathrm{min}$ ) across organs are the venousarterial (V-A) concentration difference times plasma flow.

To calculate substrate fluxes across the liver we used upper and lower bounds of liver fluxes using estimated portal-systemic shunting percentages of $70 \%$ and $100 \%$, 
respectively. These assumptions were used since we were unable to determine the fraction of shunting through the TIPSS, which has been reported to be approximately $90 \%$ (17). Thus,

(1) assuming $100 \%$ portal-systemic shunting. Liver flux = hepatic V-A concentration difference * liver plasma flow

(2) assuming $70 \%$ portal-systemic shunting: Liver flux = (liver plasma flow * hepatic venous concentration) $-\left[\right.$ (liver plasma flow $-0.3^{*}$ portal plasma flow) * arterial concentration $)+(0.3 *$ portal plasma flow $) *$ portal venous concentration $)]$

The substrate flux over the total hepato-splanchnic area is as follows:

(3) assuming $100 \%$ portal-systemic shunting. Hepato-splanchnic flux = PDV flux + Liver flux

(4) assuming 70\% portal-systemic shunting. Hepato-splanchnic flux $=\left(0.7^{*} \mathrm{PDV}\right.$ flux) + Liver flux.

Fractional extraction was calculated as: $100 \% *(\mathrm{~A}-\mathrm{V}$ concentration $) / \mathrm{A}$ concentration. Branched chain amino acids are calculated as the sum of valine, leucine and isoleucine. a-AN represents the sum of the total amino acids determined. Total body water, far-free body mass and body cell mass were calculated from bronide and deuterium dilution as detailed previously $(7)$.

Statistics. Results are presented as means \pm SEM. Wilcoxon's signed rank test was used to rest for differences from zero and to test for differences in ammonia production by the kidney and the PDV and splanchnic area, and ammonia uptake by leg muscle and liver. Pearson' correlation coefficients were determined between plasma concentrations and fluxes between biochemically rellated amino acids. 


\section{RESULTS}

All patients had patent TIPSS, placed on average 25.0 ( \pm 3.5 ) months before for treatment of variceal bleeding (Table 1). All catheters were inserted without complications and no untoward effects occurred as a result of the femoral arterial and venous catheterisation. The portal, splenic and renal vein could be sampled in all patients. The hepatic vein could not be sampled in one patient, the superior mesenteric vein and inferior mesenteric vein not in two, the femoral vein not in three and the left gastric vein not in thirteen, due to previous coiling of this vein during initial TIPSS placement. The mean plasma flows of the group were: portal flow $723( \pm 100) \mathrm{ml} / \mathrm{min}$, liver flow 609 $( \pm 95) \mathrm{ml} / \mathrm{min}$, kidney flow $665( \pm 78) \mathrm{ml} / \mathrm{min}$, and leg flow $650( \pm 77) \mathrm{ml} / \mathrm{min}$. These flows are comparable with literature data (18-20).

Mean hepatic extraction of ICG was $0.288 \pm 0,049$, which is higher than measured in patients with decompensated chronic and acute liver disease $(0.054 \pm 0,085$ (21)), supporting that our study population consisted of metabolically stable patients. In agreement, the body composition of the patients was similar to previous reports of patients with stable cirrhosis $(22,23)$ (Table 1).

Arterial data. Mean arterial ammonia concentration was $94( \pm 6) \mathrm{mM}$, much higher than reported for healthy controls ( $45 \mathrm{mM}$ ) (19). The acid-base status was within normal range, with a mean arterial $\mathrm{pH}$ of $7.386( \pm 0.09)$. The arterial concentration of ammonia and amino acids and their V-A concentration differences within the portal drained area are shown in table 2.

Portal drained viscera metabolism. The PDV was the major ammonia producer (see figure 1). Glutamine was the only amino acid that was taken up by the PDV (see table 3). Glutamine uptake did not correlate with the arterial glutamine concentration ( $r=-$ 0.241 . PDV uptake of glutamine was significantly correlated with PDV production of ammonia $(r=-0.809, p<0.001)$, citrulline $(r=-0.863, p<0.001)$ and weakly with production of alanine ( $r=0.444, p=0.034)$.

Substrate fluxes could not be calculated within the portal drained area because of the absence of flow measurements. However, the V-A concentration differences of ammonia (Table 2) showed that ammonia is taken up by organs draining into the left gastric vein, mostly stomach and pancreas, and that ammonia is produced in organs draining into the superior mesenteric vein, mostly small bowel and the inferior mesenteric vein, draining the left part of colon. Glutamine was extracted at all sites, except the left gastric vein (Figure 2), but the extraction did not correlate significantly with arterial glutamine concentration. The highest ammonia V-A difference was observed across the inferior mesenteric vein-draining viscera, much higher than the amount of 
Table 1: Patient characteristics (mean \pm SEM). Literature reference values (23) for healthy control subjects are for body mass index $20-25 \mathrm{~kg} / \mathrm{m} 2$; for fat-free body mass ( $\%$ body weight) $86 \%$, and for body cell mass ( $\%$ fat free body mass) $67 \%$.

Age (yrs)

Sex

Weight (kg)

Body mass index $(\mathrm{kg} / \mathrm{m} 2)$

Total body water (L)

Fat-free body mass $(\mathrm{kg})$

Fat-free body mass ( $\%$ body weight)

Extracellular water ( $\%$ total body water)

Body cell mass $(\mathrm{kg})$

Body cell mass (\% Fat-free body mass)

Aetiology

Pugh score

Portal pressure gradient (mmHG)

TIPSS (months)

Albumin (gram/L)

ALT (IU/L)

Billirubin

Creatinine $(\mathrm{mmo} / \mathrm{L})$
$49.6 \quad(2.4)$

7 female/ 17 male

$80.6 \quad(4.1)$

$29.6 \quad(1.1)$

$43.1 \quad(2.3)$

$64.3 \quad(3.4)$

$82.8 \quad(2.3)$

$45.0 \quad(2.7)$

$26.4 \quad(2.0)$

$40.3 \% \quad(2.0)$

18 alcoholic liver disease

2 primary sclerosing cholangitis

1 primary biliary cirrhosis

1 cryptogenic cirrhosis

1 hepatitis $\mathrm{C}$

1 autoimmune hepatitis

$6.5 \quad(0.3)$

$5.3 \quad(0.8)$

$25.0 \quad(3.5)$

$36.0 \quad(1.0)$

$37.0 \quad(2.7)$

$47.3 \quad(6.6)$

$83.0 \quad(2.3)$ 
กำ

in

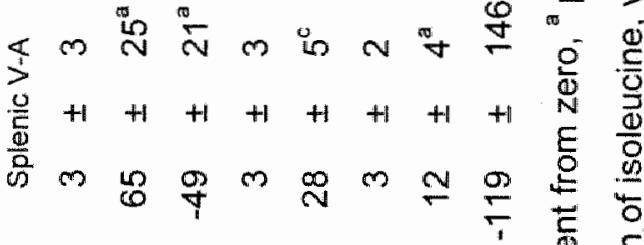

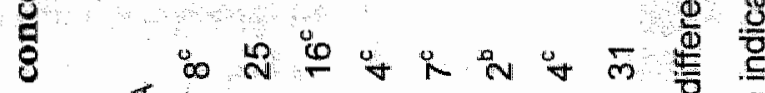

. $\frac{5}{8}+1+1+1+1+1+\frac{4}{5}$

¿

当 जल

틀

\%

8 递 $+1+1+1+1+1+1+1+\sum_{\frac{1}{0}}$

焉

$\frac{3}{5}$ 
g"utamine extracted, suggesting that ammonia release in this area was only partly related to glutamine metabolism. Urea was not extracted across the inferior mesenteric vein. Interestingly, we observed net urea release across the superior mesenteric vein and the splenic vein (Table 2).

Liver metabolism. The liver produced urea in quantities of about $800 \mathrm{nmol} / \mathrm{kg}$ $\mathrm{bw} / \mathrm{min}$. The liver consumed ammonia (Figure 1) and most amino acids, with glutamine and alanine representing $50 \%$ of total a-AN uptake (Table 3). Ammonia uptake by the liver was only weakly correlated with arterial and portal concentrations of ammonia $(r=-0.476(p=0.022)$ and $r=-0.380(p=0.073)$ for the $100 \%$ shunting estimate, and $r=-0.449(p=0.032)$ and $r=-0.397(p=0.061)$ for $70 \%$ shunting $)$.

Hepatic urea production did not correlate with hepatic uptake of ammonia $(\mathrm{r}=$ 0.269 for $100 \%$ shunting and $r=-0.202$ for $70 \%$ shunting), but it correlated strongly with hepatic uptake of alanine $(r=-0.656, p<0.001$ for $100 \%$ shunting and $r=-0.781$, $p<0.001$ for $70 \%$ shunting), arginine ( $r=-0.531, p=0.009$ for $100 \%$ shunting and $r=-$ $0.571, \mathrm{p}=0.004$ for $70 \%$ shunting) and glutamine $(\mathrm{r}=-0.506, \mathrm{p}=0.014$ for $100 \%$ shunting and $r=-0.541, p=0.008$ for $70 \%$ shunting). Hepatic urea production was weakly related with arterial and portal alanine concentrations, but showed no correlation with concentrations of ammonia, glutamine, hydrogen ion, bicarbonate and total a-AN.

Hepato-splanchnic ammonia metabolism. Net ammonia production across the hepato-splanchnic area was only significant in the $100 \%$ portasystemic shunting estimate $(350 \pm 109 \mathrm{nmol} / \mathrm{kg} \mathrm{bw} / \mathrm{min}$ versus $-38 \pm 64 \mathrm{nmol} / \mathrm{kg} \mathrm{bw} / \mathrm{min}$ for the $70 \%$ shunting estimate) (Figure 1).

Muscle metabolism. The skeletal muscle of one leg removed a similar amount of ammonia from the circulation as the liver in these cirrhotic patients (Figure 1 and Table 3). Muscle ammonia uptake did not correlate with the arterial ammonia concentration $(r=0.274)$ or with the observed muscle glutamine production $(r=-0.092)$. Muscle ammonia uptake was not correlated to fat-free body mass or body cell mass $(r=0.296$ and $\mathrm{r}=0,305$, respectively).

Renal metabolism. The kidneys produced ammonia, although significantly less than produced by the PDV $(\mathrm{p}<0.001)$ and splanchnic area in case of $100 \%$ portal systemic shunting ( $p=0.036$ ) (Figure 1). Unfortunately, we were only able to collect urine samples from 5 patients. Table 4 shows the total renal ammonia production of the individual patients and the amount of ammonia released into the systemic circulation and excreted into the urine. Urinary ammonia excretion exceeded the amount of ammonia released systemically in two patients. Correlations were not determined since total renal substrate metabolism could only be determined in 5 patients. 


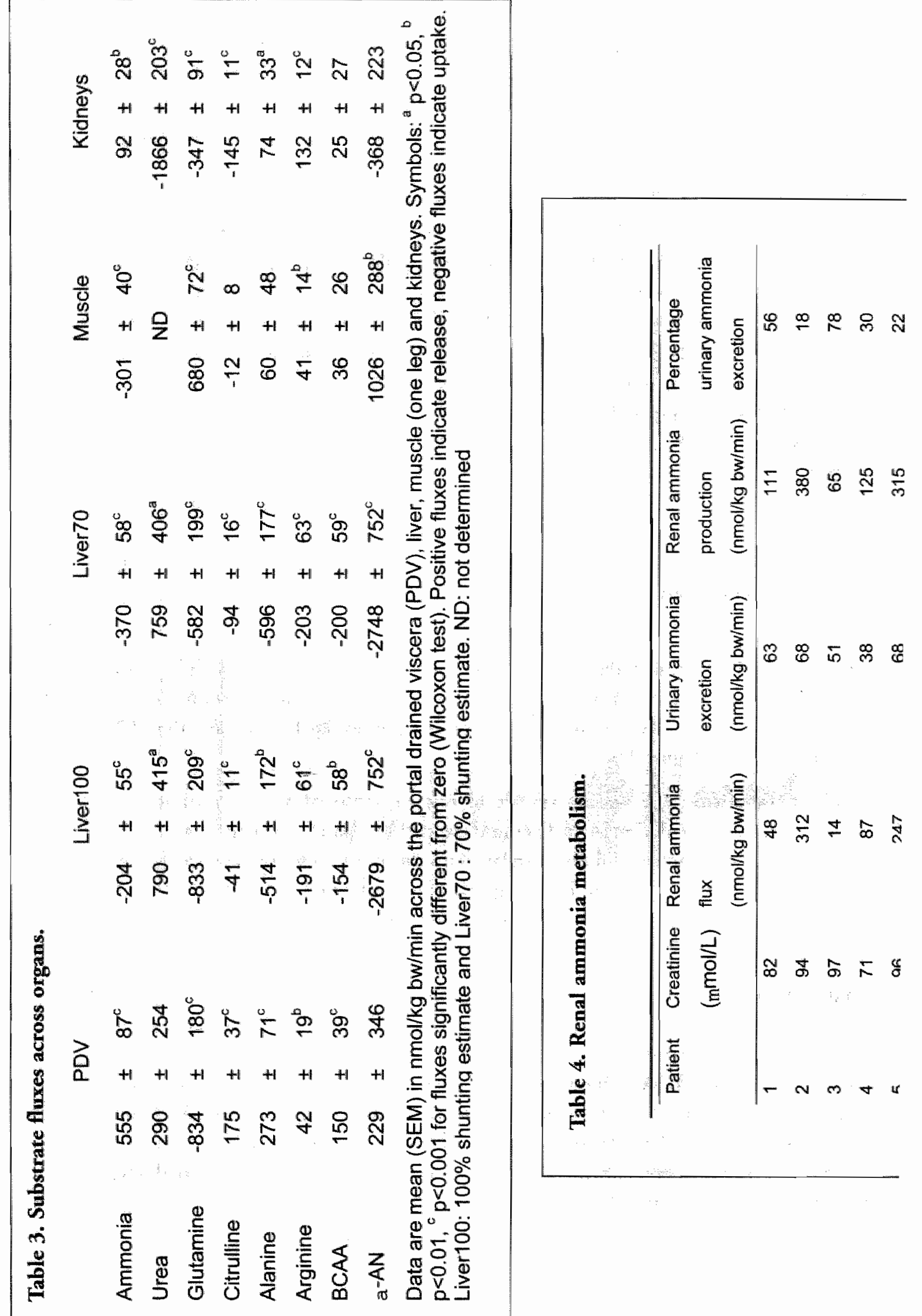




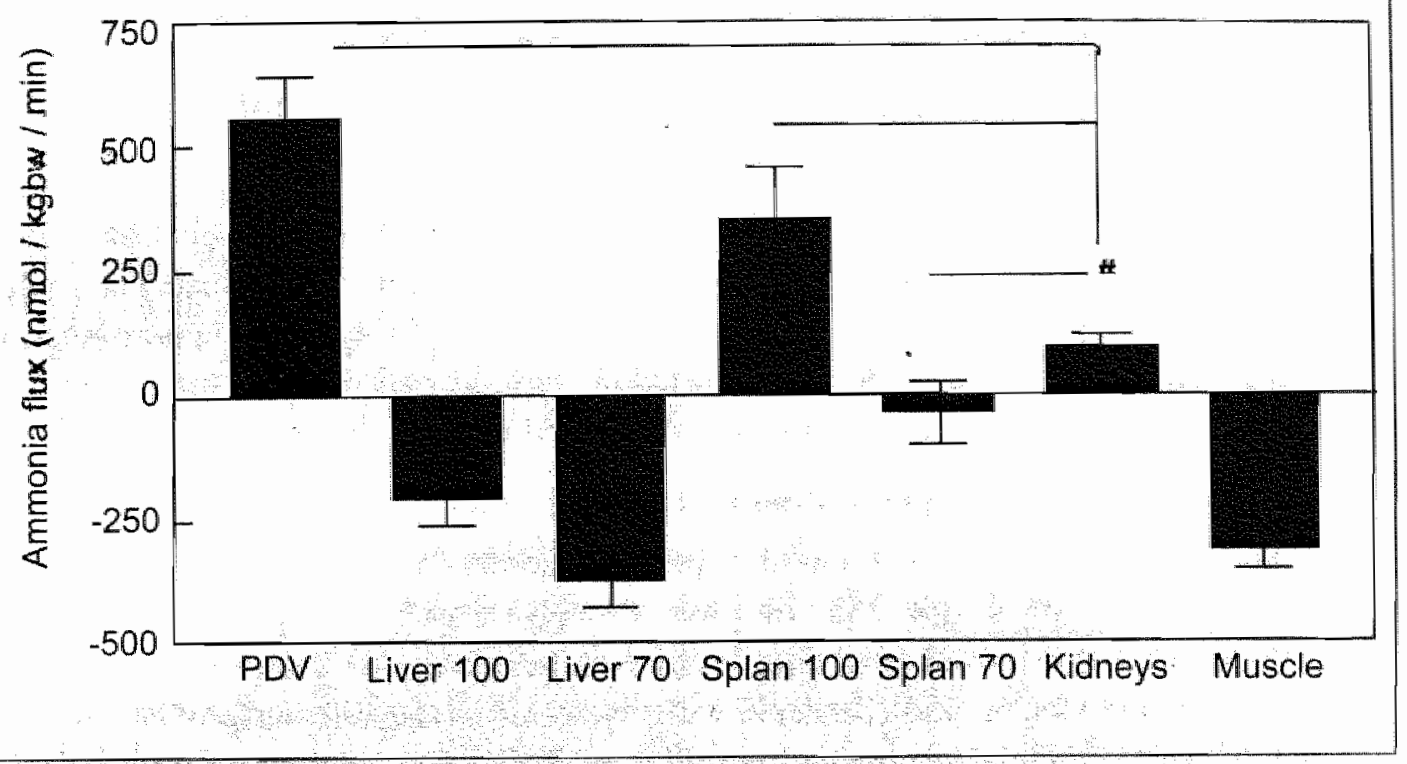

FiguRE 1 (ABOVE), Ammonia fluxes (nnol/kg bw/min) across the Portal Drained Viscera (PDV), Muscle (one leg), Kidneys, Liver and Total Hepato-splanchnic area (both expressed as upper bounds with $70 \%$ portal-systemic shunting and lower bounds with $100 \%$ portal-systemic shunting). Positive flux represents production, negative flux production of ammonia. Only the Hepato-splanchnic (70\%) ammonia production was not significantly different from zero. PDV ammonia production was significantly greater than renal production ( $\mathrm{p}<0.001$ ), as was the Hepato-splanchnic $100 \%$ estimate $(p=0.018)$. However, renal ammonia production exceeded the Hepato-splanchnic $70 \%$ estimate $(\mathrm{p}=0.048)$. Ammonia uptake by the skeletal muscle of one leg did not differ significantly from both liver estimates.

FIGURE 2 (BELOW). Fractional extraction of glutamine across the portal drained viscera (PDV), the splenic vein, the left gastric vein (LGV), and the superior (SMV) and inferior mesenteric vein (IMV).

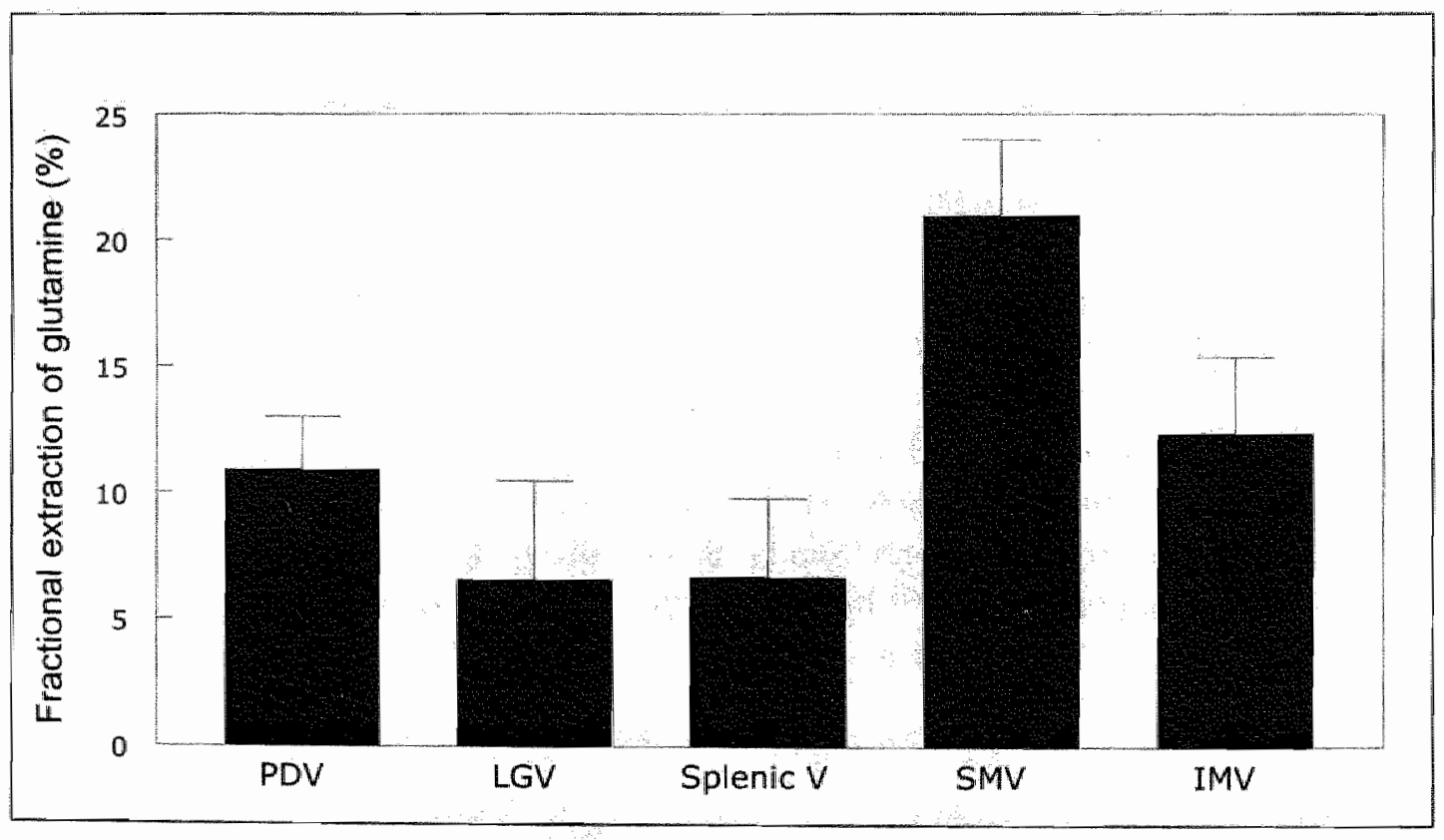




\section{DISCUSSION}

The present study is the first to quantify ammonia and amino acid metabolism of the major ammonia producing and consuming organs in the same individual. The study could be performed because this patient population offered the unique opportunity to access the PDV and was ethically supported because the patients were attending for a routine clinical procedure, which involved catheterisation of the portal vein.

\section{Ammonia production: portal drained viscera}

The PDV was the major ammonia producer, confirming previous observations in experimental animals $(5,24)$, and was strongly relared with glutamine uptake. Glutamine was extracted at all sites in the portal-drained area, except the left gastric vein draining viscera, and correlated with release of its degradation products in the enterocyte: ammonia, alanine and citrulline (25). The fractional extraction of glutamine was higher in the superior mesenteric vein (21\%) compared with inferior mesenteric vein draining viscera $(12 \%)$, which is in agreement with the observed fractional extractions across the jejunum (24\%), ileum $(9 \%)$ and colon ( $8 \%$ ) of patients undergoing elective abdominal surgery (26).

In early clinical and experimental literature it is suggested that PDV released ammonia solely originating from urea breakdown by urease-containing bacteria located in the colon $(27,28)$. However, in the present study we could not detect net urea uptake across the PDV or a net extraction of blood-derived urea across the inferior mesenteric vein draining viscera. The amount of ammonia that was released into the inferior mesenteric vein exceeded the amount of glutamine extracted, suggesting that the colon produces ammonia partly from breakdown of glutamine and parrly from breakdown of nitrogen containing material within the lumen. Weber and Veach (24) showed in post-absorptive healthy dogs that half of the ammonia produced by the colon was derived from breakdown of nitrogen containing material within the colonic lumen and $9 \%$ from uptake of arterial glutamine. In contrast to our findings, $42 \%$ of the ammonia produced by the colon of healthy dogs was derived from uptake of urea from arterial blood. Whether this discrepancy is caused by inter-species differences or because of altered colonic metabolism due to the chronic hyperammonemic state of the TIPSS patients remains unclear and demands further research.

Currently, all standard clinical approaches aim to diminish colonic (bacterial) ammonia production (1), however, the present study shows that the majority of PDV produced ammonia originates from breakdown of glutamine. Diminishing this source of ammonia production will be difficult since glutamine is essential for the intestine (25). 


\section{Ammonia production: kidneys}

The kidneys contributed to the hyperammonemic state by releasing ammonia into the circulation. Renal ammonia release is about a third of the ammonia released by the hepato-splanchnic area in case of $100 \%$ portasystemic shunting, but exceeds hepatosplanchnic ammonia release in case of $70 \%$ shunting. Renal ammonia release in patients with cirrhosis has already been described in early $A-V$ concentration difference studies $(13,29,30)$. The kidneys extracted a significant amount of glutamine, in agreement with previous studies that suggested that glutamine was the principle precursor for renal ammoniagenesis (31).

Unfortunately, we were only able to collect urine samples from 5 patients. The present data show a scatter in percentage ammonia released into the urine among these patients. In two patients urinary ammonia excretion exceeded the amount of ammonia released into the systemic circulation, thereby acting as a net ammonia removing organ. The kidneys acted as net ammonia producers to the body in the other patients. To our knowledge there is only one other study in patients with cirrhosis that studied this and reported exactly the same results in their 5 patients (13). Urinary ammonia excretion is an important way of releasing circulating ammonia and/or nitrogen during liver failure, as we demonstrated earlier $(14,32)$. In post-absorptive healthy rats, $70 \%$ of total renal ammoniagenesis is released inro the systemic circulation, whereas in rats with acute and chronic liver failure $70 \%$ of total renal ammoniagenesis was excreted into urine and $30 \%$ released into the renal vein (14). This adaptive mechanism of the kidneys to increase urinary ammonia excretion could be valuable target for clinical therapy, as it will have a major effect on net whole body ammonia removal in patients with cirrhosis of the liver.

\section{Ammonia removal: liver}

As expected the cirrhotic liver removed ammonia from the circulation, but our data suggest diminished ammonia clearance by the liver since the hepatic venous ammonia concentration was still high $(70 \pm 7 \mathrm{mM})$, compared with concentrations observed in healthy volunteers ( $41 \pm 10 \mathrm{mM})(19)$. To our best knowledge, the present study is the first to report the urea production across the liver by the organ balance method. It is difficult to compare these data with values derived from urea excretion of whole body stable isotope estimations. The present data should be interpreted with care since they are influenced by the shunting of portal blood via the TIPSS in these patients, which has been reported to be approximately $90 \%(17)$, and the patients were measured after an overnight fast, a state were the amount of circulating nitrogen is at it's lowest. Portacaval shunting results in bypassing of the peri-portally located hepatocytes that are specialized in urea synthesis via the ornithine-cycle and also contain glutaminase, which produce ammonia that serves the ornithine cycle (33). In patients with cirrhosis of the liver, the activity of hepatic glutaminase is 4 to 6 fold increased to maintain a 
normal flux through the ornithine cycle $(34,35)$. This will lead to more apparent glutamine uptake by the liwer, which is also shown in the present study. Urea synthesis was strongly related with hepatic uptake of glutamine and also alanine, in agreement with in vitro observations $(36,37)$.

\section{Ammonia removal: muscle}

This is the first study that quantifies the role of skeletal muscle in ammonia metabolism in patients with cirrhosis. Skeletal muscle from the leg removes more ammonia from the circulation than the cirrhotic liver. It is difficult to estimate the contribution of one leg to whole body skeletal muscle mass in parients with cirrhosis of the liver, because of altered body composition (23). Moreover, leg skeletal ammonia metabolism does not necessarily reflect metabolism in other muscle. In healthy volunteers, ammonia was taken up in leg skeletal muscle $(29,38)$ but not in forearm muscle $(39,40)$. To our knowledge, no solid explanation exists for these differences. Additionally, there are remarkable inter-species differences in muscle ammonia handling, since we could not show muscle ammonia uptake in experimental portacaval shunted rats $(41,42)$. The huge inter-species difference in the glutamine concentration gradient between the arterial and muscle glutamine pool could be an explanation, but further research is necessary to address this question.

In the present study we could not demonstrate a relation between arterial ammonia concentrations and ammonia uptake by muscle, reproducing previous observations in patients with stable cirrhosis $(39,40)$.

Ammonia uptake by muscle, leading to glutamine synthesis and glutamine release from muscle does not necessarily indicate net ammonia detoxification. In the present study the amount of nitrogen that was taken up by skeletal muscles in the form of ammonia was much less than the amount of nitrogen that was released in the form of glutamine. This observation suggest that muscle glutamine release is only partly accounted for by ammonia detoxification, but also caused by net protein catabolism, as is suggested by the release of total a-AN from muscle (43). Glutamine released from skeletal muscle can be taken up in the PDV and in the kidneys where it can be metabolised to ammonia, thereby abolishing the effect of muscle ammonia uptake on whole body ammonia removal. Moreover, increased muscle glutamine release because of protein catabolism could have a similar feed forward effect on ammoniagenesis. Therefore, it is important to avoid net protein catabolism and muscle wasting in cirrhotic patients, and therapy should focus on maintaining muscle mass by adequate nutritional intake.

\section{Peripheral urea synthesis}

In the present study net release of urea was observed from the spleen and from the superior mesenteric vein draining viscera. We could not quantify the amount of urea 
produced at these sites, because of the lack of blood flow meaturements, but the sig nificance of this observation can be estimated if we use Alow data avaliable in the literature. Nishida and colleagues (44) found that splenie vein flow was at least $50 \%$ of portal How in liver pationts and Burkart and colleagues (18) reported that the superior mesenteric flow was $40 \%$ of portal flow in healthy volunters. If we use these estimates, the spleen $(345 \mathrm{nmol} / \mathrm{kg} \mathrm{bw/min})$ and the superior mesenteric vein draining viscera (325nmol/kg bw/min) would produce together approximately $80 \%$ of hepatic urea production. Urea production at these sites (and in other peripheral tissues by arginase activity (45)) could explain the observed difference in hepatic urea production (about $800 \mathrm{nmol} / \mathrm{kg} \mathrm{bw} / \mathrm{min}$ ) and the amount of urea excreted by the kidneys (1800 nmol/kg $\mathrm{bw} / \mathrm{min}$ ) in the present study. However, the observed urea flux across the PDV did not reach significance. This is likely to be due to the lack of urea release from the other portal viscera draining veins, thereby diminishing the $V$-A concentration difference of urea but increasing the blood flow. The observed portal V-A concentration difference of 32 $\mathrm{mM}$ is less than $1 \%$ of the arterial urea concentration, and reaches the detection limit of the urea determination method and therefore gives rise to large variation.

Splenic urea release can be explained by arginase activity in macrophages and monocytes (46). The arginine used in this reaction is probably derived from glutamine via conversion to ornithine and cirrulline (47). The fact that the superior mesenteric vein draining viscera released both urea and arginine suggests that the arginine that was broken down via arginase to produce urea was furnished by breakdown of ammonia and glutamine, as has recently been demonstrated in enterocytes of postweaning pigs $(48,49)$. It could be hypothesized that peripheral urea production is an important route of detoxifying ammonia in cirrhotic patients with a TIPSS. Further research is necessary to determine the importance of this observation.

\section{Summary}

The hyperammonemia that is observed in the present study is caused by a combination of portasystemic shunting of PDV produced ammonia and renal ammoniagenesis (as is summarized in Figure 3). PDV ammonia production is mainly caused by glutamine metabolism, and only partly by breakdown of nitrogenous sources within the colonic lumen. This observation can explain the low therapeutic success rate of the present clinical therapies, since they all aim to lower colonic ammonia production. Preliminary data suggest that the kidneys can act as net ammonia disposers in some of these patients. Net ammonia disposal was also observed in the cirrhotic liver, although our findings suggest a diminished hepatic ammonia detoxification capacity. Skeletal muscle removes more ammonia from the circulation than the cirrhotic liver. However, muscle releases excessive amounts of the non-toxic nitrogen carrier glutamine, which can lead to ammonia production in the PDV and kidneys, abolishing the effect on net whole body ammonia removal. 


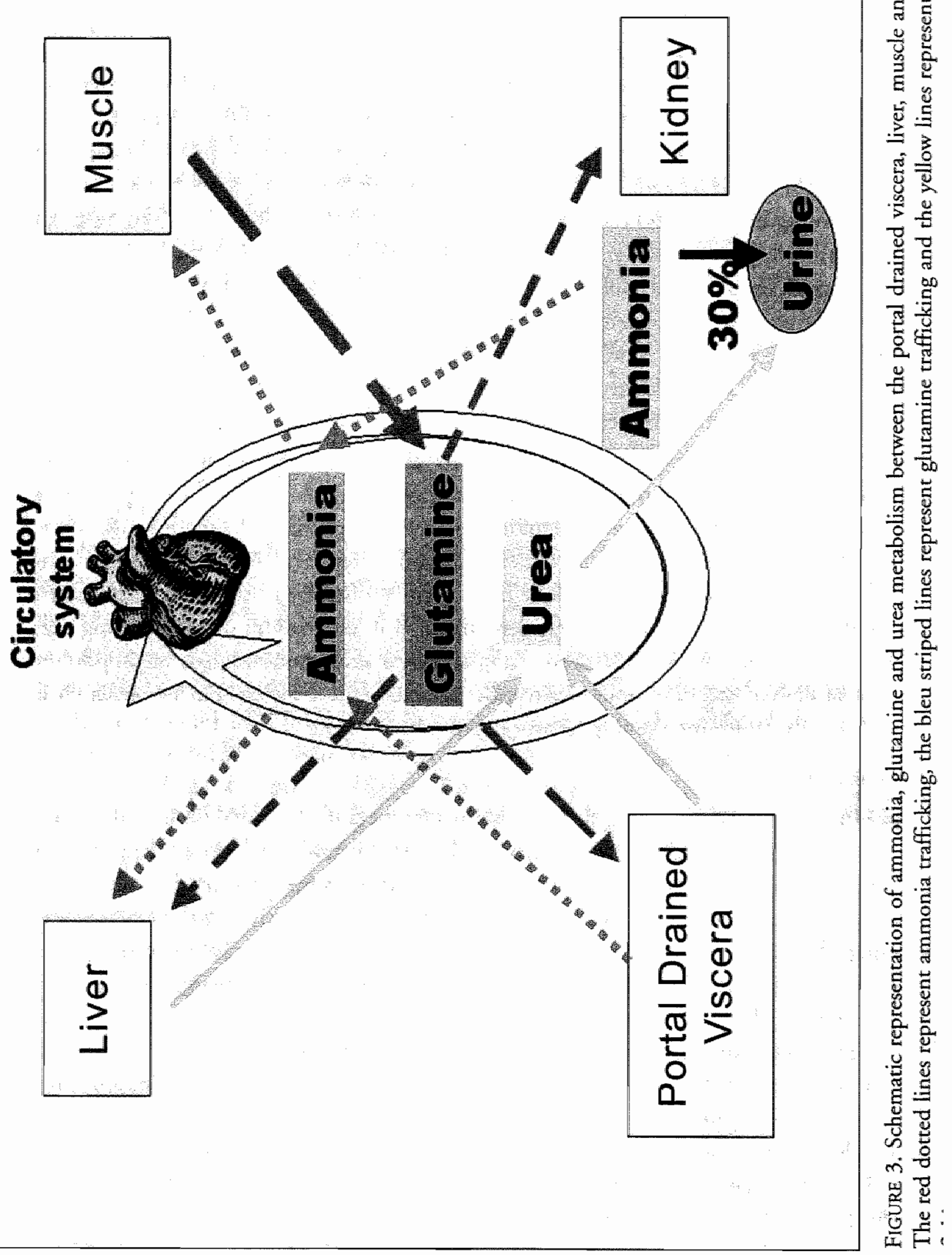




\section{REFERENCES}

1. Jalan R, Hayes PC. Hepatic encephalopathy and ascites. Lancet 1997;350:1309-15.

2. Stanley AJ, Jalan R, Forrest EH, Redhead DN, Hayes PC. Longterm follow up of transjugular intrahepatic portosystemic stent shunt (TIPSS) for the treatment of portal hypertension: results in 130 patients. Gut 1996;39:479-85.

3. Meijer AJ, Lamers WH, Chamuleau RA. Nitrogen metabolism and ornithine cycle function. Physial Rev 1990;70:701-48.

4. Lockwood AH, McDonald JM, Reiman RE, Gelbard AS, Laughlin JS, Duffy TE, et al. The dynamics of ammonia metabolism in man. Effects of liver disease and hyperammonemia. J Clin Invest 1979;63:449-60.

5. Deutz NEP, Dejong $\mathrm{CHC}$, Soeters $\mathrm{PB}$. Interorgan ammonia and glutamine exchange during liver failure. In: Record CO, Mardini HA, editors. Advances in Hepatic Encephalopathy \& metabolism in liver disease. Newcastke upon Tyne: University of Newcastle upon Tyne; 1996: 87-99.

6. Dejong CHC, Deutz NEP, Soeters PB. Ammonia and glutamine metabolism during liver insufficiency: the role of kidney and brain in interorgan nitrogen exchange. Scand J Gastroenterol Suppl 1996;218:61-77.

7. Cox-Reijven PL, Soeters PB. Validation of bio-impedance spectroscopy: effects of degree of obesity and ways of calculating volumes from measured resistance values. Int J Obes Relat Metab Disord 2000;24:271-80.

8. Owen OE, Reichle FA, Mozzoli MA, Kreulen T, Patel MS, Elfenbein IB, et al. Hepatic, gut, and renal substrate flux rates in patients with hepatic cirrhosis. I Clin Invest $1981 ; 68: 240-52$.

9. Ten Have GAM, Bost MCF Suyk-Wierts JCAW, van den Bogaard AEJM, Deutz NEP. Simultaneous measurement of metabolic flux in portally-drained viscera, liver, spleen, kidney and hindquarter in the conscious pig. Lab Anim 1996;30:347-58.

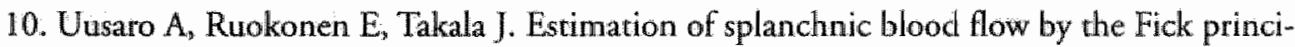
ple in man and problems in the use of indocyanine green. Cardiovasc Res 1995;30:106-12.

11. Selkurt EE. Measurement of renal blood flow. Meth Med Res 1948;1:191-9.

12. Granger DN, Bulkley GB. In: Measurement of blood flow. Applications to the splanchnic circulation. Baltimore/London: Williams\& Wilkins; 1981:1-507. .

13. Owen EE, Tyor MP, Flanagan JF, Berry JN. The kidney as a source of blood ammonia in patients with liver disease: the effect of acetazolamide. J Clin Invest 1960;39:288-94.

14. Dejong CHC, Deutz NEP, Soeters PB. Renal ammonia and glutamine metabolism during liver insufficiency-induced hyperammonemia in the rat. J Clin Invest 1993;92:283440.

15. Jalan R, Plewris JN, Jalan AR, Finlayson ND, Hayes PC. A pilot study of indocyanine green clearance as an early predictor of graft function. Transplantation 1994;58:196-200.

16. van Eijk HIM, Rooyakkers DR, Deutz NE. Rapid routine determination of amino acids in plasma by high-performance liquid chromatography with a 2-3 microns Spherisorb ODS II column. I Chromatogr 1993;620:143-8.

17. Walser EM, Harris VM, Harman JT, Park HM, Siddiqui AR. Quantification of intrahep- 
atic portosystemic shunting after placement of a transjugular intrahepatic portosystemic shunt. I Vasc Interv Radiol 1996;7:263-7.

18. Burkart DJ, Johnson CD, Ehman RL. Correlation of arterial and venous blood flow in the mesenteric system based on MR findings. Am J Roentgenol 1993;161:1279-82.

19. Clernmesen JO, Kondrup I, Otr P. Splanchnic and leg exchange of amino acids and ammonia in acute liver failure. Gastroenterology 2000;118:1131-9.

20. Jalan R, Forrest EH, Redhead DN, Dillon JF, Hayes PC. Reduction in renal blood flow following acute increase in the portal pressure: evidence for the existence of a hepatorenal reflex in man? Gut 1997;40:664-70.

21. Clemmesen JO, Tygstrup N, Ott P. Hepatic plasma flow estimated according to Fick's principle in patients with hepatic encephalopathy: evaluation of indocyanine green and Dsorbitol as test substances. Hepatology 1998;27:666-73.

22. Mullen KD, Denne SC, MoCullough AI, Savin SM, Bruno D, Tavill AS, et al. Leucine metabolism in stable cirrhosis. Hepatology 1986;6:622-30.

23. McCullough AI, Mullen KD, Kalhan SC. Defective nonoxidative leucine degradation and endogenous leucine flux in cirrhosis during an amino acid infusion. Hepatology 1998;28:1357-64.

24. Weber FL, Jr., Veach GL. The importance of the small intestine in gut ammonium production in the fasting dog. Gastroenterology 1979;77:235-40.

25. Windmueller HG. Glutamine utilization by the small intestine. Adv Enzyme Regul 1982:53:210-37.

26. van der Hulst RR, von Meyenfeldt MF, Deutz NE, Soeters PB. Glutamine extraction by the gut is reduced in depleted patients with gastrointestinal cancer. Ann Surg $1997: 225: 112-21$.

27. Wolpert E, Phillips SF, Summerskill WH. Ammonia production in the human colon. Effects of cleansing, neomycin and acetohydroxamic acid. N Engl J Med 1970;283:15964.

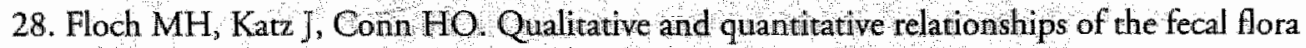
in cirthotic patients with portal systemic encephalopathy and following portacaval anastomosis. Gastroenterology 1970;59:70-5.

29. Tyor MP, Owen EE, Berry JN, Flanagan JF The relative role of extremity, liver and kidney as ammonia receivers and donors in pacients with liver disease. Gastroenterology $1960 ; 39: 420-4$.

30. Berry JN, Flanagan JF, Owen EE Tyor MP. The kidney as a source of blood ammonia in resting and hyperventilated cirrhotics. Clin Res 1959:7:154-5.

31. Pitts RF, DeHaas J, Klein J. Relation of renal amino and amide nitrogen extraction to ammonia production. Am J Physiol 1963;204:187-91.

32. Dejong $\mathrm{CH}$, Deutz NE, Soeters PB. Metabolic adaptation of the kidney to hyperammonemia during chronic liver insufficiency in the rat. Hepatology 1993;18:890-902.

33. Haussinger $\mathrm{D}$. Hepatocyte heterogeneity in glutamine and ammonia metabolism and the role of an intercellular glutamine cyde during ureogenesis in perfused rat liver. Eur J Biochem 1983:133:269-75.

34. Kaiser S, Gerok W, Haussinger D. Ammonia and glutamine metabolism in human liver slices: new aspects on the parhogenesis of hyperammonaemia in chronic liver disease. Eur $\mathrm{J}$ Clin Invest $1988 ; 18: 535-42$. 
35. Haussinger D. Regulation of hepatic ammonia metabolism: the intercellular glutamine cycle Adv Enzyme Regul 1986;25:159-80.

36. Ochs RS. Glutamine metabolism of isolated rat hepatocytes. Evidence for carecholamine activation of alpha-ketoglutarate dehydrogenase. J Biol Chem 1984;259:13004-10.

37. Krebs HA, Hems R, Lund P, Halliday D, Read WW. Sources of ammonia for mammalian urea synthesis. Biochem J 1978;176:733-7.

38. Warter JM, Brandt C, Marescaux C, Rumbach L, Micheletti G, Chabrier G, et al. The renal or gin of sodium valproate-induced hyperammonemia in fasting humans. Neurology $1983 ; 33: 1136-40$.

39. Ganda OP, Ruderman NB. Muscle nitrogen metabolism in chronic hepatic insufficiency. Metabolism 1976:25:427-35.

40. Webster LT, Gabuzda GJ. Ammonium uptake by the extremities and brain in hepatic coma. J Clin Invest 1958;37:414-24.

41. Dejong CHC, Kampman MT, Deurz NEP, Soeters PB. Altered glutamine metabolism in rat portal drained viscera and hindquarter during hyperammonemia. Gastroenterology $1992 ; 102: 936-48$.

42. Dejong CHC, Deutz NEP, Soeters PB. Muscle ammonia and glutamine exchange during chronic liver insufficiency in the rat. J Hepatol 1994;21:299-307.

43. Charlton MR. Protein metabolism and liver disease. Baillieres Clin Endocrinol Metab $1996 ; 10: 617-35$.

44. Nishida O, Moriyasu F, Nakamura T, Ban N, Miura K, Sakai M, et al. Interrelationship between splenic and superior mesenteric venous circulation manifested by transient splenic arterial acclusion using a balloon catheter. Hepatology 1987;7:442-6.

45. Aminlari $M$, Vaseghi $\mathbb{T}$. Arginase distribution in tissues of domestic animals. Comp Biochem Physiol 1992;103:385-9.

46. Featherston WR, Rogers QR, Freedland RA. Relarive importance of kidney and liver in synthesis of arginine by the rat. Am J Physiol 1973;224:127-9.

47. Murphy $C$, Newsholme P. Importance of glutamine metabolism in murine macrophages and human monocytes to L-arginine biosynthesis and rates of nitrite or urea production. Clin Sci 1998;95:397-407.

48. Wu G. Urea synthesis in enterocytes of developing pigs. Biochem J 1995;312:717-23.

49. Davis $P K_{\text {, Wul }}$.. Comparmentation and kinetics of urea cycle enzymes in porcine enterocytes. Comp Biochem Physiol B Biochem Mol Biol 1998;119:527-37. 



\section{CHAPTER 2}

THE KIDNEY PLAYS A MAJOR ROLE IN THE HYPERAMMONEMIA SEEN AFTER SIMULATED OR ACTUAL GI BLEEDING IN PATIENTS WITH CIRRHOSIS

Steven WM Olde Damink ${ }^{1,2}$, Rajiv Jalan ${ }^{2,6}$, Nicolaas EP Deutz ${ }^{1}$, Doris N Redhead ${ }^{3}$, Cornelis HC Dejong ${ }^{1,4}$, Paula Hynd ${ }^{5}$, Rosy A Jalan ${ }^{3}$, Peter C Hayes ${ }^{2}$, Peter B Soeters ${ }^{1}$

${ }^{1}$ Department of Surgery, Maastricht University, Maastricht, The Netherlands, ${ }^{2}$ Liver Unit, ${ }^{3}$ Department of Radiology, ${ }^{4}$ Surgery and 5 Dietetics, Royal Infirmary of Edinburgh, Edinburgh, Scotland, UK and GInstitute of Hepatology, Royal Free and University College Medical School, University College London, 69-75 Chenies Mews, London WC1E 6HX

Published in Hepatology 2003; 37: 1277-1285 


\section{INTRODUCTION}

Ammonia plays a central role in the hypothesis regarding the pathogenesis of hepatic encephalopathy $(1,2)$. Upper gastrointestinal (UGI) bleeding in patients with cirrhosis of the liver is known to induce hyperammonemia and is one of the most common precipitating factors of hepatic encephalopathy. The ammoniagenic potential of an UGI bleed is mainly related to the absorption of haemoglobin protein (3). Classical experiments in dogs with an Eck fistula (4) led to the development of the current hypothesis that encephalopathy induced by a nitrogenous meal is caused by portasystemic shunting of increased amounts of ammonia, produced in the portal drained (PDV) viscera. Half of this ammoniagenesis originates from amino acid metabolism (mainly glutamine) in the mucosa of the small bowel and the other half from bacterial splitting of urea in the colon (5).

Normally, ammonia is detoxified by urea synthesis in the liver, but in patients with cirrhosis of the liver this capacity is diminished, due to liver dysfunction and intra- and extra-hepatic portasystemic shunting $(6,7)$. An alternative, temporary pathway of ammonia detoxification is via glutamine synthesis and in this respect muscle has been hypothesised to be of great importance (8). In addition to the PDV, the kidney has the capacity to produce ammonia. Recently we described the interorgan metabolism of ammonia and related amino acids in patients with cirrhosis of the liver (9) as is summarized in figure 3 of chapter 1. The changes in interorgan ammonia metabolism induced by UGI bleeding in humans are unknown. Therefore, the aim of the present study was to quantify the contribution of the main ammonia producing and consuming organs to whole body ammoniagenesis during an UGI bleed. In a first series of studies we quantified ammonia metabolism of the PDV, liver, kidney and muscle after a simulated UGI bleed in patients with cirrhosis of the liver. This study enabled us to look at the pure metabolic effects of blood protein in the gut, without potential confounding effects of altered haemodynamics, absorption of haem,_effects of blood transfusion and the effects of treatment of the bleed itself. In a subsequent series of studies we quantified ammonia production of the kidneys and the total splanchnic area during an acute UGI bleed in patients with cirrhosis of the liver. 
Patients. Initially, eight, overnight fasted, metabolically stable patients with biopsyproven cirrhosis of the liver who underwent portography to check the patency of their transjugular intrahepatic portasystemic stent-shunt (TIPSS) were studied to evaluate the effects of a simulated UGI bleed (Table 1). Parients were excluded if they had severe ascites, pitting peripheral oedema, hepatic encephalopathy, active alcohol abuse, diabetes, clinically evident cardiovascular disease or renal dysfunction (serum creatinine $>100 \mathrm{mmol} / \mathrm{L}$ or $>88 \mathrm{mg} / \mathrm{dL}$ ), malignancy, pregnancy or shunt dysfunction (portal pressure gradient $>12 \mathrm{mmHg}$ ).

Subsequently, nine patients with cirrhosis of the liver that underwent urgent TIPSS placement for therapy of ongoing active variceal bleeding were studied in part 2 (Table 1). Patients had the first evidence of bleeding 18 hours before (see Table 1), but had been endoscoped a median of 4 hours before the TIPSS, where evidence of active bleeding was shown. At the time of the TIPSS the bleeding had been controlled with a Sengstaken-Blakemore tube. Furthermore, all patients had presence of melaena at the time TIPSS insertion was performed. Patients were excluded if they had diabetes, clinically evident cardiovascular disease, pregnancy or malignancy. The Lothian Ethics Research Committee approved both studies and written informed consent was obtained from each subject, or from their next of kin.

\begin{tabular}{|lll|}
\hline & Simulated bleed & Acute bleed \\
\hline Age (yrs) & $45.4(4)$ & $48.5(3)$ \\
Sex & 2 female, 6 male & 4 female, 5 male \\
Weight (kg) & $83.7(7)$ & $71.2(6)$ \\
Body mass index (kg/m2) & $30.9(2)$ & $23.0(2)$ \\
Aetiology & 7 Alcoholic liver disease & 8 Alcoholic liver disease \\
& 1 Primary biliary cirrhosis & 1 hepatitis C \\
Pugh-Childs score & $6.4(1)$ & $12.4(1)$ \\
Portal pressure gradient (PPG) & $5.4(1)$ & \\
PPG pre-TIPSS (mmHg) & & $19(3)$ \\
PPG post-TIPSS (mmHg) & & $8.9(1)$ \\
Time after bleed (hrs) & & $18.3(6)$ \\
Estimated blood loss (units) & $:$ & $7.9(1)$ \\
Months after TIPSS placement & $30.7(7)$ & \\
Albumin (mmol/L) & $36.1(2)$ & $24.0(1)$ \\
Alanine aminotransferase & $32.0(2)$ & $38.7(6)$ \\
Creatinine & $81(3)$ & $173(6)$ \\
60\% daily protein intake (q) & $46.0(4)$ & \\
\hline
\end{tabular}


Simulation of an upper gastrointestinal bleed. The metabolic consequences of a UGI bleed were simulated by a tailor made solution (Nutricia Cuijk, The Netherland: prod nr 24143) that mimics the composition of the haemoglobin molecule (10) (tabl 2). The solution was freshly prepared and xanthan gum was added (0.1 gram per 10 ml solution) to prevent sedimentation. The amount administered equalled $60 \%$ a daily nitrogen intake estimated using 3-day food history and dietetic consultation (using "Microdiet" computer programme, Salford University, UK). The amino-acii solution was infused continuously over a 4 hour period through the nasogastric tube a a mean rate of $115( \pm 10) \mathrm{ml} / \mathrm{hr}$.

Table 2: Amino acid composition of the simulated bleed (10).

\begin{tabular}{llllll}
\hline Leucine & 99.8 & Glutamate & 33.3 & Aspartate & 41.6 \\
Isoleucine & 0 & Asparagine & 27.7 & Alanine & 99.8 \\
Valine & 85.9 & Glutamine & 11.1 & Cysteine & 8.3 \\
Glycine & 55.4 & Methionine & 8.3 & Serine & 44.4 \\
Tryptophan & 8.3 & Arginine & 16.6 & Phenylalanine & 41.6 \\
Threonine & 44.4 & Tyrosine & 16.6 & Histidine & 52.7 \\
Lysine & 61.0 & Proline & 38.8 & & \\
\hline
\end{tabular}

Expressed as mmol per 100 gram amino acid solution

Insertion of catheters. Studies in patients with a simulated bleed were started between 6.00 and 9.00 A.M. after an overnight fast. The following catheters were inserted after routine portography under direct $\mathrm{x}$-ray screening (9). A naso-gastric tube (Medicinia, UK) was inserted. The right femoral artery was cannulated using an $18 \mathrm{G}$ needle (Vygon leader company, France). A sheath with a sampling port (6F, Cordis company, NL) was inserted through the right femoral vein. A 5F catheter (Terumo, femoral-visceral angiographic catheter, Belgium) was introduced through the sheath and positioned in the right renal vein. A Swan-Ganz catheter (Baxter catheter 131HF7, USA) was modified such that the distance between the distal (infusion) and the proximal (sampling) port 
was $10.5 \mathrm{~cm}$. This catheter was inserted through the $10 \mathrm{~F}$ sheath in the right internal jugular vein (used for TIPSS portography) and the proximal port was positioned in the trunk of the portal vein upstream of the shunt. There were no visual impressions of large portal tributaries close to the proximal trunk during the angiography. Patients were transported to the metabolic research ward after placement of the catheters.

In patients with a real variceal bleed undergoing urgent TIPSS insertion, directly before the TIPSS was inserted, a 5F sampling catheter (Terumo, femoral-visceral angiographic catheter, Belgium) was introduced through the jugular venous sheath (used for the TIPSS procedure) and positioned into a hepatic vein and the right renal vein. Subsequently, we took a blood sample from the portal vein during the TIPSS procedure, immediately after the liver parenchyma was punctured and the portal vein was reached, so before the actual shunt was inserted.

Sampling and measurement of plasma flow. Organ plasma flow was determined using a slight modification of the protocol used by Owen et al (11) as published recently (9). Kidney, leg and portal flow were determined using a primed $(0.56 \mathrm{ml} / \mathrm{kg}$ bw), continuous infusion $(50 \mathrm{ml} / \mathrm{h})$ of para-aminohippuric acid. (PAH, 20\% solution, MSD, NL) and liver plasma flow was determined using a primed (12 mg), continuous infusion (1 $\mathrm{mg} / \mathrm{min}$ ) of indocyanine green (Cardiogreen, Becton-Dickinson Microbiology Systems, USA). Both solutions were infused into a dorsal hand vein. $\mathrm{PAH}$ infusion was started three hours and indocyanine green one hour before the portography to ensure steady state concentrations $(12,13)$. To measure portal flow, PAH infusion was switched from the dorsal hand vein to the distal port of the Swan-Ganz catheter to ensure proper mixing of PAH in the portal vein. Sampling for PAH was performed through the proximal port of the Swan-Ganz catheter (for a detailed description see (14)). Renal plasma flow was determined by measurement of PAH in the arterial and renal venous blood. Plasma flow in a single leg was measured by switching the infusion of PAH from the Swan-Ganz catheter to the femoral artery, and after a stabilising period of 5 minutes, femoral venous blood was sampled for PAH. Portal, renal and leg plasma flow were measured before the simulation of the bleed ( 0 hours) and 2 , 3 , and 4 hours thereafter. Hepatic plasma flow was measured by simultaneous sampling of arterial and hepatic venous blood prior to and 4 hours after the simulated bleed when the position of the catheters were checked using fluoroscopy. Samples were taken from the middle or right hepatic vein that did not contain the TIPSS to prevent mixing of hepatic and portal venous blood.

In the patients group with a real variceal bleed renal and hepatic plasma flow was measured using the same PAH and indocyanine green protocol.

Analyses. Blood samples were collected on ice for the measurement of ammonia, amino-acids and acid base status at the times of measurement of plasma flow. Blood electrolyte concentrations were directly measured using a CO-oximeter (IL 282, 
Instrumentation Laboratories, USA). Blood samples were processed as described previ ously (15) and stored at $-80^{\circ} \mathrm{C}$. Ammonia, urea, PAH and indocyanine green wer determined spectrophotometrically (15), amino acids were determined using HPLC (16).

Calculations. Plasma flow rate ( $\mathrm{mL} / \mathrm{kg}$ bw/min) of the portal vein, liver, kidneys anc one leg were calculated using formulae based on the method of indicator dilution anc Fick's principle $(11,15,17,18)$. The PAH determined blood flow was converted tc plasma flow using the haematocrit. Substrate fluxes across organs are callculated as the venous-arterial concentration difference times plasma flow.

During the simulated UGI bleed, leg and renal plasma flow and substrate fluxes represent metabolism of a single leg and two kidneys. To calculate substrate fluxes across the liver we had to estimate the amount of portal-systemic shunting through the TIPSS, since this could not be determined (9). In a previous study, Walser (19) showed that the shunted fraction of portal blood via a TIPSS is on average $93 \%$. In the calculations we used the maximum fraction that can be shunted (100\%) as the upper bound: liver flux is not influenced by flux of substrates via the portal vein:

\section{- Assuming 100\% portal-systemic shunting.}

Liver flux $=$ hepatic venous minus arterial concentration difference $*$ liver plasma flow

Furthermore, we used an imaginary boundary of $70 \%$ as the lowest reasonable fraction of shunting through the TIPSS. In that case, $70 \%$ of the portal flux is shunted via the TIPSS and $30 \%$ is delivered to the liver:

\section{- Assuming $70 \%$ portal-systemic shunting.}

Liver flux = (liver plasma flow * hepatic venous concentration) $-[$ (liver plasma flow $-0.3 *$ portal plasma flow $)$ arterial concentration $)+(0.3 *$ portal plasma flow) * portal venous concentration)]

We have chosen upper and lower bounds of portacaval shunting to present the reader with a sort of confidence interval for the true liver flux.

Subsequently, the substrate flux over the cotal splanchnic area was calculated as follows:

-assuming $100 \%$ portal-systemic shunting: Splanchnic flux = PDV flux + Liver flux

—assuming 70\% portal-systemic shunting. Splanchnic flux $=(0.7 *$ PDV flux $)$ + Liver flux.

Positive fluxes reflect substrate release; negative fluxes uptake. Branched chain amino acids (BCAA) are calculated as the sum of valine, leucine and isoleucine. 
In the patients with a real variceal bleed undergoing TIPSS insertion, renal flux was calculated as detailed above. Furthermore, we calculated total splanchnic area flux as liver plasma flow *(hepatic venous concentration - arterial concentration). We could not determine the amount of portal blood that escaped liver clearance via extra-hepatic portasystemic collaterals. Therefore, ammonia flux from the total splanchnic area was estimated by multiplying the portal venous minus arterial concentration difference by various shunt flow estimates (see Figure 3).

Statistics. Statistical analysis was performed using the Statisticall Package for the Social Sciences, version 9.0 for Windows (SPSS, Chicago, Ill., USA). Wilcoxon's signed rank test was used to test for differences from zero. To test for differences in time oneway ANOVA with Bonferroni post hoc analysis was used for the arterial, PDV, renal and femoral data, and Wilcoxon's signed rank test for the hepatic data. The Mann-Whitney $U$ test was used to test for differences in ammonia production by the kidneys and the PDV, and ammonia uptake by the muscle and liver Data are expressed as mean and SEM. 


\section{RESULTS}

Simulated UGI bleed. All patients studied during a simulated UGI bleed had patent TIPSS shunts indicated by a portal pressure gradient of $<12 \mathrm{mmHg}$ and catheters were inserted without complication. A mean of 46 (4) grams of amino acids was administered, representing the haemoglobin content of $300 \mathrm{ml}$ whole blood. There were no clinically observable changes in mental state following the administration of the amino acid solution in the patients. One patient did not tolerate the nasogastric tube and the study was stopped after 2,5 hours.

Flow measurements. The plasma flow across organs did not change between $0,2,3$ and 4 hours (data in $\mathrm{mL} / \mathrm{kg}$ bw/min at $\mathrm{t}=0$ hours and the mean of $\mathrm{t}=3$ and 4 hours respectively: PDV flow: 9.3 (2.0) versus $11.6(3.0)$; flow single leg: $7.3(1.6)$ versus 9.5 (2.2); flow, two kidneys: $7.8(1.7)$ versus $9.0(2.4)$ and liver flow: 6.7 (1.5) versus 7.9 (2.6)). Apart from the hepatic plasma flow, the portal, leg and renal flows are comparable with reference values of normal subjects found in the literature: liver 20 $\mathrm{ml} / \mathrm{kg} / \mathrm{min}$, portal $12 \mathrm{ml} / \mathrm{kg} / \mathrm{min}$, leg $8 \mathrm{ml} / \mathrm{kg} / \mathrm{min}$, renal $8 \mathrm{ml} / \mathrm{kg} / \mathrm{min}(20-22)$. The lack of a statistical significant change in portal and liver flow after the simulated bleed is in agreement with the observation of blunted postprandial splanchnic flow in patients with cirrhosis of the liver (23-25). The hepatic extraction of ICG in the present study was $0,219 \pm 0,057$ at $\mathrm{t}=0$ hours and $0,170 \pm 0,079$ at $\mathrm{t}=4$ hours. These extractions were higher than the ICG extractions measured in patients with decompensated chronic and acute liver disease (26), supporting the notion that our study population consisted of metabolically stable patients with cirrhosis of the liver. Blood flow determinations using ICG in patients with cirthosis of the liver has been shown valid if the hepatic ICG extraction is above $0,10(26)$.

Baseline metabolism. In the postabsorptive state, ammonia was produced by the PDV and taken up by both liver and muscle in similar quantities (see figure 1). Ammonia production by the kidneys was not significantly different from zero $(\mathrm{p}=0.09)$. In the PDV, glutamine was taken up in stoichiometric relationship with the production of ammonia $(r=0.89, p<0.001)$. The PDV also produced alanine and branched chain amino acids (table 3 and 4). Both the liver and the kidneys consumed glutamine, the liver produced urea, which was taken up by the kidneys and subsequently released into the urine.

Metabolic consequences of simulated UGI bleed. The simulated UGI bleed resulted in a significant increase in arterial ammonia concentration (see figure $1, p=0.002$ ), but no change in arterial urea concentrations ( $p=0.44$ ) was observed. Ammonia production by the PDV did not change significantly during the simulated bleed ( $p=0.624$, 
see Figure 1) nor by the total splanchnic area (data in $\mathrm{nmol} / \mathrm{kg}$ bw/min at 0 and 4 hours respectively: 255 (156) versus 819 (429), $\mathrm{p}=0.25$ for the $100 \%$ shunting estimate; and $-23(79)$ versus $175(214), p=0.25$ for the $70 \%$ shuncing estimate). There was a significant increase in alanine and BCAA release by the PDV. Ammonia production by the kidneys increased 6-fold during the experiment $(p=0.006)$. At the end of the study, ammonia production by the kidneys equalled ammonia production by the PDV and by both estimates of total splanchnic area. Renal uptake of alanine increased during the simulated bleed $(p=0.004)$ but no differences were observed in renal fluxes of glutamine $(p=0.28)$ and BCAA $(p=0.17)$. The uptake of ammonia by the liver did not change during the simulated UGI bleed ( $\mathrm{p}=0.84$ for the $100 \%$ shunting estimate and $\mathrm{p}=0.29$ for the $70 \%$ shunting estimare) nor was there enhanced urea production by the liver $(p=0.23$ for both the $100 \%$ shuning estimate and $p=0.30$ for the $70 \%$ shunting estimate). The removal of ammonia by the leg, representing leg skeletal muscle metabolism, increased during the simulated bleed $(p=0.002)$, and there was a tendency towards enhanced glutamine efflux across muscle $(p=0.08)$. At the end of the study, muscle of one leg removed a similar amount of ammonia from the systemic circulation as the liver. The dynamics of interorgan ammonia metabolism in the post-absorptive and stimulated state are illustrated in Figure 1.

There was no change in arterial hydrogen ion concentration or $\mathrm{pH}\left(\mathrm{H}^{+} .41 .1\right.$ $\mathrm{nmol} / \mathrm{L}$ or $\mathrm{pH} 7.386$ at 0 hours and $\mathrm{H}^{+} 40.9 \mathrm{nmol} / \mathrm{L}$ or $\mathrm{pH} 7.338$ at 3 hours) and bicarbonate concentration $(22.5 \mathrm{mmol} / \mathrm{L}$ at 0 hours and $22.0 \mathrm{mmol} / \mathrm{L}$ at 3 hours) or in their organ fluxes (data not shown).

Metabolic consequences of acute variceal bleed. The following plasma flows were found in the parients with an acute variceal bleed undergoing TIPSS insertion: liver plasma flow of $6.8(1.7) \mathrm{mL} / \mathrm{kg}$ bw/min and renal plasma flow of $11.0(1.5) \mathrm{mL} / \mathrm{kg}$ $\mathrm{bw} / \mathrm{min}$. The arterial substrate concentrations were: ammonia $164(17) \mathrm{mM}$, urea 9905 (2127) $\mathrm{mM}$, glutamine $1090(170) \mathrm{mM}$ and alanine $479(81) \mathrm{mM}$. The ammonia concentration in the portal vein $(194(23) \mathrm{mM}$ ) was higher than in the hepatic vein (152 (16) $\mathrm{mM}, \mathrm{p}=0.028$ ), and was of the same magnitude as the renal venous concentration (219 (32) $\mathrm{mM}, \mathrm{p}=0.866$ ).

The kidneys produced a significant amount of ammonia $(572(184) \mathrm{nmol} / \mathrm{kg}$ $\mathrm{bw} / \mathrm{min}$ ) during the variceal bleed, whereas no ammonia was released from the splanchnic area $(-121(87) \mathrm{nmol} / \mathrm{kg}$ bw/ min, see Figure 2). The clinical relevance of this finding is emphasized in the calculared model of figure 3 , which shows that the ammonia production from the total splanchnic area would not even come close to the renal ammonia production if we assume that the portasystemic shunt flow is $1000 \mathrm{ml}$ per minute. The kidneys consumed glutamine $(-722(175) \mathrm{nmol} / \mathrm{kg} \mathrm{bw} / \mathrm{min})$ and produced alanine (186 (99) nmol/ $\mathrm{kg}$ bw/min) during the acute bleed. 

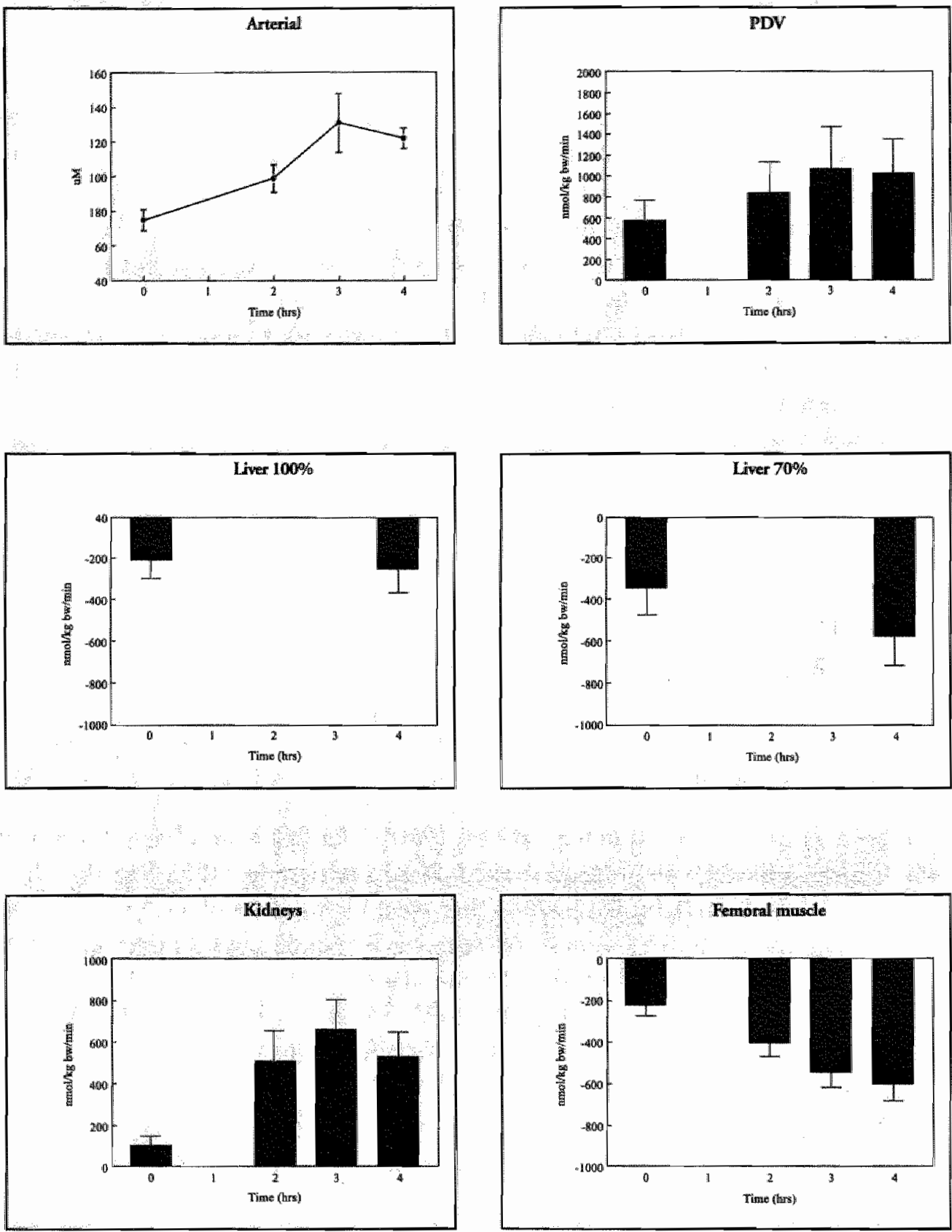

FIGURE 1: Ammonia: arterial concentrations (upper panel left; $\mu \mathrm{M}$ ) and fluxes across organs (nmol/ $\mathrm{kg}$ bw/min) during a simulated UGI bleed in parients with cirrhosis of the liver: Portal drained viscera (upper panel right), Liver 100\% shunring estimate (left panel middle), Liver $70 \%$ shunting estimate (right panel middle), Kidneys (wwo kidneys, lower panel left) and Muscle (two legs, lower panel right). Data are means \pm SEM. Positive fluxes indicate release, negative fluxes indicate uptake. 


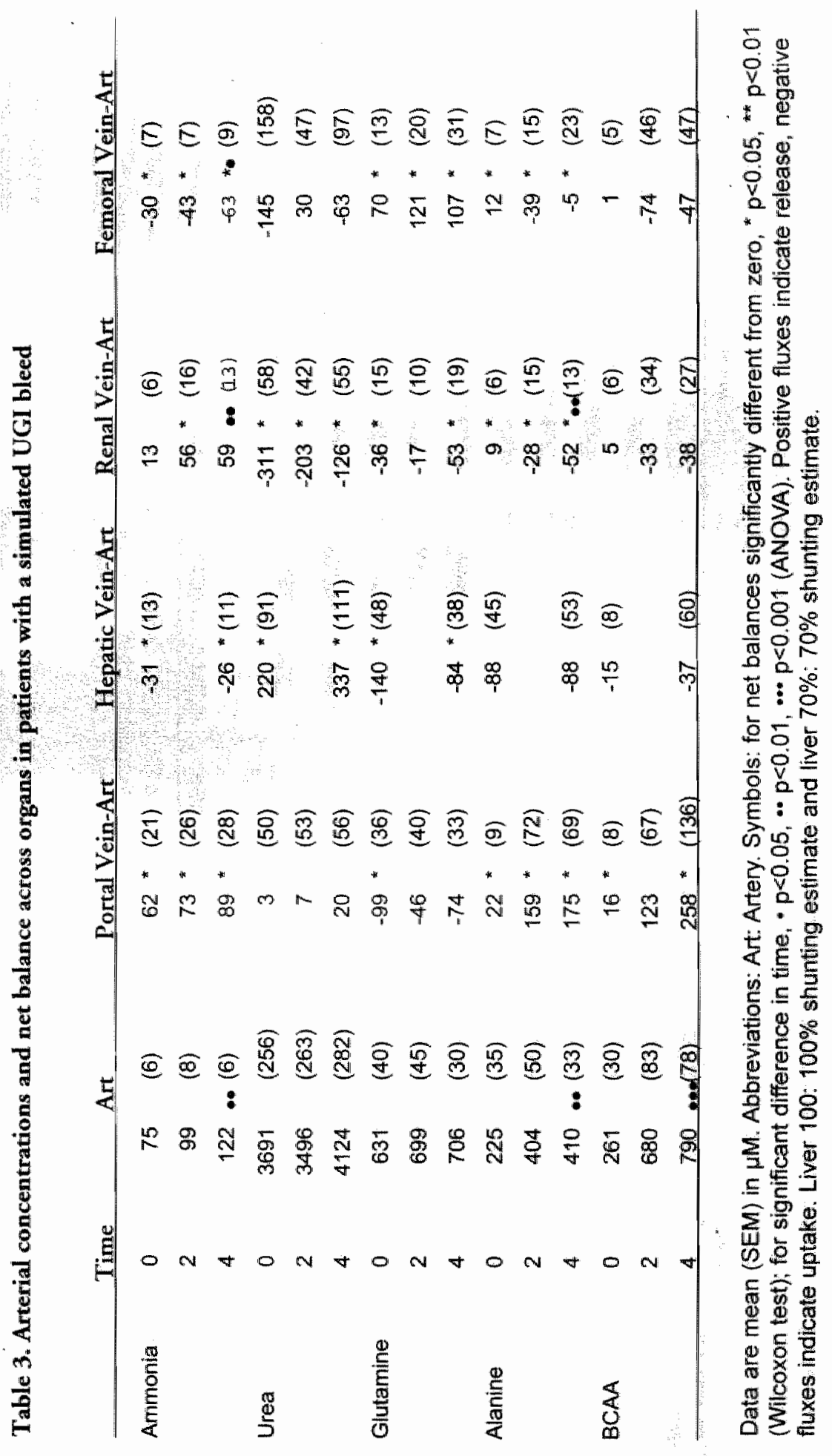




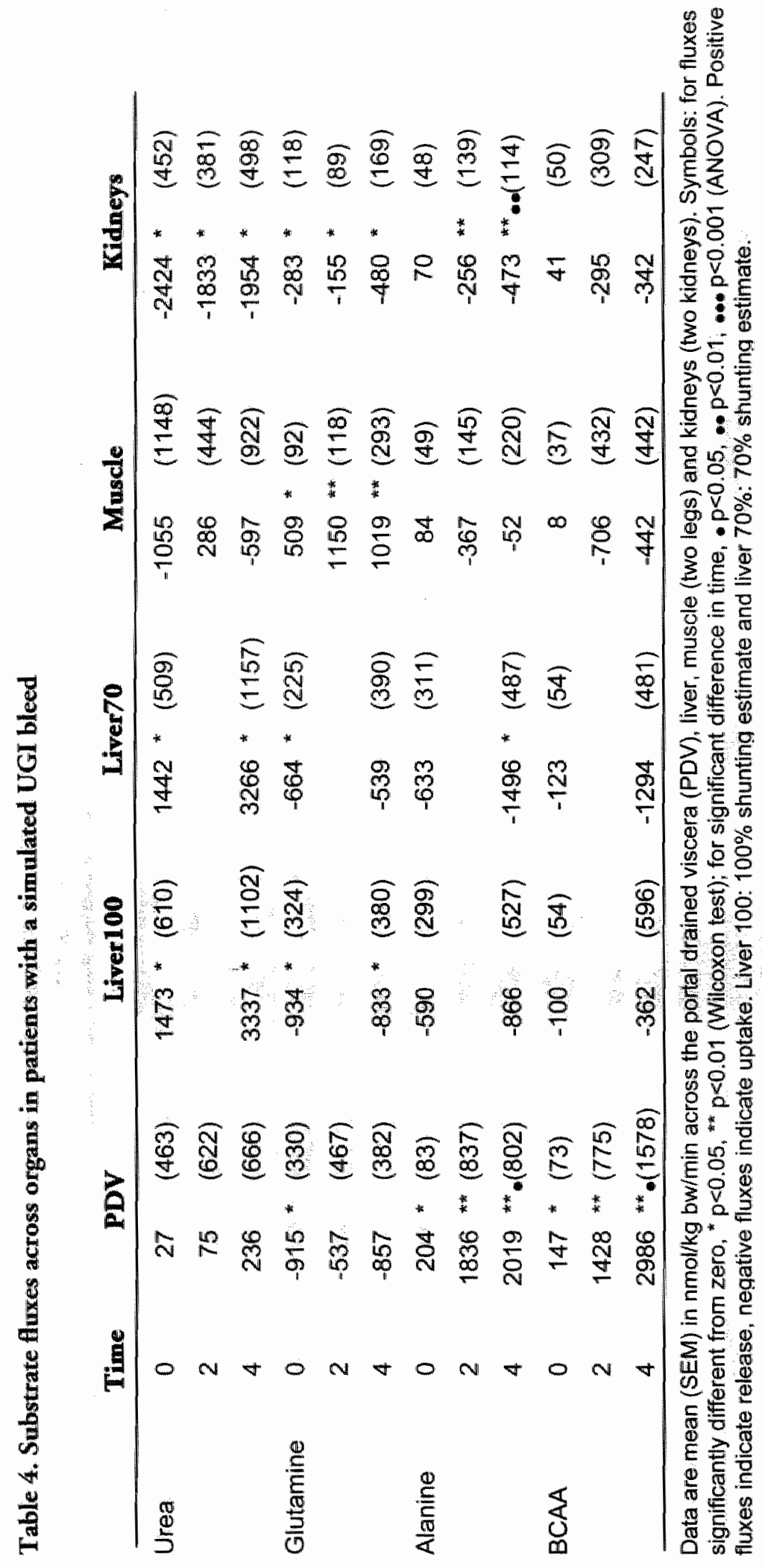




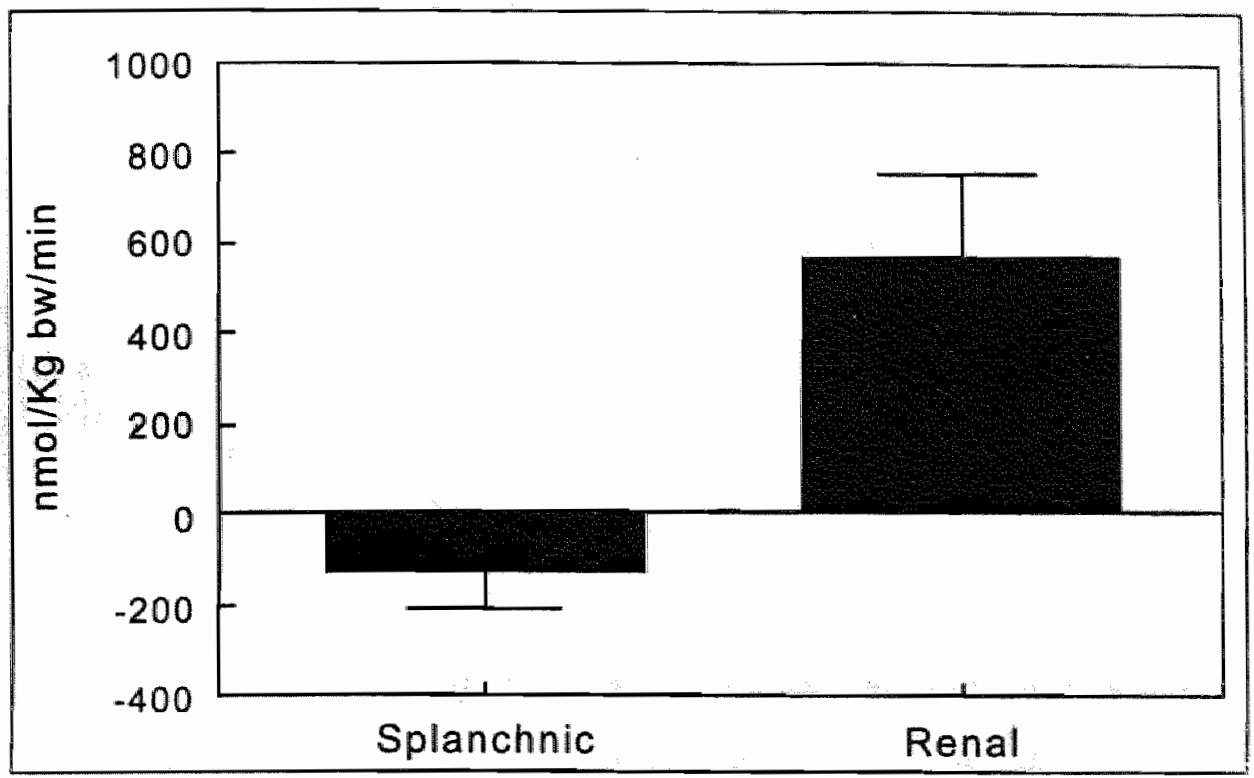

Figure 2 (ABOVE). Ammonia fluxes across the splanchnic area and the kidneys (two kidneys) $(\mathrm{nmol} / \mathrm{kg} \mathrm{bw} / \mathrm{min})$ during a real wariceal bleed in patients with cirrhosis of the liver. Data are means \pm SEM. Positive fluxes indicate release, negative fluxes indicate uptake.

FIGURE 3 (BELOw). This figure shows the theoretical relation between total splanchnic ammonia production versus the absolute flow through the extrahepatic portasystemic collaterals) in patients with an acute variceal bleed undergoing urgent TIPSS insertion. Ammonia production was calculated using the measured ammonia concentrations in the artery and, the hepatic and portal veins immediately after the portal vein was punctured during the TIPSS insertion. The model suggests that the total flow via shunts (both intraand extra-hepatic) would have to increase to above $1000 \mathrm{ml} / \mathrm{min}$ to match renal ammonia production.

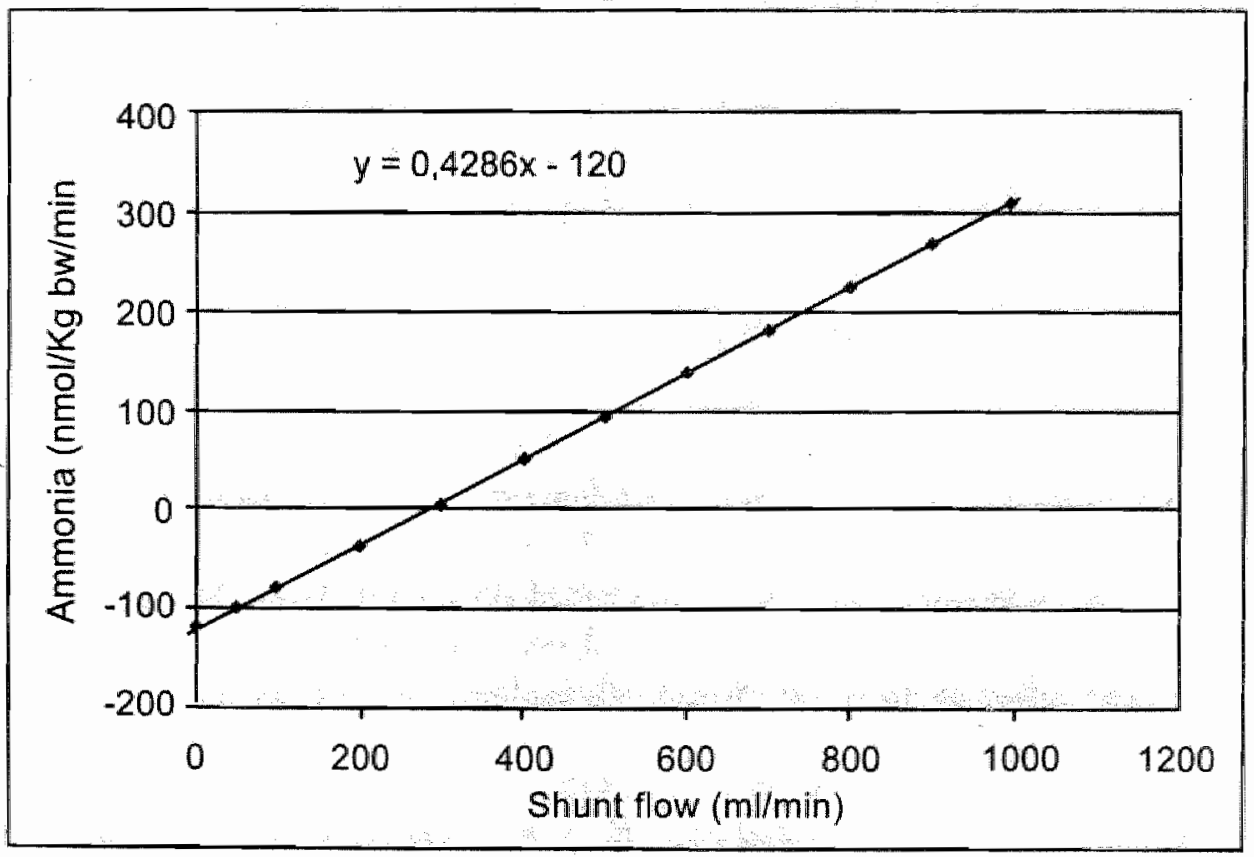




\section{DISCUSSION}

This study shows that hyperammonemia resulting from a (simulated) UGI bleed in patients with cirrhosis of the liver is caused by enhanced renal ammonia production. The apparent increase in ammonia release by the splanchnic area was of similar magnitude to the increase in renal ammoniagenesis, suggesting that the kidneys are at least as important as the splanchnic area in explaining postbleeding hyperammonemia. In patients with cirrhosis, the liver is unable to increase ammonia detoxification following a simulated UGI bleed, making muscle the major ammonia removing organ.

\section{Postabsorptive ammonia and amino acid metabolism}

Ammonia is toxic at elevated levels (1) and must be detoxified by urea synthesis in the liver $(6,7)$. Alternatively, ammonia can be removed by glutamine synthesis via amidation of glutamate to form glutamine. Consistent with previous observations (27), postabsorptive arterial ammonia concentrations were higher than those reported for healthy volunteers. In keeping with previous animal studies (28), the findings in the cirrhotic patients in which the UGI bleed was simulated suggest that post-absorptive hyperammonemia during chronic liver insufficiency is not caused by increased intestinal glutamine uptake and ammonia release. Instead, inadequate ammonia detoxification by the liver, due to reduced capacity of the liver to synthesise urea and glutamine $(6,7)$, and systemic shunting (intra- and extra hepatic portacaval shunting) of ammonia that is produced in the PDV seem to play a crucial role in determining post-absorptive arterial ammonia concentrations. This observation could have been confounded by the fact that we studied patients with cirrhosis of the liver and a TIPSS in situ, which have marked shunting of portal blood via the TIPSS. Unfortunately, the degree to which this confounds the results is difficult to estimate, but it is likely that TIPSS will probably underestimate the contribution of the liver in ammonia removal. In patients without a TIPSS the efflux of ammonia from the splanchnic area will probably be less, which will hypothetically make the role of the kidney in these patients even more important as was shown in the patients with a real variceal bleed.

\section{Metabolic consequences of a (simulated) UGI bleed in patients with cirrhosis of the liver}

The results of the present studies are against the prevailing hypothesis that ammonia produced after an UGI bleed originates solely from the PDV. Traditionally, it has been thought that ammonia is produced in the PDV partly by colonic bacteria that split urea and partly from the breakdown of amino acids by the intestinal wall (5). Ammonia produced by the PDV was stoichiometrically related with uptake of glutamine, reproducing observations made in vitro (29) and in vivo with experimental animals $(5,30)$. At 
present, clinical ammonia-lowering therapeutic strategies aim at these intestinal production sites, e.g. antibiotics, whole bowel lavage and non-absorbable disaccharides. In the present study we were unable to detect a significant increase in ammonia production by the PDV or the splanchnic area estimates during the simulated bleed. This was probably the result of the variation of the data and represents a type 2 error. It is therefore likely that an increase in ammonia production by the PDV and the splanchnic area would have been found if more patients were included in the study. However, even if the change in ammonia release by the splanchnic area using the $100 \%$ shunting estimate would reach statistical significance, the results of the present study would suggest that the kidneys are at least of equal importance to the splanchnic area in explaining hyperammonemia that follows a simulated UGI bleed. Moreover, the amount of ammonia that is produced by the kidneys during the acute bleed would not be equalled by the splanchnic area even if the portasystemic shunt flow was estimated to be 1000 $\mathrm{mL} / \mathrm{min}$. Recently, Debatin and colleagues (31) estimated the amount of portasystemic shunting prior to TIPSS insertion in patients with cirrhosis of the liver. They found that the flow in the azygos vein, a surrogate marker of portasystemic shunting, was about $50 \%$ of portal venous flow (425 versus $1000 \mathrm{~mL} / \mathrm{min}$ ).

\section{Renal ammonia metabolism}

Post absorptive ammonia production by the kidneys in metabolically stable patients with cirrhosis of the liver prior to the administration of the simulated UGI bleer did not reach statistical significance in the present study. Under normal post-absorotive conditions, about $70 \%$ of total renal ammonia production is released into the renal vein and the remainder excreted with urine (see figure 3, chapter 1) (32). Puring hyperammonemia and portal systemic shunting this is reversed to $70 \%$ excretion in urine and $30 \%$ release into the systemic circulation, making the kidney an organ of net ammonia removal during hyperammonemia $(32,33)$. Total renal ammonia production and the percentage of ammonia released into the renal vein are known to be acutely influenced by changes in acid-base status, ammonia load and amino acid administration $(33,34)$. During acute ammonia or amino acid loading, the percentage of total renal ammonia production that is released into the urine is increased. Therefore, although we did not measure urinary ammonia excretion, the results of our study suggest that total renal ammonia production increases markedly during a simulated UGI bleed.

Hyperammonemia following a simulated UGI bleed was mainly caused by enhanced renal ammonia production and proved to be a good reflection of observed ammonia metabolism during an acute UGI bleed where similar quantities of ammonia were released into the circulation by the kidney. The model to simulate the metabolic consequences of an acute UGI bleed was chosen since it enabled us to study interorgan ammonia trafficking caused by the absorption of blood protein without possible con- 
founding effects of altered haemodynamics, absorption of haeme etc. Furthermore, it enabled us to study the dynamics of whole body ammoniagenesis prior to and following a (simulated) UGI bleed, which is impossible to study during an acute variceal bleed.

The kidneys extracted a significant amount of glutamine during the acute bleed, in agreement with previous studies that suggested that glutamine was the principle precursor for renal ammoniagenesis (35-37). Glutamine is metabolised within the proximal convoluted tubule (38) and both nitrogen moieties of glutamine can serve renal ammoniagenesis (reviewed in (39)). Renal glutamine consumption was also observed at baseline in the post-absorptive patients of the simulated bleed study. However, during the simulated bleed renal glutamine uptake did not change significantly $(p=0.284)$. In contrast, renal alanine uptake increased significantly $(p=0.002)$, suggesting that alanine was the substrate for enhanced renal ammoniagenesis in these post-absorptive patients with stable cirrhosis and a TIPSS. These findings are in agreement with recent observations in multi-catheterised pigs with normal liver function (40). The mechanism of enhanced ammonia production in the kidney from alanine is not clear, but could well be linked to an increased role of renal gluconeogenesis in these patients. The possible biochemical pathway could be via transamination of alanine to a-ketoglutarate to form glutamate, which then could release one molecule of ammonia. After splitting of the amino group from alanine, the resulting pyruvate could be transformed into lactate or glucose (41), and released into the renal vein or pyruvate may be oxidised. It can be hypothesised from our data that enhanced alanine uptake by the kidneys is a resultant of increased alanine release by the PDV and impaired alanine uptake by the liver. This results in increased arterial alanine concentrations, which regulate alanine uptake by the kidneys (42). In healthy humans, alanine is taken up by the liver to produce glucose, since this pathway seems to be hampered in patients with cirrhosis of the liver it could well be that enhanced alanine uptake by the kidneys serves to increase the total gluconeogenesis in these patients.

\section{Ammonia removal during a (simulated) UGI bleed}

The results of the simulated bleed study clearly show that the cirrhotic liver is unable to increase ammonia uptake or urea production following a simulated bleed. This is in agreement with results obtained by Fabbri et al (43) who reported that urea synthesis in cirrhotic patients is refractory to stimulation by alanine, a known strong stimulus for urea synthesis. In patients with cirrhosis, muscle is the major ammonia removing organ in the body. This emphasises the importance of muscle mass for patients with cirrhosis of the liver and the danger of muscle wasting during liver disease. The increased uptake of ammonia by muscle without any increase in glutamine production suggests an increase in muscle glutamine pool, which would serve as a temporary ammonia buffer (8). 


\section{Conclusions}

The results of the present study show that enhanced renall ammonia production has an important role in the hyperammonemia resulting from both a simulated UGI bleed and an actual UGI bleed in patients with cirrhosis of the liver. Ammonia production from the total splanchnic area was not observed in the patients with an acute UGI bleed and did not significantly increase during the simulated UGI bleed, probably due to the large variance of the PDV measurements. At the end of the simulated bleed, renal ammonia production equalled total splanchnic area ammonia production in the cirrhotic parients which had a TIPSS in situ. Based on these findings, we propose that the classical hypothesis concerning the aetiology of post-bleeding hyperammonemia should be altered into a new concept in which renal ammoniagenesis also has an important role. During hyperammonemia in these patients, muscle is the major site of anmonia removal. New therapeutic strategies that aim to diminish ammonia production after an UGI bleed in patients with cirrhosis should alter our current strategy and also aim at renal ammonia production. 


\section{REFERENCES}

1. Butterworth RF. The neurobiology of hepatic encephalopathy. Semin Liver Dis 1996;16:235-244.

2. Basile $A S_{3}$ Jones EA Ammonia and GABA-ergic neurotransmission: interrelated factors in the pathogenesis of hepatic encephalopathy. Hepatology 1997;25:1303-1305.

3. Bessman $A N$, Hawkins $R$. The rellative effects of enterically administered plasma and packed cells on circularing blood ammonia. Gastroenterology 1963;45:368-373.

4. Nencki M, Pawlow JP, Zaleski J. Ueber den Ammoniakgehalt des Blutes und der Organe und die Harnstoffbildung bei den Saugethieren. Arch exp Pathol Pharmakol 1896;37:26-51.

5. Weber FL, Jr., Veach GL. The importance of the small intestine in gut ammonium production in the fasting dog. Gastroenterology 1979;77:235-240.

6. Meijer AJ, Lamers WH, Chamuleau RA. Nitrogen metabolism and ornithine cycle function. Physiol Rev 1990;70:701-748.

7. Atkinson DE. Functional roles of urea synthesis in vertebrates. Physiol Zoology 1992;65*243-267.

8. Lockwood AH, McDonald JM, Reiman RE, Gelbard AS, Laughlin JS, DuffyTE, Plum F. The dynamics of ammonia metabolism in man. Effects of liver disease and hyperammonemia. J Clin Invest 1979;63:449-460.

9. Olde Damink SWM, Jalan R, Redhead DN, Hayes PC, Deutz NEP, Soeters PB. Interorgan ammonia and amino acid metabolism in metabolically stable patients with cirrhosis and a TIPSS. Hepatology 2002;36:1163-1171.

10. Hill RJ, Koningsberg W. The structure of human hemoglobin. J Biol Chem 1962;237:3151-3156.

11. Owen OE, Reichle FA, Mozzoli MA, Kreulen T, Patel MS, Elfenbein IB, Golsorkhi M, et al. Hepatic, gur, and renal substrate flux rates in patients with hepatic cirrhosis. J Clin Invest $1981 ; 68: 240-252$.

12. Uusaro A, Ruokonen E, Takala J. Estimation of splanchnic blood flow by the Fick principle in man and problems in the use of indocyanine green. Cardiovasc Res 1995;30:106-112.

13. Selkurt EE. Measurement of renal blood flow. Meth Med Res 1948;1:191-199.

14. Granger DN, Bulkdey GB. In. Measurement of blood flow. Applications to the splanchnic circulation. Baltimore/London: Williams\& Wilkins, 1981 p 1-507.

15. Dejong CHC, Kampman MT, Deutz NTE, Soeters PB. Altered glutamine metabolism in rat portal drained viscera and hindquarter daring hyperammonemia. Gastroenterology $1992 ; 102: 936-$ 948.

16. van Eijk HM, Rooyakkers DR, Soeters PB, Deutz NEP. Determination of amino acid isotope enrichment using liquid chromatography-mass spectrometry. Anal Biochem 1999;271:8-17.

17. Owen EE, Tyor MP, Flanagan JF, Berry JN. The kidney as a source of blood ammonia in patients with liver disease the effect of acetazolamide. J Clin Invest 1960;39:288-294.

18. Ten Have GAM, Bost MCF, Suyk-Wierts JCAW, wan den Bogaard AEJM, Deutz NEP. Simultaneous measurement of merabolic flux in portally-drained viscera, liver, spleen, kidney and hindquarter in the conscious pig. Lab Anim 1996;30:347-358.

19. Walser EM, Harris VM, Harman JT, Park HM, Siddiqui AR. Quantification of intrahepatic portosystemic shunting after placement of a transjugular intrahepatic portosystemic shunt. IVasc Interv Radiol 1996;7:263-267.

20. Carter P, Welbourne TC. Glutamate transport asymmetry in renal glutamine metabolism. Am J Physiol 1998;274:E877-884.

21. Gelfand RA, Glickman MG, Castellino P, Louard RJ, DeFronzo RA. Measurement of L-[114Cilleucine kinerics in splanchnic and leg tissues in humans. Effect of amino acid infusion. Diabetes 1988;37:1365-1372. 
22. Gaiani S, Bolondi L, Li Bassi S, Santi V, Zironi G, Barbara L. Effect of meal on portal hemody namics in healthy humans and in patients with chronic liver disease. Hepatology 1989:9:815-819.

23. de Vries PJ, de Hooge P; Hoekstra JB, van Hattum J. Blunted postprandial reaction of portal venous flow in chronic liver disease, assessed with duplex Doppler: significance for prognosis. J Hepatol 1994;21:966-973.

24. Ludwig D, Schwarting $K$, Korbel CM, Bruning A, Schiefer B, Stange EF. The postprandial portal flow is related to the severity of portal hypertension and liver cirthosis. J Hepatol 1998;28:631 638.

25. Miyake M, Harada M, Taoka $Y$, Nishitani H. Evaluation by MR imaging of the velocity and volume of pre- and postprandial portal blood flow in the presence or absence of liver cirrhosis. Nippon Igaku Hoshasen Gakkai Zasshi 1997;57:244-248.

26. Clemmesen JO, Tygstrup $N$, Ott P. Hepatic plasma flow estimated according to Ficks principle in patients with hepatic encephalopathy: evaluation of indocyanine green and D-sorbitol as test substances. Hepatology 1998;27:666-673.

27. Cooper AJ, Plum F. Biochemistry and physiology of brain ammonia. Physiol Rev 1987;67:440519.

28. Dejong CHC, Deutz NEP, Soeters PB. Intestinal glutamine and ammonia metabolism during. chronic hyperammonaemia induced by liver insufficiency: Gut 1993;34:1112-1119.

29. Windmueller HG, Spaeth AE. Uprake and metabolism of plasma glutamine by the small intestine. J Biol Chem 1974;249:5070-5079.

30. Deutz NEP, Reijwen PL, Athanasas G, Soeters PB. Post-operative changes in heparic, intestinal, splenic and muscle fluxes of amino acids and ammonia in pigs. Clin Sci 1992;83:607-614.

31. Debatin JF, Zahner B, Meyenberger C. Romanowrki B, Schopke W, Marincek B, Fuchs WA . Azygos blood flow: phase contrast quantitation in volunteers and patients with portal hypertension pre- and postintrahepatic shunt placement. Hepatology 1996;24:1109-1115.

32. Dejong CHC, Deutz NEP, Soeters PB. Renal ammonia and glutamine metabolism during liver insufficiency-induced hyperammonemia in the rat. J Clin Invest 1993;92:2834-2840.

33. Owen EE, Johnson JH, Tyor MP. The effect of induced hyperammonemia on renal ammonia metabolism. J Clin Invest 1961;40:215-221.

34. Nathans D, Fahey JL, Ship AG. Sites of origin and removal of blood ammonia formed during glycine infusion; Effect of L-arginine. I Lab Clin Med. 1958;51:123-128.

35. Pitts RF, DeHiaas J, Klein ]. Relation of renal amino and amide nitrogen extraction to ammonia production. Am J Physiol 1963;204:187-191.

36. Owen EE, Robinson RR. Amino acid extraction and ammonia metabolism by the human kidney during prolonged administration of ammonium chloride. J Clin Invest 1963;42:263-276.

37. Tannen RL. Ammonia metabolism. Am J Physiol 1978;235:F265-F277.

38. Good DW, Burg MB. Ammonia production by individual segments of the rat nephron. I Clin Invest 1984:73:602-610.

39. Halperin ML, Kamel KS, Ethier JH, Stinebaugh BJ, Jungas RL. Büochemistry and Physiology of ammonium excretion. In: The Kidney: Physiology and pathophysiology. Ed: Seldin DW, Giebisch G. Raven Press, Lrd, New York 1992.

40. Weiters CF, Deutz NEP, Dejong CHC, Soeters PB. Enhanced renal vein ammonia efflux after a protein meal in the pig. I Hepatol 1999;31:489-496.

41. Newsholme EA, Leech AR. Biochemistry for the Medical sciences. 1 ed. New York: John Willey $\&$ Sons; 1983.

42. Pitts RF, Stone WI. Renal metabolism of alanine J Clin Invest. 1967;46:530-538.

43. Fabbri A, Marchesini $G$, Bianchi $G$, Bugianesi $E$, Bortoluzzi L, Zoli M, Pisi E. Unresponsiveness of hepatic nitrogen metabolism to glucagon infusion in patients with cirrhosis: dependence on liver cell failure. Hepatology 1993;18:28-35. 

CHAPTER 3A

THE KIDNEY PLAYS A MAJOR ROLE IN AMMONIA HOMEOSTASIS AFTER PORTASYSTEMIC SHUNTING IN PATIENTS WITH CIRRHOSIS

Steven WM Olde Damink ${ }^{1,2}$, Cornelis HC Dejong ${ }^{1,3}$, Nicolaas EP Deutz, Doris N Redhead ${ }^{4}$, Peter C Hayes ${ }^{2}$, Peter B Soeters ${ }^{1}$, Rajiv Jalan ${ }^{2,5}$

IDepartment of Surgery, Maastricht University, Maastricht, The Netherlands, ${ }^{2}$ Liver Unit, ${ }^{3}$ Department of Surgery and ${ }^{4}$ Radiology, Royal Infirmary of Edinburgh, Edinburgh, Scotland, UK and 5Liver Failure Group, Institute of Hepatology, Royal Free and University College Medical School, University College London. 


\section{INTRODUCTION}

About $15-20 \%$ of patients with cirthosis of the liver develop hepatic encephalopathy after insertion of a transjugular intrahepatic portasystemic stent shunt (TIPSS). This has been attributed to the induction of hyperammonemia by an acute increase of portasystemic shunting of portal drained viscera generated ammonia (1). Occasionally, TIPSS insertion results in fatal increases in intracranial pressure in patients with cirrhosis of the liver (2), which may be induced by hyperammonemia (3). Present ammonia lowering therapies are predominantly directed at intestinal ammonia production, but therapeutic results are modest. We have recently shown that the kidneys play a major role in the hyperammonemia that follows upper gastrointestinal haemorrhage (4). Interventions such as altering the intravascular volume status or acid/base balance can alter renal ammonia metabolism acutely (5), making the kidney a potential target organ for therapy. In experimental animals it has been shown that the kidney is able to switch from net ammonia production into net ammonia removal from the body following hyperammonemia induced by portacaval shunting (6). The present study was performed to evaluate whether this metabolic adaptation to portacaval shunting was present in patients with cirrhosis of the liver. For this purpose we measured arterial ammonia and amino acid concentrations, and splanchnic, renal and leg ammonia and amino acid fluxes, in patients that underwent TIPSS insertion. 


\section{METHODS}

Studies were undertaken with the approval of the Lothian Research Ethics Committee and after obtaining written informed consent from each patient or next of kin. Patients were eligible to participate in the study if they had cirrhosis of the liver and needed treatment with TIPSS for the management of variceal bleeding. Patients were excluded if they had diabetes, clinically evident cardiovascular disease, malignancy or pregnancy.

Patients. Thirteen patients were studied following admission to the Royal Infirmary of Edinburgh with an acute variceal bleed that was initially controlled with endoscopic variceal band ligation (Table 1). Patients were studied after an overnight fast.

Table 1: Patient characteristics (mean and SEM).

\begin{tabular}{|l|l|}
\hline Age (yrs) & $54.4(3.3)$ \\
\hline Sex & 6 female, 7 male \\
\hline Weight (kg) & $70.4(4.4)$ \\
\hline Body mass index (kg/m2) & $26.0(3.3)$ \\
\hline Aetiology & $\begin{array}{l}9 \text { Alcoholic liver disease, 1 PSC, 2 autoimmune } \\
\text { hepatitis, 1 hepatitis C }\end{array}$ \\
\hline Pugh score & $9.9(0.9)$ \\
\hline Time after bleed (hrs) & $30.8(5.6)$ \\
\hline Packed cells infused (units) & $7.3(1.0)$ \\
\hline Heart rate (min) & $79(5)$ \\
\hline Mean arterial pressure (mmHg) & $95(4)$ \\
\hline Urine output (mL/hr) & $95(19)$ \\
\hline $\begin{array}{l}\text { Portal pressure gradient (mmHg) } \\
\text { pre-TIPSS } \\
\text { post-TIPSS }\end{array}$ & $17.8(1.5)$ \\
\hline Prothrombin time (sec) & $8.9(0.8)$ \\
\hline Albumin (g/L) & $15.9(1.3)$ \\
\hline ALAT (IU/L) & $25.5(1.6)$ \\
\hline Creatinine (umol/L) & $60.2(8.4)$ \\
\hline
\end{tabular}


TIPSS procedure and catheter placement. Before the TIPSS procedure was started, the femoral artery was cannulated using a 18 gauge needle (Vygon leader company, Ecoven, France). A single interventional radiologist (DNR) performed all the TIPSS procedures. Procedures were performed in an angiography suite under sedation. For TIPSS insertion, the right internal jugular vein was punctured and a $10 \mathrm{~F}$ sheath (William-Cook, Bjaeverskov, Denmark) was introduced over a guide wire into the inferior vena cava. The right or middle hepatic vein was selected using a stiff hydrophilic guide wire (Terumo, Hatagaya, Tokyo, Japan). Hereafter, a 16 gauge curved transjugular needle (William-Cook, Bjaeverskov, Denmark) was introduced over the guidle wire into the heparic vein and used to guide a fine stylet anteriomedially towards a branch of the portal vein. Aspiration of blood was followed by delineation of the portal system by injection of contrast medium. Hereafter, an Amplatz wire (Medi-Tech, Boston scientific corporation, Watertown, Massachussetts, USA) and an angioplasty balloon catheter were used to dilate the parenchymal tract before the stent $(10 \mathrm{~mm}$ Wallstent, Schneider, Bulach, Switzerland) was inserted. Pressure measurements and portography were performed at the time of portal vein catheterisation and after shunt creation to ensure adequate shunting (portal pressure gradient below $12 \mathrm{mmHg}$ ).

Sampling. Directly prior to and 1 hour after TIPSS insertion, a 5F catheter (Terumo, femoral-visceral angiographic catheter, Leuven, Belgium) was introduced through the sheath in the right internal jugular vein to enable blood sampling in the right renal vein, a hepatic vein and the portal vein. The portal vein sample was taken directly after puncturing the portal vein, before the TIPSS was inserted (pre-TIPSS) and 1 hour after the TIPSS was inserted (post-TIPSS). Blood was collected from the middle or right hepatic vein that did not contain the TIPSS to prevent mixing of hepatic and portal venous blood. In the last 4 patients additional measurements were made to obtain preliminary information on muscle ammonia handling, and ammonia excretion by the kidneys in the last 3 . In these patients blood samples were also collected from the right femoral vein, urine samples were collected from an indwelling urine catheter and urine output was measured.

Blood flow measurements. To enable determination of renal and liver plasma flow, patients received a primed $(0.56 \mathrm{ml} / \mathrm{kg} \mathrm{bw})$, continuous infusion $(50 \mathrm{ml} / \mathrm{h})$ of paraaminohippuric acid (PAH, Aminohippurate Sodium 20\% solution, Merck Sharp and Dohme, Haarlem, The Netherlands) and a primed $(12 \mathrm{mg})$, continuous infusion (1 $\mathrm{mg} / \mathrm{min}$ ) of indocyanine green (Cardiogreen, Becton Dickinson Microbiology Systems, Cockeysville, USA) into a dorsal hand vein. PAH infusion was started at least three hours and indocyanine green at least one hour before TIPSS insertion to ensure steady state concentrations $(7,8)$. Renal and liver plasma flow measurements were made before the TIPSS was inserted (pre-TIPSS) and 1 hour after TIPSS (post-TIPSS) by simultaneous sampling of arterial, renal and hepatic venous blood for determination of PAH and ICG respectively. 
Sampling and analytical procedures. Blood samples were collected on ice for the measurement of ammonia, amino-acids and acid base status at the times of measurement of blood flow. Urine samples were collected on ice and acid $(6 \mathrm{~N} \mathrm{HCl})$ to prevent evaporation of ammonia (6). Blood electrolyte concentrations were directly measured using a CO-oximeter (IL 282, Instrumentation Laboratories, Boston, USA). Blood and urine samples were processed as described previously (9) and stored at $-80^{\circ} \mathrm{C}$. Plasma and urine ammonia, plasma urea, $\mathrm{PAH}$ and indocyanine green were determined spectrophotometrically as detailed previously $(9,10)$, plasma amino acids were determined using HPLC (11).

Calculations. Renal and liver plasma flow were calculated according to Fick's principle, using standard equations (9). Renal ammonia release or uptake was calculated as the venous minus arterial concentration difference times plasma flow. The hepatic venous minus arterial concentration difference multiplied by the liver blood flow reflects the substrate release or uptake across the non-shunted splanchnic region. Positive values reflect substrate release and negative fluxes substrate uptake. In the present study we were unable to measure the degree of portasystemic shunting and therefore we were unable to determine the ammonia, that was released from the splanchnic region via portasystemic shunts (like the TIPSS) and thereby escapes clearance by the liver. This 'shunted' splanchnic region release was estimated using portasystemic flow data from the literature and the measured portal venous minus arterial ammonia differences of the present study (detailed in the discussion section).

Total renal ammonia production (urinary excretion + renal vein release) could only be calculated in those three patients in whom urinary ammonia excretion was calculated. Hepatic extraction of ICG was calculated as: the arterial minus hepatic venous concentration divided by the arterial concentration.

BCAA represents the sum of the branched chain amino acids (leucine, valine and isoleucine), TEAA represents the sum of the essential amino acids, TNEAA represents the sum of the non-essential amino acids and TAA represents the sum of all amino acids measured.

Statistics. Data are expressed as mean and SEM. Statistical analysis was performed using the Statistical Package for the Social Sciences, version 10.0 for Windows (SPSS, Chicago, Ill., USA). Non-parametric statistics were used: the Wilcoxon's signed rank test for differences between pte- and post-TIPSS values and to test for differences from zero. The Mann-Whitney $\mathrm{U}$ test was used to test for differences between renal and nonshunted splanchnic region ammonia handling. Significance was considered present at $\mathrm{p}<0.05$. 


\section{RESULTS}

Patients. All patients included in the study underwent successful TIPSS insertion. The mean portal pressure gradient before TIPSS was $17.8( \pm 1.5) \mathrm{mmHg}$ and this was successfully reduced to $8.9( \pm 0.8) \mathrm{mmHg}$. At the time of TIPSS insertion the patients were haemodynamically stable (see Table 1).

Plasma flow. Hepatic and renal plasma flow did not change significantly following TIPSS insertion (liver: $8.0 \pm 2.0$ versus $6.5 \pm 1.0 \mathrm{ml} / \mathrm{kg}$ bw/min; renal: $11.5 \pm 0.9$ versus $12.1 \pm 2.3 \mathrm{ml} / \mathrm{kg} \mathrm{bw} / \mathrm{min})$. The mean hepatic extraction of ICG did not change after the TIPSS placement $(0.26 \pm 0.05$ pre-TIPSS and $0.25 \pm 0.04$ post-TIPSS $)$ and was sufficiently high to allow a valid determination of plasma flow across the liver (12).

Arterial data. Table 2 shows the concentrations of ammonia, urea and amino acids in arterial plasma, and the venous minus arterial concentration differences across the liver, kidneys and portal bed during the TIPSS procedure. Interestingly, TIPSS insertion did not change arterial ammonia concentrations, but resulted in a significant increase in arterial alanine, TNEAA and TAA concentrations.

Renal metabolism. Ammonia, urea and amino acid release or uptake across the kidneys and the non-shunted splanchnic region are shown in Table 3. Prior to TIPSS, the kidneys produced significant amounts of ammonia, but TIPSS insertion reduced renal ammonia release into the circulation $(p<0.05)$ to values that were not significantly different from zero. Interestingly, the uptake of the ammoniagenic amino acids glutamine and glycine were not influenced by TIPSS insertion. Furthermore, there was a significant uptake of urea and citrulline by the kidneys that was not altered by TIPSS. The kidneys released significant amounts of glutamate, alanine, arginine and tyrosine which was not influenced by TIPSS. After TIPSS the net efflux of methionine, lysine, BCAA, TEAA, TNEAA and TAA became significant.

Table 4 shows the total renal ammonia production of the three patients in whom urine samples were collected and the amount of ammonia released into the systemic circulation and excreted into the urine. Total renal ammonia production decreased markedly in all whereas urinary ammonia excretion increased in all patients after TIPSS insertion. Urinary ammonia excretion exceeded renal release of ammonia to the circulation in only one patient, turning the kidney into a net ammonia removing organ. Interestingly, this was the patient with the highest arterial ammonia concentration preTIPSS. After TIPSS insertion, arterial ammonia concentration decreased slightly in this patient, which was associated with a reduction in total renal ammonia production and renal glutamine uptake. 
Portal drained viscera metabolism. Ammonia concentration in the portal vein was significantly higher than the arterial concentration, and the resulting venous-minusarterial concentration difference did not change significantly after the TIPSS insertion. There was a significant negative net balance of glutamine across the portal drained viscera, which did not change significantly following TIPSS.

Splanchnic metabolism. There was no significant release or uptake of ammonia by the non-shunted splanchnic region before and after the TIPSS insertion. Apart from a net release of urea, all other substrates were taken up by the non-shunted splanchnic region. Ammonia release into the systemic circulation by the kidneys was significantly greater than that by the non-shunted splanchnic region prior to TIPSS. After TIPSS insertion, systemic ammonia release by the kidneys was reduced to values that were not significantly different from zero and similar to that in the non-shunted splanchnic region.

Muscle metabolism. Prior to TIPSS, ammonia was taken up by leg muscle in all the 4 patients studied. The femoral venous-minus-arterial ammonia concentration difference increased after TIPSS insertion in all 4 patients $(\mathrm{p}=0.065)$. The lack of statistical significance reflects the low statistical power of this sub-group. Glutamine was released by skeletal muscle in all 4 patients before TIPSS and did not change after TIPSS insertion. Of all other amino acids determined the direction of change after TIPSS was not similar for the 4 patients, and therefore a trend towards uptake or release could not be shown.

Acid-Base balance. Insertion of TIPSS did not change hydrogen ion concentration in the artery $(39 \pm 2$ versus $38 \pm 2 \mathrm{nmol} / \mathrm{L})$, the hepatic vein $(39 \pm 1$ versus $40 \pm 1 \mathrm{nmol} / \mathrm{L})$ or the renal vein $(38 \pm 1$ versus $39 \pm 2 \mathrm{nmol} / \mathrm{L}$ ), which were all within the normal physiological range. 


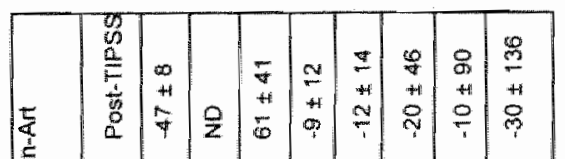

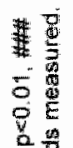

業

E

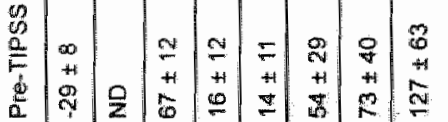

w. 응

b

*

迹

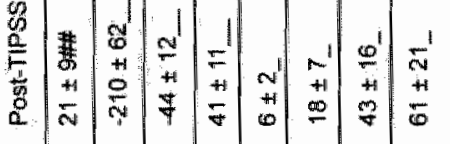

梙

a

咅

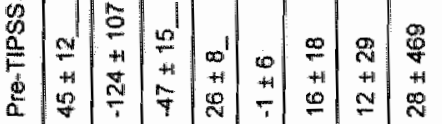

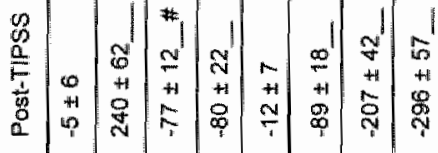

5

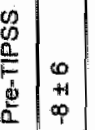

热

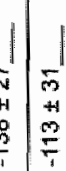

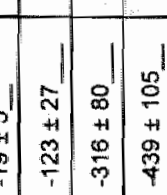

들

돈

동다

क.

$8 \frac{1}{2}$

\&

誉

훌

a

भุ:

욤

g $E$

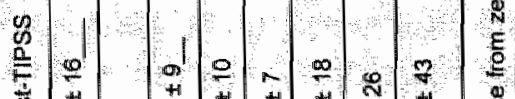

E 3

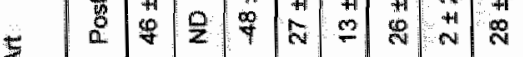

क्.

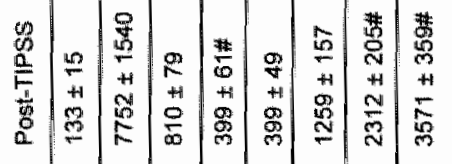

需 
Table 3. Splanchnic and renal release or uptake of ammonia and amino acids before and after TIPSS placement.

\begin{tabular}{|c|c|c|c|c|c|c|c|c|}
\hline \multirow[b]{3}{*}{ Ammonia } & \multicolumn{6}{|c|}{ Non-shunted splanchnic region } & \multicolumn{2}{|c|}{ Renal } \\
\hline & \multicolumn{2}{|c|}{ Pre-TIPSS } & \multicolumn{2}{|c|}{ Post-TIPSS } & \multicolumn{2}{|c|}{ Pre-TIPSS } & \multicolumn{2}{|c|}{ POSt-TIPSS } \\
\hline & -70 & \pm 50 & -34 & \pm 43 & 491 & \pm 118 & $18 \%$ & $\pm 104 \#$ \\
\hline Urea & 2938 & \pm 2161 & 2111 & \pm 740 & -1723 & \pm 1067 & -2638 & \pm 719 \\
\hline Glutamine & -1290 & \pm 478 & -566 & \pm 122 & -519 & \pm 166 & -487 & \pm 175 \\
\hline Alanine & -1098 & \pm 561 & -583 & \pm 179 & 259 & \pm 77 & 466 & \pm 111 \\
\hline Glycine & -426 & \pm 190 & -258 & \pm 72 & -206 & \pm 67 & -191 & \pm 40 \\
\hline Glutamate & 277 & \pm 60 & 249 & \pm 60 & 94 & \pm 42 & 193 & \pm 84 \\
\hline Histidine & -150 & \pm 44 & -81 & \pm 20 & 8 & \pm 19 & -9 & \pm 8 \\
\hline Citrulline & 21 & \pm 16 & -5 & \pm 13 & -132 & \pm 27 & -143 & \pm 20 \\
\hline Taurine & 1 & \pm 13 & 14 & \pm 9 & -43 & \pm 27 & -9 & \pm 11 \\
\hline Arginine & -238 & \pm 107 & -158 & \pm 44 & 125 & \pm 35 & 145 & \pm 20 \\
\hline Tyrosine & -135 & \pm 34 & -74 & \pm 15 & 53 & \pm 25 & 84 & \pm 15 \\
\hline Valine & -67 & \pm 28 & -8 & \pm 31 & 7 & \pm 28 & 28 & \pm 7 \\
\hline Methionine & -69 & \pm 36 & -40 & \pm 12 & 38 & \pm 19 & 13 & \pm 6 \\
\hline Isoleucine & -23 & \pm 9 & -8 & \pm 13 & -15 & \pm 14 & 16 & \pm 11 \\
\hline Phenylalanine & -159 & \pm 48 & -80 & \pm 16 & -26 & \pm 19 & -25 & \pm 15 \\
\hline Tryptophan & -26 & $\pm 10_{-}$ & -10 & \pm 13 & 0 & \pm 17 & 0 & \pm 43 \\
\hline Leucine & -77 & \pm 29 & -46 & \pm 23 & 0 & \pm 17 & 19 & \pm 16 \\
\hline Ornithine & 153 & \pm 82 & 67 & $\pm 46 \#$ & -3 & \pm 25 & -21 & \pm 27 \\
\hline Lysine & -276 & \pm 118 & .174 & \pm 36 & 19 & \pm 15 & 124 & \pm 63 \\
\hline BCAA & -167 & \pm 63 & -62 & \pm 65 & -11 & \pm 68 & 64 & $\pm 30_{-}$ \\
\hline TEAA & -1133 & \pm 410 & -624 & \pm 140 & 169 & \pm 217 & 206 & \pm 63 \\
\hline TNEAA & -3152 & \pm 1407 & -1547 & \pm 378 & 75 & \pm 319 & 459 & \pm 140 \\
\hline TAA & -4284 & \pm 1810 & -2171 & \pm 480 & 244 & \pm 523 & 665 & \pm 185 \\
\hline
\end{tabular}

Data are mean (SEM) in nmol/kg bw/min. Symbols: for statistically significant difference from zero: $P<0.05$, $\quad p<0.01$, $p<0.001$ and significance between pre- and post-TIPSS fluxes: : $\# p<0.05$, \# $p<0.01$ (both Wilcoxon test). BCCA: sum of the branched chain amino acids, TEAA: sum of the essential amino acids, TNEAA: sum of the non-essential amino acids, TAA: sum of all amino acids measured. Positive value indicates release, negative value indicates uptake. 


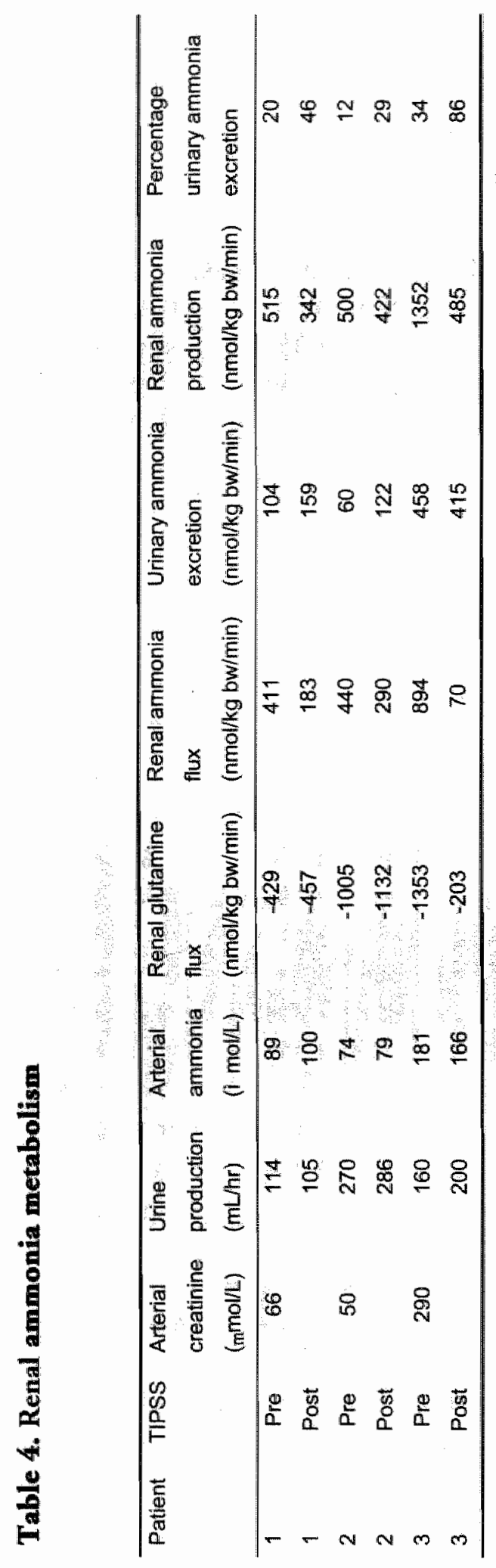




\section{DISCUSSION}

The present study confirms our recent observation (4) that the kidney plays a major role in the hyperammonemia that is observed in patients with cirrhosis of the liver. As we have shown previously, systemic ammonia release from the kidneys exceeded that from the non-shunted splanchnic region. We also confirm that the main ammonia removing organs in this situation are the liver and muscle. The most important observation of our study was the demonstration that renal ammonia release was acutely diminished after TIPSS insertion, despite the marked increase in shunting of portal drained viscera produced ammonia after TIPSS. Our findings also suggest increased uptake of ammonia by the leg (muscle) after TIPSS, whereas the non-shunted splanchnic region uptake of ammonia did not change. Our data suggest that the reduced renal ammonia production and increased muscle ammonia uptake are responsible for the observed maintenance of arterial ammonia concentration after TIPSS insertion in patients with cirrhosis. These results again underline the important role of the kidneys in ammonia metabolism.

TIPSS insertion reduced renal ammonia release into the circulation to values that were not significantly different from zero. Recently, we showed that renal ammonia production is negligible in metabolically stable patients with cirrhosis of the liver who had a TIPSS inserted on average 25 months previously (13). This suggests that the observed instantaneous adaptation of the kidneys to TIPSS induced alterations in ammonia metabolism is not a transient phenomenon and is probably still active 2 years after TIPSS insertion. The hyperammonemia observed in patients with a previously inserted TIPSS was slightly less than that observed in the present study $(95 \mathrm{mM}$, versus $45 \mathrm{mM}$ for healthy controls (14)) and was also related to the shunting of ammonia production in the portal drained viscera (13).

Creation of a portacaval shunt in rats resulted in a reduction in ammonia production by the kidneys despite markedly elevated arterial ammonia concentrations $(6,15)$. Renal glutamine consumption was reduced concomitantly with reduced ammonia production. In addition, $70 \%$ of total ammonia produced in the kidney was excreted into urine and $30 \%$ was released into the renal vein, resulting in a negative renal venous minus arterial ammonia concentration difference (6), making the kidney an organ of net ammonia removal during hyperammonemia. This observation could not be fully confirmed by our study in the three patients in whom we measured urinary ammonia excretion. Although total renal ammonia production decreased and urinary ammonia excretion increased in all patients, to even $86 \%$ of total renal ammonia production excreted via the urine in one patient, the net renal ammonia flux remained positive in all three. In the whole group, TIPSS insertion reduced renal ammonia release into the circulation to values that were not significantly different from zero. 
TIPSS insertion was not associated with significant differences in the uptake or release of the ammoniagenic amino acids, glutamine and glycine (16) by the kidneys, despite a reduction in systemic ammonia release. This abservation suggests unaltered renal ammoniagenesis. If renal ammoniagenesis remains unchanged but the systemic release of ammonia is reduced, urinary ammonia excretion must be increased (as was observed in all 3 patients in whom urine samples were available). This issue needs further confirmation in future studies.

In the present study, the change in renal ammonia metabolism was not related to changes in acid-base homeostasis. Induction of hyperammonemia in healthy volunteers by intravenous administration of ammonium lactate was associated with a prompt decrease in renal vein ammonia release and with net uptake of ammonia from the circulation by the kidneys (17). Acidosis induces enhanced renal glutamine extraction and increases ammonia release into the urine at the expense of urea excretion, whereas total urinary nitrogen excretion remains constant $(18,19)$. During acidosis the fraction of ammonia excreted in the urine rises to $70 \%$, but the exact underlying mechanism is unknown.

Renal ammonia excretion into the urine cannot be explained simply as $\mathrm{pH}$ dependent (the 'diffusion-trapping' system), but several other mechanisms (e.g. tubular urine flow, several ion exchangers and the ammonia countercurrent system) appear to be more important than $\mathrm{pH}$ in the complex regulation of urinary ammonia excretion $(16,20)$. To be excreted in urine, ammonia must be synthesized by proximal tubular cells, secreted into the proximal tubular fluid, reabsorbed by the medullary thick ascending limb, to be accumulated in the medullary interstitium, and finally secreted in medullary collecting ducts. Each step of this renal pathway is highly regulated and, in addition to acute events, mediated by peptide hormones, like angiotensin II (see for review (21)). The most important step is the absorption of ammonia by the medullary thick ascending limb (21), which occurs by active transcellular transport (60-70\%) and by passive paracellular diffusion (ref in 22). Current data suggest that the majority of the active transport is via the apical $\mathrm{Na}^{+}-\mathrm{K}^{+}-2 \mathrm{Cl}^{-}$cotransporter $(50-65 \%)$ and the $\mathrm{K}^{+} / \mathrm{NH}_{4}{ }^{+}\left(\mathrm{H}^{+}\right)$antiport system (21). Interestingly, the activity of the apical $\mathrm{Na}^{+}-\mathrm{K}^{+}$ $2 \mathrm{Cl}^{-}$cotransporter is sensitive to furosemide and bumetanide, which can eliminate total ammonia absorption by the medullary thick ascending limb (22). This could be an explanation for the often observed diuretic induced hepatic encephalopathy in patients with cirrhosis of the liver. Furthermore, the apical $\mathrm{Na}^{+}-\mathrm{K}^{+}-2 \mathrm{Cl}^{-}$cotransporter and the $\mathrm{K}^{+} / \mathrm{NH}_{4}{ }^{+}\left(\mathrm{H}^{+}\right)$antiport system can be regulated by angiotensin II via activation of protein kinase $\mathrm{C}$ (23), and via production of 20-hydroxyeicosatetraenoic acid which in turn inhibits the $\mathrm{Na}^{+}-\mathrm{K}^{+}-2 \mathrm{Cl}^{-}$cotransporter via unknown mechanisms (21).

Our paper does not allow conclusions about the molecular mechanism for the observed switch in renal ammonia handling. We propose two possible mechanisms. First, TIPSS insertion could lead to a transient increase in ammonia delivery to the kidneys and the proximale tubule (detailed in the following paragraph), which increases 
ammonia absorption by the loop of Henle and could therefore increase ammonia excretion via the urine (24-26). Second, as we have shown recently, acute changes in the wolume status of patients with cirrhosis produced an increase in urinary excretion of ammonia and a decrease in plasma ammonia concentration (5) which was closely related to decreased plasma angiotensin II concentrations. As detailed above, decreased angiotensin II levels result in increased ammonia absorption by the medullary thick ascending limb. There are no data available on the angiotensin II levels in patients undergoing acute TIPSS insertion. Patients with advanced cirrhosis depict classical haemodynamic characteristics of splanchnic vasodilation and consequent activation of the renin-aldosterone-angiotensin axis. The creation of a portasystemic shunt increases pre-load acutely resulting in haemodynamic and neurohumoral changes which may be similar to those observed following acute volume expansion described above (27). This hypothesis needs further study.

In the present study we were unable to measure the degree of portasystemic shunting and have therefore presented substrate handling across the non-shunted splanchnic region. However, ammonia release via the portasystemic shunts can be estimated using flow data from the literature and the measured portal venous minus arterial ammonia differences of the present study. Azygos venous blood flow is an accepted measure of extra-hepatic shunt flow and is reduced immediately after TIPSS insertion (from 450 $\mathrm{ml} / \mathrm{min}$ pre-TIPSS to $300 \mathrm{ml} / \mathrm{min}$ post-TIPSS $(28,29))$. In a study by Lotterer and colleagues, shunting of portal blood via the TIPSS was shown to be approximately $1400 \mathrm{ml} / \mathrm{min}$ immediately after TIPSS insertion (29). The measured liver plasma flow in their study was similar to our findings $(6 \mathrm{ml} / \mathrm{kg} \mathrm{bw} / \mathrm{min})$. In figure 1 we display a pre- and post-TIPSS model of estimated portasystemic ammonia production versus a wide range of shunt flows. Using the pre-TIPSS portasystemic shunting estimate of $450 \mathrm{ml} / \mathrm{min}$, the portasystemic ammonia production would be about $300 \mathrm{nmol} / \mathrm{kg}$ $\mathrm{bw} / \mathrm{min}$ which is lower than the observed pre-TIPSS renal ammonia release $(500$ $\mathrm{nmol} / \mathrm{kg} \mathrm{bw} / \mathrm{min}$ ). These data are similar to the data that we published recently (4). After TIPSS insertion, the estimated portasystemic ammonia production would increase to about $1100 \mathrm{nmol} / \mathrm{kg} \mathrm{bw} / \mathrm{min}$ (using an estimated azygos flow of $300 \mathrm{ml} / \mathrm{min}$ and an estimated TIPSS flow of $1400 \mathrm{ml} / \mathrm{min}$ ). Interestingly, TIPSS insertion did not change arterial ammonia levels in the present study. Our data therefore support the hypothesis that the increased release of ammonia into the circulation through enhanced shunting of portal drained blood after TIPSS does not influence arterial ammonia levels because of diminished renal ammonia release and probably increased muscle ammonia uptake.

In conclusion, the present study shows that the kidneys play a very important role in the instantaneous adaptation to portasystemic shunting in patients with cirrhosis by reducing ammonia efflux into the circulation. In view of the fact that this adaptation appears to be long-lasting (4), attention to the role of the kidneys in ammonia homeostasis may provide new treatment options for hyperammonemia. 


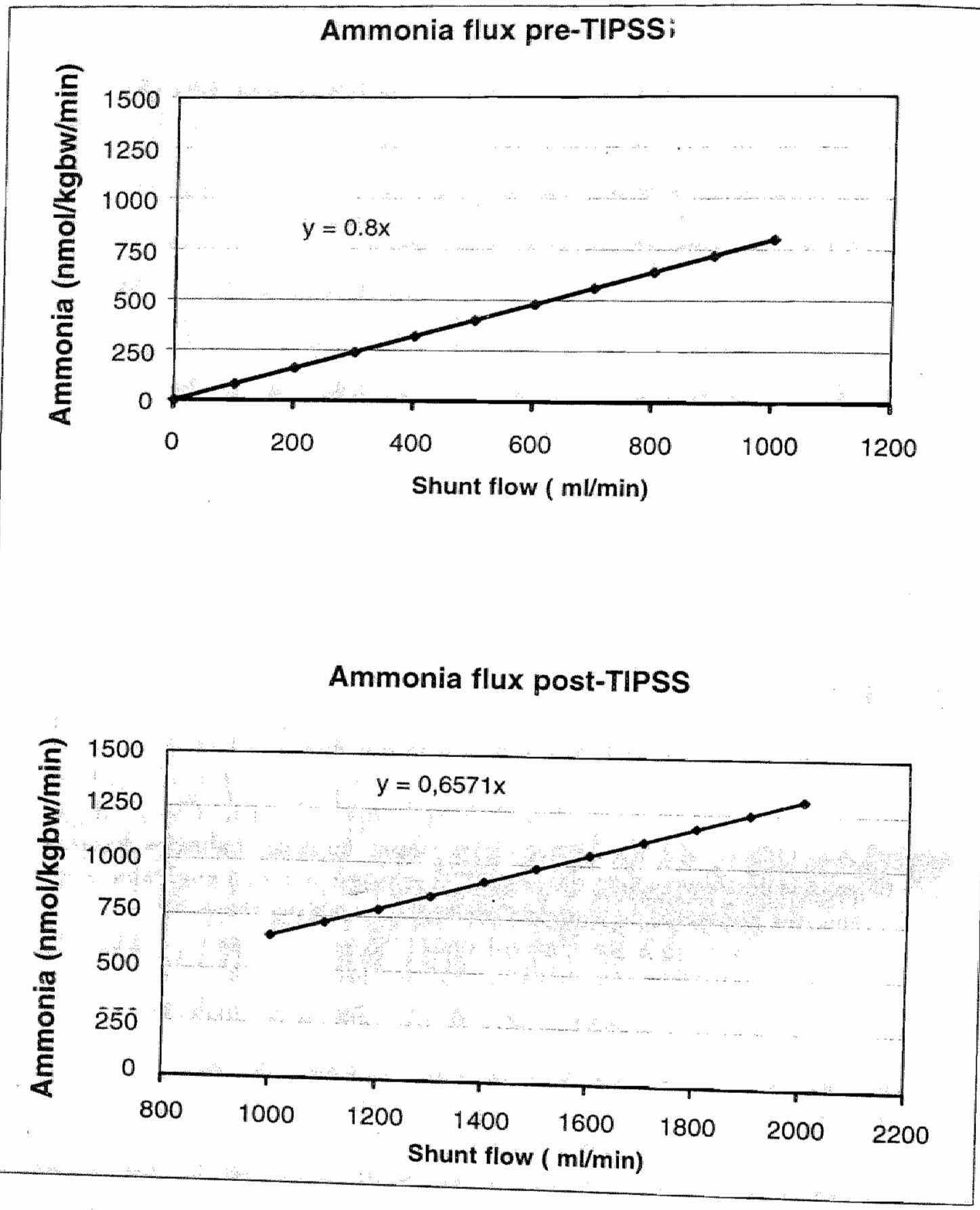

FIGURE 1: Estimated ammonia flux ( $\mathrm{nmol} / \mathrm{kg}$ bw $/ \mathrm{min}$ ) via portasystemic shunts before and after TIPSS
derived from the measured blood flow through portasystemic shunts. Literature ammonia concentration differences multiplied by $\mathrm{ml} / \mathrm{min}$ and $1700 \mathrm{ml} / \mathrm{min}$, respectively (post-TIPSS: values of pre- and post-TIPSS shunt flow are 450 cussion) $(28,29)$ ). 


\section{REFERENCES}

1. Jalan $\mathbb{R}$, Hayes PC. Hepatic encephalopathy and ascites. Lancet 1997;350:1309-15.

2. Jalan $R$, Dabos K, Redhead DN, Lee A, Hayes PC. Elevation of incractanial pressure following transjugular intrahepatic portosystemic stent-shunt for variceal haemorrhage. $\int \mathrm{He}$ patol 1997;27:928-33.

3. Clemmesen JO, Larsen FS, Kondrup J, Hansen BA, Ott P. Cerebral herniation in patients with acute liver failure is correlated with arterial ammonia concentration. Hepatology 1999;29:648-53.

4. Olde Damink SWM, Jalan R, Deutz NEP, Redhead DN, Dejong CHC, Hynds P, er al. The kidney plays a major role in the hyperammonemia seen after simulated or actual $\mathrm{GI}$ bleeding in parients with cirrhosis. Hepatology 2003;37:1277-1285.

5. Jallan R, Kapoor D. Enhanced renal ammonia excretion following volume expansion in patients with well compensated cirrhosis of the liver. Gut 2003;52:1041-5.

6. Dejong CHC, Deutz NEP, Soeters PB. Renal ammonia and glutamine metabolism during liver insufficiency-induced hyperammonemia in the rat. J Clin Invest $1993 ; 92 * 2834-2840$.

7. Uusaro A, Ruokonen E, Takala J. Estimation of splanchnic blood flow by the Fick principle in man and problems in the use of indocyanine green. Cardiovasc Res 1995;30:106-12.

8. Selkurt EE. Measurement of renal blood flow: Meth Med Res 1948;1:191-199.

9. Dejong CHC, Kampman MT, Deutz NEP, Soeters PB. Altered glutamine metabolism in rat portal drained viscera and hindquarter during hyperammonemia. Gastroenterology 1992;102:936-948.

10. Jalan R, Plevris JN, Jalan AR, Finlayson ND, Hayes PC. A pilot study of indocyanine green clearance as an early predictor of graft function. Transplantation 1994;58:196-200.

11. van Eijk HM, Rooyakkers DR, Soeters PB, Deutz NE. Determination of amino acid isotope enrichment using liquid chromatography-mass spectromerry. Anal Biochem $1999 ; 271: 8-17$.

12. Clemmesen JO, Tygstrup N, Ott P. Hepatic plasma flow estimated according to Fick's principle in patients with heparic encephalopathy: evaluation of indocyanine green and Dsorbitol as test substances. Hepatology 1998;27:666-73.

13. Olde Damink SWM, Jalan R, Redhead DN, Hayes PC, Deutz NEP, Soeters PB. Interorgan ammonia and amino acid metabolism in metabolically stable patients with cirrhosis and a TIPSS. Hepatology 2002;36:1163-1171.

14. Clemmesen JO, Kondrup J, Ott P. Splanchnic and leg exchange of amino acids and ammonia in acute liver failure. Gastroenterology 2000;118:1131-9.

15. Dejong CHC, Deutz NEP, Soeters PB. Metabolic adaptation of the kidney to hyperammonemia during chronic liver insufficiency in the rat. Hepatology 1993;18:890-902.

16. Halperin $M L$, Kamel KS, Ethier $\mathbb{J H}$, Stinebaugh BJ, Jungas RL. Biochemistry and 
Physiology of ammonium excretion. In: The Kidncy: Physiology and parhophysiology. Ed: Seldin DW, Giebisch G. Raven Press, Ltd, New York 1992.

17. Owen EE, Johnson JH, Tyor MP. The effect of induced hyperammonemia on renal ammonia metabolism. I Clin Invest 1961;40:215-221.

18. Vinay $P$, Allignet $E$, Pichette $C$, Watford $M$, Lemieux $G$, Gougoux A. Changes in renal metabolite profile and ammoniagenesis during acute and chronic metabolic acidosis in dog and rat. Kidney Int. 1980;17:312-325.

19. Welbourne TC, Childress D, Givens $G$. Renal regulation of interorgan glutamine flow in metabolic acidosis. Am J Physiol 1986;251:R858-R866.

20. Nath KA, Hostetter MK, Hostetter TH. The role of ammonia in progressive renal injury. Basel: Karger; 1991.

21. Karim Z, Artmane-Elakeb A, Bichara M. Renal handling of NH4+ in relation to the con-

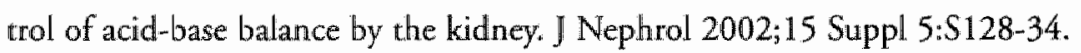

22. Good DW, Knepper MA, Burg MB. Ammonia and bicarbonate transport by thick ascending limb of rat kidney. Am J Physiol 1984;247:F35-44.

23. Amlal H, LeGoff C, Vernimmen $C$, Soleimani $M$, Paillard M, Bichara M. ANG II controls $\mathrm{Na}(+)-\mathrm{K}+(\mathrm{NH} 4+)-2 \mathrm{Cl}$ - corransport via $20-\mathrm{HETE}$ and $\mathrm{PKC}$ in medullary thick ascending limb. Am J Physiol 1998;274:C1047-56.

24. Buerkert J, Marcin D, Trigg D. Ammonium handling by superficial and juxtamedullary nephrons in the rat. Evidence for an ammonia shunt between the loop of Henlle and the collecting duct. J Clin Invest 1982;70:1-12.

25. Sajo IM, Goldstein MB, Sonnenberg H, Stinebaugh BJ, Wilson DR, Halperin ML. Sites of ammonia addition to tubular fluid in rats with chronic metabolic acidosis. Kidney Int $1981,20,353-8$.

26. Simon $\mathrm{E}$, Martin D, Buerkert]. Contribution of individual superficial nephron segments to ammonium handling in chronic metabolic acidosis in the rat. Evidence for ammonia disequilibrium in the renal cortex. J Clin Invest 1985;76:855-64.

27. Azoulay D, Castaing D, Dennison A, Martino W, Eyraud D, Bismuth $H$. Transjugular intrahepatic portosystemic shunt worsens the hyperdynamic circulatory state of the cirrhotic patient: preliminary report of a prospecrive study. Hepatology 1994;19:129-32.

28. Debatin JF, Zahner B, Meyenberger C Romanowski B, Schopke W, Marincek B, et al. Azygos blood flow: phase contrast quantitation in volunteers and parients with portal hypertension pre- and postintrahepatic shunt placement. Hepatology 1996;24:1109-15.

29. Lotterer $E_{3}$ Wengert A, Fleig WE. Transjugulat intrahepatic portosystemic shunt: shortterm and long-term effects on hepatic and systemic hemodynamics in patients with cirthosis. Hepatology 1999;29:632-9. 


\section{LINK TO CHAPTER 3B}

The work of the following chapter was initiated by a study in which we observed increased renal ammonia excretion following volume excrerion. We studied sixteen well compensated cirrhotic patients (mean Pugh score 6.7 (SEM 0.4)) after an overnight fast. One litre of $0.9 \%$ salline was administered to the patients intravenously over one hour. Plasma and urinary ammonia and sodium, renal plasma flow, glomerular filtration rate, plasma rennin activity and angiotensin II were measured before, during, and two hours after saline infusion. The results showed that saline infusion resulted in a significant reduction in plasma ammonia $93( \pm 7)$ to $56( \pm 4) ? \mathrm{~mol} / \mathrm{l} ; \mathrm{p}<0.05)$ and renal plasma flow and glomerular filtration rate increased ( $p<0.05$ for each). The change in ammonia excretion correlated directly with the change in urinary ammonia excretion $(p<0.007)$, angiotensin II $(p<0.002)$ and plasma renin activity $(p<0.01)$. The mean increase in urinary ammonia excretion during the observation period was $1.08 \mathrm{mmol}$. Assuming a volume of distribution of 45 litres, the corresponding change in whole body ammonia during the same period was $1.67 \mathrm{mmol}$. The results of this study suggested that wolume expansion reduces plasma ammonia concentration by increasing ammonia excretion and reducing ammoniagenesis.

Published as $R$. Jalun and Kapoor D. Enhanced renal ammonia excretion following volume expansion in patients with well compensated cirrhosis of the liver. Gut 2003;52;1041-45 



\section{CHAPTER 3B}

REVERSAL OF DIURETIC-INDUCED HEPATIC ENCEPHALOPATHY WITH INFUSION OF ALBUMIN BUT NOT COLLOID

Rajiv Jalan and Dharmesh Kapoor,

Liver Failure Group, Institute of Hepatology, University College London Medical School, UK,

Published in Clinical Science 2004; 106: 467-474 


\section{INTRODUCTION}

In patients with cirrhosis, ammonia is thought to be central in the pathogenesis hepatic encephalopathy $(\mathrm{HE})(1,2)$. The organs that are thought to be primar responsible for the homeostasis of ammonia are the gut and the liver (3). Recent stu ies in patients with cirrhosis have indicated an important role for the muscle and $t$ kidneys in maintaining ammonia levels $(4,5)$. The kidneys dispose ammonia followi its production from glutamine through secretion (proximal convoluted tubule), real sorption and concentration through counter-current multiplication (ascending limb loop of Henle and medullary interstitium) and finally, secretion into the collectir ducts (6). In the physiological state, most of the ammonia generated by the kidney returned to the systemic circulation and a smaller proportion is excreted in the urir $(7,8)$. This ratio is reversed in patients with cirrhosis, as the kidney becomes net ammo nia 'disposer' $(\mathcal{T}, 8)$. Acidosis promotes renal ammonia production and transport $(9$. Chronic hyperkalaemia decreases ammonium production in the proximal tubule an whole kidney, inhibits absorption of $\mathrm{NH} 4+$, reduces medullary interstitial concentra tions of $\mathrm{NH}_{4}+$ and $\mathrm{NH} 3$, and decreases entry of $\mathrm{NH} 4+$ and $\mathrm{NH} 3$ into the medullar collecting duct (10.11). Other potent stimuli modulating ammonia synthesis, trans port and excretion by the kidneys include renal blood flow, tubular cell $\mathrm{pH}$ and rena tubular lumen $\mathrm{pH}$, luminal flow rate and angiotensin II (ANG II) levels $(12,13)$.

One of the common precipitating factors for the development of $\mathrm{HE}$ in patient with cirrhosis is diuretic-induced dehydration. The mechanisms by which dehydration induces $\mathrm{HE}$ is unclear but a contraction of the intravascular volume and its redistribution may reduce renal blood flow and therefore renal ammonia excretion. Renal ammoni: excretion may be further compromised by activation of the renin-aldosterone angiotensin axis impacting upon renal ammonia excretion $(12,13)$. Furthermore, dehy. dration induces oxidative stress, which may exacerbate the neuropsychological effect: of hyperammonemia (14-16).

We have recently shown that, volume expansion in cirrhotic patients results in a reduction in plasma ammonia concentrations and an increase in urinary ammonia excretion (17). The aims of the present study were to determine the effect of volume expansion on the mental state, plasma ammonia and urinary ammonia excretion and, a marker of oxidative stress in cirrhotic patients with diuretic-induced HE. We studied 2 groups of patients consecutively. The first group received volume expansion with $4.5 \%$ human albumin solution (HAS, Baxter Bioscience, Newbury, UK) (referred to as HAS group) and the second group received colloid (Gelofusine, Braun Medical Ltd, Aylesbury, UK) (referred to as Colloid group). 


\section{METHODS}

This prospective, controlled study was conducted with informed and written assent from next-of-kin, with the approval of the local research ethics committee, and in accordance with the Declaration of Helsinki (1989) of the World Medical Association.

Patients. Fifteen patients with alcoholic liver disease $(10 \mathrm{M}, 5 \mathrm{~F})$ and diuretic-induced HE of grade 2 or more were enrolled. The patients had stable cirrhosis at their last outpatient follow up and had been receiving standard diuretic therapy, which included Spironolactone and Frusemide. The first 8 patients were treated with 4.5\% HAS for volume expansion and the subsequent 7 patients had colloid (Gelofusine). There were no significant differences in the patient characteristics between the HAS and Colloid groups. (Table 1).

Table 1. Patient Characteristics.

HAS Group ( $(n=8)$

$47.3(4.4)$

$6 \mathrm{M}, 2 \mathrm{~F}$

Alcohol 7

Alcohol $+\mathrm{HCV} 1$

Severity of Liver Disease

Bilirubin (umol/L)

Prothrombin Time (sec)

Albumin $(\mathrm{g} / \mathrm{L})$

Severity of Ascites

Diuretics on admission

Spironolactone (mg)

Frusemide

Severity of HE on admission

Grade 3

Grade 2
Child Class B: 1

Child Class C: 7

$56.3(5.2)$

$16.7(2.4)$

$27.1(3.2)$

Moderate: 4

Severe: 4

$250(150-400)$

$40(20-80)$

3

5
Colloid Group $(n=7)$

$50.1(6.1)$

$4 \mathrm{M}, 3 \mathrm{~F}$

Alcohol 5

Alcohol + HCV 2

Child Class B: 1

Child Class C: 6

$61.9(6.8)$

$15.9(4.6)$

$29.1(3.3)$

Moderate: 5

Severe: 2

$200(100-400)$

$60(20-120)$

2

5

Data expressed as mean (SEM) 
End Point for Volume Expansion. The amount of fuid (HAS or Gelofusine) admin istered was guided by the central venous pressure and urine output. The aim was t, administer fluids intravenously until the central venous pressure could be sustained a between $7-10 \mathrm{mmHg}$.

Inclusion criteria: Patients were included into this study if they had (i) HE of Grade I or higher (West Haven criteria) (18), (ii) HE was precipitated by dehydration (diuretic usage, oliguria, clinical evidence of dehydration and low central venous pressure).

Exclusion criteria: The patients were excluded if they had evidence of pre-admission renal dysfunction (haematuria/proteinuria), cardiac impairment or focal neurological abnormalities; any symptoms or signs of alcohol withdrawal; hepatic or extraheparic malignancy; presence of other known precipitants of HE such as sepsis, gastrointestinal bleeding, use of sedative-narcotics and constipation; administration of any specific therapy for HE such as lactulose or bowel enemas prior to enrolment.

Study Design and Management. If the patients fulfilled criteria, they were recruited into the study within 6 hours of admission (median 6 (range 4.5-8) hours) and managed according to a standardised protocol. Prior to inclusion into the study, both groups of patients had already started to receive intravenous fluids (HAS group 330 (220-500) ml Gelofusine; Colloid group: 410 (180-580) $\mathrm{ml}$ Gelofusine). The patients were managed in a high-dependency environment. All the diuretics were discontinued; a nasogastric tube was passed into the stomach for providing a pre-fixed volume of water during the experiment and kept in place for subsequent enteral nutrition. Peripheral venous cannula and a central venous catheter (Arrow, Arrow Int Inc., Reading, PA, USA) were inserted. An arterial blood gas sample was obtained prior to randomisation (CO-Oximeter 482, Instrumentation Laboratory, UK). Cardiovascular monitoring included measurement of blood pressure (Dynamap, Critikon, Australia) and heart rate hourly. Blood urea nitrogen, serum creatinine, plasma sodium and potassium were measured using an auto-analyser (Olympus Reply Autoanalyser, Olympus Optical Ltd., Tokyo, Japan). Requisite cultures (blood, urine and ascites) were taken at the bedside to establish the contribution of a possible 'occult' infection to the HE. All patients were fed through the NG tube with an enteral meal providing $35 \mathrm{kCal} / \mathrm{kg}$, $1 \mathrm{gm} / \mathrm{kg}$ of protein, $100 \mathrm{mEq}$ of sodium, $40 \mathrm{mEq}$ of potassium and $60 \mathrm{ml} / \mathrm{hr}$ of fluid. This meal was given over a period of 20 hours and stopped 1 hour prior to the study on Day 1 ( 24 hours) and Day 3 ( 72 hours). The study protocol is schematically depicted in Figure 1. Each study period was of 3-hour duration and all tests- clinical, biochemical, neuropsychiatric and haemodynamic were repeated after 24 and 72 hours of
admission.

Neurological Assessment. Neurological assessment was performed at the time of inclusion into the study and then at 24 and 72 hours. Severity of HE was assessed using the West Haven criteria (18). Tests of neuropsychiatric function (Trails B Test; the test 


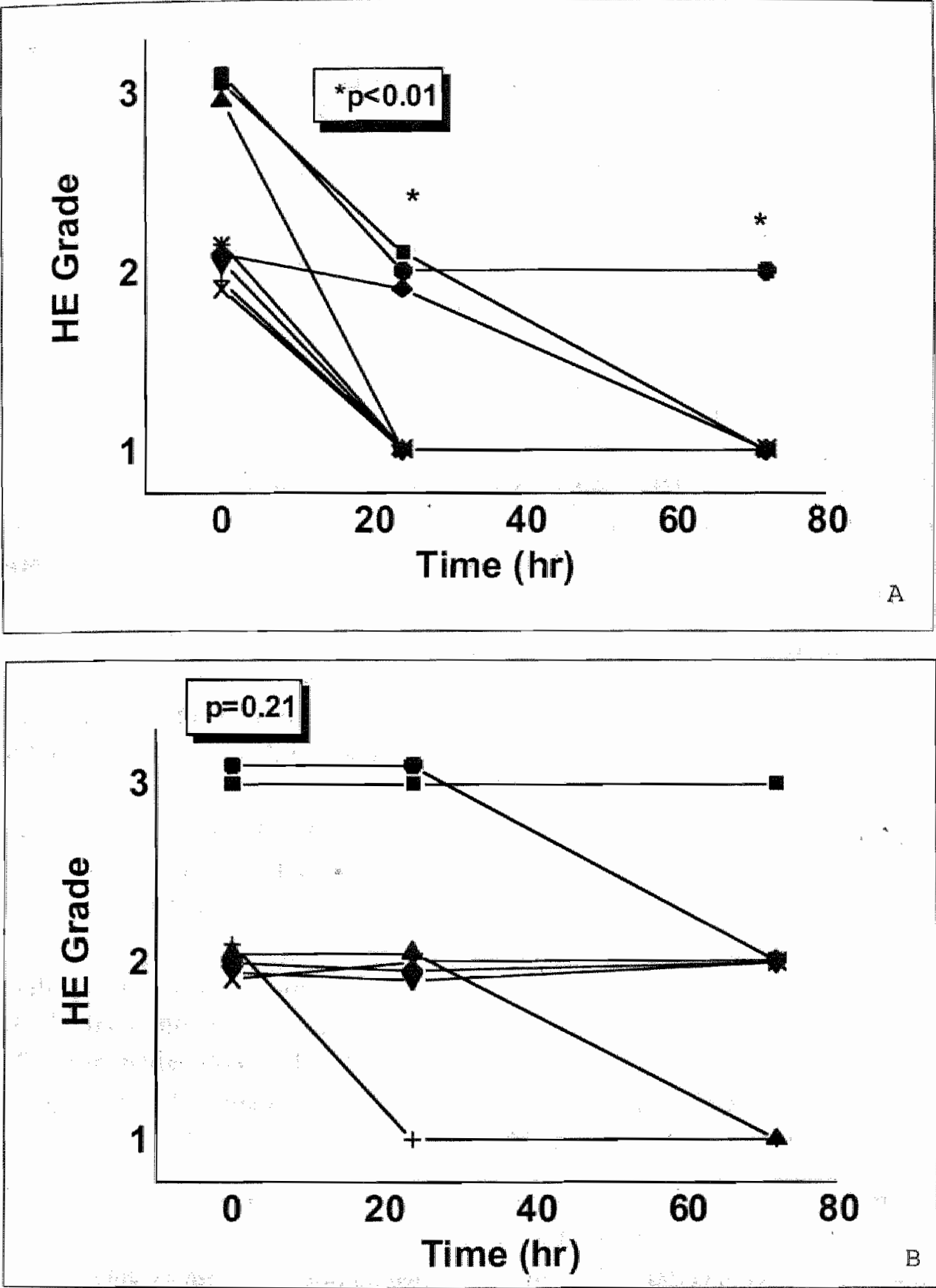

Figure 1. Change in the clinical grade of hepatic encephaloparhy following volume repletion in patients receiving (A) $4.5 \%$ Human albumin solution (HAS) and (B) Gelofusine (Colloid). Time 0 refers to the time the patients were entered into the study. 
had to be completed within 420 seconds) and digital symbol substitution test, (Dss the test score was the number of symbols correctly substituted in 90 seconds) we administered by a single investigator and scored as described elsewhere $(19,20)$.

Glomerular Filtration Rate and Renal Plasma Flow. Primed, continuous infusion c Inulin (Inutest, 25\%; Laevosan-Gesellschaft, Linz, Austria) and para-amino hippuri acid (PAH) (Merck Sharpe and Dohme, Sydney, Australia) were used as markers c glomerular filtration rate (GFR) and renal plasma flow (RPF) respectively $(17,21)$.

Sampling and Measurements. Plasma samples for circulating neurohormone (angiotensin II (ANG II) and plasma renin activity, (PRA)) and plasma ammonia wer taken at the time points shown (Figure 1). Blood was collected from a peripheral veir into pre-cooled tubes. Plasma was separated and the samples stored at $-7000 \mathrm{C}$ for analy. sis at a later date. Urine was collected in pre-cooled bottle which was maintained acid. ified with $2 \mathrm{ml}$ of $6.0 \mathrm{~N} \mathrm{HCl}$ in the urine collector as described previously for meas. urement of ammonia, inulin and para-amino hippuric acid $(17,21,22)$. Inulin concentration was measured using spectrophotometrically, and PAH using high performance liquid chromatography $(21,22)$.

Plasma renin activity. The radioimmunoassay for measurement of PRA was based on the principle that angiotensin I is generated by the action of renin on its substrate angiotensinogen. An in house antibody for angiotensin I was used. The coefficient of variation for the assay was $5.2 \%$. The reference range for PRA was $1.6(1.5) \mathrm{ng} / \mathrm{ml} / \mathrm{h}$ (17,21).

Angiotensin II. Samples of blood were obtained in ANG II inhibitor. ANG II values were measured by radioimmunoassay with an in house rabbit antibody R6B4. The coefficient of variation for the assay was $3.2 \%$. The reference range for ANG II was 3.2 (1) $\mathrm{pg} / \mathrm{ml}(1721)$.

Plasma and urinary ammonia. Samples for ammonia estimation were kept on ice during processing. Ammonia was measured using standard enzymatic method, as described previously (22). The co-efficient of variation for all determinations was $<4 \%$ Malonyldialdebyde. Malodialdehyde was determined using a modified TBARS assay as described (23). Control values for our laboratory were $0.9(0.2) \mu \mathrm{mol} / \mathrm{L}$

Calculations. Urinary sodium, urinary ammonia and urinary volume values were used to derive the following parameters as follows:

- ammonia excretion (UNH3V, mmol/hr): Urinary ammonia $x$ urinary vol.;

-Fractional excretion of ammonia (FENH3, \%): (UNH3V/Fi Na load) X 100

-Ammonia clearance $(\mathrm{NH} 3 \mathrm{Cl}, \mathrm{ml} / \mathrm{min})$ : UNH3V/plasma ammonia

Statistical Analysis. All the data are expressed as mean \pm standard error. The significance of changes within the study group was tested using One-Way ANOVA during 
each of the three periods of observation ( $T=0, T=24$ hours and $T=72$ hours). A post-hoc analysis was done assuming unequal variance, using the Dunnetr's $C$ test. Difference between groups was tested using two-way ANOVA. Relationship between variables was tested using linear regression. A $p$ value $<0.05$ was taken to be statistically significant. 


\section{RESULTS}

\section{Systemic haemodynamics and Electrolytes}

Both groups of patients showed evidence of severe dehydration indicated by tachycardia, hypotension, low central venous pressure and poor urine outpur. They had elevated urea and creatinine, reduced serum sodium and potassium and showed evidence of mild metabolic acidosis. Volume resuscitation resulted in significant and sustained increase in the central venous pressure in both groups. In the 1 st 24 hours the target central venous pressure $(7-10 \mathrm{mmHg})$ was attained and sustained with $2.5(0.8) \mathrm{L}$ of HAS in the HAS group and 2.9 (1.2) L of Gelofusine in the Colloid group ( $\mathrm{p}=0.19$ between groups). In the following $48 \mathrm{hrs}$, a further 0.9 (0.2) L of HAS was administered to the HAS group and 1.4 (0.6) L of Gelofusine to the Colloid group to sustain the increase in central venous pressure. Volume resuscitation was associated with a significant and sustained increase in mean arterial pressure, serum sodium and serum potassium, a decrease in the heart rate, and correction of the metabolic acidosis. Serum albumin increased significantly from $27.1(3.2) \mathrm{g} / \mathrm{L}$ at baseline to $30.1(2.6) \mathrm{g} / \mathrm{L}$ at 24 hr to $28.8(2.5)(\mathrm{p}<0.05)$ at $72 \mathrm{hr}$ in the HAS group. There was an insignificant reduction in serum albumin in the Colloid group (Table 2).

\section{Severity of Hepatic Encephalopathy}

HE Grade: Both groups of patients had similar severity of $\mathrm{HE}$ at baseline. There was a significant improvement in the HE grade in the patients in the HAS group both at 24 and at 72 hours $(p<0.01)$, which was not observed in the patients in the Colloid group $(\mathrm{p}=0.21)$. HAS group: 3 patients had Grade $3 \mathrm{HE}$, which improved to Grade 2 in 2 patients and Grade 1 in 1 patient at 24 hrs and further to Grade 1 in 1 patient at 72 hr. One patient remained with Grade $2 \mathrm{HE}$ at 72 hrs. Of the 5 patients with Grade 2 $\mathrm{HE}$, improvement to Grade 1 occurred in 4 patients at $24 \mathrm{hrs}$ and at $72 \mathrm{hrs}$ in one patient (Figure 2a). Colloid Group: Of the 2 patients with Grade $3 \mathrm{HE}$, improvement to Grade $2 \mathrm{HE}$ occurred in one patient at $72 \mathrm{hr}$. The other patient remained in Grade $3 \mathrm{HE}$. Of the 5 patients with Grade $2 \mathrm{HE}$, improvement to Grade 1 occurred in 1 patient at 24 hours and at $72 \mathrm{hr}$ in one. The other 3 remained in Grade 2 HE (Figure 2b).

Neuropsychological Tests: At baseline, it was not possible to perform the neuropsychological tests in 3 patients in the HAS group and in 2 parients in the Colloid group because of the severity of $\mathrm{HE}$. In the rest of the patients, the performance of the patients for Trails B test and Dsst were similar. There was significantly greater improvement in the Trails B test and also in the Dsst in the HAS group compared with the Colloid group ( $\mathrm{p}<0.01$ using two-way ANOVA) (Figure $3 \mathrm{a}$ and $3 \mathrm{~b}$ ). 
Table 2. Changes in biochemistry, renal haemodynamics, plasma ammonia and urinary ammonia excretion after volume repletion

\begin{tabular}{|c|c|c|c|c|c|c|}
\hline & \multicolumn{2}{|c|}{ HAS GROUP $(n=8)$} & & \multicolumn{3}{|c|}{ COLLOID GROUP ( $n=7)$} \\
\hline & $\mathbb{T}=0$ Ohours & $T=24 \mathrm{hrs}$ & $\mathrm{T}=72 \mathrm{Hth}$ & $T=$ ohours & $\mathrm{T}=24 \mathrm{hrsi}$ & $T=T 2 h r s$ \\
\hline Heart Rathe (min) & $96(3)$ & $83(4)^{*}$ & $772)^{*}$ & $101(3 \pi)$ & $79(3.2)^{2}$ & $81(29)^{*}$ \\
\hline Mean arthenial Pressure (mmitgi) & $70(2)$ & $81(2)^{4}$ & $B=(2)^{* *}$ & $73(3.4)$ & $04(29)^{2}$ & $85(39)^{*}$ \\
\hline Central Venous Pressure (mmHg) & $1.1(0.5)$ & $9.3(1.2)^{n=}$ & $9.9(1.3)^{m *}$ & $.1(4,1)$ & $7.8(1.2)^{*}$ & $9.3(2.1)^{\circ}$ \\
\hline Serum Sodium (mmoll) & $125(9.8)$ & $130(3.1)$ & $130(1.2)^{\circ}$ & $128(34)$ & 13129 & $13.2(2.2)^{\circ}$ \\
\hline Sexum Potassium (mmoth) & $3.2(0.1)$ & $3.6(0.1)^{4}$ & $39(0.1)^{+*}$ & $3.5(0: 3)$ & $4.990 .2 y^{*}$ & $3.9(0.3)^{4}$ \\
\hline Albumirn $(g h)$ & $27.1(3.2)$ & $30.9(2.6)^{*}$ & $28.8(2.5)^{*}$ & $29.1(3.3)$ & $27.2(4.3)$ & $27.4(4.5)$ \\
\hline "Blowd Gases & & & $=8$ & & & \\
\hline pH & $7.36(0.1)$ & & $7.43(0.1)$ & $7.38(0.1)$ & & $7.43(0.4)$ \\
\hline $\mathrm{HCOS}$ & $20.3(0.7)$ & & $23.1(0.4)^{*}$ & $18.4(0.4)$ & & $22.1(0.3)^{x}$ \\
\hline Urea (mmolith) & $20.3(23)$ & $13.2(1.1)$ & $11.2(1.9)^{\circ}$ & $18.4(2.4)$ & $9.5 \cdot(3.11)^{\circ}$ & $7,6(3,3)^{4}$ \\
\hline Creatinine (umoll L) & $197.5+(24.5)$ & $134.3(14.3)$ & $99(9.7)^{*}$ & $183: 3(18)$ & $121(11,3)^{2}$ & $10.4(13.5)^{*}$ \\
\hline RPF (milmin) & $221.4(213)$ & $362 . \theta(29.2)^{* *}$ & $325.3(40)^{* *}$ & $197(14.7)$ & $357.0(35.5)^{*}$ & $380.4(23.8)^{\circ *}$ \\
\hline GFR (moll/min) & $15.7(2.5)$ & $37.4(3.7)^{*}$ & $39.6(2.3)^{\mathrm{m}}$ & $93.7(4.4)$ & $38.3(4.4)^{64}$ & $45.4(2.5)^{*}$ \\
\hline UNaW (mmonolhr) & $14.4(1.9)$ & $33.3\left(\theta_{1} 3\right)^{*}$ & $49(160.1)^{* *}$ & $19.2(4)$ & $39: * 15.5)^{*}$ & $41.1(9.1)^{*}$ \\
\hline Pitesma ammonia (wonoll) & $98.0(7.3)$ & $64.6(4.2)^{\text {th }}$ & $52.7(4.9)^{+* 11}$ & $89.1(0.1)$ & $70.9(20)$ & $51.7(3.4)=$ \\
\hline Urimary wolume (mi/hr) & $10.111)$ & $48.6(6)^{* 4}$ & $55.7(13)^{\cdots}$ & $13.1(2.0)$ & $55.1(4.2)^{* *}$ & $63.0(2.0) *$ \\
\hline 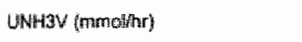 & $0.4(0.04)$ & $1.2(0.3\}^{* *}$ & $1.2(0.5)^{4 * *}$ & $0.3(0.10)$ & $4.1(0.1)^{4}$ & $1,3(0,1)^{*}$ \\
\hline FeNHH $(\%)$ & $385.3(32.9)$ & $895.1(49.6)^{*}$ & $1050.18(187.9)^{0.4}$ & $345.5(70.7)$ & $735.4(109.8)^{n i}$ & $934.7(113.06)^{* 4}$ \\
\hline $\mathrm{NH} 3 \mathrm{Cl}$ (mithin) & $63.7(8.7)$ & $3129(36.7)^{\mathrm{rm}}$ & $403.1(61.5)^{n-k}$ & $46.8(9.3)$ & $264.6(26.8)^{\circ}$ & $424.4(59)^{* *}$ \\
\hline Afl rogs/min) & $325.2(20.5)$ & $167.5(19.1)^{2 *}$ & $143.7(19,0)^{* * *}$ & $229.3(4.2 .1)$ & $185.6(18.2)^{\prime \prime}$ & 93: $1(24.2)^{*:}$ \\
\hline PRA (ongfisilhur) & $30(3)$ & $\ln (3)^{* *}$ & $10(1)^{n+x}$ & $26.145 .6)$ & $41,2(2.8)^{*}$ & $\theta .2(2.9)^{* *}$ \\
\hline
\end{tabular}

All values are expresed as Mean (SEM). BUN: blood urea nitrogen; UNH 3 Vi urinary ammonia excretion; UneV: Lrinary sodium excretion; FeNH 3 ; fractional excretion of ammonia; $\mathrm{NH}_{3} \mathrm{Cl}$ : emmonia clearance; $\mathrm{ANG}$ HI: anglotensin II; PRA; Plasma rennin activity "p<0.05; * $p<0.01$; ** $p<0.001$ (by one-way ANOWA); Blood gases measurements were available in all patients at baseline but in only 4 patients in each group all 72 hours. 


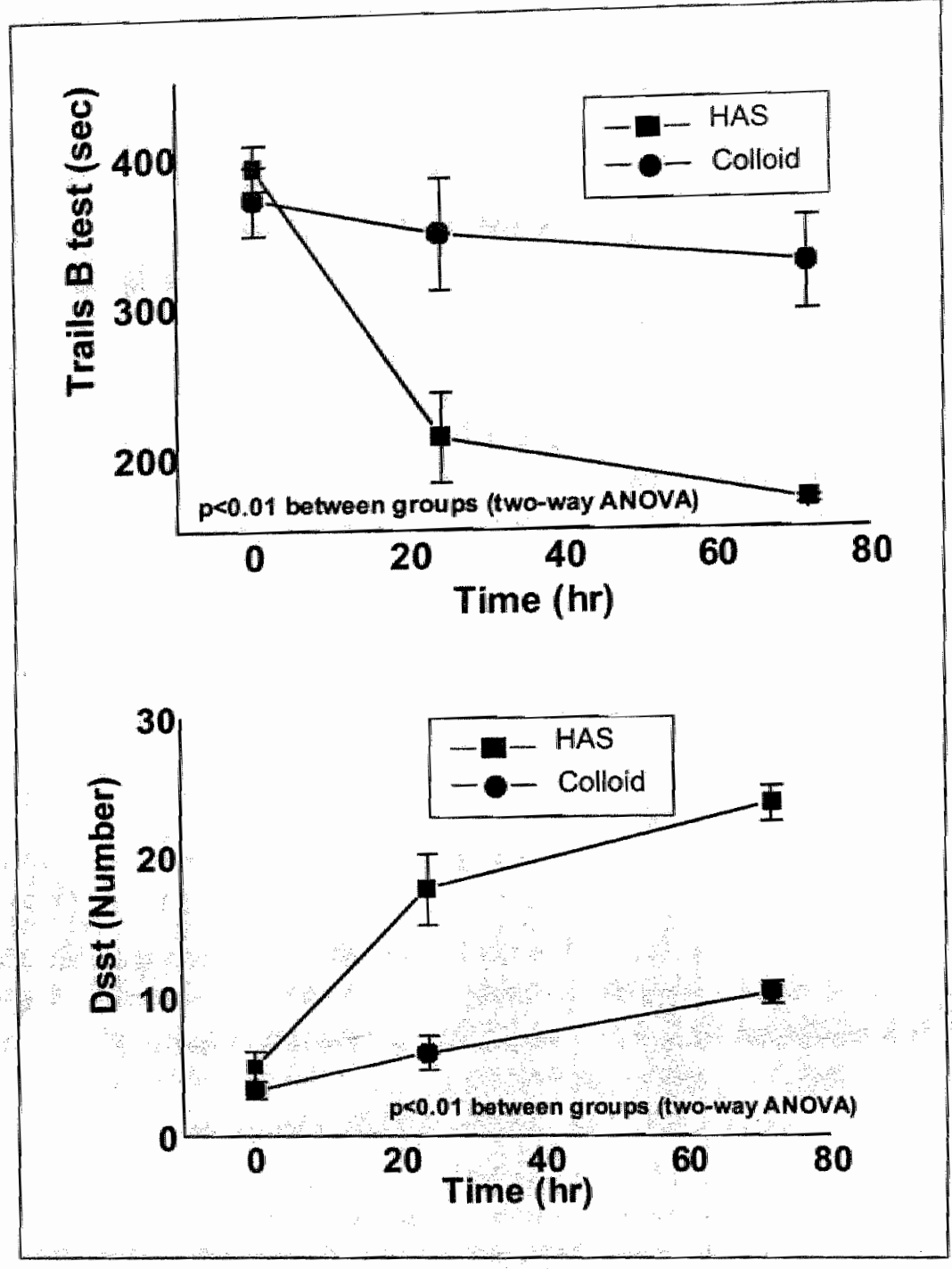

FiguRe 2. Changes in Neuropsychological tests, Trails B rest and Digit Symbol Substitution Test (Dsst) following volume repletion in the patients receiving $4.5 \%$ Human albumin solution (HAS) or Gelofusine (Colloid). Data expressed as mean and standard error. Time 0 refers to the time the patients were entered into the study. Normal values from an age and sex matched group: Dsst: 44 (range 36-52) Trails B Test: median 84 (range 61-92). 


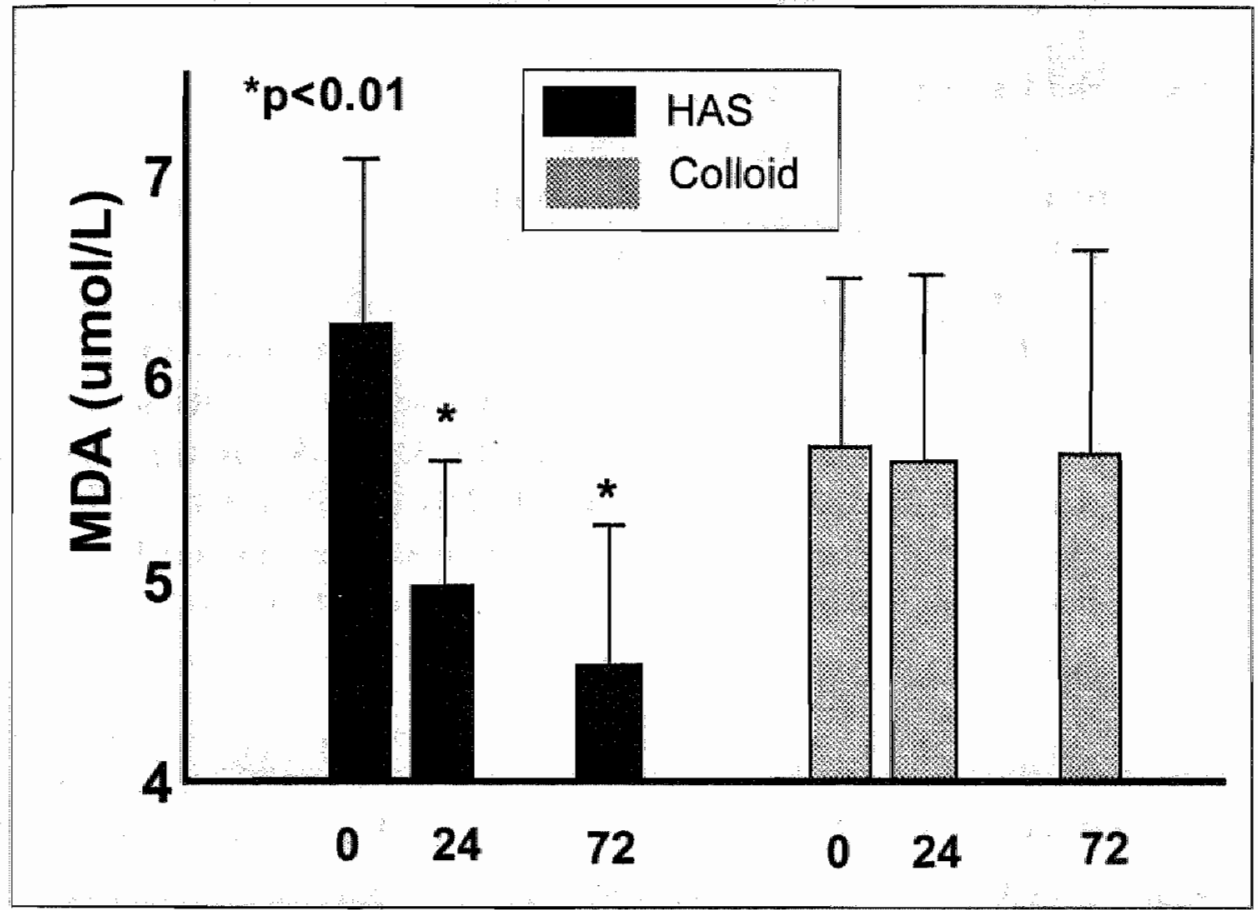

FIGURE 3. Plasma concentration of malonaldehyde (MDA) (mean and standard error) in the patients receiving $4.5 \%$ Human albumin solution (HAS) or Gelofusine (Colloid) at the time of entry into the study $(0)$ and then $24(24)$ and $72(72)$ hours afterwwards. 
Change in renal function tests, renal haemodynamics and circulating neurohormones

Volume resuscitation resulted in similar and significant increases in urine output at 24 and 72 hours in both groups. The increase in urine output was possibly due to increased RPF and GFR, both of which increased similarly and significantly in both groups. This resulted in a significant and sustained reduction in serum urea and creatinine in both groups, which were not significantly different. PRA and ANG II decreased significantly following volume repletion in both groups at 24 and at 72 hours. The reduction was similar in the 2 groups. The increase in the RPF correlated with the decrease in ANG II $(r=0.61, p=0.01)$ but not with that of PRA $(r=0.44, p=$ 0.07). Similarly, the increase in GFR correlated with the decrease in ANG II ( $r=0.75$, $\mathrm{p}=0.0 \mathrm{~g} 2)$ and PRA $(\mathrm{r}=0.89, \mathrm{p}<0.001)$.

\section{Plasma and Urinary Ammonia}

At baseline, plasma ammonia concentration was high in both groups of patients and not significantly different from each other. With volume repletion, plasma ammonia was reduced significantly in both groups. This reduction in plasma ammonia was observed at 24 hours but sustained at 72 hours and was similar in both groups, which was associated with significant increase in urinary ammonia excretion and in ammonia (Table 2). The decrease in plasma ammonia levels correlated with the increase in urinary ammonia excretion ( $r=0.97, p=0.001)$. The change in plasma ammonia levels also correlated with the decrease in the levels of ANG II $(r=0.75, p=0.002)$ and PRA $(r=0.79, p=0.001)$. The change in urinary ammonia excretion correlated with the decrease in the levels of ANG II $(r=0.79, p=0.001)$ and PRA $(r=0.82, p=0.001)$.

\section{Plasma Malonylaldehyde}

Plasma MDA levels were similar and elevated in both groups of patients. Following volume repletion, significant reduction was noted only in the patients in the HAS group. This reduction was evident at 24 hours and sustained at 72 hours. No significant change was seen in the Colloid group (Figure 4). 


\section{DISCUSSION}

The present study is the first to explore the pathophysiological basis and the role of volume expansion in the treatment of patients with diuretic-induced HE. The result of this study highlights two important observations. Volume expansion resulted in significant and sustained reduction in plasma ammonia, which was associated with a significant increase in urinary ammonia excretion. Of significant interest was the observation that although the improvement in systemic and renal haemodynamics and, reduction in plasma ammonia were similar in the patients treated with HAS and Gelofusine, the improvement in HE was significantly more marked in the patients treated with HAS. The significantly greater reduction in MDA levels in the patients treated with HAS points to the role of oxidative stress as an important adjunct to ammonia in the pathogenesis of diuretic-induced $\mathrm{HE}$.

Diuretic-induced dehydration is a common precipitating factor for $\mathrm{HE}(24,25)$. The present study was conceived following our initial observation that volume expansion with saline in patients with well-compensated cirrhosis resulted in a decrease in plasma ammonia and an increase in urinary excretion of ammonia (17). In keeping with our previous observation, volume repletion resulted in an increase in urinary ammonia excretion with consequent decrease in plasma ammonia levels. It is likely that the change in ammonia homeostasis following volume expansion is likely to be due to an increase in luminal flow rate mediated by improved renal perfusion (26), the cessation of diuretic therapy (6) which is known to inhibit the transporters involved in the excretion of ammonia and/or a reduction in renal ammoniagenesis mediated by a decrease in ANG II, which is an important modulator of the ammonia that is synthesized by the proximal tubule $(11,27-31)$. In the current study, the significant correlation between a decrease in ANG II and decrease in plasma ammonia level on one hand and increase in urinary ammonia excretion on the other support this hypothesis, but does not prove a cause-effect relationship unequivocally.

The most important resull of the study was the observation that there was significantly greater improvement in $\mathrm{HE}$ in the patients that were treated with HAS compared with those treated with Gelofusine, despite similar reduction in the plasma ammonia concentrations. This implies that the mechanism by which albumin infusion resulted in an improvement in $\mathrm{HE}$ is likely to be more than that produced by volume expansion alone. Human albumin is a $66 \mathrm{kDa}$ molecule, constituting $50 \%$ of the plasma proteins in healthy individuals. It has been used essentially as a plasma volume expander in the treatment of liver disease (32-34). Although albumin infusion was associated with significant increases in the concentration of albumin, the increment was modest. This may reflect the fact that albumin is distributed very widely in the 
body and the relatively large volume of distribution in cirrhotic patients due to the concomitant ascites. Albumin has the ability to bind to a range of different molecules, and act as a scavenger. It has a strong negative charge and binds reversibly to borh cations and anions. These include a large number of metabolites, including fatty acids, bilirubin, bile salts, amino acids and nitric oxide. It is also the major extracellular source of reduced sulphydryl groups. These scavenge reactive oxygen and nitrogen species, especially superoxide, hydroxyl and peroxynitrite radicals. Albumin can also limit the production of these reactive species by binding free $\mathrm{Cu}^{2+}$, which is known to be important in accelerating the production of free radicals (32-34). Interestingly, albumin administration in sepsis patients led to significantly increased levels of both plasma albumin and total plasma thiols (35). Albumin may thus influence the redox balance and reduce oxidarive stress $(33,34)$. In keeping with this hypothesis, we were able to show a significant reduction in the MDA levels, which is a well-recognised marker of oxidative stress. This reduction in MDA was nor observed in the patients in the Colloid group. It is interesting to note that both groups of patients had markedly increased MDA levels at baseline, which may either reflect the effect of dehydration and/or severe cirrhosis as increased oxidative stress is well-described in both situations. The results of our study point to an important role of factors that act in concert with ammonia in the pathogenesis of diuretic-induced HE (14-16). Clearly, these factors are likely to have an important albumin-binding component and as we have shown in this study, one such factor may be reactive oxygen species. There is emerging evidence suggesting that oxidative stress plays an important role in the development of $\mathrm{HE}$ (36-39), possibly by causing mitochondrial damage, including oxidation of membrane phospholipids and various enzymes involved in energy metabolism (39-42). Alternatively, the effects of albumin infusion may be through its effects on cerebral blood flow. Several studies have suggested that in patients with cirrhosis, there is a redistribution of cerebral blood flow, particularly in the frontal cortex (43-44). Ginsberg et al. (45) have shown that albumin infusion can increase cerebral blood flow. Another possible explanation, which is more difficult to substantiate is that gelatin infusion may in some ill-defined manner have interfered with recovery from $\mathrm{HE}$. In future studies, control arms should include other fluids such as saline. Our data question the role of conventional ammonia lowering strategies such as lactulose, non-absorbable antibiotics and bowel enemas in the treatment of diuretic-induced HE. Simple intervention with fluid therapy results in significant and substantial reduction in plasma ammonia concentration without any conventional ammonia lowering strategy by simply targeting the kidneys supporting our recent studies suggesting that the kidneys are important in ammonia homeostasis $(4,15,17)$.

The use of albumin infusion in critically ill patients is a subject of considerable debate following the recently published metanalysis suggesting poor outcome of critically-ill patients that were treated with albumin compared with other colloids (46). 
This metanalysis has been criticised and the issue of the use of albumin as a volume expander in the critically ill patients is being explored in controlled clinical trials (4749). In patients with liver disease, the use of albumin infusion as a volume expander has been studied in considerable detail. Studies have shown that albumin infusion following paracentesis is associated with lower incidence of renal dysfunction compared with other colloids, in patients with spontaneous bacterial peritonitis, the addition of albumin infusion to antibiotics improves survival compared with antibiotics alone and; in patients with hepatorenal syndrome, the addition of albumin infusion to the vasoconstrictor terlipressin improves survival compared with terlipressin. These data emphasise both the safety and also the efficacy of albumin in cirrhotic patients $(32,50,51)$.

The present study was terminated 72 hours after entry into study because it was designed to look at the pathophysiological mechanisms. This study can be criticised because of its non-randomised design. The patients in the HAS group were studied first and we were surprised at the rapid improvement in the severity of HE. The patients were recruited into the Colloid group to clarify whether the effects of HAS were due to volume expansion allone or due to a non-oncotic property of albumin. We do however, believe the results are nevertheless important and robust given that both groups of patients were well-matched for the severity of the underlying liver disease and $\mathrm{HE}$, the degree of dehydration, their response to volume expansion, the reduction in circulating ammonia levels with volume expansion and the increase in urinary ammonia excretion.

In conclusion, the results of our srudy suggest that volume expansion in patients with diuretic-induced $\mathrm{HE}$ results in significant reduction in plasma ammonia levels due to increased renal ammonia excretion. The significantly greater improvement in the severity of HE in the HAS group compared with the Gelofusine group indicates either a positive effect of albumin or a negative effect of Gelofusine on the mental state. The association of the greater improvement in the severity of HE in the HAS group with the greater reduction in a marker of oxidative stress support the notion that protein-bound substances such as reactive oxygen species are important adjuncts to ammonia in the parhogenesis of diuretic-induced $\mathrm{HE}$ and a possible role for HAS as the preferred volume expander in the treatment of such patients. These data advocate the need for a suitably powered randomised controlled clinical trial, using albumin infusion as an adjunct to conventional ammonia lowering strategy in $\mathrm{HE}$. 


\section{REFERENCES}

1. Butterworth RF. The neurobiology of hepatic encephalopathy. Semin Liver Dis $1996 ; 16: 235-44$.

2. Norenberg MD. Astrocytic-ammonia interactions in hepatic encephalopathy. Semin Liver Dis $1996 ; 16: 245-53$.

3. Nencki M, Pawlow JP, and Zalleski J. Ueber den Ammoniakgehalt des Blutes und der Organe und die Harnstoffbildung bei den Saugethieren. Archiv Fuer Experimentelle Pathologie und Pharmakologie 1896;37:26-51.

4. Olde Damink SWM, Jalan R, Redhead DN, Hayes PC, Deutz NEP, and Soeters PB. Interorgan ammonia and amino-acid metabolism in metabolically stable patients with cirrhosis and a TIPSS. Hepatology 2002;36:1163-71.

5. Olde Damink SWM, Jalan R, Deutz NEP, Redhead DN, Dejong CHC, Hynd P Jalan RA, Hayes $P C$, and Soeters PB. The kidney plays a major role in the hyperammonemia seen after simulated or actual GI bleeding in parients with cirmosis. Hepatology 2003;37:127785.

6. Knepper MA. NH4, transport in the kidney. Kidney Int 1.991;33:595-102.

7. Halperin ML, Kamel KS, Ethier JH, Stinebaugh BJ, and Jungas RL. Biochemistry and physiology of ammonia excretion. In: The Kidney: Physiology and Patbopbysiology. Seldin DW, Giebisch G. (editors). Raven Press Lud., New York, 1992; pp. 2645 -79.

8. Dejong $\mathrm{CHC}_{*}$ Deutz NEP, and Soeters PB. Renal ammonia and glutamine metabolism during liver insufficiency-induced hyperammonemia in the rat. J Clin Invest 1993;92:2834-40.

9. Schoolwerth AC. Regulation of renal ammoniagenesis in metabolic acidosis. Kidney Int $1991 ; 40,961-73$

10. Nagami GT. Enhanced ammonia secretion by proximal rubules from mice receivung NH(4)Cl: role of angiocensin II. Am J Physiol 2002;282:[4772-7.

11. DuBose TD Jr. Molecular and pathophysiologic mechanisms of hyperkalemic metabolic acidosis. Trans Am Clin Climatol Assoc 2000;111:122-33.

12. Lemieux $G$, Vinay $P$, and Cartier $P$. Renal hemodynamics and ammoniagenesis: characteristics of the antiluminal site for glutamine extraction. J Clin Invest 1974;53:884-94.

13. Hamm LL, and Simon EE. Ammonia transport in the proximal rubule. Miner Electrolyte Metab 1990;16:283-90.

14. Schliess $\mathrm{F}$, and Haussinger D. The cellular hydration state: a critical determinant for cell death and survival: Biol Chem 2002,383,577-83.

15. Alravilla D, Saitta A, Guarini S, Galeano M, Squadrito G, Cucinotta D, Santamaria LB; Mazzeo AT, Campo GM, Ferlito M, Minutoli L, Bazzani C, Bertolini A, Caputi AP, and Squadrito. Oxidative stress causes nuclear factor-kappaB activation in acute hypovolemic hemorrhagic shock. Free Radic Biol Med 2001;30:1055-66.

16. Arteel GE. Oxidants and antioxidants in alcohol-induced liver disease. Gastroenterology $2003 ; 24: 778-90$.

17. Jalan $\mathbb{R}$, and Kapoor D. Enhanced renal ammonia excretion following volume expansion in patients with well compensated cirrhosis of liver. Gut 2003;52:1041-5 
18. Atterbury CE, Maddrey WC, and Conn HO. Neomycin-sorbitol and lactulose in the treatment of acute portal-systemic encephalopathy. A controlled, double-blind dinical trial. Am I Dig Dis 1978; 23:398-406.

19. Davies $\mathrm{AD}$. The influence of age on trail making test performance. I Clin Psychol $1968 ; 24: 96-8$.

20. Hindmarch I. Psychomotor function and psychoactive drugs. Br I Clin Pharmacol 1980;10:189-209.

21. Jalan R, and Hayes PC. Sodium handling in patients with well-compensated cirrhosis is dependent on the severity of liver disease and portal pressure. Gut 2000;46:527-33.

22. Dejong CHC, Deutz NEP, and Soeters PB. Metabolic adaptation of the kidney to hyperammonemia during chronic liver insufficiency in the rat. Hepatology 1993;18:890-902.

23. Lapenna D, Ciofani $G$, Pierdomenico SD, Giamberardino MA, and Cuccurulio F. Reaction conditions affecting the relationship between thiobarbituric acid reactivity and lipid peroxides in human plasma. Free Radic Biol Med 2001;31:331-5.

24. Jalan R, and Hayes PC. Hepatic encephalopathy and ascites. Lancet 1997:350:1309-15.

25. Blei AT and Cordoba J. Hepatic Encephalopathy. Am J Gastroenterol 2001,96:1968-76.

26. Good DW, and DuBose TD Jr. Ammonia transport by early and late proximal convoluted tubule of rat. J Clin Invest 1987;79:684-91.

27. Good DW, and Knepper MA. Ammonia transport in the mammalian kidney. Am J Physiol $1985 ; 248: F 459-F 471$.

28. Wall SM. Ammonium transport and the role of the Na,K-ATPase. Miner Electrolyre Metab 1996;22:311-7.

29. Gesek FA. and Schoolwerth AC. Hormonal interactions with the proximal Na-H exchanger. Am I Physiol 1990; 258:F514-F521.

30. Chobanian MC and Julin CM. Angiotensin II stimulates ammoniagenesis in canine renal proximal tubule segments. Am J Physiol 1991; 260:F19-F26.

31. Nagami GT. Enhanced ammonia secretion by proximal tubules from mice receiving ammonium chloride: role of angiotensin II. Am J Physiol 2001;282:F472-7.

32. Arroyo V. Review article: albumin in the treatment of liver diseases-new features of a classical treatment. Aliment Pharmacol Ther 2002;16 Suppl 5:1-5.

33. Sen S, Mookerjee RP, Davies NA, Williams R, and Jalan R. Review article: the molecular adsorbents recirculating system (MARS) in liver failure. Aliment Pharmacol Ther 2002;16 Suppl 5:32-8.

34. Evans TW. Review article: albumin as a drug-biological effects of albumin unrelated to oncotic pressure. Aliment Pharmacol Ther 2002;16 Suppl 5:6-11.

35. Quinlan GJ, Margarson MP, Mumby S, Evans, TW, Gutteridge JM. Administration of albumin to patients with sepsis syndrome: a possible beneficial role in plasma thiol repletion. Clin Sci 1998;95:459-65.

36. Murphy MG, Jollimore C, Crocker JF, and Her H. Beta-oxidation of (1-14C) palmitic acid by mouse astrocytes in primary culture: effects of agents implicated in the encephalopathy of Reyexs syndrome. J Neurosci Res 1992;33:445-54.

37. Murthy CR, Rama Rao KV, Bai G, and Norenberg MD. Ammonia-induced production of free radicals in primary cultures of trat astrocytes. J Neurosci Res 2001;66:282-8.

38. Norenberg MD. The role of astrocytes in hepatic encephallopathy. Neurochem Pathol 1987:6:13-33. 
39. Rao KV and Norenberg MD. Cerebral energy metabolism in hepatic encephalopathy an hyperammonemia. Metab Brain Dis 2001;16:67-78.

40. Stewart VC, Sharpe MA, Clark JB, and Heales SJ. Astrocyte-derived nitric oxide cause both reversible and irreversible damage to the neuronal mitochondrial respiratory chain. Neurochern 2000; 5:694-700.

41. Heales SI, Bollanos JP, Stewart VC, Brookes PS, Land JM, and Clark JB. Nitric oxide mitochondria and neurological disease. Biochim Biophys Acta 1999;1410:215-28.

42. Mitchell JA, Kohlhaas $\mathrm{KL}_{\text {, }}$ Sorrentino $\mathrm{R}$, Warner TD, Murad F, and Vane JR. Inductior by endotoxin of nitric oxide synthase in the rat mesentery: lack of effect on action of vaso constrictors. Br T Pharmacol 1993;109:265-70.

43. O Carroll RE, Hayes PC, Ebmeier KP, Dougall N, Murray C, Best IJ, Bouchier LA, anc Goodwin GM."Regional cerebral blood flow and cognitive function in parients with chronic liver disease. Lancet 1991;337:1250-3.

44. Jalan R, Olde Damink SWM, Lui HF, Glabus M, Deutz NEP, Hayes PC, and Ebmeier K Oral amino acid load mimicking hemoglobin results in reduced regional cerebral perfusion and deterioration in memory tests in patients with cirrhosis of the liver. Metab Brain Dis 2003; 18:37-49.

45. Huh PW, Belayew L, Zhao W, Busto R, Saul I, and Ginsberg MD. The effect of high-dose albumin therapy on local cerebral perfusion after transient focal cerebral ischemia in rats. Brain Res 1998;31;804:105-13.

46. Schierhout $\mathrm{G}$, and Roberts 1. Fluid resuscitation with colloid or crystalloid solutions in critically ill patients: a systematic review of randomised trials. BMJ 1998;316:961-964.

47. McClelland B. Albumin: don't confuse us with the facts. BMJ 1998;317: 829-830.

48. Revell M, Porter $K$, and Greaves I. Fluid resuscitation in prehospital trauma care: a consensus view. J Accid Emerg Med 2002;19:494-498.

49. Gosling P. Salt of the earth or a drap in the ocean? A parhophysiological approach to fluid resuscitation. I Accid Emerg Med 2003;20:306 - 315.

50. Sort P, Navasa M, Arroyo V, Aldeguer X, Planas R, Ruiz-del-Arbol L, Castells L, Vargas V, Soriano $G_{i}$ Guevara M, Gines P, and Rodes I. Effect of intravenous albumin on renal impairment and mortality in patients with cirrhosis and spontaneous bacterial peritonitis. N Engl J Med 1999;341:403,9.

51. Ortega R, Gines P, Uriz J, Cardenas A, Calahorra B, De Las Heras D, Guevara M, Bataller R, Jimenez W, Arroyo $V$, and Rodes $J$. Terlipressin therapy with and without albumin for patients with hepatorenal syndrome: results of a prospective, nonrandomized study. Hepatology 2002;36:941-8. 


\section{LINK TO CHAPTER 4}

The work of the following chapter was initiated by an experimental animal study in which we showed behavioural changes and ammonia accumulation in the cerebral cortex of portacaval shunted rats following repeated blood gavages. Rats were studied 14 days after portacaval shunt or sham surgery. Rats received $3 \mathrm{~mL}$ bovine erythrocytes or saline at $t=0,1,2$, and 3 h via a previously placed gastrostomy catheter. At $t=0,2,4$, 6 and $8 \mathrm{~h}$ arterial blood and at $t=8 \mathrm{~h}$ cerebral cortex were sampled for determination of ammonia and amino acids. Control rats were sampled without previous surgery.

Repeated intragastric blood administration increased the already elevated arteriall ammonia levels in portacaval shunted rats further. This resulted in higher cerebral cortex ammonia and glutamine levels after blood administration. Despite the accumulation of ammonia and glutamine, cerebral cortex glutamate concentrations remained unaltered. Portacaval shunted rats became more encephalopathic after blood gavages. Interestingly, cerebral cortex concentrations of GABA, tyrosine and phenylalanine were markedly increased. The observations led us to conclude that the model of a simulating an upper gastrointestinal bleeding in portacaval shunted rats, could be used a suitable, clinically valid model for future research regarding hepatic encephalopathy.

Published as Olde Damink SWM, Dejong CHC, Deutz NEP, Soeters PB. Effects of simulated upper gastrointestinal baemorrbage on ammonia and related amino acids in blood and brain of chronic portacatal-shunted rats. Metabolic Brain Disease 1997; 12: 121-35. 



\section{CHAPTER 4A}

ORAL AMINO ACID LOAD MIMICKING HAEMOGLOBIN RESULTS IN REDUCED REGIONAL CEREBRAL PERFUSION AND DETERIORATION IN MEMORY TESTS IN PATIENTS WTTH CIRRHOSIS OF THE LIVER

1,2Rajiv Jalan, 2,5Steven WM Olde Damink, ${ }^{2}$ Hock F Lui, 3Mike Glabus, 5 Nicolaas EP Deutz, 2 Peter $\mathrm{C}$ Hayes, ${ }^{3,4}$ Klaus Ebmeier

Institute of Hepatology, University College London Medical School, London ${ }^{4}$ ${ }^{2}$ Departments of Medicine and ${ }^{3}$ Medical Physics, Royal Infirmary of Edinburgh, and 4Psychiatry, Royal Edinburgh Hospital, Edinburgh, UK, and 5 Department of Surgery, Maastricht University, Maastricht, the Netherlands

Published in Metabolic Brain Disease 2003; 18: 37-49 


\section{INTRODUCTION}

Hepatic encephalopathy (HE) is a neuropsychiatric syndrome with potential for full reversibility that occurs in patients with significant liver dysfunction and cannot be attributed to other causes. Two distinct forms can be identified in patients with cirrhosis of the liver: the 'overt' form, which is easy to diagnose, and the 'minimal' form $(1,2)$ with subtle neuropsychological changes. The prevalence of minimal HE in cirrhosis varies from $30-84 \%$ and is defined by abnormalities in electrophysiological and psychomerric tests. Minimal HE is an important cause of occupational and psychosocial morbidity, and its severity correlates with poor quality of life that is a characteristic feature of patients with cirrhosis (3).

Patients with cirrhosis and $\mathrm{HE}$ cepict widespread impairment in regional cerebral blood How (rCBF) compared with healthy volunteers (4-9). Most consistently, the rCBF pattern of the frontal cortex and the basal ganglia are altered. However, both increased as well as decreased $\mathrm{rCBF}$ alterations are reported. These discrepancies may reflect heterogeneity of the clinical variables among the patient groups enrolled in the various studies, as well as methodological differences and the choice of the reference region for semi-quantitative assessments. Furthermore, it is unclear whether changes in rCBF are the cause or the effect of $\mathrm{HE}$. To overcome these problems a recent report studied the same cirrhotic patients, both before and after liver transplantation and reported normalisation of the observed decreased $\mathrm{rCBF}$ of brain regions after liver transplantation (8). This observation suggests that the reduced $\mathrm{rCBF}$ is the result of a liver related disorder but it does not allow determination of putative pathogenic mechanisms in the development of $\mathrm{HE}$ and results may be confounded by effects of immunosuppressive medication and altered nutritional status.

Ammonia is considered the most important factor in the genesis of HE (10). Induction of hyperammonemia has been used widely in experimental animal models to study the pathophysiology of HE. Recently, feeding of the amino acid glutamine has been used to induce hyperammonemia and study neuropsychological alterations in parients with cirrhosis (11), but the clinical relevance of this model can be questioned. It is well known that an upper gastrointestinal (UGI) bleed in cirrhotic patients produces hyperammonemia and precipitates HE. Simulating the metabolic effects of an UGI bleed by oral administration of a specifically prepared amino acid solution that is identical to the amino acid profile of haemoglobin has been shown to induce hyperammonemia and behavioural disturbance in portacaval shunted rats (12). Oral administration of a mixture of amino acids mimicking the composition of haemoglobin to patients with cirnhosis of the liver could be a clinically more valid model. The aim of the present study was to determine the changes in regional cerebral perfusion and neuropsychological function as a consequence of an oral amino acid load mimicking haemoglobin. 


\section{METHODS}

Approval was obtained from the Ethics Committee of the Lothian Hospitals, Edinburgh and the Administration of Radioactive Substances Advisory Committee (ARSAC) at the Department of Health. All subjects provided written informed consent.

Patients. Eight, overnight fasted, metabolically stable male patients [(median age 51 years (range 42-68)], were studied. Their Child classes were: A-3, B-3, C-2; the median Pugh-score was 8 (range 6-10)(13); median body mass index was 27 (range 25-32). All were suffering from alcoholic liver disease with biopsy proven cirrhosis of the liver. Patients were excluded if they had severe ascites, pitting peripheral oedema, overt $\mathrm{HE}$, diabetes, clinically evident cardiovascular disease or renal dysfunction (serum creatinine $>100 \mathrm{mmol} / \mathrm{L}$ ), malignancy, or pregnancy. Patients had to be abstinent from alcohol and benzodiazepines for at least 3 months prior to the study.

Oral amino acid load. The metabolic consequences of an UGI bleed were simulated by oral bolus administration of 75 gram of a specifically prepared amino acid solution (Nutricia Cuijk, The Netherlands, Product Number: 24143) that mimics the amino acid composition of the haemoglobin molecule (14). The solution was freshly made and xanthan gum was added to prevent sedimentation.

Measurement of neuropsychological function. Detailed neurological examination was performed on all the patients to exclude evidence of clinically overt $\mathrm{HE}$ and any focal neurology. A construct-driven neuropsychological test battery was designed. The critical factor in developing this battery was the total time period needed to complete the battery, so it could be used as a tool to assess changes in neuropsychological function in response to the simulated UGI bleed. The following cognitive domains were tested: concentration, memory, visuospatial-construction skills and motor function. The neuropsychological test battery consisted of the Trails B Test (15), the Digit Symbol Substitution Test (DSST) (16), choice reaction time (17) and the repeating numbers and, immediate and delayed story recall subtests of the Randt test (18). Total time to perform this battery was 20 minutes.

Trails B test: This test is a derivative of the Trail Making Test and measures visual conceptual and visuomotor tracking. Patients were asked to connect subsequent numbers with letters. The test has to be completed within 420 seconds (15).

The Digit Symbol Substitution Test: The DSST is part of the Wechsler Adult Intelligence Schedule (16). This test is used to assess visuomotor coordination and vigilance. The subject substituted a symbol for a digit from a code that was visible 
throughout the test. A different code was used for each test to control for the effect of learning. The test score was the number of symbols correctly substituted in 90 seconds.

Reaction time: This test is part of the Continuous Performance Task and measures motor function, sustained concentration and the ability to suppress inappropriate responses. The patients saw a sequence of letters $(X, M, T, E)$ on a computer display at the rate of one letter every 1,5 seconds. The task was to press the space bar of the computer as quickly as possible every time the letter $E$ appeared, except when it was immediately preceded by the letter $\mathrm{X}$. Presented were 10 Es requiring a response and 10 Es preceded by Xs. The average reaction time was recorded (17).

\section{Repeating numbers, Immediate and Delayed Story recall subtests of the Randt} memory test: These tests are subtests of the Randt memory test and measure various aspects of memory function and include rote, associative, discourse and incidental memory as well as recall (18).

Repeating Numbers: Numbers of increasing length were given up to a maximum of 9 digits forward followed by a maximum of 8 digits backwards. The largest number of digits recalled before 2 failures were recorded.

Story recall: This test measures recall of twenty words from a paragraph that has an emotionally charged substance and includes fire and disaster. This single presentation takes about 15 seconds. The patient was asked to recall the short story immediately after it had been read to them. After this the gist of the story was requested both immediately and also after 20 minutes. One point was awarded for each word that was recalled immediately after presentation (acquisition). For the gist score, credit was given for any block in which the precise word had been retrieved.

Visual analogue scale for Fatigue: A $10 \mathrm{~cm}$ scale extending from 'wide awake' to "very sleepy' was used to monitor the degree of fatigue during the study period.

The test battery was performed immediately before administration of the oral amino acid load and 2 and 3,5 hours afterwards (except Trails B, only at 0 and 3,5 hours), so that the patients finished 10 minutes before the final scan was taken. All the neuropsychological tests were performed by one of two investigators (HFL or SWMOD), in which each patient was assessed by the same investigator. All patients had one practice session of each test. All the tests have been well validated and parallel forms were used to reduce practice effects $(15,17,18)$. Minimal HE in this study was defined by abnormalities in at least 2 neuropsychological tests, defined as performance more than two standard deviations below the mean of age and education adjusted controls.

Single Photon Emission Computerised Tomography Scanning (SPECT). All subjects were imaged with a single slice twelve detector head scanner with an in slice and $\mathrm{z}$ axis resolution of $8.5 \mathrm{~mm}$ full width half maximum (FWHM) using the intermediate 
572 hole collimators (Neuro 900, Strichman Medical Equipment Inc., Boston, USA). The sensitivity of the scanner is 15000 counts $/ \mathrm{s} / \mathrm{mCi} / \mathrm{ml}$, using intermediate 572 -hole collimators. A catheter was inserted into a dorsal hand vein for the administration of $250 \mathrm{MBq}$ of technetium-99m hexamethyl propylamineoxime (99m Tc-HM-PAO) as part of a split-dose scanning procedure $(19,20)$. During and for five minutes after the injection, patients were required to lie still and silent with eyes patched and ears unplugged and environmental noise was kept to a minimum. The tracer was administered over a 30 second period prior to and 4 hours after the simulated bleed. The subject's head was then placed in a moulded head-holder and aligned with the help of two crossed light beams, and fixed with pressure pads over the zygomatic arches. Slices were acquired parallel to the orbito-meatal plane, starting at a level approximately $2 \mathrm{~cm}$ above the orbito-meatal line and at $1 \mathrm{~cm}$ intervals above this level. Further details of the method have been described previously (21). Images were reconstructed using software supplied by Strichman Medical Equipment (SME) for the Apple Macintosh. The SME reconstruction algorithm selects an enveloping ellipse, derived from an oversmoothed image of the brain. This, together with the absorption length parameter $(95 \mathrm{~mm})$, determines the Chang like attenuation correction, which was done with one iteration. Count distributions were deconvoluted into the radioisotope concentrations responsible, using a Wiener filter with a correlation length of $6 \mathrm{~mm}$.

The SPECT data were analysed using an automated, i.e. objective, procedure described in detail previously (21). In short, images were exported in binary format using the scanner software (Strichmann Medical Equipment, Neuro 900, version 2.92). For the computation of brain activity due to the second injection, the first image was subtracted from the second image after decay correction. The data were converted into ANALYZE format (Bioimaging Resources, Mayo Foundation) and spatially normalised against a standard positron emission tomography (PET) template using a 12 point affine transformation to standard 'Talairach' space. We previously compared normalisation against the PET cerebral blood flow template with a self-generated SPECT template. The slightly higher resolution of the PET template appeared to result in better alignment of scans and was therefore preferred. For the region of interest (ROI) analysis two transverse slices approximately 40 and $60 \mathrm{~mm}$ above the orbito-meatal line were chosen. A standard template was prepared by drawing ROIs over corresponding brain atlas slices (22). The ROIs included, in the lower transwerse slice, frontal, anterior and posterior cingulate, anterior temporal, posterior temporal, calcarine and occipital cortex, as well as caudate, putamen and thalamus. The ROIs of the higher transverse slice contained frontal, anterior and posterior cingulated, parietal and occipital cortex. Regional count densities were normalised by proportional scaling to whole blood brain blood flow. Regional activity was normalised by dividing raw values by global brain activity (global scaling). Detailed information about template processing and reliability of this ROI analysis are published previously (19). For the voxel-based 
analysis, a Gaussian smoothing kernel of $12 \mathrm{~mm}$ was employed to increase signal to noise ratio. The normalisation method used was scaling to whole brain activity. A within-subjects statistical analysis was then performed, using Statistical Parametric Mapping (SPM 96, Wellcome Department of Cognitive Neurology, London, UK) (23).

Blood Sampling and analysis. Blood samples were collected on ice from a cannula inserted into a peripheral vein prior to the simulated bleed ( 0 hours), and 2 and 4 hours thereafter. Plasma was obtained by centrifugation and was deproteinised with trichloroacetic acid $(50 \% \mathrm{w} / \mathrm{v})$ prior to storage $\left(-80^{\circ} \mathrm{C}\right)$, for spectrophotometric determination of ammonia by standard enzymatic methods (CobasMiraS, HoffmanLaRoche, Switzerland) and amino acids by HPLC technique (24).

Statistics. All the clinical and biochemical data were expressed as median and range. We tested for changes in ammonia concentration and neuropsychological function by ANOVA with Bonferroni correction (2-tailed, $\mathrm{p}<0.05$ ). ROI analyses were done with planned (repeated measures) $t$-tests $(P<0.05,2$-tailed) and for the SPM analysis using peak $z$-values with p-values corrected for multiple comparisons. SPM significance at cluster level was based on simultaneous consideration of local effect magnitudes ( $\mathrm{z}$-values), the spatial extent of changes, the overall image smoothness and the total volume examined, and using the theory of Gaussian fields (23). Only regions significant at both voxel and cluster level were accepted. P-values reported are corrected for multiple comparisons. 


\section{RESULTS}

All patients completed the study successfully. None of the patients showed any overt change in the mental state during or after the study period. Significant deterioration was observed in the immediate and delayed story recall subtests of the Randt battery. No significant changes were observed in the Trails B Test, DSST, reaction time or repeating numbers subtests of the Randt test battery (Table 1 ). There was no significant differences in the visual analogue scale for fatigue (Table 1).

Plasma ammonia increased in all patients from a median of 87 (range 67-94) $\mathrm{mmol} / \mathrm{L}$ to $133(122-148) \mathrm{mmol} / \mathrm{L}$ at 2 hours and $105(98-112) \mathrm{mmol} / \mathrm{L}$ at 4 hours after the simulated bleed (Figure 1). The plasma concentrations of most amino acids increased after the simulated bleed (Table 2), with most marked increases in plasma leucine and valine concentrations (approximately 300\%). Only isoleucine concentration decreased after the simulated bleed $(-50 \%)$, resembling the amino acid pattern observed after acute UGI bleeding in patients with cirrhosis of the liver (25).

The ROI analysis of the SPECT data (Figure 2, Table 3) showed significant reduction in regional cerebral perfusion in the region of the left posterior cingulate gyrus $(\mathrm{t}=2.4, \mathrm{p}<0.05)$, the left thalamus $(\mathrm{t}=2.5, \mathrm{p}<0.05)$ and the right anterior cingulate gyrus $(t=2.5, p<0.05)$. Using SPM, significant reduction in regional cerebral perfusion were observed in the right parietal region ( $p=0.02$, corrected for multiple comparisons), left superior temporal gyrus ( $p=0.04$, corrected), left and right temporal lobe ( $p=0.01$, corrected) and the right anterior and posterior cingulate gyrus. There were similarities between the statistical parametric map and region of interest data, as can be seen from Table 3. 


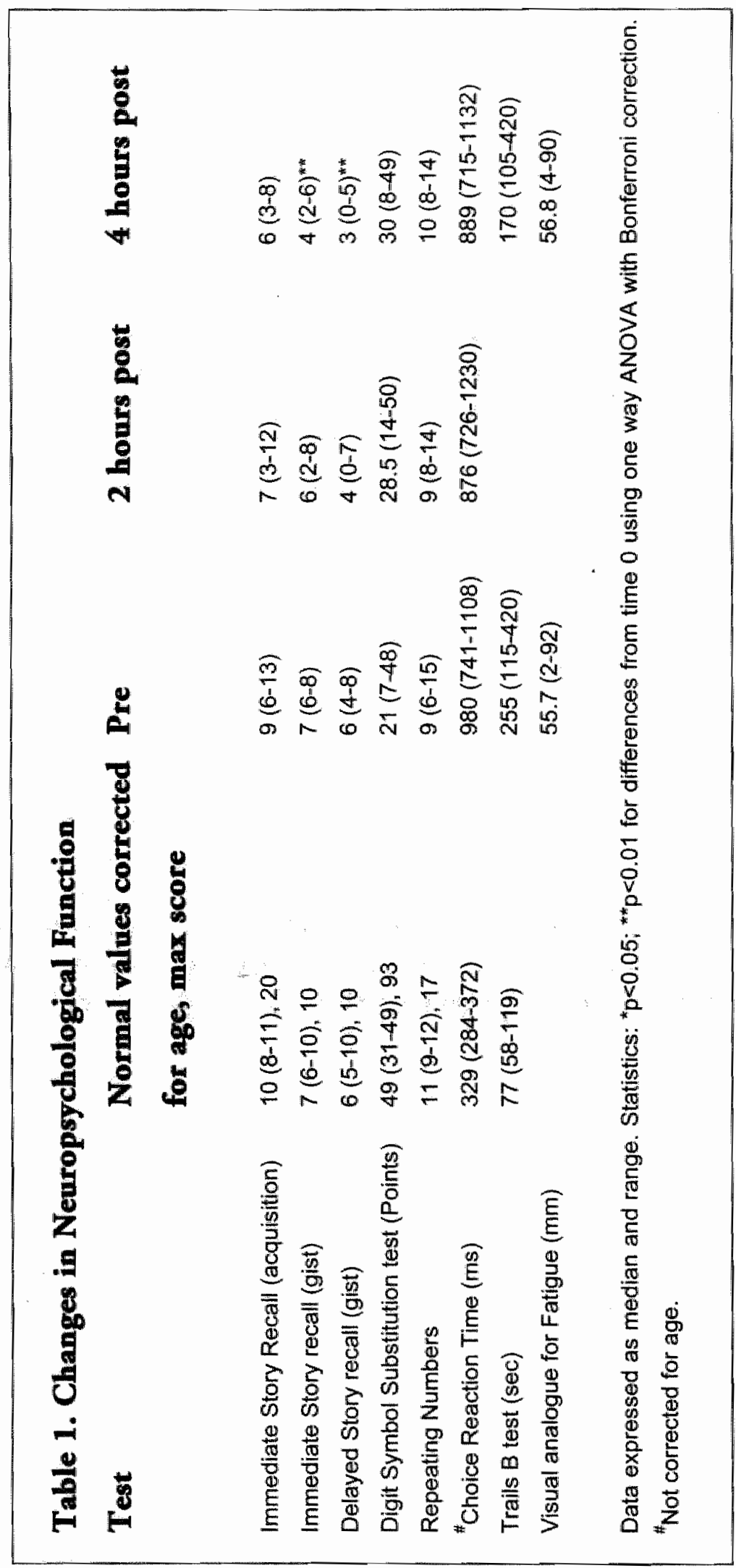


Table 2: Changes in amino acid concentrations following the simulated bleed

$\begin{array}{lccc}\text { Amino acid } & \text { Pre } & \mathbf{t}=\mathbf{2} \text { hours } & \text { t- 4 hours } \\ \text { Glutamate } & 70(58-77) & 86(69-99) & 75(64-85)^{*} \\ \text { Asparagine } & 80(44-91) & 133(97-187) & 87(75-100)^{* *} \\ \text { Serine } & 151(51-199) & 281(190-392) & 215(153-255)^{*} \\ \text { Glutamine } & 524(455-742) & 714(633-958) & 739(610-892)^{*} \\ \text { Histidine } & 90(52-149) & 282(157-346) & 173(173-214)^{* * *} \\ \text { Glycine } & 226(96-354) & 431(331-562) & 326(282-390)^{* *} \\ \text { Threonine } & 205(122-246) & 375(221-497) & 325(299-373)^{* *} \\ \text { Citrulline } & 51(23-72) & 72(21-93) & 79(73-84) \\ \text { Arginine } & 128(85-186) & 188(88-286) & 168(112-218) \\ \text { Alanine } & 314(230-377) & 643(387-915) & 421(299-529)^{* *} \\ \text { Taurine } & 45(25-58) & 41(33-53) & 32(25-36) \\ \text { Tyrosine } & 134(57-163) & 196(147-252) & 212(170-252)^{* *} \\ \text { Valine } & 131(90-387) & 481(336-623) & 421(351-531)^{* * *} \\ \text { Methionine } & 41(23-56) & 65(37-91) & 52(40-64) \\ \text { Isoleucine } & 67(63-75) & 38(36-42) & 35(32-41)^{* * *} \\ \text { Phenylalanine } & 140(99-212) & 292(220-356) & 284(249-327)^{* * *} \\ \text { Tryptophan } & 50(23-65) & 109(76-168) & 113(92-139)^{* *} \\ \text { Leucine } & 77(46-184) & 304(197-375) & 244(182-306)^{* * *} \\ \text { Ornithine } & 88(65-94) & 154(70-166) & 165(135-202)^{* * *} \\ \text { Lysine } & 221(87-279) & 404(304-506) & 304(225-371)^{* *}\end{array}$

Amino acid concentration are in $\mu \mathrm{M}$, Data expressed as median and range. Statistics: ${ }^{*} p<0.05,{ }^{* *} p<0.01,{ }^{* * *} p<0.001$. 
Table 3: ROI data before and after the simulated bleed and significance of difference.

Left

Frontal

Frontal $^{\star}$

Anterior Cingulate

Anterior Cingulate

Anterior Temporal

Middle Temporal

Parietal"

Occipital

Occipital"

Calcarine

Posterior Cingulate

Posterior Cingulate

Caudate

Putamen

Thalamus

Right

Frontal

Frontal

Anterior Cingulate

Anterior Cingulate

Anterior Temporal

Middle Temporal

Parietal ${ }^{\star}$

Occipital

Occipital

Calcarine

Posterior Cingulate

Posterior Cingulate

Caudate

Putamen

Thalamus

\section{Before}

Uptake ratio

$m e a n$ ( D)

$1.03(0.1)$

$1.02(0.1)$

$1.03(0.1)$

$0.98(0.3)$

$1.05(0.1)$

$1.17(0.1)$

$1.11(0.04)$

$1.13(0.1)$

$1.13(0.1)$

$1.20(0.2)$

$0.99(0.2)$

$1.26(0.1)$

$1.19(0.2)$

$1.42(0.1)$

$1.30(0.1)$

\section{Before}

Uptake rati

mean (SD)

$1.05(0.04)$

$1.02(0.1)$

$1.00(0.1)$

$1.06(0.2)$

$1.10(0.1)$

$1.17(0.1)$

$1.12(0.05)$

$1.14(0.1)$

$1.11(0.1)$

$1.13(0.2)$

$0.95(0.2)$

$1.27(0.1)$

$1.26(0.1)$

$1.34(0.1)$

$1.20(0.1)$
After

T-test

vptake ato

m ean (SD)

Pvalue

$1.06(0.1)$

0.42

$1.04(0.1)$

0.48

$0.98(0.1)$

0.43

$0.99(0.2)$

0.87

$1.05(0.1)$

0.97

$1.13(0.1)$

0.12

$1.14(0.1)$

0.33

$1.14(0.1)$

0.88

$1.11(0.1)$

0.64

$1.18(0.2)$

0.61

$0.89(0.2)$

$0.047^{*}$

$1.35(0.1)$

0.21

$1.09(0.3)$

0.11

$1.38(0.1)$

0.43

$1.17(0.1)$

$0.04^{*}$
T-test

Uptake rato

mean $(\mathrm{SD}$ )

P value

$1.04(0.1) \quad 0.84$

$1.03(0.1) \quad 0.17$

$1.01(0.1) \quad 0.81$

$0.96(0.2) \quad 0.04^{*}$

$1.06(0.1) \quad 0.07$

$1.12(0.1) \quad 0.07$

$1.11(0.1) \quad 0.68$

$1.11(0.1) \quad 0.33$

$1.12(0.1) \quad 0.93$

$1.24(0.2) \quad 0.16$

$0.99(0.3) \quad 0.44$

$1.28(0.2) \quad 0.95$

$1.20(0.2) \quad 0.20$

$1.38(0.1) \quad 0.38$

$1.17(0.1) \quad 0.61$

"Upper transaxial slice (60 $\mathrm{mm}$ above orbital-meatal line); all unmarlked ROIs are from transaxial slice at $40 \mathrm{~mm}$ above the orbital-meatal line. Statistics: " $p<0.05$. 


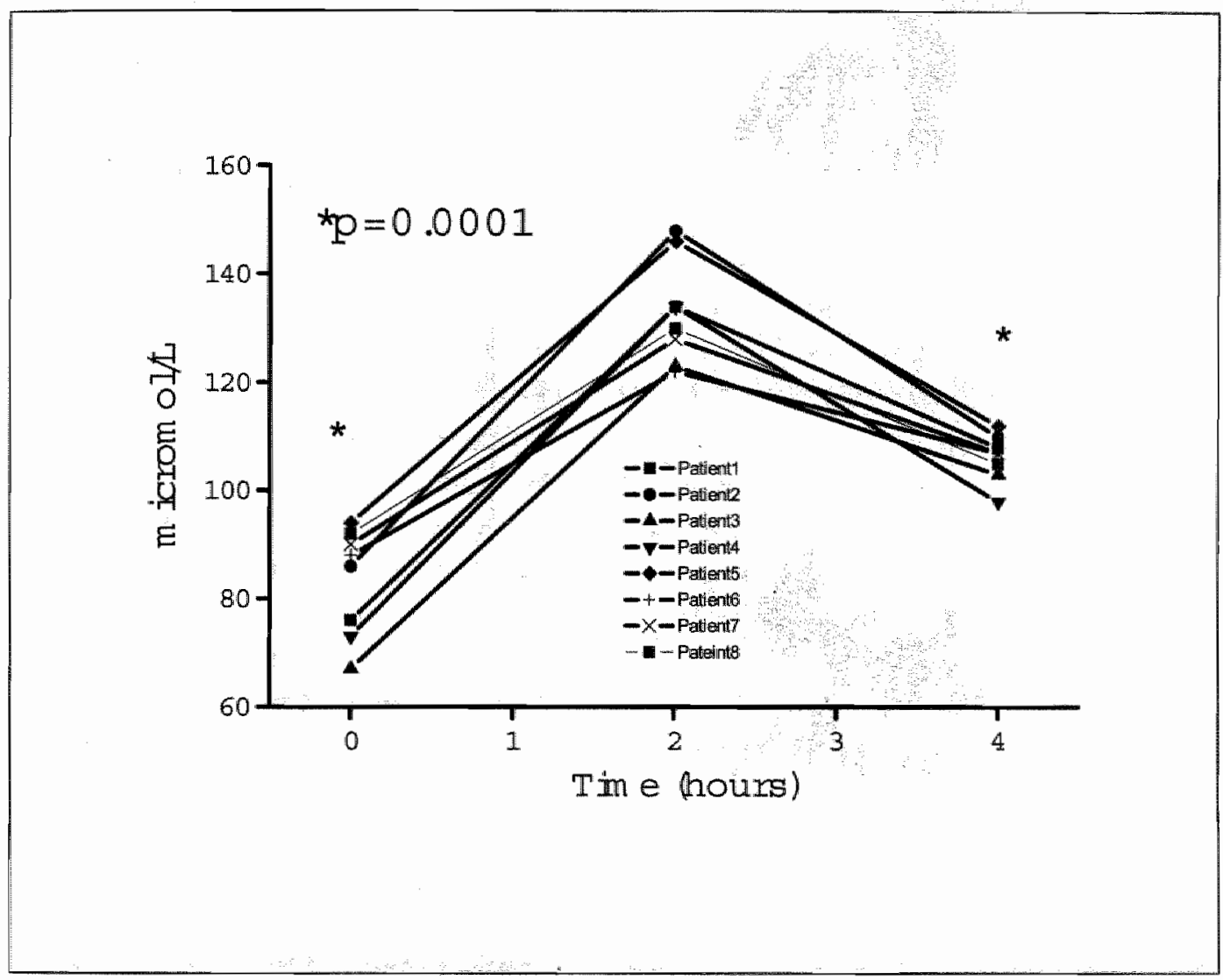

FIGURE 1. Changes in plasma venous ammonia concentrations following the simulated bleed. Statistics * $\mathrm{p}<0.05$. 


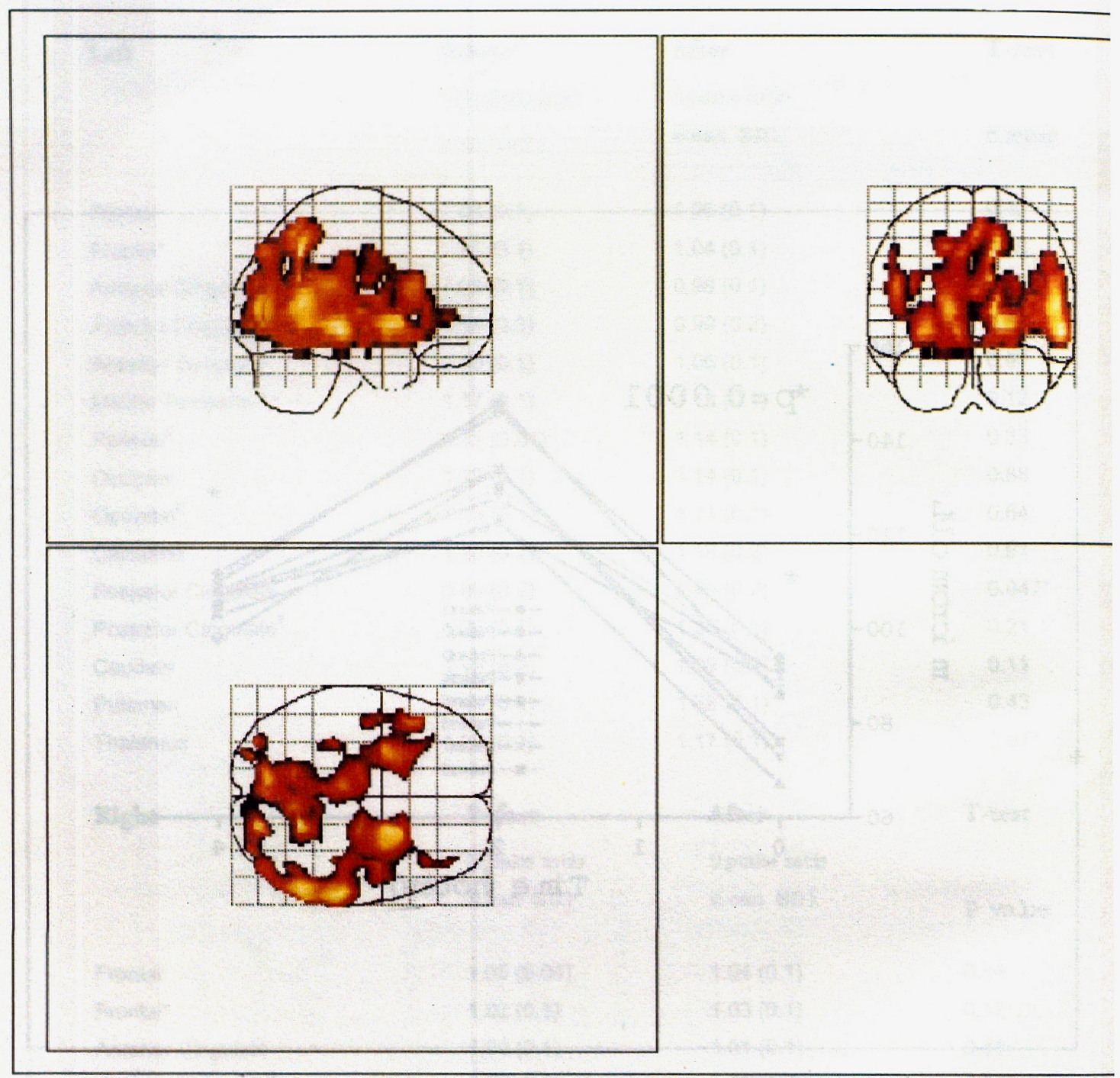

FIGURE 2. Maximum intensity projection of voxels with $Z>2.33(\mathrm{p}<0.01)$ for the contrast of (higher) pre-c to (lower) post-drink values. Four significant clusters of $8.5 \mathrm{~cm} 3,3 \mathrm{~cm} 3,31 \mathrm{~cm} 3$ and $26 \mathrm{~cm} 3$ emerged. regions affected were right parietal lobe $(22,-42,40)$, left middle frontal gyrus $(-28,12,48)$, right post-ce gyrus (58,-28, 43), and right anterior cingular gyrus (2, 20, 40). SPM: Statistical Parametric Mapping; $H_{1}$ threshold $\{u\}=2.33, p<0.01$; Extent threshold $\{k\}=125$ voxels, $p<0.01$ (corrected); [effectively $<0.001$ (ur rected)], Expected voxels per cluster, $E(n)=125.1$; Expected number of clusters, $E(m)=1.4$; Volume $(S)=61$ voxels or 114.7 Resels; Degrees of freedom due to error $=7.0$; Smoothness $(\mathrm{mm})=19.8,21.0,20.4$; Voxels $10.5,5.1$ 


\section{DISCUSSION}

This study clearly shows that administration of an oral amino acid load mimicking haemoglobin to simulate the metabolic consequences of an upper gastrointestinal bleed in parients with cirrhosis is followed by hyperammonemia and hypoisoleucinemia and results in a reduction in regional cerebral perfusion and neuropsychological deterioration.

In patients with cirrhosis, ingestion of erythrocytes produces a larger increase in ammonia than ingestion of an isonitrogenous amount of whole blood, which produces greater increase in ammonia than ingestion of an isonitrogenous amount of blood plas$\mathrm{ma}$ (26). These findings are explained by the amino acid composition of the haemoglobin protein (27), which is a protein of very low biologic value. Absorbed (blood) amino acids cannot be used for protein synthesis and are degraded producing ammonia, which is released into the systemic circulation (27). Enhanced ammoniagenesis and hypoisoleucinemia was observed in this study as is suggested by the above hypothesis. Although we measured ammonia concentration at 2 and 4 hours after the bolus administration of the simulated bleed, we know from pilot studies that peak ammonia levels may be reached already at 1 hour after the administration of the amino acid solution (28). The simulated UGI bleed produced significant deterioration in the immediate and delayed story recall subtests of the Randt test battery, which tests memory function. This module reflects the ability of the patient to integrate and store new information for immediate and later recall thereby possibly involving semantic aspects of secondary memory. The deterioration in the memory test occurred only at 4 hours with minimal changes at 2 hours after the administration of the amino acid solution suggesting that this may be the result of a prolonged effect of accumulation of ammonia rather than an immediate or peak level effect. These are consistent with the observation that mild hyperammonemia in patients with liver disease increases the permeabilitysurface area product to ammonia and enhances the entry of ammonia into the brain (29). This enhanced uptake might account for the hypersensitivity of cirrhotic patients to ammoniagenic conditions e.g. UGI bleeding, as well as for the presence of cerebral dysfunction in some patients with near-normal ammonia levels.

Oral administration of glutamine has also been shown to induce hyperammonemia and changes in neuropsychological test and electroencephalography (11). In that study, changes in choice reaction time were observed which is at variance with our results. This discrepancy may reflect the different patient population studied, as Oppong et. al. (11) studied patients with more advanced liver disease and all of whom had a history of previous HE. Furthermore, a substantial proportion of these parients had primary biliary cirrhosis and all the patients were being assessed for liver transplantation (11). 
Ideally, the time course of changes in cerebral perfusion should be studied continuously after administration of the amino acid solution but this is not possible because of limitations to the dose of radiation that can be administered to a single patient. The choice of 4 hours was dictated by pilot studies which showed that neuropsychological deterioration was apparent at 4 hours after the simulated bleed and that prolonging the experiment further introduces the confounding effects of disinterest and fatigue on neuropsychological function. Indeed, the lack of any significant changes in the visual analogue scale for fatigue suggests that the observed changes in memory function were independent of fatigue. Nevertheless, the change in regional cerebral perfusion at 4 hours after administration of the amino acid solution, and simultaneous deterioration in neuropsychological function is consistent with the hypothesis that hyperammonemia produces changes in regional cerebral perfusion, which in turn results in neuropsychological deterioration.

The multifocal pattern of hypoperfusion is clearly shown in the SPM figure, which shows the specific cerebral sites of abnormal perfusion after corrections are made for multiple comparisons. This abnormal flow partern support the view that $\mathrm{HE}$ is multifocal (5) as opposed to a global disorder of corrical function. It is notable that significant reduction in perfusion was noted in the cingulate gyrus where previous studies of cerebral perfusion and metabolism have consistently demonstrated abnormalities (4-6). In the study of Catafau et. al., (9), the anterior cingulated gyrus was not separately studied but was included in the prefrontal ROI, which showed a significantly reduced flow pattern. Interestingly, in both SPECT studies from our group the right anterior cingulate gyrus was affected, as opposed to the left (6). The cingulate gyrus plays an important role in the attentional system (30). The pattern of increased blood flow and metabolism in basal ganglia reported in circhotics with various degrees of encephalopathy could not be sliown in the present study. However, the hypoperfusion of the thatamus could influence the function of the basal ganglia-thalamocortical circuits. There is no model of absolute regional cerebral blood flow using HM-PAO SPECT; as compared with e.g. H2O-PET. It is, therefore not possible to comment on absolute changes in blood fow, but only changes in perfusion pattern.

It is important to recognise that this study is in patients with well compensated cirrhosis and the change in plasma ammonia concentration is modest and within the parhophysiological range for patients with moderately severe chronic liver disease, because changes in cerebral blood flow with acute ammonia infusion in rats with portacaval shunts or the hyperammonemia present in patients with acute liver failure produces the opposite result, i.e. increased cerebral blood flow (31-37). It is likely that pathophysiological changes occurring in the acute and the chronic situations are different. The possible mechanism of the reduced regional cerebral perfusion observed in the present study may be due to the inhibitory effect of glutamine on nitric oxide synthesis. According to this hypothesis, ammonia is detoxified in the astrocytes by gluta- 
mine synthetase to glutamine. Increase in brain ammonia and glutamine have been observed in rats with portacaval shunt given an erythrocyte meal (12). Although glutamine is a neutral amino acid with no known vascular receptors, it might exert indirect effects. In cultured endorhelial cells, glutamine but not ammonia inhibits agonistinduced nitric oxide release by interacting with arginine in the signal transduction pathway linked to nitric oxide synthase (38), or possibly by inhibiting citrulline uptake (39), and recycling of citrulline to arginine (40-42). In vitro studies using pial arteries from pigs have suggested that the dilator nerves that release nitric oxide may play a predominant role in regulating porcine pial vessel tone (43). Regional variations in cerebral perfusion observed in the current study are predicted by this hypothesis, as patients with HE have differential glutamine concentrations in different brain regions (44).

In conclusion, the results of this study support the hypothesis that hyperammonemia due to administration of an oral amino acid load mimicking haemoglobin to simulate the metabolic consequences of an upper gastrointestinal bleed produces neuropsychological deterioration that is associated with reduced regional cerebral perfusion. Further studies should address the mechanism by which ammonia may alter regional cerebral perfusion, determine whether these changes are dose-dependent and whether these changes can be reversed by treatment. 


\section{REFERENCES}

1. Quero JC, Schalm SW. subclinical Hepatic Encephaloparhy. Seminar in Liver Disease $1996 ; 16: 343-328$.

2. Hazel AS, Butterworth RF. Hepatic encephalopathy. Proc Soc Exp Biol Med $1999 ; 222: 99-112$.

3. Groeneweg M, Quero JC, De Bruijn I, Hartmann IJ, Essink-bot ML, Hop WC, et al. Subclinical hepatic encephalopathy impairs daily functioning. Hepatology 1998;28:459.

4. Lockwood AH, Yap EW, Rhoades HM, Wong WH. Altered cerebral blood flow and glucose metabolism in parients with liver disease and minimal encephalopathy. I Cereb Blood Flow Metab 1991;11:331-6.

5. Lockwood AH, Murphy BW, Donnelly KZ, Mahl TC, Perini S. Positron-emission tomographic localization of abnormalities of brain merabolism in patients with minimal heparic encephalopathy. Hepatology 1993;18:1061-8.

6. O'Carroll RE, Hayes PC, Ebmeier KP, Dougall N, Murray C, Best JJ, et al. Regional cerebral blood flow and cognitive funcrion in patients with chronic liver disease. Lancet $1991 ; 337: 1250-3$.

7. Trzepacz PT, Tarter RE, Shah A, Tringali R, Faett DG, Van Thiel DH. SPECT scan and cognitive findings in subclinical hepatic encephalopathy. I Neuropsychiatry Clin Neurosci 1994:6:170-5.

8. Dam M, Burra P, Tedeschi U, Cagnin A, Chierichetti F, Ermani M, et al. Regional cerebral blood flow changes in parients with cirrhosis assessed with $99 \mathrm{~m}$ Tc-HM-PAO singlephoton emission computed tomographyt effect of liver transplantation. J Hepatol $1998 ; 29: 78-84$

9. Catafau AM, Kulisevsky J, Berna L, Pujol J, Martin JC Otermin $\mathbb{C}_{*}$ et al. Relationship berween cerebral perfusion in frontal-limbic-basal ganglia circuits and neuropsychologic impaiment in partents with subclinical hepatic encephalopathy. I Nucl Med 2000;41:405-10.

10. Butterworth RF. The neurobiology of hepatic encephalopathy. Semin Liver Dis $1996 ; 16: 235-44$.

11. Oppong KN, Al-Mardini H, Thick M, Record CO. Oral glutamine challenge in cirthotics pre- and post-liver transplantation: a psychometric and analyzed EEG study. Hepatology 1997;26:870-6.

12. Olde Damink SWM, Dejong CHC, Deutz NEP, Soeters PB. Effects of simulated upper gastrointestinal hemorrhage on ammonia and related amino acids in blood and brain of chronic portacaval-shunted rats. Metabolic Brain Disease 1997;12:121-135.

13. Pugh RN, Murray-Lyon IM, Dawson JL, Pietroni MC, Williams R. Transection of the oesophagus for bleeding oesophageal varices. Br J Surg 1973;60:646-9. 
14. Hill RJ, Koningsberg W. The strucrure of human hemoglobin. the Journal of Biological Chemistry 1962;237:3151-3156.

15. Davies $\mathrm{AD}$. The influence of age on trail making test performance. J Clin Psychol 1968;24:96-8.

16. Hindmarch I. Psychomotor function and psychoactive drugs. Br J Clin Pharmacol 1980;10:189-209.

17. Frith CD, Leary J, Cahill C, Johnstone EC. Performance on psychological tests. Demographic and clinical correlates of the results of these tests. Br J Psychiatry Suppl $1991: 26-9,44-6$.

18. Randt CT, Brown ER, Osborne DPJ. A memory test for longitudinal measurement of mild to moderate defects. Clinical Neuropsychology 1980;1I:184-197.

19. Ebmeier KP, Dougall NJ, Austin MP, al. e. The split-dose technique for the study of psychological and pharmacological activarion with the cerebral blood flow marker exametazine and single photon emission computed tomography (SPECT): reproducibility and reliability. International Journal of Methods in Psychiatric Research 1991;i:27-38.

20. Ebmeier KP, Cavanagh JT, Moffoot AP, Glabus MF, O'Carroll RE, Goodwin GM. Cerebral perfusion correlates of depressed mood. Br J Psychiatry 1997;170:77-81.

21. Ebmeier KP, Steele JD, MacKenzie DM, O'Carroll RE, Kydd RR, Glabus MF, et al. Cognitive brain potentials and regional cerebral blood flow equivalents during two- and three-sound auditory "oddball tasks". Electroencephalogr Clin Neurophysiol $1995 ; 95: 434-43$.

22. Talairach J, Tournoux P. Co-planar stereotaxic atlas of the human brain. Stuttgart: George Thieme Verlag 1988.

23. Ebmeier KP Glabus MF, Prentice N, Ryman A, Goodwin GM. A voxel-based analysis of cerebral perfusion in dementia and depression of old age. Neuroimage 1998;7:199208.

24. van Eijk HM, Rooyakkers DR, Soeters PB, Deutz NE. Determination of amino acid isotope enrichment using liquid chromatography-mass spectrometry. Anal Biochem $1999 ; 271: 8-17$.

25. Dejong CHC, Meijerink WJHJ, van Berlo CLH, Deutz NEP, Soeters PB. Decreased plasma isoleucine concentracions after upper gastrointestinal haemorrhage in humans. Gut 1996;39:13-17.

26. Bessman $A N_{*}$ Hawkins $R$. The relative effects of enterically administered plasma and packed cells on circullating blood ammonia. Gastroenterology 1963:45:368-373.

27. Olde Damink SWM, Dejong CHC, Deutz NEP, van Berlo CLH, Soeters PB. Upper gastrointestinal bleeding: an ammoniagenic and catabolic event due to the total absence od isoleucine in the haemoglobin molecule. Medical Hypotheses 1999;52:515-519.

28. Olde Damink SWM, Jalan $R$, Deutz NEP, Hayes PC, Soeters PB. Simulation of an upper gastrointestinal bleed in patients with cirrhosis of the liver: a model to study the metabolism of hepatic encephalopathy. Hepatology 1998;28:544A (abstract). 
29. Lockwood AH, Yap EW, Wong WH. Cerebral ammonia metabolism in patients wit severe liver disease and minimal hepatic encephalopathy. J Cereb Blood Flow Metal $1991 ; 11: 337-41$.

30. Posner MI, Driver J. The neurobiology of selective artention. Curr Opin Neurobio $1992: 2: 165-9$.

31. Dempsey RJ, Kindt GW. Experimental acute hepatic encephalopathy: relationship of pathological cerebral vasodilation to increased intracranial pressure. Neurosurgery $1982 ; 10 \% 737-41$.

32. Larsen FS, Adel Hansen B, Pott F, Ejlersen E, Secher NH, Paulson OB, et al. Dissociated cerebral vasoparalysis in acute liver faillure. $A$ hyporhesis of gradual cerebral hyperaemia. J Heparol 1996;25:145-51.

33. Durham S, Yonas H, Aggarwal S, Darby J, Kramer D. Regional cerebral blood flow and CO2 reactivity in fulminant hepatic failure. J Cereb Blood Flow Metab 1995;15:329-35.

34. Wendon JA, Harrison PM, Keays R, Williams R. Cerebral blood flow and metabolism in fulminant liver failure. Hepatology 1994;19:1407-13.

35. Aggarwal S, Kramer D, Yonas H, Obrist W, Kang Y, Martin M, et al. Cerebral hemodynamic and metabolic changes in fulminant hepatic failure: a retrospective study. Hepatology 1994;19:80-7.

36. Jalan R, Olde Damink SWM, Deutz NEP, Lee A, Hayes PC. Moderate hypothermia for uncontrolled intracranial hypertension in acute liver failure. Lancet 1999;354:1164-8.

37. Master S, Gottstein J, Blei AT. Cerebral blood flow and the development of ammoniainduced brain edema in rats after portacaval anastomosis. Hepatology 1999;30:876-80.

38. Amal JF, Munzel T, Venema RC, James NL, Bai CL, Mitch WE, et al. Interactions between L-arginine and L-glutamine change endothelial NO production. An effect independent of NO synthase substrate availability. J Clin Invest 1995,95.2565-72.

39. Wu $\mathrm{G}$, Methinger $\mathrm{CJ}$, Regulation of L-arginine synthesis from L-citrulline by L-glutamine in endothelial cells. Am J Physiol 1993,265:H1965-71.

40. Hecker M, Mitchell JA, Swierkosz TA, Sessa WC, Vane JR. Inhibition by L-glutamine of the release of endothelium-derived relaxing factor from cultured endothelial cells. Br J
Pharmacol $1990 ; 101: 237-9$.

41. Hecker M, Sessa WC, Harris H], Anggard EE, Vane JR. The metabolism of L-arginine and its significance for the biosynthesis of endothelium-derived relaxing factor: cultured endothelial cells recycle L-citrulline to L-arginine. Proc Nat Acad Sci U S A
1990;87:8612-6.

42. Sessa WC, Hecker M, Mitchell JA, Vane JR. The metabolism of L-arginine and its significance for the biosynthesis of endothelium-derived relaxing factor: L-glutamine inhibits the generation of L-arginine by cultured endothelial cells. Proc Natl Acad Sci U
$S$ A 1990;87:8607-11.

43. Ishine $T, Y u$, J, Asada $Y$, Lee TJ. Nitric oxide is the predominant mediator for neurogenic vasodilation in porcine pial veins. I Pharmacol Exp Ther 1999;289:398-404 
44. Taylor-Robinson SD, Sargentoni J, Marcus CD, Morgan MY, Bryant DJ. Regional variations in cerebral proton spectroscopy in patients with chronic hepatic encephalopathy. Metabolic Brain Disease 1994;9:347-359. 



\section{CHAPTER 4B}

INDUCED HYPERAMMONEMLA ALTERS NEUROPSYCHOLOGY, BRAIN MR SPECTROSCOPY AND MAGNETIZATION TRANSFER IN CIRRHOSIS

${ }^{1}$ Sherzad Balata, ${ }^{1,2}$ Steven WM Olde Damink, ${ }^{3}$ Karen Ferguson, ${ }^{3}$ Ian Marshall, 1 Peter C Hayes, ${ }^{2}$ Nicolaas EP Deutz, ${ }^{5}$ Roger Williams, ${ }^{3}$ Joanna Wardlaw, 1,5 Rajiv Jalan ${ }^{1}$ Liver Unit, Royal Infirmary of Edinburgh, Edinburgh, ${ }^{2}$ Dept of Surgery, Academic Hospital, Maastricht University, The Netherlands, ${ }^{3}$ Dept of Medical \& Radiological Sciences, Western General Hospital, Edinburgh, ${ }^{4}$ Department of Medical Physics, University of Edinburgh, 5 Liver Failure Group, Institute of Hepatology, University College London Medical School and University College London Hospitals, UK

Published in Hepatology 2003; 37: 931-939 


\section{INTRODUCTION}

Ammonia is thought to be central in the pathogenesis of hepatic encephalopathy (HE) (1-4). It is well known that an upper gastrointestinal (UGI) bleed in cirrhotic patients produces hyperammonemia and precipitates HE (1-6). Simulating the metabolic effects of an UGI bleed by oral administration of erythrocytes has been shown to induce hyperammonemia and behavioural disturbance in portacaval shunted rats (7). In an uncontrolled study, we showed that oral administration of an amino acid (aa) solution that is identical to the amino acid profile of haemoglobin results in deterioration in memory tests suggesting that this may be a clinically valid model to study the pathogenesis of $\mathrm{HE}(8)$.

Although the data relating arterial ammonia concentration to the severity of $\mathrm{HE}$ are controversial there is growing evidence that higher arterial ammonia concentrations are observed in more severe states of $\operatorname{HE}(3,4,9)$. Indeed in a recent study in patients with acute liver failure, an arterial concentration of ammonia of greater than $150 \mathrm{mM}$ was predictive of future brain herniation in patients with acute liver failure (3). Recently, administration of the amino-acid glutamine has been used to induce hyperammonemia and study neuropsychological alterations in patients with cirrhosis, but the clinical relevance of this model is difficult to determine (10).

$\mathrm{HE}$ is characterized neuropathologically by Alzheimer Type II astrocytosis and the finding that similar changes can be induced experimentally in astrocytes in culture following exposure to ammonia suggests that HE is primarily due to a dysfunction of these cells (11). Astrocytes are the site of ammonia detoxification in the brain and eliminate ammonia by the synthesis of glutamine through amidation of glutamate (12). The ammonia-glutamine-brain swelling hypothesis of HE suggests that the accumulation of glutamine in the astrocytes induced by hyperammonemia produces an osmotic stress and causes astrocytes to swell and dysfunction $(13,14)$.

Overt brain swelling and increases in intracranial pressure are characteristic of patients with acute liver failure but have also been reported to occur in the context of cirrhosis of the liver (15). Magnetization transfer imaging is an application of magnetic resonance imaging designed as a means of exploring characteristics of non-water components in tissue. Compared with conventional MR imaging, where differences in brightness on images reflect differences in observed relaxation times, magnetization transfer imaging theory incorporates the influence of additional parameters, specifically those reflecting the exchange of protons between molecules of water and molecules of more-solid, structural components. Thus, magnetization transfer imaging offers the potential of a window on tissue structure, and structural components that are normally not resolvable with MRI (16-19). This technology has been extensively used to study 
patients with multiple sclerosis and is based upon the principle that myelin is a rigid macromolecular structure and that macromolecular protons can be saturated by using off-resonance $(18,19)$. These protons then exchange with free-water protons and produce a decrease in signal intensity of the free-water protons. Thus, stimuli such as changes in the state of activation, blood flow, oxygenation, neuronal polarity or brain water may change magnetization ratio rapidly (16-20). Indeed, in experimental animals, a reduction in magnetization transfer ratio (MTR) can be induced within a half hour of induction of a stress that alters the tissue-to-water interface $(21,22)$. Cordoba et al. have recently demonstrated that patients with minimal HE have reduced MTR and suggest that this may represent increased brain water (13).

The present study was performed to determine whether induction of hyperammonemia by oral administration of an amino-acid solution mimicking the composition of the haemoglobin molecule would produce neuropsychological deterioration, an increase in brain glutamine (using proton magnetic resonance spectroscopy (MRS)) and a reduction in MTR (indicating an increase in brain water). 


\section{METHODS}

Studies were undertaken with the approval of the local research ethics committee, the written informed consent from each patient, and in accordance with the Declaration of Helsinki (1989) of the World Medical Association.

Patients. Patients were recruited for two separate studies, which were performed consecutively. Patients were included in the study if they had biopsy proven cirrhosis and no history of 'overt' HE. Patients were excluded if they had clinical evidence of HE (G), diabetes, clinically evident cardiovascular disease, renal dysfunction (serum creatinine $>100 \mathrm{mmol} / \mathrm{L}$ ), any other neurological illness, malignancy, or pregnancy. Patients had to be abstinent from alcohol and benzodiazepines for at least 1 month prior to the study. All the patients were metabolically stable and were studied after an overnight fast. Patient details are summarised in Table 1 .

Table 1. Patient Characteristics.

\begin{tabular}{lll}
\multicolumn{2}{c}{ STUDY 1 } & STUDY 2 \\
PART 1 & PART 2 & \\
Amino-acid $(\mathrm{N}=24)$ & Placebo $(\mathrm{N}=24)$ & Amino-acid $(\mathrm{N}=7)$
\end{tabular}

Age (years)

Sex

Aetiology of cirrhosis

Alcoholic cirrhosis

Hepatitis C Virus

Cryptogenic

PSC

PBC

Pugh score

Severity

Child A

Child B

Child $C$

Previous HE

Ascites

Body Mass Index

Oesophageal Varices

Previous portosystemic

shunt (TIPSS)
$53.7(2.5)$

$16 \mathrm{M} / 8 \mathrm{~F}$

18

3

1

2

$8.5(0.4)$

2

16

6

None

20

$27(1.2)$

22

3

$50.7(2.4)$

$49(2.9)$

$18 \mathrm{M} / 6 \mathrm{~F}$

$5 \mathrm{M} / 2 \mathrm{~F}$

6

1

2

1

2

$8.2(0.3)$

$7.9(0.4)$

4

17

5

32

None None

$18 \quad 5$

$26.1(0.9)$

$29.2(1.4)$

20

All

4

1 


\section{STUDY 1}

Part 1. Forty-eight patients were randomised into two groups; one group received the amino-acid solution and the other placebo. Changes in neuropsychological function prior to and following administration of the aa solution or placebo were measured in all the patients. For our power calculation we assumed a $40 \%$ difference in neuropsychological function between the groups administered the amino-acid-solution and those administered placebo. Using this assumption we would need to randomise 48 patients if the $a$ is 0.05 and the $b$ is 0.10 .

Part 2. The first 16 of the patients that were randomised in study 1 also underwent proton MR spectroscopy. Eight patients received the aa solution and 8 the placebo solution.

Randomisation of Study 1 was performed by the closed envelope method in batches of 16 , which was generated by random numbers. Both the investigator and also the patient were blinded to the content of the solution that was administered.

\section{STUDY 2}

Seven cirrhotic patients were studied in this second study and underwent measurement of MTR to determine brain water in specified cerebral regions. MTR was measured prior to and 4 hours after administration of the aa solution. The patient characteristics are described in Table 1. The baseline data were compared with those obtained from 10 age-matched healthy volunteers [mean age $46( \pm 4.3)$ ] years.

Oral amino acid load. Simulation of the UGI bleed was by administration of an oral bolus of 75 gram of a specifically prepared solution (Nutricia Cuijk, The Netherlands, Product Number:24143) that mimics the amino acid composition of the haemoglobin molecule [Table 2] (23). The solution was freshly made in $200 \mathrm{ml}$ of water and xanthan gum was added to prevent sedimentation. The placebo solution comprised of water and xanthum gum.

Table 2: Amino acid composition of the simulated bleed

\begin{tabular}{llllll}
\hline Leucine & 99.8 & Glutamate & 33.3 & Aspartate & 41.6 \\
Isoleucine & 0 & Asparagine & 27.7 & Alanine & 99.8 \\
Valine & 85.9 & Glutamine & 11.1 & Cysteine & 8.3 \\
Glycine & 55.4 & Methionine & 8.3 & Serine & 44.4 \\
Tryptophan & 8.3 & Arginine & 16.6 & Phenylalanine & 41.6 \\
Threanine & 44.4 & Tyrosine & 16.6 & Histidine & 52.7 \\
Lysine & 61.0 & Proline & 38.8 & & \\
\hline
\end{tabular}

Expressed as mmol per 100 gram amino acid solution 
Measurement of neuropsychological function. Detailed clinical neurological examination was performed to exclude evidence of clinically overt $\mathrm{HE}$ and any focal neurology. A construct-driven neuropsychological test battery was designed. A critical factor in developing this battery was the total time period needed to complete the battery, so it could be used as a tool to assess changes in neuropsychological function in response to the simulated UGI bleed. The following cognitive domains were tested: concentration, memory, visuospatial-construction skills and motor function. The neuropsychological test battery consisted of the Trails B Test (24), the Digit Symbol Substitution Test (25), the immediate story recall subtest of the Randt test battery (26) and choice reaction time (27). Total time to perform this battery was less than 20 minutes.

Trails B test. This test is a derivative of the Trail Making Test and measures visual conceptual and visuomotor tracking. The test has to be completed within 420 seconds (24).

The Digit Symbol Substitution Test. The test is part of the Wechsler Adult Intelligence Schedule (25). This test is used to assess visuomotor coordination and vigilance. The test score was the number of symbols correctly substituted in 90 seconds.

Reaction time: This test is part of the Continuous Performance Task (26) and measures motor function, sustained concentration and the ability to suppress inappropriate responses. The task was to press the space bar of the computer as quickly as possible every time the letter $\mathrm{E}$ appeared, except when it was immediately preceded by the letter X. The mean reaction time, the standard deviation for the mean reaction time and the number of observations were recorded.

Immediate Story recall subtest of the Randt memory test. This subtests measures immediate memory function (27). This subject is asked to recall twenty words from a paragraph read to him that has an emotionally charged substance and includes fire and disaster. One point was awarded for each word that was recalled immediately after presentation (acquisition).

The test battery was performed immediately before the administration of the oral aa load and 2 and 4 hours afterwards (except Trails B test, only at 0 and 4 hours), so that the patients finished 20 minutes before the final scan was taken. All the neuropsychological tests were performed by one of two investigators (SB, RJ), in which the same investigator assessed each parient. All patients had one practice session of each test. All the tests have been well validated and parallel forms were used.

Magnetic Resonance. Magnetic resonance imaging and spectroscopy were carried out on an Elscint Prestige scanner (GE Medical, Haifa, Israel) in the SHEFC Brain Imaging Research Centre for Scotland, operating at 1.9 Tesla.

Magnetic Resonance Spectroscopy. The patients underwent proton magnetic resonance spectroscopy immediately prior to and four hours after administration of the aa 
solution or placebo in order to determine changes in the brain osmolytes glutamine and myoinositol. Part of the reason for using the randomised design was to counteract the inherent variability of the technique. We also acquired MRS data prior to and after the administration of the aa solution (instead of just after) to further counter the variability effects. Using T2-weighted axial images for positioning, we acquired PRESS-localized spectra from $15 \mathrm{~mm}$ cubical volumes of interest (VOIs) in the left basal ganglia and the left occipital cortex. The repetition time was $1500 \mathrm{~ms}$, and the echo time was $36 \mathrm{~ms}$, the shortest that our scanner would support. Following localized shimming and water suppression calibration for each VOI, 200 acquisitions with water suppression were collected. Eight acquisitions without water suppression were also collected to serve as a phase reference. The VOI positions within the head coil were noted, as was the scanner radiofrequency calibration figure to allow for the effects of head coil loading. Spectroscopy data were transferred to a Sun workstation for analysis. Analysis consisted of phase correction using the water reference data (28), and removal of the residual water signal using Hankel-Lanczos singular value decomposition (HLSVD; Boogaart). Spectral peak areas were quantified using the AMARES method within the Magnetic Resonance User Interface software (MRUI, Lyon, France) (29). A model consisting of 12 Gaussian peaks was developed with reference to in vitro measurements on metabolites, in vivo measurements on healthy volunteers, and literature values for peak assignments. Three observers, who were blinded for the treatment protocol, studied the fitted results independently. The observers discussed any peak assignments on which they did not all agree. In this way, a consensus was reached for all the spectra in the study. The following cerebral metabolites were quantified: ( $\mathrm{N}$-acetyl aspartate (NAA), Choline (Cho), Creatine (Cr), Myoinositol (mI), Glutamine and Glutamate (Glx). Finally, peak areas for each subject were corrected for head coil loading and VOI position within the head coil. The resulting "institutional" units enabled intersubject comparisons.

Magnetization Transfer Ratio. The magnetization transfer study was performed in transversal plane with a two-dimensional gradient-echo pulse sequence (714/12/20/1=TR/TE/flip angle/acquisitions) as has been described previously (30, $31)$. In the patients and the healthy volunteers, MTR values of the normal-appearing white matter were measured in four different supratentorial locations, one in each of the frontal and parietal lobes of each hemisphere. The mean value of the MTRs within selected areas was obtained by averaging the pixel values in the regions of interest on the MTR map $(30,31)$.

Blood Sampling and Analysis. Blood samples were collected on ice from a cannula inserted into a peripheral vein prior to administration of the aa solution or the placebo ( 0 hours), and 2 and 4 hours thereafter. Plasma was obtained by centrifugation and was deproteinised with trichloroacetic acid $(50 \% \mathrm{w} / \mathrm{v})$ for ammonia determination and 
sulphosalicylic acid for determination of amino acids. Plasma was stored at $-80^{\circ} \mathrm{C}$ for spectrophotometric determination of ammonia (CobasMiraS, Hoffman-LaRoche, Switzerland) and HPLC measurement of amino acids, at a later date $(32,33)$.

Statistics. All the clinical and biochemical data are expressed as mean and standard error. Within the group; we tested for changes in the concentration of variables that were measured repeatedly (ammonia, amino acids, neuropsychological function) using one-way analysis of variance with Bonferroni correction; and the MRS and MTR data using the paired t-test. Difference between groups was tested using 2 way ANOVA. A p-value of $<0.05$ was considered statistically significant. 


\section{RESULTS}

\section{STUDY 1}

There were no clinically observable changes either in the mental state or neurological function in any of the patients in the two groups studied. The amino-acid solution was well tolerated in all except one patient who had nausea requiring administration of an anti-emetic. One patient complained of claustrophobia and therefore did not complete the magnetic resonance spectroscopy at 4 hours. Patients recruited to both parts of the study were well matched with respect to severity of liver disease [Table 1].

Ammonia and amino acids. Ammonia concentrations at baseline were similar in the two groups and increased in the patients administered the amino-acid solution from a mean of $76 \pm 7.3$ to $121 \pm 6.4 \mathrm{mmol} / \mathrm{L}$ at 2 hours and $104( \pm 6.3) \mathrm{mmol} / \mathrm{L}$ at 4 hours ( $p<0.001)$ afterwards. No significant changes were observed in the patients administered the placebo solution (baseline: $83 \pm 3.3 ; 2$ hours: $78 \pm 2.9 ; 4$ hours: $75( \pm 1.3)$; $\mathrm{p}=0.34$ ). The change in ammonia concentration was significantly greater in those patients administered the amino-acid solution $(\mathrm{p}<0.001)$ [Figure $\mathbb{1} \mathrm{A}$ ].

In the group administered the amino-acid solution, there was a three-folld reduction in the concentration of isoleucine $(\mathrm{p}<0.001)$ [Figure $1 \mathrm{~B}$ ] and a three-fold increase in the concentrations of leucine $(p<0.001)$ [Figure $1 C$ ] and valine $(p<0.001)$. The concentration of all other amino acids also increased bur was statistically significant only for asparagine, serine, hisridine, glycine, threonine, lysine, tryptophan ( $\mathrm{p}<0.05$ for each), and phenylalanine ( $<<0.001)$. No significant changes in the concentration of any of the amino acids were observed in the placebo group [Table 3].

Neuropsychological function. None of the patients entered into the study showed any evidence of altered mental state. There were no significant differences at baseline between the 24 patients that received the aa solution and the 24 patients that were given the placebo in any of the neuropsychological tests. There was no significant change in the Trails B test in the aa solution group but significant improvement was observed in the placebo group ( $p<0.03$ ). The difference between the groups at 4 hours was significant using two-way ANOVA $(\mathrm{p}<0.01)$, [Figure $2 \mathrm{~A}$ ]. There was no significant change in the Digit Symbol Substitution Test in the aa solution group but significant improvement was observed in the placebo group $(\mathrm{p}<0.02)$. The difference between the groups at 4 hours was significant using two-way ANOVA $(\mathrm{p}<0.05)$ [Figure $2 \mathrm{~B}$ ]. There was a significant deterioration in the immediate story recall subtest of the Randt battery in the amino-acid group $(\mathrm{p}<0.03)$. No significant change was observed in the placebo group. The difference between the groups was significant using two-way ANOVA $(\mathrm{p}<0.01)$ [Figure 2C]. 


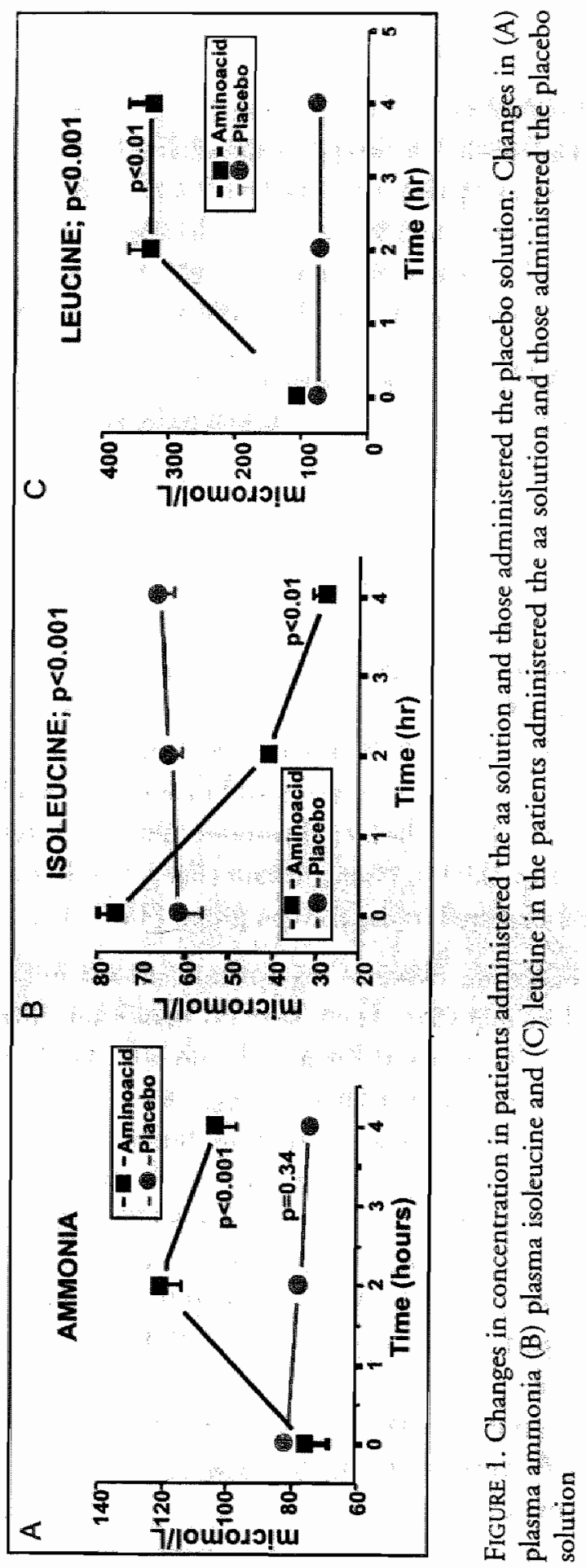




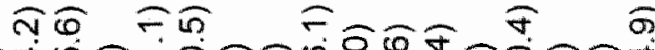

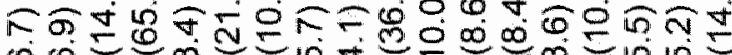

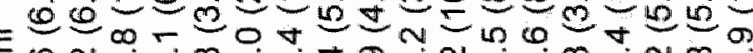
f 0 N

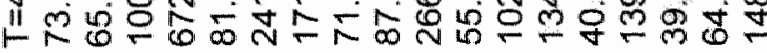

तิ की के की สิรตำ $\therefore$ - 5 mo

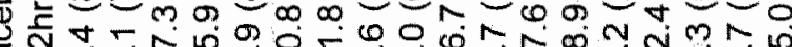

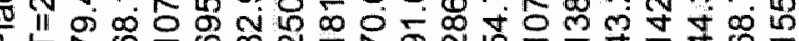

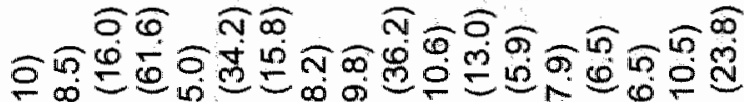
¿क तr

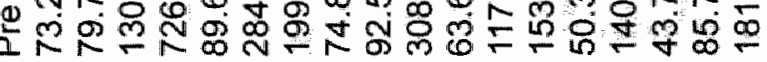

$\operatorname{sengs}$

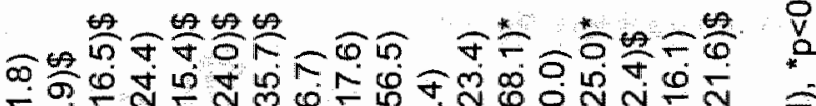
8 Ev

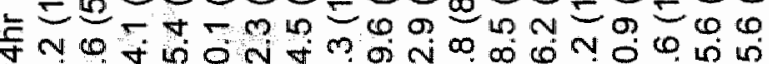

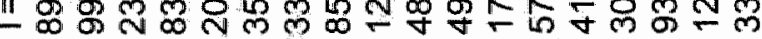

$\frac{0}{0}$

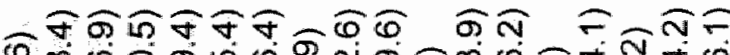
ต ต

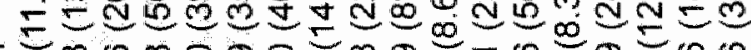

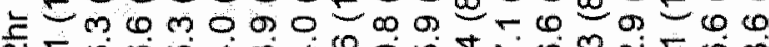

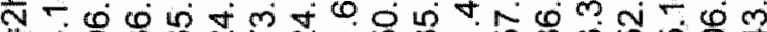
III Uv $\infty 6 \%$ m

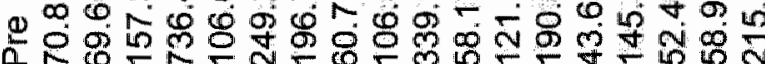
परा 


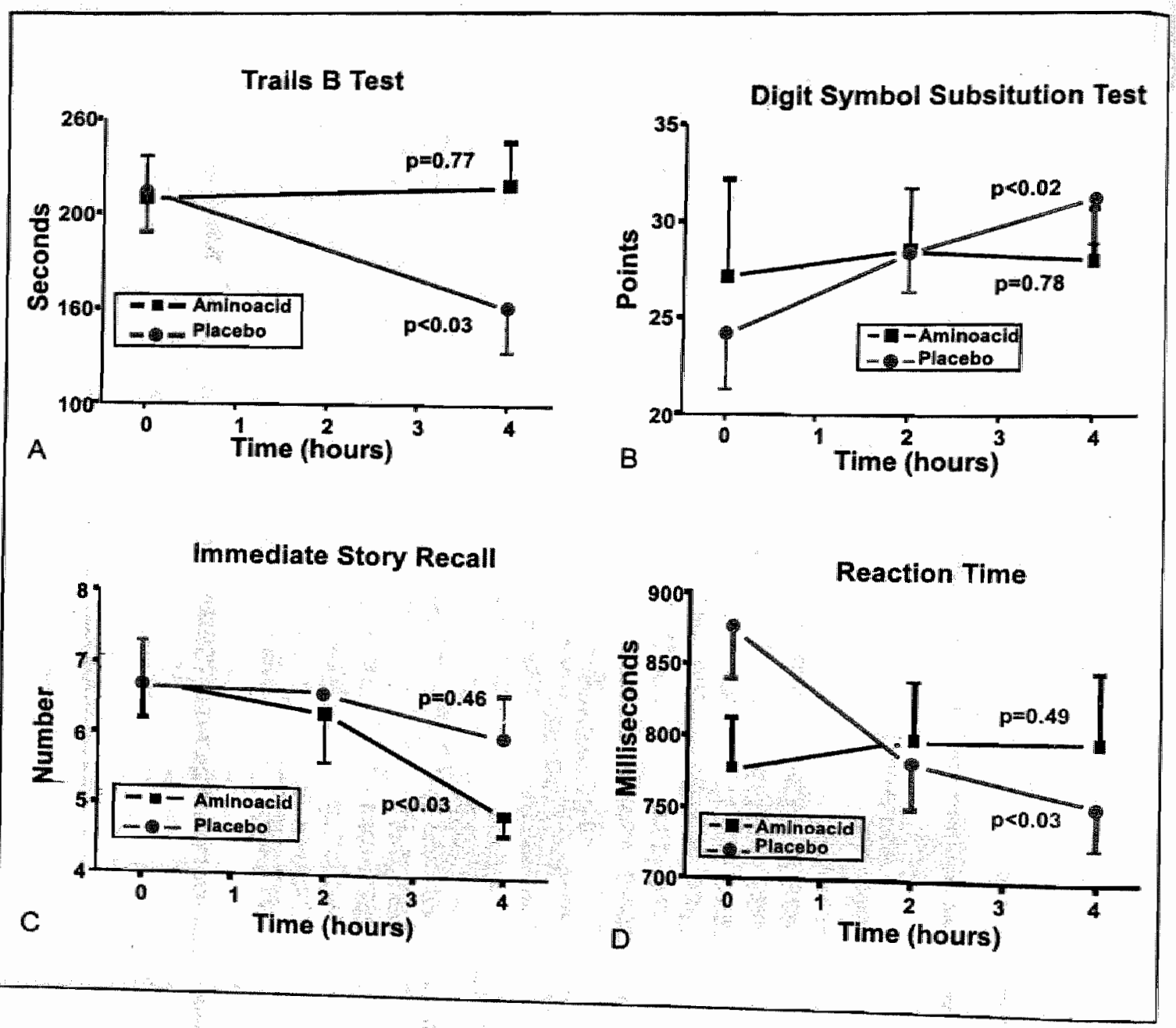
Story Recall (D) Reaction Time. There were no significt (B) Digit Symbol Substitution Test (C) Immediate B Test: $\mathrm{p}=0.56$; Digit Symbol Substitution Test $\mathrm{p}=0 . \mathrm{c}$, Trails $p=0.12$ ). The $p$-values refer those obtained using $p=0.45$; Immediate Story Recall: $p=0.32$; Reaction Time: between groups was tested using two-way ANOV one-way ANOVA for the individual groups. Differences $\mathrm{p}<0.05$; Immediate Story Recall: $\mathrm{P}<0.01$; Reaction Time: $\mathrm{p}<0.05$. 
The standard deviation for the mean reaction at baseline for the placebo group was \pm 268 and for the amino acid group was \pm 310 . The mean numbers of observations for the placebo group were similar to the amino acid group both prior to and after the simulated bleed [placebo: pre: $8.2( \pm 1.1), 2$ hr: $9.1(2.4), 4 \mathrm{hr}: 9.0(2.1)$; aa: $7.9(1.9), 2$ hr: $8.1(1.9), 4 \mathrm{hr}: 8.3(2.1)]$. There was no significant change in the mean reaction time in the amino-acid group but significant improvement was observed in the placebo group $(p<0.03)$. The difference between the groups at 4 hours was significant using two-way ANOVA $(\mathrm{p}<0.05)$ [Figure 2D].

Proton Magnetic Resonance Spectroscopy. There were no significant differences in any of the measured ratios between the amino acid and the placebo groups at baseline. $\mathrm{Glx} / \mathrm{Cr}$ ratios increased significantly both in the basal ganglia and also in the temperoparietal cortex in the amino-acid group ( $\mathrm{p}<0.05$ each), which was not observed in the placebo group [Figure $3 \mathrm{~A}$ ]. $\mathrm{ml} / \mathrm{Cr}$ ratio decreased significantly in the basal ganglia in the amino-acid group but did not change significantly in the placebo group [Figure $3 B$ ]. No significant changes in the $\mathrm{Cho} / \mathrm{Cr}$ or NAA/Cr ratios were observed in either group in both areas (data not shown).

\section{STUDY 2}

Magnetization Transfer Ratio. MTR was significantly lower in the patients at baseline compared with age-matched healthy controls (controls 33.2 ( \pm 3.1 ); patients: 29.6 $( \pm 1.7) ; \mathrm{p}<0.05)$. There was a significant reduction 4 hours after administration of the aa solution to $23.3( \pm 1.7)$ in all the patients $(p<0.01)$ [Figure 4]. The measured values of MTR were similar in the frontal and the parietal areas both prior to and after administration of the aa solution; $\mathrm{p}=0.35$ and $\mathrm{p}=0.16$ respectively. 


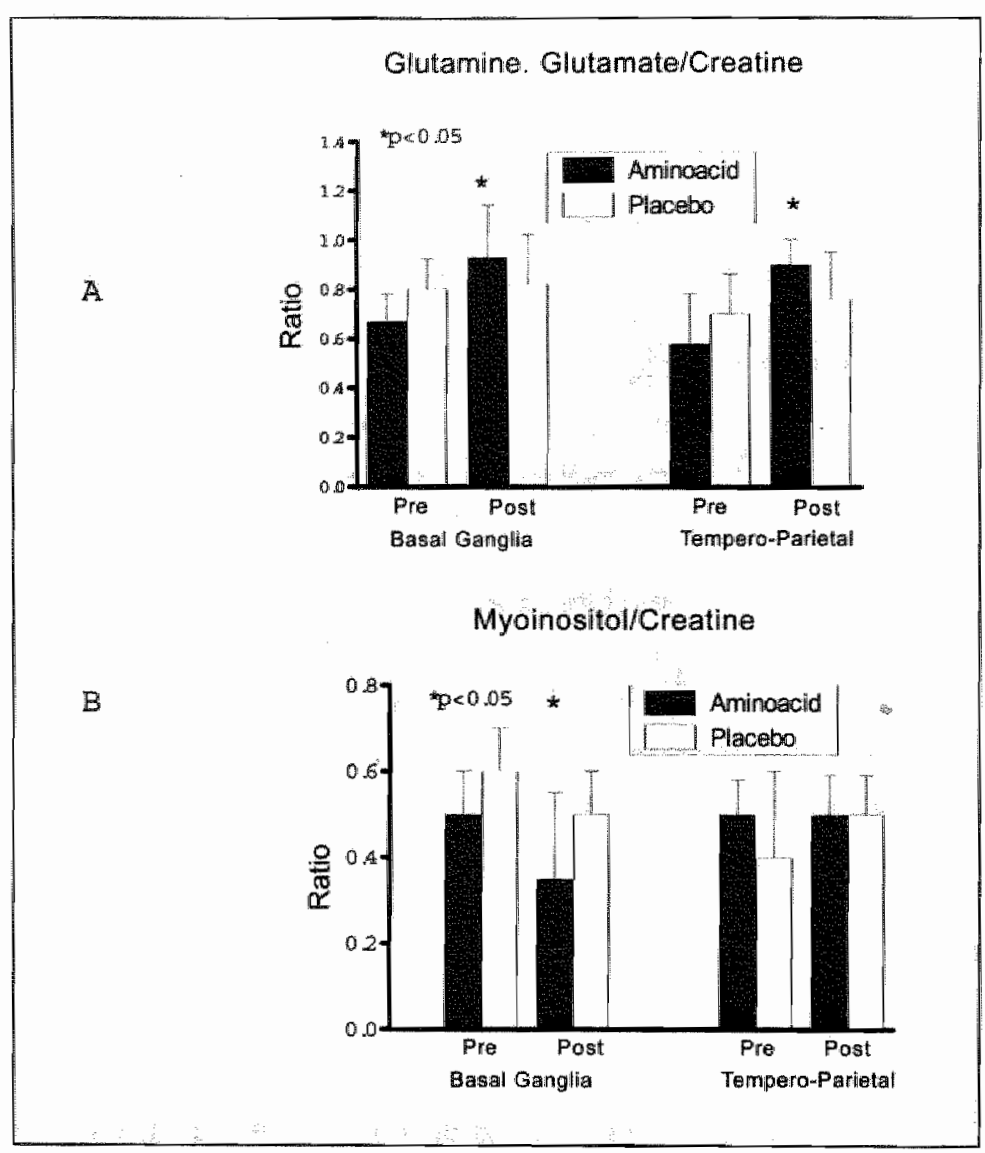

Figure 3 (ABOvE). Changes in (A) Glutamine.glutamate/ $\mathrm{Cr}$ ratio and (B) myoinositol in the basal ganglia and Tempero-parietal region in the parients administered the a solution and those administered the placebo solution. * Refers to comparison with the value obtained at baseline.

FiGURE 4 (BELOW). Changes in the Magnetization Transfer Ratio in the patients administered the aa solution.

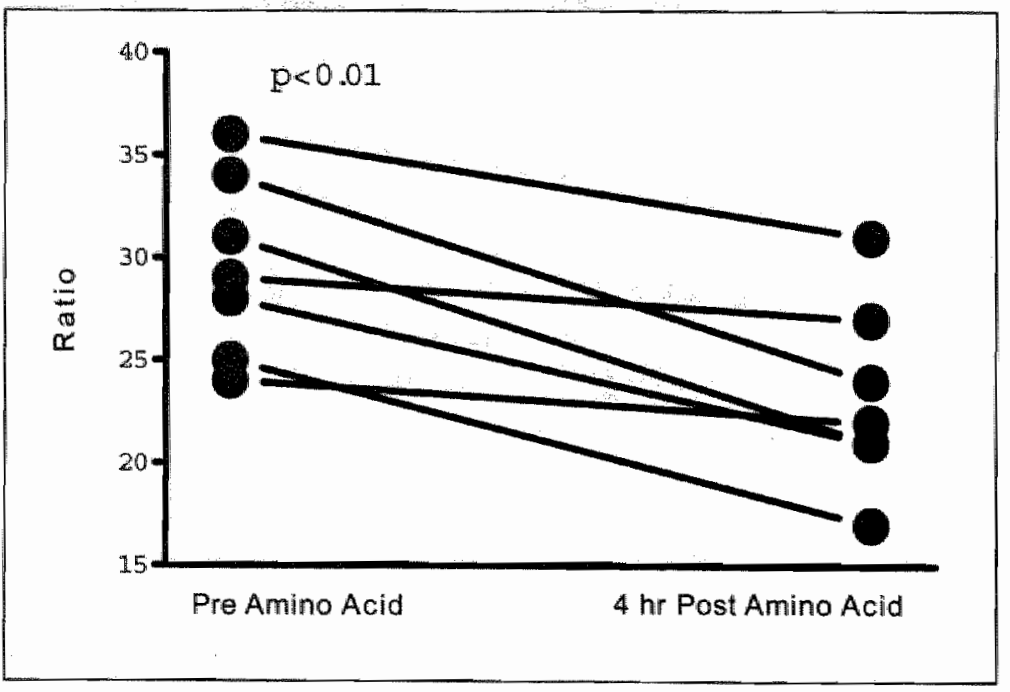




\section{DISCUSSION}

In a randomised controlled evaluation, the results of our study have shown an improvement in neuropsychological tests following administration of the placebo solution which was not observed following induction of hyperammonemia by oral administration of an aa solution mimicking the composition of the haemoglobin molecule. In addition, in the patients administered the aa solution there was a significant increase in brain $\mathrm{Glx} / \mathrm{Cr}$ ratio that was not observed in the placebo group. According to the ammonia-glutamine-brain swelling hypothesis, we also showed a reduction in MTR following the aa solution suggesting an increase in brain water following induction of hyperammonemia. These data provide the first direct evidence in patients with liver disease that induced hyperammonemia results in changes in neuropsychological function, increase in brain glutamine and a reduction in MTR suggesting an increase in brain water.

In patients with cirrhosis, ingestion of erythrocytes produces a larger increase in ammonia than ingestion of an isonitrogenous amount of whole blood, which produces greater increase in ammonia than ingestion of an isonitrogenous amount of blood plasma (5). These findings are explained by the amino acid composition of haemoglobin, which is a protein of very low biologic value (6,34). Absorbed (blood) amino acids cannot be used for protein synthesis and are degraded producing ammonia, which is released into the systemic circulation (34). As has been demonstrated previously, the administration of the aa solution resulted in enhanced ammoniagenesis and hypoisoleucinemia $(7,8)$.

Although data in the literature do not generally show a direct relationship between the severity of $\mathrm{HE}$ and plasma ammonia concentrations, more severe forms of liver disease and $\mathrm{HE}$ are associated with higher concentrations of arterial ammonia (9). The arterial ammonia concentration in healthy volunteers is about $45 \mathrm{mM}(3), 60 \mathrm{mM}$ in chronic liver failure and proven cirrhosis (3), $80 \mathrm{mM}$ in cirrhotic patients who are treated with a transjugular intrahepatic portosystemic stent shunt (35), 90-120 mM in acute-on-chronic liver failure and 150 to $180 \mathrm{mM}$ in acute liver failure and $340 \mathrm{mM}$ in those with uncontrolled intracranial hypertension $(3,36)$. Our data clearly show that the patients administered the placebo solution, showed a significant improvement in all the neuropsychological function except the memory test suggesting a learning effect. In contrast the patients who were administered the aa solution and developed hyperammonemia were unable to learn the tests and in case of the memory test, showed a significant deterioration. Therefore, the patients administered the aa solution had significantly worse neuropsychological performance compared with the placebo group. It is not clear why the memory tests deteriorated significantly but the other 3 
neuropsychological tests did not change significantly following induced hyperammonemia. This observation may confirm that the different neuropsychological tests used in this study tests different functional components and that the pathways subserving memory and learning are more significantly affected by induced hyperammonemia. The mechanism of this inability to learn is unclear but may reflect that hyperammonemia induces an attention deficit which is crucial both for learning and has been suggested as being central to the development of minimal $\mathrm{HE}(37,38)$. Patients who have minimal HE are likely to have marked abnormalities of attention that includes all the domains such as working memory, alertness, selective attention, attention shift and, visual and acoustic vigilance $(37,38)$. Those with cirrhosis but without evidence of minimal HE had normal attention tests. Although the battery of tests used by us require intact 'attention', they do not specifically test 'attention' and suitable studies will need to be performed to address this question. Our results are at variance with those reported by the Newcastle group who used a glutamine challenge to produce hyperammonemia and showed deterioration in reaction time (10). Similarly, they showed that the administration of an aa solution similar in composition to ours was also followed by deterioration in neuropsychological tests (39). We cannot explain the differences in these observations but suggest that differences may be due to different population under study and bias due to the lack of a blinded control group.

In keeping with the ammonia-glutamine-brain swelling hypothesis, our MR spectroscopy data shows an increase in the $\mathrm{Glx} / \mathrm{Cr}$ ratio in the parients that were hyperammonemic following administration of the aa solution. Hyperammonemia increases the delivery of ammonia to the brain when cerebral blood flow remains constant. Since the blood-brain barrier is freely permeable to ammonia, elevated ammonia levels lead to enhanced cerebral ammonia uptake and subsequent incorporation of ammonia into glutamine via the glutamine synthetase reacrion and glutamine release into the blood (40). However, our data do not allow quantification of the fractional extraction of ammonia in the brain due to the semi-quantitative nature of our measurements of Glx. Such unidirectional brain ammonia uptake has been repeatedly observed in various hyperammonemic states $(40,41)$. Studies of cerebral ammonia metabolism in patients with liver disease have shown a fractional extraction of ammonia ranging from $11 \%$ to $20 \%$. In general, the fractional extraction increased with increasing severity of encephalopathy (42-45).

In keeping with the ammonia-glutamine-brain swelling hypothesis of $\mathrm{HE}$, we observed a reduction in $\mathrm{ml} / \mathrm{Cr}$ ratio in those administered the aa solution $(13,46)$. Accordingly, with hyperammonemia there is an increase in glutamine, which increases astrocytic osmolality, and in order to maintain cellular homeostasis, $\mathrm{mI}$ is extruded from the cell. We were however surprised by the rapidity of this homeostatic mechanism such that significant reduction in $\mathrm{mI} / \mathrm{Cr}$ was observed within 4 hours of administration of the aa solution $(47,48)$. 
Our observation of reduction MTR following induction of hyperammonemia is likely to indicate an increase in brain water. This is supported by the observation that the degree of MTR reduction was mild and occurred diffusely in several normal appearing areas (on MRI). These are characteristic fearures of increase in brain water $(30,31)$. In addition, hyperammonemia was associated with an increase in $\mathrm{Glx} / \mathrm{Cr}_{\text {, }}$ which supports the ammonia-glutamine-brain swelling hypothesis. It is however, notable that the reduction in $\mathrm{mI}$ was unable to compensate for the increase in Glx. Our data are consistent with those of Iwasa et al. (49) and Cordoba (13). Indeed, Cordoba et al. reported an inverse correlation between $\mathrm{Glx} / \mathrm{Cr}$ and MTR values supporting the above hypothesis. Further development in technology and the use of higher strength magnets would allow separation of the glutamine/glutamate residues and more accurate correlations between changes in brain water and accumulation of glutamine. Alternative explanations for the reduction in MTR following hyperammonemia may be due to the effect of ammonia upon the polarity, effects upon cerebral blood flow and glucose metabolism, which may impact upon the state of activation. However, these effects of ammonia are seen at millimolar concentrations (11-13).

These data provide supporting evidence in favour of the ammonia-glutamine-brain swelling hypothesis of HE that hyperammonemia induced by oral administration of an aa solution leads to significant alteration in neuropsychological function and an increase in brain glutamine. The reduction in MTR may indicate that the operative mechanism for the deterioration in neuropsychological tests is an increase in brain water. Furthermore, we introduce a model of HE that is safe, well tolerated, reproducible and clinically relevant. It could therefore be a potent new research tool to study the pathophysiology of $\mathrm{HE}$ in cirrhosis. 


\section{REFERENCES}

1. Hahn M, Massen O, Nencki M, Pawlow J. Die Eck'sche fistel zwischen der unteren hohlvene und der pfortader und thre folgen für den organismus. Arch Exper Path Pharm $1893 ; 32: 161-210$

2. Jalan R, Hayes PC. Hepatic encephalopathy and ascites. Lancet 1997;350:1309-15.

3. Clemmesen JO, Larsen $\mathbb{E S}$, Kondrup J, Hansen BA, Ott P. Cerebral herniation in patients with acure liver fallure is correlated with arterial ammonia concentration. Hepatology 1999;29:648-53

4. Kramer L, Tribl B, Gendo A, Zauner C, Schneider B, Ferenci P, Madl C. Partial pressure of ammonia wersus ammonia in hepatic encephalopathy. Hepatology 2000;31:30-4

5. Bessman AN, Hawkins R. The relative effects of enterically administered plasma and packed cells on circulating blood ammonia. Gastroenterology 1963;45:368-73.

6. Riordan SM, Williams $R$, Treatment of hepatic encephalopathy. $N$ Engl I Med $1997 ; 337: 473-9$.

7. Olde Damink SWM, Dejong CHC, Deutz NEP, Soeters PB. Effects of simulated upper gastrointestinal hemorrhage on ammonia and related amino acids in blood and brain of chronic portacaval-shunted rats. Metabolic Brain Disease 1997;12:121-35.

8. Jalan R, Olde Damink SWM, Lui HF, Glabus M, Deutz NEP, Hayes PC, Ebmeier KP. Oral amino acid load mimicking haemoglobin results in reduced regional cerebral perfusion and deterioration in memory tests in patients with cirrhosis of the liver. Metabolic Brain Diseases 2003; 18:37-49.

9. Olde Damink SWM, Deutz NEP, Dejong CHC, Soeters PB, Jalan R. Interorgan ammonia metabolism in liver failure. Neurochem Int. 2002;41:177-88.

10. Oppong $\mathrm{KN}, \mathrm{Al}$-Mardini $\mathrm{H}$, Thick $\mathrm{M}$, Record $\mathrm{CO}$. Oral glutamine challenge in cirrhotics pre- and post-liver transplatutation: a psychometric and analyzed EEG study. Hepatology 1997;26:870-6.

11. Butterworth RF. The neurobiology of hepatic encephalopathy. Semin Liv Dis $1996: 16: 235-44$.

12. Norenberg MD. Astrocytic-ammonia interactions in hepatic encephalopathy: Semin Liv Dis $1996 ; 16: 245-53$.

13. Cordaba J, Alonso J, Rovira A, Jacas C, Sanpedro F, Castells L, Vargas V, Margarit C, Kulisewsky J, Esteban R, Guardia J. The development of low-grade cerebral edema in cirrhosis is supported by the evolution of (1)H-magnetic resonance abnormalities after liver transplantation. J Hepatol 2001;35:598-604.

14. Martinez-Hernandez A, Bell KP, Norenberg MD. Glutamine synthetase: glial localization in brain. Science $1977: 25 ; 195: 1356-8$.

15. Jalan R, Dabos K, Redhead DN, Lee A, Hayes PC. Elevation of intracranial pressure following transjugular intrahepatic portosystemic stent-shunt for variceal haemorrhage. J Hepatol $1997 ; 27: 928-33$ 
16. McGowean JC. The physical basis of magnetization transfer imaging. Neurology $1999 ; 53$ (supple 1):S9-S18.

17. van Buchem MA. Magnetization transfer: applications in neuroradiology. J Comput Assist Tomog 1999;23 (supple 1): S9-S18.

18. Grossman RI, Magnetization transfer in multiple sclerosis. Ann Neurol 1994;36 Suppl:597-9

19.Henkelman RM, Stanisz GJ, Graham SJ. Magnerization transfer in MRI: a review. NMR Biomed. 2001; 14:57-64

20. Stanisz GJ, Yoon RS, Joy ML, Henkelman RM. Why does MTR change with neuronal depolarization? Magn Reson Med. 2002;47:472-5

21. Makela HI, Kettunen MI, Grohn OH, Kauppinen RA. Quantitative T(1rho) and magnetization transfer magnetic resonance 1 maging of acute cerebral ischemia in the rat.) Cereb Blood Flow Metab. 2002;22:547-58

22. Kettunen MI, Grohn $\mathrm{OH}$, Silvennoinen MJ, Pentonen M, Kauppinen RA Effects of intracellular $\mathrm{pH}$, blood, and tissue oxygen tension on $\mathrm{T} 1$ tho relaxation in rat brain. Magn Reson Med. 2002;48:470-7

23. Hill RJ, Koningsberg W. The structure of human hemoglobin. the Journal of Biological Chemistry 1962;237:3151-6.

24. Davies $\mathrm{AD}$. The influence of age on trail making test performance. J Clin Psychol 1968;24:96-8.

25. Hindmarch I. Psychomotor function and psychoactive drugs. Br J Clin Pharmacoll 1980;10:189-209.

26. Randt CT, Brown ER, Osborne DPJ. A memory test for longitudinal measurement of mild to moderate defects. Clinical Neuropsychology 1980;1I:184-97.

27. Frith CD, Leary J, Cahill C, Johnstone EC. Performance on psychological tests. Demographic and clinical correlates of the results of these tests. Br J Psychiatry Suppl $1991: 26-9,44-6$.

28. Ordidge RJ, Cresshull ID. The correction of transient $B_{0}$ field shifts following the application of pulsed gradients by phase correction in the time domain. I Magn Reson $1986 ; 69: 151-5$.

29. Mierisova S, van den Boogaart A, Tkac I, van Hecke P, Vanhamme L, Liptaj T. New approach for quantificarion of short echo time in vivo ${ }^{1} \mathrm{H} M R$ sprectra of brain using AMARES. NMR Biomed 1998;11:32-9.

30. Rovira A, Alonso J, Cucurella G, Nos C, Tintore M, Pedraza S et al.. Evolution of multiple sclerosis lesions on serial contrast-enhanced T1-weighted and magnetization-transfer MR images. Am J Neuroradiol 1999;20:1939-45.

31. Grossman RI, Gomori JM, Ramer KN, Lexa FJ, Schnall MD. Magnetization transfer: theory and clinical applications in neuroradiology. Radiographics 1994;14:279-90.

32. Deutz NEP, Reijven PL, Bost MC, van Berlo CL, Soeters PB. Modification of the effects of blood on amino acid metabolism by intrawenous isoleucine. Gastroenterology $1991 ; 101: 1613-20$. 


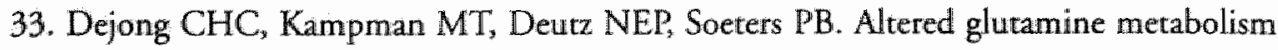
in rat portal drained viscera and hindquarter during hyperammonemia. Gastroenterology 1992;102:936-948.

34. Olde Damink SWM, Dejong CHC, Deutz NEP, van Berlo CLH, Soeters PB. Upper gastrointestinal bleeding: an ammoniagenic and catabolic event due to the total absence of isoleucine in the hemoglobin molecule. Medical Hypotheses 1999;52:515-9.

35. Jalan R, Gooday R, O'Carroll RE, Redhead DN, Elton RA, Hayes PC. A prospective evaluation of changes in neuropsychological and liver function tests following transjugular intrahepatic portosystemic stent-shunt. J Hepatol 1995;23:697-705.

36. Jalan R, Olde Damink SWM, Deutz NEP, Lee A, Hayes PC. Moderate hypothermia for uncontrolled intracranial hypertension in acute liver failure. Lancet 1999;354:1164-8.

37. Amodio P, Marchetti P, Del Piccolo F, Campo G, Rizzo C, Iemmolo RM, Gerunda G, Caregaro L, Merkel C, Gatta A. Visual attention in cirrhotic patients: a study on covert visual attention orienting. Hepatology. 1998;27:1517-23

38. Weissenborn $\mathrm{K}$, Heidenreich S, Ennen J, Ruckerr N, Hecker $H$. Artention deficits in. minimal hepatic encephalopathy. Metab Brain Dis. 2001;16(1-2):13-9

39. Douglass A, Al Mardini H, Record C. Amino acid challenge in patients with cirrhosis: a model for the assessment of treatments for hepatic encephalopathy. I Hepatol 2001;34:658-64.

40. Cooper AJ, Plum F. Biochemistry and physiology of brain ammonia. Physiol Rev 1987;67:440-519.

41. Cooper AJ, Mora SN, Cruz NF, Gelbard AS. Cerebral ammonia metabolism in hyperammonemic rats. J Neurochem 1985;44:1716-23.

42. Fazekas JF, Ticktin WR, Ehrmantraut WR, Alman RW. Cerebral metabolism in hepatic insufficiency. Am J Med 1956;21:843-9.

43. Bessman SP, Bradley JE. Uptake of ammonia by muscle. Its implications in ammoniagenic coma. N Engl J Med 1955;253:1143-7.

44. Webster LT, Gabuzda GJ. Ammonium uptake by the extremities and brain in hepatic coma. J Clin Invest 1958;37:414-24.

45. Bessman SP, Bessman AN. The cerebral and peripheral uptake of ammonia in liver disease with an hypothesis for the mechanism of hepatic coma. J Clin Invest 1955;34:6228.

46. Haussinger D, Laubenberger J, vom Dahl S, Ernst T, Bayer S, Langer M, Gerok W, Hennig J. Proton magnetic resonance spectroscopy studies on human brain myo-inositol in hypo-osmolarity and hepatic encephalopathy. Gastroenterology 1994;107:1475-80.

47. Ross BD, Jacobson S, Villamil F, Korula J, Kreis R, Ernst T, Shonk T, Moats RA. Subclinical hepatic encephalopathy: proton MR spectroscopic abnormalities. Radiology 1994;193:457-63.

48. Seery JP, Taylor-Robinson SD. The application of magnetic resonance spectroscopy to the study of hepatic encephalopathy. J Heparol 1996;25:988-98. 
49. Iwasa M, Kinosada Y, Nakatsuka A, Watanabe S, Adachi X. Magnetization transfer contrast of various regions of the brain in liver cirrhosis. AJNR Am J Neuroradiol $1999 ; 20: 652-4$. 



\section{SECTION 2}

\section{CHAPTER 5}

MEDICAL HYPOTHESIS: UPPER GASTROINTESTINAL BLEEDING: AN AMMONIAGENIC AND CATABOLIC EVENT DUE TO THE TOTAL ABSENCE OF ISOLEUCINE IN THE HAEMOGLOBIN MOLECULE

Steven W.M. Olde Damink, Cornelis H.C. Dejong, Nicolaas E.P. Deutz, Charles L.H. van Berlo, Peter B. Soeters

Department of Surgery, Academic Hospital Maastricht, Maastricht University, Maastricht, and \#Department of Surgery, Sint Maartensgasthuis, Venlo, The Netherlands.

Published in Medical hypotheses 1999; 52: 515-519 


\section{INTRODUCTION}

Fifry percent of patients with cirrhosis have oesophageal varices and a third of these patients will bleed from them within 2 years following diagnosis (1). The risk of death from the first variceal haemorrhage depends mainly on the severity of their liver disease estimated using Child-Pugh classification (2). Three to ten per cent of patients with Child-Pugh class A, $5-25 \%$ of Child-Pugh class B and 15-30\% with Child-Pugh class $\mathrm{C}$ disease will die from the effects of the bleed (3). These deaths occur despite adequate controll of bleeding in up to $90 \%$ of patients (4). Deaths occur due to the metabolic consequences of this upper gastrointestinal (UGI) haemorrhage, which precipitates life-threatening complications as spontaneous bacterial peritonitis, sepsis, renal failure and hepatic encephalopathy ( $\mathrm{HE}$ ) (3-6). HE is probably the result of the metabolic consequences of the blood protein load in the digestive tract (7). Whereas UGI bleeding increases plasma urea concentrations in patients with normal liver function, an UGI bleed in patients with liver failure results in an increased plasma ammonia concentration, which has been proposed as a central factor in the development of HE (7-9).

The first evidence for this 'toxic', probably ammonia-related potential of a protein load in the gut during liver failure was the 'meat intoxication' syndrome observed by Pavlov's group more than a century ago (10). They described the development of stupor in dogs with an Eck fistula following raw meat ingestion, and reversal of these symptoms after switching to a milk and bread diet. Since then a hierarchy in the ammoniagenic potential of different proteins has been described. The least toxic protein, with the lowest coma inducing potential is vegetable protein, whereas blood protein is the most toxic $(11,12)$.

\section{Ammonia liberating effect of blood}

For a long time, it was believed that the increased ammonia production after an UGI bleeding was solely caused by digestion of intraluminal blood by colonic bacteria. Therefore, at present, most ammonia-lowering therapeutic straregies such as bowel cleansing, non-absorbable antibiotics or lactulose focus on this production site (7). However, studies in dogs with a decontaminated digestive tract showed that ammonia production after blood enemas was reduced, but that ammonia production after blood meals persisted (13). In agreement with this, blood meals administered to germ-free Eck-fistula dogs resulted in raised peripheral blood ammonia concentrations comparable to those observed in animals colonised with bacteria (14). These studies clearly showed that micro-organisms are not the major ammonia producers of the gut after UGI bleeding and that other mechanisms are also involved.

The basis of our hypothesis relies upon two elegant studies by Bessman and coworkers $(11,12)$. They showed that a meal of whole blood administered to patients 
with liver failure caused a much greater rise in blood ammonia than an iso-nitrogenous casein hydrolysate meal (12). In another study (11), cirrhotic patients received an isonitrogenous amount of plasma, whole blood and packed cells in a cross-over design. The increase in blood ammonia levels after whole blood was more than after plasma, but less than that after ingestion of packed cells. They hypothesised that the amount of protein ingested was not the determining factor in ammoniagenesis, but the nature of the protein. With respect to the specific ammonia liberating effect of blood in the gut they stated: "In view of the fact that about 95 percent of the protein of the erythrocyte is haemoglobin, we are probably dealing with a specific effect of this protein" (11). However, the nature of this speciffc effect is just starting to become clear.

\section{Development of the hypothesis}

For the past 10 years, we have been studying the effects of enteral administration of blood products to humans and experimental animals. We have developed a technique that allows measurement of amino acid fluxes in non-stressed, unrestrained, conscious pigs. As in Bessman's study, the administration of an enteral meal of iso-nitrogenous amounts of packed cells, whole blood, plasma and pig feed produced an increase in ammonia production that was most pronounced after the erythrocyte meal (15). Most interestingly, this meal also resulted in extremely low plasma isoleucine concentrations ( $25 \%$ of normal fasting levels) and in hyperaminoacidemia of almost all other amino acids compared with values obtained in animals receiving iso-nitrogenous amounts of pig feed. Furthermore after ingestion of packed cells, ammonia production by the gut and plasma urea concentrations were significantly higher than in controls (15).

These findings led us to study the amino acid composition of haemoglobin, since $98 \%$ of the erythrocyte protein is haemoglobin. This revealed that the haemoglobin molecule is totally devoid of the amino acid isoleucine $(16,17)$ (see Table 1) and contains large amounts of the two other branched chain amino acids (BCAAs) valine and leucine. In addition, plasma protein, mostly consisting of albumin and globulin, also contains only small amounts of isoleucine (18). Therefore, blood protein is severely deficient in the essential amino acid, isoleucine.

In a similar experiment, we showed that a blood meal in healthy human volunteers induced a reduction in plasma isoleucine concentration to less than $50 \%$ of normal fasting values. However, the concentration of all other amino acids increased (19). In patients with normal and impaired liver function presenting with an UGI bleed we have also demonstrated a reduction in isoleucine concentration by $60-70 \%(20)$. The plasma isoleucine concentration in these patients remained low for up to $50 \mathrm{~h}$ after the onset of the bleed. Moreover, we confirmed that this decrease in plasma isoleucine concentration is associated with a decreased isoleucine concentration in tissues in rats with normal and impaired liver function (21). These experiments in pigs, rats and humans provide evidence that the reduction in the concentration of isoleucine after an (simulated) 
Table 1. Amino acid composition of the haemoglobin molecule $(14,15)$.

\begin{tabular}{llllll}
\hline Leucine & 72 & Glutamate & 24 & Aspartic acid & 30 \\
Isoleucine & 0 & Asparagine & 20 & Alanine & 72 \\
Valine & 62 & Glutamine & 8 & Cysteine & 6 \\
Glycine & 40 & Methionine & 6 & Serine & 32 \\
Tryptophan & 6 & Arginine & 12 & Phenylalanine & 30 \\
Threonine & 32 & Tyrosine & 12 & Histidine & 38 \\
Lysine & 44 & Proline & 28 & & \\
\hline
\end{tabular}

Total amino acids: 574

UGI bleed is not a phenomenon restricted to certain animal species, but occurs universally.

The above studies suggest that supplementation of isoleucine during an UGI bleed reduces ammoniagenicity. To confirm this hypothesis, we administered erythrocytes enterally to unrestrained conscious pigs and intravenously infused isoleucine or saline (control) randomly (22). The amount of isoleucine was calculated to supplement the quantity of isoleucine lacking in the red cells administered. In the pigs receiving isoleucine we showed a normal postprandial plasma isoleucine concentration and significantly lower concentrations of other amino acids compared with the control group. Furthermore, the production of ammonia and total ?-amino nitrogen by the gut and urea by the liver was significantly lower in the isoleucine supplemented animals. These experiments show that the ammoniagenicity of blood protein can be reduced by concomitant infusion of isoleucine which is lacking in the haemoglobin molecule.

\section{Hypothesis}

These series of experiments was the basis of the hypothesis that the ammoniagenic potential of (simulated) UGI bleed is the result of to the complete absence of the amino acid isoleucine in the hatemoglobin molecule. As isoleucine is an essential amino acid, the UGI bleed presents the organism with protein of very low 'biological value' (23). This can explain why most of the amino acids and ammonia show a significantly greater rise in the portal and systemic circulation when blood protein is taken up by the gut than after an iso-nitrogenous protein load of normal composition. Increased production of ammonia by the gut in patients with normal liver function and portal circulation will be almost completely cleared by the liver, thereby keeping systemic 
ammonia concentrations within normal limits (24). However, in patients with compromised liver function and portasystemic shunting the inability to detoxify this ammonia may manifest as $\mathrm{HE}(24,25)$.

The simple absence of isoleucine cannot explain the decrease in plasma and tissue isoleucine concentrations by 60-70\%. An explanation for this marked decrease is probably the presence of high amounts of the other two BCAAs, leucine and valine, in the haemoglobin molecule. This imbalance between the BCAAs can lead to a phenomenon called BCAA antagonism (see Fig. 1), which presumably results from the common pathway of transport and degradation. As a result of BCAA antagonism, the high plasma leucine concentration that results from an UGI bleed stimulates BCAA oxidation, irrespective of the level of the other BCAA. Consequently, isoleucine oxidation will be stimulated, resulting in a further depression of plasma and tissue isoleucine concentrations, which could diminish protein synthesis (see Fig. 2). The rapid occurrence of BCAA antagonism was demonstrated by Block and Harper (26), who showed that the reduction of plasma isoleucine and valine pools occur only 10-30 min after intragastric administration of leucine.

\section{Diminished protein synthesis}

Our hypothesis that the low plasma and tissue isoleucine concentrations will diminish protein synthesis is consistent with other teports concerning impaired protein synthesis, DNA synthesis, cell proliferation and cell function in an isoleucine deficient state (27-30). Lecavalier et al. (27) showed that even a short period ( 8 hours) of hypoisoleucinemia impaired whole-body protein synthesis in humans. Impaired protein synthesis could shift the balance between protein synthesis and breakdown towards a net catabolic state. Apart from high urea concentrations and hyperammonemia, other effects of isoleucine deficiency may become clinically apparent at an early point in time, notably diminished synthesis of proteins with short half-lives (as clotting-factors VII and II), synthesis of proteins with a high isoleucine content or rapidly dividing cells (as immune cells). At present, clotting abnormalities, inadequate response to injury and high incidence of infection after an UGI bleed in patients with liver failure are thought to result from loss or use of clotting-factors and acute phase proteins. Our hypothesis suggests that, as a result of the perculiar composition of the haemoglobin molecule, diminished protein synthesis may also play an important role in interfering with the production of these proteins. This may increase the risk of further bleeding, infection and catabolism.

\section{Therapy}

The hypothesis suggests simple relief for the adverse metabolic effects of UGI bleeding. Parenteral infusion of isoleucine, in amounts calculated to balance the BCAA pattern may transform blood protein in a balanced protein and stimulate the utilization of amino acids for protein synthesis. Stimulation of protein synthesis in the gut wall will 


\section{BCAA antagonism}

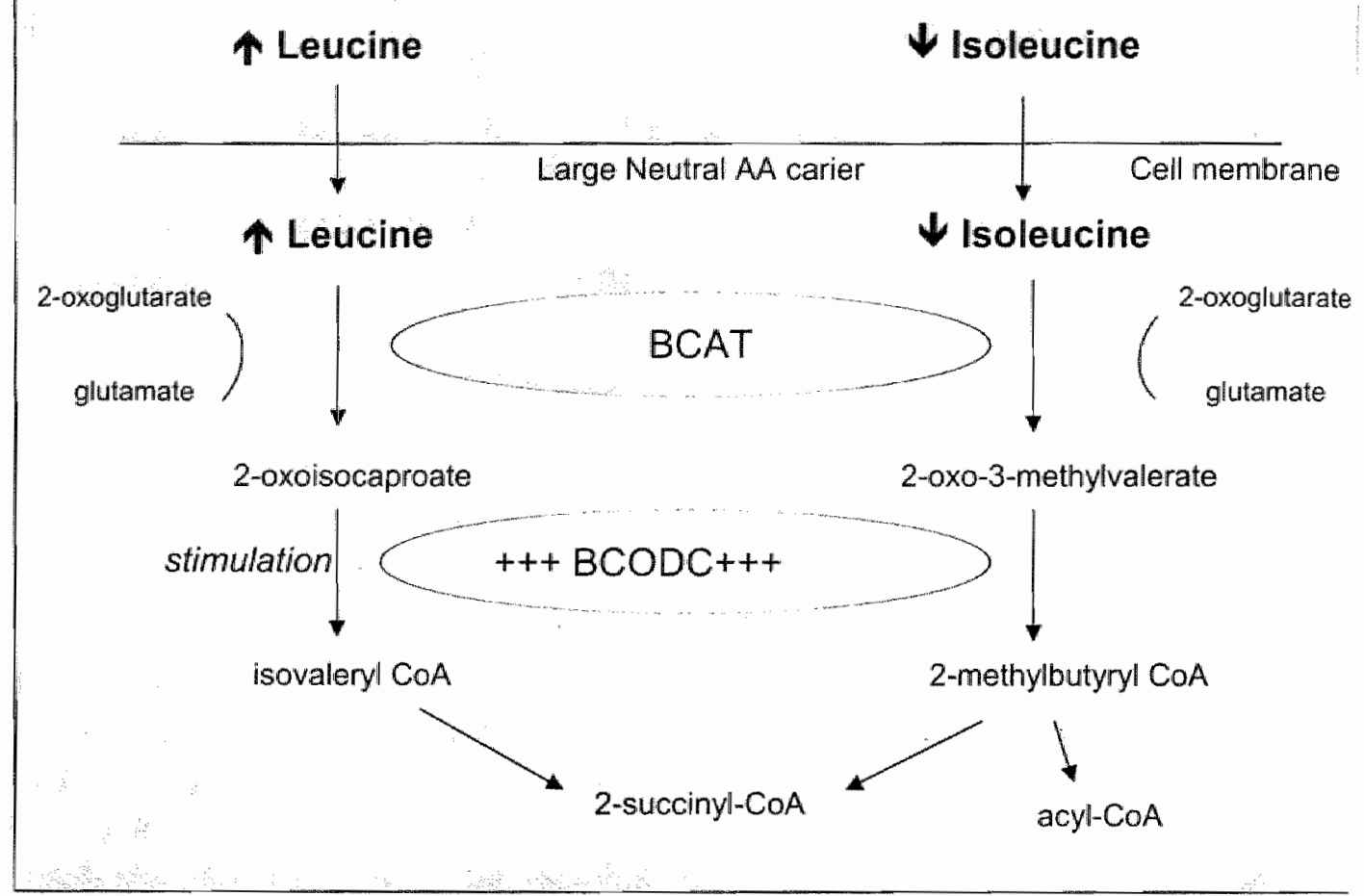

FIGURE 1. The proposed mechanism of BCAA-antagonism between leucine and isoleucine.

Firstly, increased plasma concentrations of leucine and valine could compete with the decrease isoleucine concentrations for transport across the cell membrane via their shared transport carrie termed the large neutral amino-acid carrier (31). This might result in a significantly greater reductio in isoleucine concentration within cells than in plastna (32).

Another, more likely, explanation for the reduction in isoleucine concentration is that all th BCAA have a common degradation pathway $(32,33)$. Within the cell, the initial step is a reversibl concentration-dependent, transamination reaction by BCAA aminotransferase (BCAT, EC 2.6.1.42 The rate of transamination is concentration-dependent and BCAT is widely distributed among tissue with high activity in the skeletal muscles and low activity in the liver and intestine $(33,34)$.

After this transamination reaction, the resulting 2-oxoacids undergo an irreversible oxidati decarboxylation reaction by the branched chain 2-oxoacid dehydrogenase complex (BCODC, $\mathrm{E}$ 1.2.4.4), the rate limiting step in BCAA oxidation $(32,33)$. The activity of the BCODC comple shows large differences between various organs because it is present in active, dephosphorylated form (liver) and in inactive, phosphorylated forms (muscle and intestines) $(32,35)$. The BCODC comple is activated by inhibition of a specific regulatory kinase of the complex (IEC 2.7.1.115); most potent by the oxo-acid of leucine: 2-oxoisocaproate or also called alpha-ketoisocaproate $(36,37)$. The reactic products of the oxidative decarboxylation of the individual BCAA follow their own catabolic pathw: to, e.g., the Krebs-cycle. 
Figure 2. Schematic representation of the hypothesis.

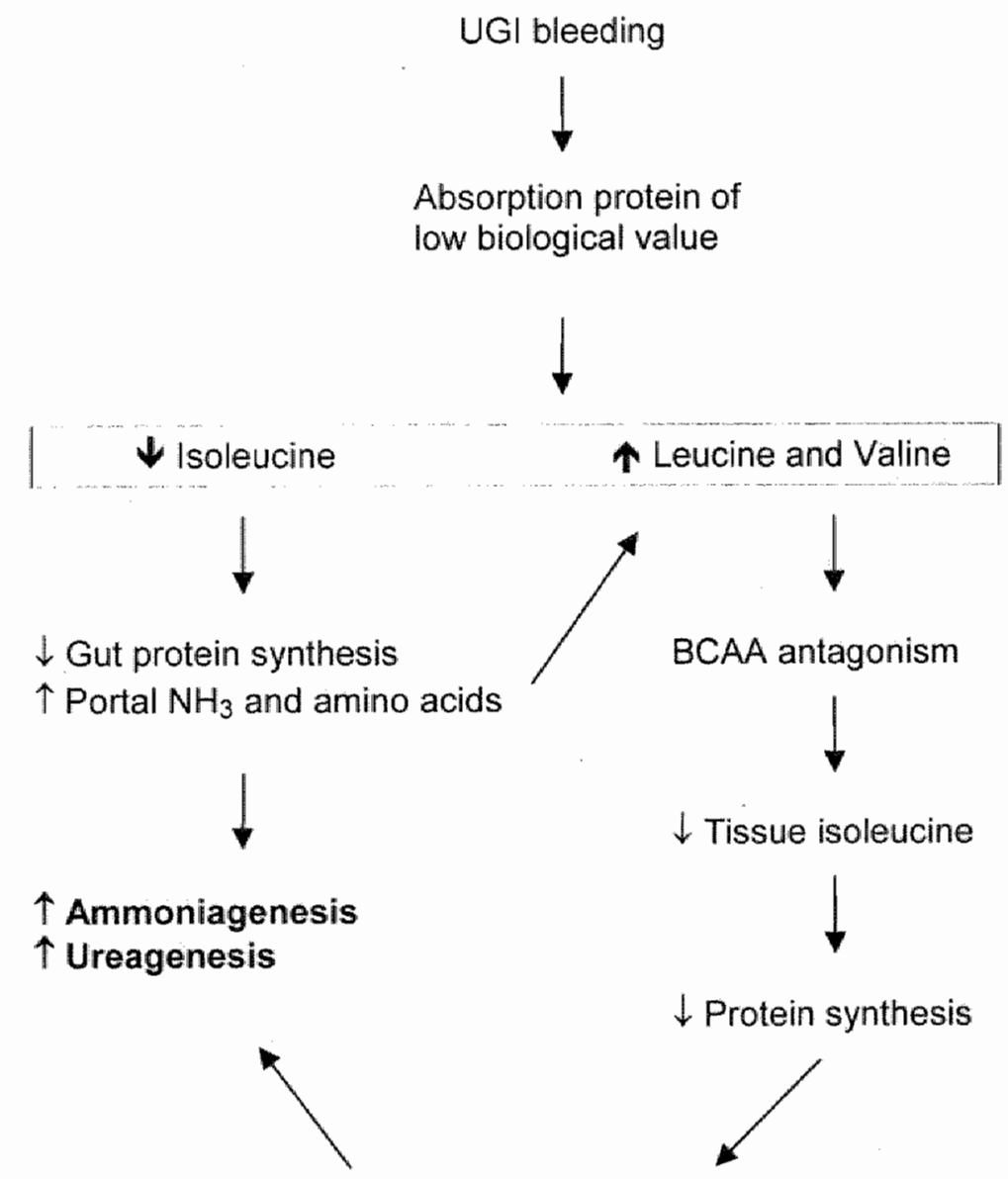

Net catabolic state 
reduce the release of ammonia and total ?-amino nitrogen in the portal vein, resulting in only a short-lived rise in ammonia and urea production. Infusion of isoleucine during or after an UGI bleed could hypothetically reduce the incidence of $\mathrm{HE}$ in patients with impaired liver function and uraemia in patients with normal liver function.

It is expected that, if this hypothesis is correct, simple and cheap intervention with the infusion of isoleucine during an UGI bleed would transform the outcome of these patients. 


\section{REFERENCES}

1. Grace ND. Prevention of initial variceal hemorrhage. Gastroenterol Clin North Am $1992 ; 21: 149-61$.

2. Pugh RN, Murray-Lyon IM, Dawson JL, Pietroni MC, Williams R. Transection of the oesophagus for bleeding oesophageal varices. Br J Surg 1973;60:646-9.

3. Teran JC, Imperiale TF, Mullen KD, Tavill AS, McCullough AJ. Primary prophylaxis of variceal bleeding in cirrhosis: a cost- effectiveness analysis. Gastroenterology $1997 ; 112: 473-82$.

4. Garden OJ, Motyl H, Gilmour WH, Utley RJ, Carter DC. Prediction of outcome following acute variceal haemorrhage. Br J Surg 1985;72:91-5.

5. Pauwels A, Mostefa-Kara N, Debenes B, Degoutte $E$, Levy VG, Systemic antibiotic prophylaxis after gastrointestinal hemorrhage in cirrhotic patients with a high risk of infection. Hepatology 1996;24:802-6.

6. Bleichner $G$, Boulanger $R$, Squara $P$, Sollet JP, Parent $A$. Frequency of infections in cirrhotic patients presenting with acute gastrointestinal haemorrhage. $\mathrm{Br} J$ Surg 1986;73:724-6.

7. Riordan SM, Williams $R$. Treatment of hepatic encephalopathy. $N$ Engl I Med 1997;337:473-9.

8. Jalan R, Hayes PC. Hepatic encephalopathy and ascites. Lancet 1997;350(9087):1309-15.

9. Basile AS, Jones EA. Ammonia and GABA-ergic neurotransmission: interrelated factors in the pathogenesis of hepatic encephalopathy. Hepatology 1997;25:1303-5.

10. Hahn M, Massen O, Nencki M, Pawlow J. Die Eck'sche fistel zwischen der unteren hohlvene und der pfortader und ihre folgen für den organismus. Arch F Exper Path u Pharm $1893 ; 32: 161-210$.

11. Bessman AN, Hawkins R. The relative effects of enterically administered plasma and packed cells on circulating blood ammonia. Gastroenterology 1963;45:368-373.

12. Bessman AN, Mirick GS, Hawkins R. Blood ammonia levels following the ingestion of casein and whole blood. J Clin Invest 1958;37:990-999.

13. Sugarbaker SP, Revhaug A, Wilmore DW. The role of the small intestine in ammonia production after gastric blood administration. Ann Surg 1987;206:5-17.

14. Nance FC, Batson RC, Kline DG. Ammonia production in germ-free Eck fistula dogs. Surgery 1971;70:169-74.

15. van Berlo $C L$, van de Bogaard $A E$, van der Heijden MA, van Eijk HM, Janssen MA, Bost $\mathrm{MC}$, et al. Is increased ammonia liberation after bleeding in the digestive tract the consequence of complete absence of isoleucine in hemoglobin? A study in pigs. Hepatology $1989 ; 10: 315-23$.

16. Hill RJ, Koningsberg W. The structure of human hemoglobin. the Journal of Biological Chemistry 1962;237:3151-3156.

17. Lawn RM, Efstratiadis A, O'Connell C, Maniatis T. The nucleotide sequence of the human beta-globin gene. Cell 1980;21:647-51. 
18. Diem K. Lentner C. Wissenschaftliche tabellen. 7 ed: Ciba-Geigy Basel; 1976.

19. wan Berlo CLH, Dejong CHC, Meyerink WJ J, won Meyenfeldt MF, Fick TE, Deutz NEP, et al. A decrease in plasma isoleucine levels after simulated upper digestive tract bleeding in human volunteers. Clinical Nutrition 1991;10(suppl 2):O56, abstract.

20. Dejong CHC, Meijerink WJH], van Berlo CLH, Deutz NEP, Soeters PB. Decreased plasma isoleucine concentrations after upper gastrointestinal haemorrhage in humans. Gut $1996 ; 39: 13-17$.

21. Olde Damink SW, Dejong CH, Deutz NE, Soeters PB. Decreased plasma and tissue isoleucine levels after simulated gastrointestinal bleeding by blood gavages in chronic portacaval shunred rats. Gut 1997;40:418-24.

22. Deutz NE, Reijwen PL, Bost MC, van Berlo CL, Soeters PB. Modification of the effects of blood on amino acid metabolism by intravenous isoleucine. Gastroenterology $1991 ; 101: 1613-20$.

23. Newsholme EA, Leech AR. Biochemistry for the Medical sciences. 1 ed. New York: John Wiley \& Sons; 1983.

24. Rudman D, DiFulco TJ, Galambos JT, Smith RBd, Salam AA, Warren WD. Maximal rates of excretion and synthesis of urea in normal and cirrhotic subjects. I Clin Invest 1973;52:2241-9.

25. McDermott WV, Adams RD. Episodic stupor associated with an Eck Fistula in the human with particular reference to the metabolism of ammonia. JClinInvest 1954:33:1-9.

26. Block KP, Harper AE. Valine metabolism in vivo: effects of high dietary levels of leucine and isoleucine. Merabolism 1.984;33:559-66.

27. Lecavalier L, De Feo P, Haymond MW. Isolated hypoisoleucinemia impairs whole body but not hepatic protein synthesis in humans. Am I Physiol 1991;261:E578-86.

28. Kohn A. Differential effects of isoleucine deprivation on cell motility, membrane transport and DNA synthesis in NIL 8 hamster cells. Exp Cell Res 1975;94:15-22.

29. Tobey RA, Ley KD. Isoleucine-mediated regulation of genome repliction in various mammalian cell lines. Cancer Res 1971;31:46-51.

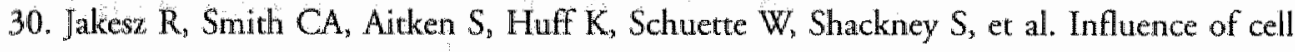
proliferation and cell cycle phase on expression of estrogen receptor in MCF-7 breast cancer cells. Cancer Res 1984;44:619-25.

31. Shotwell MA, Kilberg MS, Oxender DL. The regulation of neutral amino acid transport in mamnatian cells. Biochim Biophys Acta 1983;737:267-84.

32. Harper AE, Miller RH, Block KP. Branched chain amino acid metabolism. Annu Rev Nutr 1984;4:409-454.

33. Wagenmakers AJM, Soeters PB. Metabolism of branched-chain amino acids. Boca Raton, FL: CRC Press; 1995: 67-87.

34. Ooiwa T, Goto H. Tsukamoto $Y$, Hayakawa $T$, Sugiyama $S$, Fujisuka N, et al. Regulation of valine catabolism in canine tissues: tissue distributions of branched-chain aminotransferase and 2-oxo acid dehydrogenase complex, methacrylyl-CoA hydratase and 3-hydroxybutyryl-CoA hydrolase. Biochim. Biophys.Acta 1995;1243:216-220. 
35. Harris RA, Goodwin GW, Paxton R, Dexter P, Powell SM, Zhang B, et al. Nutritional and hormonal regulation of the activity state of hepatic branched-chain alpharketo acid dehydrogenase complex. Ann N Y Acad Sci 1989;573:306-13.

36. Paxton R, Harris RA. Regulation of branched-chain alpha-ketoacid dehydrogenase kinase. Arch Biochem Biophys 1984;231:48-57.

37. Aftring RP, Block KP, Buse MG. Leucine and isoleucine activate skeletal muscle branchedchain alpha- keto acid dehydrogenase in vivo. Am J Physiol $1986 ; 250: E 599-604$. 

CHAPTER 6

PROTEIN SYNTHESIS IS SEVERELY DIMINISHED FOLLOWING A SIMULATED UPPER GI BLEED IN PATIENTS WITH CIRRHOSIS

Steven WM Olde Damink ${ }^{1,2}$, Rajiv Jalan ${ }^{2,3}$, Nicolaas EP Deutz ${ }^{1}$, Peter C Hayes ${ }^{2}$, Peter B Soeters ${ }^{1}$

'Department of Surgery, Maastricht University, Maastricht, The Netherlands, 2Liver Unit, Royal Infirmary of Edinburgh, Edinburgh, Scotland, UK and 3 Liver Failure Group, Institute of Hepatology, Royal Free and University College Medical School, University College London, 69-75 Chenies Mews, London WC1E 6HX 


\section{INTRODUCTION}

In patients with cirthosis of the liver, an upper gastrointestinal (GI) bleed is known to increase the susceptibility to life-threatening complications such as spontaneous bacterial peritonitis, sepsis, and hepatic encephalopathy (1-4). These complications are thought to result in part from the metabolic consequences of the blood protein load in the digestive tract (5), including in severe hypoisoleucinemia in the post-bleeding period. Hypoisoleucinemia has been shown to impair whole body protein synthesis in humans (6) and may induce catabolism (5).

In a series of studies, isoleucine concentrations were shown to decrease markedly after an actual or a simulared upper GI bleed, whereas the concentration of most other amino acids increased (7-11). Post-bleeding hypoisoleucinemia has been shown to occur in experimental animals $(7,10,11)$, in healthy volunteers $(8)$, and in patients with impaired and normal liver function (9). We hyporhesized that decreased plasma and tissue isoleucine concentrations could impair protein synthesis (5), and therefore influence the function of rapidly dividing cells (e.g. immune cells) and short half-life proteins (e.g. clotting-factors). This may increase the risk of further bleeding, infection and catabolism in post-bleeding patients with cirrhosis of the liver.

Analysis of the haemoglobin molecule shows that it is devoid of the essential branched chain amino acid (BCAA) isoleucine and contains large amounts of the two other BCAA's, valine and leucine $(12,13)$. In addition, plasma proteins, like albumin and globulin also contain only small amounts of isoleucine (14). Therefore, whole blood is severely deficient in the essential amino acid isoleucine. We hyporhesised that the mere absence of isoleucine is not the only explanation of post-bleeding hypoisoleucinemia but that the peculiar imbalance in BCAA's leads to BCAA antagonism (5). BCAA antagonism is resulting from the shared transport and degradation pathways of all BCAA"s (15), whereby elevated leucine concentrations induce oxidation of all BCAA's as a group via activation of the branched chain 2-oxoacid dehydrogenase $(B C O D)$ complex. This means that isoleucine oxidation will also be induced despite the fact that its plasma concentrarions are already low. Within cells, BCAA have two fates, incorporation into protein or oxidation. Under conditions of BCAA deprivation, the $B C O D$ complex activity is suppressed (16-19) limiting the catabolism of the $B C A A$, preserving as much BCAA as possible for protein synthesis (16). However, we hypothesize that during a upper GI bleed this mechanism is deficient, and that the elevated leucine concentration that normally results from a bleed, will prevent isoleucine oxidarion to be decreased, or would even increase it.

The aim of the present study was to investigate the hypothesis that the peculiar amino acid composition of haemoglobin would lead to BCAA antagonism and diminished protein synthesis. The study was performed in patients with cirrhosis of the liver, since an upper GI bleed has the highest morbidity in this group of patients. 


\section{METHODS}

Studies were undertaken with the approval of the Lothian Research Ethics Committee and after obtaining written informed consent from each patient. Patients were eligible to participate in the study if they were clinically and metabolically stable and had biopsy proven cirrhosis of the liver. Patients were recruited from the outpatient clinic of the Liver Unit of the Royal Infirmary of Edinburgh. Exclusion criteria were the presence of a transjugular intrahepatic systemic stent shunt, severe ascites, pitting peripheral oedema, clinical evidence of hepatic encephalopathy, active alcohol abuse, diabetes, clinically evident cardiovascular disease or renal dysfunction (serum creatinine $>100 \mathrm{mmol} / \mathrm{L}$ ), malignancy or pregnancy.

Patients. Five overnight fasted patients ( 1 female, 4 male; median age 60 (39-62) yrs) with cirrhosis of the liver participated in the study (table 1). Patients were asked to continue their normal diet until the day they were studied.

Table 1: Patient characteristics (medlian and range).

\begin{tabular}{|l|l|}
\hline Age (yrs) & $60(39-62)$ \\
\hline Sex & 1 female; 4 male \\
\hline Weight (kg) & $64(57-116)$ \\
\hline Body mass index (kg/m2) & $24,7(20,2-43,7)$ \\
\hline Aetiology & 3 Alcoholic liver disease, 2 cryptogenic \\
\hline Pugh-Childs score & $8(6-9)$ \\
\hline Prothrombin time (sec) & $1,4(1,3-1,5)$ \\
\hline Albumin (mmol/L) & $34(28-39)$ \\
\hline ALAT & $46(12-68)$ \\
\hline
\end{tabular}

Study protocol. The protocol started after an overnight fast at 9.00 A.M. with the insertion of a naso-gastric tube (Medicinia, UK), a radial artery catheter ( $V$ ygon leader company, France) and a peripheral venous catheter for infusion of stable isoropes. A blood sample was collected and expired air was sampled before the start of the infusion of the stable isotopes for the measurement of background isotope enrichment. Immediately hereafter $(\mathrm{t}=0 \mathrm{hr})$, the patients received a primed (hourly dose), continuous intravenous infusion of $[1-13 \mathrm{C}]$-isoleucine $(1 \mathrm{mg} / \mathrm{kg} \mathrm{bw} / \mathrm{h})$ until the end of the experiment ( $\mathrm{t}=480 \mathrm{~min}$, see figure 1 ) using a calibrated IVAC pump. The bicarbonate pool was primed with an intravenous bolus of sodium $\left[{ }^{13} \mathrm{C}\right]$-bicarbonate $(0.08 \mathrm{mg} / \mathrm{kg}$ 
bw). Tracers were purchased from Cambridge Isotope Laboratories (99 atom\%, Woburn, Massachusetts, USA).

Figure 1. Experimental design of the study.

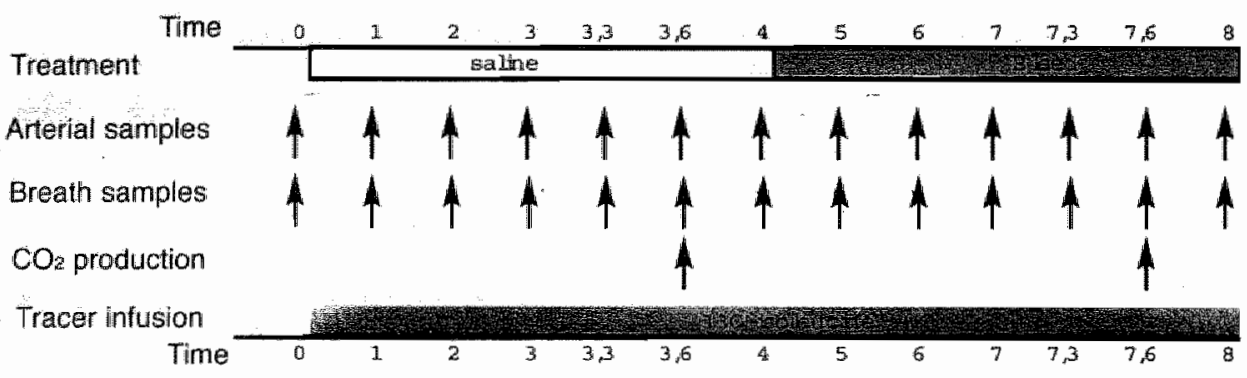

Intragastric infusions. The protocol consisted of a 4 hour period of intragastric saline infusion followed by a 4 hour period in which the metabolic consequences of an upper GI bleed was simulated by intragastric infusion of an amino acid solution that mimicked haemoglobin (prod nr 24143, Nutricia Cuijkk, The Netherlands) (table 2). The amino acid solution was freshly prepared and xanthan gum was added ( 0.1 gram per $100 \mathrm{ml}$ solution) to prevent sedimentation and infused at a rate of $100 \mathrm{ml} / \mathrm{hr}$. Subjects were not allowed to eat or drink (except water) during the study period.

To ensure that all patients received a comparable nitrogen load we administered a quantity that equalled $60 \%$ of daily nitrogen intake. Daily nitrogen intake was estimated using a 3 day food history and dieteric consultation (using the "Microdiet" computer programme, Salford University, UK).

Table 2. Amino acid composition of the simulated bleed.

\begin{tabular}{llllll}
\hline Leucine & 99.8 & Glutamate & 33.3 & Aspartate & 41.6 \\
Isoleucine & 0 & Asparagine & 27.7 & Alanine & 99.8 \\
Valine & 85.9 & Glutamine & 11.1 & Cysteine & 8.3 \\
Glycine & 55.4 & Methionine & 8.3 & Serine & 44.4 \\
Tryptophan & 8.3 & Arginine & 16.6 & Phenylalanine 41.6 \\
Threonine & 44.4 & Tyrosine & 16.6 & Histidine & 52.7 \\
Lysine & 61.0 & Proline & 38.8 & & \\
\hline
\end{tabular}

Expressed as mmol per 100 gram amino acid solution 
Sampling. Blood and expired air samples were collected at hourly intervals during the first 3 hours of the two infusion periods and at 20 minutes intervals in the final hour (see Figure 1). Blood samples were collected in pre-cooled heparinised syringes and immediately placed on ice. Expired air samples were obtained by having the patient breathe normally for 3 minutes into a $6.75 \mathrm{~L}$ mixing chamber (20), after which a 20 $\mathrm{mL}$ vacutainer was filled with a sample of the mixed expired air. In the final hour of both infusion periods, the patients were asked to rest on a bed so that their $\mathrm{CO}_{2}$ and $\mathrm{O}_{2}$ production rates and their resting energy expenditure (REE) could be determined by means of a computerized open-circuit ventillated hood (Datex, Engstrom, Helsinki, Finland)(20).

Analyses. Plasma for the measurements of amino acids was obtained by centrifugation of heparinised whole blood at $8,900 \mathrm{~g}$ at $4^{\circ} \mathrm{C}$ for 5 minutes, after which $500 \mathrm{~mL}$ of plasma was deproteinised by mixing with $20 \mathrm{mg}$ dry sulfosalicylic acid, placed into liquid nitrogen and stored at $-80^{\circ} \mathrm{C}$ for analysis at a later date. Plasma isoleucine and [1$13 \mathrm{Cl}$-isoleucine enrichments were measured using a fully automated liquid chromatography mass spectrometry system (Thermoquest LCQ, Veenendaal, the Netherlands) using pre-column derivatisation with ortho-phthaldialdehyde $(21),[1-13 \mathrm{C}]-\mathrm{CO}_{2}$ enrichment in expired air was measured with a GC continuous flow-isotope ratio mass spectrometer (Finnigan MAT-252, San Jose, California, USA) (20).

Calculations. Calculations of isoleucine kinetics were performed as described by Wolfe (22). In short, isoleucine flux was measured from the dilution of $\left[1-1{ }^{13} \mathrm{C}\right]$-isoleucine in plasma at isotopic steady state:

(1) Isoleucine flux or whole-body rate of appearance $=I / T T R_{A}$ where $I$ is the $\left[1-{ }^{13} \mathrm{C}\right]$-isoleucine infusion rate $(\mu \mathrm{mol} / \mathrm{kg} \mathrm{bw} / \mathrm{min})$ and $T T R_{A}$ is the arterial tracer to tracee ratio. At isotopic steady state, whole-body rate of appearance (which represents the rate at which isoleucine is released from protein in $\mathrm{nmol} / \mathrm{kg}$ bw/min) equals whole-body rate of disappearance (which represents the rate of incorporation into protein and the rate of isoleucine oxidation). Isoleucine oxidation was calculated from total $\mathrm{CO}_{2}$ production $\left(\mathrm{VCO}_{2}\right)$, ${ }^{13} \mathrm{CO}_{2}$ enrichment (TTR) at steady state and plasma $\left[1-{ }^{13} \mathrm{C}\right]$-isoleucine enrichment:

(2) Oxidation $(\mu \mathrm{mol} / \mathrm{kg} \mathrm{bw} / \mathrm{min})=\left(\mathrm{VCO}_{2}{ }^{*} \mathrm{TTRCO}_{2}\right) /\left(\mathrm{TTR}_{\mathrm{A}}{ }^{*} \mathrm{c}\right)$

where TTRCO ${ }_{2}$ is the tracer to tracee ratio of $\mathrm{CO}_{2}$, and $c$ is the correction factor (0.75) that accounts for the fact that not all ${ }^{13} \mathrm{CO}_{2}$ that is produced at cellular level is excreted in the breath (22). The non-oxidative portion of isoleucine flux was calculated as isoleucine flux minus isoleucine oxidation, and represents the rate at which isoleucine is incorporated into protein. 
Statistics. Data are expressed as median and range. Statistical analysis was performed using the Statistical Package for the Social Sciences, version 10.0 for Windows (SPSS, Chicago, Ill., USA). All the results represent the mean values of the final hour of each infusion period when isotopic plateau conditions were obtained (23). Wilcoxon's signed rank test was used to test for significant differences. Tracer steady state was defined as absence of a slope significantly different from 0 and was tested using oneway ANOVA. Significance was considered present at $\mathrm{p}<0.05$. 


\section{RESULTS}

All patients studied tolerated the insertion of the catheters, naso-gastric tube and amino acid administration without complications. A median of 65 (range 45-80) grams of amino acid solution was administered, representing an upper GI bleed of about $450 \mathrm{ml}$ whole blood. There were no clinical signs of changes in mental state following administration of the amino acid solution in the patients.

The 'simulated upper GI' bleed resulted in a significant decrease $(\mathrm{p}<0.05)$ in arterial isoleucine concentration to a median of $26 \%$ of the values of the saline infusion period. The decrease in isoleucine concentration showed a plateau during the final hour of amino acid infusion period (figure 2). Following the simulated bleed the concentration of leucine $(400 \%)$ and valine $(350 \%)$ increased significantly $(\mathrm{p}<0.05)$.

Figure 2. Average arterial isoleucine concentration $(\mu \mathrm{mol} / \mathrm{L}$ ) during the last hour of saline and amino acid infusion.

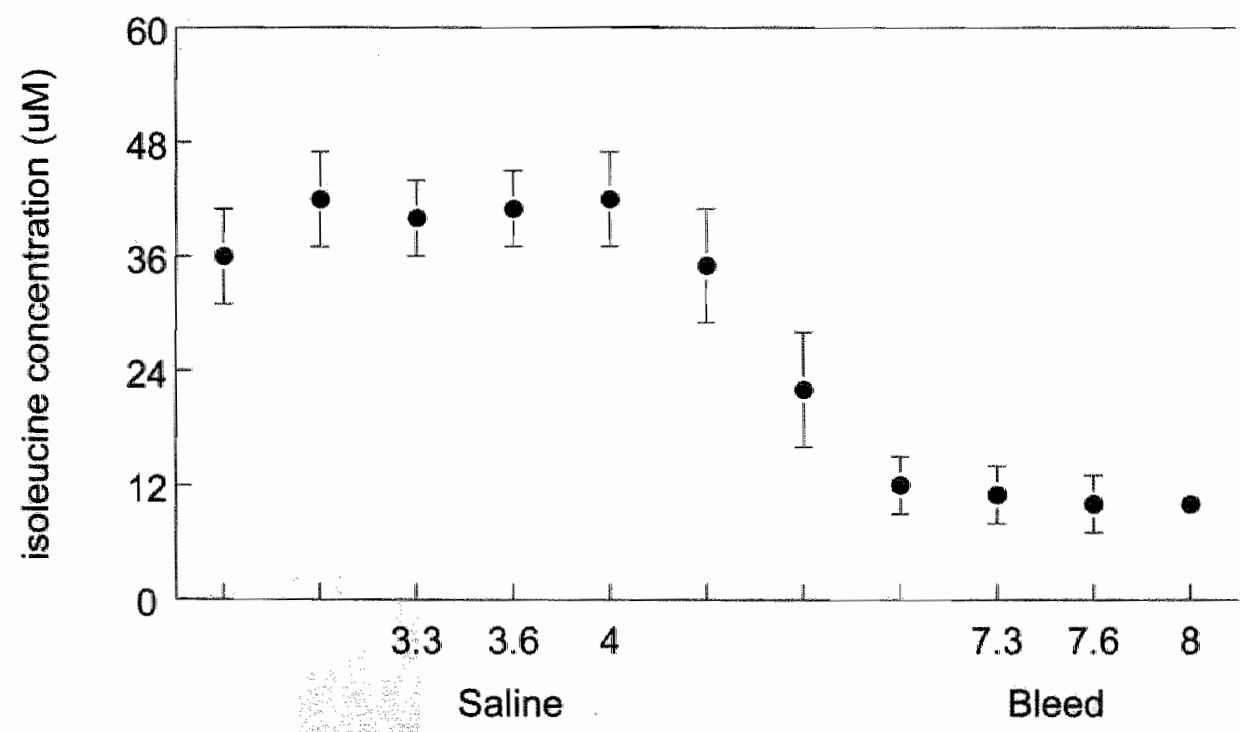


Total $\mathrm{CO}_{2}$ and $\mathrm{O}_{2}$ production increased significantly after administration of the 'simulated bleed $\left(\mathrm{VCO}_{2}\right.$ : median 2.5 (range $1.8-3.1$ ) to 2.7 (range $2.1-3.8$ ) $\mathrm{ml} / \mathrm{kg}$ bw/min; $\mathrm{VO}_{2}$ : median 3.1 (range $2.3-3.9$ ) to 3.2 (range $2.5-4.5$ ) $\mathrm{ml} / \mathrm{kg} \mathrm{bw} / \mathrm{min}$, both $\mathrm{p}<0.05$ ). Consequently, REE increased similarly after the amino acid load from a median of 21.0 (range $15.3-26.4$ ) to 22.8 (range $17.0-31.7$ ) $\mathrm{kcal} / \mathrm{kg}$ bw/day $(\mathrm{p}<0.05)$.

The tracer infusion protocol resulted in isotopic steady state of the $\left[1-{ }^{13} \mathrm{C}\right]$ isoleucine tracer during the final hour of both infusion periods Figure 3, $>0.05$ ). The simulated bleed resulted in a significant reduction of the whole body isoleucine flux tc a third of the saline infusion values (figure 4). Concomitantly, isoleucine oxidation decreased in a similar amount (Figure 4). The fraction of isoleucine flux used for oxidation was similar during the saline and the amino acid infusion periods with mediar. values of $30 \%$ (range $28-42$ ) and $24 \%(21-34)$ respectively ( $p>0.05$ ). Consequently the non-oxidative portion of isoleucine flux, representing protein synthesis decreasec similarly between the two periods, from a median of $206 \mathrm{nmol} / \mathrm{kg} \mathrm{bw} / \mathrm{min}$ (range 11s -425 ) during the saline infusion to a median of $80 \mathrm{nmol} / \mathrm{kg} \mathrm{bw} / \mathrm{min}$ (range $56-120$ $p<0.05$ ) during the amino acid infusion, respectively. 
Figure 3. Average values of the arterial tracer to tracee ratio of ${ }^{13} \mathrm{C}$-isoleucine during the final hour of the saline and simulated upper GI bleed administration.

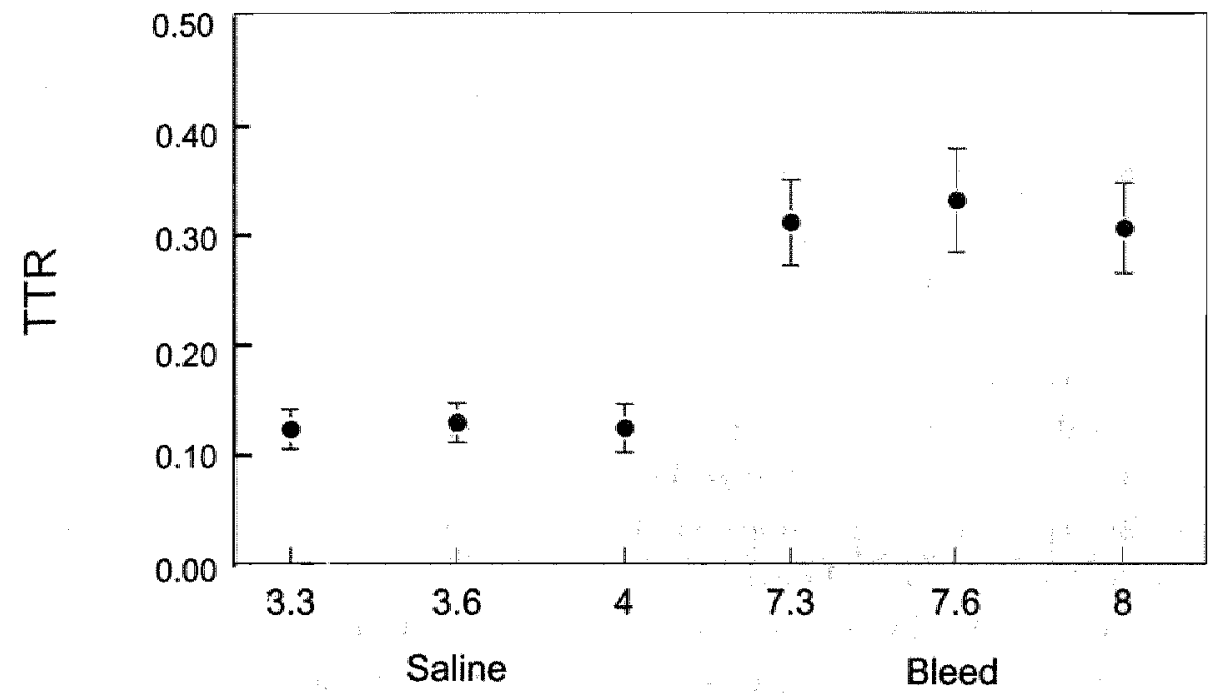

Figure 4. Awerage whole body $\mathrm{Ra}$ of isoleucine and isoleucine oxidation during the last hour of saline (black bars) and amino acid (striped bars) infusions (values in mean $\pm S E M$; represents $p<0.05$ ).

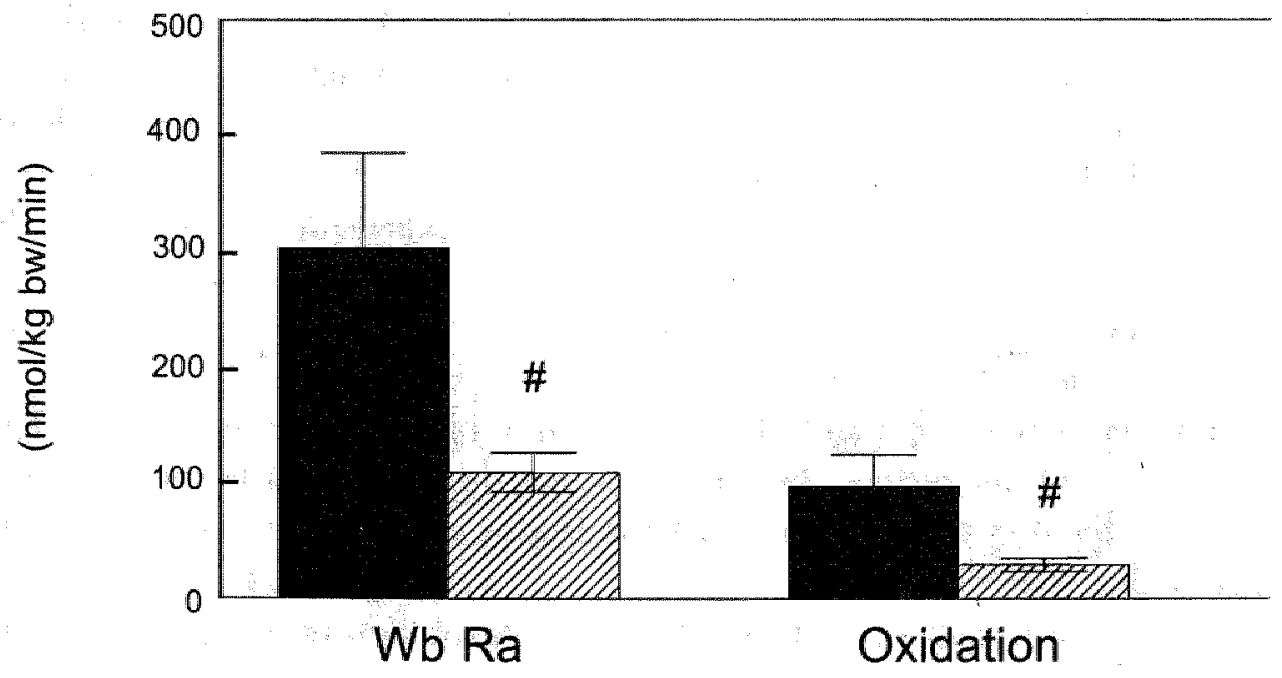




\section{DISCUSION}

The present study shows that simulating an upper GI bleed in patients with cirrhosis resulted in severe hypoisoleucinemia and decreased whole body isoleucine flux to a third of initial values. Isoleucine oxidation and the non-oxidative fraction of isoleucine flux decreased similarly, indicating a significant decrease in the rate of protein synthesis. The fact that the fraction of isoleucine flux used for oxidation did not diminish as a consequence of the hypoisoleucinemic state points to the occurrence of BCAA antagonism.

The most remarkable observation of the present study is the rapid decrease in protein synthesis that occurred after simulation of the upper GI bleed to a third of initial values. In agreement with our findings, Lecavalier et al (6) showed that a short period ( 8 hours) of hypoisoleucinemia impaired whole body protein synthesis by 10 percent in healthy human volunteers. Impaired protein synthesis has the potential to influence the function of rapidly dividing cells and could therefore increase the susceptibility to life-threatening complications in patients with cirrhosis during and after the bleed. The isoleucine content of proteins involved with acute phase response and haemostasis is between 3 and 5 percent and their synthesis could well be influenced by a period of hypoisoleucinemia (table 5). Therefore, the absorption of blood protein may increase the risk of further bleeding, infection and catabolism in post-bleeding patients with cirrhosis of the liver.

In in-vitro studies it has been shown that low isoleucine impaired protein synthesis, DNA synthesis, cell proliferation and cell function $(6,24-26)$. Recently, it has been shown that cells undergo apoptosis when deprived of isoleucine (27). Parenteral infusion of isoleucine, in amounts calculated to balance the BCAA pattern may transform the relative deficiency of circulating amino acids to balanced levels that are appropriate to facilitate protein synthesis (6). In a recent, unpublished study we show that parenteral infusion of isoleucine during a simulated upper GI bleed in parients with cirrhosis of the liver stimulates protein synthesis of the liver and muscle (28). Improving liver protein synthesis during an acute upper GI bleed in patients with cirrhosis of the liver could have major clinical effects, since liver protein synthesis is of pivotal importance in generating adequate cellular immunity, an acute phase response and normal clotting function. However, in the above mentioned study simulation of a upper GI bleed did not result in diminished incorporation of phenylalanine into protein. This discrepancy with the present observation suggests that hypoisoleucinemia influences protein synthesis of protein with a higher isoleucine content more. This observation needs further research.

In the present study we could not show a significant decrease in the proportion of 
isoleucine flux that is used for oxidation, despite the markedly decreased isoleucine concentration. We therefore conclude that this finding supports the hypothesis that $\mathrm{BCAA}$ antagonism plays a role in post-bleeding hypoisoleucinemia. BCAA antagonism was first described following the observation that feeding animals a low-protein diet supplemented with leucine inhibited growth. Harper and colleagues (29) found that adding 3\% leucine to a low-protein diet caused anorexia and greatly inhibited the growth of mature rats. These abnormalities were not observed when an excess of valine was given (15). Subsequent studies showed that excess dietary leucine reduces the concentrations of valine and isoleucine in plasma and tissue of rats $(30,31)$. Block and Harper (32) reported that rats fed a single meal of $9 \%$ protein supplemented with leucine substantially increased ${ }^{14} \mathrm{CO}_{2}$ release from $\mathrm{L}-\left[1-{ }^{14} \mathrm{C}\right]$ valine. In contrast, intravenous leucine infusion in healthy male volunteers did not increase ${ }^{13} \mathrm{CO}_{2}$ release from $\mathrm{L}-\left[1-{ }^{13} \mathrm{C}\right]$ valine. The mechanism of $\mathrm{BCAA}$ antagonism has been related to the common pathway of transport and degradation of the BCAA (32).

First, increased plasma concentrations of leucine and valine could compete with the decreased isoleucine concentrations for transport across the cell membrane via their shared transport carrier (33). This might result in a significantly greater reduction in isoleucine concentration within cells than in plasma (15).

Second, the three BCAA's have a common degradation pathway (15). Within the cell, the initial step is a reversible, concentration dependent, transamination reaction by BCAA aminotransferase (BCAT). The rate of transamination is concentration dependent and BCAT is widely distributed among tissues, with high activity in skeletal muscle and low activity in the liver and intestine (34). After this transamination reaction the resulting 2-oxoacids undergo an irreversible oxidative decarboxylation reaction by the BCOD-complex, the rate limiting step in BCAA oxidation (15). The activity of this complex exhibits large differences in various organs and is activated most potently by the oxo-acid of leucine: alpha-ketoisocaproate $(35,36)$. The reaction products of the oxidative decarboxylation of the individual BCAA follow their own catabolic pathway e.g. to the Krebs-cycle.

Within cells, BCAA have two fates, incorporation into protein or oxidation. Under conditions of BCAA deprivation, the BCOD complex activity is suppressed (17-19) limiting the catabolism of the BCAA, preserving as much BCAA as possible for protein synthesis. In a recent study, Doering and colleagues (16) showed that cultured cells that were deprived of BCAA decrease BCOD-activity by 50\%, and therefore prevented intracellular protein loss. In respect to this, we therefore interpret the observation of the present study that there was no change in the proportion of isoleucine flux being oxidized, despite the severe hypoisoleucinemia, as a confirmation of our hypothesis (5), that the high plasma leucine concentration that results from an upper GI bleed stimulates $\mathrm{BCAA}$ oxidation via $\mathrm{BCAA}$-antagonism. 
In conclusion, simulating an upper GI bleed in patients with cirrhosis resulted in severe hypoisoleucinemia and markedly decreased whole body protein synthesis to a third of initial values. The fraction of isoleucine flux used for oxidation did not change after the 'simulated bleed' despite the marked hypoisoleucinemia, pointing to occurrence of $B C A A$ antagonism.

Table 5. Isoleucine content in proteins involved in acute phase response and haemostasis (expressed as isoleucine per total amino acids and \%) (SWISS-PROT)

Serum albumin

Ceruloplasmin

C-reactive protein

$\alpha_{1}$-antitrypsin

Haptoglobin-2

$\alpha_{2}$-Macroglobin

Alpha-1- antichymotrypsin

Complement C3

Haptoglobin-1

TNF- $\alpha$

IL-6

Fibrinogen $\alpha$ and $\alpha-E$

Fibrinogen Bchain

Coagulation factor II

Coagulation factor III

Coagulation factor $\mathrm{VI}$

Coagulation factor VII

Coagulation factor VIII

Coagulation factor IX

Coagulation factor $X$

Coagulation factor $X I$

Coagulation factor XII

Coagulation factor XIII
9/609

$57 / 1065$

$12 / 224$

$20 / 418$

$18 / 406$

$54 / 1474$

$20 / 423$

$83 / 1663$

$16 / 347$

$11 / 233$

$9 / 212$

$26 / 866$

$16 / 491$

$21 / 622$

$15 / 295$

$119 / 2224$

$17 / 466$

107/2351

$24 / 461$

$17 / 488$

$8 / 615$

$33 / 661$

$37 / 731$
$(1,48 \%)$

(5,35\%)

$(5,37 \%)$

$(4,78 \%)$

$(4,43 \%)$

$(3,66 \%)$

$(4,73 \%)$

$(4,99 \%)$

$(4,61 \%)$

$(4,27 \%)$

$(4,25 \%)$

$(3,00 \%)$

(3,26\%)

$(3,38 \%)$

$(5.08 \%)$

(5.35\%)

(3,65\%)

(4.55\%)

$(5.2 \%)$

(3.48\%)

(4.99\%)

$(5.06 \%)$ 


\section{REFERENCES}

1. Teran JC, Imperiale TF, Mullen KD, Tavill AS, McCullough AJ. Primary prophylaxis of variceal bleeding in cirrhosis: a cost- effectiveness analysis. Gastroenterology 1997;112:473-82.

2. Garden OJ, Motyl H, Gilmour WH, Utley RJ, Carter DC. Prediction of outcome following acute variceal haemorrhage. Br J Surg 1985;72:91-5.

3. Pauwels A, Mostefa-Kara N, Debenes B, Degoutte E, Levy VG. Systemic antibiotic prophylaxis after gastrointestinal hemorrhage in cirrhotic patients with a high risk of infection. Hepatology 1996;24:802-6.

4. Bleichner G, Boulanger R, Squara P, Sollet JP, Parent A. Frequency of infections in cirrhotic parients presenting with acute gastrointestinal haemorrhage. $\mathrm{Br}$ J Surg 1986;73:724-6.

5. Olde Damink SWM, Dejong CHC, Deutz NEP, van Berlo CLH, Soeters PB. Upper gastrointestinal bleeding: an ammoniagenic and catabolic event due to the total absence of isoleucine in the haemoglobin molecule. Medical Hyporheses 1999:52:515-519.

6. Lecavalier L, De Feo P, Haymond MW. Isolated hypoisoleucinemia impairs whole body but not hepatic protein synthesis in humans. Am J Physiol 1991;261:E578-86.

7. van Berlo CL, van de Bogaard AE, van der Heijden MA, van Eijk HM, Janssen MA, Bost $\mathrm{MC}$, et al. Is increased ammonia liberation after bleeding in the digestive tract the consequence of complete absence of isoleucine in hemoglobin? A study in pigs. Hepatology $1989 ; 10: 315-23$.

8. van Berlo CLH, Dejong CHC, Meyerink WJHJ, von Meyenfeldt MF Fick TE, Deutz NEP, et al. A decrease in plasma isoleucine levels after simulated upper digestive tract bleeding in human volunteers. Clinical Nutrition 1991;10:056

9. Dejong CHC, Meijerink WJHJ, van Berlo CLH, Deurz NEP, Soeters PB. Decreased plasma isoleucine concentrations after upper gastrointestinal haemorrhage in humans. Gut 1996;39:13-17.

10. Deutz NE, Reijven PL, Bost MC, van Berlo CL, Soeters PB. Modification of the effects of blood on amino acid metabolism by intravenous isoleucine. Gastroenterology 1991; 101:1613-20.

11. Olde Damink SW, Dejong CH, Deutz NE, Soeters PB. Decreased plasma and tissue isoleucine levels after simulated gastrointestinal bleeding by blood gavages in chronic portacaval shunted rats. Gut 1997;40:418-24.

12. Hill RJ, Koningsberg W. The structure of human hemoglobin. the Journal of Biological Chemistry 1962;237:3151-3156.

13. Lawn RM, Efstratiadis A, O'Connell C, Maniatis T. The nucleotide sequence of the human beta-globin gene. Cell 1980;21:647-51.

14. Diem K, Lentner C. Wissenschaftliche tabellen. 7 ed: Ciba-Geigy Basel; 1976.

15. Harper AE, Miller RH, Block KP. Branched-chain amino acid metabolism. Annu Rev Nutr 1984;4:409-54.

16. Doering CB, Danner DJ. Amino acid deprivation induces translation of branched-chain alpha-ketoacid dehydrogenase kinase. Am J Physiol 2000;279:C1587-94.

17. Beggs M, Shaw JM, Randle PJ. Longer-term regulation of branched-chain-2-oxoacid 
dehydrogenase complex studied in rat hepatocytes in culture. Biochem ] 1989;257:271-5.

18. Espinal ], Beggs M, Patel H, Randle PJ. Effects of low-protein diet and starvation on the activity of branched-chain 2-oxo acid dehydrogenase kinase in rat liver and heart. Biochem 1986;237:285-8.

19. Miller RH, Eisenstein RS, Harper AE. Effects of dietary protein intake on branched-chain keto acid dehydrogenase activity of the rat. Immunochemical analysis of the enzyme complex. J Biol Chem 1988;263:3454-61.

20. Pannemans DLE, Wagenmakers AJM, Westerterp KR, Schaafsma G, Halliday D. The effect of an increase of protein intake on whole-body protein turnover in elderly women is tracer dependent. Am J Clin Nutr 1997;127.

21. van Eijk HM, Rooyakkers DR, Soeters PB, Deutz NE. Determination of amino acid isotope enrichment using liquid chromatography-mass spectrometry. Anal Biochem 1999;271:8-17.

22. Wolfe RR. Radioactive and stable isotope tracers in biomedicine. Principles and practice of kinetic analysis. New York: Wiley-Liss; 1992.

23. Matthews IN, Alman DG, Campbell M], Royston P. Analysis of serial measurements in medical research. BMJ 1990;300:230-5.

24. Kohn A. Differential effects of isoleucine deprivation on cell motility, membrane transport and DNA synthesis in NLL 8 hamster cells. Exp Cell Res 1975;94:15-22.

25. Tobey RA, Ley KD. Isoleucine-mediated regulation of genome repliction in various mammalian cell lines. Cancer Res 1971;31:46-51.

26. Jakesz R, Smith CA, Aitken S, Huff K, Schuette W, Shackney S, et al. Influence of cell proliferation and cell cycle phase on expression of estrogen receptor in MCF-7 breast cancer cells. Cancer Res 1984;44:619-25.

27. Yen $\mathrm{CL}, \mathrm{Mar} M \mathrm{MH}$, Craciunescu $\mathrm{CN}$, Edwards LJ, Zeisel SH. Deficiency in methionine, tryptophan, isoleucine, or choline induces apoptosis in cultured cells. I Nutr 2002; 132:1840-7.

28. Olde Damink SWM, Jalan R, Deutz NEP, Dejong CHC, Redhead DN, Hynds P, et al. Isoleucine enhances liver and muscle protein synthesis in cirrhosis during simulated upper GI bleeding: Submitted 2004.

29. Harper $A E$, Benton DA, Winje $M$, Elvehjem $C A$. Leucine and isoleucine antagonism in rat. Arch Biochem Biophys 1954;51:523-524.

30. Tannous RI, Rogers QR, Harper AE. Effect of leucine--isoleucine antagonism on the amino acid pattern of plasma and tissues of the rat. Arch Biochem Biophys 1966;113:35661.

31. Clark AJ, Yamada C, Swendseid ME. Effect of L-leucine on amino acid levels in plasma and tissue of normal and diabetic rats. Am J Physiol 1968;215:1324-8.

32. Block KP, Harper AE. Valine metabolism in vivo: effects of high dietary levels of leucine and isoleucine. Metabolism 1984;33:559-66.

33. Shotwell MA, Kilberg MS, Oxender DL. The regulation of neutral amino acid transport in mammalian cells. Biochim Biophys Acta 1983;737:267-84.

34. Ooiwa T, Goto H, Tsukamoto Y, Hayakawa T, Sugiyama S, Fujisuka N, et al. Regulation of valine catabolism in canine tissues: tissue distributions of branched-chain aminotransferase and 2-oxo acid dehydrogenase complex, methacrylyl-CoA hydratase and 3-hydroxybutyryl-CoA hydrolase. Biochim Biophys Acta 1995;1243:216-220. 
35. Paxton R, Harris RA. Regulation of branched-chain alpha-ketoacid dehydrogenase kinase. Arch Biochem Biophys 1984;231:48-57.

36. Aftring RP, Block KP, Buse MG. Leucine and isoleucine activate skeletal muscle branchedchain alpha- keto acid dehydrogenase in vivo. Am J Physiol 1986;250:E599-604. 


\section{CHAPTER 7}

ISOLEUCINE INFUSION DURING SIMULATED UPPER GASTROINTESTINAL. BLEEDING IMPROVES LIVER AND MUSCIE PROTEIN SYNTHESIS IN CIRRHOTICS

Steven WM Olde Damink ${ }^{1,2}$, Rajiv Jalan ${ }^{2,6}$, Nicolaas EP Deutz, Cornelis HC Dejong $^{1,3}$, Doris N Redhead ${ }^{4}$, Paula Hynd ${ }^{5}$, Peter C Hayes ${ }^{2}$, Peter B Soeters ${ }^{1}$

${ }^{1}$ Department of Surgery, Maastricht University, Maastricht, The Netherlands, ${ }^{2}$ Liver Unit, ${ }^{3}$ Department of Surgery, Radiology, ${ }^{4}$ Surgery and 5 Dietetics, Royal Infirmary of Edinburgh, Edinburgh, Scotland, UK and GInstitute of Hepatology, Royal Free and University College Medical School, University College London, London, UK 


\section{INTRODUCTION}

Fifty percent of patients with cirrhosis of the liver have oesophageal varices and a third of these patients will bleed from them within 2 years of diagnosis (1). The upper gastrointestinal (GI) bleed precipitates life-threatening complications such as spontaneous bacterial peritonitis, sepsis, renal failure and encephalopathy (2-5), resulting in the death of about $30 \%$ of patients despite adequate control of bleeding (1). These complications are thought to result in part from the metabolic consequences of the blood protein load in the digestive tract (6).

Seventy five percent of whole blood protein is haemoglobin, which comprises $98 \%$ of the erythrocyte protein. Haemoglobin is unique because it is totally devoid of the essential branched-chain amino acid (BCAA) isoleucine (6). Moreover, albumin and globulin have also an extremely low isoleucine content (6). Therefore, an upper GI bleed presents the gut with protein of very low biologic value and consequently the absorbed amino acids from blood protein cannot be used for protein synthesis.

A simulated (oral administration of a mixture of amino acids mimicking the amino acid composition of haemoglobin) or actual upper GI bleed results in extremely low isoleucine concentrations in experimental animals, healthy volunteers and patients with normal and impaired liver function (7-9). We hypothesized that decreased plasma and tissue isoleucine concentrations could impair protein synthesis (6), and therefore influence the function of rapidly dividing cells (e.g. immune cells) and short half-life proteins (e.g. clotting-factors). This may increase the risk of further bleeding, infection and catabolism in post-bleeding patients with cirrhosis of the liver. Theoretically, supplying isoleucine in this situation would be beneficial. In accordance with this, in a pig model, the catabolic cascade following a simulated bleed was significantly blunted in the group that received isoleucine supplementation intravenously compared to control animals (10).

The purpose of the present study was to test the hypothesis that simulating an upper GI bleed in patients with cirrhosis of the liver impairs organ protein synthesis which can be restored by intravenous infusion of isoleucine. In the present study we quantified protein kinetics of the portal-drained-viscera (PDV), liver, muscle and kidney during a simulated upper GI bleed with or without isoleucine infusion in patients with cirrhosis of the liver. 


\section{METHODS}

Patients. Sixteen metabolically stable patients with biopsy-proven cirrhosis of the liver who underwent portography to check the patency of their transjugular intrahepatic portasystemic stent-shunt (TIPSS) were studied (table 1). Patients were excluded if they had severe ascites, pitting edema, hepatic encephalopathy, active alcohol abuse, diabetes, clinically evident cardiovascular disease or renal dysfunction (serum creatinine $>100 \mathrm{mmol} / \mathrm{L}$ ), malignancy, pregnancy or shunt dysfuncrion (portal pressure gradient $>12 \mathrm{mmHg}$ ). Patients did not use lactulose, diuretics, or antibiotics. To avoid metabolic changes due to recent modifications of the diet, patients were included that had eaten their usual diet during the week preceding the study. The study was approved by the Lothian Research Ethics Committee and written informed consent was obtained from each subject. Severity of liver disease was assessed by the Pugh-Child scoring system (11).

Table 1: Patient characteristics (mean and SEM).

\begin{tabular}{lll}
\hline Characteristics & SB-saline & SB-isoleucine \\
\hline Age (yrs) & $43(4)$ & $51(4)$ \\
Sex & 2 female.6 male & 4 female, 4 male \\
Weight (kg) & $83.7(6.7)$ & $75.8(6.6)$ \\
Body mass index (kg/m²) & $31.4(2.9)$ & $28.8(2.1)$ \\
Total body water (L) & $48.6(4.5)$ & $43.7(3.4)$ \\
Fat-free body mass (kg) & $66.4(6.1)$ & $59.7(4.6)$ \\
Fat-free body mass (\% body weight) & $83.1(3.7)$ & $79.7(3.4)$ \\
Extracellular water (\% total body water) & $45.2(1.3)$ & $40.9(2.7)$ \\
Body cell mass (kg) & $36.7(4.0)$ & $35.5(3.5)$ \\
Body cell mass (\% Fat-free body mass) & $54.9(1.3)$ & $59.1(2.7)$ \\
Aetiology & 7 alcaholic liver & 6 alcohollic liver \\
& disease & disease \\
& 1 primary & 1 primary biliary cirrhosis \\
& sclerosing & 1 cryptogenic \\
Pugh-Child's score & cholangitis & \\
Portal pressure gradient (mm Hg) & $6.4(0.5)$ & $7.0(0.3)$ \\
Months after TIPSS placement & $5.4(1.2)$ & $5.4(1.4)$ \\
Albumin (g/L) & $30.7(6.7)$ & $25.9(6.2)$ \\
Alanine aminotransferase (IU/L) & $36.1(1.6)$ & $36.0(1.2)$ \\
INR & $32.0(3.1)$ & $38.8(6.0)$ \\
Creatinine ( $\mu$ mol/L) & $1.4(0.1)$ & $1.3(0.1)$ \\
60\% daily protein intake (gram) & $81.4(2.8)$ & $84.9(4)$ \\
\hline
\end{tabular}

Note: literature reference values (26) for healthy control subjects are: body mass index 20.25

$\mathrm{kg} / \mathrm{m}^{2}$; fat-free body mass ( $\%$ body weight) $66 \%$; bady cell mass (\% fat free body mass) $67 \%$. 
Simulation of an upper gastrointestinal bleed. An upper GI bleed was simulated by administration of a tailor made solution that was identical to the amino acid composition of the haemoglobin molecule (14) and consisted of eight essential amino acids (data in mmol per 100 gram solution: isoleucine, 0; leucine, 99.8; valine, 85.9 ; tryptophan, 8.3; threonine, 44.4; lysine, 61.0; methionine, 8.3; phenylalanine, 41.6; histidine, 52.7) and eleven non-essential amino acids (glycine, 55.4; glutamate, 33.3; asparagine, 27.7; glutamine, 11.1; arginine, 16.6; tyrosine, 16.6; proline, 38.8; aspartate, 41.6 ; alanine, 99.8 ; cysteine, 8.3 ; serine, 44.4). The solution was freshly prepared and xanthan gum was added to prevent sedimentation. To ensure that patients received a comparable nitrogen load that would lead to a moderate elevation of plasma ammonia we administered an amino acid quantity that equaled $60 \%$ of daily nitrogen intake. Daily nitrogen intake was estimated using 3-day food history and dietetic consultation (using "Microdiet" computer program, Salford University, UK). The amino acid solution was infused continuously over the study period through the nasogastric tube at a mean rate of $115( \pm 10) \mathrm{ml} / \mathrm{h}$.

Infusion of isoleucine or placebo. At the same time as the amino acid infusion was started ( $t=0$ hrs, see figure 1 ), a continuous intravenous infusion of an iso-osmotic solution containing $40 \mathrm{mg} / 1$ of isoleucine (Clinical Pharmacy, Academic Hospital Maastricht, Maastricht, The Netherlands) or saline was commenced using an IVAC pump. The amount of isoleucine infused was calculated to equal half the amount of leucine administered in the amino acid solution in order to reflect the normal leucine to isolleucine ratio in average protein (15). Patients were randomly assigned to one of the two study groups which will be referred to as SB-saline and SB-isoleucine respectively.

\section{Figure 1: Experimental set-up}

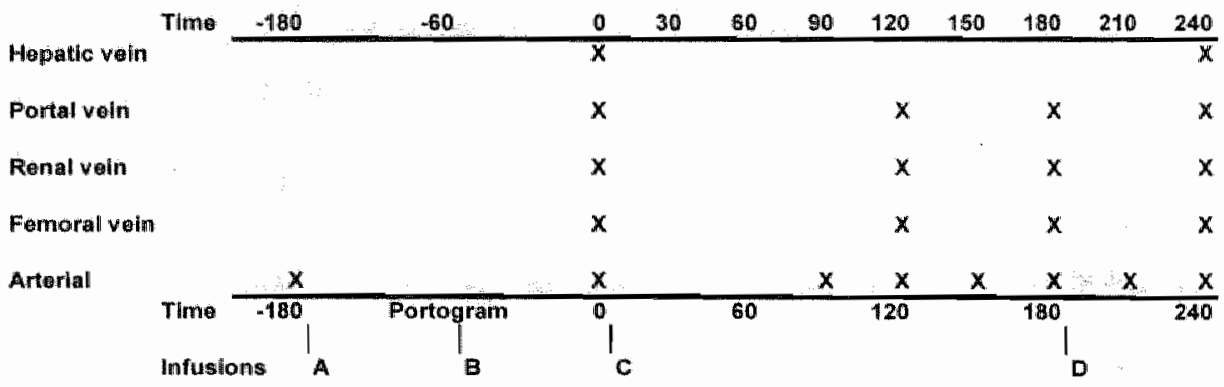

Experimental sel-up: blood samples are indicated as $X$, time is expressed in minutes. Infusion $A$ : D5-Phenylalanine, D4-Tyrosine, D2-Tyrosine, PAH. Infusion B and D: ICG. Infusion C: simulated bleed and therapy or placebo and infusion of $1-{ }^{43} \mathrm{C}$ Leucine, Sodium ${ }^{13} \mathrm{C}$ Bicarbonate and D3Leucine. 
Administration of stable isotopes. Studies were started between 6.00 and 9.00 A.M. after an overnight fast, three hours before the portography $(t=-180 \mathrm{~min})$ was planned (see flow chart figure 1). The protocol began after a catheter was inserted in a dorsal hand vein for infusion of stable isotopes and para-aminohippuric acid (PAH). A venous blood sample was drawn before the start of stable isotope infusion for measurement of background amino acid enrichment. Patients drank a mixture of 20 gram deuterium oxide dilution (99.9 atom\%, D-4501, Sigma, Boston, MA, USA) and $30 \mathrm{ml}$ of 150 mM sodium bromide (Clinical Pharmacy, Academic Hospital Maastricht, The Nerherlands) to determine total body water and extracellular water (12). Hereafter, the following stable isotopes were administered: a primed (hourly dose), continuous infusion of a mixture of $\mathrm{L}-\left[\right.$ ring- ${ }^{2} \mathrm{H}_{5}$ ]phenylalanine $([\mathrm{D} 5]-\mathrm{Phe}, 0,5 \mathrm{mg} / \mathrm{kg} \mathrm{bw} / \mathrm{h}), \mathrm{L}-[$ ring${ }^{2} \mathrm{H}_{4}$ ]tyrosine ([D4]-Tyr, only prime: $0.08 \mathrm{mg} / \mathrm{kg}$ bw) and $\mathrm{L}$-[ring- $\left.{ }^{2} \mathrm{H}_{2}\right]$ tyrosine ([D2]Tyt, $0.1 \mathrm{mg} / \mathrm{kg} \mathrm{bw} / \mathrm{h})$ using IVAC pumps. Tracer infusion was maintained until the end of the experiment $(\mathrm{t}=240 \mathrm{~min})$. The tracers were purchased from Cambridge Isotope Laboratories (Woburn, MA, USA). At the same time as the start of the infusion of the amino acid mixture through the nasogastric tube, $(t=0$ hrs, see figure 1) a primed (hourly dose), continuous infusion of $[1-13 \mathrm{C}]$ leucine $(1 \mathrm{mg} / \mathrm{kg} \mathrm{bw} / \mathrm{h}$, intravenously) and $\left[{ }^{2} \mathrm{H}_{3}\right]$ leucine ([D3]-Leu, $1 \mathrm{mg} / \mathrm{kg} \mathrm{bw} / \mathrm{h}$, intragastrically, no priming dose) was infused. The bicarbonate pool was primed with sodium $\left[{ }^{13} \mathrm{C}\right]$ bicarbonate $(0.08 \mathrm{mg} / \mathrm{kg}$ bw, intravenously). Leucine tracers were administered to determine the rate of synthesis of the plasma proteins albumin and apo-lipoprotein B100, which will be described elsewhere.

Arterial blood samples were taken until $\mathrm{t}=0 \mathrm{~min}$ and starting at $\mathrm{t}=90$ minutes with 30 minutes intervals during the infusion of the simulated bleed to assess the achievement of steady state in isotope enrichments. Steady state was defined as absence of a slope significantly different from 0 .

Insertion of catheters. Catheters were inserted under strict asepric conditions after routine portography was performed under fluoroscopic control as described in detail recently (13). In short, a naso-gastric tube was inserted, the right femoral artery was cannulated and a sheath with a sampling port was inserted in the right femoral vein. A sampling catheter was introduced through this sheath and positioned in the right renal vein. A Swan-Ganz catheter with a distal (infusion) and proximal (sampling) port was inserted through the sheath in the right internal jugular vein (used for TIPSS portography) and the proximal port was positioned in the trunk of the portal vein distal to the shunt. During catheter insertion, care was taken to position the sampling port away from any feeding tributaries to prevent improper mixing of $\mathrm{PAH}$. Patients were transported to the metabolic research ward after insertion of the catheters.

Sampling and measurement of blood flow. Organ blood flow was determined as detailed recently $(13,14)$. Kidney, leg and portal flow were determined using a primed, 
continuous infusion of para-aminohippuric acid (PAH, MSD, Haarlem, The Netherlands) and liver plasma flow was determined using a primed, continuous infusion of indocyanine green (Cardiogreen, Becton-Dickinson Microbiology Systems, Aalst, Belgium). Borh solutions were infused into a dorsal hand vein. PAH infusion was started 180 minutes and indocyanine green 60 minutes before the portography to achieve steady state concentrations $(13,14)$. To measure portal flow, $\mathrm{PAH}$ infusion was switched from the dorsal hand vein to the distal port of the Swan-Ganz catheter. Sampling for PAH was performed through the proximal port of the Swan-Ganz catheter (for a detailed description see (16)). Portal flow was also calculated using the portal venous concentration of the intragastrically administered [D3]-Leu isotope and the assumption that in the TIPSS patient all the portal blood is shunted via the TIPSS (13). Since the infusion rate of [D3]-Leu is known, correction for the splanchnic extraction of [D3]-Leu $(17,18)$ will automatically yield the flux of [D3]-Leu in the portal vein. Renal blood flow was determined by measurement of PAH in the arterial and renal venous blood $(13,14)$. Blood flow in a single leg was measured by switching the infusion of PAH from the Swan-Ganz catheter to the femoral artery $(13,14)$. Portal, renal and leg blood flow were measured immediately before simulation of the bleed $(0 \mathrm{~min})$ and 120,180 , and 240 minutes thereafter. ICG infusion was discontinued after baseline sampling and restarted at $180 \mathrm{~min}$ to obtain steady state concentrations during the final sample. Hepatic blood flow was measured by simultaneous sampling of arterial and hepatic venous blood prior to and 240 minutes after the simulated bleed when the position of the catheters was checked using fluoroscopy (see figure 1) $(13,14)$. Samples were taken from the middle or right hepatic vein that did not contain the TIPSS to prevent mixing of hepatic and portal venous blood.

Analytical procedures. Blood samples were collected from the femoral artery, the portal, renal, femoral and hepatic veins at the times of measurement of blood flow. A blood sample for deuterium and bromide analysis was taken 4 hours afrer the ingestion. Blood samples were taken with pre-cooled heparinised syringes and immediately placed on ice. Blood electrolyte concentrations were directly measured using a CO-oximeter (IL 282, Instrumentation Laboratories, USA), except for the hepatic venous blood sample. For PAH determinations, $300 \mathrm{~mL}$ heparinised blood was added to $600 \mathrm{~mL}$ of $0.7 \mathrm{mmol} / \mathrm{L}$ trichloroacetic acid (TCA) solution, thoroughly mixed, centrifuged at $8,900 \mathrm{~g}$ at $4{ }^{\circ} \mathrm{C}$ for 5 minutes, put into liquid nitrogen and stored at $-80^{\circ} \mathrm{C}$. Plasma for ammonia, urea, amino acids, bromide, deuterium and ICG was obtained by centrifugation of heparinised whole blood at $8,900 \mathrm{~g}$ at $4^{\circ} \mathrm{C}$ for 5 minutes. Plasma for bromide, deuterium and ICG was put into liquid nitrogen and stored at $-80^{\circ} \mathrm{C}$. For ammonia and urea determination, $500 \mathrm{~mL}$ of plasma was mixed with $50 \mathrm{~mL}$ of 3.5 $\mathrm{mmol} / \mathrm{L}$ TCA solution, put into liquid nitrogen and stored at $-80^{\circ} \mathrm{C}$. For amino acid analysis $500 \mathrm{~mL}$ of plasma was deproteinised by mixing with $20 \mathrm{mg}$ dry sulfosalicylic acid, put into liquid nitrogen and stored at $-80^{\circ} \mathrm{C}$. 
Plasma ammonia, urea, PAH and ICG were determined spectrophotometrically as described before (13). Bromide concentrations were determined by a fully automated HPLC system (12). Deuterium was analysed with an isotope ratio mass spectroscope (12). Plasma amino acid concentrations and tracer amino acid enrichments were measured using a fully automated liquid chromatography mass spectrometry system (Thermoquest LCQ, Veenendaal, The Netherlands) using pre-column derivatisation with ortho-phthaldialdehyde (19).

Calculations. Plasma PAH concentrations were calculated from whole blood concentrations using the haematocrit. Plasma flow was calculated according to Fick's principle, using standard equations (13). Substrate fluxes across organs were calculated as venous minus arterial concentration difference times plasma flow. Liver fluxes were calculated assuming that all the portal venous blood is shunted through the TIPSS (for a detailed description see (14)). Renal and leg plasma flow and substrate fluxes are expressed per two kidneys and for one leg. Amino acid enrichments were calculated as tracer to tracee (TTR) ratios. Branched chain amino acids (BCAA) were calculated as the sum of valine, leucine and isoleucine. TAA represents the sum of all amino acids, TEA the sum of all essential amino acids and TNEA the sum of non-essential amino acids determined. Total body water, fat-free body mass and body cell mass were calculated from bromide and deuterium dilution as detailed previously (12).

\section{Tracer kinetics}

Whole body kinetics. The steady-state rates of whole-body phenylalanine rate of appearance ( $\mathrm{Ra} ; \mathrm{nmol} / \mathrm{kg}$ body cell mass $/ \mathrm{min}$ ) were calculated by dividing the [D5]phenylalanine infusion rate by the plasma [D5]-phenylalanine entichment expressed as tracer-tracee ratio"s (TTR)(20). Whole-body Ra of phenylalanine was used as an indication of whole body protein breakdown because this amino acid cannot be synthesized de novo. The rate of whole-body phenylalanine hydroxylation was calculated from the conversion of [D5]-phenylalanine to [D4]-tyrosine:

(1) Whole-body phenylalanine hydroxylation $=$ Whole-body $\operatorname{RaTYR}_{\mathrm{TY}} \mathrm{x}$ $\mathrm{TTR}_{\mathrm{A}[\mathrm{D} \text { 4 }]-\mathrm{TYR}} / \mathrm{TTR}_{\mathrm{A}[\mathrm{D} 5]-\mathrm{PHE}}$

where whole-body $\mathrm{Ra}_{\mathrm{TYR}}$ is calculated with the use of [D2]-tyrosine (20). The whole-body rate of disappearance of [D5]-phenylalanine is a combination of the whole-body rate of protein synthesis and whole-body phenylalanine hydroxylation:

(2) Whole-body protein synthesis = whole-body protein breakdown - wholebody phenylalanine hydroxylation

Organ kinetics. Net organ protein Balance (protein synthesis minus breakdown) was determined by dilution of stable isotopes across the PDV, liver, kidney and leg using a two-compartment model as described by Wolfe (21). In this model, disposal (nmol/ $/ \mathrm{kg}$ 
body cell mass/miny is the total rate of metabolism of the amino acid across an orga (incorporation into protein plus degradation), whereas production ( $\mathrm{nmol} / \mathrm{kg}$ body ce mass $/ \mathrm{min}$ ) represents the rate of amino acid release from protein breakdown across th: organ. In the current study disposal and production of phenylalanine is calculated.

The Ner Balance across an organ (NB in nmol/ $/ \mathrm{kg}$ body cell mass $/ \mathrm{min}$ ) was calcu lated by multiplying plasma flow by the venous minus arterial concentration differenc of the amino acid. The net balance of the tracer ( $\mathrm{nb}, \mathrm{nmol} / \mathrm{kg}$ body cell mass $/ \mathrm{min}$ ) wa calculated similarly, using arterial and venous TTR's, corrected for background TTR

(3) $\mathrm{NB}=$ Flow $_{\text {plasma }} \mathrm{x}([\mathrm{Ven}]-[$ Art $])$

(4) $\mathrm{nb}=$ flow $\times\left\{\left([\right.\right.$ Art $\left.] \times T T R_{\text {Art }}\right)-\left([\right.$ Ven $\left.\left.] \times T T R_{Y_{e n}}\right)\right\}$

(5) Disposal $=\mathrm{nb} / \mathrm{TTR} \mathrm{R}_{\mathrm{V}}$

where TTR represents venous phenylalanine enrichment for the phenylalanin isotope since this is thought to approach best the intracellular enrichment (precurso pool) of the organ (21). Because the NB of an amino acid across an organ is the ne difference between producrion and disposal, production is represented as

(6) Production $=\mathrm{NB}+$ Disposal

In muscle and gut, disposal and production of phenylalanine reflect protein synthesi and protein breakdown, respectively, as these organs have relatively low phenylalanin hydroxylase activity (22). Disposal of phenylalanine in liver and kidney is a combina tion of protein synthesis and hydroxylation of phenylalanine to tyrosine (22). The rat of phenylalanine hydroxylation, or conversion of [D5]-phenylalanine into [D4]-tyrosin was calculated by conventional formulas (see for details) (23), using the direct estimat of tyrosine $\mathrm{Ra}$ with the [D2]-tyrosine isotope. The rate of phenylalanine disposal inte protein synthesis was corrected for this hydroxylation.

Statistics. Results are presented as means \pm SEM. Statistical analysis was performec using the Statistical Package for the Social Sciences, version 9.0 for Windows (SPSS Chicago, Ill., USA). Results are presented as baseline values ( $t=0$ hours) and study enc values (the mean values of the final hour of the amino acid infusion, when isotopic plateau conditions were obtained for all the isotopes employed in the study as well a: for isoleucine and ammonia. Wilcoxon's signed rank test was used to test for differences from zero and to test for significant changes in arterial values. Because of missing data in the organ flux and protein synthesis data the Mann-Whitney $U$ test was used to test for differences between the basal and study-end data within the groups. Significance was considered present at $\mathrm{p}<0.05$. 


\section{RESULTS}

General. There were no significant differences in patient characteristics between the groups (see table 1). All patients had patent TIPSS shunts indicated by a portal pressure gradient of $<12 \mathrm{mmHg}$ and catheters were inserted without complications. A mean of $46( \pm 4)$ grams and $42( \pm 4)$ grams of amino acids were administered in the SBsaline and the SB-isoleucine group respectively, representing the approximate haemoglobin content of $300 \mathrm{ml}$ whole blood. One patient did not tolerate the nasogastric tube and the study was stopped after 2,5 hours. There were no clinical signs of changes in mental state following administration of the amino acid solution in the patients.

Mean hepatic extraction of ICG was not significantly different between the two groups $(0.22 \pm 0.06$ and $0.30 \pm 0.07$ in the SB-saline and the SB-isoleucine group respectively), and was considerably higher than literature data for patients with decompensated chronic and acute liver disease (0.05 \pm 0.09 ) (24). Body composition of the patients did not differ between groups and was similar to previous reports of patients with stable cirrhosis $(25,26)$ (Table 1$)$.

Table 2. Arterial concentrations of isoleucine and ammonia.

\begin{tabular}{|c|c|c|c|}
\hline & Time & Isoleucine & Ammonia \\
\hline \multirow[t]{7}{*}{ SB-saline } & 0 & $38.0 \pm 3.0$ & $75 \pm 6$ \\
\hline & 90 & $30.2 \pm 4.2$ & $93 \pm 10$ \\
\hline & 120 & $26.1 \pm 4.3$ & $98 \pm 8$ \\
\hline & 150 & $22.5 \pm 2.7$ & $118 \pm 14$ \\
\hline & 180 & $16.2 \pm 1.6$ & $131 \pm 17$ \\
\hline & 210 & $15.0 \pm 1.8$ & $127 \pm 9$ \\
\hline & 240 & $14.1 \pm 1.1$ & $122 \pm 6$ \\
\hline \multirow[t]{7}{*}{ SB-lle } & 0 & $36.5 \pm 3.6$ & $82 \pm 6$ \\
\hline & 90 & $161.3 \pm 11.6$ & $91 \pm 9$ \\
\hline & 120 & $177.8 \pm 11.6$ & $117 \pm 10$ \\
\hline & 150 & $176.8 \pm 11.6$ & $124 \pm 15$ \\
\hline & 180 & $174.6 \pm 10.1$ & $143 \pm 11$ \\
\hline & 210 & $173.3 \pm 8.4$ & $144 \pm 16$ \\
\hline & 240 & $180.9 \pm 10.2$ & $137 \pm 13$ \\
\hline
\end{tabular}

Data are mean \pm SEM in $\mu \mathrm{m}$ and time in minutes. 
Arterial data. The administration of the amino acid solution resulted in a significant decrease in arterial isoleucine concentration to $39 \%$ of initial value in the saline group, with a plateau at $\mathrm{t}=180 \mathrm{~min}$ (table 2 ). The ammonia concentration increased significantly, with a plateau between $\mathrm{t}=180$ and $240 \mathrm{~min}$, that was not affected by isoleucine infusion (table 2). Following the simulated bleed in the SB-saline group all other amino acids increased. The most significant increase was in leucine $(400 \%)$, valine $(335 \%)$ and alanine (200\%), resulting in an elevation of $170 \%$ of initial value in the sum of all amino acids measured (see table 3). Supplementation of isoleucine in the SB-isoleucine group prevented the fall in isoleucine levels resulting in a significantly higher isoleucine concentration that was equivalent to $37 \%$ of valine and $52 \%$ of leucine concentrations at study end. The change in isoleucine concentration was significantly different between the two groups $(\mathrm{p}<0.001)$. No other significant differences in the concentration of leucine, valine and the sum of all amino acids measured was observed between the groups. Plasma urea concentration increased significantly and the magnitude of the increase was not significantly different between groups.

Table 3. Arterial substrate concentrations

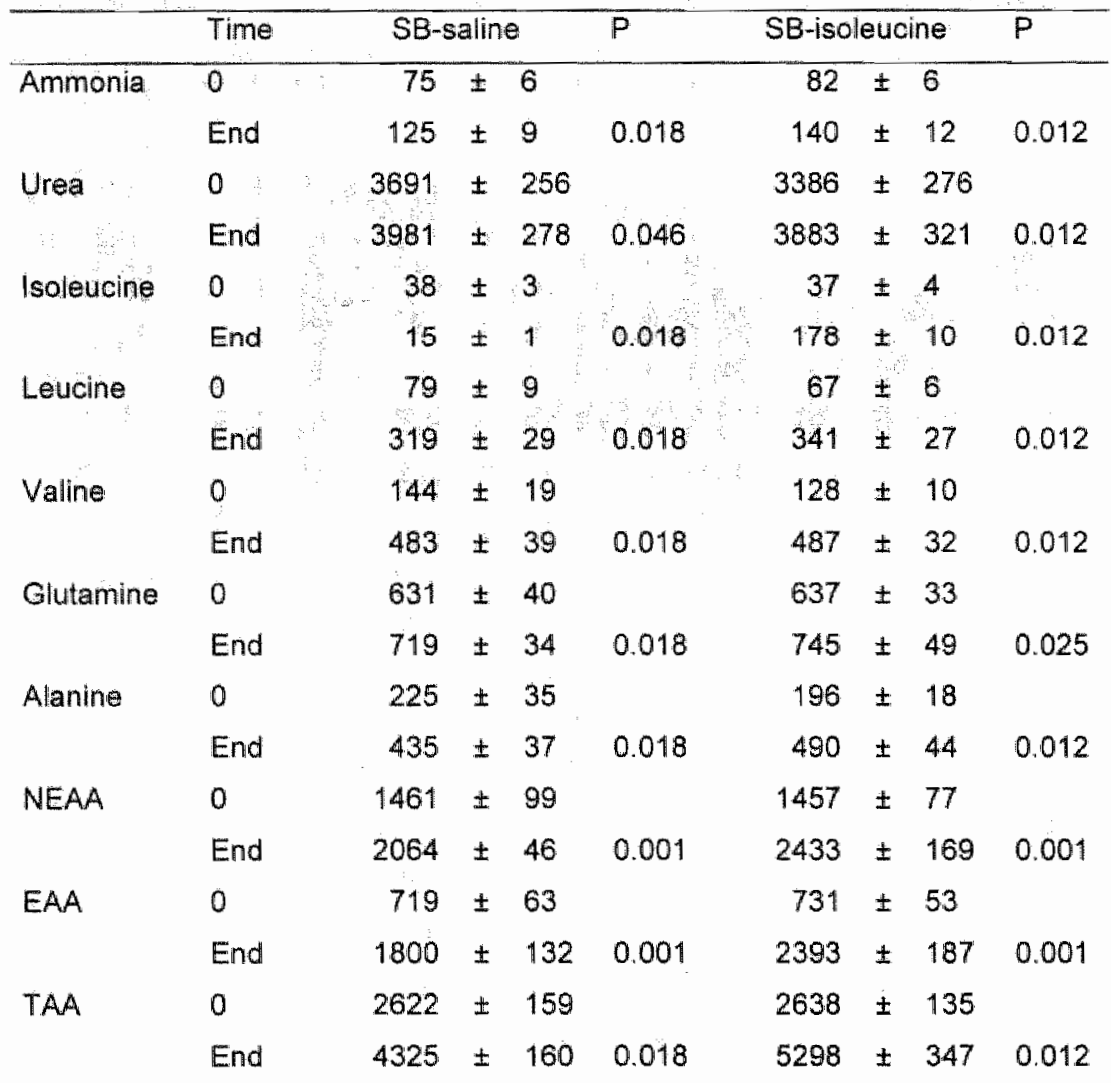

Data are mean $\pm S E M$ in $\mu M$. End values represent the mean values of the final hour of the amino acid infusion. Significance between group differences: only in isoleucine $=0.001$. 
Tracer steady state. The infusion protocol resulted in isotopic steady state of all stable isotopes used. Tracer steady state was reached at baseline and $180 \mathrm{~min}$ after the amino acid solution administration was started. The tracer to tracee ratio of all infused isotopes as below $6 \%$ (data not shown).

Whole body protein kinetics. A simulated bleed significantly increased phenylalanine whole body $\mathrm{Ra}$ (SB-saline: $1375 \pm 99$ to $2193 \pm 135 \mathrm{nmol} / \mathrm{kg}$ body cell mass $/ \mathrm{min}$; SBisoleucine: $1305 \pm 98$ to $2206 \pm 149 \mathrm{nmol} / \mathrm{kg}$ body cell mass $/ \mathrm{min}, \mathrm{p}<0.01 \mathrm{in}$ both groups) and whole body phenylalanine hydroxylation in both groups (SB-saline: $150 \pm$ 29 to $200 \pm 21 \mathrm{nmol} / \mathrm{kg}$ body cell mass $/ \mathrm{min}$; SB-isoleucine: $107 \pm 8$ to $184 \pm 27$ $\mathrm{nmol} / \mathrm{kg}$ body cell mass $/ \mathrm{min}, \mathrm{p}<0.01$ in both groups). Consequently, no significant differences between whole body estimates for protein synthesis were found (WbRa minus Wb-Hydroxylation, SB-saline: $1225 \pm 86$ to $1993 \pm 138 \mathrm{nmol} / \mathrm{kg}$ body cell mass $/ \mathrm{min}$; SB-isoleucine $1197 \pm 96$ to $2022 \pm 133 \mathrm{nmol} / \mathrm{kg}$ body cell mass $/ \mathrm{min})$.

Portal drained viscera metabolism. PDV plasma flow did not change significantly during the simulated bleed (SB-saline $19.6 \pm 4.8$ to $25.5 \pm 5.5 \mathrm{ml} / \mathrm{kg}$ body cell mass $/ \mathrm{min}$ and SB-isoleucine $26.8 \pm 5.5$ to $32.4 \pm 3.7 \mathrm{ml} / \mathrm{kg}$ body cell mass $/ \mathrm{min}$ ). Plasma flows determined with the PAH dilution and stable isotopes extraction method yielded similar results. The baseline amino acid fluxes across the PDV were comparable between the two groups (table 4). The PDV produced ammonia in post absorptive patients with cirrhosis of the liver. PDV ammonia production increased after administration of the amino acid solution, however due to the large SEM's this did not reach statistical significance in both groups. Isoleucine infusion during the simulated bleed resulted in a significantly enhanced uptake of isoleucine by the PDV. Consequently, the change in isoleucine uptake by the PDV during the experiment was significantly greater in the SB-isoleucine group $(\mathrm{p}<0.05)$. There was significant release of leucine and valine in the SB-saline group (both $\mathrm{p}=0.029$ ). This could not be demonstrated in the SB-isoleucine group, probably due to the large SEM's. Alanine uptake was significantly increased in both groups.

Portal drained viscera protein kinetics. The PDV were in a net catabolic state after the overnight fast, evidenced by negative net balances of phenylalanine at $\mathbf{t}=0$ hours and protein breakdown estimates that were higher than protein synthesis estimates (table 5). The net balance of phenylallanine did not change significantly during the protocol in both groups, but the rate of protein turnover was increased, as evidenced by elevated protein synthesis and protein breakdown in both groups (table 8).

Liver metabolism. Liver plasma flow did not change significantly during the administration of the amino acid solution (liver flow: $14.1( \pm 2.9)$ to $16.9( \pm 4.9)$ and $9.9( \pm 3.0$ ) to $10.8( \pm 2.0) \mathrm{ml} / \mathrm{kg}$ body cell mass $/ \mathrm{min}$ for the SB-saline and the SB-isoleucine group respectively). Basal ammonia and amino acid metabolism was comparable between 
Table 4. Portal Drained Viscera Fluxes

\begin{tabular}{|c|c|c|c|c|c|c|c|c|c|}
\hline \multirow{2}{*}{ Ammonia } & \multirow{2}{*}{$\frac{\text { Time }}{0}$} & \multicolumn{3}{|c|}{ SB-saline } & \multirow[t]{2}{*}{$P$} & \multicolumn{3}{|c|}{ SB-lle } & \multirow[t]{2}{*}{$\mathrm{P}$} \\
\hline & & 1217 & \pm & $412 \#$ & & 1454 & \pm & $278 \#$ & \\
\hline & End & 2274 & \pm & $783 \#$ & 0.281 & 2142 & \pm & $223 \#$ & 0.083 \\
\hline \multirow[t]{2}{*}{ Isoleucine } & 0 & 46 & \pm & 31 & & 55 & \pm & $23 \#$ & \\
\hline & End & 40 & \pm & 33 & 1.0 & -397 & \pm & $75 \#$ & 0.000 \\
\hline \multirow[t]{2}{*}{ Leucine } & 0 & 169 & \pm & $58 \#$ & & 174 & \pm & $42 \#$ & \\
\hline & End & 2739 & \pm & $1307 \#$ & 0.029 & 2368 & \pm & 1208 & 0.442 \\
\hline \multirow[t]{2}{*}{ Valine } & 0 & 95 & \pm & 79 & & 122 & \pm & 86 & \\
\hline & End & 2765 & \pm & $1445 \#$ & 0.029 & 1638 & \pm & 1248 & 0.382 \\
\hline \multirow[t]{2}{*}{ Glutamine } & 0 & -1936 & \pm & $698 \#$ & & -2001 & \pm & $581 \#$ & \\
\hline & End & -2181 & \pm & $857 \#$ & 0.955 & -2540 & \pm & $530 \#$ & 0.721 \\
\hline Alanine & 0 & 432 & \pm & $176 \#$ & & 518 & \pm & $192 \#$ & \\
\hline 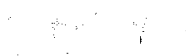 & End & 5694 & \pm & $2610 \#$ & 0.001 & 4686 & \pm & $1521 \#$ & 0.000 \\
\hline \multirow[t]{2}{*}{ TNEAA } & 0 & -304 & \pm & 520 & & -865 & \pm & 937 & \\
\hline & End & 7568 & \pm & 3768 & 0.002 & 6506 & \pm & $2481 \#$ & 0.038 \\
\hline \multirow[t]{2}{*}{ TEAA } & 0 & 739 & \pm & 407 & & 207 & \pm & 340 & \\
\hline & End. & 13281 & \pm & 6234 & 0.006 & 9749 & \pm & 4614 & 0.382 \\
\hline \multirow[t]{2}{*}{ TAA } & 0 & 614 & \pm & $911 \#$ & & -805 & \pm & 1378 & \\
\hline & End & 20781 & \pm & $9954 \#$ & 0.002 & 16173 & \pm & 7049 & 0.234 \\
\hline
\end{tabular}

Data are mean \pm SEM in nmol/ $\mathrm{kg}$ body cell mass $/ \mathrm{min}$. End values represent the mean valonly in isoleucine $(p=0.001)$. symbol acid infusion. Significance between group differences 


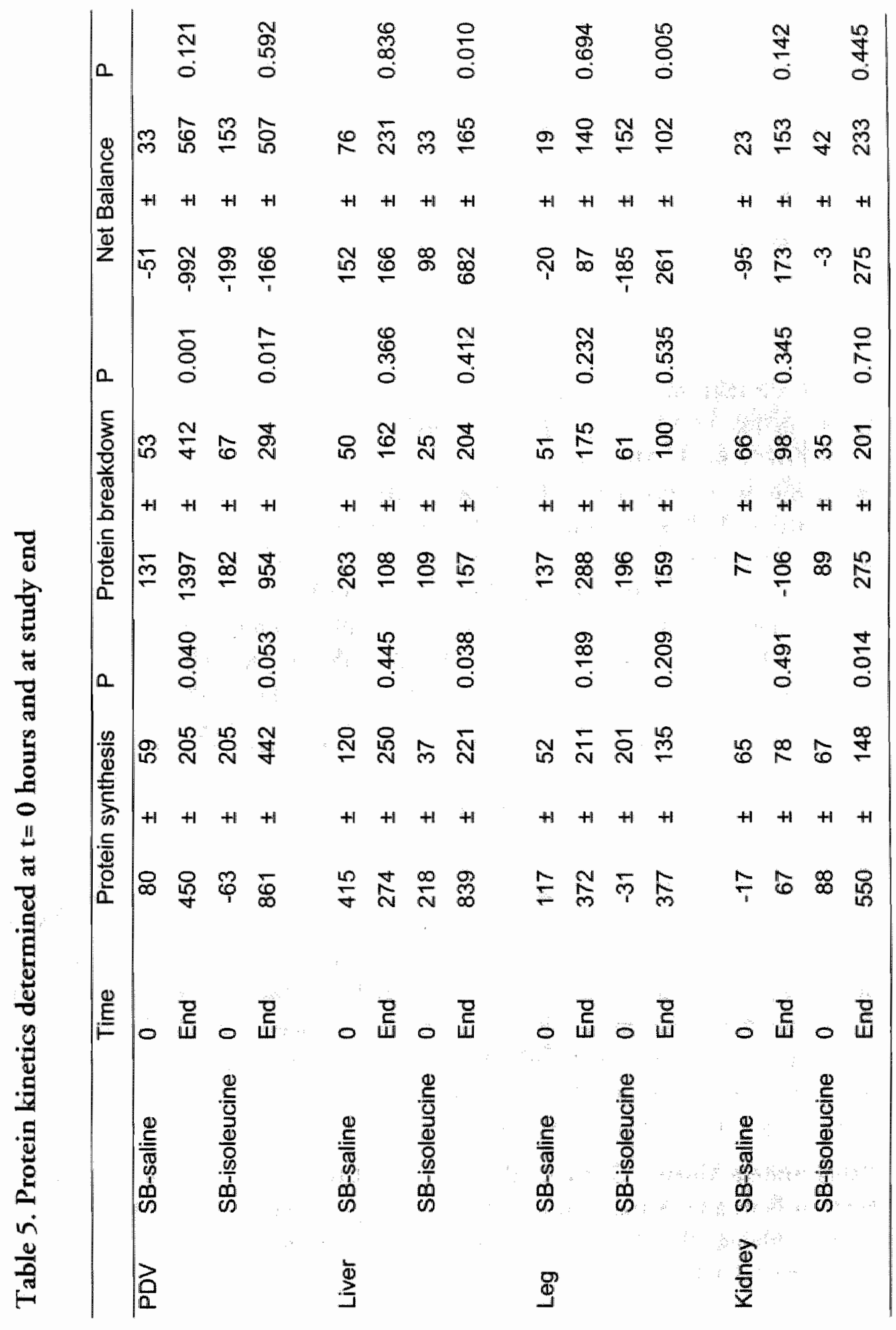


groups and did not differ significantly (see table 6). The simulated upper GI bleed resulted in the SB-saline group in an increased uptake of isoleucine by the liver, but did not result in a change in uptake of ammonia or amino acids. Isoleucine infusion during the simulated upper GI bleed increased the uptake of most amino acids by the liver, including isoleucine. The change in uptake of isoleucine was significantly greater in the SB-isoleucine group. Isoleucine infusion did not alter liver removal of ammonia.

Liver protein kinetics. Administration of the amino acid solution did not change hepatic protein kinetics in the SB-saline group, but protein synthesis was stimulated in the SB-isoleucine group resulting in an increased Net Balance of phenylalanine at study end (see table 5). No differences were observed in protein breakdown estimates.

Muscle metabolism. Plasma flows did not change significantly during the simulated bleed: leg flow $15.3( \pm 5.1)$ to $21.3( \pm 5.1)$ and $22.4( \pm 3.9)$ to $13.3( \pm 3.2) \mathrm{ml} / \mathrm{kg}$ body cell mass/min for the SB-saline and the SB-isoleucine group respectively. The apparent decrease in leg plasma flow in the SB-isoleucine group approached statistical significance ( $\mathrm{p}=0.063)$. Baseline ammonia and amino acid fluxes across the muscle were comparable between groups (table 7). Muscle removed ammonia and released glutamine. The administration of the amino acid solution resulted in increased ammonia uptake and glutamine release in the SB-saline group, in contrast with the $\mathrm{SB}$-isoleucine group where no differences could be observed. The $S B$ resulted in increased uptake of valine, leucine and isoleucine in the SB-isoleucine group, but not in the SB-saline group.

Muscle protein kinetics. At the start of the study, muscle protein kinetics did not differ between the two groups (table 5). Administration of the amino acid solution did not change the Net Balance in the $S B$-saline group, whereas it increased significantly in the SB-isoleucine infused group, resulting in a net anabolic state (table 8 ).

Renal metabolism. Renal plasma flows across organs did not change significantly during the administration of the amino acid solution: $18.0( \pm 4.8)$ to $21.1( \pm 6.7)$ and 21.1 $( \pm 5.4)$ to $21.9( \pm 5.5) \mathrm{ml} / \mathrm{kg}$ body cell $\mathrm{mass} / \mathrm{min}$ for the SB-saline and the SB-isoleucine group respectively. Baseline flux data were similar in both groups (see table 8). Administration of the amino acid solution resulted in elevated renal ammoniagenesis in both groups ( $P=0.065$ in the SB-isoleucine group, see table 8 ). Renal uptake of alanine during the simulated bleed increased in both groups $(\mathrm{p}=0.083$ in the SBisoleucine group). No major alterations in other amino acids were observed.

Renal protein kinetics. The net balance of phenylalanine did not differ significantly between both groups (table 5) and did not change during the experiment in borth groups, although the SB-isoleucine group showed an increase in protein synthesis by the kidney $(\mathrm{p}=0.014)$. 
Table 6. Liver Fluxes

\begin{tabular}{|c|c|c|c|c|c|c|c|c|c|}
\hline \multirow{2}{*}{ Ammonia } & \multirow{2}{*}{$\begin{array}{l}\text { Time } \\
0\end{array}$} & \multicolumn{3}{|c|}{ SB-Saline } & \multirow[t]{2}{*}{$P$} & \multicolumn{3}{|c|}{ SB-lle } & \multirow[t]{2}{*}{$P$} \\
\hline & & -439 & \pm & $186 \#$ & & -138 & \pm & 101 & \\
\hline & End & -431 & \pm & 191\# & 0.945 & -140 & \pm & 128 & 0.755 \\
\hline \multirow[t]{2}{*}{ Urea } & 0 & 3112 & \pm & $1288 \#$ & & 1492 & \pm & $870 \#$ & \\
\hline & End & 5662 & \pm & $1871 \#$ & 0.295 & 4223 & \pm & $1224 \#$ & 0.093 \\
\hline \multirow[t]{2}{*}{ Isoleucine } & 0 & -11 & \pm & 16 & & -41 & \pm & $14 \#$ & \\
\hline & End & -51 & \pm & $9 \#$ & 0.046 & -398 & \pm & $62 \#$ & 0.001 \\
\hline \multirow[t]{2}{*}{ Leucine } & 0 & -111 & \pm & 58 & & -112 & \pm & $35 \#$ & \\
\hline & End & -311 & \pm & 421 & 0.731 & -1070 & \pm & $336 \#$ & 0.001 \\
\hline \multirow[t]{2}{*}{ Valine } & 0 & -89 & \pm & $41 \#$ & & -126 & \pm & $44 \#$ & \\
\hline & End & -253 & \pm & 605 & 0.366 & -1299 & \pm & $479 \#$ & 0.003 \\
\hline \multirow[t]{2}{*}{ Glutamine } & 0 & -1973 & \pm & $685 \#$ & & -979 & \pm & 314 & \\
\hline & End & -1413 & \pm & $645 \#$ & 0.445 & -2260 & \pm & $539 \#$ & 0.053 \\
\hline \multirow[t]{2}{*}{ Alanine } & 0 & -1246 & \pm & 633 & & -483 & \pm & 209 & \\
\hline & End & -1470 & \pm & 894 & 0.731 & -2648 & \pm & 583\# & 0.008 \\
\hline \multirow[t]{2}{*}{ NEAA } & 0 & -4549 & \pm & $1882 \#$ & & -2114 & \pm & $917 \#$ & \\
\hline & End & -5519 & \pm & $2675 \#$ & 0.945 & -7774 & \pm & $1270 \#$ & 0.005 \\
\hline \multirow[t]{2}{*}{ EAA } & 0 & -1325 & \pm & $632 \#$ & & -988 & \pm & $377 \#$ & \\
\hline & End & -3511 & \pm & 2584 & 0.366 & -7577 & \pm & 1433\# & 0.001 \\
\hline \multirow[t]{2}{*}{ TAA } & 0 & -5777 & \pm & $2504 \#$ & & -3374 & \pm & 1431\# & \\
\hline & End & -9218 & \pm & 5109 & 0.534 & -15451 & \pm & $2401 \#$ & 0.001 \\
\hline
\end{tabular}




\section{Table 7. Muscle Fluxes}

\begin{tabular}{|c|c|c|c|c|c|c|c|c|c|}
\hline \multirow[b]{2}{*}{ Ammonia } & \multirow{2}{*}{$\frac{\text { Time }}{0}$} & \multicolumn{3}{|c|}{ SB-saline } & \multirow[t]{2}{*}{$P$} & \multicolumn{3}{|c|}{ SB-lle } & \multirow[t]{2}{*}{$P$} \\
\hline & & -458 & \pm & $109 \#$ & & -843 & \pm & $146 \#$ & \\
\hline & End & -1259 & \pm & $106 \#$ & 0.001 & -950 & \pm & $56 \#$ & 0.798 \\
\hline \multirow[t]{2}{*}{ Isoleucine } & 0 & 5 & \pm & 12 & & 32 & \pm & 25 & \\
\hline & End & 2 & \pm & 29 & 0.867 & -257 & \pm & $55 \#$ & 0.002 \\
\hline \multirow[t]{2}{*}{ Leucine } & 0 & 35 & \pm & 24 & & 77 & \pm & 42 & \\
\hline & End & -535 & \pm & 327 & 0.189 & -497 & \pm & $127 \#$ & 0.001 \\
\hline \multirow[t]{2}{*}{ Valine } & 0 & -24 & \pm & 60 & & 92 & \pm & 71 & \\
\hline & End & -553 & \pm & 373 & 0.336 & -537 & \pm & 264 & 0.065 \\
\hline \multirow[t]{2}{*}{ Glutamine } & 0 & 1068 & \pm & 193\# & & 1784 & \pm & $380 \#$ & \\
\hline & End & 2364 & \pm & $467 \#$ & 0.029 & 1723 & \pm & $246 \#$ & 0.878 \\
\hline Allanine & 0 & 176 & \pm & 104 & & -15 & \pm & 206 & \\
\hline \multirow[t]{2}{*}{ NEAA } & $\begin{array}{l}\text { End } \\
0\end{array}$ & $\begin{array}{r}-87 \\
1526\end{array}$ & \pm & $\begin{array}{l}140 \\
448 \#\end{array}$ & 0.694 & $\begin{array}{c}-261 \\
2354\end{array}$ & $\begin{array}{l} \pm \\
\pm\end{array}$ & $\begin{array}{l}102 \# \\
1093 \#\end{array}$ & 0.005 \\
\hline & End & 1757 & \pm & 1437 & 1.00 & 1569 & \pm & $667 \#$ & 0.721 \\
\hline \multirow[t]{2}{*}{ EAA } & 0 & 205 & \pm & 198 & & 1120 & \pm & 681 & \\
\hline & End & -1694 & \pm & 1589 & 0.613 & -1990 & \pm & $718 \#$ & 0,010 \\
\hline \multirow[t]{2}{*}{ TAA } & 0 & 1781 & \pm & $616 \#$ & $t$ & 3285 & \pm & $1361 \#$ & \\
\hline & End & 30 & \pm & 2892 & 0.397 & -205 & \pm & 1341 & 0.161 \\
\hline
\end{tabular}


Table 8. Renal Fluxes

\begin{tabular}{|c|c|c|c|c|c|c|c|c|c|}
\hline \multirow[b]{2}{*}{ Ammonia } & \multirow{2}{*}{$\frac{\text { Time }}{0}$} & \multicolumn{3}{|c|}{ SB-saline } & \multirow[t]{2}{*}{$P$} & \multicolumn{3}{|c|}{ SB-Hle } & \multirow[t]{2}{*}{$P$} \\
\hline & & 234 & \pm & 104 & & 251 & \pm & 125 & \\
\hline & End & 1351 & \pm & 233\# & 0.001 & 1127 & \pm & $329 \#$ & 0.065 \\
\hline \multirow[t]{2}{*}{ Urea } & 0 & -5611 & \pm & $1045 \#$ & & -4704 & \pm & 1068\# & \\
\hline & End & -4893 & \pm & $1351 \#$ & 0.943 & -4496 & \pm & $816 \#$ & 0.708 \\
\hline \multirow[t]{2}{*}{ Isoleucine } & 0 & 5 & \pm & 19 & & -14 & \pm & 21 & \\
\hline & End & -34 & \pm & 29 & 0.228 & -213 & \pm & $99 \#$ & 0.161 \\
\hline \multirow[t]{2}{*}{ Leucine } & 0 & 68 & \pm & $32 \#$ & & 59 & \pm & 32 & \\
\hline & End & -282 & \pm & 302 & 0.345 & -65 & \pm & 302 & 0.959 \\
\hline \multirow[t]{2}{*}{ Valine } & 0 & 20 & \pm & 75 & & 14 & \pm & 64 & \\
\hline & End & -205 & \pm & 411 & 0.573 & -182 & \pm & 296 & 0.505 \\
\hline \multirow[t]{2}{*}{ Glutamine } & 0 & -655 & \pm & $274 \#$ & & -937 & \pm & $264 \#$ & \\
\hline & End & -1117 & \pm & 411 & 0.414 & -747 & \pm & 331 & 0.382 \\
\hline \multirow[t]{2}{*}{ Alanine } & 0 & 163 & \pm & 111 & & 181 & \pm & 222 & \\
\hline & End & -1091 & \pm & $238 \#$ & 0.001 & -816 & \pm & 385\# & 0.083 \\
\hline \multirow[t]{2}{*}{ NEAA } & 0 & -604 & \pm & 457 & & -894 & \pm & 885 & \\
\hline & End & -3627 & \pm & $1011 \#$ & 0.029 & -2029 & \pm & 1172 & 0.645 \\
\hline \multirow[t]{2}{*}{ EAA } & 0 & 279 & \pm & 278 & & -108 & \pm & 200 & \\
\hline & End & -826 & \pm & 1660 & 0.491 & -509 & \pm & 1176 & 0.328 \\
\hline \multirow[t]{2}{*}{ TAA } & 0 & -179 & \pm & 702 & & -1097 & \pm & 1181 & \\
\hline & End & -4483 & \pm & 2483 & 0.108 & -2399 & \pm & 2401 & 0.645 \\
\hline
\end{tabular}

Data are mean \pm SEM in nmol/kg body cell mass $/ \mathrm{min}$. End values represent the mean values of the final hour of the amino acid infusion. Significance between group differences only in isoleucine $(p=0.001)$. Symbol \# for fluxes significantly different from zero $(p<0.05)$. 


\section{DISCUSSION}

This study demonstrates that isoleucine infusion alone during intragastric administration of an amino acid solution that resembles the amino acid pattern of haemoglobin stimulates liver and muscle protein synthesis in patients with cirrhosis of the liver and a TIPSS in situ. In the control group, this normal response to administration of an amino acid mixture $(27,28)$ was impaired. Isoleucine infusion did not diminish postbleeding hyperammonemia, nor was there a significant effect on hepatic ureagenesis.

The simulated upper GI bleed resulted in increased arterial concentrations of all amino acids except isoleucine, which was decreased markedly. This amino acid pattern resembled the changes observed in patients admitted with an acute upper gastrointestinal bleed $(7)$ and experimental animals receiving intragastric erychrocytes (8-10). The common finding of severe hypoisoleucinemia can be explained by the fact that haemoglobin does not contain isoleucine and by BCAA-antagonism (6), for which the present study supplies circumstantial evidence. The uptake of isoleucine by the liver was enhanced after the simulated bleed in the SB-saline group, although arterial isoleucine concentrations dropped. Haemoglobin comprises $98 \%$ of erythrocyte protein, is totally devoid of the BCAA isoleucine and contains large amounts of the two other BCAAs valine and leucine. Consequently, the simulated upper GI bleed resulted in a 3 to 4 fold increase in arterial valine and leucine concentration. As we have detailed previously (6), the imbalance between the BCAAs can lead to BCAA antagonism, which presumably results from their common pathway of transport and degradation (reviewed in (6)). First, increased plasma concentrations of leucine and valine could compete with decreased isoleucine concentrations for transport across the cell membrane via a shared transport carrier. Second, the high plasma leucine concentration that results from an upper GI bleed may stimulare BCAA oxidation, irrespective of the level of the other BCAA. Consequently, isoleucine oxidation may be stimulated, resulting in a further depression of plasma and tissue isoleucine concentrations (6). BCAA antagonism has been shown to occur within 10-30 minutes after intragastric leucine administration (29).

In the present study, administration of the simulated upper GI bleed amino acid mixture did not result in a positive Net Balance in any of the organs studied. This supports our hypothesis that the limired availability of isoleucine prevents the normal stimulatory effect of hyperaminoacidaemia on protein synthesis that follows after an amino acid meal $(30,31)$. It is still not known whether anabolism after amino acid administration in healthy subjects and patients with cirrhosis of the liver is achieved via inhibition of proteolysis or stimulation of protein synthesis or both (see for review (31)). In a recent study, Mc Cullough and co-workers (26) showed that suppression of 
proteolysis in response to amino acid administration was impaired in cirrhotics and anabolism was achieved by increased protein synthesis.

Short-term limited availability of a single essential amino acid during insulin and amino acid infusion impairs the synthesis of any protein requiring that amino acid (32). Impaired protein synthesis after an upper GI bleed has been hypothesized to occur (6) based on literature reports in which hypoisoleucinemia resulted in impaired protein synthesis, DNA synthesis, cell proliferation and cell function (33-35). Recently, it has been shown that cells undergo apoptosis when deprived of isoleucine (36). Lecavalier et al. (37) showed that even a short period ( 8 hours) of hypoisoleucinemia impaired whole body protein synthesis in humans. Although the present study did not show a net catabolic state in any of the organs measured after amino acid ingestion, one has to bear in mind that only a bleeding of $300 \mathrm{ml}$ was simulated. Further research is necessary to determine the metabolic consequences of an actual upper GI bleed that normally can result in several litres of blood loss (1). At present, clotting abnormalities, inadequate response to injury and high incidence of infections after an upper GI bleed in patients with liver failure are thought to result from loss or use of clotting-factors and acute phase proteins. We also hypothesize (6), that in addition to this impaired protein synthesis following an upper GI bleed may play an important role in interfering with the production of these proteins. This further increases the risk of bleeding, infection and catabolism.

The present study supports our hypothesis that parenteral infusion of isoleucine may be an effective and simple treatment for the adverse metabolic effects of upper GI bleeding (6). Isoleucine infusion balanced the BCAA pattern of arterial plasma and probably transformed the imbalanced amino acid composition of the haemoglobin molecule into a balanced protein, which can be utilized for protein synthesis in liver and muscle. In agreement with the recent report of McCullough et al (26) a net anabolic state was reached via increased incorporation of amino acids for protein synthesis and nor via inhibited proteolysis. Isoleucine infusion stimulated the uptake of most amino acids by the liver. We suggest that improving liver protein synthesis during an acute upper GI bleed in patients with cirrhosis of the liver has major clinical effects, since liver protein synthesis is of pivotal importance in generating adequate cellular immunity, an acute phase response and normal clotting function.

In the present study we could not demonstrate an increase in net protein synthesis across the PDV with infusion of isoleucine. In the saline control group there was a tendency towards a decrease in Net Balance after the simulated bleed, however this decrease was not statistically significant, probably due to the large variations observed in the fluxes measured across the PDV. Furthermore, these large SEM's prevented us from detecting a significant difference between the two groups. However, we believe that the present study provides evidence that PDV protein kinetics during a simulated bleed were positively influenced by intravenous isoleucine infusion. First, the Net 
Balance of phenylalanine remained the same in the SB-isoleucine group, whereas ther was a tendency towards a decrease in the SB-saline group. Second, in the SB-salin group there was a greater appearance of EAA in the portal vein, suggesting that ther was greater retention or utilization of essential amino acids by the gut in the SB isoleucine group during the simulated bleed, since both groups received simila amounts of EAA via the simulated bleed. Such increased retention in the gut wal would be in keeping with previous observations by our group in pigs (38) and woulc fit in the concept of a labile protein pool (38). Third, in the SB-isoleucine group ther was enhanced uptake of isoleucine by the PDV, suggesting enhanced utilization of this essential amino acid. Further research study is necessary to put these observations intc perspective. Furthermore, it would be interesting to study the effects of isoleucine intervention on PDV protein kinetics with and without the addition of a caloric source, since it has been shown that carbohydrates increase the retention of amino acids in the gut (38).

Although the kidneys have recently been recognized as organs with a very high protein turnover (39), simulation of an upper GI bleed had no influence on renal protein kinetics. As detailed recently (14), the kidneys have a key role in the hyperammonemia that follows an upper GI bleed in patients with cirrhosis. Renal ammoniagenesis after the administration of a simulated bleed is probably related to alanine uptake (14), which was not influenced by isoleucine infusion in the present study. This explains the lack of influence of isoleucine infusion on post-bleeding hyperammonemia.

In summary, we have shown that infusion of isoleucine during a simulated bleed in patients with cirrhosis of the liver restores impaired protein synthesis of liver and muscle leading to a net anabolic state in these organs. Further research is needed to evaluate the therapeutic benefit of intravenous isoleucine infusion during an actual acute upper GI bleeding in patients with cirrhosis of the liver. This research should focus on the effects on clotting function and infection rate, or surrogate endpoints like synthesis rate and function of clotting factors and rapidly dividing cells (e.g. immune cells and enterocytes). 


\section{REFERENCES}

1. Grace, N.D. 1992. Prevention of initial variceal hemorthage. Gastroenterol Clin North Am 21:149-161.

2. Teran, J.C., Imperiale, T.F., Mullen, K.D., Tavill, A.S., and McCullough, A.J. 1997. Primary prophylaxis of variceal bleeding in cirrhosis: a cost- effectiveness analysis. Gastroenterology 112:473-482.

3. Garden, O.J., Motyl, H., Gilmour, W.H., Utley, R.J., and Carter, D.C. 1985. Predictio of outcome following acure variceal haemorrhage. $\mathrm{Br} / \mathrm{Surg} 72.91-95$.

4. Pauwels, A., Mostefa-Kara, N., Debenes, B., Degoutte, E., and Levy, V.G. 1996. Systemic antibiotic prophylaxis after gastrointestinal hemorrhage in cirrhotic patients with a high risk of infection. Hepatology 24:802-806.

5. Bleichner, G., Boulanger, R., Squara, P., Soller, J.P., and Parent, A. 1986. Frequency of infections in cirrhotic patients presenting with acute gastrointestinal haemorrhage. $\mathrm{Br} J$ Surg 73:724-726.

6. Olde Damink, S.W.M., Dejong, C.H.C., Deutz, N.E.P., van Berlo, C.L.H., and Soeters, P.B. 1999. Upper gastrointestinal bleeding: an ammoniagenic and catabolic event due to the total absence of isoleucine in the haemoglobin molecule. Medical Hypotheses 52:515519.

7. Dejong, C.H.C., Meijerink, W.J.H.J., van Berlo, C.L.H., Deutz, N.E.P, and Soeters, P.B. 1996. Decreased plasma isoleucine concentrations after upper gastrointestinal haemorthage in humans. Gut 39:13-17.

8. van Berlo, C.I., van de Bogaard, A.E., van der Heijden, M.A., van Eijk, H.M., Janssen, M.A., Bost, M.C., and Soeters, P.B. 1989. Is increased ammonia liberation after bleeding in the digestive tract the consequence of complete absence of isoleucine in hemoglobin? A study in pigs. Hepatology 10:315-323.

9. Olde Damink, S.W., Dejong, C.H., Deutz, N.E., and Soerers, P.B. 1997. Decreased plasma and rissue isoleucine levels after simulated gastrointestinal bleeding by blood gavages in chronic portacaval shunted rats. Gut 40:41 8-424.

10. Deutz, N.E., Reijven, P.L., Bost, M.C. van Berlo, C.L., and Soeters, P.B. 1991. Modification of the effects of blood on amino acid metabolism by intravenous isoleucine. Gastroenterology 101:1613-1620.

11. Pugh, R.N., Murray-Lyon, I.M., Dawson, J.L., Pietroni, M.C., and Williams, R. 1973. Transection of the oesophagus for bleeding oesophageal varices. $B r /$ Surg 60:646-649.

12. Cox-Reijven, P.L., and Soeters, P.B. 2000. Validation of bio-impedance spectroscopy: effects of degree of obesity and ways of calculating volumes from measured resistance values. Int J Obes Relat Metab Disord 24:271-280.

13. Olde Damink, S.W.M., Jalan, R., Redhead, D.N., Hayes, P.C., Deutz, N.E.P, and Soeters, PB. 2002. Interorgan ammonia and amino acid metabolism in metabolically stable patients with cirrhosis and a TIPSS. Hepatology 36:1163-1171.

14. Olde Damink, S.W.M., Jalan, R., Deutz, N.E.P., Redhead, D.N., Dejong, C.H.C., Hynds, P., Jalan, R.A., Hayes, P.C., and Soeters, PB. 2003. The kidney plays a major role in the hyperammonemia seen after simulated or actual GI bleeding in patients with cirrhosis. Hepatology 37:1277-1285. 
15. Diem, K, and Lentner, C. 1976. Wissenschafiliche tabellen: Ciba-Geigy Basel.

16. Granger, D.N., and Bulkley, G.B. 1981. In Measurement of blood flow Applications to the splanchnic circulation. Baltimore/London: Williams\& Wilkins. 1-507.

17. Boirie, Y, Gachon, P., and Beaufrere, B. 1997. Splanchnic and whole-body leucine kinetics in young and elderly men. Am J Clin Nutr 65:489-495.

18. Cayol, M., Boirie, Y., Rambourdin, F., Prugnaud, J., Gachon, P., Beaufrere, B., and Obled, C. 1997. Influence of protein intake on whole body and splanchnic leucine kinetics in humans. Am J Physiol 272:E584-591.

19. yan Eijk, H.M. Rooyakkers, D.R. Soeters, P.B., and Deutz, N.E 1999 . Determination of amino acid isotope enrichment using liquid chromatography-mass spectrometry. Anal Biachem 271:8-17.

20. Tessari, P., Barazzoni, R., Zanetti, M., Vettore, M., Normand, S., Bruttomesso, D., and Beaufrere, B. 1996. Protein degradation and synthesis measured with multiple amino acid tracers in vivo. Am J Physiol 271:E733-741.

21. Wolfe, R.R. 1992. Radioactive and stable isotope tracers in biomedicine. Principles and practice of kinetic analysis. New York: Wiley-Liss. 1-471 pp.

22. Tourian, A., Goddard, J., and Puck, T.T. 1969. Phenylalanine hydroxylase activity in mammalian cells. J Cell Physiol 73:159-170.

23. Tessari, P., Barazzoni, R., Zanetri, M., Vettore, M., Normand, S., Bruttomesso, D., and Beaufrere, B. 1996. Protein degradation and synthesis measured with multiple amino acid tracers in vivo. Am.J. Pbysiol. 271:E733-E741.

24. Clemmesen, J.O., Tygstrup, N., and Ott, P. 1998. Hepatic plasma flow estimated according to Fick's principle in patients with heparic encephalopathy: evaluation of indocyanine green and $\mathrm{D}$ - sorbitol as test substances. Hepatology 27:666-673.

25. Mullen, K.D., Denne, S.C., McCullough, A.J., Savin, S.M., Bruno, D., Tavill, A.S., and Kalhan, S.C. 1986. Leucine metabolism in stable cirrhosis. Hepatology 6:622-630.

26. McCullough, A.J., Mullen, K.D., and Kalhan, S.C. 1998. Defective nonoxidative leucine degradation and endogenous leucine flux in cirrhosis during an amino acid infusion. Hepatology 28:1357-1364.

27. Tessari, P. Zanetri, M., Barazzoni, R., Biolo, G., Orlando, R., Vettore, M., Inchiostro, S., Perini, $P_{0,}$ and Tiengo, A. 1996. Response of phenylalanine and leucine kinetics to branched chain-entiched amino acids and insulin in patients with cirrhosis. Gastroenterology 111:127-137.

28. Tessari, P., Inchiostro, S., Barazzoni, R., Zanetti, M., Orlando, R., Biolo, G., Sergi, G., Pino, A., and Tiengo, A. 1994. Fasting and postprandial phenylalanine and leucine kinetics in liver cirrhosis. Am J Physiol 267:E140-149.

29. Block, K. P., and Harper, A.E 1984. Valine metabolism in vivo: effects of high dietary levels of leucine and isoleucine. Metabolism 33:559-566.

30. Svanberg, E., Moller-Loswick, A.C., Marthews, D.E., Korner, U., Andersson, M., and Lundholm, K. 1996. Effects of amino acids on synthesis and degradation of skeletal muscle proteins in humans. Am J Pbysiol 271:E718-724.

31. Charlton, M.R. 1996. Protein metabolism and liver disease. Baillieres Clin Endocrinol Metab 10:617-635.

32. Flaim, K.E, Peavy, D.E., Everson, W.V., and Jefferson, L.S. 1982. The role of amino acids in the regulation of protein synthesis in perfused rat liver. I. Reduction in rates of synthesis 
resulting from amino acid deprivarion and recovery during flow-through perfusion. J Biol Chem 257:2932-2938.

33. Kohn, A. 1975. Differential effects of isoleucine deprivation on cell motility, membrane transport and DN.A synthesis in NIL 8 hamster cells. Exp Cell Res 94:15-22.

34. Tobey, R.A., and Ley, K.D. 1971 . Isoleucine-mediated regulation of genome repliction in various mammalian cell lines. Cancer Res 31:46-51.

35. Jakesz, R., Smith, C.A., Aitken, S., Huff, K., Schuetre, W., Shackney, S., and Lippman, M. 1984. Influence of cell proliferation and cell cycle phase on expression of estrogen receptor in MCF-7 breast cancer cells. Cancer Res 44:619-625.

36. Yen, C.L., Mar, M.H., Craciunescu, C.N., Edwards, L.J., and Zeisel, S.H. 2002. Deficiency in methionine, tryptophan, isoleucine, or choline induces apoptosis in cultured cells. J Nutr 132:1840-1847.

37. Lecavalier, L., De Feo, P., and Haymond, M.W. 1991. Isolated hypoisoleucinemia impairs whole body but not hepatic protein synthesis in humans. Am J Physiol 261:E578-586.

38. Deutz, N.E.P., Ten Have, G.A.M., Soeters, P.B., and Moughan, P.J. 1995. Increased intestinal amino-acid retention from addition of carbohydrates to a meal. Clin. Nutrition 14:354-364.

39. Tessari, P., Garibotto, G., Inchiostro, S., Robaudo, C., Saffioti, S., Vettore, M., Zanetri, M., Russo, R., and Deferrari, G. 1996. Kidney, splanchnic, and leg protein turnover in humans. Insight from leucine and phenylalanine kinetics. J Clin Invest 98:1481-1492. 



\section{LINK TO CHAPTER 8}

The work in the following chapter was initiated after the observations made that insertion of a TIPPS in patients with cirrhosis of the liver can lead to increased ICP. In a series 160 patients that received a TIPSS insertion, 56 procedures were emergencies for uncontrolled variceal haemorrhage. Four of these patients developed features of acute liver failure, with marked deterioration in liver function and elevated intracranial pressure, leading to death in all. We were the first to report this important complication.

In a case report of one patient we confirmed this observation. We described the instantaneous changes in cerebral blood flow determined by intravascular ultrasound and Doppler, in a patient with cirrhosis undergoing placement of a TIPPS for uncontrolled variceal bleeding. Acute changes in $\mathrm{CBF}$ were observed during and after portasystemic shunting, which culminated in cerebral edema and cerebral herniation.

Published as Jalan $R$, Dabos K, Redhead D N, Lee A, Hayes PC. Elevation of intracranial pressure following transjugular intrahepatic portosystemic stent shunt for variceal haemorrhage. I Hepatol 1997;27:928-933 and.

Jalan R, Newby DE, Olde Damink SWM, Redhead DN, Hayes PC, Lee A. Acute changes in cerebral blood flow and metabolism during portasystemic shunting. Liver Transpl 2001; 7:274-8. 



\section{SECTION 3}

\section{CHAPTER 8}

INCREASED CEREBRAL AND PERIPHERAL VASODILATION, AND, WHOLE BODY NITRIC OXIDE PRODUCTION AFTER PORTACAVAL SHUNTING IN CIRRHOSIS

${ }^{1}$ R. Jalan, ${ }^{2}$ SWM Olde Damink, ${ }^{2} \mathrm{JC}$ ter Steege, ${ }^{3} \mathrm{DN}$ Redhead, ${ }^{3} \mathrm{~A}$ Lee, ${ }^{3} \mathrm{PC}$ Hayes, 2NEP Deutz

1Liver Failure Group, Institute of Hepatology, University College London Medical School and Hospital, London, 'Department of Surgery, Academic Hospital, Maastricht University, Maastricht, The Netherlands, ${ }^{3}$ Scottish Liver Transplantation Unit, Royal Infirmary of Edinburgh 


\section{INTRODUCTION}

The pathogenesis of hepatic encephalopathy ( $\mathrm{HE})$ in patients with cirrhosis is unclear. Present hypotheses support a central role for ammonia (1-3). In the brain, ammonia is detoxified in the astrocytes to glutamine through amidation of glutamate. The ammonia-glutamine-brain swelling hypothesis of $\mathrm{HE}$ suggests that accumulation of glutamine produces osmotic stress and causes astrocytes to swell $(4,5)$. In patients with cirrhosis, there is a low-grade brain swelling which is not associated with increased intracranial pressure (ICP) $(6,7)$. In patients with acute liver failure, where increased $1 \mathrm{CP}$ is common, several studies have suggested that in addition to ammonia an increase in cerebral blood flow (CBF) is important its pathogenesis $(8,9)$. In parients with cirrhosis, the role of alterations in $\mathrm{CBF}$ in the pathogenesis of $\mathrm{HE}$ has not been studied systematically but cirrhotic patients have been shown to have impaired CBF autoregulation (10).

A small proportion of patients with cirrhosis may under some circumstances develop intracranial hypertension $(11,12)$. There are emerging data describing cerebral edema and increased ICP in patients with cirrhosis, particularly during acute hepatic and circulatory decompensation that accompanies gastrointestinal bleeding $(11,12)$. We have recently reported deaths in 4 patients due to brain herniation from intracranial hypertension following insertion of a transjugular intrahepatic portosystemic stent-shunt (TIPSS) $(11,12)$. These patients had advanced liver disease, uncontrolled variceal bleeding, renal failure and Hyponatraemia. The TIPSS procedure provides the opportunity to be able to study the acute haemodynamic and metabolic effects of portacaval shunting in patients with cirrhosis. We have recently observed that insertion of TIPSS in a single patient resulted in an increase in CBF and brain ammonia uptake (13).

In animal models of portacaval shunting, ammonia infusion results in an increase in CBF and brain water $(14,15)$. Experiments using a glutamine synthase inhibitor suggest that activation of nitric oxide (NO) synthase in the brain may be responsible for the change in CBF (15). The aims of the present study were to evaluate the effect of TIPSS on brain ammonia and amino acid metabolism and determine whether TIPSS insertion results in an increase in CBF. Previous studies have shown TIPSS results in increases in cardiac output (CO) and, a reduction in systemic vascular resistance (SVR) and mean arterial pressure (MAP) $(16,17)$. We therefore also wanted to determine whether these changes in systemic haemodynamics were accompanied by altered nitric oxide metabolism or by a cytokine-mediated inflammatory response. 


\section{METHODS}

Studies were undertaken with the approval of the local research ethics committee, written informed consent from the next of kin of each patient, and were in accordance with the Declaration of Helsinki (1951) of the World Medical Association.

Management Protocol and Study Design. The patients were managed according to a standardized protocol, and in accordance with the UK guidelines for variceal haemorrhage (17). The decision for TIPSS insertion was made on clinical grounds, either because the patient had variceal bleeding that was uncontrolled with endoscopic therapy or because of recurrent bleeding despite endoscopic therapy. All the invasive monitoring used in this study were part of the routine clinical care apart from the procedures required for CBF measurements. No vasoconstrictor therapy such as terlipressin or octreotide was used in the management of variceal bleeding. In short, all the patients received broad spectrum antibiotics prophylactically and blood glucose levels were maintained between 5-7 mmol/1/. Electrocardiogram was monitored continuously, fluid resuscitation and red cell concentrate were administered in guidance with the central venous pressure, mean arterial pressure and the haematocrit. Once a decision to insert a TIPSS was made, consent from the patient or assent from the next of kin was obtained. At the time of TIPSS insertion, the patients were mechanically ventilated following sedation with propofol and paralysed with atracurium besylate (300$600 \mathrm{mg} / \mathrm{Kg} / \mathrm{hr}$ ). Measurements were made prior to and 1 hour after insertion of TIPSS. The study design is summarised in Figure 1.

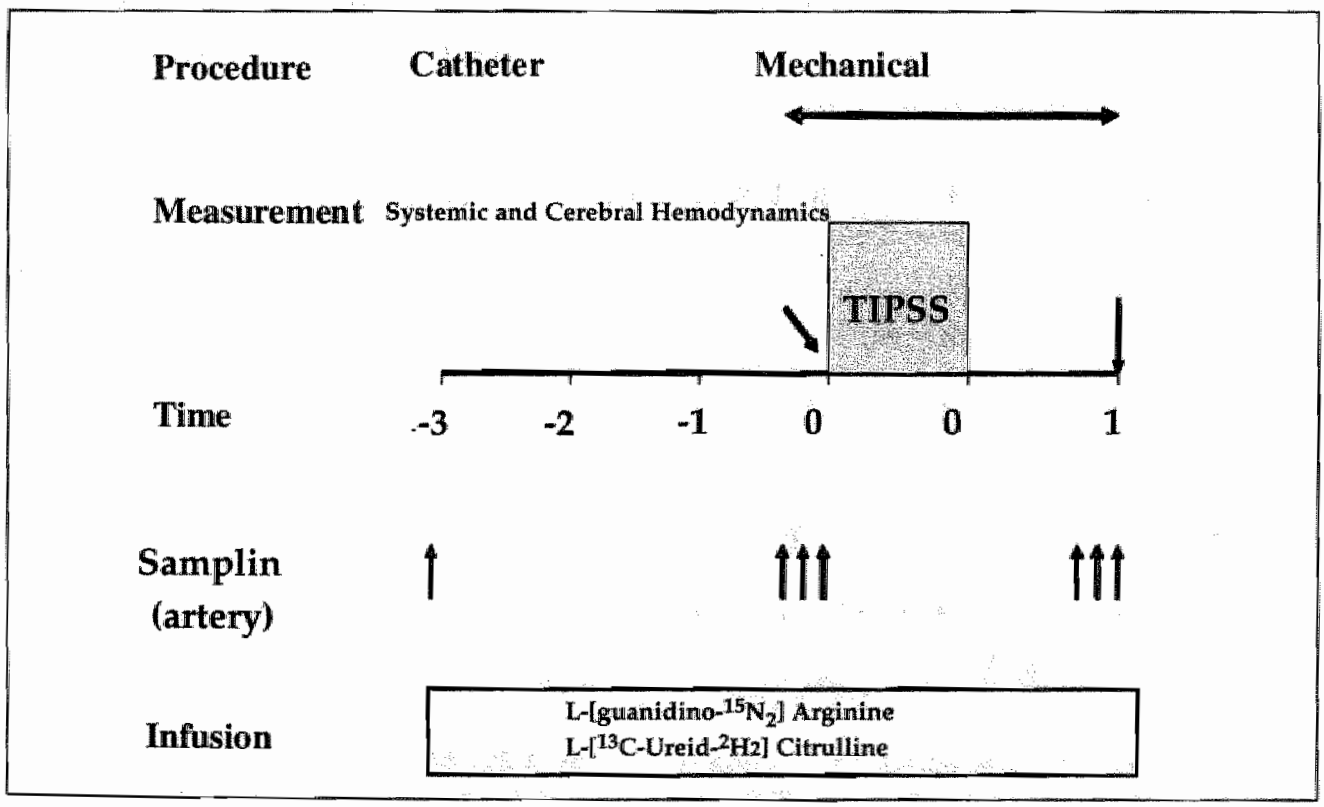

Figure 1. Study Design 
Table 1. Patient Characteristics $(\mathbf{n}=12)$

Age (years)

Sex

Body Mass Index $\left(\mathrm{Kg} / \mathrm{m}^{2}\right)$

Aetiology of Cirrhosis

*Encephalopathy > Grade 2

Ascites

Albumin ( $g / L)$

Bilirubin (umol/l)

\&Prothrombin Time (sec)

ALT (IU/L)

Urea $(g / L)$

Creatinine (umol/L)

Pugh Score

Time from presentation with bleeding to

TIPSS (hours)

Units of RCC transfused

Portal Pressure Gradient ( $\mathrm{mmH}$ )

Pre

Post
$54(3.2)$

$8 \mathrm{~m}, 4 \mathrm{f}$

$25.7(3.1)$

Alcoholic Liver Disease: 9

Autoimmune Hepatitis: 2

Primary Sclerosing Cholangitis:

6

Mild: 4

Moderate-Severe: 8

$26(1.6)$

$97.5(24)$

$15.7(1.2)$

$59(8)$

$25.6(3.1)$

$128(28)$

$10.2(1.0)$

$34.2(5.8)$

$7.2(1)$

$27.3(3.1)$

$9.3(1.1)$

"Using West-Haven criteria; \&Control PT: 11 s (at first presentation with the bleed); 
Patients. Patients were included into the study if they had clinical, biochemical or histologic evidence of cirrhosis, were undergoing TIPSS for variceal haemorrhage, were haemodynamically stable at the time of TIPSS insertion and informed consent (from the patient) or assent (from next of kin) was available. Patients were excluded from the study if they were treated with vasoconstrictor drugs, had diabetes, clinically evident cardiovascular disease, malignancy or pregnancy. The severity of $\mathrm{HE}$ was measured using the West-Haven criteria (18).

TIPSS procedure and catheter insertion. Before the TIPSS procedure was started a femoral artery was cannulated using an 18 gauge needle (Vygon leader company, Ecoven, France) and a reverse jugular catheter was inserted into the left jugular vein ( $4 \mathrm{~F}$ Opticath, U440, Abbot, USA). A single interventional radiologist performed all the TIPSS procedures in an angiography laboratory as described previously (19). In short, for TIPSS insertion, the right internal jugular vein was punctured and a $10 \mathrm{~F}$ sheath (William-Cook, Bjaeverskov, Denmark) was introduced. The right or middle heparic vein was selected using a stiff hydrophilic guide wire (Terumo, Hatagaya, Tokyo, Japan) and a fine stylet was used to puncture a branch of the portal wein. Hereafter, an angioplasty balloon catheter was used to dilate the parenchymal tract before the stent (10 $\mathrm{mm}$ Wallstent, Schneider, Bulach, Switzerland) was inserted. Then the portal venous and inferior vena caval pressures were recorded and the resulting portal pressure gradient determined (portal venous pressure minus inferior vena caval pressure.

Cerebral Blood Flow. CBF was determined using a modification of the Kety-Schmidt technique measuring the rate of uptake of nitrous oxide by the brain as detailed previously (9) immediately prior to insertion of TIPSS and 1 hour afterwards. CBF was only determined if the patient was haemodynamically stable during the measurement, defined as a difference of less than $10 \%$ in the MAP between the values at the start and at the end of CBF measurement. Ventilation was adjusted to achieve an arterial carbon dioxide tension of 4-4.5 kiloPascals, prior to the measurement of CBF.

Administration of stable isotopes. A venous blood sample was drawn before the start of the stable isotope infusion protocol for the measurement of background amino acid enrichment. The following stable isotopes were infused into the peripheral vein: a primed (hourly dose), continuous infusion of a mixture of L- [guanidino-15 $\mathrm{N}_{2}$ ] Arginine $\left(\mathrm{N}_{2}\right.$-Arginine, prime: $1 \mathrm{mg} / \mathrm{Kg}$ bw, infusion: $\left.1 \mathrm{mg} / \mathrm{Kg} \mathrm{bw} / \mathrm{h}\right)$ and L-[ureido$\left.{ }^{13} \mathrm{C} ; 5,5-{ }^{2} \mathrm{H}_{2}\right]$ Citrulline $\left(\mathrm{C}_{1} \mathrm{D}_{2}\right.$-Citrulline, prime: $0.1 \mathrm{mg} / \mathrm{Kg} \mathrm{bw}$, infusion: $0.1 \mathrm{mg} / \mathrm{Kg}$ bw /h). The tracers were purchased from Cambridge Isotope Laboratories (Woburn, MA, USA).

Blood sampling and Measurements. Blood samples were collected in pre-cooled tubes from the artery and the reverse jugular catheter prior to and 1 hour after insertion of TTPSS, for the measurement of ammonia, amino-acids, and the acid base sta- 
tus at the times of measurement of blood flow. For the determination of the isotopic steady state, 3 extra arterial blood samples were taken at 5 minute intervals prior to the above mentioned time points (Figure 1). Blood gases were directly measured using a CO-oximeter (IL 282, Instrumentation Laboratories, Boston, USA). For the measurement of ammonia, plasma was obtained by centrifugation and was deproteinised using trichloroacetic acid $(50 \% \mathrm{w} / \mathrm{v})$ prior to storage $\left(-80^{\circ} \mathrm{C}\right)$ and ammonia was measured spectrophotometrically by standard enzymatic methods (CobasMiraS, HoffmanLaRoche, Switzerland) (20). For amino acid and isotopic enrichment analysis, $500 \mu \mathrm{L}$ of plasma was deproteinised by mixing with $20 \mathrm{mg}$ dry sulfosalicylic acid, mixed, frozen in liquid nitrogen and stored at $-80^{\circ} \mathrm{C}$. Plasma amino acid concentrations and tracer amino acid enrichments were measured using a fully automated LC-MS system (LC-MS, Thermoquest LCQ, Veenendaal, the Netherlands), using pre-column derivatisation with $o$-phthaldialdehyde (21). Concentrations and enrichments of amino acids were calculated as tracer-to-tracee ratios (TTR). Serum was collected for measurement of TNFa and its receptors, interleukin (IL) $1 \mathrm{~b}$, and IL-6. These cytokines were measured by previously described methods $(22,23)$.

Calculations. BCAA represents the sum of the branched chain amino acids (leucine, valine and isoleucine), TEAA represents the sum of the essential amino acids, TNEAA represents the sum of the non-essential amino acids and TAA represents the sum of all amino acids measured. Brain ammonia and amino acid release or uptake was calculated as the venous minus arterial concentration difference times plasma flow. Positive values reflect substrate release and negative values substrate uptake. Fractional extraction of substrates were calculated as [arteriovenous difference]/[arterial]. Steady state was defined as absence of a slope significantly different from 0 , as well as of changes in concentration and enrichments greater than $5 \%$.

NO synthesis. The whole-body rate of production (in $\mu \mathrm{mol} \cdot \mathrm{kg} \mathrm{bw}^{-1} \cdot \mathrm{min}^{-1}$ ) of arginine ( $Q$ Prod $_{A R G}$ ) and citrulline ( $Q$ Prod $C I T$ ), were derived from formula (1) using the $\mathrm{N}_{2}$-arginine and the $\mathrm{C}_{1} \mathrm{D}_{2}$-citrulline isotope, respectively. I represents the tracer infused (in $\mu \mathrm{mol} \cdot \mathrm{kg} \mathrm{bw}^{-1} \cdot \mathrm{min}^{-1}$ ) and $\mathrm{TTR}_{\mathrm{A}}$ represents the rracer-to-tracee ratio of the isotopic tracer in the arterial plasma (24). TTR values were corrected for background values.

(1) $\mathrm{Q}$ Prod $=\mathbb{I} / \mathrm{TTR}_{\mathrm{A}}$

Formula (2) was used to calculate the rate of whole-body synthesis of NO $(Q$ Syn $\mathrm{NO}$ ) based upon the plasma isotope transfer from $\mathrm{N}_{2}$-arginine to $\mathrm{N}_{1}$-citrulline.

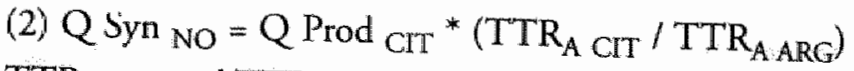

$T_{T R} R_{A C T}$ and $T T R_{A \text { ARG }}$ represent the TTR of $N_{1}$-citrulline and $N_{2}$-arginine in arterial plasma, respectively. 
Statistics. Statistical analysis was performed using the Statistical Package for the Social Sciences, version 10.0 for Windows (SPSS, Chicago, Ill., USA). Non-parametric statistics were used: the Wilcoxon's signed rank test for differences between pre- and postTIPSS values and to test for differences from zero. Relationship between variables was calculated using Pearsons correlation test. Significance was considered present at $\mathrm{p}<0.05$. 


\section{RESULTS}

Twelve patients were enrolled into the study. In two patients, placement of a reverse jugular vein catheter was not possible for technical reasons. In one other patient, the measurement of CBF failed due to technical difficulties with the automatic sampler and the mean value of CBF from the whole group was used for calculation of brain flux in this patient. The present paper therefore reports the systemic data obtained from all the 12 patients but CBF data from 9 patients and brain flux data from 10 patients.

Patients. The reason for the TIPSS procedure was variceal bleeding that was not controlled with endoscopic therapy. Variceal bleeding was controlled with a Sengstaken tube and the patients were hemodynamically stable for at least 3 hours before the TIPSS procedure, not requiring any on going fluid therapy or red cells. Six patients had clinically evident $\mathrm{HE}$ of Grade 2 or more and the other six had no clinically detectable HE. None of the patients had clinical signs of elevated ICP before or during the study (seizures, sluggish pupillary reaction to light, pupillary asymmetries, or systemic hypertension). Mean time from onset of bleeding to insertion of TIPSS was 34.2 (5.8) hours. None of the patients required inotropic support and all were mechanically ventilated during the TIPSS procedure. TIPSS insertion resulted in a significant reduction in the portal pressure gradient. Patient details are summarised in Table 1.

Systemic Haemodynamics. Prior to TIPSS insertion, the patients showed evidence of the hyperdynamic circulation of cirrhosis manifested by low MAP, high CO and low SVR. The central venous pressure and the pulmonary capillary wedge pressure were within normal range implying adequate resuscitation. TIPSS insertion resulted in a significant reduction in MAP and SVR and, a concomitant increase in cardiac output (Table 2).

Cerebral Blood Flow. Prior to TIPSS insertion, CBF was above the normal range in one patient and less than the normal range in 3 patients (normal range 45$55 \mathrm{ml} / 100 \mathrm{~g} / \mathrm{min}$ ). Of the 6 patients that had HE (grade 2 or more; CBF was successfully measured in 4 of these patients), prior to TIPSS insertion 2 were in the normal range, 1 was above the normal range and 1 was below. TIPSS insertion resulted in a significant increase in CBF in each of the patients from $53.2(2.4) \mathrm{ml} / 100 \mathrm{~g} / \mathrm{min}$ to 67.2 (3.2) $\mathrm{ml} / 100 \mathrm{gm} / \mathrm{min}, \mathrm{p}<0.01$ (Figure 2).

Ammonia and Amino acids. Arterial ammonia concentration was increased above normal levels in all patients and the lowest value was twice the upper limit of normal for our laboratory $(40 \mathrm{umol} / \mathrm{L})$. There was no significant difference in the arterial ammonia levels, its brain extraction or brain flux in the patients with and those with- 
Table 2. Arterial Vallues and Brain Flux of Ammonia and Amino acids prior to and 1 hour after TIPSS insertion

Arterial

Pre-TIPSS

Ammonia

Glutamine

Alanine

Glycine

Glutamate

Histidine

Citrulline

Taurine

Arginine

Tyrosine

Valine

Methionine

Isoleucine

Phenylalanine

Tryptophan

Leucine

Ornithine

Lysine

$B C A A$

TEAA

TNEAA

TAA

\begin{abstract}
$137 \pm 16$
\end{abstract}
$890 \pm 172$

$360 \pm 65$

$330 \pm 58$

$65 \pm 10$

$135 \pm 34$

$62 \pm 10$

$119 \pm 125$

$121 \pm 17$

$249 \pm 49$

$49 \pm 9$

$51 \pm 12$

$137 \pm 26$

$19 \pm 4$

$150 \pm 30$

$105 \pm 22$

$278 \pm 45$

$449 \pm 75$

$1280 \pm 207$

$2411 \pm 368$

$3691 \pm 570$
$57 \pm 10$
Post-TIPSS

$143 \pm 18$

$937 \pm 159$

$428 \pm 75 \#$

$358 \pm 55$

$54 \pm 6$

$141 \pm 30$

$59 \pm 9$

$61 \pm 10$

$125 \pm 9$

$129 \pm 15$

$254 \pm 43$

$55 \pm 8$

$59 \pm 13$

$144 \pm 25$

$20 \pm 3$

$161 \pm 27$

$110 \pm 21$

$303 \pm 42$

$474 \pm 68$

$1354 \pm 182$

$2572 \pm 342$

$3926 \pm 515 \#$

\section{Brain flux}

\section{Pre-TIPSS}

Post-TIPSS

$-731 \pm 205 \bullet$

$1318 \pm 331 \cdots$

$-75 \pm 253$

$-158 \pm 172$

$-207 \pm 241$

$-22 \pm 78$

$163 \pm 171$

$-33 \pm 109$

$-125 \pm 53$.

$-21 \pm 68$

$-196 \quad \pm 97$

$41 \pm 30$

$-169 \pm 48 \bullet$

$-60 \pm 48$

$-57 \pm 39$

$-473 \pm 86 \cdots$

$-123 \pm 66$

$-145 \pm 168$

$-838 \pm 195 \bullet$

$-1209 \pm 553$

$351 \pm 1033$

$-858 \pm 1493$
$-1489 \pm 385 \cdots$

$1650 \pm 678$.

$255 \pm 370$

$-207 \pm 300$

$-408 \pm 162$

$-25 \pm 139$

$-146 \pm 158$

$8 \pm 41$

$-42 \pm 62$

$44 \pm 96$

$-181 \pm 135$

$-41 \pm 38$

$-10 \pm 106$

$29 \pm 110$

$143 \pm 154$

$-438 \pm 127 \bullet$

$-248 \pm 192$

$120 \pm 228$

$-623 \pm 326$

$-591 \pm 1033$

$941 \pm 1718$

$357 \pm 2688$

Data are mean (SEM) in nmol $/ \mathrm{kg}$ bw/min. Symbols: for statistically significant difference from zero: $P<0.05$ and significance between pre- and post-TIPSS fluxes: \# $p<0.05$ (both Wilcoxon test). BCCA: sum of the branched chain amino acids, TEAA: sum of the essential amino acids, TNEAA: sum of the non-essential amino acids, TAA: sum of all amino acids measured. Positive value indicates release, negative value indicates uptake. Note: Borderline significances from zero: glutamate pre 0,093 and TEAA pre 0,059 


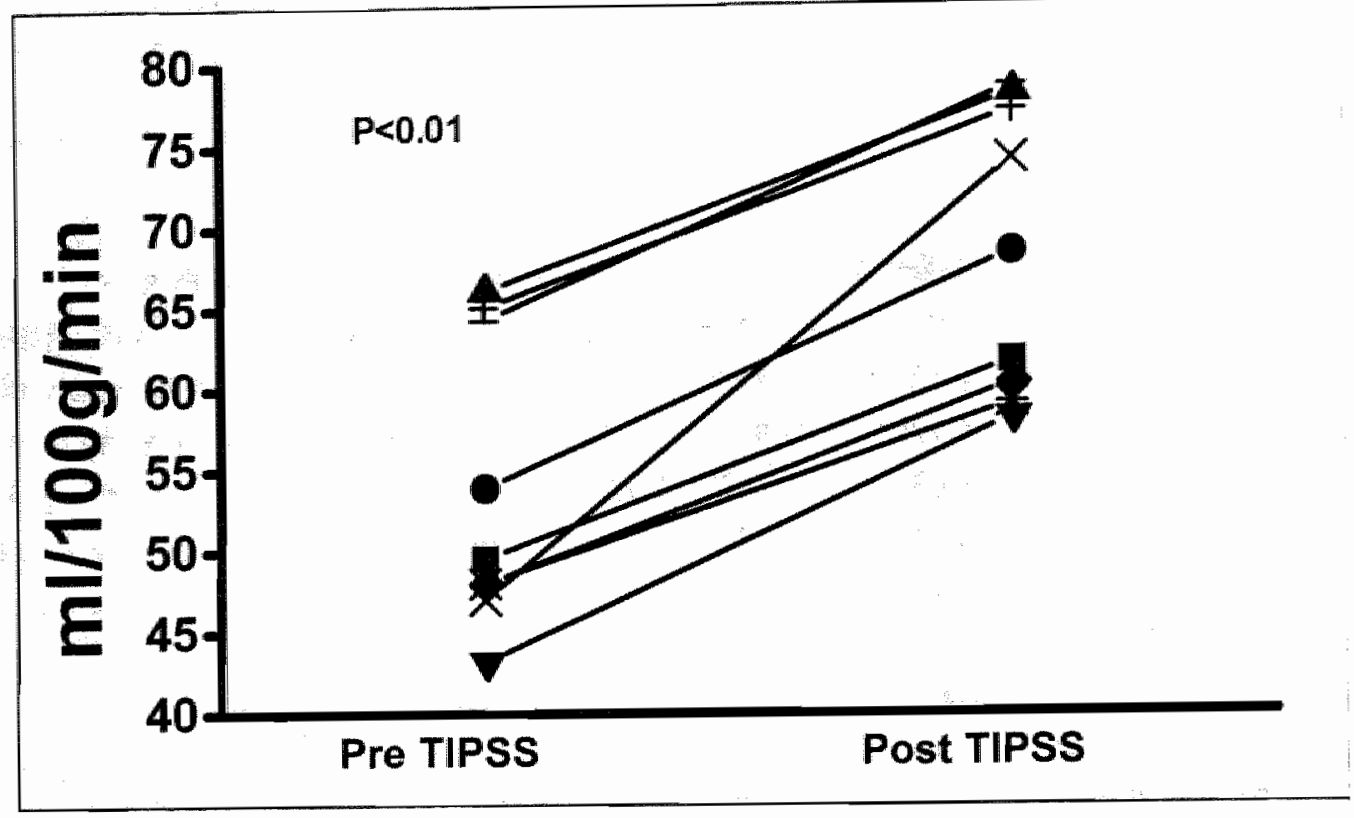

FigURE 2. Cerebral blood flow values prior to and 1 hour after TIPSS insertion.

out HE (data not shown). Insertion of TIPSS did not produce any significant change in the arterial ammonia level or its brain extraction. However, the brain flux of ammonia was significantly greater after TIPSS insertion. Prior to TIPSS, there was glutamine efflux from the brain which was about twice the brain flux of ammonia. Insertion of TIPSS resulted in no significant changes in the brain glutamine flux. TIPSS insertion resulted in a marginal but statistically significant increase in arterial alanine and total amino acids but no significant changes in their brain flux (Table 3 ).

Arginine, Citrulline and Nitric Oxide Metabolism. Artental arginine and citrulline concentrations prior to TIPSS were within normal range and did not change with TIPSS. Adequate steady state of the isotopic arginine and citrulline were achieved in all 9 patients in whom the infusions were performed (Figure $3 \mathrm{a}$ ). The rate of synthesis of arginine and citrulline did not change with TIPSS insertion (Table 4). Prior to TIPSS insertion, the whole body NO synthesis was significantly greater than the values published in the literature in each patient and increased significantly in all the patients following TIPSS (from $40.6(11.2)$ to $71.2(15.7) \mathrm{nmol} / \mathrm{kg} \mathrm{bw} / \mathrm{min}$ ) (p<0.05) (Figure 3b). Prior to TIPSS insertion, there were no significant correlations between the whole body NO synthesis and systemic or cerebral haemodynamics. However, following TIPSS insertion, significant correlations were detected between the change in NO synthesis and the change in MAP $(r=0.9 ; p<0.001)$, SVR $(r=0.8 ; p<0.008)$ and CBF $(r=0.6$; $\mathrm{p}<0.05$ ) (Figure 4). 
Cytokines. TNF alpha and IL-1B were not detected in any of the patients but the TNF receptors were elevated abour 6 times the upper limit of the normal values. IL- 6 values were about twice that detected in healthy volunteers $(22,23)$. TIPSS insertion did not produce any significant changes in the arterial levels of these cytokines. The brain flux of these cytokines were not significantly different from zero prior to TIPSS and did not change after TIPSS insertion (Table 5).

Table 3. Systemic Haemodynamics prior to and 1 hour after TIPSS insertion.

$\begin{array}{lll} & \text { Pre TIPSs } & \text { Post TIPSS } \\ \text { Heart Rate (per min) } & 89(2.8) & 93(2.7) \\ \text { Mean arterial pressure (mmHg) } & 69.5(1.3) & 61.5(2.0)^{\star *} \\ \text { Central Venous Pressure } & 9.4(0.8) & 13.8(0.9)^{\star} \\ \text { Pulmonary Capillary Wedge Pressure (mmHg) } & 11.8(2.4) & 14.2(1.9)^{*} \\ \text { Cardiac Output (L/min) } & 10.6(0.5) & 13.1(0.6)^{*} \\ \text { Systemic Vascular Resistance } & 468(30) & 300.5(32)^{\star *} \\ & & \end{array}$

Table 4. Arginine, Citrulline and Nitric Oxide Metabolism prior to and 1 hour after TIPSS insertion

\section{Pre TIPSS Post TIPSS}

Arterial Arginine (umol/L)

$119(25)$

$125(9)$

Arterial Citrulline (umol/L)

$57(10)$

$59(9)$

WBRa (Arginine) $\mathrm{nmol} / \mathrm{Kg} \mathrm{bw} / \mathrm{min}$

$701(100)$

$739(117)$

WBRa (Citrulline) nmol/Kg bw/min

$209(18)$

195 (15)

WBRa (Nitric Oxide) nmol/Kg bw/min

$41(11)$

$71(16) \#$

Data expressed as mean (SEM); WBRa: Whole body rate of appearance. \#p<0.05 

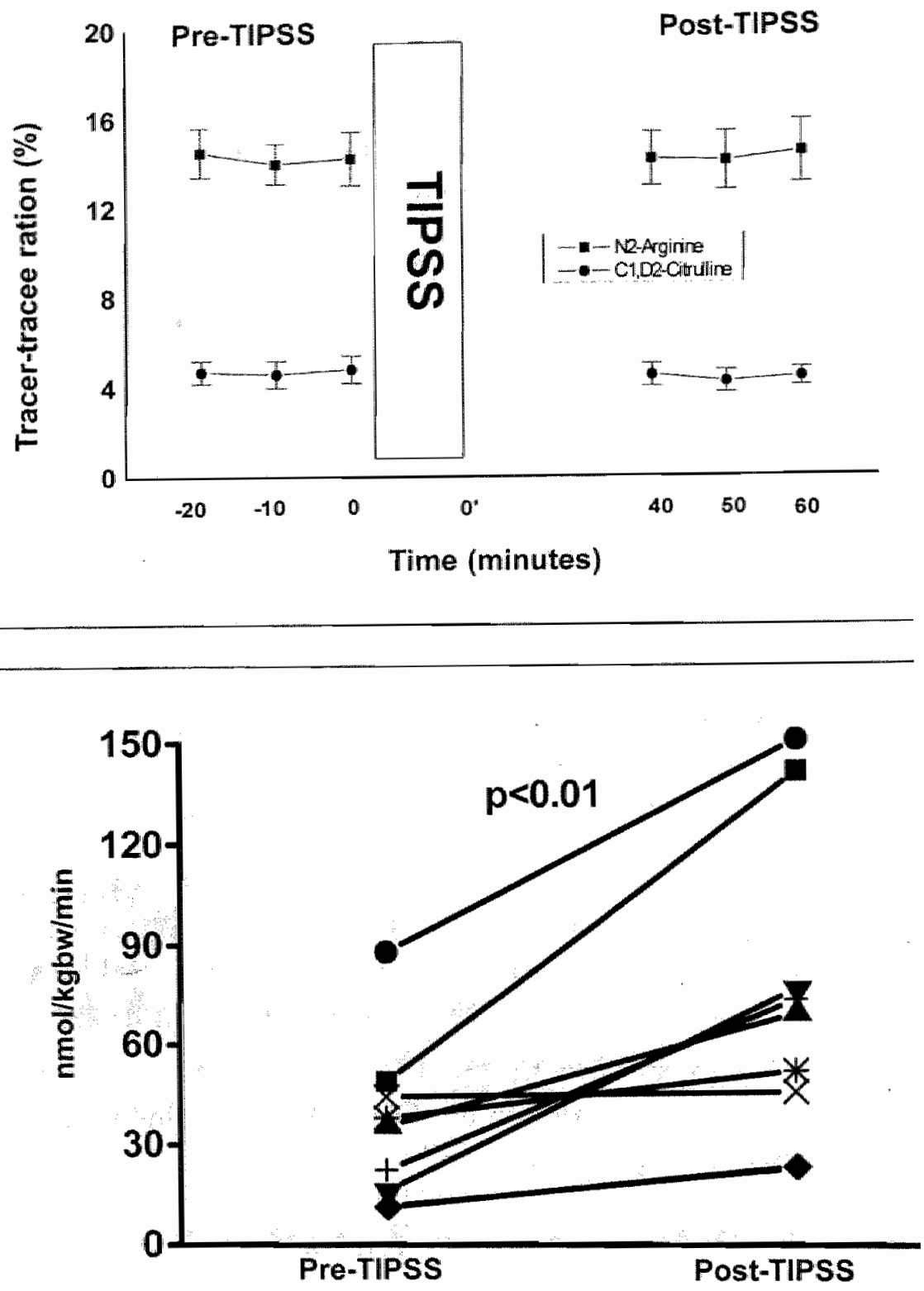

FIGURE 3. Figure depicting evidence of steady state of the infused stable isotopic tr: prior to and 1 hour after TIPSS insertion leading up to the time of the measurem of Whole body Nitric Oxide production rates prior to and 1 hour after TIPSS in tion. 

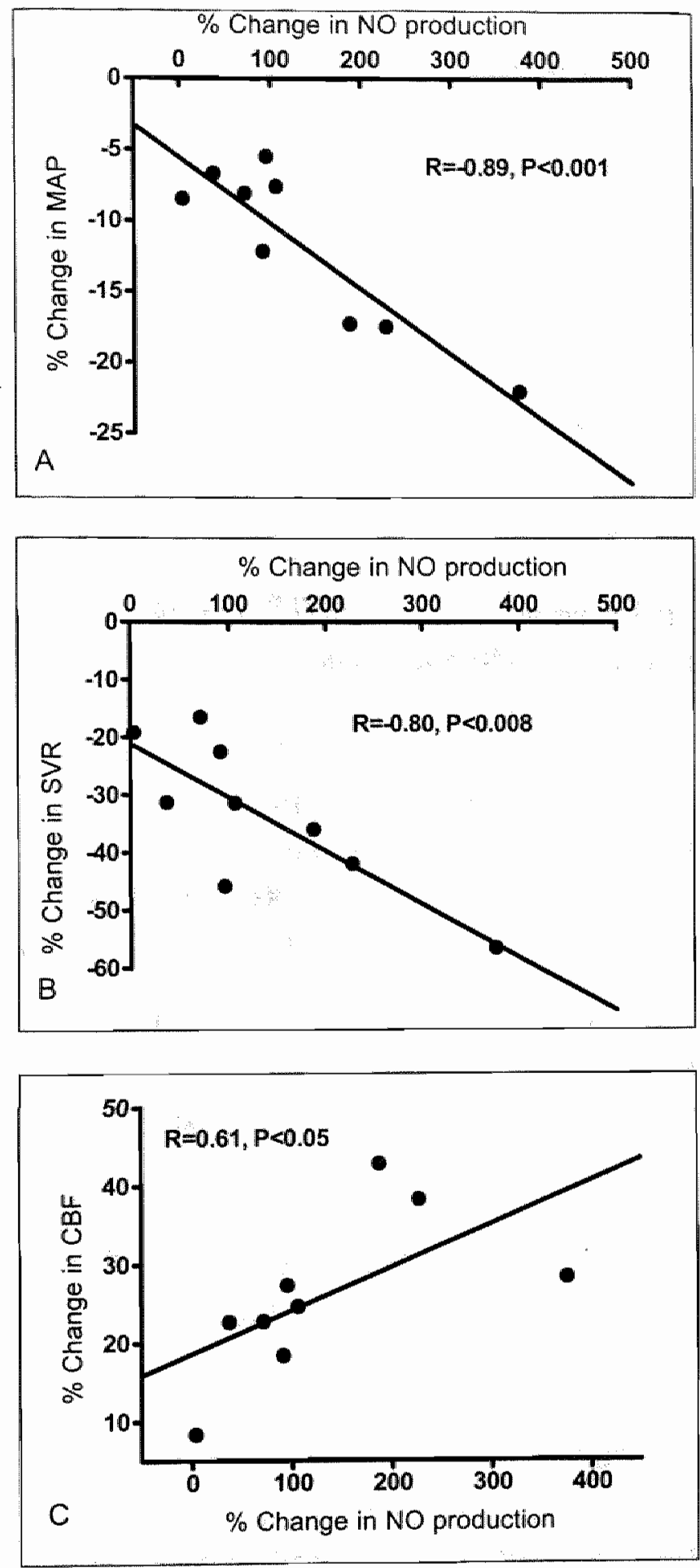

FIGURE 4. Relationship between the change in Nitric Oxide production after TIPSS insertion and the change in mean arterial pressure systemic vascular resistance and cerebral blood flow 
Table 5. Arterial values and Brain Fluxes of Pro-inflammatory cytokines prior to and 1 hour after TIPSS insertion

\author{
Arterial Brain flux
}

Pre-TIPSS Post-TIPSS Pre-TIPSS Post-TIPSS

TNF alpha ND ND ND ND

TNF R1 $12.8 \pm 2.7 \quad 16.4 \pm 4.8 \quad * 134.0 \pm 104 \quad *-108 \pm 151.6$

TNFR2 $\quad 6.3 \pm 1.1 \quad 5.8 \pm 0.9 \quad *-8.1 \quad \pm 14.4 \quad * 16.0 \quad \pm 17.9$

IL-1B ND ND ND ND

IL-6 $\quad 0.2 \pm 0.06 \quad 0.4 \pm 0.1 \quad{ }^{*}-0.7 \pm 3.3 \quad{ }^{*}-3.3 \pm 1.8$

Arterlal values expressed in ng/ml Brain fluxes expressed in $\mathrm{ng} / 100 \mathrm{~g} / \mathrm{ml}$ " *No significant differences from zero. 


\section{DISCUSSION}

The most important result of our study was the observation of an acute increase in CBF after TIPSS insertion. Our data confirm that TIPSS insertion results in an acute increase in cardiac output and a reduction in systemic vascular resistance. TIPSS increased ammonia uptake by the brain, but did not change the cerebral fractional extraction of ammonia or the efflux of glutamine from the brain suggesting that the increase in brain uptake of ammonia observed after TIPSS insertion is the result of an increase in CBF. TIPSS insertion resulted in an increase in whole body NO synthesis. This increase in NO synthesis could not be explained by changes in pro-inflammatory cytokines suggesting that this increase may possibly be due to the TIPSS induced increase in cardiac output and the consequent shear stress.

Strauss et al. were the first to show evidence of impaired CBF autoregulation in patients with cirrhosis who had severe HE and low MAP (1.0). Guevara et al showed evidence of increased cerebral vascular resistance and a concomitant decrease in $\mathrm{CBF}$ in patients with cirrhosis and low MAP (25). Philips et al. were unable to find any significant differences in CBF between cirrhotic patients with or without HE (26). It is possible that the differences in the results may be due to methods of measurement, its timing and the population of patients studied. In keeping with the data from the above mentioned studies, our study shows no correlation between the severity of $\mathrm{HE}$ and baseline CBF. However, the insertion of TIPSS was associated with a significant increase in CBF that was observed in each of the patients studied. This observation supports the hypothesis that an increase in CBF following TIPSS insertion may under appropriate circumstances result in increased ICP, as we have previously reported (11). In patients with acute liver failure, there is emerging evidence that an increase in CBF predisposes to marked rises in $\operatorname{ICP}(8,9)$. Our study also provides circumstantial evidence for the mechanisms that may underlie the TIPSS induced increase in CBF. We considered 3 possible mechanisms that may account for the observed increase in CBF with TIPSS.

First, ammonia and its brain metabolism has been suggested to be important in the increased CBF observed in animal models of liver failure $(8,9)$. Incerestingly arterial ammonia concentration did not change after TIPSS insertion. Recent data from our group suggests that ammonia homeostasis is maintained following TIPSS insertion via decreased systemic ammonia release from the kidney and via increased ammonia uptake by the muscle. In the present study, brain extraction of ammonia did not change, suggesting that the observed increase in brain uptake of ammonia following TIPSS was secondary to increase in CBF. However, increased ammonia uptake sec- 
ondary to elevated CBF can influence brain swelling; directly via increased glutamine synthesis and its accumulation $(15,27,28)$ and, secondarily by influencing intracerebral vasodilation $(14,29)$. Therefore, it is likely that the increased deliver of ammonia to the brain may further enhance cerebral vasodilatation.

Second, there is increasing body of literature in patients with acute liver failure, that systemic inflammatory response may predict progression to more advanced stages of intracranial hypertension $(30,31)$. In patients with acute liver failure, we have recently shown a correlation between circulating concentration of pro-inflammatory cytokines and CBF (32). In addition, the presence of an inflammatory response in patients with cirrhosis exacerbates the neuropsychological effects of hyperammonemia (33). We therefore, considered a cytokine-mediated mechanism as an alternative explanation for the known increase in CO that follows TIPSS insertion but were unable to show any significant changes in the arterial concentrations of the pro-inflammatory cytokines following TIPSS. In addition, the brain flux of these cytokines was not significantly different from zero, suggesting that the TIPSS induced increase in CBF is unlikely to be due to a cytokine-dependent inflammatory response.

Third, we considered whether the TIPSS induced increase in CBF was due to NO production. Some studies in animal models have suggested that NO may be an important mediator of the increased $\mathrm{CBF}$ in liver failure $(15,34,35)$, while a recent study has failed to confirm this (36). We have confirmed that TIPSS insertion results in a further increase in $\mathrm{CO}$ and a reduction in SVR, which is thought to be due to a TIPSS induced increase in cardiac pre-load that follows the shunting of the splanchnic bed into the systemic circulation through the portacaval shunt (rather than the liver in the pre-TIPSS situation). These cardiovascular changes may result in an increase in shear stress which activates endothelial NO synthase and therefore NO production $(37,38)$. To the best of our knowledge, in the present study we are the first to quantify whole body NO synthesis in patients with cirrhosis of the liver. NO has been shown to play a prominent role in mediating the hyperdynamic circulation of cirrhosis and splanchnic vasodilation as evidenced by the ability of specific NO synthase inhibitors to correct vascular hyporesponsiveness in vitro and in vivo $(39,40)$. In patients with cirrhosis, inhibition of NO synthase results in a reduction in cardiac output and an increase in systemic vascular resistance and $\operatorname{MAP}(41)$.

Most studies only report end-products of NO metabolism. NO is produced during direct conversion of arginine to citrulline (42-44). NO is rapidly metabolised, mainly to nitrite/nitrate, and finally excreted as urinary nitrate. For that reason, plasma and urinary nitrite/nitrate have been measured frequently as indicators of nitric oxide production. However, these methods only give qualitative data. Therefore, in this study we have examined plasma kinetics of arginine, citrulline and assessed the activity of 
the L-arginine-NO pathway using a technique using the stable isotope technology based upon the measurement of the rate of conversion of intravenously infused L[guanidino- ${ }^{15} \mathrm{~N}_{2}$ ] arginine to [15N] citrulline (43). This method is likely to provide the greatest sensitivity when looking at acute effects of intervention. The values of NO production observed in the patients were significantly higher than that observed in healthy volunteers using the same method (43). This is in agreement with the existing hypotheses that increased NO synthesis is responsible for the hyperdynamic circulation found in cirrhoric patients. The observed correlation between the change in MAP and SVR and, the change in NO production provides circumstantial evidence that a change in NO production may underlie the haemodynamic changes that follow TIPSS insertion. Furthermore, the relationship between the increase in NO production after TIPSS and the increase in CBF supports the idea that increased NO production may be the force driving the increases in $\mathrm{CBF}$.

The data of the present study shows net uptake of ammonia and efflux of glutamine. We and others have shown net ammonia uptake by the brain in patients with acute liver failure $(9,45)$. However, Strauss and colleagues failed to demonstrate similar uptake of ammonia in the brain in patients with well-compensated cirrhosis and healthy volunteers. The fractional extraction of ammonia was $22 \%( \pm 8)$ in patients with acute liver failure, $4 \%( \pm 23)$ in the cirrhotics and $-4 \%( \pm 18)$ in the healthy controls. It is possible that the results of our studies are at variance because of the differences in arterial ammonia concentration and the populations of patients studied. The median ammonia concentration in our patients was over twice that in the patients studied by Strauss and colleagues (45). The variance in the data in these two studies supports the results of Lockwood's studies that suggested that the uptake of ammonia may be modulated by ammonia concentrations (46). The patients in our study had more advanced liver disease and were more unstable which may alter the brain permeability to ammonia.

The significance of greater glutamine efflux from the brain than would be expected from ammonia uptake prior to TIPSS insertion is unclear but is unlikely to reflect protein breakdown as there is no net phenylalanine nor total essential amino acid efflux from the brain (47). Following TIPSS, the increased brain flux of ammonia is not reflected by a significant increase in glutamine efflux or a change in the flux of phenylalanine or total essential amino acids. It is therefore unlikely that TIPSS induces brain protein breakdown.

In conclusion, the results of our study have shown that insertion of TIPSS results in an increase in CBF, which drives increased brain ammonia uptake. Our results support the hypothesis that TIPSS induced increase in CBF is the result of increased whole body NO synthesis. Although the exact mechanism of the increase in NO synthesis is 
not entirely clear, our data support the notion that the TIPSS-induced increase in cardiac output and the consequent shear stress may be the mechanism through which the change in NO synthesis is affected. The results of our study provides the rationale for studies using intervention with NO synthase inhibition during TIPSS insertion in the patients that are at high risk of systemic and cerebral haemodynamic disturbance with TIPSS. 


\section{REFERENCES}

1. Hahn M, Massen O, Nencki M, Pawlow J. Die Ecksche fistel zwischen der unteren hohlvene und der pfortader und ihre folgen für den organismus. Arch F Exper Path u Pharm 1893;32:161-210.

2. Clemmesen JO, Larsen $F S_{x}$ Kondrup J, Hansen BA, Ott P. Cerebral herniation in patients with acute liver failure is correlated with arterial ammonia concentration. Hepatology 1999;29:648-53.

3. Jalan R, Hayes PC. Hepacic encephaloparhy and ascites. Lancet 1997;350:1309-15.

4. Haussinger D, Kircheis G, Fischer R, Schliess F, vom Dahl S. Hepatic encephalopathy in chronic liver disease: a clinical manifestation of astrocyte swelling and low-grade cerebral edema? J Hepatol 2000;32:1035-8.

5. Martinez-Hernandez A, Bell KP, Norenberg MD. Glutamine synthetase: glial localization in brain. Science 1977;195:1356-8.

6. Balata $S$, Olde Damink SW, Ferguson $K$, Marshall I, Hayes PC, Deutz NE, et al. Induced hyperammonemia alters neuropsychology, brain MR spectroscopy and magnetization transfer in cirrhosis. Hepatology 2003;37:931 -9.

7. Cordoba J, Alonso J, Rovira A, Jacas C, Sanpedro F, Castells L, et al. The development of low-grade cerebral edema in cirrhosis is supported by the evolution of (1)H-magneric resonance abnormalities after liver transplantation. J Hepatol 2001;35:598-604.

8. Aggarwal S, Kramer D, Yonas H, Obrist W, Kang Y, Martin M, et al. Cerebral hemodynamic and metabolic changes in fulminant hepatic failure: a retrospective study. Hepatology 1994;19:80-7.

9. Jalan $\mathbb{R}$, Olde Damink SWM, Deutz NEP, Lee A, Hayes PC. Moderate hypothermia for uncontrolled intracranial hypertension in acute liver failure. Lancet 1999;354:1164-8.

10. Strauss GI, Hansen BA, Herzog T, Larsen FS, Cerebral autoregulation in patients with end-stage liver disease. Eur J Gastroenterol Hepatol 2000;12:767-71.

11. Jalan R, Dabos K, Redhead DN, Lee A, Hayes PC. Elevation of intractanial pressure following transjugular intrahepatic portosystemic stent-shunt for variceal haemorrhage. J Hepatol 1997;27:928-33.

12. Donowan JP, Schafer DF, Shaw BW, Jr., Sorrell MF Cerebral oedema and increased intracranial pressure in chronic liver disease. Lancer 1998;351:719-21.

13. Jalan R, Newby DE, Olde Damink SWM, Redhead DN, Hayes PC, Lee A. Acute changes in cerebral blood flow and metabolism during portasystemic shunting. Liver Transpl $2001 ; 7: 274-8$.

14. Willard-Mack CL, Koehler RC, Hirata T, Cork LC, Takahashi H, Traystman RJ, et al. Inhibition of glutamine synthetase reduces ammonia-induced astrocyte swelling in rat. Neuroscience 1996;71:589-99.

15. Master S, Gottstein J, Blei AT. Cerebral blood flow and the development of ammoniainduced brain edema in rats after portacaval anastomosis. Hepatology 1999;30:876-80.

16. Azoulay D, Castaing D, Dennison A, Martino W, Eyraud D, Bismuth $H$. Transjugular intrahepatic portosystemic shunt worsens the hyperdynamic circulatory state of the cirrhotic patient: preliminary report of a prospective study. Hepatology 1994;19:129-32.

17. Jalan $\mathrm{R}$, Hayes PC. UK guidelines on the management of variceal haemorthage in cirrhotic patients. British Society of Gastroenterology. Gut 2000;46 Suppl 3-4:III1-15. 
18. Conn HO, Leevy CM, Vlahcevic ZR, Rodgers JB, Maddrey WC, Seeff L, et al. Comparison of lactulose and neomycin in the treatment of chronic portal-systemic encephalopathy. A double blind controlled trial. Gastroenterology 1977;72:573-83.

19. Jalan R, Redhead DN, Simpson KJ, Elton RA, Hayes PC. Transjugular intrahepatic portosystemic stent-shunt (TIPSS): long-term follow-up. Qim 1994;87:565-73.

20. Dejong CH, Deutz NE, Soeters PB. Metabolic adaptation of the kidney to hyperammonemia during chronic liver insufficiency in the rat. Hepatology 1993;18:890-902.

21. van Eijk HM, Rooyakkers DR, Soeters PB, Deurz NE. Determination of amino acid isotope enrichment using liquid chromatography-mass spectrometry. Anal Biochem $1999 ; 271: 8-17$.

22. Leeuwenberg JF Jeunhomme TM, Buurman WA. Slow release of soluble TNF receptors by monocytes in vitro. J Immunol 1994;152:4036-43.

23. Dentener MA, Bazil V, Von Asmuth E], Ceska M, Burman WA. Involvement of CD14 in lipopolysaccharide-induced tumor necrosis factor-alpha, IL-6 and IL-8 release by human monocytes and alveolar macrophages. J Immunol 1993;150:2885-91.

24. Barle H, Nyberg B, Essen P, Andersson K, McNurlan MA, Wernerman J, et al. The synthesis rares of total liver protein and plasma albumin determined simultaneously in vivo in humans. Hepatology 1997;25:154-8.

25. Guevara M, Bru C, Gines P, Fernandez-Esparrach G, Sort P, Bataller R, et al. Increased cerebrovascular resistance in cirrhotic patients with ascites. Hepatology 1998;28:39-44.

26. Philips BJ, Armstrong IR, Pollock A, Lee A. Cerebral blood flow and metabolism in patients with chronic liver disease undergoing orthotopic liver transplantation. Hepatology $1998 ; 27: 369-76$

27. Cordoba ], Crespin J, Gottstein J, Blei AT. Mild hypothermia modifies ammonia-induced brain edema in rats after portacaval anastomosis. Gastroenterology 1999;1 16:686-93.

28. Blei AT, Olafsson S, Therrien G, Burterworth RF. Ammonia-induced brain edema and intracranial hypertension in rats after portacaval anastomosis. Hepatology 1994:19:143744.

29. Takahashi H, Koehler RC, Brusilow SW, Traystman RJ. Inhibition of brain glutamine accumulation prevents cerebral edema in hyperammonemic rats. Am J Physiol $1991 ; 261: H 825-9$.

30. Rolando N, Wade J, Davalos M, Wendon J, Philpott-Howard J, Williams $R$. The systemic inflammatory response syndrome in acute liver failure. Hepatology 2000;32:734-9.

31. Vaquero J, Polson J, Chung C, Helenowski I, Schiodt FV, Reisch J, et al. Infection and the progression of hepatic encephalopathy in acute liver failure. Gastroenterology 2003; 125:755-64.

32. Jalan R, Olde Damink SWM, Deutz NEP, Davies NA, Garden OJ, Madhavan KK, et al. Moderate hypothermia prevents cerebral hyperemia and increase in intracranial pressure in patients undergoing liver transplantation for acute liver failure. Transplantation $2003 ; 75: 2034-9$.

33. Shawcross DL, Davies NA, Williams $R$, Jalan R. Systemic inflammatory response exacerbates the neuropsychological effects of induced hyperammonemia in cirrhosis. J Hepatol 2004;40:247-54.

34. Rao VL, Butrerworth RF. Neuronal nitric oxide synthase and Hepatic Encephalopathy. Metab Brain Dis 1998;13:175-89.

35. Rao VL, Audet RM, Burterworth RF. Increased neuronal nitric oxide synthase expression in brain following portacaval anastomosis. Brain Res 1997;765:169-72. 
36. Larsen FS, Gottstein J, Blei AT. Cerebral hyperemia and nitric oxide synthase in rats with ammonia-induced brain edema. J Hepatol 2001;34:548-54.

37. Furchgott RF, Vanhoutte PM. Endothelium-derived relaxing and contracting factors. Faseb ] 1989;3:2007-18.

38. Fleming 1, Busse R. Signal transduction of eNOS activation. Cardiovase Res 1999;43:532. 41.

39. Wiest R, Groszmann RJ. The paradox of nitric oxide in cirrhosis and portal hypertension: too much, not enough. Hepatology 2002;35:478-91.

40. Helmy A, Newby DE, Jalan R, Johnston NR, Hayes PC, Webb DJ. Nitric oxide mediates the reduced vasoconstrictor response to angiotensin II in patients with preascitic cirrhosis. J Hepatol 2003;38:44-50.

41. La Villa G, Barletta $G$, Pantaleo $P$, Del Bene $R$, Vizzutti $F$, Vecchiarino $S$, et al. Hemodynamic, renal, and endocrine effects of acute inhibition of nitric oxide synthase in compensated cirrhosis. Hepatology 2001;34:19-27.

42. Wu G, Morris SM, Jr. Arginine metabolism: nitric oxide and beyond. Biochem J 1998:336:1-17.

43. Castillo L, Beaumier L, Ajami AM, Young VR. Whole body nitric oxide synthesis in healthy men determined from [15N] arginine-to-[15N]citrulline labeling. Proc Natl Acad Sci U S A 1996;93:11460-5.

44. Castillo L, Sanchez M, Vogt ], Chapman TE, DeRojas-Walker TC, Tannenbaum SR, et al. Plasma arginine, citrulline, and ornithine kinetics in adults, with observations on nitric oxide synthesis. Am J Physiol 1995;268:E360-7.

45. Strauss GI, Knudsen GM, Kondrup J, Moller K, Larsen FS. Cerebral metabolism of ammonia and amino acids in patients with fulminant hepatic failure. Gastroenterology $2001 ; 121: 1109-19$.

46. Lockwood AH, Yap EW, Wong WH. Cerebral ammonia metabolism in patients with severe liver disease and minimal hepatic encephalopathy. I Cereb Blood Flow Metab $1991 ; 11: 337-41$.

47. Wolfe RR. Radioactive and stable isotope tracers in biomedicine. Principles and practice of kinetic analysis. New York: Wiley-Liss; 1992. 


\section{CHAPTER 9}

\section{PATHOGENESIS OF INTRACRANIAL HYPERTENSION IN ACUTE LIVER FAILURE: INFLAMMATION, AMMONLA AND CEREBRAL BLOOD FLOW}

${ }^{1,3}$ Rajiv Jalan, ${ }^{2,3}$ Steven WM Olde Damink, 3Peter C Hayes, ${ }^{2}$ Nicolaas EP Deutz, ${ }^{3}$ Alistair Lee

${ }^{1}$ Liver Failure Group, Institute of Hepatology, Royal Free and University College London Medical School and University College London Hospitals, UKK

2Dept of Surgery, Academic Hospital, Maastricht University, The Netherlands ${ }^{3}$ Scottish Liver Transplantation Unit, Royal Infirmary of Edinburgh, Edinburgh 


\section{INTRODUCTION}

Raised intracranial pressure (ICP) is common in patients with acute liver failure (ALF) and a major cause of mortality (1-3). The pathophysiology of this elevated ICP is not completely unravelled, but current hypotheses suggest a role for hyperammonemia, cerebral hyperaemia and mediators of systemic inflammatory response (SIRS) (4-8). In patients with ALF, an arterial ammonia concentration of greater than $150 \mathrm{mmol} / \mathrm{L}$ predicts subsequent cerebral herniation, with emerging evidence of active ammonia uptake in the brain (9-11).

There is increasing evidence that increased cerebral blood flow (CBF) may underlie the pathogenesis of elevated ICP in $\operatorname{ALF}(6,10,12,13)$ but studies in patients with ALF have shown wide variations in the measured values of $C B F$ (14-17). A part of the problem in assessing the role of altered CBF in patients with ALF and how they relate to changes in ICP is the absence of longitudinal studies with serial measurements in the same patient, the effect of therapeutic intervention and lack of simultaneous measurements of ICP and CBF in the same patient. Recent studies suggest that mediators of SIRS play a role in the development of intracranial hypertension. In a large series of patients with ALF, the presence of markers of inflammation or overt infection was associated with more severe encephalopathy and the likelihood of developing intracranial hypertension $(18,19)$. We have recently confirmed that a reduction in the pro-inflammatory cytokines following hepatectomy reduces $\mathrm{CBF}$ and $\operatorname{ICP}(20,21)$, and presence of SIRS increases the deleterious neuropsychological effect of hyperammonemia (22).

Studies in patients with head injury and meningitis have suggested that proinflammatory cytokines may increase CBF and ICP and the reduction in their concentration reduces $\mathrm{CBF}$ and $\operatorname{ICP}(3,23-27)$. Treatment of ALF with hypothermia results in a reduction in the ICP, inflammatory response, CBF and ICP (10). Taken together, the above studies support the notion that increases in CBF may be the critical event that produces a transition from minimally increased ICP to the advanced stages and mediators of inflammation may be the trigger that increases CBE. The aim of this longitudinal study in patients with ALF was to evaluate the role of the inflammation and its mediators in the pathogenesis of intracranial hypertension and, its interplay with brain ammonia metabolism and CBF. 


\section{METHODS}

Studies were undertaken with the approval of the local research ethics commitree and the written informed consent/assent from the next of kin of each parient.

Patients. Twenty-one consecutive patients with ALF who were admitted to a single tertiary referral centre and fulfilled the Kings College Criteria for poor prognosis (1) were studied (table 1). Patients were enrolled into the study from the time they were admitted to the Intensive Care Unit with ALF and grade IV hepatic encephalopathy (HE), requiring mechanical ventilation until they needed specific treatment for increased ICP. The following patients were excluded; lack of informed consent/assent from next of kin; criteria for poor prognosis not fulfilled; specific therapy for increased ICP before entry into the study; failure to insert ICP monitor.

Table 1. Patient characteristics of those that required treatment (Group 1)

for increased ICP compared with those that did not (Group 2).

\begin{tabular}{|c|c|c|}
\hline & Group $1(n=8)$ & Group $2(n=13)$ \\
\hline Age (years) & $24(20-29)$ & $27(18-37)$ \\
\hline Sex & $3 M, 5 F$ & $5 \mathrm{M}, 8 \mathrm{~F}$ \\
\hline \multicolumn{3}{|l|}{ Aetiology of AHF } \\
\hline Paracetanot overdose & 7 & 11 \\
\hline Non A-Non B & 1 & $\Uparrow$ \\
\hline Drug Related & & 1 \\
\hline Kings College criteria for poor prognosis & 8 & 13 \\
\hline Highest Prothrombin Time (sec) & $104 \cdot(78-121)$ & $100(91-132)$ \\
\hline Bilirubin (pumol/L) & $291(267-345)$ & $321(289-432)$ \\
\hline Creatinine (umol/L) & $278(254-376)$ & $3 \pi 1(298-432)$ \\
\hline White Cell Count $\left(\times 10^{9} / \mathrm{L}\right)$ & $13.3(10.2-20)$ & $8.9(6.9-11.2)^{\mathrm{t}}$ \\
\hline SRS components & $3(1-4)$ & $1(1-2)^{\text {s }}$ \\
\hline C-reactive protein (mg/L) & $82(57-156)$ & $47(23-98))^{*}$ \\
\hline \& Need fior Remal Support & 8 & 13 \\
\hline Inotropes & 7 & 11 \\
\hline Mechanical Ventillation & 8 & 13 \\
\hline $\begin{array}{l}\text { Time between onset of symptoms and encephalopathy } \\
\text { (days) }\end{array}$ & $3(2-6)$ & $4(2-13)$ \\
\hline Candidates for Liver Transplantation & 6 & 6 \\
\hline Resting Energy Expenditure (kCal/24hr) & $1290(1761-2530)$ & $1356(1124 \cdot 4456$ \\
\hline Arterial Carbondyoxide (Kpa) & $4.1(3.9-4,2)$ & $4.0(3.804 .2)$ \\
\hline Arterial hydrogen ion concentration (nmol/c) & $46(36-58)$ & $45(32-51)$ \\
\hline Propofol dosage (mg/hr) & $70(50-170)$ & $60(50-140)$ \\
\hline
\end{tabular}


Management Protocol. All patients were managed according to a standardised protocol as described previously (10). In short, all the patients were mechanically ventilated following sedation with propofol and paralysis with atracurium besylate. ICP was continuously monitored using a subdural fibre-optic system (Camino, Camino Laboratories, San Diego, USA). Cardiovascular haemodynamics were continuously monitored and noradrenaline was used to keep the cerebral perfusion pressure above 50 $\mathrm{mmHg}$ and/or a mean arterial pressure greater than $80 \mathrm{mmHg}$.

Study Design. Measurements were made prior to any specific treatment for increased ICP. The measurement of CBF was made immediately prior to insertion of the ICP monitor. In those patients who were studied longitudinally and developed increases in ICP, this was treated with removal of $500 \mathrm{ml}$ of fluid using the veno-venous haemofilter before the measurement of CBF. The initial ICP was used to define two patients groups (Figure 1). If the initial ICP was greater than $20 \mathrm{mmHg}$ or the cerebral perfusion pressure was less than $50 \mathrm{mmHg}$, the patients were enrolled in Group 1. These patients were treated with $20 \%$ mannitol $(1 \mathrm{~g} / \mathrm{Kg})$ and no furcher measurements of CBF were performed. If the initial ICP was $£ 20 \mathrm{mmHg}$, the patients were enrolled in Group 2. In these patients CBF was measured at the beginning of the study and repeated at 8 hour intervals or when the ICP surged to values of $>20 \mathrm{mmHg}(5,10)$.

Measurement of cerebral blood flow. CBF was only determined if the patient was haemodynamically stable during the measurement, defined as a difference of less than $10 \%$ in the mean arterial pressure between the values at the start and at the end of CBF measurement. Ventilation was adjusted to achieve an arterial carbon dioxide tension of 4-4.5 kiloPascals ( $\mathrm{kPa}$ ), and the $\mathrm{pH}$ was corrected, prior to the measurement of CBE. CBF was measured using a modification of the Kety-Schmidt technique $(27,28)$ measuring the rate of uptake of nitrous oxide by the brain as detailed previously $(10,29)$.

Measurement of Ammonia and Cytokines. Immediately after each measurement of CBF, blood samples were simultaneously collected from the femoral artery and the jugular bulb carheters for the measurement of blood gases and ammonia. Ammonia was measured spectrophotometrically (CobasMiraS, Hoffman-LaRoche, Switzerland). IL-1b, TNFa and IL-6 were measured using commercially available ELISA (R\&D systems, Minneapolis, MN) at time 0 in all patients. In the Group 2 patients, the measurement of TNFa was repeated at either at 24 hours or when the patients were treated for elevated ICP which ever was earlier.

Calculations. Cerebral metabolic rate for ammonia was calculated as CBF times arterial-jugular venous concentration differences. Fractional excraction of ammonia was calculated as arterial-jugular venous concentration difference divided by the arterial concentration (30).

Statistical Analysis. Difference between groups was calculated using the MannWhitney test. Relationships berween variables were calculated using linear regression. 


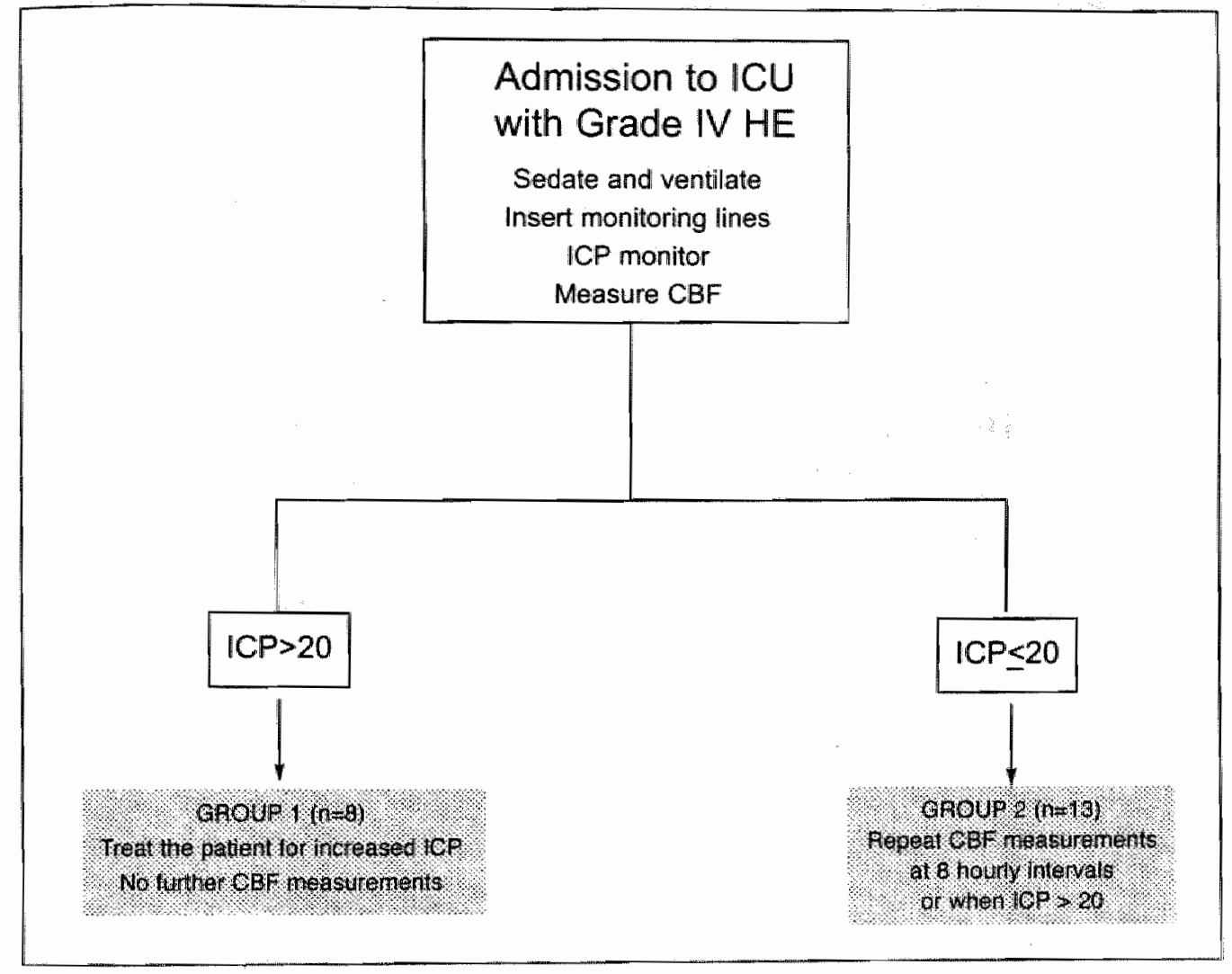

Figure 1. Study Design 


\section{RESULTS}

Patients. Twenty-one patients were enrolled in the study. All patients fulfilled the criteria for poor prognosis and required haemofiltration. Eighteen patients required inotropic support (Table 1).

Intracranial Pressure. Eight patients were allocated to Group 1; the ICP at initial measurement was a median of $32(28-54) \mathrm{mmHg}$. Thirteen patients were allocated to Group 2; the ICP at initial measurement was $15(10-20) \mathrm{mmHg}$ [Figure 2]. There were no significant differences between the time from onset of first symptoms or in the amount of propofol that was administered to the two groups (Table 1). Pupillary dilatation $(>6 \mathrm{~mm})$ and sluggish reaction to light were evident in 3 patients in Group 1 which resolved following treatment of the increased ICP. None of the patients in Group 2 showed pupillary abnormalities. Nine patients in Group 2 did not require treatment for increased ICP during the course of the study because the ICP remained less than $20 \mathrm{mmHg}$ throughout. However, during follow up, 4 patients went on to have surges of high ICP requiring treatment after a median of $32(15-112)$ hours from the time they were enrolled in the study.

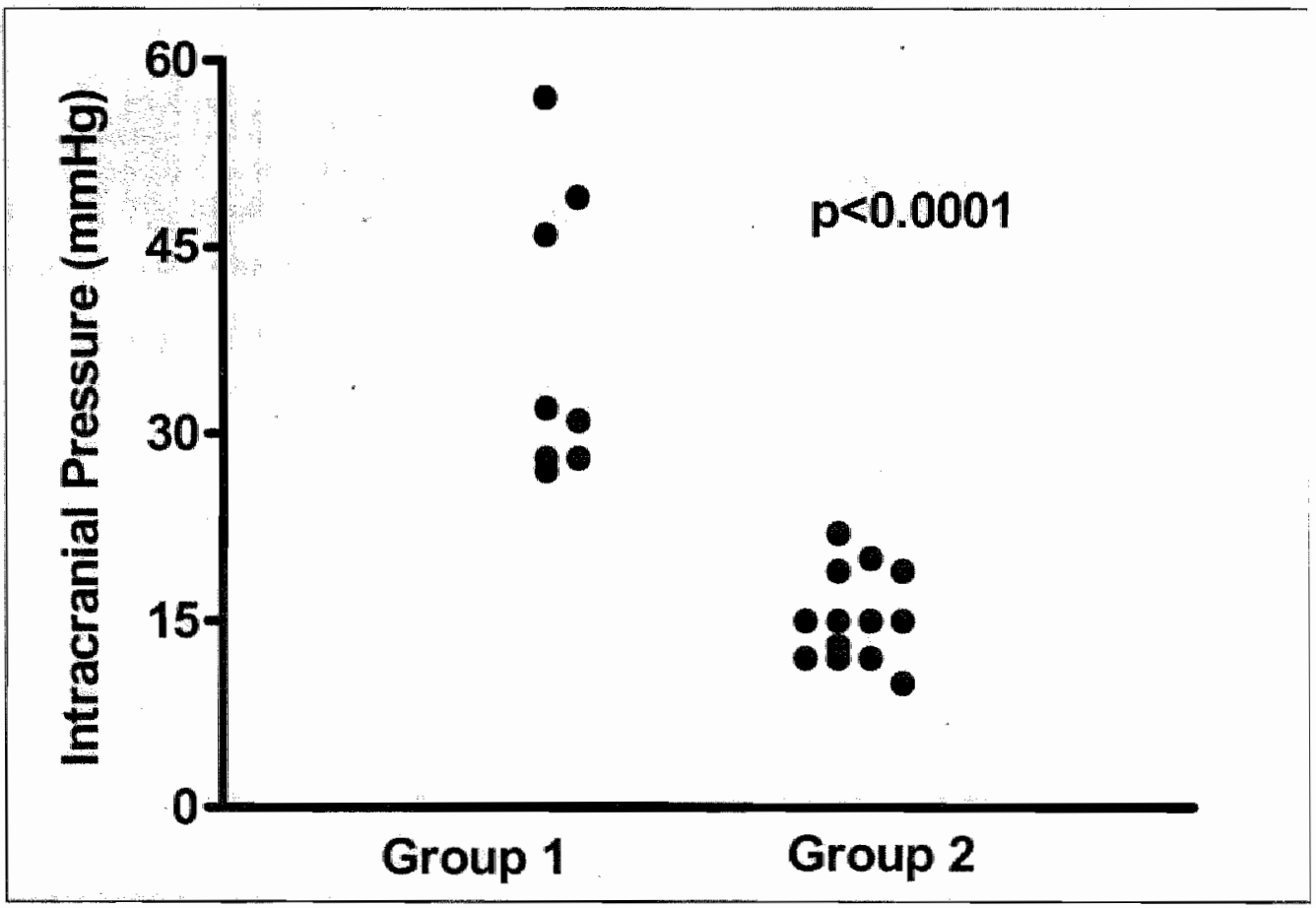

Figure 2. Intracranial Pressure of individual patients in the two Groups (Group 1: ICP>20 and or cerebral perfusion pressure $<50 \mathrm{mmHg}$; Group 2: ICP $<20$ and or cerebral perfusion pressure $>50 \mathrm{mmHg}$. 
Cardiovascular Haemodynamics. The parients in Group 1 had significantly higher cardiac output $(p<0.05)$ and cerebral perfusion pressure $(p<0.05)$, lower systemic vascular resistance $(p<0.01)$. and the noradrenaline requirement $(p<0.05)$ for the maintenance of mean arterial pressure $(p=0.09)$ was also greater (Table 2$)$.

Table 2. Haemodynamic parameters for the patients that required treatment (Group 1) for increased ICP compared with those that did not (Group 2).

Intracranial Pressure (mmHg)

Cerebral Blaod Flow (ml/100g/min)

Heart rate (beats/miti)

Mean arterial pressure (mmHig)

Systemic vascular resistance

(dyn.sec. $\mathrm{cm}^{-5} / \mathrm{m}^{2}$ )

Cardiac Index $\left(\mathrm{L} / \mathrm{min} / \mathrm{m}^{2}\right)$

Noradrenaline requirement ( $\mu \mathrm{g} / \mathrm{Kg} / \mathrm{mmin}$ )

Cerebral perfusion pressure $(\mathrm{mm} \mathrm{Hg}$ )
GROUP $1(n=8)$

$32(28-54)$

$85(24-134)$

$101(84-118)$

$90(64-111)$

924

$(739-1131)$

$9.4(7.7-13.1)$

$0.8(0.5-1.1)$

$45(41-62)$
GROUP $2(n=13)$

$15(10-22)^{* *}$

$45(23-56)^{* *}$

$89(58-105)^{*}$

$84(74-148)$

111.2

$(756-1231)^{*}$

$7.9(6-12.3) *$

$0.5(0.3-0.9)^{*}$

$59(51-78)^{*}$

Data expressed as median (range), Mann Whitney U test: "p<0.05; ${ }^{*} p<0.01$

Outcome. Five of the 6 patients who were considered suitable for orthotopic liver transplantation (OLT) from Group 1 were successfully transplanted. The $6^{\text {th }}$ patient died while waiting for a suitable donor organ. All 6 patients from Group 2 that were considered suitable for OLT were successfully transplanted. The rest of the patients in both groups who were considered unsuitable for OLT died (reason for unsuitability for OLT: Psychosocial reasons: 7; history of significant substance abuse: 2 ).

Cerebral Blood Flow. Only one CBF measurement was performed in each of the Group 1 patients. In Group 2 patients, a total of $53 \mathrm{CBF}$ measurements were performed [median no. of measurements, 4 (range 2-7)]. At the time of insertion of the ICP monitor, the CBF in the Group 1 patients was significantly higher compared with the Group 2 patients. CBF values correlated directly with arterial ammonia concentrations $(r=0.78 ; p<0.02)$ and with the ICP $(r=0.74, p<0.02)$ [Figure $3 a$ and $3 b$ ]. In the patients in Group 2, there was no significant change in the CBF in the 9 patients during the repeated measurements [Figure $4 \mathrm{a}$ ] that did not develop surges of increased ICP to values greater than $20 \mathrm{mmHg}$. However, in the 4 patients that went on to develop surges of increased ICP, there was a concomitant increase in CBF [Figure 4b].

Inflammatory markers. The patients in Group 1 showed significantly higher SIRS score (Table 1). Furthermore, the concentrations of IL-1b, TNF $a$ and IL-6 were also 

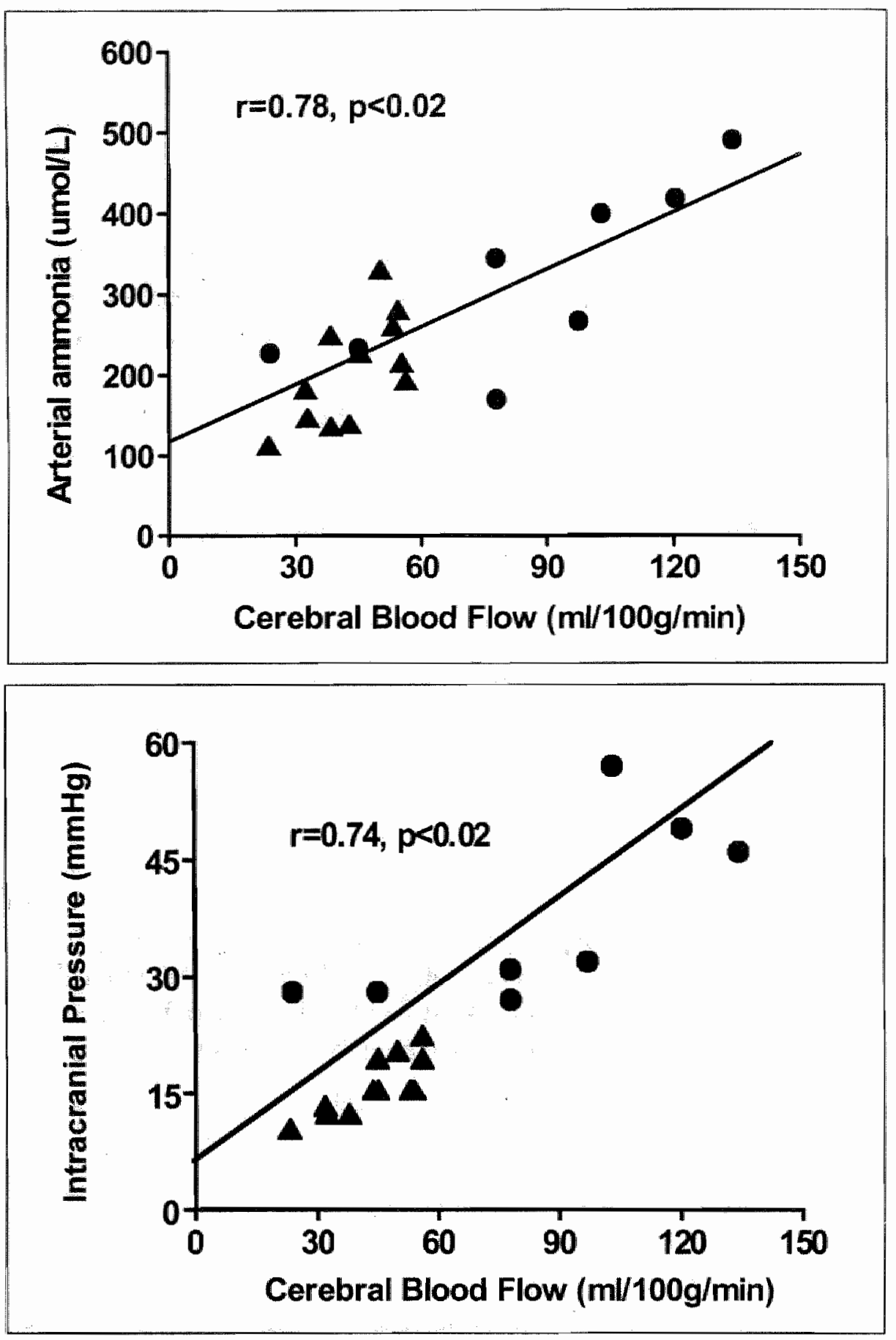

FIGURE 3 TOP. (a) This figure shows the relationship between cerebral blood flow and (a) arterial ammonia concentrations and BELOW (b) intracranial pressure. 

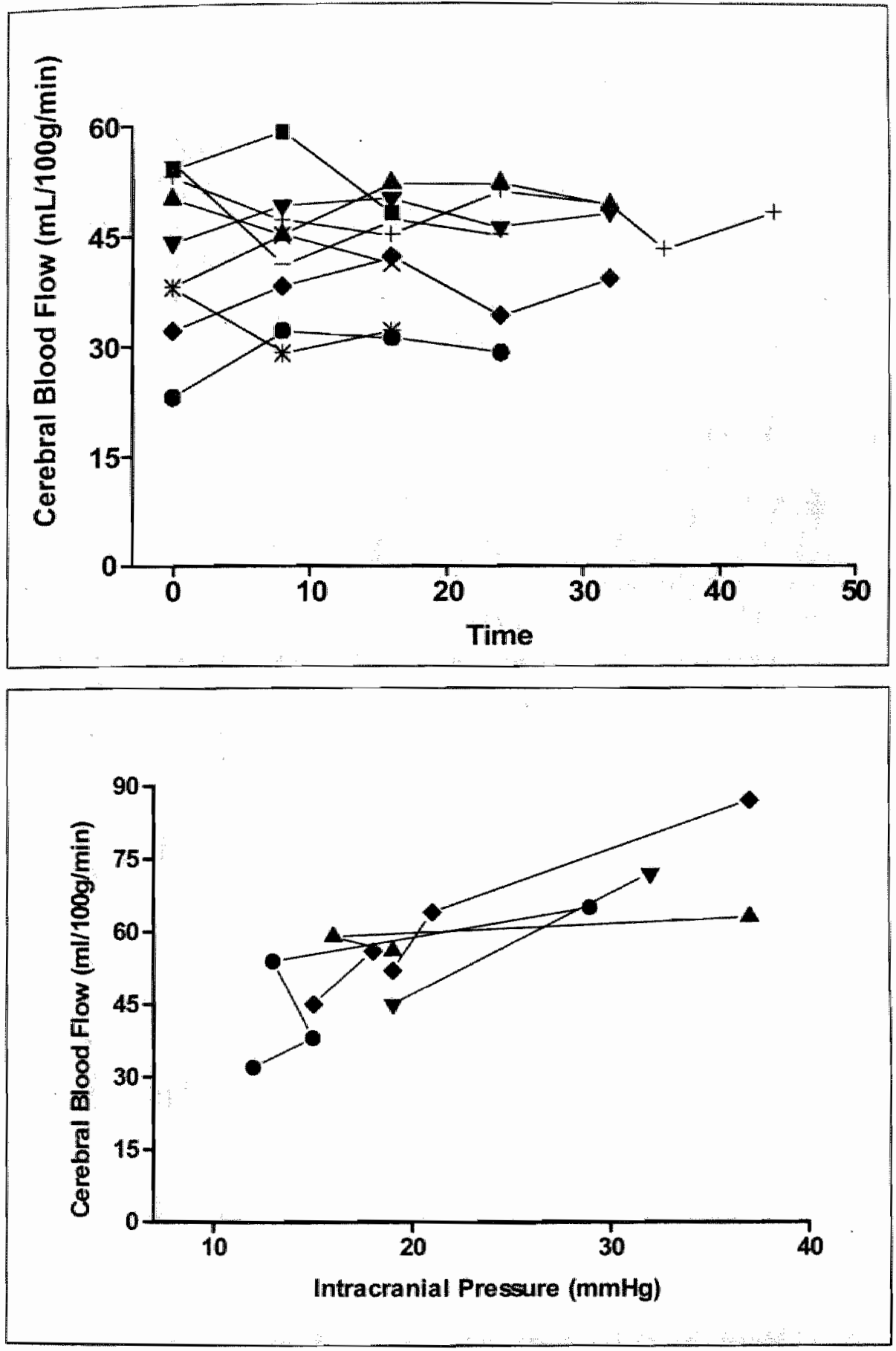

FIGURE 4 TOP. (a) Cerebral blood flow in the 9 patients in Group 2 in whom the ICP remained less than $20 \mathrm{mmHg}$ during follow up. The figure shows that the measured values of cerebral blood flow during this period did not change significantly ( $\mathrm{p}=0.32)$.

BELOW. (b) This figure shows the retationship between cerebral blood flow and ICP in the sub-group of 4 patients in Group 2 who went onto develop bursts of increased. ICP requiring treatment. The symbols tepresent individual patients. 
significantly greater in the Group 1 patients ( $<<0.01, p<0.05$ and $p<0.05$ respectively) [Figure 5a]. TNF $a$ correlated directly with $\mathrm{CBF}(\mathrm{r}=0.80 ; \mathrm{P}<0.01)$ [Figure 5b]. No significant correlations were noted between IL-1b or IL-6 and ICP or CBF. In the patients in Group 2, no significant changes in the C-reactive protein (CRP), SIRS or TNFa levels were detected in the 9 patients in whom there was no change in the ICP. However, in the other 4 patients that went onto develop increases in ICP there were marked increases in the CRP, SIRS score, white cell count and TNFa levels (Table 3) when the measurements were repeated at 24 hours (i.e even before the elevation in ICP was observed).

Ammonia Metabolism. The arterial ammonia concentration was higher in Group 1 patients compared with those in group $2(\mathrm{p}<0.01)$ but the ammonia delivery to the brain was 4 -fold higher, $p<0.01$, [Figure 6a and 6b]. Since there were no significant differences in the brain extraction of ammonia in the 2 groups the cerebral metabolic rate for ammonia was also higher in Group $1(p<0.005)$ [Figure $6 \mathrm{c}$ and $6 \mathrm{~d}$ ]. There was no significant change in the arteriall ammonia concentration in the 4 patients in Group 2 at 24 hours after insertion of the ICP monitor, who went onto develop increased ICP and CBF. However, as would be expected the ammonia delivery to the brain increased secondary to the increase in CBF. Brain extraction of ammonia remained unchanged (Table 4). 

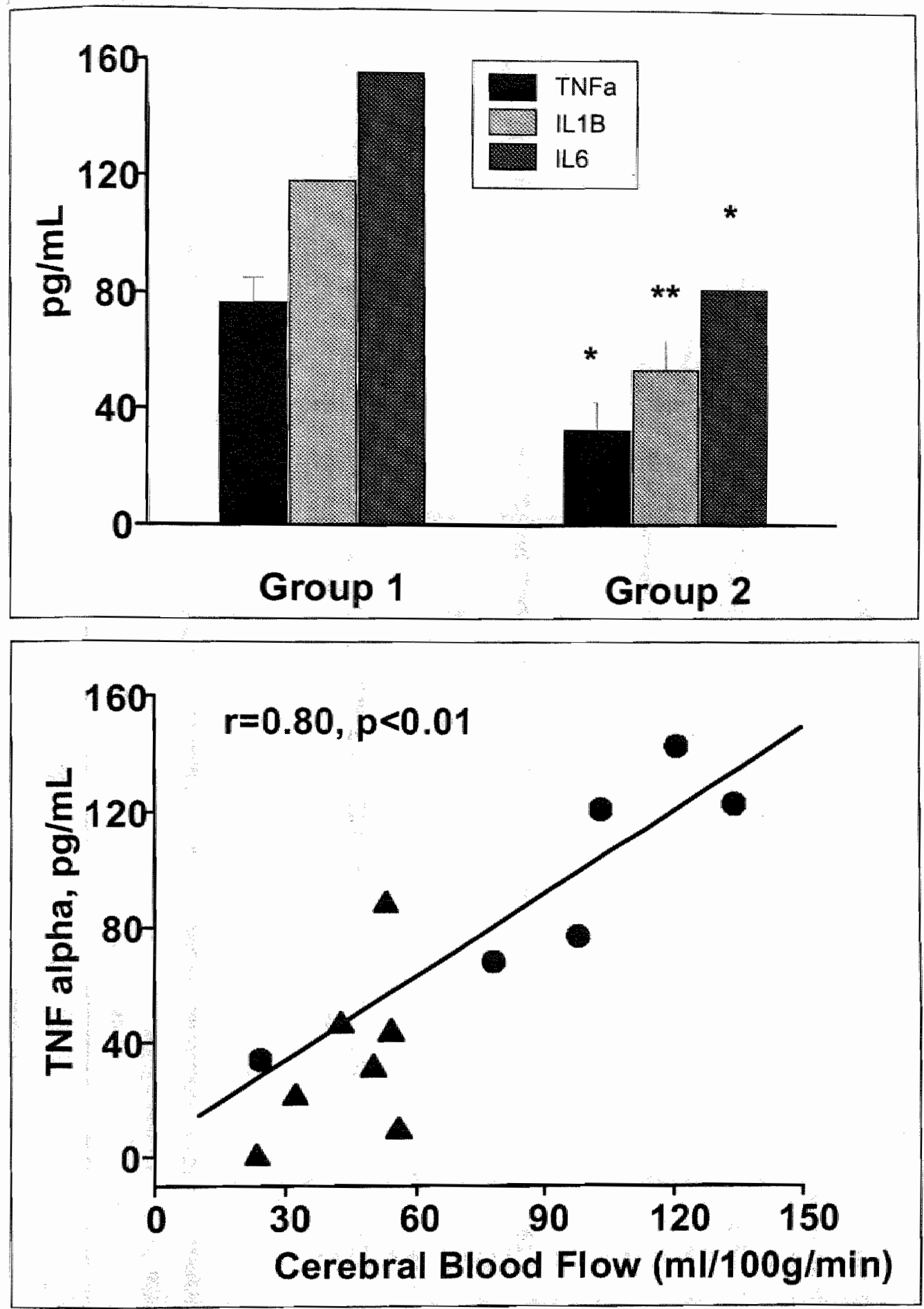

FIGURE 5 TOP (a) This figure shows mean (SEM) concentration of the pro-inflammatory cytokines TNFa, IL- $1 b$ and $I L-6$ in the patients in the two groups. ${ }^{*} p<0.05 * 0<0.01$. BELOW. (b) This shows the relationship between the measured values of TNFa and cerebral blood flow. 


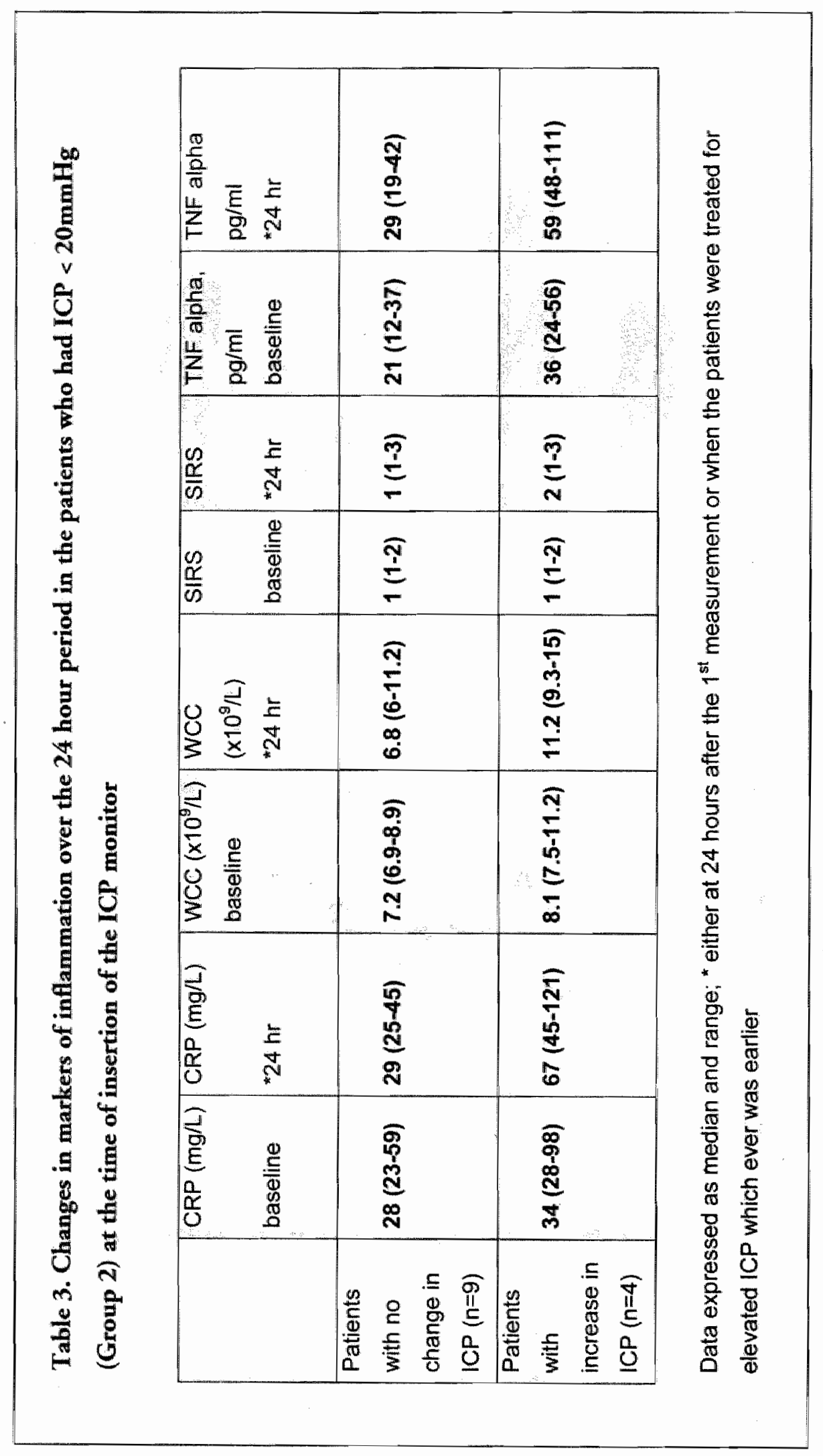



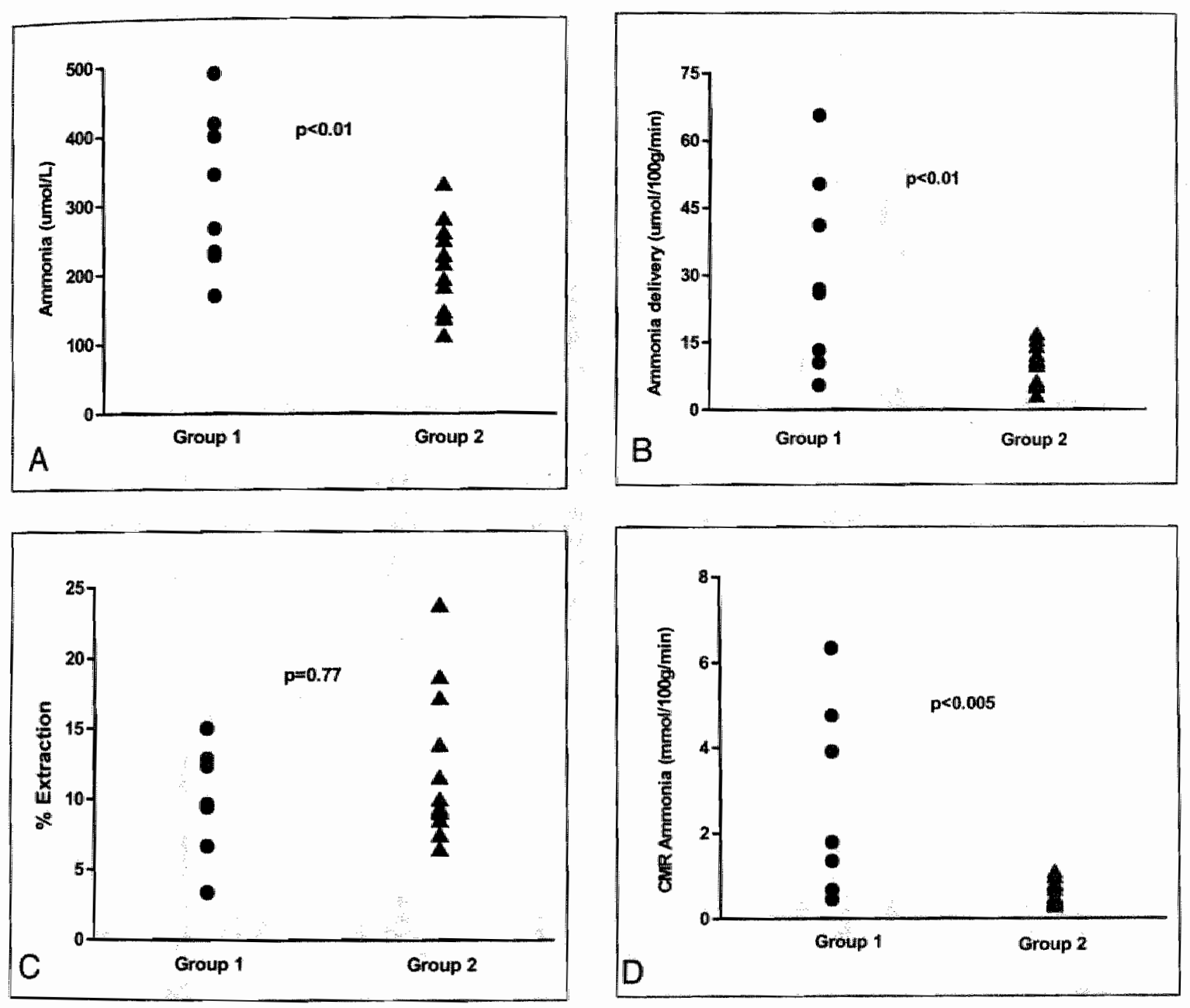

FIGURE 6. Ammonia metabolism in the two groups. (a) Arterial ammonia concentration (b) Brain ammonia delivery, (c) \%Extraction of ammonia by the brain and (d) Cerebral metabolic rate of ammonia. (Group 1: $I C P>20$ and or cerebral perfusion pressure $<50 \mathrm{mmHg}$; Group 2: ICP $<20$ and or cerebral perfusion pressure $>50$ $\mathrm{mmHg}$. 


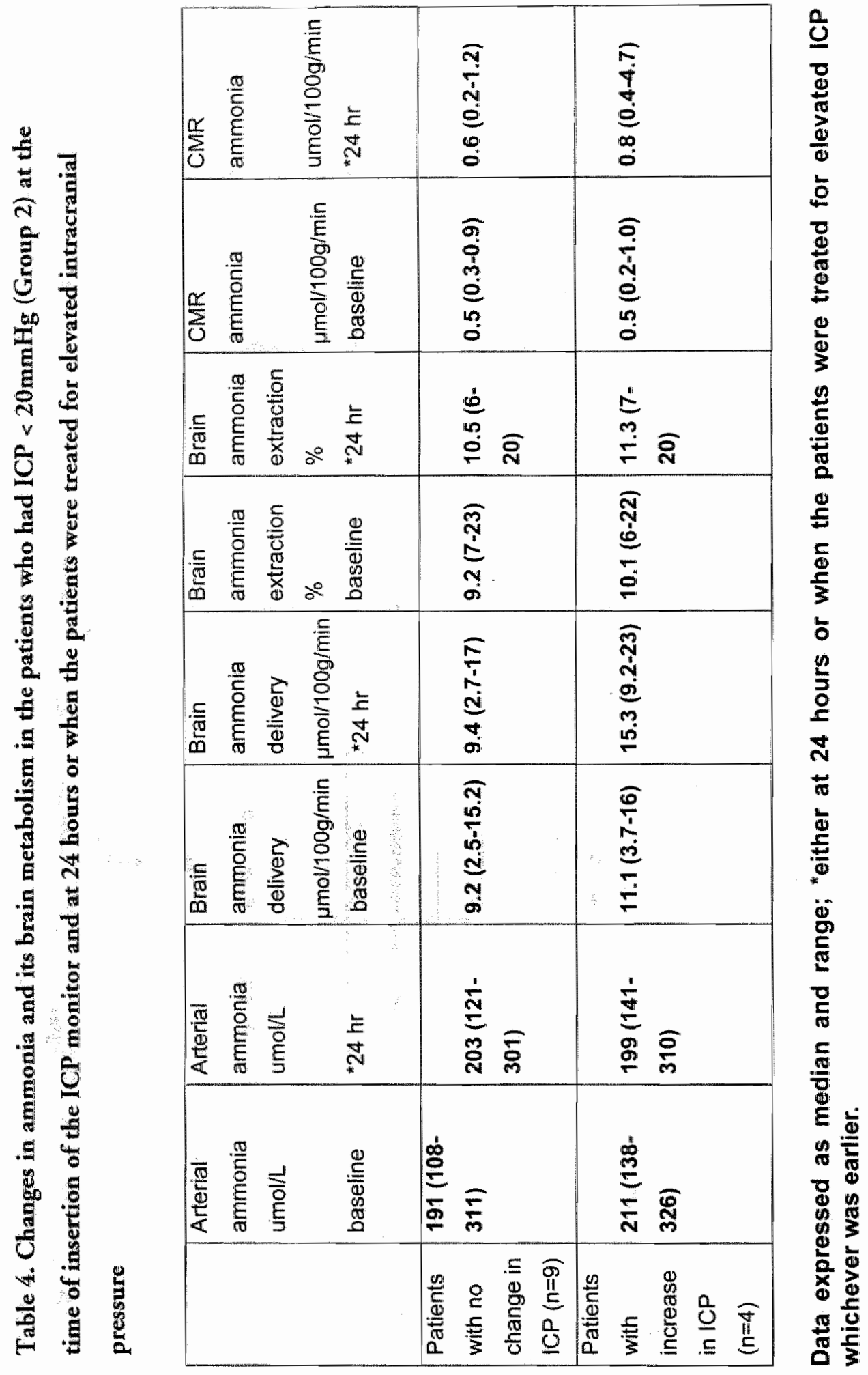




\section{DISCUSSION}

In this longitudinal study in patients with ALF, we have shown that inflammation plays an important synergistic role in the pathogenesis of intracranial hypertension in patients with ALF. We have also shown that there is a close relationship between the ICP and CBF suggesting that cerebral hyperaemia may be important in the surges of ICP observed in these patients. We also confirm the previous observations that ammonia and its brain metabolism are likely to be important in the pathogenesis of this syndrome. The relationship between CBF and, markers of SIRS and TNFa suggests that inflammation may exert its effects on ICP through alterations in $\mathrm{CBF}$.

The results of our study confirm and extend the previous observations in patients with ALF, suggesting the possible role of inflammation in progression to more advanced stages of encephalopathy $(18,19)$. We showed that the patients with high ICP requiring specific therapy had evidence of more marked inflammatory response that was evidenced by a higher SIRS score, CRP and the pro-inflammatory cytokines, TNFa, IL-6 and IL-1b. Of greater interest was the observation of increases in the SIRS components, CRP and TNF $a$ in the 4 patients in Group 2 who went onto develop surges of increased ICP. Such changes in markers of inflammation were not observed in the patients in whom the ICP remained below $20 \mathrm{mmHg}$. It is likely that the effects of the SIRS are mediated by pro-inflammatory cytokines. However in ALF, the blood brain barrier remains intact and should therefore prevent the entry of systemic cytokines into the cerebral circulation (31). The circulating or endothelial cytokines can however, transduce signals to the brain through vaso-active agents such as nitric oxide or prostanoids that are synthesized by inducible enzymes such as the nitric oxide synthases and the cycloxygenases, the transcription of which can be increased by the pro-inflammatory cytokines, which can also generate oxidative stress (32-34).

Arterial concentrations of ammonia, its delivery to the brain and its metabolic rate were significantly higher in the patients with the high ICP confirming the important role of ammonia in the pathogenesis of intracranial hypertension. There was however, an overlap in the values of arterial ammonia concentration in the patients that required and those that did not require specific treatment for increased ICP. In principle, our data are consistent with those of Clemmesen et al. (9) in that we believe that ammonia is important in the development of increased ICP in ALF but do not support the observation that an absolute cut-off value of $150 \mathrm{mmol} / \mathrm{L}$ predicts cerebral herniation. The delivery of ammonia to the brain, is likely to be more important considering that this was 4 fold higher in the Group 1 patients. This is further supported by the observation that although the brain extraction of ammonia was similar in the two groups, its metabolic rate was substantially higher in the Group 1 patients suggesting that the effects of 
hyperammonemia on the brain may be accentuated by increased CBF. Recently, Strauss and colleagues (11) reported cerebral metabolic rates for ammonia in 16 patients with ALF, of which 11 fulfilled criteria for poor prognosis, values which are similar to those in our Group 2 patients. Interestingly, the cerebral metabolic rate for ammonia was twice as high in a subgroup of 6 patients that died of brain herniation, on average 4 days after the measurements were performed. However, the lack of concomitant ICP measurements, do not allow any conclusion on the relationship between ICP and brain ammonia uptake. 11

An important observation of our study was that CBF values correlated directly with ICP implicating cerebral hyperaemia as an important pathogenic mechanism in the increased ICP. It is however, important to note that there were 2 patients that had either low or normal CBF (Figure 2). The importance of cerebral hyperaemia is evident from observations in Group 2. All 4 patients that developed surges of intracranial hypertension had increased CBF and those that did not require any treatment for increased ICP, continued to show values of CBF in the normal range. The values of CBF in ALF reported in the literature are very variable. Ede et al. (14) studied patients with ALF in the late stage of the disease but before the development of cerebral herniation and observed a high CBF. Almdal et al. (15) observed the opposite in patients in the more advanced stages of encephalopathy and reported low CBF. Wendon and colleagues (16) studied 30 patients in various stages of $\mathrm{HE}$ and suggested that the $\mathrm{CBF}$ was likely to be low. Aggarwal et al. (17) studied 33 patients but reported simultaneous measurement of ICP and CBF in 8 patients. Patients with an ICP of greater than $24 \mathrm{mmHg}$ had high CBF. The major difference between these studies and ours is the differences in the technique of the measuring $\mathrm{CBF}$; measurements at a single time point and not longitudinally, the effects of treatment for elevated ICP prior to the measurement of CBF; and the lack of relationship with ICP. Our observation is consistent with data from studies in experimental animals in which a rise in CBF was shown to parallel the increase in ICP (35).

Although the exact mechanisms that leads to increases in CBF has not been established but the data supports the hypothesis that inflammation may be important. The good correlation between CBF and 'TNFa and, the observation of increases in the markers of inflammation and $\mathrm{CBF}$ in the Group 2 patients suggests that inflammation may be an important mediator of increased CBF. The increase in ICP in the 4 patients was associated with increases in $\mathrm{CBF}^{\mathrm{F}}$ with evidence of more marked inflammatory activity. In contrast other 9 patients in this Group showed no increase in CBF, ICP and inflammatory markers. This notion of inflammation leading to increased CBF is consistent with data from patients with meningitis and head injury, which have increased $\mathrm{CBF}$, which is thought to be associated with increased brain inflammatory activity (2325). In both cases, reduction of this inflammatory state with hypothermia results in alleviation in $\mathrm{CBF}$ and a reduction in ICP (23-26). These observations indicating a 
possible relationship between inflammation and CBF, are also borne out in patients with ALF, in whom a reduction in systemic and brain cytokine production by hepatectomy was associated with a reduction in $\operatorname{CBF}$ and also $\operatorname{ICP}(20,21)$. The reduction in CBF by induction of hypothermia in patients with ALF and uncontrolled increase in ICP support the notion that inflammation may be a critical factor determining increases in $\mathrm{CBF}$ (1.0). It is noteworthy therefore, that Moller et al. failed to detect any significant changes in CBF in healthy volunteers administered endotoxin that produced an increase in TNFa (36). The seemingly discordant results may indicate that other factors such as hyperammonemia may be important co-factors in modulating the cerebral effects of inflammatory cytokines. Increased ammonia concentration and its brain metabolism may provide the alternative explanation to the observed increase in CBF. In an animal model of ammonia induced cerebral oedema, a link between glutamine accumulation and an increase in CBF has been suggested (6). Our results show a good correlation between arterial ammonia concentration and CBF that is consistent with the data from animal studies. The data from the patients in Group 2 do not support a central role for ammonia as providing the initiating stimulus for increases in CBF. There was no significant change in arterial ammonia or its brain extraction in the 4 patients who developed increases in CBF. The increase in the brain delivery of ammonia observed was obviously secondary to the increase in CBF.

In conclusion, our data support a multiple-hit hyporhesis for the development of intracranial hypertension in patients with ALF. Accordingly, brain ammonia uptake is possibly the initial stimulus that leads to cerebral oedema but the bursts of increased ICP is the result of cerebral hyperaemia which may be consequent on the action of putative vasodilators which are the result of the systemic inflammatory response and/or due to ammonia-induced glutamine accumulation. The validity of this hypothesis should the tested in future studies. 


\section{REFERENCES}

1. O'Grady JG, Alexander GIM, Hayllar KM, Williams R. Early indicators of prognosis in fulminant hepatic failure. Gastroenterology 1989;97:439-45.

2. Ascher NL, Lake JR, Emond JC, Roberts JP. Liver transplantation for fulminant hepatic failure. Arch Surg 1993;128:677-82.

3. Makin AJ, Wendon J, Williams R. A 7-year experience of severe acetaminophen induced hepatotoxicity (1987-1993). Gastroenterology 1995;109:1907-16.

4. Blei AT. Infection, inflammation and hepatic encephalopathy, synergism defined. J Hepatol 2004; $40: 327-330$

5. Jalan R. Intractanial hypertension in acute liver failure: parhophysiological basis of rational management. Semin Liver Dis 2003; 23: 271-282.

6. Master S, Gottstein J, Blei AT. Cerebral blood flow and the development of ammoniainduced brain edema in rats after portacaval anastomosis. Hepatology 1999;30:876-80.

7. Rose C, Michalak A, Rao KV, Quack G, Kircheis G, Butterworth RF. L-ornithine-L-aspartare lowers plasma and cerebrospinal fluid ammonia and prevents brain edema in rats with acute liver failure. Hepatology 1999;30:636-40.

8. Sass JO, Sperl W, Bachmann C. Treatment of neonatal hyperammonaemia. Lancet 2001;358:1727-8.

9. Clemmesen JO, Larsen FS, Kondrup J, Hansen BA, Ott P. Cerebral herniation in patients with acute liver failure is correlated with arterial ammonia concentration. Hepatology 1999;29:648-53.

10. Jalan R, Olde Damink SWM, Deutz NEP, Lee A, Hayes PC. Treatment of uncontrolled intracranial hypertension in acute liver failure with moderate hypothermia. Lancet 1999;354:1164-8.

11. Strauss GI, Knudsen GM, Kondrup J, Moller K, Larsen FS. Cerebral metabolism of ammonia and amino acids in patients with fulminant hepatic failure. Gastroenterology 2001;121:1109-19.

12. Larsen FS. Cerebral circulation in liver failure: Ohms law in force. Semin Liver Dis $1996 ; 16: 281-92$.

13. Jalan R, Olde Damink SWM, Deutz NEP, Hayes PC, Lee A. Restoration of cerebral blood flow autoregulation and reactivity to carbon dioxide in acute liver failure by moderate hypothermia. Hepatology 2001;34:50-4.

14. Ede R], Williams R. Heparic encephalopathy and cerebral edema. Semin Liv Dis 1986;6:107-18.

15. Almdal T, Schroeder T, Ranek L. Cerebral blood flow and liver function in patients with encephalopathy due to acute and chronic liver diseases. Scand J Gastroenterol 1989;24:299303.

16. Wendon JA, Harrison PM, Keays R, Williams R. Cerebral blood flow and metabolism in fulminant liver failure. Hepatology 1994;19:1407-13.

17. Aggarwal S, Kramer D, Yonas H, Obrist W, Kang Y, Martin M, Policare R. Cerebral hemodynamic and metabolic changes in fulminant hepatic failure: a retrospective study. Hepatology 1994;19:80-7.

18. Vaquero J, Polson J, Chung C, Helenowski I, Schiodt FV, Reisch J, Lee WM, Blei AT. Infection and the progression of hepatic encephalopathy in acure liver failure. Gastroenterology 2003;125:755-64. 
19. Rolando $\mathrm{N}$, Wade J, Davalos $\mathrm{M}$, Wendon J, Philpott-Howard J, Williams R. The systemic inflammatory response syndrome in acute liver failure Hepatology 2000;32:734-9.

20. Jalan R, Pollok $A$, Shah $S$, Madhavan $K$, Simpson $K$. Liver derived pro-inflammarory cytokimes may be important in producing intracranial hypertension in acure liver failure. ] Hepatol 2002;37:536.

21. Jalan R, Olde Damink SW, Deutz NE, Davies NA, Garden OJ, Madhavan KK, Hayes PC, Lee A. Moderate hypothermia prevents cerebral hyperemia and increase in intracranial pressure in patients undergoing liver transplantation for acute liver failure. Transplantation 2003 Jun 27; 75: 2034-2039.

22. Shawcross DL, Davies NA, Williams R, Jalan R. Systemic inflammatory response exacerbates the neuropsychological effects of induced hyperammonemia in cirrhosis. Hepatology $2004: 40: 247-54$.

23. Marion DW, Penrod LE, Kelsey SF, Obrist WD, Kochanek PM, Palmer AM, Wisniewski SR, DeKosky ST. Treatment of traumatic brain injury with moderate hypothermia. N Engl J Med 1997:336:540-6.

24. Muller M, Schwerdtfeger K, Maier B, Mautes A, Schiedat T, Bianchi O, Marzi I; Homburg Traumaric Brain Injury Group. Cerebral blood flow velocity and inflammatory response after severe traumatic brain injury. Eur J Ultrasound 2001;12:203-8.

25. Angstwurm K, Reuss S, Freyer D, Arnold G, Dirnagl U, Schumann RR, Weber JR. Induced hypothermia in experimental pneumococcal meningitis. I Cereb Blood Flow Metab 2000;20:834-8

26. Marion DW. Moderate hypothermia delays proinflammarory cytokine production of human peripheral blood mononuclear cells Crit Care Med. 2002;30:1499-502.

27. Jacobsen M, Enevoldsen E. Retrograde catheterization of the right internal jugular vein for serial measurements of cerebral venous oxygen content. J Cereb Blood Flow Merab 1989;9:717-20.

28. Kety SS, Schmidt CF. The nitrous oxide method for the quantitative determination of cerebral bllood flow in man: Theory, procedure and normal values. J Clin Invest 1947;27:47683.

29. Phillips BJ, Armstrong IR, Pollock A. Lee A. Cerebral blood flow and metabolism in patients with chronic liver disease undergoing orthotopic liver transplantation. Hepatology $1998 ; 27: 369-76$.

30. Wolfe RR. (1992) Radioactive and stable isotope tracers in biomedicine. Principles and practice of kinetic analysis. Wiley-Liss, New York.

31. Kato M, Hughes RD, Keays RT, Williams R. Electron microscopic study of brain capillaries in cerebral edema from fulminant hepatic failure. Heparology 1992;15:1060-6.

32. Licinio J, Wong ML. Pathways and mechanisms for cytokine signaling of the central nervous system. I Clin Invest 1997;100:2941-7.

33. Gorg B, Foster N, Reinehr R, Bidmon HJ, Hongen A, Haussinger D, Schliess F.Benzodiazepine-induced protein tyrosine nitration in rat astrocytes. Hepatology $2003 ; 37: 334-42$.

34. Norenberg MD. Oxidative and nitrosative stress in ammonia neurotoxicity. Hepatology 2003;37:245-8.

35. Larsen FS, Gottstein J, Blei AT. Cerebral hyperaemia and nitric oxide synthase in rats with ammonia-induced brain edema. J Hepatol 2001;34:548-54.

36. Moller K, Strauss GI, Qvist ], Fonsmark L, Knudsen GM, Larsen FS, Krabbe KS, Skinhoj P, Pedersen BK. Cerebral blood flow and oxidative metabolism during human endotoxemia. J Cereb Blood Flow Metab 2002;22:1262-70. 


$$
\text { - }
$$




\section{LINK TO CHAPTER 10}

The work of the following chapter was initiated by the observations made in a patient with paracetamol induced acute liver failure who fulfilled criteria for poor prognosis and was waiting for a liver to become available for transplantation. Because of severe uncontrolled intracranial hypertension she underwent a hepatectomy that resulted in stabilization of her systemic and cerebral haemodynamics. She remained anhepatic for 14 hours and was successfully bridged to liver transplantation. The removal of the liver was associated with a sharp and sustained reduction in the circulating pro-inflammatory cytokine concentration suggesting that liver derived pro-inflammatory cytokines may be important in the pathogenesis of intracranial hypertension in patients with acute liver failure.

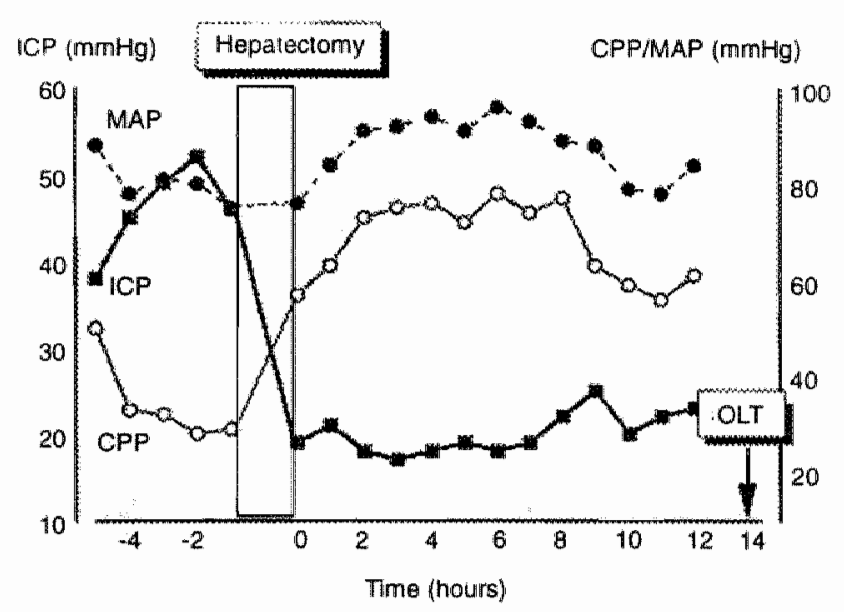

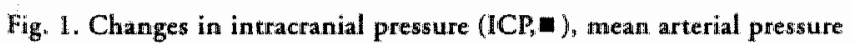
(MAP, ) and cerebral perfusion pressure (CPP, $O$, mean arterilal pressim ure-ICP) prior to and after hepatectomy.

Published as Jalan R, Pollok A, Shah SHA, Madbavan KK, Simpson KJ. Liver derived proinflammatory cytokines may be important in producing intracranial hypertension in acute liver failure. J Hepatology 2002; 37; 536-8. 

CHAPTER 10

TREATMENT OF UNCONTROLLED INTRACRANIAL HYPERTENSION IN ACUTE LIVER FAILURE WITH MODERATE HYPOTHERMIA

Rajiv Jalan ${ }^{1}$, Steven WM Olde Damink ${ }^{1,3}$, Nicolaas EP Deutz ${ }^{3}$, Alistair Lee ${ }^{2}$, Peter C Hayes $^{1}$

${ }^{1}$ Liver Unit and Scotrish Liver Transplantation Unit, Royal Infirmary of Edinburgh, 2Department of Anaesthetics and the Intensive Care Unit, Royal Infirmary of Edinburgh, ${ }^{3}$ Department of Surgery, Maastricht University, Maastricht, The Netherlands

Published in the Lancet 1999; 354: 1164-1168 


\section{INTRODUCTION}

Encephalopathy in acute liver failure (1) (ALF) is characterised by rapid deterioration in the level of consciousness, increased intracranial pressure (ICP), reduced cerebra perfusion pressure (CPP) and a mortality rate of about $90 \%$ in patients who fulfil cri teria for poor prognosis (2). With orthotopic liver transplantation (OLT), survival rate of up to $80 \%$ can be achieved (3-5). However, 30-40\% patients with ALF die whil waiting for a donor organ to become available, primarily due to the deleterious effect: of increased ICP (2-5).

As therapy for increased ICP, hyperventilation delays the onset of cerebellar herni. ation but does not prevent the development of cerebral edema and is therefore not routinely recommended (6). Mannitol may improve survival in patients with ALF and is useful in reducing elevated ICP but its usefulness is limited in patients with associated renal failure (7). Thiopentone controls ICP in patients who fail to respond to mannitol (8), but produces haemodynamic instability and is therefore not routinely used. Relapses of increase in ICP after treatment with mannitol, hyperventilation or thiopentone varies from $71-80 \%$ (5).

Therefore, other modalities of treatment to reduce increased ICP in ALF are needed. In experimental models of ALF the role of hypothermia for the treatment of cerebral edema has shown promising results and suggests its application for treating intracranial hypertension in ALF (9-11).

The aims of the present study were to evaluate the efficacy and safety of moderate hypothermia in the management of patients with ALF and raised ICP that is resistant to conventional treatment. 


\section{METHODS}

Between June 1997 and November 1998, 27 patients with ALF and grade III-IV hepatic encephalopathy were admitted to our Unit. Patients were enrolled if they had ALF (1), fulfilled criteria for poor prognosis (2) and had raised ICP that was refractory to conventional treatment (2). Uncontrolled intracranial hypertension was defined as persistently elevated ICP of $>25 \mathrm{mmHg}$ for 1 hour or more despite 2 separate treatments with mannitol $(1 \mathrm{~g} / \mathrm{Kg}$ body weight over $20 \mathrm{~min})$ and removal of $500 \mathrm{ml}$ of fluid by continuous veno-venous hemofiltration. Ethical permission for this study was obtained from the Lothian ethics committee. Written informed consent was obtained from the next of kin. Twenty patients were excluded; because of increased ICP that was responsive to treatment with mannitol and/or ultrafiltration $(\mathrm{n}=9)$ and/or did not fulfil criteria for poor prognosis $(n=8)$, paracetamol overdose with concomitant alcoholic hepatitis $(n=6)$, failure to obtain informed consent $(n=3)$ or cardiac arrest $(n=1)$.

All patients were mechanically ventilated following sedation with propofol and paralysis with atracurium besylate $(300-600 \mathrm{mg} / \mathrm{Kg} / \mathrm{hr}$ ). Following mechanical ventilation, a pulmonary artery catheter, a right atrial catheter and an arterial catheter were inserted for monitoring. Patients were managed according to a standardised protocol. $\mathrm{N}$-acetylcysteine was commenced and/or continued $(150 \mathrm{mg} / \mathrm{Kg} / 24 \mathrm{hr})(12)$; broad spectrum antibiotics and fluconazole were administered prophylactically; blood glucose levels were maintained between $5-7 \mathrm{mmol} / 1$; intravascular volume was maintained by keeping pulmonary capillary wedge pressures between 8 and $14 \mathrm{mmHg}$; continuous veno-venous hemofiltration (Gambro Laboratories, USA) was instituted in all patients for established renal failure (blood flow of $200 \mathrm{ml} / \mathrm{min}$ and 1 litre cycle exchanges).

ICP was monitored continuously using a subdural fibre-optic system (Camino, Camino Laboratories, San Diego, USA) that was inserted after correction of coagulation abnormalities with fresh frozen plasma and platelets. Noradrenaline was used to keep the CPP above $50 \mathrm{mmHg}$ and/or a mean arterial pressure of greater than 90 mmHg. Patients with raised ICP ( $>20 \mathrm{mmHg}$ for 10 minutes) were initially treated with 2 boluses of mannitol ( $1 \mathrm{~g} / \mathrm{Kg}$ body weight over $20 \mathrm{~min}$ ) and removal of $500 \mathrm{ml}$ of fluid. If this regime failed to keep the ICP $<25 \mathrm{mmHg}$ moderate hypothermia was instituted.

Study Design. Two groups of patients were studied with different protocols. Group A consisted of patients who were considered unsuitable candidates for OLT based on a history of multiple episodes of self harm and lack of compliance with previous medical therapy (Patients 1-3). These patients were cooled for a period of 8 hours and then gradually rewarmed over a $1 \mathrm{hr}$ period to the baseline temperature of $37^{\circ} \mathrm{C}$. Group B consisted of patients who were suitable candidates for OLT and were on the "superur- 
gent' transplant waiting list (Patients 4-7). These patients were cooled until and through the OLT procedure using cooling blankets that were placed above and below the patient (during OLT, below the patient and on the legs) (Blanketrol II, Cincinnati Sub-Zero, Cincinnati) to maintain the blood temperature at $32-33^{\circ} \mathrm{C}$. This temperature was chosen because this has been shown to be safe and successfully reduces ICP both in animal models and head injured patients $(10,13)$.

Detailed neurological examination was performed before and after institution of hypothermia with particular attention to pupillary size and its reaction to light. The ICP values that have been reported represent the highest recorded ICP that persisted for $>10 \mathrm{~min}$ in the previous hour.

Cerebral blood flow measurements. To measure cerebral blood flow (CBF), another arterial catheter was inserted into the femoral artery and a jugular bulb catheter inserted into the left internal jugular vein (4F Opticath, U440, Abbot, USA). Correct positioning of the jugular bulb catheter was confirmed with a lateral head and neck radiograph (14). CBF was only determined if the parient was haemodynamically stable, defined as a difference of less than $10 \%$ in the mean arterial pressure. Ventilation was adjusted to achieve an arterial carbon dioxide tension of 4 kiloPascals ( $\mathrm{pa}$ ), and was not altered again during the study to prevent $\mathrm{PaCO}_{2}$ as a confounding factor of $\mathrm{CBF}$. CBF was measured using a modification of the Key-Schmidt technique (15) measuring the rate of uptake of nitrous oxide by the brain as detailed previously (16).

Measurement of metabolic parameters. Resting energy expenditure was measured continuously during the study period using indirect calorimetry (Deltatrac, Datex, Helsinki, Finland). Immediately after each measurement of $\mathrm{CBF}$, blood samples were simultaneously collected from the femoral artery and the jugular bulb catheters. Percentage oxygen saturation of haemoglobin was directly measured (CO-Oximeter 482, Instrumentation Laboratory, $\mathrm{UK}$ ). Plasma was obtained by centrifugation and was deproteinised using trichloroacetic acid $(50 \% \mathrm{w} / \mathrm{v})$ prior to storage $\left(-80^{\circ} \mathrm{C}\right)$, for spectrophotometric determination by standard enzymatic methods (CobasMiraS, Hoffman-LaRoche, Switzerland) of glucose, lactate and ammonia (17).

Calculations and statistical analysis. All the data were expressed as median and range. Fractional extraction of metabolites was calculated as arterial-jugular venous concentration difference divided by the arterial concentration. Cerebral metabolic rate for oxygen, glucose, lactate, and ammonia were calculated as CBF times arterial-jugular venous concentration differences.

Mean pre-cooling data from each patient ( 3 time points, -2 to 0 hour) and mean post cooling data for each patient ( 8 time points, 1 to 8 hour) were calculated and tested for difference using the Wilcoxon signed-ranks test for variables that were measured continuously (temperature, intracranial pressure, cardiac index, noradrenaline 
requirement, heart rate, mean arterial pressure, systemic vascular resistance, $\mathrm{CPP}$, pulmonary capillary wedged pressure and resting energy expenditure). For all the other variables Wilcoxon signed-ranks test was used to compare pre and post cooling (time $=4$ hours) observations. To determine whether the median of the distribution of the pre and post cooling values of oxygen, glucose, lactate and ammonia data were different from zero, the Wilcoxon signed-ranks test was used. Relationship between the change in ICP with the change in CBF and cardiac index were measured using the Spearman's correlation coefficients. 


\section{RESULTS}

Clinical Outcome. Patient characteristics are summarized in Table 1. Even before starting cooling, the temperature of Patient 6 was $33.5^{\circ} \mathrm{C}$. All 4 patients who were candidates for OLT survived to OLT. The transplantation procedure was uncomplicated in 3 (Patients 4,6,7) and these patients were neurologically unimpaired after the transplantation operation. During the dissection/mobilisation phase of OLT, Patient 6 developed a sudden increase in ICP that peaked at $71 \mathrm{mmHg}$ and during this time the mean CPP was between 20 and $30 \mathrm{mmHg}$. This patient survived the OLT but died with brain death. All 3 patients who were unsuitable candidates for OLT died from the effects of multiorgan failure 3,6 and 11 hours after rewarming. There was no evidence of clinically significant bleeding during the cooling period. Patient 4 had staphylococcal septicaemia after OLT which was successfully treated with antibiotics.

Changes in neurological parameters. Pupillary dilatation $(>6 \mathrm{~mm})$ and sluggish reaction to light were evident in Patient 2, Patient 6 and Patient 7 before cooling. The pupils remained dilated and poorly reactive to light despite reduction in ICP in Patient 2. Pupil size and its reaction to light returned to normal in Patients 6 and 7 following cooling. Following rewarming, pupillary dilatation and loss of reaction to light became evident in Patient 3.

Changes in haemodynamic and metabolic parameters. These are summarised in Table 2. Following cooling there was significant reduction in ICP and CBF (see Figure, Table 2). In Patient 5, CBF was low $(25 \mathrm{ml} / 100 \mathrm{~g} / \mathrm{min})$ and did not change significantly $(24 \mathrm{~m} / 100 \mathrm{~g} / \mathrm{min})$. Despite optimisation of the volume status all patients required noradrenaline prior to cooling and this requirement was significantly reduced after cooling ( $45 \%, \mathrm{p}<0.05)$. Cerebral vascular resistance (CPP divided by CBF) increased significantly after cooling $(300 \%, \mathrm{p}<0.05)$. There was no significant correlation between the change in cerebral vascular resistance with the change in systemic vascular resistance. There was no significant correlation between the change in cardiac index or CBF with the change in ICP (correlation coefficients: -0.29 and 0.26 respectively, $\mathrm{p}=\mathrm{ns})$.

Resting energy expenditure was reduced with cooling. Prior to cooling, there was net uptake of ammonia by the brain and this was reduced to values that were not significantly different to zero after cooling. There was a significant reduction in the extraction of ammonia and glucose with cooling but there was no significant difference in the extraction of oxygen or lactate. After cooling, the median distribution of the extraction of both ammonia and glucose were not significantly different from zero. 
Table 2. Changes in hacmodynamic and metabolic parameters.

\begin{tabular}{|c|c|c|}
\hline Heart rate (beats/min) & $114(94-148)$ & $81(64-118) \pi$ \\
\hline Mean arterial pressure $(\mathrm{mmHg})$ & $94(58-105)$ & $90(64-111)$ \\
\hline $\begin{array}{l}\text { Pulmonary Capillary Wedge Pressure } \\
(\mathrm{mmHg})\end{array}$ & $14(7-18)$ & $12(5-17)$ \\
\hline \multicolumn{3}{|l|}{ Systemic vascular resistance } \\
\hline$(d y n . s e c . c m-5 / m 2)$ & $924(739-1131)$ & $1436(1231-1676) \pi$ \\
\hline $\begin{array}{l}\text { Cerebral perfusion pressure } \\
(\mathrm{mmH})\end{array}$ & $45 \quad(37-56) 70$ & $(60-78) \pi$ \\
\hline Cerebral blood flow $(\mathrm{m} / / 100 \mathrm{~g} / \mathrm{min})$ & $103(25-134)$ & $44(24-75)$ \\
\hline $\begin{array}{l}\text { Cerebral Vascular resistance } \\
\text { (Wood units } 1100 \mathrm{~g} \text { ) }\end{array}$ & 0.4 (range $0.3-1.8)$ & 1.6 (range $0.9-2.5$ ) \\
\hline Resting Energy Expenditure (kCal/24hr) & $1890(1761-2530)$ & \multirow[t]{2}{*}{$1356(1121-1456) \pi$} \\
\hline \multicolumn{2}{|l|}{ Oxygen } & \\
\hline Arterial (mllod) & $15.8(14.4-17.5)$ & $15.7(13.2-17.1)$ \\
\hline Jugular venous (m//oll) & $12.7(9.7-14.3)$ & $(10-13.7)$ \\
\hline$\%$ Extraction & $24.3(9.0-38.7) \S$ & $(8.2-34.1) 5$ \\
\hline Cerebral melabolic rate $(\mathrm{m} / / 100 \mathrm{~g} / \mathrm{min})$ & $0.4(0.1-0.8) \S$ & $(0.1-0.2) \S \pi$ \\
\hline \multicolumn{3}{|l|}{ Glucose } \\
\hline Arterial (mmollo) & $6.4 \quad(5.2-8.7)$ & $(5.8-10.4)$ \\
\hline Jugular venous (mmol/i) & $6.0 \quad(4.7-8.4)$ & $(5.8-11.3)$ \\
\hline$\%$ Extraction & $6.3 \quad(2.4-13.3) \S$ & $(-9.7-4.2) \pi$ \\
\hline Cerebral metabolic rate (mmol/100g/min) & $35.1(7.8-88.6) \S$ & $(-36.5-21.9) \pi$ \\
\hline \multicolumn{3}{|l|}{ Lactate } \\
\hline Arterial (mmold) & $(3.7-9.3)$ & $(3.3-14.8)$ \\
\hline Jugular venous (mmoll) & $8.5 \quad(3.6-9.3)$ & $(3.4-14.5)$ \\
\hline$\%$ Extraction & $2.5 \quad(-2.3-3.8)$ & $(-18.6-5.9)$ \\
\hline Cerebral melabolic rate (mmol/ $100 \mathrm{~g} / \mathrm{min})$ & $12.0(19.2-33.8)$ & $(-40,6-24.6)$ \\
\hline \multicolumn{3}{|l|}{ Ammonia } \\
\hline Artenial (mimol/i) & $343 \quad(109-490)$ & $(100-453) \pi$ \\
\hline Jugular venous (mmol/h) & $305 \quad(49-477)$ & $(85-527)$ \\
\hline$\%$ Extraction & $11.0(1.0-15.0) \S$ & $(-16.3-15.4) \pi$ \\
\hline Cerebral metabolic rate (mmol/100g/min) & $2.6 \quad(0.6-6.3) \S$ & $-0.3(-3.1-1.4) \pi$ \\
\hline Arterial Carbondioxide (Kpa) & $4.1(3.9-4.2)$ & $4(3.8-4.2)$ \\
\hline Arterial hydrogen ion concentration $(\mathrm{nmol} / \mathrm{L})$ & $48(36-58)$ & $44.6(3.2-51)$ \\
\hline Propofol dosage (mg/hr) & $70(50-170)$ & $60(50-140)$ \\
\hline
\end{tabular}

Data expressed as median and range. Statistics: Wilcoxon signed-ranks test; level of significance: $\pi P<0.05$. For $\%$ extraction and cerebral metabolic rate data: Wilcoxon signed-ranks test for differences from zero: $\$ \mathrm{P}<0.05$. Range of normal cerebral blood flow: $45-60 \mathrm{ml} / 100 \mathrm{~g} / \mathrm{min}$; cerebral perfusion pressure $=$ mean arterial pressure-intracranial pressure; cerebral vascular resistance $=$ cerebral perfusion pressure/cerebral blood flow. 
Patients who dus not hakt oli
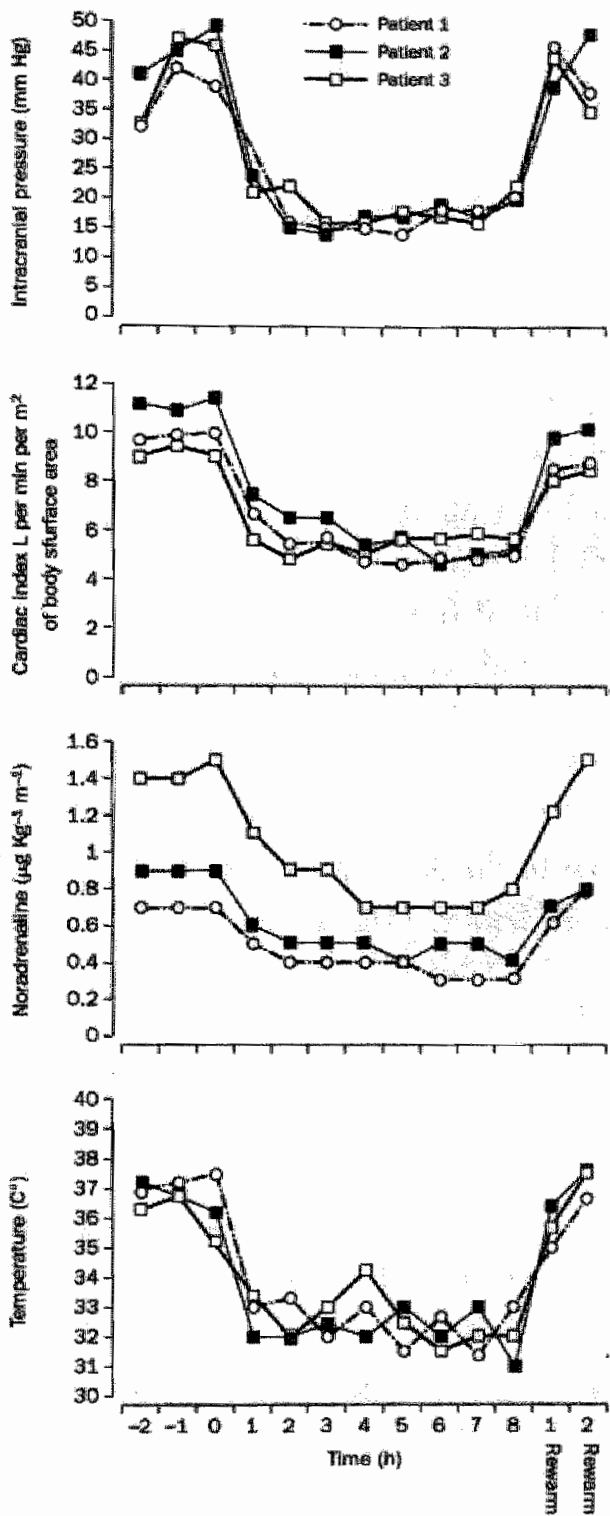

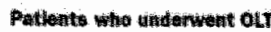
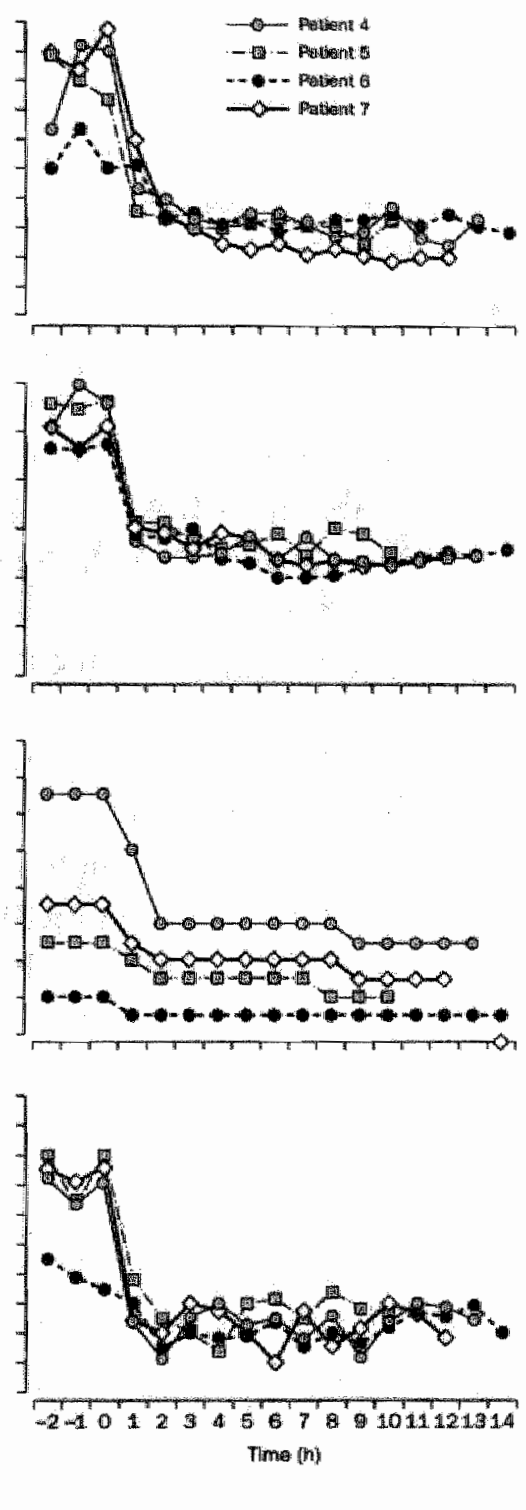

Figure 1: Changes in Temperature, Noradrenaline requirement, Cardiac Index and Intracranial pressure in individual patients following cooling. Group A (Patients 1,2 and 3) were not suitable candidates for liver transplantation. Group B (Patients 4, 5, 6 and 7) were candidares for liver transplantation and all were successfully bridged to OLT . Changes in Temperature, Noradrenaline requirement, Cardiac Index and Intracranial pressure were statistically significant $(p<0.02)$. 


\section{DISCUSSION}

Over $90 \%$ patients die from the effects of increased ICP within 12 hours if this is not controlled by repeated mannitol treatments and ultrafiltration (2). Even a relatively short increase of 12-24 hours for which these patients can be supported may allow a significant number of patients to be maintained until a liver for transplantation becomes available. In this study, we have shown that moderate hypothermia as a treatment for uncontrolled increase in ICP is easy to institute and safe, reduces ICP, CBF, cardiac index and noradrenaline requirements, and increases CPP. Moderate hypothermia successfully 'bridged' all 4 transplant candidates to OLT.

The parients in Group A were terminally ill and were treated with hypothermia to determine the efficacy of hypothermia on reducing ICP. To establish whether this reduction in ICP was an effect of hypothermia, rewarming was built into the protocol. With hindsight, these patients may have benefited from cooling for a longer period. Group B comprised patients waiting for OLT and received livers within a median of 13 hours of hypothermia for uncontrolled increase in ICP, with a median time from ventilation for grade 3-4 encephalopathy to receiving an organ for OLT of 23.5 hours. This reflects the average time for receiving a liver on the 'super urgent' list in the UK.

Ammonia has been thought to be central in the development of increased ICP in ALF which is detoxified in the astrocyte to glutamine (18). Several studies in animal models and patients with ALF have shown an increase in brain glutamine (19-21). Glutamine accumulation in the astrocyte increases intracellular osmolality, causing movement of water and cerebral edema $(19,22)$. Inhibition of glutamine syntherase during hyperammonemia reduces astrocytic swelling (19) and prevents increase in ICP (22).

Prior to cooling all patients had markedly elevated arterial ammonia that was significantly reduced with hypothermia. As a consequence of this and the decreased CBF, there was a 3 -fold decrease in ammonia delivery to the brain. In this study, we have demonstrated a significant extraction and net uptake of ammonia by the brain prior to cooling which decreased to levels that were not significantly different from zero after cooling. This effect of hypothermia upon ammonia metabolism may be an important mechanism by which hypothermia reduces ICP. The mechanism of reduced concentration of ammonia is unclear but may be a consequence of reduction in overall metabolism.

Although a study showed reduced $\mathrm{CBF}$ in $\mathrm{ALF}^{23}$ others showed increased $\mathrm{CBF}$ $(24,25)$. Studies in patients with ALF have demonstrated a loss of autoregulation of CBF. Therefore the relationship between metabolism and blood flow may become interrupted (25). In this study, CBF was increased, and cerebral metabolic rate for oxy- 
gen and glucose were reduced compared with normal values and in cirrhosis $(15,16)$. The mechanism of this dysregulation of $\mathrm{CBF}$ is unclear but may be related to astrocytic glutamine accumulation during hyperammonemia (26). The observed reduction in CBF following cooling may be the result of reduced cerebral metabolism or decreased astrocytic glutamine synthesis (26) as is suggested by reduced ammonia uptake by the brain. However, the effect of hypothermia upon endorhelium dependent mechanisms such as nitric oxide, prostanoids and endothelin requires further investigation (27-29).

CPP is critical in determining outcome and in this study hypothermia improved $\mathrm{CPP}$ in all patients (5) without changing jugular venous oxygen saturation and cerebral extraction of oxygen. Even prior to cooling the cerebral metabolic rate for oxygen was low and this was reduced even further. Although a value of over $1.3 \mathrm{ml} / 100 \mathrm{~g} / \mathrm{min}$ has been suggested as being important for the maintenance of brain viability (30), this and another study (23) have confirmed neurologic recovery following OLT despite lower cerebral metabolic rates for oxygen. The substantial reduction in CBF without any significant change in oxygen extraction substantiates the previously described phenomenon of 'luxury perfusion' in patients with ALF and increased ICP (25). In a few patients we observed a negative cerebral metabolic rate for glucose which may reflect the efflux of glucose from the brain glucose pool following disturbance in the cellular homeostasis due to cooling. This has also been observed during the reperfusion phase of orthotopic liver transplantation (16). The significance of this observation is not clear.

In conclusion, moderate hypothermia appears useful in treating uncontrolled increase in ICP in this selected group of patients with ALF while an organ for transplantation is awaited. However, a randomised controlled study is necessary for determining the place of moderate hypothermia in the treatment of patients with ALF. The mechanism of reduction in ICP by hypothermia in ALF may be through reduction in brain uptake of ammonia and modulation of CBF. 


\section{REFERENCES}

1. Trey C, Davidson CS. The management of fulminant hepatic failure. In: Popper $\mathrm{H}$, Schaffner $F$, eds. Progress in liver disease, Volume III. New York: Grune and Stratton, 1970:282-298.

2. O'Grady JG, Alexander GM, Hayllar KM, Williams R. Early indicators of prognosis in fulminant hepatic failure. Gastroenterology 1989;97:439-445.

3. Ascher NL, Lake JR, Emond JC, Roberts JP. Liver transplantation for fulminant hepatic failure. Arch Surg 1993,128:677-682.

4. Makin AI, Wendon ], Williams R. A 7-year experience of severe acetaminophen-induced hepatotoxicity (1987-1993). Gastroenterology 1995;109:1907-16.

5. Hoofnagle JH, Carithers RL, Chapiro C, Ascher NL. Fulminant hepatic failure: Summary of a Workshop. Hepatology 1995:21:240-252.

6. Ede R., Gimson AE, Bihari D, Williams R. Controlled hyperventilation in the prevention of cerebral oedema in fulminant hepatic failure. J Hepatol 1986;2:43-51.

7. Canallese J, Gimson AES, Davis C, Mellon PJ, Davis M, Williams R. Controlled trial of dexamethasone and mannitol for the cerebral oedema of fulminant hepatic failure. Gut $1982 ; 23: 625-629$.

8. Forbes A, Alexander GJ, O'Grady JG, Keays R, Gullan R, Dawling S, Williams R. Thiopental infusion in the treatment of intracranial hypertension complicating fulminant hepatic failure. Hepatology 1989; 10:306-10.

9. Peignoux M, Bernuau J, Benhamou JP. Total hepatectomy and vascular exclusion in the rat. A comparison, with special reference to the influence of body temperature. Clin Sci 1982:62:273-7.

10. Traber P, DalCanto M, Ganger D, Blei AT. Effect of body temperature on brain edema and encephalopathy in the rat after hepatic devascularization Gastroenterology $1989 ; 96: 885-91$.

11. Eguchi S, Kamlot A, Ljubimova J, Hewint WR, Lebow LT, Demetriou AA, Rozga J. Fulminant heparic failure in rats: survival and effect on blood chemistry and liver regeneration. Hepatology 1996;24:1452-9.

12. Keays R, Harrison PM, Wendon JA, Forbes A, Gove G, Alexander GJ, Williams R. Intravenous acetylcysteine in paracetamol induced fulminant hepatic failure: a prospective controlled trial. BMJ 1991;303:1026-9.

13. Marion DW, Penrod LE, Kelsey SF, Obrist WD, Kochanek PM, Palmer AM, Wisniewski SR, DeKosky ST. Trearment of traumatic brain injury with moderate hypothermia. N Eng. J Med 1997;336:540-46.

14. Jacobsen M, Enevoldsen E. Retrograde catheterization of the right internal jugular vein for serial measurements of cerebral venous oxygen content. J Cereb Blood Flow Metab 1989;9:717-720.

15. Kety SS, Schmidt CF "The nitrous oxide method for the quantitative determination of cerebral blood flow in man: Theory, procedure and normal values. J Clin Invest $1947 ; 27: 476-483$.

16. Philips BJ, Armstrong IR, Pollock A, Lee A. Cerebral blood flow and metabolism in parients with chronic liver disease undergoing orthotopic liver transplantation. Hepatology $1998 ; 27: 369-76$. 
17. Dejong CHC, Deutz NEP, Soeters PB. Renal ammonia and glutamine metabolism during liver insufficiency-induced hyperammonemia in the rat. I Clin Invest 1993;92:283440.

18. Cooper AI, Plum E. Biochemistry and physiology of brain ammonia. Physiol Rev $1987 ; 67: 440-519$.

19. Takahashi H, Koehler RC, Brusilow SW, Traystman RJ. Inhibition of brain glutamine accumulation prevents cerebral edema in hyperammonemic rats. Am J Physiol 1991;261:H825-9.

20. Olafsson $S$, Gottstein J, Blei AT. Brain edema and intracranial hypertension in tats after total hepatectomy. Gastroenterology 1995;108:1097-103.

21. McConnell JR, Antonson DL, Ong CS, Chu WK, Fox I], Heffron TG, Langnas AN, Shaw BW. Proton spectroscopy of brain glutamine in acute liver failure Hepatology 1995;22:69-74.

22. Willard-Mack CL, Koehler RC, Hirata T, Cork LC, Takahashi H, Traystman RJ, Brusilow SW. Inhibition of glutamine synthetase reduces ammonia-induced astrocyte swelling in rat. Neuroscience 1996;71:589-99.

23. Wendon JA, Harrison PM, Keays $\mathrm{R}$. Williams R. Cerebral blood flow and merabolism in fulminant liver failure. Hepatology 1994;19:1407-13.

24. Aggarwal S, Kramer D, Yonas H, Obrist W, Kang Y, Martin M, Policare R. Cerebral hemodynamic and metabolic changes in fulminant hepatic failure: a retrospective study. Hepatology 1994; 19:80-7.

25. Larsen FS Cerebral circulation in liver failure: Ohm's law in force. Semin Liver Dis $1996 ; 16: 281-92$.

26. Hirata T, Kaehler RC, Kawaguchi T, Brusilow SW, Traystman RJ. Impaired pial arteriolar reactivity to hypercapnia during hyperammonemia depends on glutamine synthesis. Stroke $1996 ; 27: 729-36$.

27. Clemmesen JO, Hansen BA, Larsen FS. Indomethacin normalizes intracranial pressture in acute liver failure: a twenty-three-year-old woman treated with indomethacin. Hepatology 1997;26:1423-5.

28. Rao VL, Butterworth RF. Neuronal nitric oxide synthase and Hepatic Encephalopathy. Metab Brain Dis 1998;13:175-89.

29. Ejlersen E, Larsen FS, Pott F, Gyrtrup HI, Kirkegaard P, Secher NH. Hepatectomy corrects cerebral hyperperfusion in fulminant hepatic failure. Transplant Proc 1994 26:17941795

30. Powers WJ, Grubb RL Iry Darrier D, Raichle ME. Cerebral blood flow and cerebral metabolic rate of oxygen requirements for cerebral function and viability in humans. J Cereb Blood Flow Metab 1985;5:600-8. 
RESTORATION OF CEREBRAL BLOOD FLOW AUTOREGULATION AND REACTIVITY TO CARBON DIOXIDE IN ACUTE LIVER FAILURE BY MODERATE HYPOTHERMIA

${ }^{1,2}$ Rajiv Jalan, ${ }^{2,3}$ Steven WM Olde Damink, ${ }^{3}$ Nicolaas EP Deutz, 2 Peter C Hayes, ${ }^{4}$ Alistair Lee

${ }^{1}$ Institute of Hepatology, University College London Medical School, London UK, ${ }^{2}$ Liver Unit and Scottish Liver Transplantation Unit, Royal Infirmary of Edinburgh, UK, ${ }^{3}$ Department of Surgery, Maastricht University, Maastricht, The Netherlands, ${ }^{4}$ Department of Anaesthetics and the Intensive Care Unit, Royal Infirmary of Edinburgh, UK

Published in Hepatology 2001; 34: 50-54 


\section{INTRODUCTION}

Encephalopathy in acute liver failure (ALF) is characterised by rapid deterioration in the level of consciousness, increased intracranial pressure (ICP), reduced cerebral perfusion pressure (CPP) and a mortality rate of about $90 \%$ in patients who fulfil criteria for poor prognosis $(1,2)$. Over $90 \%$ of the patients die from the effects of increased ICP within 12 hours if this is not controlled by repeated mannitol treatments and ultrafiltration (1-3). We have recently shown that moderate hypothermia $(32 \infty \mathrm{C})$ as a treatment for uncontrolled increase in ICP is easy to institute and safe, reduces ICP, cardiac index and noradrenaline requirements, increases CPP and can be used as a bridge to liver transplantation. Although we showed that arterial concentrations of ammonia and its uptake by the brain were reduced, the mechanism by which hyporhermia reduces ICP is not clear (4).

Within the range of mean arterial pressure (MAP) of $60-160 \mathrm{mmHg}$ or a CPP of $50-150 \mathrm{mmHg}$ there is little variation in cerebral blood flow (CBF) and this homeostatic mechanism is termed 'autoregulation'. This is maintained by direcr variation of cerebrovascular resistance with the perfusion pressure. In addition, the $\mathrm{PaCO}_{2}$ closely regulates $C B F$ and within a range of $25-55 \mathrm{mmHg}, \mathrm{CBF}$ varies linearly with $\mathrm{PaCO}_{2}$ changing at about $3 \%$ per $\mathrm{mmHg}(5,6)$. Studies in both patients and also animal models of ALF suggest that the CBF is increased in patients with ALF and increased ICP (4, 7-13). The mechanism of this increase in CBF has been suggested to result from events that follow glutamine accumulation in the brain and increased activity of neuronal nitric oxide synthase has been suggested as a possible cause $(8,14,15)$. Studies have also shown that $\mathrm{CBF}$ autoregulation is lost in patients with ALF and this may be due to cerebral hyperaemia that is characteristic of this condition because the disturbed autoregulation is corrected by hyperventilation which induces arteriolar vasoconstriction (16). However, the relationship between the increase in CBF following increase in mean arterial pressure (MAP) and its effects upon ICP are unknown. The purpose of this study was to test the hypothesis that moderate hypothermia reduced ICP by restoring $\mathrm{CBF}$ autoregulation. 


\section{METHODS}

Studies were undertaken with the approval of the local research ethics committee, the written informed consent from the next of kin of each patient, and in accordance with the Declaration of Helsinki (1989) of the World Medical Association. The next of kin of the patients were informed of the potential dangers associated with the invasive monitoring, particularly insertion of the intracranial pressure monitors. All the invasive monitoring described in the ensuing methods section apart from the measurement of $\mathrm{CBF}$ is routine in the management of patients with ALF and severe hepatic encephalopathy who require mechanical ventilation.

Patients. Nine patients (median age 32 (range 22-46); 2M, 7F) with uncontrolled intracranial hypertension (see further) and ALF (due to paracetamol overdose, 7; drug induced, 1; non A, non B hepatitis, 1) who fulfilled criteria for poor prognosis were studied (highest median prothrombin time 123 [101-172] sec; creatinine 331 [221435] $\mathrm{mmol} / \mathrm{L}$ (1). Two of these patients formed a part of our previous report (4). All the patients were mechanically ventilated following sedation with propofol and paralysis with atracurium besylate $(300-600 \mathrm{mg} / \mathrm{Kg} / \mathrm{hr})$. ICP and cardiovascular haemodynamics were continuously recorded using a subdural fibre-optic system (Camino, Camino Laboratories, San Diego, USA), pulmonary artery catheter, a right atrial catheter and an arterial catheter. CPP was calculated as the difference between the MAP and the ICP. Patients were managed according to a standardised protocol as described previously (4). Noradrenaline was used to keep the CPP above $50 \mathrm{mmHg}$ and/or a MAP of greater than $90 \mathrm{mmHg}$. Patients with raised ICP ( $>20 \mathrm{mmHg}$ for 10 minutes) were initially treated with 2 boluses of mannitol $(1 \mathrm{~g} / \mathrm{Kg}$ body weight over 20 $\mathrm{min}$ ) and removal of $500 \mathrm{ml}$ of fluid. If this regime failed to keep the ICP $<25 \mathrm{mmHg}$, the patients were defined as having uncontrolled intracranial hypertension and moderate hypothermia was instituted using a cooling blanket (Blanketrol II, Cincinnati SubZero, Cincinnati) (4). CBF autoregulation was measured in 6 patients and reactivity to carbon dioxide was measured in 5 patients. Although we would have liked to perform both sets of experiments in the same patients, this was difficult because a single measurement of CBF takes a minimum of $20 \mathrm{~min}$. Therefore, simultaneous measurements of $\mathrm{CBF}$ autoregulation and reactivity to carbon dioxide could only be performed in 2 patients.

Measurement of cerebral blood flow. A second arterial catheter was inserted into the right-femoral artery (115.11, Vygon, Ecouen, France), and a jugular bulb catheter inserted into the left-internal jugular vein (4F Opticath, U440, Abbot Laboratories, Queensborough, UK) through a $5 \mathrm{~F}$ haemostasis introducer (Fast Cath, Daig 
Corporation, Minnetonka, MN). CBF was calculated using a modification of the Kety-Schmidt method measuring the rate of uptake of nitrous oxide $\left(\mathrm{N}_{2} \mathrm{O}\right)$ by the brain as described previously $(4,17-19)$. Briefly, following baseline blood sampling to determine background concentration, $\mathrm{N}_{2} \mathrm{O}(5 \%)$ was administered to the parient, and blood was withdrawn simultaneously from the arterial and jugular bulb catheters into 20-mL syringes using a Harvard parallel withdrawal pump (Harvard Apparatus, Inc., Natick, MA) at a rate of $0.824 \mathrm{~mL} / \mathrm{min}$. After 20 minutes, the pump was turned off and $1-\mathrm{mL}$ samples were taken from the arterial and jugular bulb catheters. Five $1-\mathrm{mL}$ aliquots were taken from each syringe and levels of $\mathrm{N}_{2} \mathrm{O}$ were measured in the gaseous phase using an infrared $\mathrm{N}_{2} \mathrm{O}$ analyser ( $A D C 7000$ gas analyser calibrated for $\mathrm{N}_{2} \mathrm{O}$, range $0-225$ parts per million). $\mathrm{CBF}$ was calculated from the formulas:

$$
\mathrm{CBF}=\left[\mathrm{S} /\left(\mathrm{V}_{\mathrm{E}} / 100\right)\right] /\left[\left(\mathrm{A}_{\mathrm{I}}-\mathrm{V}_{\mathrm{I}}\right] / \mathrm{t}\right] \mathrm{ml} / 100 \mathrm{~g} / \mathrm{min}
$$

where $V_{E}=$ end venous sample; $A_{I}=$ integrated arterial sample; $V_{I}=$ integrated venous sample; $t=$ time (minutes); and $\mathrm{S}=$ blood/brain partition coefficient (for $\mathrm{N}_{2} \mathrm{O}=1.0$ ). CBF measurements were only accepted if the sample at time zero was devoid of $\mathrm{N}_{2} \mathrm{O}$, and if the end venous and arterial values were within $10 \%$ of each other.

Measurement of cerebral blood flow autoregulation and carbondioxide reactivity. CBF was only measured for the autoregulation studies with the $\mathrm{PaCO}_{2}$ maintained between $4.0-4.5 \mathrm{kPa}$ and ensuring that the arterial hydrogen ion concentrations were not different between the measurements. In 6 patients, CBF autoregulation was evaluated before and 4 hours after cooling, by measuring the $\mathrm{CBF}$ prior to and after elevating the resting MAP by $20-30 \mathrm{mmHg}$ by infusion of noradrenaline. An elevation in $\mathrm{CBF}$ by $10 \%$ or more with the change in CPP and MAP was taken as the evidence for lost autoregulation (5).

In 5 subjects, the reactivity of the cerebral vasculature to altered tensions of arterial $\mathrm{CO}_{2}$ was assessed both before and after cooling. $\mathrm{CBF}$ was measured with the $\mathrm{PaCO}_{2}$ set between 4.0 and $4.5 \mathrm{kPa}$ and then after it was increased to between 5.5 and $6.0 \mathrm{kPa}$, both confirmed by arterial blood gas analysis. A minimum of 30 minutes was allowed between the CBF measurements to allow for the clearance of $\mathrm{N}_{2} \mathrm{O}$ and the establishment of the new $\mathrm{PaCO}_{2}$. Carbon dioxide reactivity was assessed only in those patients who maintained haemodynamic stability for the required period.

Analysis. Statistical analysis was performed using the Statistical Package for the Social Sciences, version 9.0 for Windows (SPSS, Chicago, Ill., USA). All the data were expressed as median and range. Wilcoxon signed-ranks test was used to compare pre and post cooling (time $=4$ hours) observations. Spearman rank correlation was used to test the relationship between variables. 


\section{RESULTS}

All the studies were completed successfully and no complications were observed related with either the monitoring or with the measurement of $\mathrm{CBF}$ autoregulation.

Median temperature prior to cooling was $36.4 \infty \mathrm{C}$ (range 35.5-37.3) and this was successfully reduced to $33.1 \infty \mathrm{C}$ (range $31.8-33.5$ ) within 1 hour of starting cooling $(p<0.01)$. Significant reductions were observed in the ICP [median 46 (range 27-54) $\mathrm{mmHg}$ to 19 (range 15-22) $\mathrm{mmHg}, \mathrm{p}<0.01$ ]; heart rate [median 119 (range 88-128) to 86 (range 64-106), $\mathrm{p}<0.01$ ]; cardiac index [median 10.8 (range 8.5-12.1) to 5.6 (range 4.9-6.2) L/min $/ \mathrm{m}^{2}, \mathrm{p}<0.01$ ] and CBF [median 111 (range 69-134) to 56 (range $38-67) \mathrm{ml} / 100 \mathrm{~g} / \mathrm{min}, \mathrm{p}<0.05]$. MAP was unchanged but the noradrenaline requirement was significantly reduced [median 0.9 (range 0.6-1.4) to 0.4 (range 0.1-0.6) $\mathrm{mg} / \mathrm{Kg} / \mathrm{min}, \mathrm{p}<0.05$ ]. Significant increases were observed in the CPP [median 48 (range 35-61) to 66 (range 53-74) $\mathrm{mmHg}, \mathrm{p}<0.05$ ] systemic vascular resistance [median 765 (range 538-893) to 1246 (range 1032-1431) dyn.sec.cm5 $5 / \mathrm{m}^{2}, \mathrm{p}<0.05$ ] and cerebral vascular resistance [median 0.3 (range 0.2-1.6) Wood units to 1.4 (range 0.7 2.2) Wood units, $\mathrm{p}<0.05]$. There was no significant change in the hydrogen ion concentration [median $38(32-56) \mathrm{nmol} / \mathrm{L}$ to $44(31-52) \mathrm{nmol} / \mathrm{L}, \mathrm{p}=\mathrm{ns}$ ], $\mathrm{PaO}_{2}$. [median 13.4 (9.9-16.4) $\mathrm{kPa}$ to $14.1(10.3-15.2) \mathrm{kPa}, \mathrm{p}=\mathrm{ns})$ or in $\mathrm{PaCO}_{2}$ with cooling [3.9 (range $3.8-4.1$ ) $\mathrm{kPa}$ to 4 (range $3.9-4.2$ ) $\mathrm{kPa}, \mathrm{p}=\mathrm{ns}$ ].

All six patients showed evidence of defective $\mathrm{CBF}$ autoregulation prior to institution of hypothermia evidenced by an increase in CBF following increased MAP (Figure 1). Following cooling, $\mathrm{CBF}$ autoregulation was restored in all 6 patients (Figure 2). The percentage change in $\mathrm{CBF}$ with the increase in MAP was significantly less following cooling [pre cooling: median 21.8 (range 10.8-60.8)\%; post cooling: median 5 (range $-7.0-8.0) \%, \mathrm{p}<0.04]$. In addition, the increase in CBF induced by the increased MAP was associated with an increase in ICP prior to cooling [median 32 (range 27-54) to 40 (range 34-59) $\mathrm{mmHg}, \mathrm{p}<0.04$ ] (Figure 3). No significant changes in ICP were observed during increased MAP following cooling [median 18 (range 15-22) to 20 (range 15-22) $\mathrm{mmHg}, \mathrm{p}=0.7]$.

The amount of noradrenaline required to produce this increase in blood pressure was significantly lower in the cooled patients [pre cooling: median 0.6 (range 0.4-1.8) $\mathrm{mg} / \mathrm{Kg} / \mathrm{min}$; post cooling median 0.2 (range $0.1-0.5$ ) $\mathrm{mg} / \mathrm{Kg} / \mathrm{min} ; \mathrm{p}<0.05]$. No significant correlation was detected between the change in the MAP with the change in ICP either before or after cooling. Similarly, there was no significant correlation between the change in systemic vascular resistance and the change in cerebral vascular resistance.

There was no significant change in $\mathrm{CBF}$ with the increase in $\mathrm{pCO}_{2}$ in all 5 patients prior to cooling but significant increase in $\mathrm{CBF}$ was noted after cooling [pre cooling: 

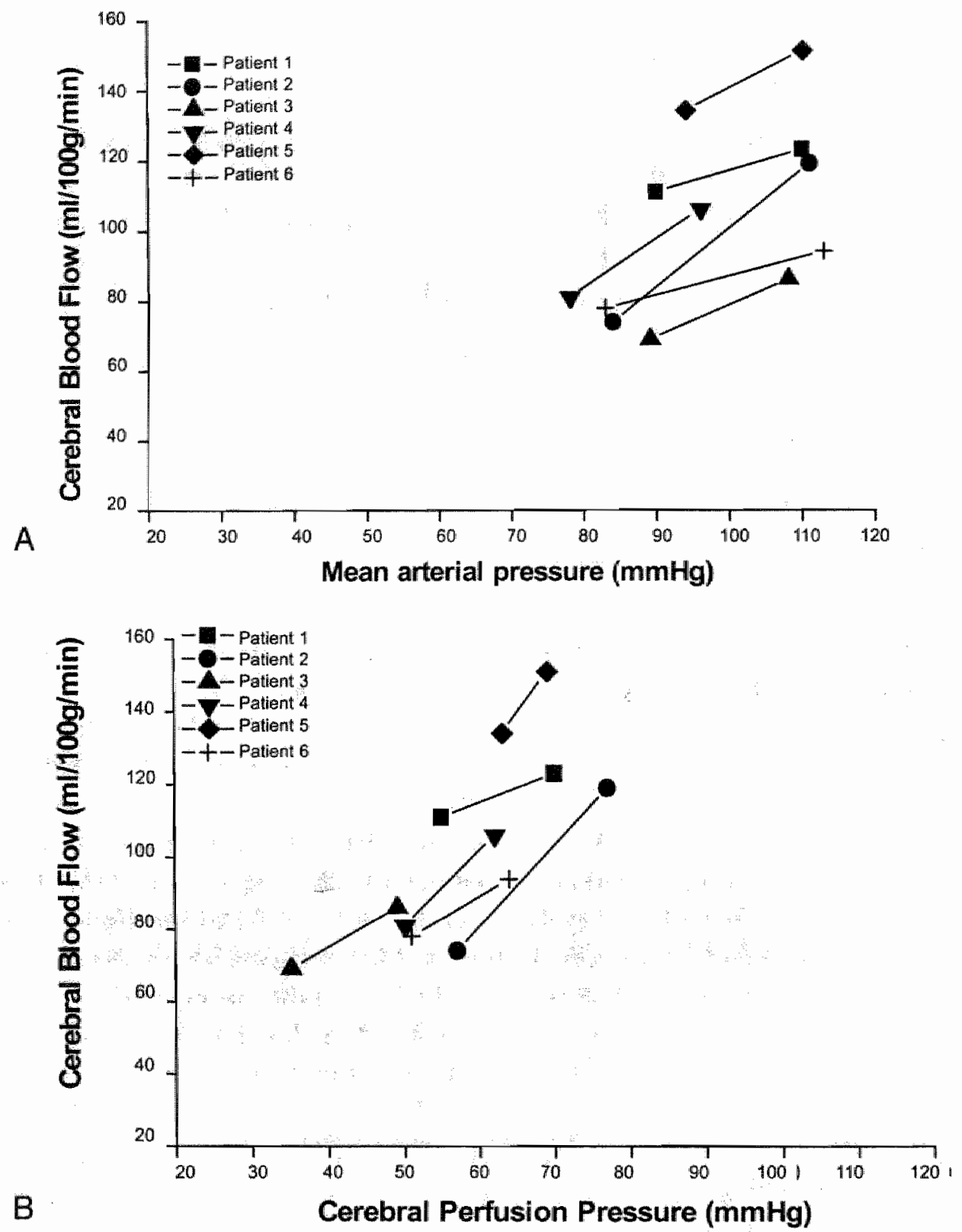

FIGURE 1: Relationship between cerebral blood flow and (a) mean arterial pressure and (b) cerebral perfusion pressure in patients with acute liver failure before cooling. The figures show that cerebral blood flow autoregulation is lost as evidenced by increase in blood flow with the increase in mean arterial pressure and cerebral perfusion pressure. Median temperature prior to cooling was $36.4^{\circ} \mathrm{C}$ (range $35.5-37.3$ ) and $33.1^{\circ} \mathrm{C}$ (range $31.8-33.5$ ) after cooling. 


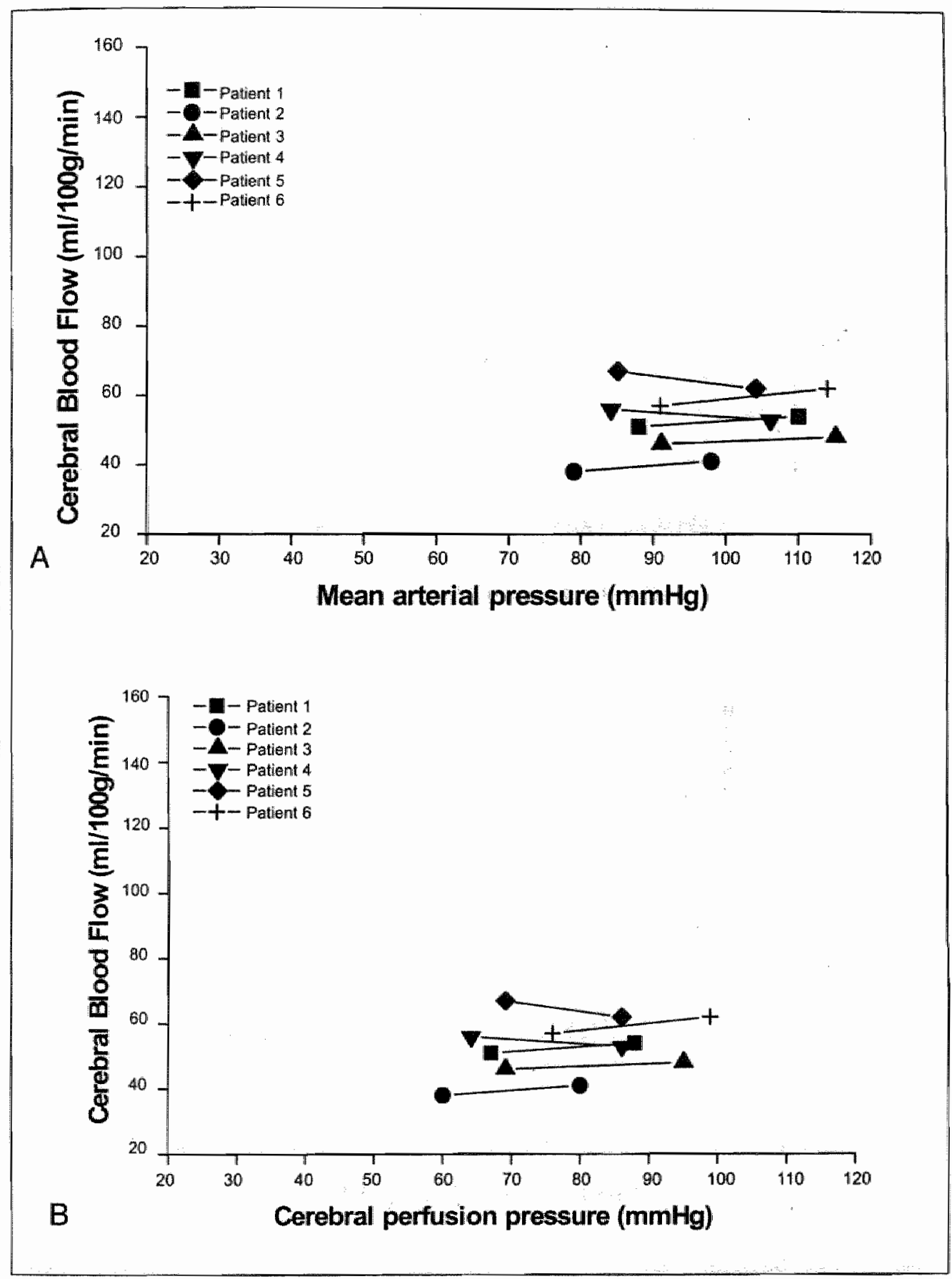

FIGURE 2: Relationship between cerebral blood flow and (a) mean arterial pressure and (b) cerebral perfusion pressure in patients with acute liver failure four hours after cooling $\left(32^{\circ} \mathrm{C}\right)$. The figures show that cerebral blood flow autoregulation is restored as evidenced by no significant changes in cerebral blood flow with increase in mean arterial pressure cerebral perfusion pressure. Median temperature prior to cooling was $36.4^{\circ} \mathrm{C}$ (range $35.5-37.3$ ) and $33.1^{\circ} \mathrm{C}$ (range 31.8-33.5) after cooling. 

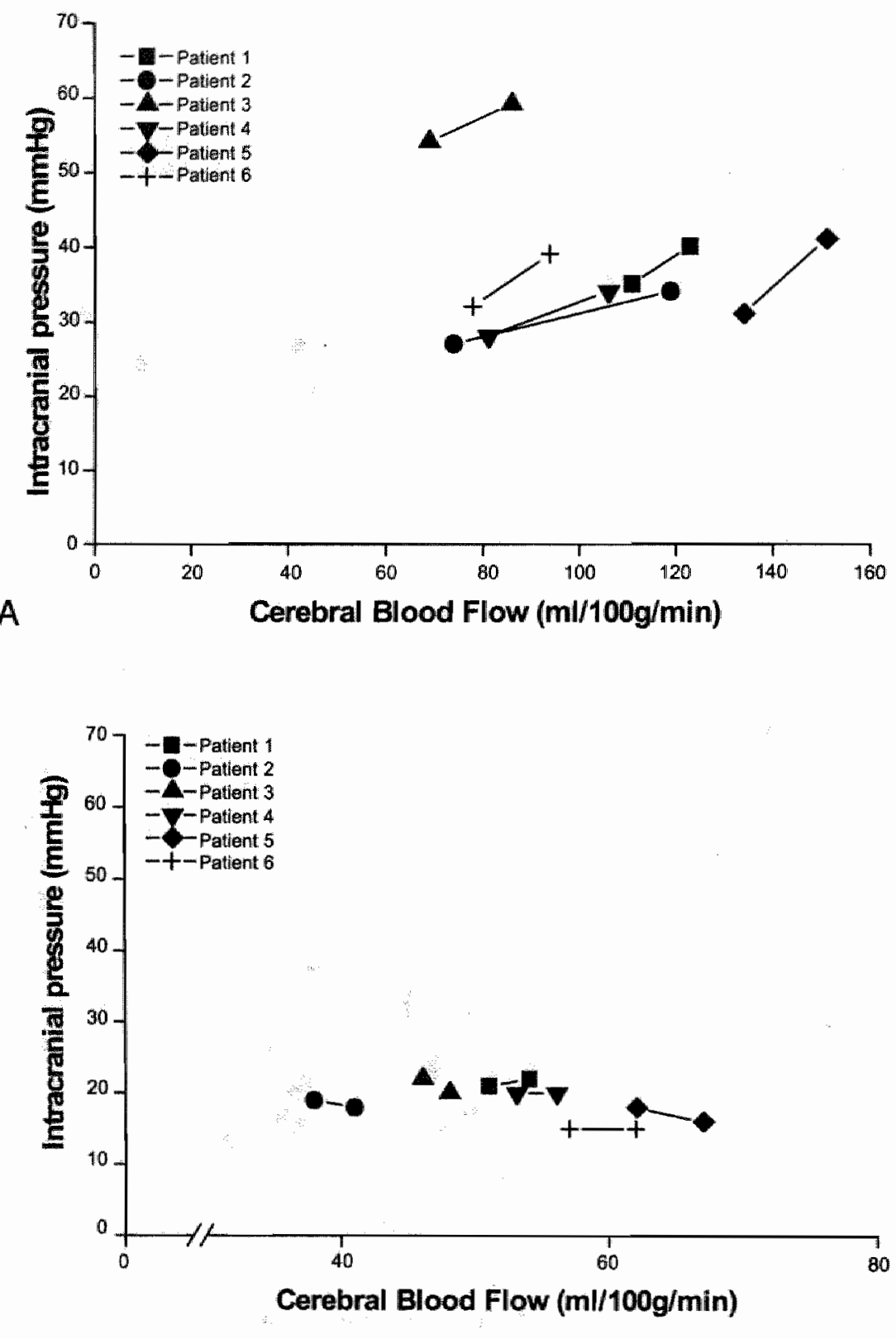

FIGURE 3: Changes in intracranial pressure with the change in cerebral blood flow in patients with acute liver failure that is produced by infusion of noradrenaline (a) before cooling and (b) after cooling. Median temperature prior to cooling was $36.4^{\circ} \mathrm{C}$ (range $35.5-37.3$ ) and $33.1^{\circ} \mathrm{C}$ (range 31.8-33.5) after cooling. 
median CBF, 69 (range 74-78) $\mathrm{ml} / 100 \mathrm{~g} / \mathrm{min}$ at a median $\mathrm{PaCO}_{2}$ of 3.9 (range 3.84.1), and 70 (range 69-80) $\mathrm{ml} / 100 \mathrm{~g} / \mathrm{min}$ at median $\mathrm{PaCO}_{2}$ of $6.3 \mathrm{kPa}$ (range 5.9-6.4), $\mathrm{p}=0.4$; post cooling: median $\mathrm{CBF}, 46$ (range $38-57$ ) $\mathrm{ml} / 100 \mathrm{~g} / \mathrm{min}$ at a median $\mathrm{PaCO}_{2}$ of $4 \mathrm{kPa}$ (range $3.9-4.2$ ), and $56 \mathrm{kPa}$ (range $57-63$ ) $\mathrm{ml} / 100 \mathrm{~g} / \mathrm{min}$ at a median $\mathrm{PaCO}_{2}$ of $6.1 \mathrm{kPa}$ (range 5.8-6.2), $\mathrm{p}<0.05$ ]. Prior to cooling, the increase in carbon dioxide concentration was associated with a small increase in the ICP [median ICP, $46 \mathrm{mmHg}$ (range 31-54) at a median $\mathrm{PaCO}_{2}$ of $3.9 \mathrm{kPa}$ (range 3.8-4.1) to, median ICP, 49 $\mathrm{mmHg}$ (range 33-55) at a median $\mathrm{PaCO}_{2}$ of $6.3 \mathrm{kPa}$ (range 5.9-6.4), $\mathrm{p}=0.3$ ]. After cooling, the ICP did not change significantly with the increase in $\mathrm{PaCO}_{2}$ (median ICP, $19 \mathrm{mmHg}$ (range 17-22) at a median $\mathrm{PaCO}_{2} 4 \mathrm{kPa}$ (range 3.9-4.2) to, median ICP, 21 $\mathrm{mmHg}$ (range 14-22) at a median $\mathrm{PaCO}_{2}$ of $6.1 \mathrm{kPa}$ (range 5.8-6.2), $\mathrm{p}=0.3$ ]. The percentage change in $\mathrm{CBF}$ with the increase in $\mathrm{PaCO}_{2}$ was significantly greater following cooling [pre cooling: median 2.5 (range -0.2-7.8)\%; post cooling: 19 (range 10.5$23.6) \%, \mathrm{p}<0.04]$. Individual patient data are presented in Table 1. 


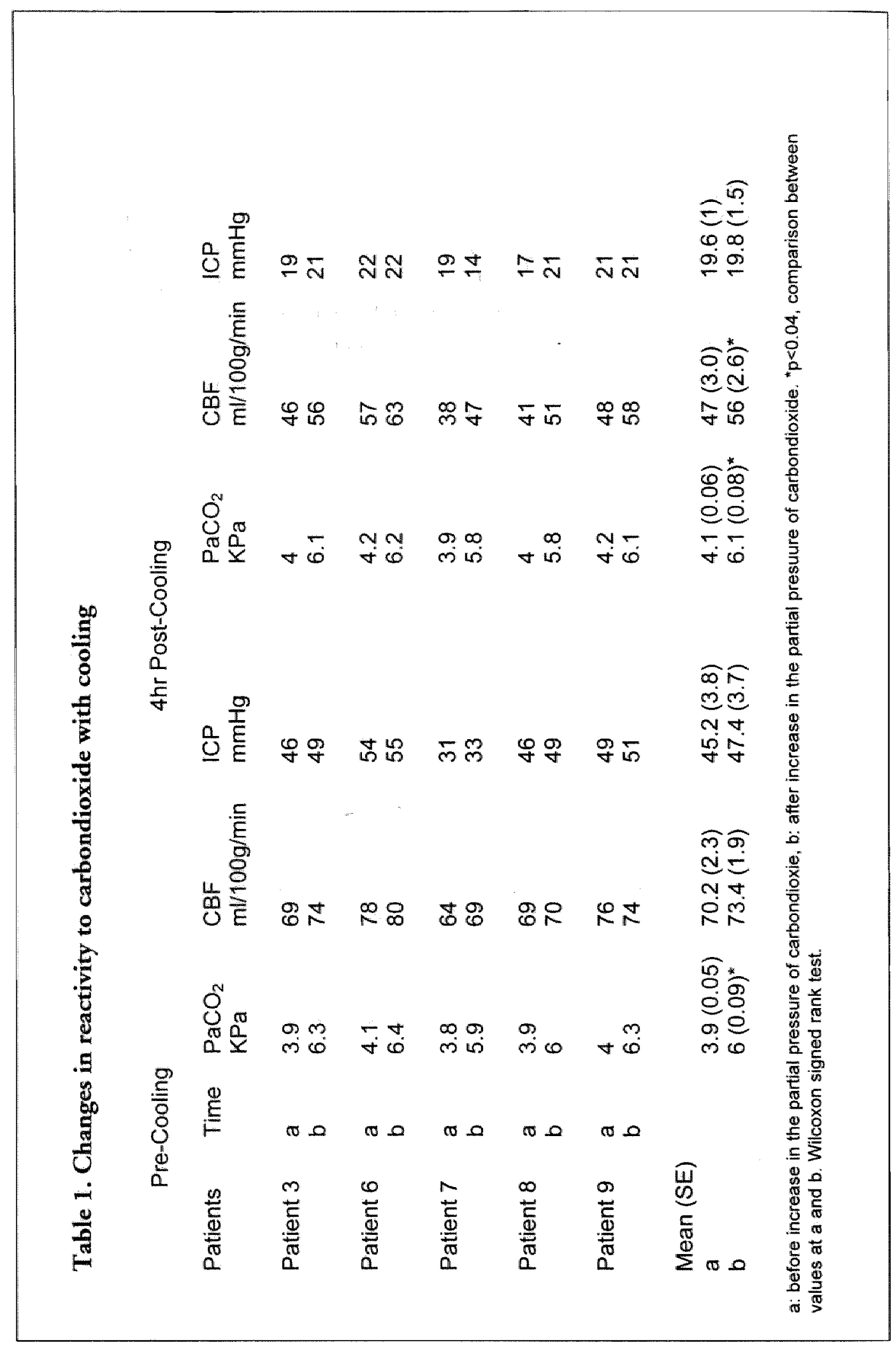




\section{DISCUSSION}

The results of this study confirms our previous observation that hypothermia successfully reduces intracranial hypertension and has beneficial effects upon the cardiovascular factors such as heart rate, cardiac output and noradrenaline requirements. Although we have previously shown that hypothermia reduces arterial ammonia concentration and its uptake by the brain, the mechanisms by which it reduces ICP are not clear (4). This study clearly shows that hypothermia successfully reduced $\mathrm{CBF}$, restored $\mathrm{CBF}$ autoregulation and reactivity to carbon dioxide.

We have used a modification of the Kety Schmidt method, employing an integrated blood sampling technique $(4,17-19)$. This technique measures global CBF $(\mathrm{mL} / 1.00 \mathrm{~g}$ perfused brain/min) without providing any information on regional perfusion. Although a previous study showed reduced CBF in ALF (12) others have shown increased $\mathrm{CBF}$ as was observed in this study $(4,9-11)$. In portacaval shunted rats, ammonia infusion produces an increase in $\mathrm{CBF}$ and which precedes cerebral oedema (13). In agreement with our observation, hypothermia in this animal model prevents cerebral hyperaemia and reduces brain oedema (13).

Most studies that have demonstrated dysregulation of CBF in patients with ALF have used transcranial Doppler to determine the mean velocity in the middle cerebral artery $(7,8,16)$. Recently published data suggest that this dysregulation of CBF may extend to involve the area of the brain subserved by the anterior cerebral artery (20). Our results are consistent with these previous studies that suggest a loss of CBF autoregulation in patients with ALF. The rapidity of restoration of CBF with cooling is unique to ALF because re-establishment of CBF autoregulation in other conditions such as head trauma and inflammatory conditions of the brain and meninges may talke days $(21,22)$. Similar rapid restitoration of CBF autoregulation has been shown with liver transplantation (7). Even before cooling, all the 6 patients thad an MAP of $>60 \mathrm{mmHg}$ which is above the normal lower limit of CBF autoregulation. In this study, $\mathrm{CPP}$ was also measured which was $>50 \mathrm{mmHg}$ in 5 of the 6 patients indicating that they were within the range of $\mathrm{CBF}$ autoregulation confirming that $\mathrm{CBF}$ autoregulation was truly lost prior to cooling and restored subsequently $(5,6)$.

Prior to cooling, the patients showed a lack of $\mathrm{CBF}$ reactivity to $\mathrm{CO}_{2}$ which was restored after cooling. It is possible that the lack of response to $\mathrm{CO}_{2}$ prior to cooling may be the result of the limited further capacity for cerebral vasodilatation at the upper end of the response curve and cooling induces cerebral vasoconstriction thereby restoring the response curve $(5,6)$.

The correction of both $\mathrm{CBF}$ autoregulation and reactivity to $\mathrm{CO}_{2}$ with hypothermia suggests that a common mechanism 1 rust underlie the dysregulated CBF that is 
characteristic of ALF. The mechanisms of derangements in CBF autoregulation is not clear but is possibly mediated by some unknown toxic factor that is produced by the necrotic liver, because hepatectomy in patients with ALF is followed by rapid improvement both in the intracranial pressure and also in $\mathrm{CBF}$ autoregulation $(23,24)$. It is possible that moderate hypothermia may restore $C B F$ autoregulation by reducing the production of this presumed toxic substance through reduction in the metabolic rate as evidenced by its effects upon the resting energy expenditure (4). Studies in portacaval shunted rats undergoing ammonia infusion have shown that administration of a glutamine synthase inhibitor reduces cerebral hyperaemia and increase in brain water (15). Experiments in healthy rats undergoing ammonia infusion showed that the responsiveness to $\mathrm{CO}_{2}$ was restored if glutamine synthase was inhibited prior to ammonia infusion $(25,26)$. These experiments suggest that dysregulation of $\mathrm{CBF}$ and its responsiveness to $\mathrm{CO}_{2}$ follow the accumulation of glutamine. We have previously shown that hypothermia reduces both the arterial concentrations of ammonia and also its uptake by the brain suggesting that it may modulate the improvement in autoregulation and reactivity to carbondioxide through reducing glutamine synthesis (4). Alternatively, or in addition, hypothermia may produce the beneficial effects upon cerebral circulation through a reduction in the excitatory neurotransmitter glutamate or nitric oxide production $(14,15,27,28)$. Our study does not allow any firm conclusion on this point.

Although previous studies in patients with ALF have demonstrated that CBF changes in parallel with arterial pressure $(7,16)$ the direct effect of increased CBF upon ICP has been unclear because concomitant measurement of CBF, ICP and MAP in the same patients has been lacking. Our study demonstrates that the increase in MAP increases $C B F$ which results in an increase in ICP and suggests a critical pathogenic role for the increased CBF in the causation of intracranial hypertension in ALF. This lack of autoregulation and the increase in ICP produced by an increase in MAP and CPP suggests the need to exercise caution when treating ALF patients with increasing doses of vasopressors without monitoring the ICP. This relationship supports the hypothesis that increased ICP in patients with ALF is a two stage process (8). The first stage refers to brain swelling as a result of increased glutamine synthesis due to the effects of ammonia. This produces alterations in the compliance of the brain and acute changes in ICP that is characteristic of ALF may be the result of increases in CBF (8).

In this study we also show that patients require significantly less inotropic support following cooling. The pathogenesis of circulatory disturbances in ALF is not clear but is manifested by a markedly dilated peripheral and splanchnic circulation $(29,30)$. In addition to restoring CBF to normal values and correcting its autoregulation, cooling reduced the cardiac index and increased systemic vascular resistance. It is also interesting to note that the hypothermic patients required significantly less amounts of noradrenaline to achieve the same increase in MAP suggesting that hypothermia may have 
profound effects upon correcting the vascular hyporesponsiveness that is characteristic of patients with liver failure $(31,32)$. Although cooling increased both the systemic vascular resistance and cerebral vascular resistance, no direct correlation between these have been found suggesting that the mechanisms of cerebral vasodilatation are different to the mechanism of systemic vasodilatation. Alternatively, the lack of demonstrable relationship may be due to the relatively small sample size.

It is important to state that although there were no complications related directly with the invasive monitoring that was used in the patients in this study, questions about the level of monitoring that patients with ALF should undergo remains a point of discussion. Intensive studies of the sort described in this paper are difficult to perform without an adequate team of people and three experienced medical doctors and 2 nurses were directly involved in performing each study.

In conclusion, the results of our study suggest that the improvement in ICP observed with hypothermia may be due to its effects upon $\mathrm{CBF}$ autoregulation and provides a tool to explore the mechanisms associated with the deranged autoregulation that is important in the pathogenesis of intracranial hypertension in ALF. 


\section{REFERENCES}

1. O'Grady JG, Alexander GJM, Hayllar KM, Williams R. Early indicators of prognosis in fulminant hepatic failure Gastroenterology 1989;97:439-445.

2. Hoofnagle JH, Carithers RL, Chapiro C Ascher NL. Fulminant hepatic failure: Summary of a Workshop. Hepatology 1995:21:240-252.

3. Ascher NL, Lake JR, Emond JC, Roberts JP. Liver transplantation for fulminant hepatic fallure Arch Surg 1993; 128:677-682.

4. Jalan R, Olde Damink SWM, Deutz NEP, Lee A, Hayes PC. Treatment of uncontrolled intracranial hypertension in acute lrver failure with moderate hypothermia. Lancet 1999:354:1164-8.

5. Aaslid R, Lindegaard KF, Sorteberg WT, Nornes $\mathrm{H}$. Cerebral autoregulation dynamics in humans. Stroke 1989;20:45-52.

6. Iassen NA. Normal average vallue of cerebral blood flow in younger adults is $50 \mathrm{~mL} / 100$ g/min. J Cereb Blood Flow Metab 1985; 5: 347-349.

7. Strauss $G$, Adel Hansen B, Kirkegaard P, Rasmussen A, Hjortrup A, Larsen FS Liver function, cerebral blood flow autoregulation, and hepatic encephalopathy in fulminant hepatic failure. Hepatology 1997;25:837-9.

8. Blei AT, Larsen FS Pathophysiology of cerebral edema in fulminant hepatic failure. I Hepatology 1999;31:771-6.

9. Aggarwal S, Kramer D, Yonas H, Obrist W, Kang Y, Martin M, Policare R. Cerebral hemodynamic and netabolic changes in fulminant hepatic failure: a retrospective study. Hepatology 1994;19:80-7.

10. Dempsey, RJ, Kindt GW. Experimental acute hepatic encephalopathy: relationship of pathological cerebral vasodilatation to increased intracranial pressure. Neurosurgery $1982 ; 10: 737-41$.

11. Larsen FS, Adel Hansen B, Pott F, Ellersen E, Secher NH, Paulson OB, Knudsen GM. Dissociated cerebral wasoparalysis in acute liver failure. A hypothesis of gradual cerebral hyperaemia. J Hepatol 1996;25:145-51.

12. Wendon JA, Harrison PM, Keays R, Willians R. Cerebral blood flow and metabolism in fulminant liver fallure. Hepatology 1994:19:1407-13.

13. Cordoba J, Crespin J, Gottstein ], Blei AT Mild hypothermia modifies ammonia-induced brain edema in tats after portacaval anastomosis. Gastroenterology 1999;116:686-93.

14. Rao VL, Butterworth RF. Neuronal nitric oxide synthase and Hepatic Encephalopathy. Metab Brain Dis 1998;13:175-89.

15. Master S, Gottstein J, Blei AT Cerebral blood flow and the development of ammoniainduced brain edema in rats after portacaval anastomosis. Hepatology 1999;30:876-80.

16. Strauss G1, Hogh P, Moller K. Knudsen GM, Hansen BA, Larsen FS Regional cerebral blood flow during mechanical hyperventilation in patients with fulminant hepatic failure. Hepatology 1999;30:1368-73.

17. Kety SS, Schmidt CF. The nitrous oxide method for the quantitative determination of cerebral blood flow in man: Theorys procedure and normal values. I Clin Invest $1947 ; 27: 476-483$. 
18. Scheinberg P, Stead EA. The cerebral blood flow in male subjects as measured by the nitrous oxide technique, normal values for blood flow, oxygen utilization, glucose utilization and peripheral resistance with observations on the effect of tilting and anxiety. I Appl Plyysiol 1949; 28: 1163-1171.

19. Philips BJ, Armstrong IR, Pollock A, Lee A Cerebral blood flow and metabolism in patients with chronic liver disease undergoing orthotopic liver transplantation. Hepatology $1998 ; 27: 369-76$.

20. Larsen FS, Strauss G, Moller K, Hansen BA. Regional cerebral blood flow autoregulation in patients with fulminant hepatic failure. Liver Transplantation 2000;6:795-800.

21. Paulson $\mathrm{OB}$, Strandgaard S, Edvinsson L Cerebral autoregulation. Cerebrovasc Brain Metab Rev 1990;2:161-92.

22. Tureen JH, Dworkin RJ, Kennedy SL, Sachdeva M, Sande MA Loss of cerebrovascular autoregulation in experimental meningitis in rabbits. J Clin Invest 1990;85:577-81.

23. Ejlersen E, Larsen FS, Pott F, Gyrtrup H], Kirkegaard P, Secher NH Hepatectomy corrects cerebral hyperperfusion in fulminant hepatic failure. Transplant Proc 1994;26:1794-5.

24. Ringe $B$, Lubbe $N$, Kuse $E_{y}$ Frei U, Pichlmayr R Total hepatectomy and liver transplantation as two-stage procedure. Ann Surg 1993;218:3-9.

25. Takahashi $\mathrm{H}$, Koehller $\mathbb{R C}$, Brusilow SW, Traystman RJ. Inhibition of brain glutamine accumulation prevents cerebral edema in hyperammonemic rats. Am I Physiol 1991;261:H825-9.

26. Takahashi H, Koehller RC, Hirata T, Brusilow SW, Traystman RJ. Restoration of cerebrovascular $\mathrm{CO} 2$ responsivity by glutamine synthesis inhibition in hyperammonemic rats. Circ Res 1992;71:1220-30.

27. Rose C, Michalak A, Pannunzio M, Chatauret N, Rambaldi A, Butterworth RF Mild hypothermia delays the onset of coma and prevents brain edema and extracellular brain glutamate accumulation in rats with acute liver failure. Heparology 2000;31:872-7.

28. Fujisawa H, Koizumi $H$, Ito $\mathbb{H}$, Yamashita K, Maekawa T Effects of mild hypothermia on the cortical release of excitatory amino acids and nitric oxide synthesis following hypoxia. J Neurotrauma 1999;16:1083-93.

29. Trewsby PN, Williams R. Pathophysiology of hypotension in patients with fulminant hepatic failure. Gut 1977;18:1021-1026.

30. Clemmesen JO, Gerbes A, Hansen BA, Larsen FS, Skak C, Tygstrup N, Ott P. Hepatic blood flow and metabolism in patients with fulminant hepatic failure before and after high volume plasmapheresis. Hepatology 1999;29:347-355.

31. MacGilchrist AJ, Sumner D, Reid JL Impaired pressor reactivity in cirrhosis: evidence for a peripheral vascular defect. Hepatology 1991;13:689-94.

32. Helmy $A$, Jalan $R$, Newby DE, Hayes PC, Webb DJ Role of angiotensin II in regulation of basal and sympathetically stimulated vascular tone in early and advanced cirrhosis. Gastroenterology 2000;118:565-72. 


\section{CHAPTER 12}

MODERATE HYPOTHERMIA PREVENTS CEREBRAL HYTEREMIA AND INCREASE IN INTRACRANIAL PRESSURE IN PATIENTS UNDERGOING LIVER TRANSPLANTATION FOR ACUTE LIVER FAILURE

1,3Rajiv Jalan, ${ }^{2}$ Steven WM Olde Damink, ${ }^{2}$ Nicolaas EP Deutz, 1 Nathan A Davies, ${ }^{3}$ Oliver J Garden, ${ }^{3}$ Krishna K Madhavan, ${ }^{3}$ Peter $\mathrm{C}$ Hayes, ${ }^{3}$ Alistair Lee.

${ }^{1}$ Liver Failure Group, Institute of Hepatology, Royal Free and University College London Medical School, London, 2Department of Surgery, Academic Hospital, Maastricht University, Maastricht, The Netherlands, ${ }^{3}$ Scottish Liver Transplantation Unit and Intensive Care Unit, Royal Infirmary of Edinburgh.

Published in Transplantation 2003; 75: 2034-2039 


\section{INTRODUCTION}

A $90 \%$ mortality rate can be expected for patients with acute liver failure (ALF) wh fulfil criteria for poor prognosis (1), but survival rates of up to $80 \%$ can be achieve with orthotopic liver transplantation (OLT) (2). However, OLT is associated with well described systemic and pulmonary circulatory changes. Reperfusion of the donor live is usually accompanied by a decrease in mean arterial pressure (MAP), an increase i pulmonary arterial pressure, and an increase in the cardiac index. Occasionally, thes haemodynamic alterations are profound and are referred to as the Post-Reperfusio Syndrome (3). In addition to the systemic haemodynamic effects, patients with AL] undergoing OLT show significant increases in intracranial pressure (ICP) and a reduc tion in cerebral perfusion pressure (CPP), both during the dissection (pre-clamp) an reperfusion phases of the operation $(4,5)$. This period of intra-operative intracrania hypertension may, in some patients, have catastrophic consequences leading to brail death. The mechanism of increased ICP during the dissection and reperfusion phase of OLT is not clear, although increases in cerebral blood flow (CBF) and loss of it autoregulation have been suggested as possible mechanisms (6-8).

We have recently shown that moderate hypothermia $(32 \infty \mathrm{C})$ can be used as: treatment for uncontrolled increase in ICP in patients with ALF. It is easy to institut and safe. It reduces ICP; cardiac index; and noradrenaline requirements; with a con comitant increase in CPP (9). In addition, moderate hypothermia restored CB1 autoregulation (10). The current study was designed to test the hypothesis that, it patients with ALF, moderate hypothermia would prevent the increases in ICP that ar observed during OLT. We measured changes in ICP and global CBF, using a methor based on the Kety-Schmidt technique (11) to determine cerebral haemodynamic changes during the different phases of OLT. Three groups of parients were studied patients who were treated with moderate hypothermia as therapy for uncontrollec increased ICP before OLT, patients who had ALF and, either did not require treatmen1 for intracranial hypertension, or had controlled ICP prior to OLT and were maintained normothermic. 


\section{METHODS}

Ethical permission for this study was obtained from the Lothian ethics committee. Written informed assent was obtained from the next of kin.

Patients. We have studied 16 consecutive patients with ALF who underwent OLT (median age 29 (range 28-54), 12 females, 4 males); due to paracetamol (acetaminophen) overdose (13), other drug (1) and non-A non-B hepatitis (2). All the patients fulfilled the King's College criteria for poor prognosis. Patient details are summarised in Table 1. The patients were divided into 3 groups according to their ICP prior to OLT. Group I $(n=6)$ comprised patients who had an ICP of $<15 \mathrm{mmHg}$ during the course of their illness, did not require any treatment for the increased ICP and underwent OLT with the body temperature maintained at a median of $35.9^{\circ} \mathrm{C}$ (35.5-37.1). Group II $(n=5)$ comprised patients who had episodes of increased ICP that was controlled on treatment with mannitol to $<15 \mathrm{mmHg}$ and underwent OLT with the body temperature maintained at a median of $36.4{ }^{\circ} \mathrm{C}(35.8-37.3)$. Group $I I I \quad(n=5)$ comprised patients who had uncontrolled intracranial hypertension (defined as an ICP of $>25$ $\mathrm{mmHg}$ for at least 1 hour despite 2 treatments with mannitol and removal of $500 \mathrm{ml}$ of fluid with CVVH) prior to OLT for which they received moderate hypothermia, with their mean body temperature set at a median of $33.4^{\circ} \mathrm{C}(31.9-33.8)$.

Monitoring. The electrocardiogram, heart rate, arteriall pressure (left-radial artery), core and peripheral temperatures were monitored continuously. Blood electrolytes, coagulation and gases were monitored according to our established protocol for OLT.

Cerebrovascular haemodynamics: Clinical neurological examination during the OLT involved attention to pupillary size and its reaction to light. ICP was monitored continuously using a subdural fibre-optic system (Camino, Camino Laboratories, San Diego, USA). The changes in ICP that have been reported represent the highest recorded ICP that persisted for $>10 \mathrm{~min}$ in that hour. CPP was measured as the difference of MAP and ICP. CBF was measured as described previously using a modification of the Kety-Schmidt method which is based upon the rate of uptake of nitrous oxide by the brain $(9,10,11)$. Briefly, a second arterial catheter was inserted into the right femoral artery (115.11, Vygon, USA) and a jugular bulb catheter inserted into the left internal jugular vein (4F Opticath, U440, Abbot, USA) through a $5 \mathrm{~F}$ haemostasis introducer (Fast Cath, Daig, USA). Correct positioning of the jugular bulb catheter was confirmed with a lateral head and neck radiograph. A CBF measurement was only determined if the patient was haemodynamically stable at that time. It was defined as a difference of less than $10 \%$ between pre and post $\mathrm{CBF}$ measurement haemodynamic variables, with the $\mathrm{PaCO}_{2}$ fixed at between 4 and $5 \mathrm{KPa}$. These cerebral haemodynamic measurements were made after induction of anaesthesia, during the dissection, anhepatic, and reperfusion phases of OLT and in addition, 2 hours post OLT. 


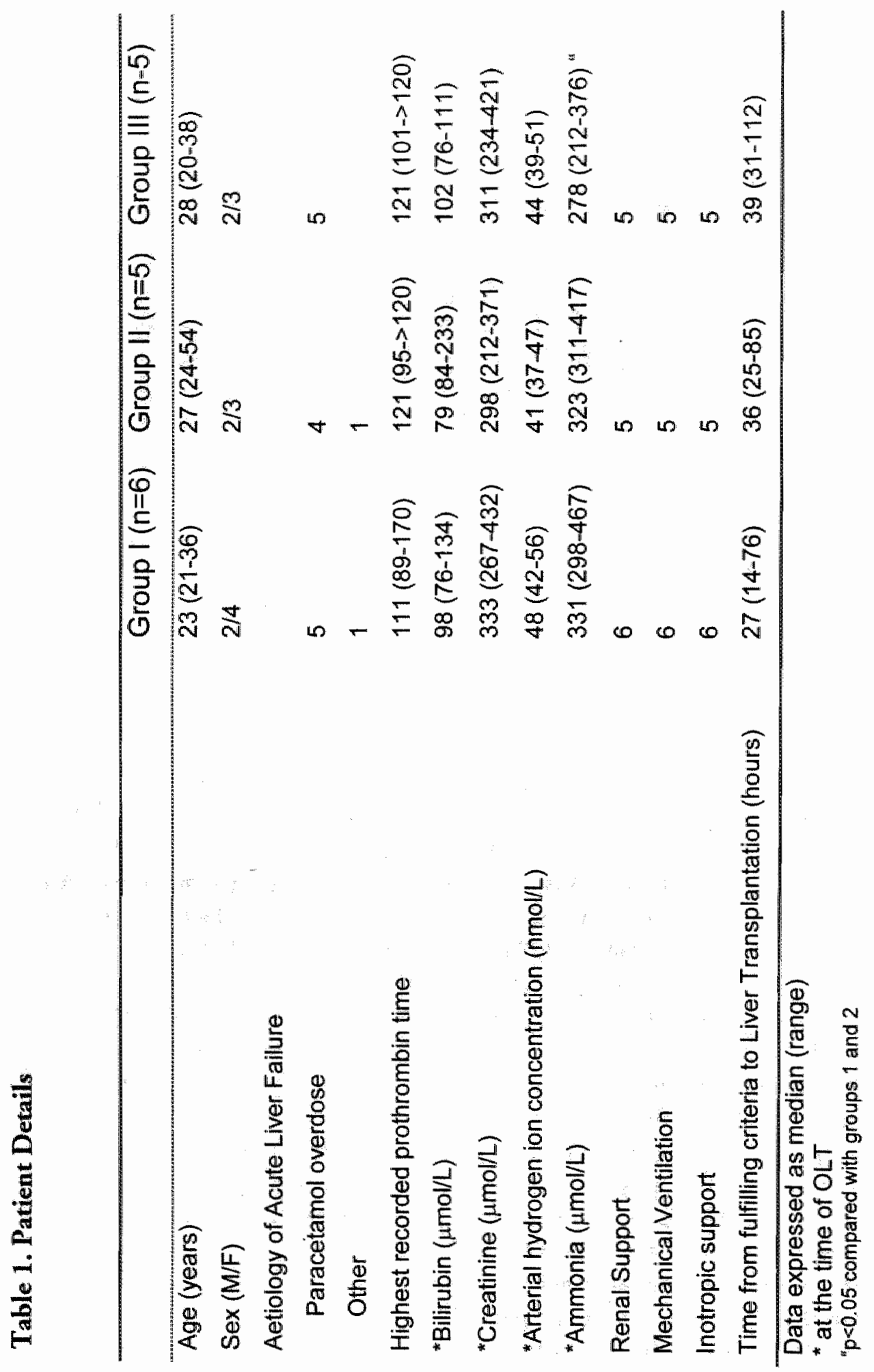


Moderate Hypothermia. All patients in Groups I and II were normothermic prior to surgery. Target body temperature was achieved using cooling blankets (Blanketrol II, Cincinnati Sub-Zero, Cincinnati, Vickers Medical) placed below the patient and on the legs such that blood temperature was maintained at $32-33^{\circ} \mathrm{C}$ in the hypothermic group. Post surgery the patients were gradually re-warmed over a period of 24 hours.

Anaesthesia and Surgery. Anaesthesia was induced with propofol (1-3 mg/kg), and maintained with an oxygen/air/isoflurane mix, with infusions of alfentanil, midazolam, and atracurium. A bag in bottle ventilator was used and gases were administered to the patient through a circle system with a $\mathrm{CO}_{2}$ absorber. Ventilation was adjusted to initially achieve an arterial carbon dioxide tension $\left(\mathrm{PaCO}_{2}\right)$ of $4-5 \mathrm{kPa}$, and was not altered again for the duration of the study. All patients received dopamine ( 3 $\mu \mathrm{g} / \mathrm{kg} / \mathrm{min}$ ), and calcium chloride when required to maintain ionized calcium within the normal range. Blood was transfused to maintain a haemoglobin value between 8 and $10 \mathrm{~g} / \mathrm{dl}$. Intravascular volume was maintained using crystalloids, colloids or red cells as appropriate to maintain the pulmonary capillary wedge pressure between 8 and $14 \mathrm{mmHg}$. Noradrenaline was used if required to keep the CPP above $50 \mathrm{mmHg}$ and/or a MAP of greater than $90 \mathrm{mmHg}$. Blood glucose was maintained between 5-7 $\mathrm{mmol} / \mathrm{l}$ using a continuous infusion of $50 \%$ Dextrose. The goal of treatment was to maintain the ICP below $20 \mathrm{mmHg}$. Patients with raised ICP ( $>20 \mathrm{mmHg}$ for $10 \mathrm{~min}$ ) were initially treated with mannitol $(1 \mathrm{~g} / \mathrm{Kg}$ body weight over $20 \mathrm{~min})$; and subsequently thiopental sodium, if necessary. OLT was performed without veno-venous bypass, with caval preservation using the piggyback technique.

Sampling and measurement of IL-1b. Arterial and venous (jugular bulb) blood was collected in pre-cooled, heparinised syringes, in 6 patients from Groups I and II (3 patients in each group) and from 5 patients in Group III. Samples for cytokine analysis were not collected from all patients, as this technique was not available at the start of the study; all patients were included thereafter. Plasma was separated and stored at $-70 \infty \mathrm{C}$ for analysis at a later date. IL-1b was measured using a commercially available ELISA assay (R\&D systems, Minneapolis). Venous-arterial differences for IL-1b were calculated, a positive value was considered to represent brain production and a negative value uptake of this cytokine.

Analysis. Data are expressed as median and range unless otherwise stated. Changes in variables with time were measured using one-way analysis of variance with Bonferroni post-hoc correction. The percentage changes from baseline were described (pre-transplant values) and differences between the groups were calculated using the MannWhitney test. For the IL-1b data the values for the patients from Groups I and II were compared with those from Group $\mathrm{HI}$ using the Mann-Whitney test. Differences from 0 in values of $\mathrm{IL}-1 \mathrm{~b}$ were tested using the Mann-Whitney test. 


\section{RESULTS}

Patients. One patient in each of the groups died during the same hospital admission from multi-organ failure, possibly related to sepsis. All the patients in Group I, 3 of Group II and 4 patients from Group III had evidence of post-operative infection requiring antibiotics. There was no evidence of fungal infection in any of the patients. None of the patients that were transplanted (successfully) were reported as having developed any neurological defects following OLT and have been followed for a median of 17 months (range 6-37 months). There were no significant difference in the amount of coagulation factors, fluids or red cells transfused during the OLT [Red cell concentrate: Group I, 4 (2-7) units, Group II (4 (2-6) units, Group III, 4 (3-8) units; Platelet concentrate: Group I, 2 (1-3) units, Group II 2 (1-3) units and Group III 3 (23); Fresh Frozen Plasma: Group I, $600(300-1200) \mathrm{ml}$, Group II, $900(300-1200) \mathrm{ml}$, Group III 1200, (600-1500) ml].

Changes in ICP. Changes in ICP prior to, during the dissection, anhepatic, and reperfusion phases and post OLT are shown in Figure 1. At the start of the operation, there was no significant difference in ICP between the 3 groups. In Group I, a significant increase in ICP was observed during the dissection $(\mathrm{p}<0.001)$ and reperfusion phases $(\mathrm{p}<0.003)$ requiring treatment with thiopentone in 4 of the 6 patients. In Group II, there was significant increase in ICP during the dissection $(\mathrm{p}<0.006)$ and reperfusion phases $(p<0.01)$ with thiopentone treatment required in all patients. In Group III there was no significant increase in ICP during the OLT, although, there was a transient peak increase in ICP in one of the patients to values of $71 \mathrm{mmHg}$ during the dissection phase, which resolved spontaneously. The measured ICP, during dissection and reperfusion, was significantly greater in patients from Groups I and II compared with those in Group III (dissection: $p<0.03$ and $p<0.03$ and reperfusion: $p<0.009$ and $p<0.03$ respectively). The rise in $\mathrm{ICP}$ was not significantly different between patients in Group II compared with Group I $(p=0.48)$. There was a clear reduction in ICP in Groups I and II during the anhepatic phase and following OLT. There were no significant differences in the measured ICP between the 3 groups during this anhepatic phase and for 2 hours post OLT.

Changes in CBF. Changes in CBF prior to the OLT, during the dissection, anhepatic, and reperfusion phases and 2 hours post OLT are shown in Figure 2. It is possible that the modified Kety-Schmidt method employed in this study may cause a slight overestimation of the CBF measurements, any source of error would be consistent between the three groups allowing direct comparison. At the start of the operation, there was no significant difference in CBF between the 3 groups. In Group I, a significant increase 


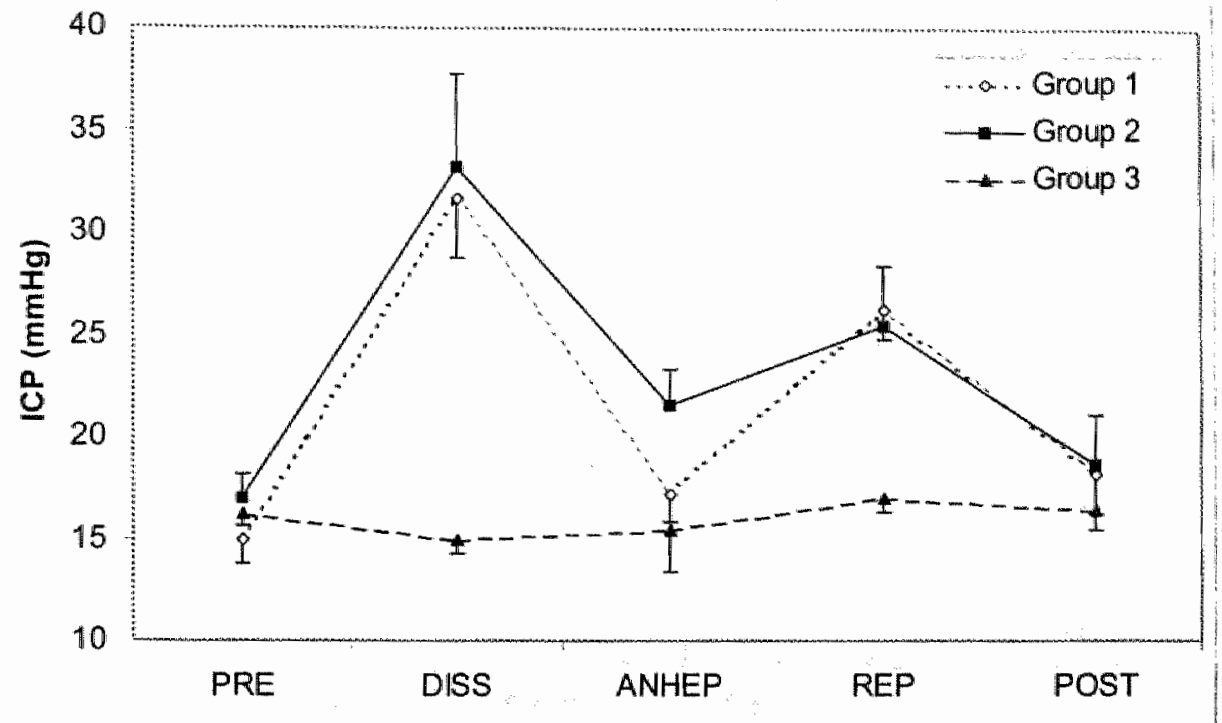

FIGURE 1. Changes in the intracranial pressure during the Liver Transplantation operation in the parients that were: normothermic (Groups I and II (closed squares)) and those that were maintained hypothermic (Group III (closed triangles)).

Group I (open diamond): Intracranial pressure increased significantly during the dissection $(p<0.001)$ and reperfusion phases $(p<0003)$.

Group II (closed square): Intracranial pressure increased significantly during the dissection $(\mathrm{p}<0.006)$ and reperfusion phases $(\mathrm{p}<0.01)$.

Group III (closed triangle): No significant increase in intracranial pressure during Liver Transplantation.

Values shown are mean \pm S.E.

in CBF was observed in all parients during the dissection $(p<0.02)$ and reperfusion phases $(\mathrm{p}<0.02)$. In Group II, there was significant increase in CBF during the dissection $(p<0.001)$ and reperfusion phases $(p<0.003)$. In Group III, no significant change in CBF during OLT was observed. The increase in CBF during dissection and reperfusion was significantly greater in Groups I and II compared with Group III (dissection: $p<0.02$ and $p<0.008$ and reperfusion: $p<0.05$ and $p<0.02$ respectively). The rise in $\mathrm{CBF}$ was not significantly different between Group II compared with Group I $(\mathrm{p}=0.37)$. There was a reduction in CBF in Groups I and II during the anhepatic phase and 2 hours post OLT, such that there was no significant difference in the CBF between the 3 groups during this phase. 


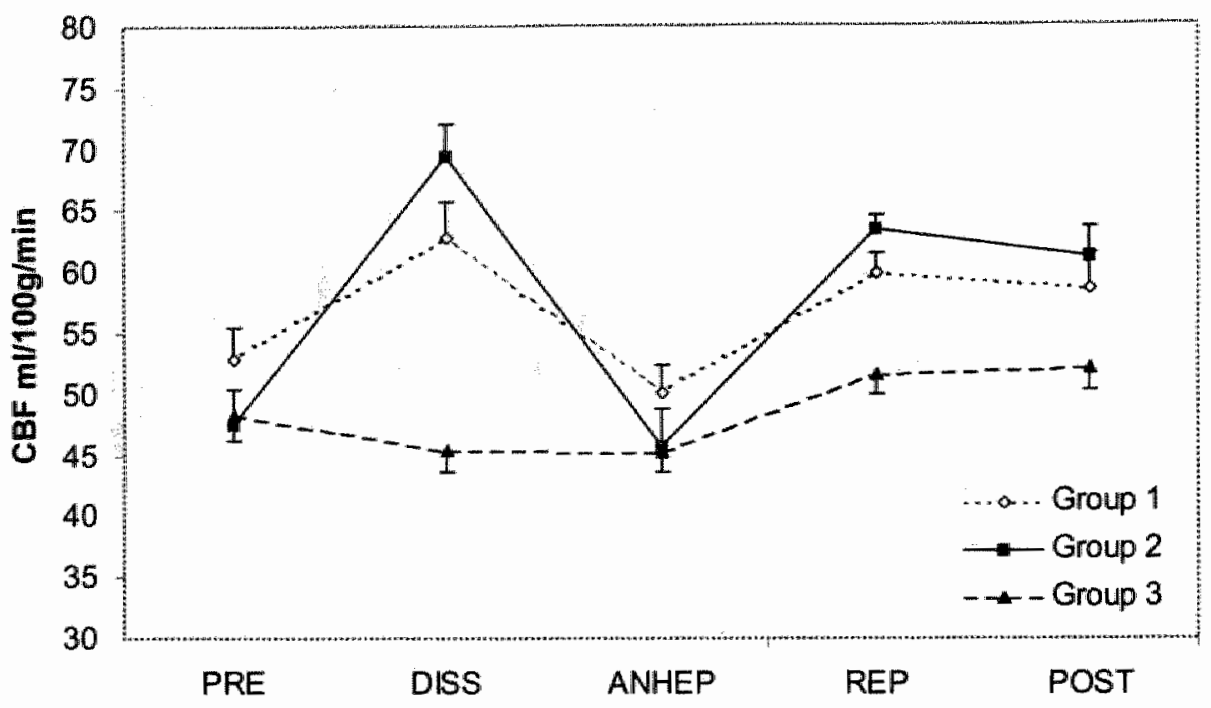

Figure 2. Change in the cerebral blood flow during the Liver Transplantation operation in the patients that were normothermic (Groups I and II) and those that were maintained hypothermic (Group III). Group I(open diamond): Cerebral blood flow increased significantly during the dissection $(p<0.02)$ and reperfusion phases $(p<0.02)$. Group II (closed square): Cerebral blood flow increased significantly during the dissection $(p<0.001)$ and reperfusion phases $(p<0.003)$. Group III (closed diamond): No significant increase in cerebral blood flow during Liver Transplantation. Values shown are mean \pm S.E.

Changes in mean arterial pressure, heart rate, cerebral perfusion pressure and noradrenaline requirement. Changes in these parameters are illustrated in Figure 3 (ad). Despite the increase in ICP in Groups I and II during the dissection and reperfusion phases, no significant changes in CPP or the MAP were observed. It is important to observe that during the dissection and also the reperfusion phases, the noradrenaline requirement was significantly greater in Groups I and II compared with Group III (dissection: $\mathrm{p}<0.05$ and $\mathrm{p}<0.05$; reperfusion: $\mathrm{p}<0.02$ and $\mathrm{p}<0.03$ respectively). The inotrope levels were titrated upwards to maintain adequate CPP. The increases in heart rate observed in groups I and II during the dissection phase were not observed in Group III $(\mathrm{p}<0.03, \mathrm{p}<0.01$ respectively).

Changes in IL-1b. Prior to OLT, veno-arterial difference in IL-1b was larger in the normothermic groups compared to the hypothermic patients of Group III indicating brain production. In the hypothermic patients IL-1b values did not change signifi- 
cantly throughout the entire time course of the operation. In this group the IL-1b level remained low, near the detection limit of the assay throughout all the phases. However, changes were observed in the normothermic patients, whose arterial IL-1b levels were found to be significantly higher than the hypothermic patients $(p<0.01)$ overall. In the normothermic patients there was a significant increase in both the arterial levels $(p<0.001)$ and also the veno-arterial difference $(p<0.05)$ of IL-1b during the dissection phase of the operation. During the anhepatic phase there was a significant reduction in both the arterial concentration and veno-arterial difference of IL- $1 \mathrm{~b}$. Two hours post OLT, there was a significant reduction in the arterial and veno-arterial difference of IL$\mathrm{Ib}$ in the normothermic patients to values that were not significantly different to zero. These data are summarised in Table 2.

Figure 3. Change in the: (A) Mean Arterial Pressure; (B) Cerebral Perfusion Pressure (mean arterial pressure-intracranial pressure); (C) Heart rate; and (D) Noradrenaline requirement during Liver Transplantation. The patients were normothermic (Groups I and II), and those that were maintained hypothermic (Group III). There were no significant changes in the mean arterial pressure and cerebral perfusion pressure during the operation but the heart rate increased significantly during the dissection phases of the operation in Groups I and II ( $p<0.03, p<0.01$ respectively). The noradrenaline requirement increased significantly during the dissection and reperfusion phases of the operation for the normothermic groups (dissection: $\mathrm{p}<0.01$ and $p<0.001$ and reperfusion: $p<0.02$ and $p<0.01$ respectively). No such increase in noradrenaline requirement was observed in patients in Groups III.

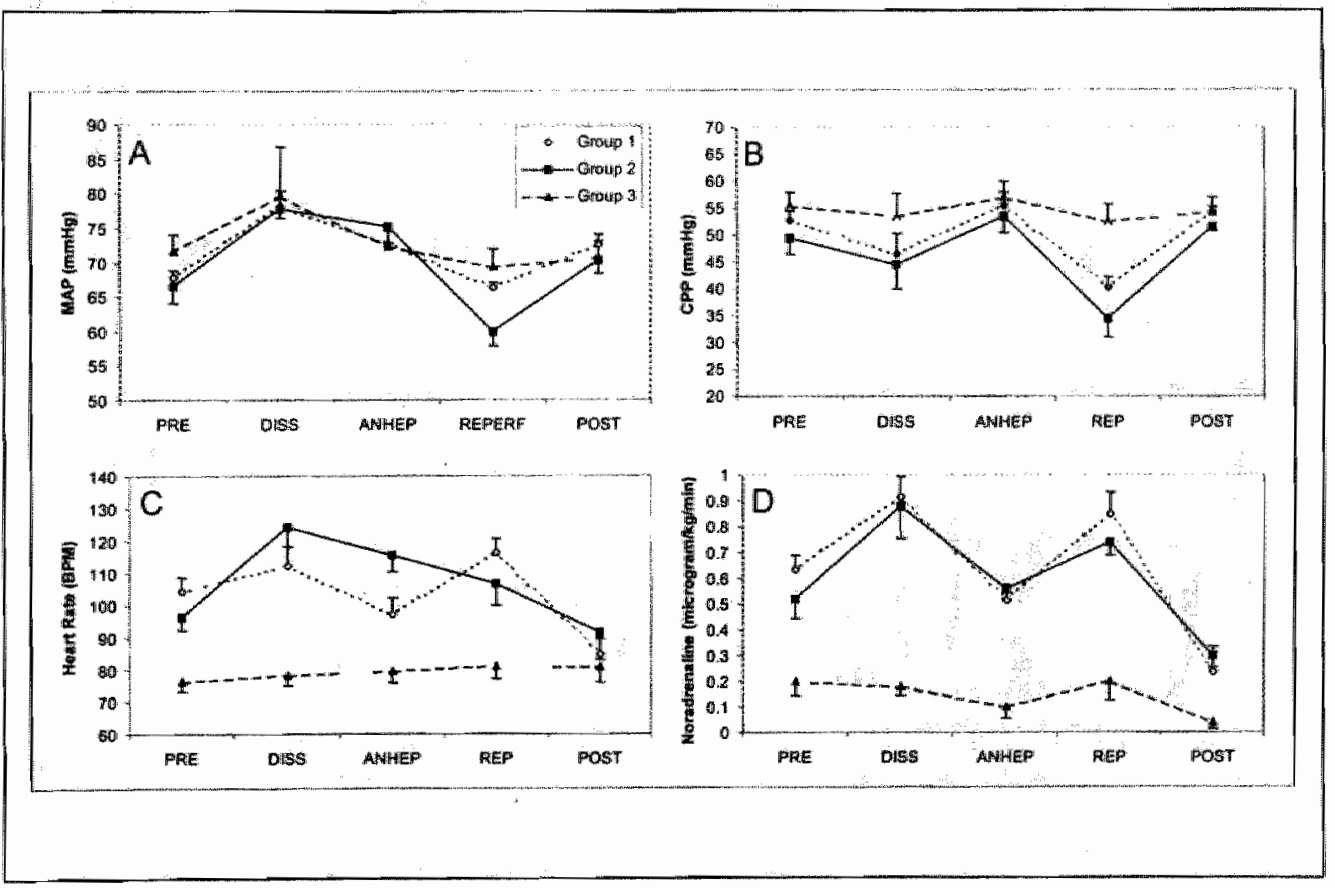




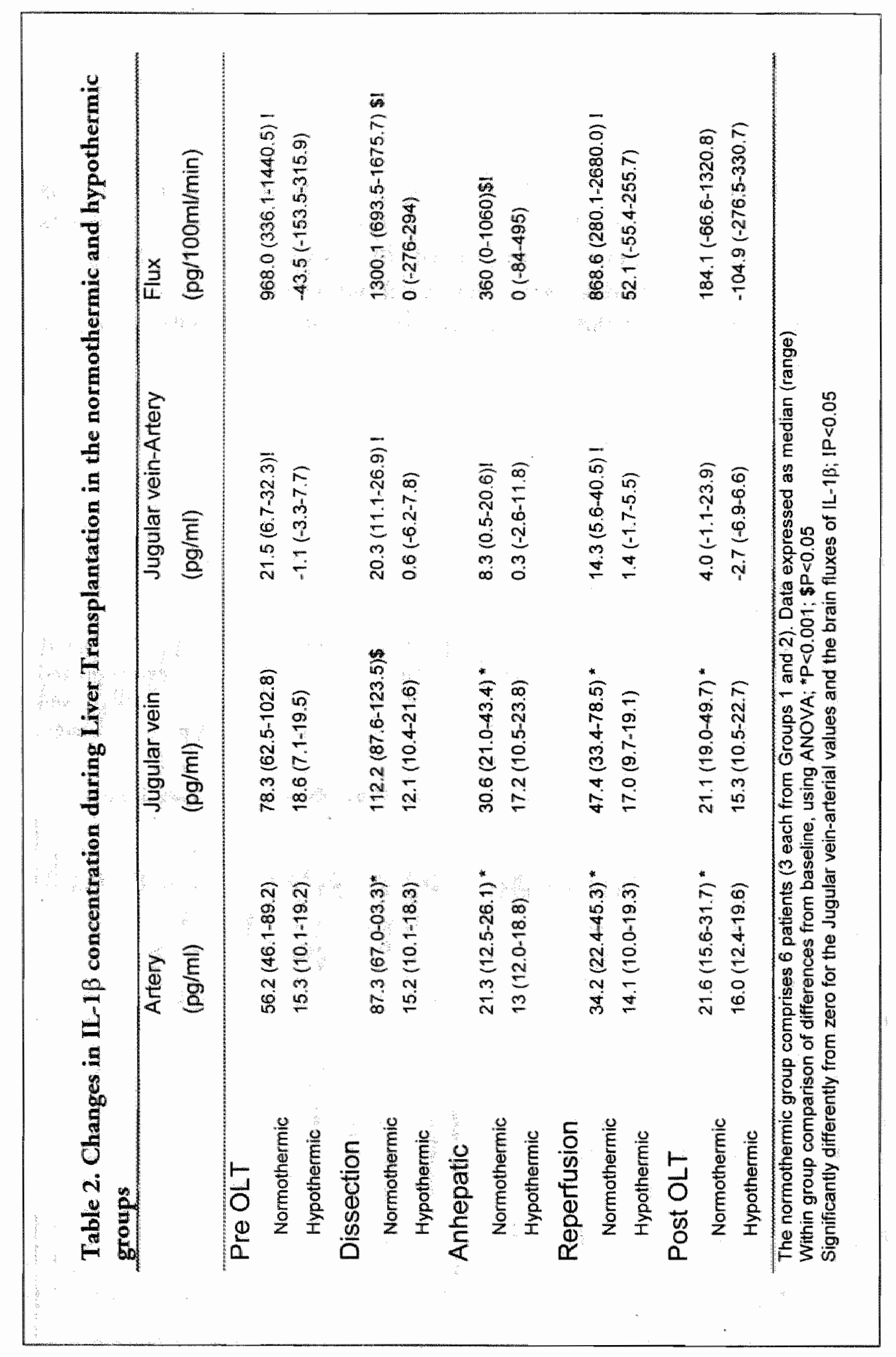




\section{DISCUSSION}

The results of this study show that moderate hypothermia is safe and successfully prevents the rise in ICP during the dissection and the reperfusion phases of the operation. In the normothermic patients, significant increases in ICP in patients with ALF undergoing OLT that were observed during the dissection and the reperfusion phases were abolished during the anhepatic phase.

About $10 \%$ of the patients that are successfully transplanted for ALF show evidence of severe postoperarive neurological disturbances and/or postoperative brain death (2). The results of our study confirm previous observations that patients with ALF undergoing OLT have significant increases in ICP during the dissection and reperfusion phases of the operation. Keays et al. (4) reported a similar pattern of ICP changes in 6 parients with ALF undergoing OLT, but Detry er al. (5) in a later study, observed that the increases in ICP were most frequently observed in those patients that had intracranial hypertension prior to OLT. All patients in the normothermic groups had increases in ICP during OLT, the majority requiring treatment with thiopentone. The most important observation of our study was that moderate hypothermia prevented the increase in ICP during OLT. It is also important to note that the hypothermic group comprised of patients with the poorest prognosis with uncontrolled ICP prior to cooling. There was howeyer, a sudden, transient unexplained increase in ICP to values of $71 \mathrm{mmHg}$ in one of the patients during the dissection phase of OLT that resolved spontaneously and was not associated with relapses. It is probable that this was an instrument artefact.

We observed significant increases in CBF in the normothermic patients that were temporally related to changes in the ICP during the dissection and the reperfusion phases of OLT (measurements were made with the $\mathrm{PCO}_{2}$ at $4-5 \mathrm{kPa}$ ). We believe that these changes in CBF were causally related to increases in ICP. Although a previous study showed reduced CBF in ALF (12) others have shown increases in agreement with our observations $(9,10,13,14)$. Current hypotheses suggest a critical role of the disturbed CBF autoregulation in the intracranial hypertension in ALF patients. Furthermore, previous studies have shown increases in CBF during OLT that is thought to be related to disturbed cerebrovascular autoregulation (6-8). Interestingly, these $\mathrm{CBF}$ changes were not observed during the dissection and the reperfusion phases in the hypothermic patients. It is possible that the lack of any deleterious changes in CBF during OLT, and therefore increases in $\mathrm{CP}$ may be the result of a restoration in $\mathrm{CBF}$ autoregulation by hypothermia as we have previously demonstrated (10).

It is interesting to note that there was reduction in ICP in the normothermic groups during the anhepatic phase that was also associated with a normalisation of 
CBF, confirming the observation of Ejlersen et al. (15), Rozga et al. (16), and Ringe et al. (17) who have previously shown temporary improvement in systemic and cerebral haemodynamics after hepatectomy in patients with ALF suggesting that the products of the 'necrotic' liver may have deleterious consequences for both systemic and cerebral haemodynamics, and its removal causes an improvement (18). The mechanism of how the "necrotic" liver influences CBF and ICP is uncertain. It is interesting to hypothesize that the 'necrotic' liver may release or induce the production of pro-inflammatory substances causing cerebral hyperaemia and cerebral oedema. The lack of significant differences in ICP between the hypothermic and normothermic patients during the anhepatic phase, suggests that the beneficial effects of hyporhermia may be due to a reduction in the release of such proinflammatory mediators from the liver $(9,19)$.

Previous studies have suggested that both the systemic concentration and liver expression of pro-inflammatory cytokines are increased in ALF $(20,21)$. In a single case we have shown that removal of the liver from a patient with ALF results in a reduction in the proinflammatory cytokines TNFa, IL- 6 and IL- $1 b(22,23)$. In the present study we chose to measure IL-1b, which is an important pro-inflammatory cytokine, that has been linked with altered cerebral haemodynamics in head injury patients (24). The beneficial cerebral haemodynamic effect of hypothermia in patients with head injury was associated with a reduction in IL-1b. This effect has been previously examined in animal models (25). We have shown that both the arterial concentration and also the veno-arterial concentration difference across the brain were significantly greater in patients with ALF that were normothermic than those that were cooled. Furthermore, there were significant increases in the concentration of this cytokine during both the dissection and the reperfusion phases of OLT. However, during the anhepatic phase of the operation, there was a significant reduction in both the arterial concentration and also in the veno-arterial difference across the brain of IL-1b. This observation suggests that the necrotic liver is the likely source of the circulating IL-Ib. In the hypothermic patients, there were no significant changes in either the arterial levels or in the brain production of $I L-1 b$ suggesting that the protective effect of hypothermia may at least partially be mediated by reduced production in this cytokine and also its action in the brain.

In ALF, the blood brain barrier is thought to be anatomically intact and should therefore not allow cytokines from the systemic circulation to enter the brain (28). However, it is possible for them to exert an effect on the brain by direct signalling via peripheral or autonomic nerves that innervate the liver. Signalling can also occur through the brain vasculature by production of endothelial factors such as nitric oxide (NO) or prostanoids. It is also possible that direct action by cytokines could be achieved after crossing the blood brain barrier, which could become porous as a consequence of a raised ICP (29). It is likely that either or both of the first two mechanisms may be occurring as the blood brain barrier is preserved in patients with ALF (26). 
Additionally, the cell in the brain that is central in mediating pathophysiology of intracranial hypertension in ALF is the astrocyte (27). These cells belong to the microglial lineage and have extensive repertoire to modulate an inflammatory response (28). Therefore the reduction in CBF following hepatectomy suggests that the circulating pro-inflammatory cytokines may be important in the regulation of CBF, possibly through their action upon mediators such as nitric oxide (30). The role of cytokines and their inter-relationship with putative mediators that may alter cerebral haemodynamics require more investigation.

Although there were no significant difference between the groups in the mean arterial pressure and cerebral perfusion pressure, patients that were maintained hypothermic required significantly less noradrenaline for maintenance of CPP. This is consistent with our previous observation $(9,10)$ and suggests that hypothermia may have profound effects upon correcting the vascular hyporesponsiveness that is characteristic of patients with liver failure.

There were no specific complications related directly to hypothermia and there was no increased evidence of bleeding complications. The overall requirements for clotting factors/blood products were similar in the 3 groups. There were no differences in the post-operative infection rates in the 3 groups and the neurological recovery in all the groups in which the patients survived were similar.

In conclusion, the results of this study suggest that hypothermia prevents the increase in ICP that is encountered in patients with ALF undergoing OLT, particularly during the dissection and the reperfusion phases of the operation. These protecrive effects of hypothermia may be mediated by decreased production and end-organ effects of the products of the 'necrotic' liver. 


\section{REFERENCES}

1. O'Grady JG, Alexander GJM, Hayllar KM, Williams R. Early indicators of prognosis in fulminant hepatic failure. Gastroenterology $1989 ; 97: 439-445$.

2. Hoofnagle $\mathrm{JH}$, Carthers RL Ir Shapiro C, Ascher N. Fulminant heparic failure: summary of a workshop: Heparology 1995;21:240-52.

3. Aggarwal S, Kang Y, Freeman JA, Fortunato FL Jr, Pinsky MR. Postreperfusion syndrome: hypotension after reperfusion of the transplanted liver. I Crit Care 1993;8:154-60.

4. Keays R, Potter D, O'Grady J, Peachey T, Alexander G, Williams R. Intracranial and cerebral perfusion pressure changes before, during and immediately after orthotopic liver transplantation for fulminant hepatic failure. QJ Med 1991,79:425-33.

5. Detry O, Arkadopoulos N, Ting P, Kahaku E, Margulies J, Arnaout W, Colquhoun SD, Rozga J, Demetriou. AA. Intracranial pressure during liver transplantation for fulminant hepatic failure. Transplantation 1999;67:767-70.

6. Doblar DD, Frenette L, Poplawski S, Gelman S, Boyd G, Ranjan D, Halsey JH. Middle cerebral artery transcranial Doppler velocity monitoring during orthotopic liver transplantation: changes at reperfusion-a report of six cases. IClin Anesth 1993;5:479-85.

7. Aggarwal S, Kang Y, DeWolf A, Scott V, Martin M, Policare R. Transcranial Doppler: monitoring of cerebral blood flow velocity during liver transplantation. Transplant Proc $1993 ; 25: 1799-800$.

8. Pott F Larsen FS, Ejlersen $E_{\text {, Linkis }} \mathrm{P}_{*}$ Jorgensen LG, Secher NH. Cerebral perfusion during human liver transplantation. Clin Physiol 1995;15:119-30.

9. Jalan $\mathbb{R}$, Olde Damink SWM, Deutz NEP, Lee A, Hayes PC. Treatment of uncontrolled intracranial bypertension in acute liver failure with moderate hypothermia Lancet $1999 ; 354,11648$

10. Jalan R, Olde Damink SW, Deutz NE, Hayes PC, Lee A. Restoration of cerebral blood flow autoregulation and reactivity to carbon dioxide in acute liver failure by moderate hypothermia. Hepatology 2001;34:504.

11. Kety $\mathrm{SS}$, Schmidt CF. The nitrous oxide method for the quantitative determination of cerebral blood flow in man: Theory, procedure and normal values. J Clin Invest $1947 ; 27: 476-483$.

12. Wendon JA, Harrison PM, Keays R, Williams R. Cerebral blood flow and metabolism in fulminant liver failure. Hepatology 1994;19:1407-13.

13. Aggarwal $S$, Kramer D, Yonas H, Obrist W, Kang Y, Martin M, Policare R. Cerebral hemodynamic and metabolic changes in fulminant hepatic failure: a retrospective study. Hepatology 1994:19:80-7.

14. Larsen FS, Adel Hansen B, Pott F, Ejlersen E, Secher NH, Paulson OB, Knudsen GM. Dissociated cerebral vasoparalysis in acute liver failure. A hypothesis of gradual cerebral hyperaemia. J Hepatol $1996 ; 25: 145-51$.

15. Ejlersen $E_{*}$ Larsen FS, Pott F, Gyrtrup HJ, Kirkegaard $\mathbb{P}_{3}$ Secher NH. Hepatectomy corrects cerebral hyperperfusion in fulminant hepatic failure. Transplant Proc 1994:26:17945.

16. Rozga J, Podesta L, LePage E, Hoffman A, Morsiani E, Sher L, Woolf GM, Makowka L, 
Demetriou AA. Control of cerebral oedema by total hepatectomy and extracorporeal liver support in fulminant hepatic failure. Lancet. 1993;342:898-9.

17. Ringe B, Lubbe N, Kuse E, Frei U, Pichlmayr R. Total hepatectomy and liver transplantation as two-stage procedure. Ann Surg. 1993;218:3-9.

18. Olafsson $S_{x}$ Gottstein J, Blei AT. Brain edema and intracranial hypertension in rats after total hepatectomy. Gastroenterology 1995;108:1097-103.

19. Walsh TS, Wigmore SJ, Hopton P, Richardson R, Lee A. Energy expenditure in acetaminophen-induced fulminant hepatic failure. Crit Care Med 2000;28:649-54.

20. Wolf D, Hallmann R, Sass G, Sixt M, Kusters S, Fregien B, Trautwein C, Tiegs G. TNFalpha-induced expression of adhesion molecules in the liwer is under the control of TNFR1-relevance for concanavalin A-induced hepatitis. J Immunol 2001;166:1300-7.

21. Iwai $H$, Nagaki $M$, Naito 'T, Ishiki $Y$, Murakami $N$, Sugihara J, Muto $Y$, Moriwaki $H$. Removal of endotoxin and cytokines by plasma exchange in patients with acute hepatic failure. Crit Care Med 1998;26:873-6.

22. Hecht N, Pappo O, Shouval D, Rose-John $S_{3}$ Galun E, Axelrod JH. Hyper-IL-6 gene therapy reverses fulminant hepatic failure. Mol Ther 2001;3:683-7.

23. Simpson KJ, Lukacs NW, McGregor AH, Harrison DJ, Strieter RM, Kunkel SL. Inhibition of tumour necrosis factor alpha does not prevent experimental paracetamolinduced hepatic necrosis. J Pathol 2000;190:489-94.

24. Marion DW, Penrod LE, Kelsey SF, Obrist WD, Kochanek PM, Palmer AM, Wisniewski SR, DeKosky ST. Treatment of traumatic brain injury with moderate hypothermia. N Engl J Med 1997:336:540-6.

25. Kato A, Singh S, McLeish KR, Edwards MJ, Lentsch AB. Mechanisms of hypothermic protection against ischemic liver injury in mice. Am J Physiol. 2002; 282:G608-16.

26. Kato M, Hughes RD, Keays RT, Williams R. Electron microscopic study of brain capillaries in cerebral edema from fulminant hepatic failure. Hepatology 1992;15:1060-6.

27. Norenberg MD. Astroglial dysfunction in hepatic encephalopathy. Metab Brain Dis 1998;13:319-35.

28. Sternberg EM. Neural-immune interactions in health and disease. J Clin Invest 1997; $100: 2641-7$.

29. Licinio J, Wong ML. Pathways and mechanisms for cytokine signaling of the central neryous system. J Clin Invest 1997;100:2941-7

30. Tureen J. Effect of recombinant human tumor necrosis factor-alpha on cerebral oxygen uptake, cerebrospinal fluid lactate, and cerebral blood flow in the rabbit: role of nirric oxide. J Clin Invest 1995;95:1086-91. 


\section{MODERATE HYPOTHERMIA IN PATIENTS WITH ACUTE LIVER FAILURE AND UNCONTROLLED INTRACRANIAL HYPERTENSION}

1,3Rajiv Jalan FRCPE, ${ }^{2,3}$ Steven WM Olde Damink MD, ${ }^{2}$ Nicolaas EP Deutz PhD, 3Peter C Hayes, ${ }^{3}$ Alistair Lee FRCA

${ }^{1}$ Liver Failure Group, Institute of Hepatology, University College London Medical School and University College London Hospitals, UK; 2Dept of Surgery, Academic Hospital, Maastricht Universiry, The Netherlands; ${ }^{3}$ Scottish Liver Transplantation Unit, Royal Infirmary of Edinburgh, Edinburgh

Published in Gastroenterology 2004; 127: 133846 


\section{INTRODUCTION}

The occurrence of hepatic encephalopathy ( $\mathrm{HE}$ ) in patients with acute liver injury is the key event defining their outcome (1-3). The encephalopathy of acute liver failure (ALF) is characterized by cerebral edema, which culminates in increased intracranial pressure (ICP). Treatment options for increased ICP in patients with ALF are limited and about $30 \%$ patients with ALF die from cerebral herniation while waiting for a suitable donor organ $(2,4)$. Indeed, in patients that have uncontrolled intracranial hypertension with conventional therapy, death from brain herniarion commonly occurs within a matter of hours. In a pilot study, we showed that treatment of patients with ALF and uncontrolled intracranial hypertension using moderate hypothermia $(32 \infty \mathrm{C})$ resulted in a reduction in ICP and allowed all the 4 patients that were suitable candidates for orthotopic liver transplantation (OLT) to be transplanted (5).

The mechanism underlying the observed reduction in ICP with hypothermia is uncertain. Studies in animal models have suggested that the beneficial effects of hypothermia may impact on the critical factors that underlie the pathogenesis of intracranial hypertension in ALF such as ammonia and its brain metabolism, cerebral blood flow (CBF), extracellular glutamate and brain lactate production (6-10). In a pilot study, we observed a reduction in arterial ammonia concentration and $\mathrm{CBF}$ when patients with uncontrolled increase in ICP were cooled to $3200 \mathrm{C} \mathrm{(5).} \mathrm{There} \mathrm{is} \mathrm{increas-}$ ing evidence that in addition to ammonia and alteration in CBF, systemic inflammatory response $(11,12)$, oxidative stress (13) and nitric oxide (14) may play a role in the pathogenesis of increased ICP in patients with $\operatorname{ALF}(2,15,16)$.

This study was designed as a follow up to our initial observation to extend and confirm the data from our pilot study (5) and evaluate the clinical effects of moderate hypothermia in patients with ALF who were candidates for OLT and had uncontrolled intracranial hypertension that was refractory to medical therapy. Moreover we wanted to study the effects of moderate hypothermia on the proposed pathophysiological pathways that are thought to be important in the pathogenesis of increased ICP in ALF; namely ammonia and its brain metabolism, CBF, mediators of inflammation and oxidarive stress. 


\section{METHODS}

Studies were undertaken with the approval of the local research ethics committee, the written informed consent from the next of kin of each patient, and were in accordance with the Declaration of Helsinki (1951) of the World Medical Association.

Patients. Patients were included in this study if they had ALF, uncontrolled intracranial hypertension and were waiting for a suitable organ for OLT. Patients were excluded if there was no informed consent and if the criteria for poor prognosis were not fulfilled. Over a 2 year period, fourteen patients with ALF who fulfilled the Kings College Criteria for poor prognosis (3) and the above criteria were recruited. Uncontrolled intracranial hypertension was defined as persistently elevated ICP of $>25 \mathrm{mmHg}$ for 1 hour or more despite 2 separate treatments with Mannitol ( $\mathrm{g} / \mathrm{Kg}$ body weight over 20 $\mathrm{min}$ ) and removal of $500 \mathrm{ml}$ of fluid by continuous veno-venous hemofiltration (5). The patients were cooled to $320 \mathrm{C}$ from the time of enrolment until OLT, spontaneous recovery or death. The patients were treated with boluses of Mannitol if the ICP increased to $>20 \mathrm{mmHg}$. They underwent measurement of CBF at the time of enrolment into the study, 4 hours after cooling and then again at 24 hours afterwards (depending upon organ availability for OLT). In the patients that underwent OLT before 24 hours, measurements were made prior to OLT. Blood was sampled in precooled heparinised tubes from an artery and reverse jugular catheter prior to and at the time of the CBF measurements. All the analyses were performed as a single batch using plasma/serum which was kept frozen at $-80^{\circ} \mathrm{C}$.

Management Protocol. The patients were managed according to a standardized protocol as described previously $(2,5)$. All the invasive monitoring used in this study are part of the routine clinical care apart from the procedures required for $\mathrm{CBF}$ measurements. In short, all the patients were mechanically ventilated following sedation with propofol and paralysis with atracurium besylate $(300-600 \mathrm{mg} / \mathrm{Kg} / \mathrm{hr}) . \mathrm{N}$-acetylcysteine was commenced and/or continued $(150 \mathrm{mg} / \mathrm{Kg} / 24 \mathrm{hr})(2)$; broad spectrum antibiotics and fluconazole were administered prophylactically; blood glucose levels were maintained between 5-7 mmol/l (2); Cardiovascular haemodynamics were monitored with a pulmonary artery catheter, a right atrial catheter and an arterial catheter. Continuous veno-venous hemofiltration (Gambro Laboratories, USA) was instituted in all patients for established renal failure (blood flow of $200 \mathrm{ml} / \mathrm{min}$ and 1 litre cycle exchanges). Noradrenalin was used to keep the cerebral perfusion pressure (CPP) above $50 \mathrm{mmHg}$ and/or a mean arterial pressure (MAP) of greater than $80 \mathrm{mmHg}$. Cooling was performed using cooling blankets that were placed above and below the patient (Blanketrol II, Cincinnati Sub-Zero, Cincinnati) to maintain the blood temperature at $32-33 \infty \mathrm{C}(5)$. 
Clinical endpoints. Survival to OLT or death, neurological changes during therapy, survival after OLT and ICP. Neurological examination was performed at hourly intervalls with particular attention to pupillary size and its reaction to light. ICP was continuously monitored using a subdural fibre-optic system (Camino, Camino Laboratories, San Diego, USA). Patients were followed up after transplantation to assess neurological sequela of cooling. CPP was calculated as the difference between the MAP and the ICP.

\section{Pathophysiological endpoints.}

1. Ammonia: Plasma was obtained by centrifugation and was deproteinised using trichloroacetic acid $(50 \% \mathrm{w} / \mathrm{v})$ prior to storage $\left(-80^{\circ} \mathrm{C}\right)$ and ammonia was measured spectrophotometrically by standard enzymatic methods (CobasMiraS, HoffmanLaRoche, Switzerland) (17).

2. CBF: CBF was measured using a modification of the Kety-Schmidt technique (18) measuring the rate of uptake of nitrous oxide by the brain as detailed previously $(5,19)$. Another arterial catheter and a jugular bulb catheter (4F Opticath, U440, Abbot, USA) were inserted. CBF was only determined if the patient was haemodynamically stable, defined as a difference of less than $10 \%$ in the MAP. For each measurement of CBF, the arterial hydrogen ion concentrations was corrected and ventilation was adjusted to achieve an arterial carbon dioxide tension of $4-4.5$ kiloPascals (kPa), and was not altered again during the measurement to prevent $\mathrm{PaCO}_{2}$ as a confounding factor of CBE.

3. Mediators of inflammation: Serum was collected for measurement of TNFa, IL $1 \mathrm{~b}$, and IL-6. Measurements were made using standard commercially available ELISA ( $R \& D$ systems, Minneapolis, MN). The lower limit for detection of TNFa, IL $1 \mathrm{~b}$, and IL-6 was $3 \mathrm{pg} / \mathrm{ml}(20)$.

4. Markers of oxidative stress: Malonyldialdehyde (MDA) was measured as a markers of oxidative stress and determined using a modified TBARS assay as described (21). Control values for our laboratory were $0.9(0.2) \mu \mathrm{mol} / \mathrm{L}$. Nitrate/Nitrite: was measured using the HPLC as described (22). Control values for nitrate/nitrite in our laboratory was $40(3.6) \mu \mathrm{mol} / \mathrm{L}$.

Calculations. Cerebral metabolic rate was calculated as CBF times arterial-jugular venous concentration differences. Fractional extraction was calculated as arterial-jugular venous concentration difference divided by the arterial concentration.

Analysis. All the data were expressed as mean and SEM. One-way analysis of variance with Bonferroni correction was used to analyse the statistical significance of data, which were analysed at multiple time points. Wilcoxon signed ranks or t-test were used for paired data as appropriate. P-value of $<0.05$ was considered statistically significant. 


\section{RESULTS}

Patients. Patient details are summarized in Table 1 . The core temperature was reduced from a mean of $36.3^{\circ} \mathrm{C}(0.2)$ to a mean of $33.1^{\circ} \mathrm{C}(0.5)$ at within an hour of starting cooling and remained lowered ar $32.6^{\circ} \mathrm{C}(0.4)$ ar 24 hours. However, the effect of cooling on ICP was evident within the first hour of starting cooling, even before the target temperature of $32^{\circ} \mathrm{C}$ was reached. Cooling was associated with a significant and sustained increase in systemic vascular resistance $(\mathrm{p}<0.01)$, MAP $(\mathrm{p}<0.001)$ and CPP $(p<0.001)$, and a reduction in heart rate $(p<0.0001)$, cardiac output $(p<0.01)$ and noradrenalin requirement $(\mathrm{p}<0.01)$. (Table 2$)$

\section{Table 1. Patient Characteristics}

$n=14$

Age (years)

$24(3.1)$

Sex

$5 \mathrm{M}, 9 \mathrm{~F}$

Aetiology of ALF

Paracetamol overdose

13

Non A-Non B Hepatitis

1

Kings College criteria for poor prognosis

14

Highest Prothrombin Time (sec)

$114(6.5)$

Bilirubin $(\mu \mathrm{mol} / \mathrm{L})$

$213(14.1)$

Creatinine $(\mu \mathrm{mol} / \mathrm{L})$

$212(11.9)$

Continuous veno-venous haemofiltration

14

Inotropes

Mechanical Ventilation

Candidates for Liver Transplantation

14

Arterial hydrogen ion concentration ( $\mathrm{nmol} / \mathrm{L}$ )

$48(3.2)$

Propofol dosage (mg/hr) 


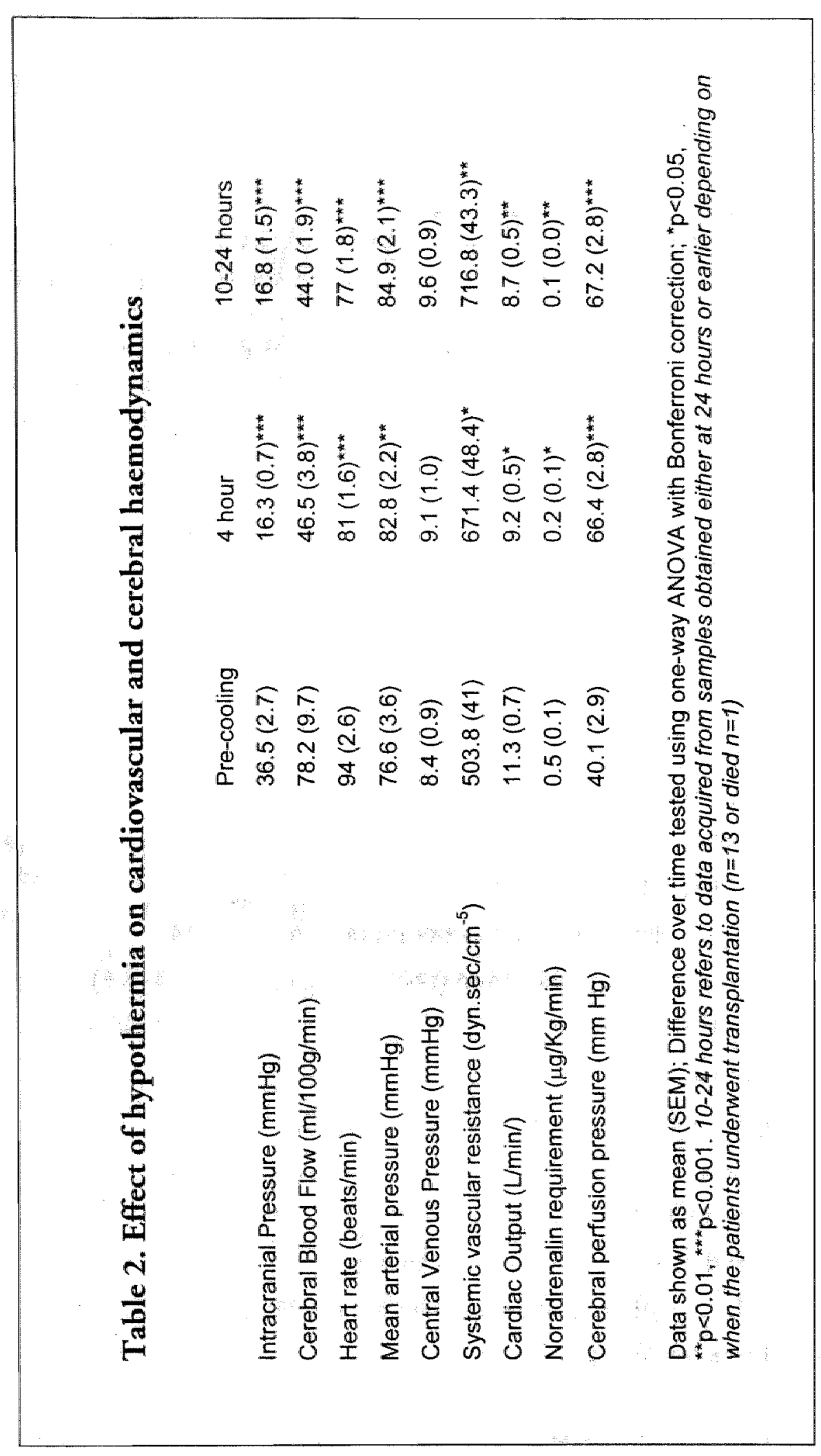




\section{Clinical endpoints.}

Survival: Thirteen of the 14 patients were successfully bridged to OLT with a mean of $31.8 \mathrm{hr}$ (9.1, range 10-118) hours of cooling. Seven patients underwent OLT between 12 and 24 hours, 2 between 24 and 48 hours, 2 between 48 and 72 hours, 1 between 72 and 96 hours and 1 at 118 hours after onset of cooling. One patient was taken off the OLT list about 10 hours after onset of cooling who subsequently died from brain herniation (see later).

Neurological changes during cooling: Prior to cooling, 8 of the 14 patients showed clinical evidence of increased ICP characterized by unequal pupil sizes. In 2 patients, no pupillary reaction to light was present. The inequality in the pupillary size persisted in 1 patient during therapy with moderate hypothermia, but was corrected with hypothermia in the others. Pupillary reaction to light was restored in both patients with cooling.

Intracranial Pressure. Prior to cooling the ICP was elevated at $36.5(2.7) \mathrm{mmHg}$ and this was reduced to $16.3(0.7) \mathrm{mmHg}$ at 4 hours, which was sustained at 24 hours $(16.8(1.5) \mathrm{mmHg})(p<0.0001)$ ICP increased to $48 \mathrm{mmHg}$ in one patient and remained elevated despite repeated doses of Mannitol, fluid removal with CVVH and 2 separate doses of Thiopentone sodium. This patient died from cerebral herniation. In the 7 patients that were treated with hypothermia for more than 24 hours, 5 developed transient increases in ICP to greater than $20 \mathrm{mmHg}$ which responded to additional treatment with Mannitol [Figure 1].

Post OLT, Safety of Hypothermia

Survival after OLT: All 13 patients underwent successful transplantation and 10 of these parients were alive 3 months after OLT. Deaths in the 3 patients after OLT were due to multiorgan failure in 2 parients (possibly from sepsis) and from primary graft non-function in 1 .

Requirement for transfusion during OLT: No episodes of bleeding were noted either due to the insertion of monitoring devices or from the effects of cooling. During the transplant operation, the hypothermia group was transfused with a median of 5 (2-8) units of red cell concentrates, $3(2-5)$ pools of platelets and $900(600-1800) \mathrm{ml}$ of fresh frozen plasma.

Infection after OLT: In the post-operative period a total of 9 episodes of infection were noticed in the 13 patients that underwent successful OLT [chest infection: 6; staphylococcus aureus septicemia: 2; urinary tract infection (E.Coli): 1]. As stated above death in one patient with staphylococcal sepsis was associated with multi-organ failure. Another patient, who was thought to have chest infection and multiorgan failure died. The rest of the infections resolved with standard antibiotic therapy.

Neurologic function after OLT: The median time to extubation following successful transplantation was $126(78-243)$ hours. All patients had normal neurological recovery apart from 1 patient who required prolonged rehabilitation for muscle weakness due to prolonged stay in the intensive care unit. 


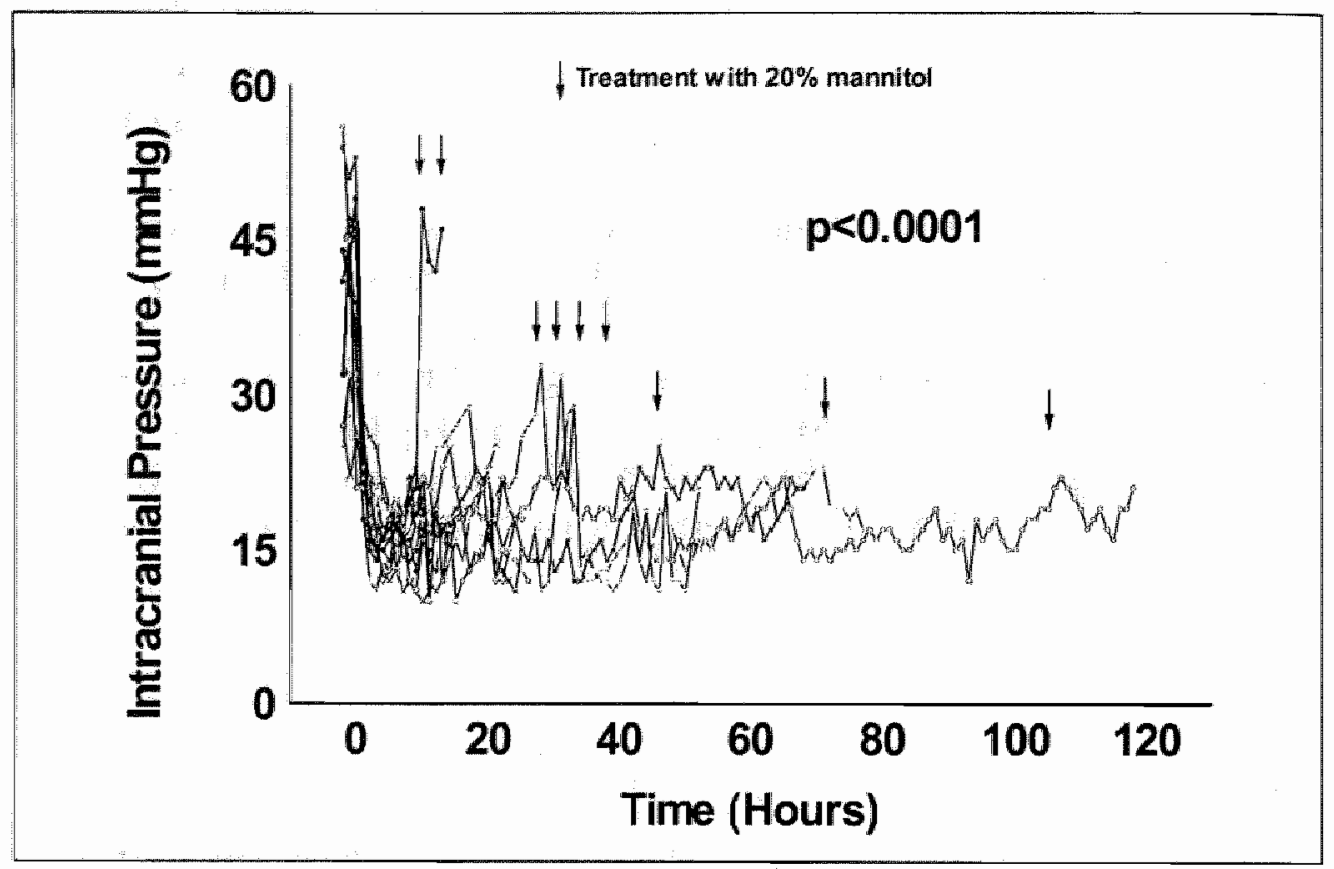

FIGURE 1. Figure showing changes in intracranial pressure in the 14 patients following cooling $\left(32^{\circ} \mathrm{C}\right)$. P-value calculated using one-way ANOVA. The arrows represent the need for administration of $20 \%$ mannitol during cooling in 5 patients. Despite cooling, death from brain herniation due to uncontrolled. increased ICP occurred in 1 patient about 10 hours after the onset of cooling, The arrows represent mannitol treatments in 5 patients who developed increases in ICP during cooling.

\section{Pathophysiological endpoints.}

Ammonia Metabolism: There was a significant reduction in arterial ammonia $(\mathrm{p}<0.05)$ concentration with cooling [Figure 2a]. Prior to cooling the brain extraction of ammonia was 17.2 (2.2) \% which was reduced to values that were not significantly different from zero after cooling. In contrast, the brain produced glutamine prior to cooling which was reduced to values that were not significantly different from zero [Figure $2 \mathrm{~b}$ and $2 \mathrm{c}$ ). Brain flux of ammonia prior to cooling was $-4.1(0.6) \mathrm{umol} / 100 \mathrm{~g} / \mathrm{min}$ and this was reduced significantly to values that were not significantly different from zero after cooling. Brain glutamine flux prior to cooling was 11.5 (3.9) umol $/ 100 \mathrm{~g} / \mathrm{min}$ and this was reduced to values that were not significantly different from zero after cooling [Figure $2 \mathrm{~d}$ ]. Prior to cooling, brain uptake of ammonia correlated with brain production of glutamine $(r=0.61, p<0.05)$.

Cerebral Blood Flow. CBF measurements were made in 12 of the 14 patients. The measured values were above the normal range $(45-55 \mathrm{~m} / 100 \mathrm{~g} / \mathrm{min})$ in 10 patients and were reduced in 2 patients. CBF was reduced from a mean of $78.2(9.7) \mathrm{ml} / 100 \mathrm{~g} / \mathrm{min}$ to $46.5(3.8) \mathrm{ml} / 100 \mathrm{~g} / \mathrm{min}$ at 4 hours after cooling and this was sustained (44 (1.9) $\mathrm{ml} / 100 \mathrm{~g} / \mathrm{min})(\mathrm{p}<0.0001)$ [Figure 3]. 

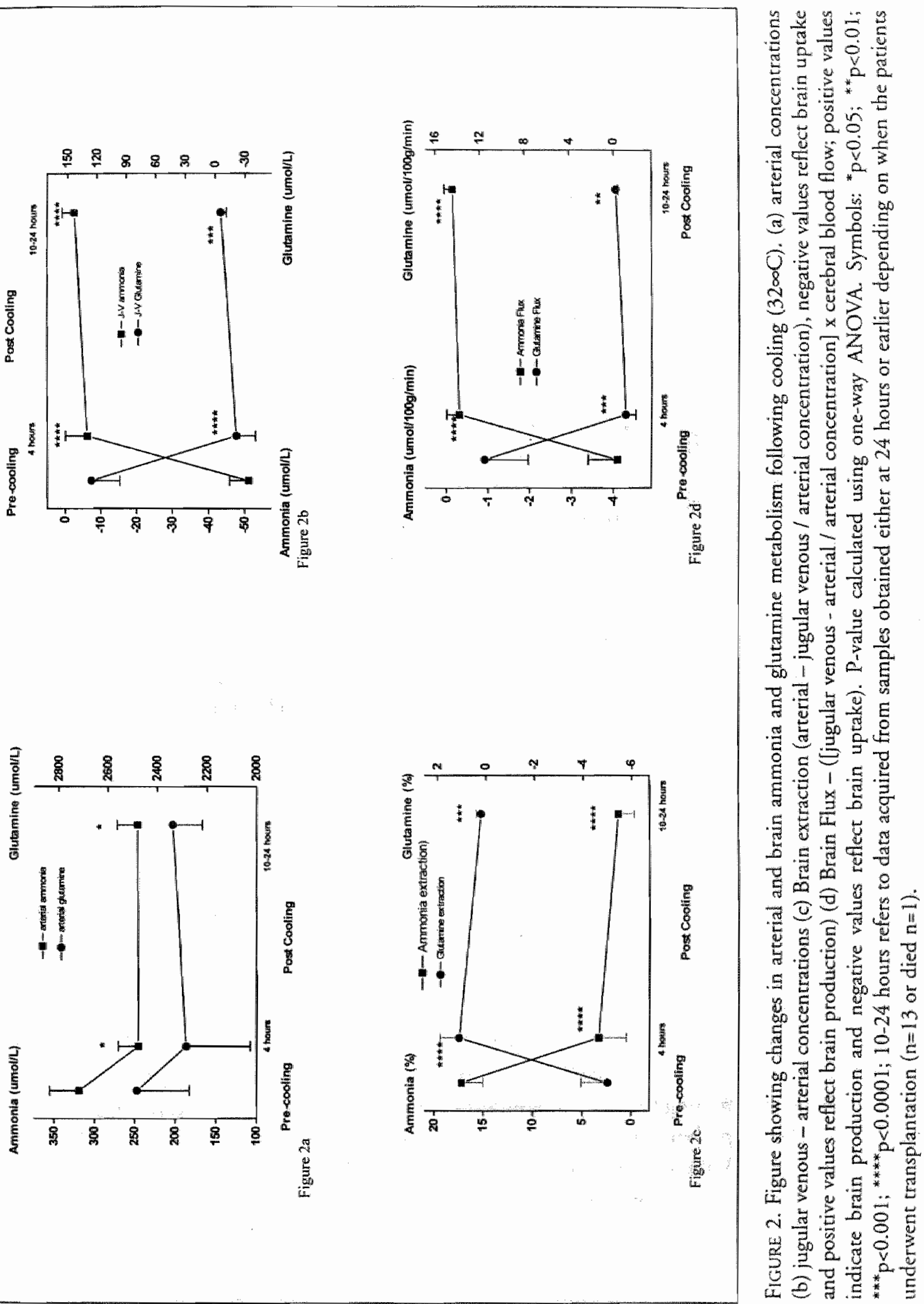


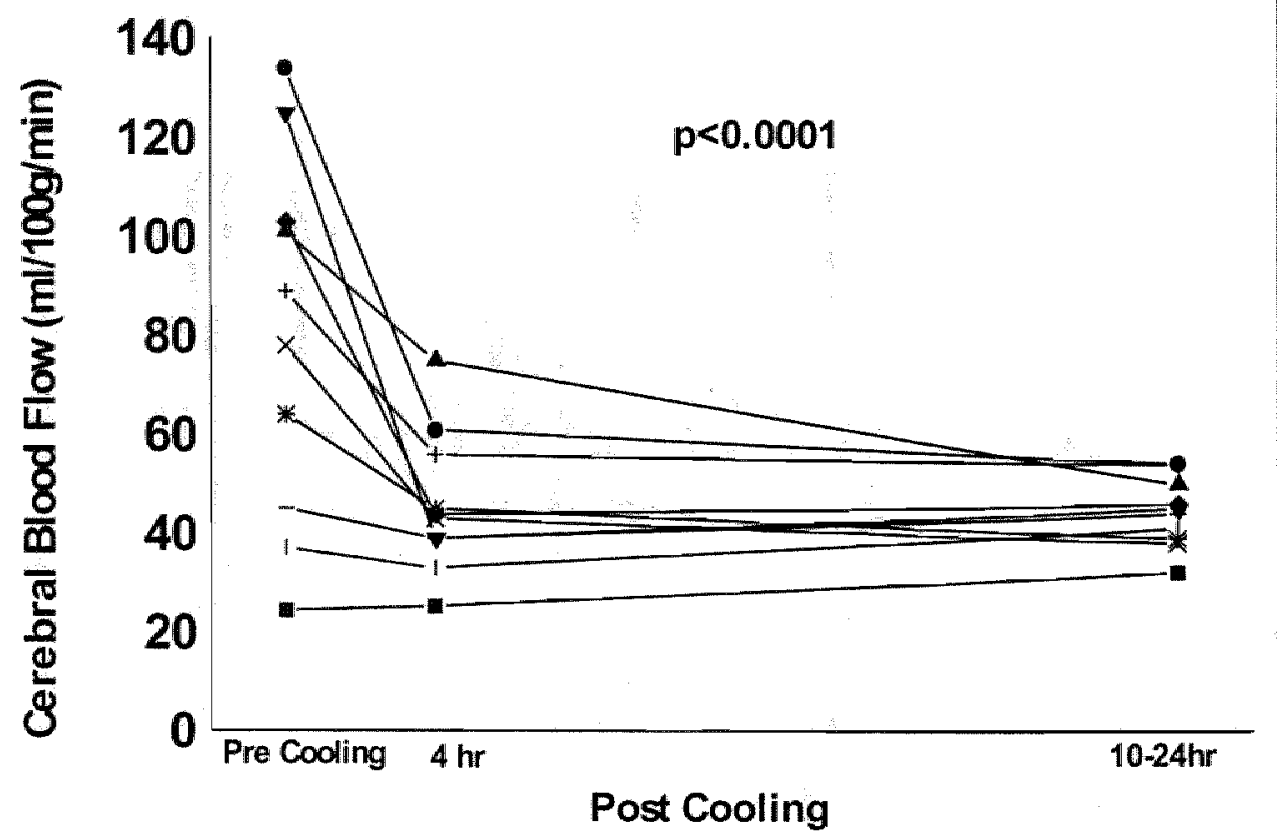

Figure 3. Changes in cerebral blood flow with cooling $(32 \infty \mathrm{C})$. Data shown for 12 of the patients in whom CBF measurements were made. P-value calculared using one-way ANOVA. 10-24 hours refers to data acquired from samples obtained either at 24 hours or earlier depending on when the patients underwent transplantation $(n=13$ or died $n=1)$.

Markers of inflammation: Patients showed markedly elevated arterial concentrations of the pro-inflammatory cytokines TNFa, $\mathrm{IL}-1 \mathrm{~b}$ and $\mathrm{IL}-6$ with evidence of brain production of these cytokines. With cooling, there was a significant reduction in the arterial concentration ( $\mathrm{p}<0.05, \mathrm{p}<0.05$ and $\mathrm{p}<0.001$ respectively) and also in the brain flux of these cytokines $(p<0.01, p<0.001$ and $p<0.05$ respectively) to values that were not significantly different from zero. (Table 3) [Figure 4]

Markers of oxidative stress: Malonylaldehyde levels were elevated about 14-fold over control values but the brain flux of MDA was not significantly different from zero. Following cooling there was a significant reduction in arterial MDA ( $\mathrm{p}<0.0001)$. (Table 3) [Figure 5] Nitrate/nitrite levels were elevated two-fold over control values but the brain flux of nitrate/nitrite was not significantly different from zero. Following cooling, there was a significant reduction in arterial nitrate/nitrite levels $(p<0.05)$. (Table 3 ) 


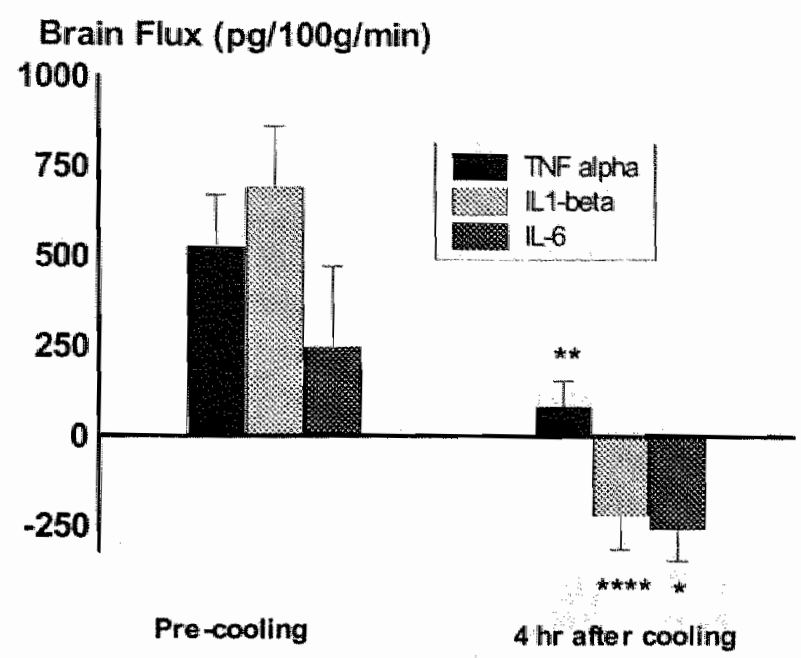

FIGURE 4 (ABOVE). Changes in brain flux of pro-inflammatory cytokines with cooling $\left(32^{\circ} \mathrm{C}\right)$. Brain Flux - ([jugular venous - arterial / arterial concentration] x cerebral blood flow); positive values indicate brain production and negative values reflect brain uptake. P-value calculated using paired r-test. Symbols: ${ }^{*} \mathrm{p}<0.05 ;{ }^{* *} \mathrm{p}<0.01$; $* * * * * 000001$

FIGURE 5 (BELOW). Changes in arterial concentration of malonyldehyde with conling $\left(32^{\circ} \mathrm{C}\right)$. P-value calculated using one-way ANOVA.*** $\mathrm{p}<0.001 .10-24$ hours refers to data acquired from samples obtained either at 24 hours or earliet depending on when the patients underwent transplantation $(n=13$ or died $n=1)$.

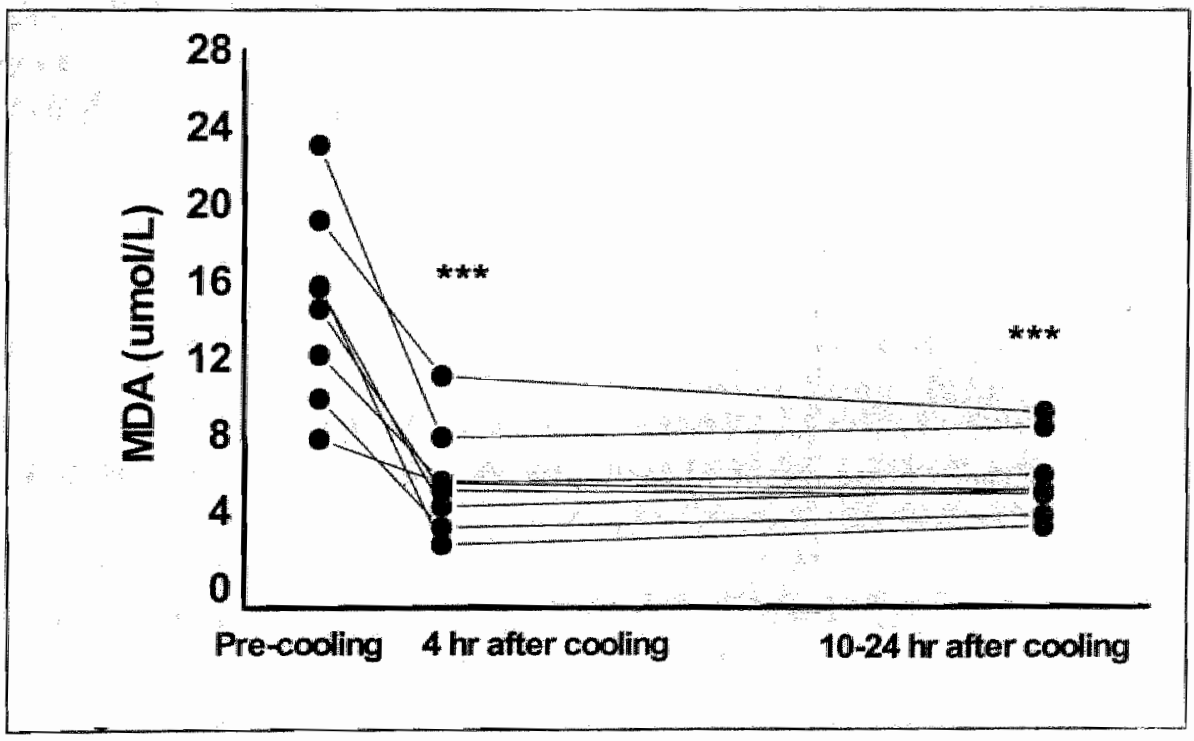


Table 3. Effect of hypothermia on Cytokines, Malonylaldehyde, and Nitrates/Nitrites

"TNFa

Arterial $(\mathrm{pg} / \mathrm{mL})$

Jugular Venous (pg/mL)

Jugular Venous - Arterial (pg/mL)

Brain Flux (ug/ $100 \mathrm{~g} / \mathrm{min}$ )

\section{" $L-1 \beta$}

Arterial $(\mathrm{pg} / \mathrm{mL})$

Jugular Venous (pg/mL)

Jugular Venous - Arterial (pg/mil)

Brain Flux (ug/100g/min)

“IL-6

Arterial (pg/mL)

Jugular Venous (pg/mL)

Jugular Venous - Arterial (pg/mL)

Brain Flux (ug/100g/min)

"Malonylaidehyde

Arterial (umol/L)

Jugular Venous (umol/L)

Jugular Venous - Arterial (umol/L)

Brain Flux (umol $/ 100 \mathrm{~g} / \mathrm{min}$ )

Mitrate/Nitrites

Arterial (umol/L)

Jugular Venous (umol/L)

Jugular Venous - Arterial (umol/L)

Brain Flux (umol $/ 100 \mathrm{~g} / \mathrm{min}$ )
Pre-cooling 4 hour

$21.2(4.8)^{*}$

$22.7(5.1)^{*}$

$1.6(1.4)^{*}$

$83.7(70.0)^{* * *}$

$5.2(1.1)$
$529.0(1.40 .4)$

$52.1(10.1)$

$60.6(11.0)$

$8.5(1.1)$

692 (169)

$110.3(13.6)$

$111.6(14.3)$

$1.3(2.9)$

$248.5(221.6)$

$14.8(1.9)$

$14.7(1.7)$

$-0.1(0.5)$

$-24.8(30.5)$

$95.9(12.9)$

$95.6(25.6)$

$-0.3(2.3)$

$299.2(1928)$
$22.1(5.9)^{*}$

$17.4(5.2)^{\star \star}$

$-4.7(2.1)^{\star * *}$

$-223(92)^{* * *}$

$29.2(4.4)^{* * * *}$

$23.8(3.3)^{* \text { k.*. }}$

$-5.4(1.5)^{*}$

$-260.6(87.1)^{*}$

$5.6(1.1)^{* * * *}$

$5.7(1.2)^{\star * *}$

$0.1(0.2)$

$2.7(9.0)$

$62.0(11.7)^{2}$

$49.6(13.6)$

$-12.4(5.2)$

$-590(247)$
$5.7(1.1)^{* \ldots x}$

$5.6(1.9)^{* \star *}$

$-0.1(0.3)$

$-3.8(10.1)$

10-24 hours

N/A

N/A

N/A

$56.5(6.8)^{*}$

$48.2(6.8)$

$-8.4(2.1)$

$-370(107)$

TABLE 3: Data shown as mean (SEM); Difference tested using "Students t-rest; One-way ANOVA with Bonferroni correction; ${ }^{*} \mathrm{p}<0.05,{ }^{* *} \mathrm{p}<0.01,{ }^{* * *} \mathrm{p}<0.001$. Brain extraction (arterial - jugular venous / arteria concentration), negative values reflect brain uptake and positive values reflect brain production); Brain Flu - (jugular venous - arterial / arterial concentration) $x$ cerebral blood flow; positive values indicate brain pro duction and negative values reflect brain uptake. 10-24 hours refers to data acquired from samples obtaine eirher at 24 hours or earlier depending on when the patients underwent transplantation $(n=13$ or died $n=1)$ 


\section{DISCUSSION}

The results of this study extend and confirm those of our pilot study suggesting that in patients with ALF who have uncontrolled intracranial hypertension and are at immediate risk of cerebral herniation, moderate hyporhermia can be used successfully and safely to control ICP, improve neurological end-points and possibly survival by bridging patients to OLT (5).

Brain herniation from the effects of elevated ICP is the immediate cause of death in 35\% ALF patients who fulfil criteria for poor prognosis and are not thought to be suitable candidates for OLT (2). Of the patients with ALF that are listed for OLT, about $15-20 \%$ die from the effects of increased ICP while waiting for a donor organ to become available $(2,4)$. In the present study we show a substantial improvement in the outcome of ALF patients with uncontrolled intracranial hypertension using moderate hypothermia, with 13 of the 14 patients being successfuily bridged to OLT It is important to note that most of these patients who had uncontrolled intracranial hypertension would have been taken off the waiting list for transplantation. Cooling produced significant neurological improvement in most of the patients who showed pupillary abnormalities. Traditionally, lack of pupillary reaction to light is taken as evidence of brain herniation and OLT is contraindicated but post-OLT recovery of such patients has been described $(23,24)$. Survival after OLT in 10 of the 13 patients successfully transplanted is not different from data in patients transplanted for ALF $(2-4,23)$. The data obtained from the patients studied do provide insights into the safety profile of using short duration of hypothermia as a bridge to transplantation. Compared with the patients transplanted with their body temperatures maintained at $37 \infty \mathrm{C}$, the induction of hypothermia did not introduce any significant complications. There were no major episodes of bleeding and the requirement of transfusion during OLT was similar to that we have described previously for normothermic patients (25). Although the rate of infection after OLT was similar to that expected in this group of patients, this aspect should be explored in more detail in future studies $(4,25,26)$. Neurological recovery after OLT was also normal in all except one of the patients who sustained muscle weakness related to prolonged ITU admission.

The effect of hypothermia on ICP was apparent within the first hour of initiation of cooling with marked reduction in the ICP. Data from studies in animal models, suggest that the beneficial effects of hypothermia may be evident at a temperatures of $350 \mathrm{C}$ and a step-wise reduction in the temperature may therefore be more logical $(6$, 7). It is important to note that despite reduction in temperature to $3200 \mathrm{C}$, death from cerebral herniation occurred in one patient who remained refractory to other medical manoeuvres. We also observed minor instances of increases in ICP in patients who had 
been cooled for longer than 24 hours, to values greater than $20 \mathrm{mmHg}$, which responded rapidly to treatment with boluses of Mannitol.

The exact mechanisms involved in the pathogenesis of intractanial hypertension in ALF is not entirely clear but it is likely that a number of inter-related factors contribute $(2,13,14)$. First, ammonia related brain edema is thought to be important $(27,28)$. Ammonia is detoxified in the astrocytes to glutamine through amidation of glutamate. The ammonia-glutamine-brain swelling hypothesis of HE suggests that this accumulation of glutamine produces an osmotic stress and causes astrocytes to swell (28). Second, CBF autoregulation is lost in patients with ALF with concomitant increase in CBF (29). Third, mediators of systemic inflammatory response may play an important modulating role in the pathogenesis of increased ICP $(10,11,15,16)$. Our studies therefore explored how hypothermia modifies these proposed pathophysiological processes.

The application of hypothermia resulted in a reduction in arterial ammonia concentration but the mechanism of this reduction is not clear (5). Our data showing a good correlation between the uptake of ammonia into the brain with glutamine efflux are consistent with those of Strauss et al. (30). The reduction in the uptake of ammonia into the brain and the consequent reduction in the brain glutamine production suggest that one of the mechanisms whereby hypothermia may reduce ICP is through reduction in astrocytic swelling according to the ammonia-glutamine-brain swelling hypothesis.

We observed increased CBF in the majority of the patients who had uncontrolled ICP and this was reduced significantly to near normal values with cooling. Accumulating data support an important role for cerebral hyperaemia in the pathogenesis of increased ICP in ALF $(5,13,14,31-34)$. The mechanism of the observed reduction in CBF with cooling is likely to be due to its vasoconstrictor effect (5). Our data showing a reduction in brain glutamine efflux with the reduction in CBF supports observations that the increase in CBF is intricately related to ammonia-induced glutamine synthesis $(35,36)$. We observed no significant brain flux of nitrate/nitrite in the patients questioning the role of nitric oxide as a primary mediator of increased CBF, an observation that is consistent with data from animal models (37).

Prior to cooling we observed brain production of TNFa, IL-6 and IL-1b providing evidence of inflammatory activity in the brain. The pathophysiological significance of this observation is debatable but may indicate proliferation/activation of the cells of the glial lineage $(38,39)$. The question of how these cytokines exit from the brain is more difficult given that the blood brain barrier in ALF is thought to be intact (40). It is possible that the blood brain barrier may breakdown in very advanced stages of cerebral edema, which may be of relevance in the occurrence of uncontrolled increase in ICP. This hypothesis needs to be tested in appropriate studies. Hypothermia resulted in a 
marked reduction in the production of these cytokines (41), to values that were not significantly different from zero. The relationship between systemic inflammatory response and how this affects the brain is not clear. Recent observations suggest that TNFa initiated signals can produce vasoactive substances and reactive oxygen species resulting in neurological dysfunction (42-45). There is emerging evidence suggesting that oxidative stress plays an important role in the development of $\mathrm{HE}$, possibly by causing mitochondrial damage and energy metabolism $(13,15,16)$. We measured MDA as reflecting lipid peroxidation. Although there were significantly elevated levels of MDA in the systemic circulation which was markedly reduced with cooling, we were not able to demonstrate a significant flux across the brain which may either reflect that there is no generation of oxidative stress across the brain or that MDA is not a sensitive enough measure.

All patients required inotropic support to maintain adequate CPP. Cooling reduced cardiac output and the requirement for noradrenalin. The reduction in cardiac output is likely to reflect a reduction in heart rate (stroke volume remained unchanged) and the increase in MAP may indicate an increase in the vascular resistance, which indicates improvement in vascular responsiveness by hypothermia. The mechanism of the observed increase in vascular responsiveness may be mediated through hypothermia induced reduction in nitrate/nitrite, which is thought to an important mediator of vascular hyporesponsiveness (46).

In conclusion, the results of this study show that mild-moderate hypothermia can be used safely to bridge patients with ALF and uncontrolled intracranial hypertension to liver transplantation. The mechanism by which hypothermia reduces ICP is likely to be multifactorial and we showed that it reduces arterial ammonia, its brain uptake and metabolism, CBF and brain flux of pro-inflammatory cytokines. These data confirm those of our pilot study and calls for an appropriately powered large clinical trial to determine the place of hypothermia in ALF. 


\section{REFERENCES}

1. Trey C, Davidson CS. The management of fulminant hepatic faillure. In: Popper $\mathrm{H}$, Schaffner $F_{s}$ eds. Progress in liver disease, Volume III. New York: Grune and Stratton, $1970: 282-98$.

2. Jalan R. Intracranial Hypertension in Acute Liver Failure: Pathophysiological basis of Rational Management. Semin Liv Dis 2003;23:271-82.

3. O'Grady JG, Alexander GJM, Hayllar KM, Williams R. Early indicators of prognosis in fulminant hepatic failure. Gastroenterology 1989;97:439-45.

4. Farmer DG, Anselmo DM, Ghobrial RM, Yersiz H, McDiarmid SV, Cao C, Weaver M, Figueroa J, Khan K, Vargas J, Saab S, Han S, Durazo F, Goldstein L, Holt C, Busuttil RW. Liver transplantation for fulminant hepatic failure: experience with more than 200 patients over a 17-year period. Ann Surg. 2003;237:666-75

5. Jalan R, Olde Damink SWM, Deutz. NEP, Lee A, Hayes PC. Treatment of uncontrolled intracranial hypertension in acute liver failure with moderate hypothermia. Lancet 1999;354:1164-8

6. Traber $\mathbb{P}$, DalCanto M, Ganger D, Blei AT. Effect of body temperature on brain edema and encephalopathy in the rat after hepatic devascularization. Gastroenterology 1989;96:88591.

7. Cordoba J, Crespin J, Gottstein J, Blei AT. Mild hypothermia modifies ammonia-induced brain edema in rats after portacaval anastomosis. Gastroenterology 1999;1 16:686-93.

8. Rose $\mathrm{C}$, Michalak A, Pannunzio M, et al. Mild hypothermia delays the onset of coma and prevents brain edema and extracellular brain glutamate accumulation in tats with acute liver failure. Hepatology $2000,31: 872-7$

9. Zwingmann $\mathrm{C}$, Chatauret N, Leibfritz D, Butterworth RF. Selective increase of brain lactate synthesis in experimental acute liver failure: Results of a $\left[{ }^{1} \mathrm{H}_{-}{ }^{13} \mathrm{C}\right]$ nuclear magnetic resonance study. Hepatology. 2003;37:420-8.

10. Rolando $\mathrm{N}$, Wade J, Davalos $\mathrm{M}$, et all. The systemic inflammatory response syndrome in acute liver failure Hepatology. 2000;32:734-9.

11. Vaquero J, Polson J, Chung C, Helenowski I, Schiode FV, Reisch J, Lee WM, Blei AT. Infection and the progression of heparic encephalopathy in acute liver failure. Gastroenterology. 2003;125:755-64.

12. Chatauret $N$, Zwingmann $C$, Rose $C$, Leibfritz D, Butterworth RF. Effects of hypothermia on brain glucose metabolism in acute liver failure: A H/C-nuclear magnetic resonance study. Gastroenterology. 2003; 125:815-24

13. Norenberg MD. Oxidative and nitrosative stress in ammonia neurotoxicity. Hepatology 2003;37:245-8. 
14. Rao VL, Audet RM, Butterworth RF. Increased neuronal nitric oxide synthase expression in brain following portacaval anastomosis. Brain Res. 1997:765:169-72

15. Vaquero J, Chung C, Cahill ME, Blei AT. Pathogenesis of hepatic encephalopathy in acute liver failure Semin. Liv Dis 2003;23:259-69

16. Butterworth RF. Molecular neurobiology of acute liver failure. Semin Liv Dis 2003:23:251-8

17. Dejong CH, Deutz NE, Soeters PB. Renal ammonia and glutamine metabolism during liver insufficiency-induced hyperammonemia in the rat. J Clin Invest 1993;92:2834-40.

18. Kety SS, Schmidt CF. The nitrous oxide method for the quantitative determination of cerebral blood flow in man: Theory, procedure and normal values. $J$ Clin Invest $1947 ; 27: 476-83$.

19. Jalan R, Olde Damink SWM, Deutz NEP, Hayes PC, Lee A. Restoration of cerebral blood flow autoregullation and reactivity to carbon dioxide in acute liver failure by moderate hypothermia. Hepatology 2001;34:50-54.

20. Mookerjee RP, Sen S, Davies NA, Hodges SJ, Williams R, Jalan R. Tumour necrosis factor alpha is an important mediator of portal and systemic haemodynamic derangements in alcoholic heparitis. Gut. 2003;52:1182-7.

21. Lapenna D, Ciofani G, Pierdomenico SD, Giamberardino MA, Cuccurullo F. Reaction conditions affecting the relationship between thiobarbituric acid reactivity and lipid peroxides in human plasma. Free Radic Biol Med 2001;31:331-5.

22. Bruins MJ, Soeters PB, Lamers WH, Meijer AJ, Deutz NEP. L-arginine supplementation in hyperdynamic endotoxemic pigs: effect on nitric oxide synthesis by the different organs. Crit Care Med. 2002;30:508-17.

23. Devlin J, O'Grady J. Indications for referral and assessment in adult liver transplantation: a clinical guideline. Gut. 1999;45 Suppl 6:VI1-VI22.

24. Davies $M H_{1}$, Mutimer D, Lowes J, et al. Recovery despite impaired cerebral perfusion in fulminant hepatic failure. Lancet. 1994;343:1329-30.

25. Jalan R, Olde Damink SWM, Deutz NEP, Davies NA, Garden OJ, Madhavan KK, Hayes $\mathrm{PC}_{\mathrm{s}}$ Lee A. Moderate hypothermia prevents cerebral hyperemia and increase in intracranial pressure in patients undergoing liver transplantation for acute liver failure. Transplantation. $2003 ; 27 ; 75: 2034-9$

26. Wade JJ, Rolando N, Hayllar K, Philpott-Howard J, Casewell MW, Williams R, Bacterial and fungal infections after liver transplantation: an analysis of 284 patients. Hepatology. $1995 ; 21: 1328-36$

27. Clemmesen $\mathbb{J O}$, Larsen FS, Kondrup J, et al. Cerebral herniation in patients with acute liver failure is correlated with arterial ammonia concentration. Hepatology. 1999;29:64853.

28. Haussinger D, Kircheis G, Fischer R, Schliess F, vom Dahl S. Hepatic encephalloparhy in 
chronic liver disease: a clinical manifestation of astrocyte swelling and low-grade cerebral edema? J Hepatol. 2000;32:1035-8.

29. Larsen FS. Cerebral circulation in liver failure: Ohm's law in force. Semin Liver Dis. $1996 ; 16: 281-92$.

30. Strauss GI, Knudsen GM, Kondrup J, Moller K, Larsen FS. Cerebral metabolism of ammonia and amino acids in patients with fulminant hepatic failure. Gastroenterology. 2001;121:1109-19.

31. Aggarwal S, Kramer D, Yonas H, Obrist W, Kang Y, Martin M, Policare R. Cerebral hemodynamic and metabolic changes in fulminant hepatic failure: a retrospective study. Hepatology 1994;19:80-7.

32. Ede RJ, Williams R. Heparic encephalopathy and cerebral edema. Semin Liv Dis 1986;6:107-18.

33. Wendon JA, Harrison PM, Keays R, Williams R. Cerebral blood flow and metabolism in fulminant liver failure. Hepatology 1994;19:1407-13.

34. Blei AT, Larsen FS. Pathophysiology of cerebral edema in fulminant hepatic failure. J Hepatol 1999;31:771-76.

35. Strauss G, Hansen BA, Knudsen GM, Larsen FS. Hyperventilation restores cerebral blood flow autoregulation in patients with acute liver failure. J Hepatol. 1998;28:199-203.

36. Master S, Gottstein J, Blei AT. Cerebral blood flow and the development of ammoniainduced brain edema in rats after portacaval anastomosis. Hepatology 1999;30:876-80.

37 Larsen FS, Gottstein J, Blei AT. Cerebral hyperemia and nitric oxide synthase in rats with ammonia-induced brain edema. J Hepatol. 2001;34:548-54

38. Carson MJ. Microglia as liaisons between the immune and central nervous systems: functional implications for multiple sclerosis, Glia. 2002;40:218-31.

39. Hanisch UK. Microglia as a source and target of cytokines. Glia. 2002;40:140-55.

40. Kato M, Hughes RD, Keays RT, Williams R. Electron microscopic study of brain capillaries in cerebral edema from fulminant hepatic failure. Heparology 1992;15:1060-6.

41. Marion DW, Penrod LE, Kelsey SF, Obrist WD, Kochanek PM, Palmer AM, Wisniewski SR, DeKosky ST. Treatment of traumatic brain injury with moderate hypothermia. N Eng J Med 1997;336:540-46.

42. Licinio J, Wong ML. Pathways and mechanisms for cytokine signaling of the central nervous system. J Clin Invest 1997;100:2941-7.

43. Gorg B, Foster N, Reinehr R, Bidmon HJ, Hongen A, Haussinger D, Schliess F Benzodiazepine-induced protein tyrosine nitration in rat astrocytes. Hepatology $2003 ; 37: 334-42$.

44. Jalan R, Williams R. The inflammatory basis of intracranial hypertension in acure liver failure. J Hepatol. 2001;34:940-2.

45. Suematsu N, Tsutsui H, Wen J, Kang D, Ikeuchi M, Ide T, Hayashidani S, Shiomi T, 
Kubota T, Hamasaki N, Takeshita A. Oxidative stress mediates tumor necrosis factor-alphainduced mitochondrial DNA damage and dysfunction in cardiac myocytes. Circulation. 2003;107:1418-23.

46. Helmy A, Newby DE, Jalan R, Johnston NR, Hayes PC, Webb DJ. Nitric oxide mediates the reduced vasoconstrictor response to angiotensin II in patients with preascitic cirrhosis. J Hepatol. 2003;38:44-50 



\section{HOOFDSTUK 11}

\section{SAMENVATTING}

Dit proefschrift is opgebouwd uit drie delen. In het eerste deel (hoofdstukken 1-4) wordt het interorgaan metabolisme van ammoniak en metabool gerelateerde aminozuren in patiënten met lever cirrhose besproken en tevens de metabole en neurospychologische gevolgen van een hoge tractus digestivus (HTD) bloeding. In het tweede gedeelte (hoofdstukken 5-7) worden de metabole gevolgen van een HTD bloeding op het vertakte keten aminozuur en het eiwitsynthese metabolisme beschreven. Het derde deel (hoofdstukken 8-13) beschrijfr de pathofysiologische basis van hepatische encephalopathie (HE) in patiënten die een transjugulaire intrahepatische portasystemische stent shunt (TIPSS) insertie ondergaan. Tevens wordt in patiënten met acuut leverfalen (ALF) de relatie tussen hersenmetabolisme en haemodynamiek beschreven en wordt de klinische en pathofysiologische basis van de therapeutische toepassing van gematigd koelen van het gehele lichaam bij deze patiënten bestudeerd.

In Hoofdstuk 1 beschrijven we het interorgaan metabolisme van ammoniak en gerelateerde aminozuren in metabool stabiele patiënten met levercirrhose en een TIPSS. De studie was ontworpen om het interorgaan ammoniak metabolisme te kwantificeren in patiënten met levercirrhose. Hierbij was er de belangrijke grondaanname dat tijdens levercirrhose de ureumsynthesecapaciteit verminderd is en dat dan de belangrijkste alternatieve ammoniak ontgiftigingsroute bestaat uit de synthese van het aminozuur glutamine uit ammoniak en glutamaat. In de 24 patiènten die we bestudeerden bleek dat ammoniak voornamelijk geproduceerd werd in de portaal drainerende organen. Daarnaast produceerden de nieren ammoniak, in hoeveelheden die gelijk was aan hetgeen in het heparo-splanchnische gebied geproduceerd werd. De skeletspieren verwijderden meer ammoniak dan de cirhotische lever, echter de hoeveelheid stikstof die werd opgenomen door de spier in de vorm van ammoniak was minder dan afgegeven in de vorm van glutamine. De portaal drainerende organen consumeerden glutamine en produceerden ammoniak, alanine en citrulline. Een opvallende observatie was dat ureum werd afgegeven aan de vena lienalis en de vena mesenterica superior, waardoor organen die hierop draineren bijdragen aan de gehele-lichaam ureagenese in deze cirrhotische pariènten. In deze studie werd geconcludeerd dat de 
hyperammonemie in metabool stabiele patiënten met levercirrhose en een TIPSS het resultaat is van de portasystemische shunting van ammoniak geproduceerd in de portaal drainerende organen en uit de nier. De spieren verwijderden meer ammoniak uit de circulatie dan de cirrhotische lever, maar produceren excessieve hoeveelheden van de niet-toxische stikstof carrier glutamine, die elders (in nier en darm) weer tot ammoniak kan worden gemetaboliseerd.

In Hoofdstuk 2 worden de metabole gevolgen van een HTD bloeding in patiènten met lever cirrhose bestudeerd. Een HTD bloeding is klinisch een van de meest voorkomende $\mathrm{HE}$ inducerende momenten in deze patiëntenpopulatie. In dit hoofdstuk worden twee studies besproken. In de eerste studie werd een HTD bloeding gesimuleerd in acht patiënten met levercirrhose en een TIPSS. De bloeding werd gesimuleerd door een intragastrische toediening van een aminozuur oplossing met de samenstelling van het hemoglobine molecuul. Bloed werd gedurende vier uren intermitterend afgenomen uit de arteria femoralis en de vena femoralis, renalis, porta en de vena hepatica. Tevens werd de plasma flow over deze organen gemeten. Door de gesimuleerde HTD bloeding steeg de arteriële ammoniak concentratie zoals verwacht significant. Er kon geen significante toename in de ammoniak productie uit de portaal drainerende organen gevonden worden, maar de ammoniak productie uit de nier nam wel 6-voudig toe. In tegenstelling tot de onveranderde ammoniak opname door de cirrhotische lever, was er wel een significante toename in ammoniak consumptie door de spier.

In de tweede studie werden 9 patiënten met levercirrhose bestudeerd die een acute HTD bloeding hadden en een acute TIPSS plaatsing ondergingen. Bloed werd afgenomen uit een arterie, de vena hepatica, renalis en de vena porta. Tevens werd de plasma flow bepaald over de lever en de nier. Het bleek dat ammoniak alleen werd geproduceerd door de nieren en dat er geen netto ammoniak productie was over het splanchnische gebied. Uit beide studies werd geconcludeerd dat een toegenomen ammoniak productie door de nieren een belangrijke rol speelt in de hyperammonemie die volgt op een HTD bloeding bij patiënten met levercirrhose. Tijdens deze situatie van hyperammonemie was de spier het belangrijkste ammoniak consumerende orgaan.

In Hoofdstuk 3A worden de metabole gevolgen beschreven van een andere $\mathrm{HE}$ inducerende klinische situatie. In deze studie wordt de belangrijke rol van de nier in ammoniak homeostase beschreven tijdens de plaatsing van een TIPSS in patiënten met levercirrhose. Dertien patiënten met levercirrhose werden bestudeerd 1 uur voor en 1 uur na plaatsing van een TIPSS. In deze patiënten werd bloed afgenomen uit de vena porta, hepatica, de rechter vena renalis en de vena femoralis. Vóór plaatsing van de TIPSS bleek de efflux van ammoniak uit de nier vene significant groter te zijn dan de hoeveelheid ammoniak die door de lever werd afgegeven. De hoeveelheid ammoniak die door de lever werd afgeven was niet significant verschillend van nul. TIPSS plaatsing veranderde de arteriële ammoniak concentratie en de hoeveelheid ammoniak die 
door de lever werd afgegeven niet, maar de hoeveelheid ammoniak die door de nier werd afgegeven reduceerde significant, tot hoeveelheden die niet significant verschilden van nul. De opname van de ammoniagene aminozuren glutamine en glycine door de nier werd niet beïnloed door de TIPSS plaatsing. Dit suggereert een toename in ammoniak excretie via de urine (hetgeen echter niet gemeten werd in deze studie). Het veneuze minus arteriële concentratie verschil van ammoniak over de spier nam toe na plaatsing van de TIPSS. Na TIPSS plaatsing was de geschatte hoeveelheid ammoniak die via porta-systemische shunts in de systemische circulatie wordt afgegeven 7 keer hoger dan de hoeveelheid die door de nier werd afgegeven. De nieren bleken in staat om snel na plaatsing van de TIPSS te veranderen van een orgaan dat netto ammoniak afgeeft aan de systemische circulatie in een orgaan dat geen ammoniak meer afgeeft. Deze aanpassing van de nier zou her mechanisme kunnen zijn waarmee de patiënt met levercirrhose de ammoniak homeostase handhaaft na portasystemische shunting.

In Hoofdstuk 3B beschrijven we de effecten van plasma volume expansie op de ernst van $\mathrm{HE}$, plasma ammoniak en urine ammoniak excretie in patiënten met diuretica-geïnduceerde HE. Vijftien patiènten met alcoholische lever cirrhose en diureticageinduceerde HE werden geincludeerd. In 8 patiënten werd humaan albumine oplossing gebruikt voor volume expansie en in 7 patiënten werden colloiden gebruikt. Een significante verbetering van $\mathrm{HE}$ gradatie trad alleen op in de groep die was behandeld mer humaan albumine. In beide groepen waren er vergelijkbare verminderingen in plasma ammoniak concentraries, plasma renine activiteit en angiotensine II en was er een vergelijkbare toename in de gemiddelde arteriële bloeddruk, nier plasma flow en ammonia uitscheiding via de urine. Plasma malondialdehyde, een merkstof voor oxidatieve stress, was initieel verhoogd in beide groepen, maar daalde in de groep patiënten die werden behandeld met humaan albumine oplossing. De resultaten van deze studie toonden aan dat plasma volume expansie leidt tot een significante verlaging van de arterièle ammoniak concentratie. Dit geschiedt waarschijnlijk via een toegenomen ammoniak excretie via de urine. Deze verlaging van de ammoniak concentratie resulteerde alleen in een verbetering van de $\mathrm{HE}$ gradering in die patiënten die met humaan albumine werden behandeld en waarin een reductie in oxidatieve stress werd gezien. Deze resultaten ondersteunen de hypothese dat andere factoren, zoals oxidatieve stress, interacteren met ammoniak in de pathogenese van diuretica-geïnduceerde $\mathrm{HE}$, en suggereren een potentiële rol voor albumine infusie bij de behandeling van deze patiënten.

In Hoofdstuk 4 beschrijven we twee studies waarin het model om een HTD bloeding te simuleren, middels een intragastrisch toegediende aminozuur oplossing die lijkt op het hemoglobine molecuul, wordt gebruikt om de pathofysiologie van $\mathrm{HE}$ te bestuderen.

In Hoofdstuk 4A testten we de hypothese dat een gesimuleerde HTD bloeding leidt tot een verminderd neuropsychologisch functioneren en een reductie in regionale cerebrale doorbloeding. Acht metabool stabiele patiënten met cirrhose van de lever 
werden bestudeerd vlak voor en 4 uur na de toediening van de oplossing. De neuropsychologische test bestond uit de Trails B Test, Digit Symbol Substitution Test, de geheugen subtest van de Randt test en een reactie tijd test. Bloed werd afgenomen voor de bepaling van ammoniak en aminozuren. Regionale cerebrale perfusie werd gemeten met behulp van een head-SPECT-scanner. De resultaten lieten zien dat een gesimuleerde HTD bloeding leidt tot hyperammonemie, hypoisoleucinemie en tot een significante vermindering in neuropsychologisch functioneren. Analyse van de SPECT gegevens liet zien dat de gesimuleerde HTD bloeding leidde tot een significant verminderde cerebrale perfusie in beide temporaal kwabben, de linker gyrus frontalis superior en de rechter gyrus parietale en cingulatus. We concludeerden uit deze studie dat een simulatie van de metabole gevolgen van een HTD bloeding in patënten met cirrhose van de lever een interessant nieuw model is om de pathofysiologie van $\mathrm{HE}$ te bestuderen.

In Hoofdstuk 4B wordt hetzelfde model gebruikt om de gevolgen met betrekking tor neurospychologische tests, intracerebrale glutamine concentratie en magnetisatie transfer ratio (MTR) te bestuderen. Achtenveertig metabool stabiele patiënten met levercirrhose die geen kenmerken vertoonden van $\mathrm{HE}$ werden gerandomiseerd tussen de gesimuleerde bloeding en placebo toediening. Metingen werden verricht vlak vóór en 4 uur na toediening van de oplossing. De neurospychologische test bestond uit de Trails B Test, Digit Symbol Substitution Test, de geheugen subtest van de Randt test en een reactie tijd test. Bloed werd afgenomen voor bepaling van ammoniak en aminozuren. In de eerste 16 patiënten werd het hersenmetabolisme bestudeerd middels proton magnetische resonantie (MR). In 7 andere pariënten werden de effecten op MTR bestudeerd. De arteriële ammoniak concentratie steeg significant na de gesimuleerde HTD bloeding en bleef gelijk in de placebo groep. Het neuropsychologisch functioneren verbeterde significant in de placebo groep, in tegenstelling tot de gesimuleerde HTD bloeding groep. Na de gesimuleerde HTD bloeding was er een significante toename van de intracerebrale glutamine/glutamate/creatine ratio en een significante afname in MTR. Dit suggereert dat een toename in intracerebraal water een van de pathofysiologische mechanismen is waardoor $\mathrm{HE}$ ontstaat in patiënten met levercirrhose. We concludeerden uit deze studie dat een gesimuleerde HTD bloeding bij patiënten met lever cirrhose leidt tot een vermindering in neuropsychologisch functioneren, waarschijnlijk tengevolge van een intracerebrale glutamine accumulatie en gerelateerde intracerebrale zwelling.

In hoofdstuk 5 wordt de hyporhese geïntroduceerd dat de ammoniagene en katabole gevolgen van een HTD bloeding worden veroorzaakt door de afwezigheid van het essentiële aminozuur isoleucine in het hemoglobine molecuul. Het hemoglobine molecuul is uniek omdat het essentiële vertakte keten aminozuur isoleucine mist en een grote hoeveelheid van de twee andere vertakte keten aminozuren, leucine en valine bevat. Een HTD bloeding heeft daardoor tot gevolg dat de darmen een eiwit van zeer 
lage biologische waarde aangeboden krijgen, hetgeen een stimulus is om een cascade van biologische processen te induceren die uiteindelijk resulteren in netro eiwit afbraak. Netto eiwit afbraak is nadelig voor het functioneren van sneldelende cellen, zoals cellen betrokken bij het immuunproces, en van eiwitten met een korte halfwaardetijd. In dit hoofdstuk onderbouwen wij deze hypothese en poneren we een oplossing om deze ammoniagene en katabole cascade te voorkomen door intraveneuze infusie van het essentiële aminozuur isoleucine.

In Hoofdstuk 6 wordt de hypothese getest dat een HTD bloeding resulteert in hypoisoleucinemie door de inductie van vertakte keten aminozuur antagonisme en daardoor resulteert in verminderde eiwitsynthese. Wij bestudeerden 5 gevaste, metabool stabiele patiënten met cirrhose van de lever. De patiënten kregen een geprimed, continue intraveneuze infusie van het stabiele isotoop $[1-13 \mathrm{C}]$ isoleucine en een priming dosis van $\left[{ }^{13} \mathrm{C}\right]$ bicarbonaat zodat het mogelijk was om isoleucine oxidatie en eiwitsynthese te bepalen. Het protocol bestond uit een 4 uur durende periode waarin intragastrisch fysiologisch zout werd gegeven gevolgd door een 4 uur durende periode waarin een HTD bloeding werd gesimuleerd door intragastrische toediening van een aminozuur mengsel met een samensteling identiek aan het hemoglobine molecuul. Deze gesimuleerde HTD bloeding resulteerde in een significante daling van de arteriële isoleucine concentratie (tot $26 \%$ van de uitgangsconcentratie) en een signifcante stijging van de arteriële concentraties van de vertakte keten aminozuren leucine $(400 \%)$ en $(350 \%)$. Zowel de flux als de oxidatie van isoleucine verminderden tot eenderde van de uitgangswaarde als gevolg van de gesimuleerde HTD bloeding. Hierdoor veranderde de fractie van de isoleucine flux, die gebruikt wordt voor oxidatie, niet tengevolge van de gesimuleerde HTD bloeding. Als gevolg hiervan daalde de niet-oxidatieve fractie van de isoleucine flux, een maat voor de eiwitsynthese, tot eenderde van de uitgangswaarde. Het feit dat dit niet leidde tor een relatieve daling van de oxidatie van isoleucine kan alleen maar verklaard worden door het optreden wan vertakte keten aminozuur antagonisme.

In Hoofdstuk 7 beschrijven we de gevolgen van een gesimuleerde HTD bloeding op de eiwit kinetiek in patiënten met cirrhose van de lever en de mogelijke therapeutische effecten van intraveneuze infusie van isoleucine tijdens deze interventie. We kwantificeerden de eiwit kinetiek over de portaal drainerende organen, de lever, de beenspieren en de nier gedurende de gesimuleerde bloeding. Zestien overnacht gevaste, metabool stabiele patiënten kregen gedurende een 4 uur durende periode een intragastrische infusie van een aminozuur mengsel met een samenstelling identiek aan het hemoglobine molecuul en werden gerandomiseerd om intraveneus fysiologisch zout of intraveneus isoleucine te krijgen. Gedurende de studie werd een mix van stabiele isotopen gegeven om op orgaan niveau eiwitsynthese en afbraak te bepalen. De gesimuleerde HTD bloeding resulteerde in hyperammonemie in beide groepen en in hypoisoleucinemie in de fysiologisch zout groep, dat werd voorkomen in de isoleucine 
interventie groep. Isoleucine infusie stimuleerde eiwit synthese in de lever en de spier. In de controle groep was deze normale respons op een aminozuur maaltijd onderdrukt. Eiwit kinetiek in de portal drainerende organen en de nier werd niet beïnvloed door het studie protocol. Isoleucine infusie had geen invloed op de post-haemorrhagische hyperammonemie, die voornamelijk werd veroorzaakt door de toegenomen ammoniak productie in de nier. Wij concludeerden uit deze studie dat infusie van isoleucine gedurende een gesimuleerde HTD bloeding in patiënten met cirrhose van de lever de verstoorde eiwitsynthese in de lever en de spier herstelt en resulteert in een netto eiwit aanmaak in deze organen. Verwolg onderzoek is nodig om de therapeutische waarde van isoleucine infusie in patiënten met een acute HTD bloeding te onderzoeken. Dit onderzoek zou zich moeten richten op de effecten van isoleucine infusie op stollingsfunctie en infectie incidencie of op substituut eindpunten als aanmaak snelheid en functie van stollingsfactoren en sneldelende cellen die betrokken zijn bij de immuuunrespons.

In Hoofdstuk 8 onderzoeken we de acute effecten van een TIPSS plaatsing op het ammoniak en aminozuur metabolisme van de hersenen van patiënten met cirrhose van de lever. Tevens bestudeerden we de effecten op de cerebrale doorbloeding en de NO productie van het gehele lichaam. Twaalf patiënten die een TIPSS insertie ondergingen vanwege bloedende oesophagus varices werden bestudeerd. Cardiovasculaire haemodynamiek werd gemonitoord middels een Swan-Ganz katheter en cerebrale bloedflow (CBF) werd gemeten met de Kety-Schmidt techniek. NO metabolisme werd gemeten met behulp van stabiele isotopen (L-[guanidino- $\left.{ }^{15} \mathrm{~N}_{2}\right]$ Arginine en $\mathrm{L}-\left[\right.$ ureido- ${ }^{13} \mathrm{C} ; 5,5$ $\left.{ }^{2} \mathrm{H}_{2}\right]$ Citrulline). TIPSS insertie resulteerde in een significante vermindering van de gemiddelde arteriële bloeddruk, een significante stijging in cardiac output en CBF. Er werden geen significante veranderingen waargenomen in de arteriële ammoniak concentratie, maar de opname van ammoniak door de hersenen nam significant toe. Tevens was er een significante toename van de productie van $\mathrm{NO}$, welke correleerde met de veranderingen in gemiddelde arteriële bloeddruk, cardiac output en CBF. We concludeerden dat een TIPSS plaatsing resulteert in een significante stijging van de CBF, welke waarschijnlijk resulteert in een toegenomen ammoniak opname door de hersenen. De data van de studie ondersteunen tevens de hypothese dat een TIPSS geïnduceerde toename van de CBF wordt gemedieerd door een toename van de NO productie. Het exacte mechanisme van de gestimuleerde NO productie is niet geheel duidelijk, maar de data van onze studie ondersteunen een rol voor een toegenomen ammoniak opname door de hersenen en een TIPSS-geïnduceerde toename van de cardiac output welke leidt tot een toename in shear stress.

Hoofdstuk 9 beschrijft de rol van ontstekingsmediatoren in de pathofysiologie van gestegen intracraniële druk (ICP) in patiënten met ALF en de correlatie met CBF en ammoniak metabolisme. 21 patiënten met ALF werden bestudeerd vanaf het moment dat ze beademd werden vanwege hepatisch coma, tot het moment dat ze specifieke 
behandeling ondergingen voor gestegen intracraniële druk. Afhankelijk van de initieel gemeten intracraniële druk werden de patiënten ingedeeld in twee groepen: patiënten die specifieke behandeling behoefden voor verhoogde intracraniële druk (ICP>20mmHg, Groep 1: $n=8$ ) en patiënten waarbij dit niet nodig was (ICP£20 $\mathrm{mmHg}$, Groep 2: $\mathrm{n}=13$ ). Patiënten in Groep 1 hadden significant verhoogde waarden van ontstekingsmediatoren en een verhoogde plasma ammoniak concentratie. In die groep was er een significante correlatie tussen TNFa concentraties en CBF ( $r=0.80$ ). Vier patiënten uit groep 2 ontwikkelden uitbraken van verhoogde intracraniële druk welke waren geassocieerd met toenamen in de CBF, ontstekingsmediatoren en TNFa. Er werd geen verandering waargenomen in deze markers, CBF en/of ICP in de andere 9 patiënten van deze groep. De resultaten van deze studie ondersteunen een "multiplehit' hypothese voor de etiologie van intracraniële hypertensie in patiënten met cirrhose van de lever. Hierbij is een toename in ammoniak opname door de hersenen de initiële stimulus, welke resulteert in cerebraal oedeem, maar worden de plotselinge stijgingen in de intracraniële druk veroorzaakt door een toename in cerebrale hyperemie. Cerebrale hyperemie wordt waarschijnlijk veroorzaakt door de geactiveerde werking van vasodilatoren welke gestimuleerd worden door de systemische inflammatoire respons en/of door een ammoniak geïnduceerde glutamine accumulatie in de hersenen.

Hoofdstuk 10 beschrijft de rol van gematigd koelen van het gehele lichaam als behandeling voor oncontroleerbaar gestegen verhoogde intracraniële druk bij patiënten met acuut leverfalen (ALF). We bestudeerden zeven achtereenvolgende patiënten met ALF welke voldeden aan de criteria van slechte prognose volgens het Kings College. De patiënten hadden verhoogde intracraniële druk welke niet reageerde op 2 behandelingen met mannitol en dialyse. Vier van deze patiënten waren tevens kandidaat voor acute orthotope levertransplantatie (OLT). De centrale lichaamstemperatuur werd verlaagd met behulp van koeldekens tot $32-33^{\circ} \mathrm{C}$. De vier patiënten welke kandidaat waren voor OLT werden succesvol behandeld tot transplantatie, waarbij ze gemiddeld 13 uur (spreiding 10-14 uur) werden gekoeld. De drie patiënten die ongeschikt werden geacht voor OLT overleden na opwarming. Koelen leidde tot een opmerkelijke daling van de ICP en CBF en een stijging van de cerebrale perfusie druk. Tijdens koelen was er geen terugval naar verhoogde intracraniële hersendrukken. Koelen leidde tevens tot een daling van de arteriële ammoniak concentratie en de opname van ammoniak door de hersenen. Er werden geen nadelige gevolgen van koelen waargenomen. We concludeerden uit deze studie dat gematigd koelen van het gehele lichaam een nuttige en veilige behandeling is voor ongecontroleerd verhoogde intracraniële druk bij patiënten met ALF en dat het kan helpen om de tijd tot OLT te overbruggen.

In Hoofdstuk 11 bestuderen we de hypothese dat gematigd koelen van het gehele lichaam bij patiënten met ALF een vermindering van de intracraniële druk geeft doordat het de autoregulatie van CBF herstelt. Negen patiënten met een oncontroleerbaar verhoogde intracraniële druk en ALF werden bestudeerd. Direct voor aanvang van het 
koelen en 4 uur nadien werd de CBF autoregulatie en de reactiviteit op $\mathrm{CO}_{2}$ bepaald. We observeerden significante verlagingen van de ICP en CBF. Voordat gekoeld werd was er geen $\mathrm{CBF}$ autoregulatie in de bestudeerde pariënten. Toepassing van koelen als therapie herstelde dit bij alle bestudeerde patiënten. De resultaten van deze studie suggereren dat de verbetering van ICP door koelen van het gehele lichaam bij patiënten met ALF mogelijk her gevolg is van het herstellen van de autoregulatie van de CBF.

In Hoofdstuk 12 beschrijven we het effect van gematigd koelen wan het gehele lichaam op de intracraniële druk van patienten met ALF welke een orthotope lever transplantatie (OLT) ondergaan. Het is bekend dat gedurende een OLT sommige pariënten met ALF een acuut verhoogde intracraniële druk krijgen. Wij onderzochten de hypothese dat dit zou kunnen worden vóórkomen door de patiënten tijdens de OLT te koelen. Zestien patiënten met ALF werden bestudeerd. Afhankelijk van de gemeten intracraniële druk vóór OLT werden de patiënten in drie groepen ingedeeld. Groep I $(n=6)$ : patiënten welke geen therapie behoefden voor verhoogde intracraniële druk $(\mathrm{ICP}<15 \mathrm{mmHg})$. Groep II $(\mathrm{n}=5)$ : patiënten welke episoden van verhoogde intracraniële druk hadden doorgemaakt, maar konden worden behandeld met conventionele therapie. De patiënten uit deze beide groepen werden normothermisch gehouden tijdens de OLT. Groep III $(n=5)$ bestond uit patiënten welke een ongecontroleerd verhoogde intracraniële druk hadden voorafgaand aan de OLT, waarvoor ze gematigd koelen als therapie ondergingen, zowel voorafgaande aan als gedurende de OLT. Er was een significante stijging van de intracraniële druk tijdens de dissectie en reperfusie fase van de OLT in de patiënten uit groep I en II. Patiënten uit groep III lieten geen verandering in intracraniële druk zien tijdens de OLT. De stijging in intracraniële druk in groep I en II was geassocieerd met een toename van de CBF, welke niet werd geobserveerd in groep IT. Tijdens de anhepaxische fase van de OLT normaliseerde de intracranele druk in groep I en II. Er was geen verschil in transfusie behoefte of infecties tussen de groepen. We concludeerden dat gematigd koelen een veilige en succesvolle methode is om stijgingen in intracraniële hersendruk tijdens een OLT bij patiênten met ALF te voorkomen, en dan metname tijdens de dissectie en reperfusie fase tijdens de operatie. De beschermende effecten van koelen worden waarschijnlijk veroorzaakt door een verminderde behoefte aan substraat en zuurstof, waardoor het substraat aanbod en/of verbruik in de hersenen beter aansluit aan de behoefte, welke verstoord is bij patiënten met ALF.

Hoofdstuk 13 besluit met een evaluatie van de klinische effecten en de pathofysiologische mechanismen van gematigd koelen van het gehele lichaam bij patiënten met ALF, welke een oncontroleerbaar verhoogde intracraniële druk ontwikkelen die resistent is tegen conventionele therapie. We bestudeerden veertien patiënten met ALF die op de wachtijst stonden voor een acute OLT en die een oncontroleerbaar verhoogde intracraniele druk hadden die niet reageerde op 2 behandelingen met mannitol en/of dialyse. De lichaamstemperatuur werd verlaagd met behulp van koeldekens 
tot $32-33^{\circ} \mathrm{C}$. Dertien patiënten overbrugden de wachtperiode tot OLT succesvol met een mediane koelingduur van 32 uur (spreiding 10-118 uur). De patiènten ondergingen de OLT zonder significante koelinggerelateerde complicaties en herstelden zonder neurologische gevolgen. De ICP voor aanvang van het koelen was $36.5(2.7) \mathrm{mmHg}$ en daalde na 4 uur koelen naar $16.3(0.7) \mathrm{mmHg}$, welke waarde gehandhaafd bleef na 24 uur $(16.8(1.5) \mathrm{mmHg})(\mathrm{p}<0.0001)$. Ondanks koelen stierf 1 patiënt aan de gevolgen van cerebrale herniatie. De gemiddelde arteriële bloeddruk en cerebrale perfusie druk steeg significant, terwijl er een significante afname was in inotropie behoefte. Gematigd koelen leidde tot een aanhoudende verlaging van de arteriële ammoniak concentratie en ammoniak opname door de hersenen. Tevens verminderde de CBF, de cerebrale cytokine productie en indicatoren van oxidatieve stress. We concludeerden uit deze studie dat gematigd koelen van het gehele lichaam een effectieve en veilige methode is om de tijd tot OLT te overbruggen bij patiënten met ALF en een ongecontroleerde verhoogde intracraniële druk welke resistent is tegen conventionele therapie. 
औ क स 


\section{SUMMARY}

This thesis comprises three major sections. The first section (Chapters 1-4) deals with the interorgan metabolism of ammonia and related amino acids in patients with cirrhosis of the liver and with the metabolic and neuropsychological consequences of an upper GI bleed. The second section (Chapters 5-7) deals with the consequences of an upper GI bleed on isoleucine and protein metabolism and the possible therapeutic role of isoleucine. In the third section (Chapters 8-13) the pathophysiological basis of HE in patients undergoing TIPSS insertion, is described with special emphasis on alterations in cerebral blood flow and nitric oxide metabolism. In subsequent chapters in this section the interrelationship is explored between cerebral metabolism and haemodynamics in patients with ALF. Furthermore, the clinical and parhophysiological basis of intervention with moderate hypothermia in these patients is investigated.

In Chapter 1 the quantitative dynamics of interorgan ammonia and amino acid metabolism are described in 24 metabolically stable patients with cirrhosis who underwent TIPSS portography. Blood was sampled and blood flows measured across portal drained viscera, leg, kidney and liver, and arterio-venous differences across the spleen and the inferior and superior mesenteric veins. We found that ammonia was predominantly produced by the portal drained viscera. The kidneys also produced ammonia, in amounts that equalled total hepato-splanchnic area production. Skeletal muscle removed more ammonia than the cirrhotic liver. However, the amount of nitrogen that was taken up by muscle in the form of ammonia was less than the amount of glutamine released. This excess in glutamine released in the circulation, can, after irs degradation, lead to ammonia production in the PDV and kidneys. The portal drained viscera consumed glutamine and produced ammonia, alanine and citrulline. Urea was released in the splenic and superior mesenteric vein, contributing to whole body ureagenesis in these cirrhotic patients. We concluded from this study that hyperammonemia in metabolically stable, overnight fasted patients with cirrhosis of the liver and a TIPSS results from portosystemic shunting and renal ammonia production.

In Chapter 2 the effects of an upper GI bleed on interorgan ammonia metabolism are described. An upper GI bleed is one of the clinically most important ammonia liberating events leading to $\mathrm{HE}$ in patients with cirrhosis of the liver. In this chapter two studies are described. In the first study an upper GI bleed was simulated in 8 patients with cirrhosis and a TIPSS, by intragastric infusion of an amino acid solution that mimics the amino acid composition of the haemoglobin molecule. We sampled blood at regular intervals from a femoral artery and from a femoral, renal, portal and hepatic vein for 4 hours during the simulated bleed and measured plasma flows across these 
organs. During the simulated bleed arterial concentrations of ammonia increased significantly. There was no change in ammonia production from the portal drained viscera but renal ammonia production increased 6-fold. In contrast to an unchanged ammonia removal by the liver, a significant increase in muscle ammonia removal was observed. In the second study we studied 9 cirrhotic patients with an acute upper GI bleed that underwent acute TIPSS insertion. Blood was sampled from an artery, a hepatic, renal and portal vein, and plasma flows were measured. We showed that ammonia was only produced by the kidneys and not by the splanchnic area. We conduded from these studies that enhanced renal ammonia release plays an important role in the hyperammonemia that follows an upper GI bleed in patients with cirrhosis. During this hyperammonemic state, muscle was the major site of ammonia removal.

In Chapter 3A the consequences of another major clinical event that induces $\mathrm{HE}$ was described. This study highlights the important role of the kidney in ammonia homeostasis after portasystemic shunting in patients with cirrhosis. Thirteen parients with cirrhosis of the liver were studied prior to and one hour after TIPSS insertion. In these patients blood was sampled from the portal vein, a hepatic vein, the right renal vein and the right femoral vein. We observed that prior to TIPSS, renal ammonia release was significantly higher than ammonia release from the hepatosplanchnic area, which was not significantly different from zero. TIPSS insertion did not change the arterial ammonia concentration or ammonia release by the hepatosplanchnic area, but reduced ammonia release in the renal vein significantly, to values that were not different from zero. The uptake of the ammoniagenic amino acids glutamine and glycine was not changed by TIPSS, suggesting that the obligatory ammonia production resulting from the uptake of glutamine and glycine is excreted in the urine. TIPSS resulted in an increased venous minus arterial ammonia concentration difference across the leg muscle. The post-TIPSS estimate of ammonia efflux via portasystemic shunts was 7 times higher than renal efflux. We concluded from this study that the kidneys have the ability to switch from an organ that produces net systemic ammonia release to an organ with no significant systemic ammonia release immediately after TIPSS insertion. This may be the mechanism by which the cirhotic patient maintains ammonia homeostasis after portasystemic shunting.

In Chapter $\mathbf{3 B}$ we describe the effects of plasma volume expansion on the severity of HE, plasma and urinary ammonia in patients with diuretic-induced $\mathrm{HE}$. Fifteen parients with alcoholic cirrhosis and diuretic-induced HE were enrolled. In 8 patients, human albumin solution was used for volume expansion and in 7 patients colloid was used. Significant improvement of HE grade was observed only in the group treated with human albumin solution. There were similar reductions in plasma ammonia concentration, plasma renin activity and angiotensin II, and an increase in mean arterial pressure, renal plasma flow and urinary ammonia excretion, in both groups. Plasma malondialdehyde, a marker of oxidative stress, was elevated in both groups but was sig- 
nificantly reduced only in the group treated with human albumin solution. The results of this study showed that plasma volume expansion results in significant reduction in plasma ammonia concentration possibly through an increase in urinary ammonia excretion. This reduction in ammonia concentration translates into an improvement in mental state only in those patients treated with human albumin solution in whom concomitant reduction in oxidative stress was observed. These data support the notion that other factors such as oxidarive stress act as adjuncts to ammonia in the pathogenesis of diuretic-induced $\mathrm{HE}$ and suggest a possible role for albumin infusion in its treatment, possibly by virtue of its potential scavenging properties.

In Chapter 4 two studies are described in which we use the model of simulating an upper GI bleed to study neuropsychological changes and intracerebral metabolic and bloodflow changes.

In Chapter 4A we tested the hypothesis that administration of an oral amino acid load mimicking haemoglobin in patients with cirrhosis of the liver produces deterioration in neuropsychological function and a reduction in regional cerebral perfusion. Eight overnight fasted, metabolically stable cirrhotic patients with no evidence of overt hepatic encephalopathy were studied prior to and 4 hours after simulating the upper GI bleed. Neuropsychological function was measured using a neuropsychological test battery and venous blood was collected for ammonia and amino acid determinations. Regional cerebral perfusion was measured using a head SPECT scanner. The results showed that the oral amino acid load mimicking haemoglobin induced hyperammonemia, hypoisoleucinemia and caused a significant deterioration in memory tests, probably due to a reduction in regional cerebral perfusion. SPECT analysis showed a significant reduction in cerebral perfusion after the simulated bleed in both temporal lobes, left superior frontal gyrus and, right parietal and cingulate gyrus. We therefore concluded that simulating the metabolic effects of an upper GI bleed in patients with cirrhosis of the liver is a useful new model to study the metabolism of $\mathrm{HE}$ and also point to the importance of ammonia-induced reduction in cerebral perfusion in the pathogenesis of HE in cirthosis.

In Chapter $\mathbf{4 B}$ the model of simularing an upper GI bleed was used to study neuropsychological changes, brain glutamine levels and brain magnetization transfer ratio (MTR). Forty-eight, metabolically stable patients with cirrhosis and no evidence of 'overt' HE were randomised to receive the amino acid solution or saline as placebo and measurements were performed prior to and 4 hours afterwards. Neuropsychological function was tested using the same test-battery as used in chapter 4a. Plasma was collected for ammonia and amino acid measurements and brain metabolism was studied using proton magnetic resonance spectroscopy in the first 16 of the randomised patients. In 7 other patients MTR were measured. Administration of the amino acid solution induced hyperammonemia, which was absent in the placebo group. The study showed an improvement in neuropsychological tests following placebo, which was not 
observed in patients that received the amino acid solution. The hyperammonemia induced, resulted in an increased brain peak of glutamate and glutamine (which could not separated), an increased the glutamate.glutamine/creatine ratio and a decrease in MTR, which indicates the presence of an increase in brain water. We concluded from this study that a simulated upper GI bleed impaired neuropsychological function by intracerebral glutamine accumulation and consequent brain swelling. This study has provided the first direct evidence in patients with cirrhosis for the ammonia-glutaminebrain swelling hypothesis of $\mathrm{HE}$.

In Chapter $\mathbf{5}$ we introduce the hypothesis that an upper GI bleed is an ammoniagenic and catabolic event due to the absence of isoleucine in the haemoglobin molecule. "The haemoglobin moleculle is unique because it lacks the essential amino acid isoleucine and has high amounts of leucine and valine. Upper GI bleeds therefore present the gut with protein of very low biologic value, which may be the stimulus to induce a cascade of events culminating in net carabolism which may influence the function of rapidly dividing cells and short half-life proteins. We hypothesise that following an upper GI bleed in a cirrhotic patient, the lack of isoleucine in blood protein is the cause of hyperammonemia and catabolism. We propose that intravenous administration of isoleucine may serve as a simple therapeutic intervention to reverse this cascade.

In Chapter 6 studies are described in which the above mentioned hypothesis is tested, that an upper GI bleed produces hypoisoleucinemia by inducing BCAA antagonism, which would lead to diminished protein synthesis. We studied five overnight fasted, metabolically stable patients with cirrhosis that received a primed, continuous intravenous infusion of $[1-13 \mathrm{C}]$ isoleucine to study isoleucine turnover and oxidation. The protocol consisted of a 4 hour period of intragastric saline infusion followed by a 4 hout period in which an upper GI bleed was simulated. We showed that the simulated upper GI bleed produced a significant reduction in arterial isoleucine concentration (26\% of initial values) and an increase in leucine ( $400 \%)$ and valine $(350 \%)$ concentrations. Isoleucine flux and isoleucine oxidation decreased to a third of initial values following a simulated bleed, but the fraction of isoleucine flux used for oxidation did not change after the simulated bleed. Consequently, the non-oxidative portion of isoleucine flux, representing protein synthesis, decreased similarly. We concluded from these results that a simulated upper GI bleed induces hypoisoleucinemia and decreases protein synthesis markedly. The fact that the percentage of isoleucine flux that was oxidized was not influenced by the hypoisoleucinemic state pointed to the occurrence of BCAA antagonism. The results of this study therefore supported the isoleucine hypothesis outlined in Chapter 5 .

In Chapter 7 we investigated the consequences of a simulated upper GI bleed on protein kinetics in patients with cirrhosis of the liver and the possible therapeutic effects of intravenous infusion of isoleucine. Portal drained viscera, liver, muscle and kidney protein kinetics were quantified. Sixteen overnight fasted, metabolically stable 
patients received a 4 hourly, intragastric infusion of the amino acid solution that mimicked haemoglobin and were randomised to receive either intravenous saline or isoleucine. A mixture of stable isotopes was administered to determine organ protein breakdown and synthesis rates. The simulated bleed resulted in hyperammonemia in both groups and in hypoisoleucinemia that was attenuated by isoleucine infusion. Isoleucine infusion stimulated liver and muscle protein synthesis. In the control group not supplemented with isoleucine, this normal response to the administration of an amino acid mixture was impaired. Protein kinetics in the portal drained viscera and the kidney were not influenced by the study protocol. Isoleucine infusion did not diminish post-bleeding hyperammonemia, which was predominantly kidney related. We concluded that infusion of isoleucine during a simulated bleed in patients with cirrhosis of the liver restores impaired protein synthesis of liver and muscle leading to a net anabolic state in these organs. Improving liver protein synthesis during an acute upper GI bleed in patients with cirrhosis of the liver may have major clinical benefits, since liver protein synthesis is of pivotal importance in generating adequate cellular immunity, an acute phase response and normal clotting function.

In Chapter 8 we investigated the acute effect of TIPSS insertion on brain ammonia and amino acid metabolism and we wanted to determine whether TIPSS insertion would result in an increase in cerebral blood flow (CBF). We also measured the rate of whole body nitric oxide (NO) production. Twelve patients with cirrhosis who were undergoing TIPSS insertion for variceal bleeding were included. Cardiovascular haemodynamics were measured using a Swan-Ganz catheter and CBF was measured using the Kety-Schmidt technique. Blood was sampled from an artery and a reverse jugular catheter for measurement of ammonia, amino acids and pro-inflammatory cytokines. NO production was measured using the stable isotope technique using a primed, continuous infusion of L- $\left[\right.$ guanidino- $\left.{ }^{15} \mathrm{~N}_{2}\right]$ Arginine and L-[ureido- ${ }^{13} \mathrm{C} ; 5,5-$ $2 \mathrm{H}_{2}$ ] Citrulline. TIPSS insertion resulted in a significant reduction in mean arterial pressure and an increase in cardiac output and CBF. No significant changes were observed in the arterial ammonia but the brain flux of ammonia increased significantly without any significant changes in brain glutamine flux. Whole body NO production increased significantly. The change in NO production correlated with the changes in mean arterial pressure, cardiac output and CBF. In conclusion, the results of our study show that insertion of TIPSS results in an increase in CBF, which drives increased brain ammonia flux. Our results support the hypothesis that TIPSS induced increase in CBF is mediated by increased NO synthesis. The exact mechanism of the increase in NO synthesis is not entirely clear, but it could be related to the increased delivery of ammonia to the brain and the TIPSS-induced increase in cardiac output.

In Chapter 9 we studied the role of inflammation in the pathogenesis of increased ICP in patients with ALF and its interplay with CBF and ammonia. Twenty-one patients with ALF were studied from the time they were ventilated for grade 4 
encephalopathy until receiving specific treatment for increased ICP. Depending upon the ICP, the patients were divided into two groups; those that required specific treatment (ICP $>20 \mathrm{mmHg}$, Group 1: $\mathrm{n}=8$ ) and those that did not (ICP£20 $\mathrm{mmHg}$, Group 2: $n=13$ ). Patients in Group 1 had significantly greater evidence of inflammation and higher arterial ammonia concentrations. TNFa levels correlated with CBF $(r=0.80)$. Four patients from Group 2 developed surges of increased ICP that were associated with increases in CBF, markers of inflammation and TNFa. There was no change in these inflammatory markers, CBF or ICP in the other 9 patients in Group 2. In conclusion, our data support a multiple-hit hypothesis for the development of intrackanial hypertension in patients with ALF. Accordingly, brain ammonia uptake is possibly the initial stimulus that leads to cerebral oedema but the bursts of increased ICP is the result of cerebral hyperaemia. This hyperaemia may be the consequence of putative vasodilators operative during the systemic inflammatory response and/or resulting from ammonia-induced glutamine accumulation.

In Chapter 10 we investigated the role of hypothermia as a treatment of uncontrolled intracranial hypertension in patients with ALF. We studied seven consecutive patients with ALF who fulfilled the Kings College criteria for poor prognosis and had increased ICP that was unresponsive to 2 treatments with mannitol and ultrafiltration. Four of the patients studied were candidates for orthotopic liver transplantation $(\mathrm{OLT})$. Core temperature was reduced to $32-33^{\circ} \mathrm{C}$ using cooling blankets. The 4 patients who were candidates for OLT were successfully bridged to transplantation with a median of 13 (range 10-14) hours of hypothermia. The three patients who were unsuitable candidates for OLT died following rewarming. ICP and CBF reduced markedly following cooling, whereas cerebral perfusion pressure increased significantly following cooling. During hypothermia there was no significant relapse of increased ICP. Arterial ammonia and cerebral uptake of ammonia were significantly reduced with cooling. No adverse effects of hypothermia were observed. We concluded from this study that moderate hypothermia is useful in the treatment of uncontrolled increase in ICP in patients with ALF and may prevent brain death in the period necessary to prepare for OLT.

In Chapter 11 we describe investigations to test the hypothesis that moderate hypothermia reduced ICP by restoring CBF autoregulation. Nine patients with uncontrolled intracranial hypertension and ALF who fulfilled criteria for poor prognosis were studied. CBF autoregulation and reactivity to carbon dioxide were evaluated before and 4 hours after cooling $\left(32^{\circ} \mathrm{C}\right)$. Significant reductions were observed in the ICP and CBF. The defective $\mathrm{CBF}$ autoregulation and the absence of reactivity to carbondioxide that was observed in all patients was restored with cooling. The results of this study suggest that the improvement in ICP observed with hypothermia may be due to its effects upon $\mathrm{CBF}$ autoregulation and provides a tool to explore the mechanisms associated with the deranged CBF autoregulation in ALF. The results further point to the subgroup of patients with intracranial hypertension that may benefit from hypothermia. 
In Chapter 12 we describe the effect of hypothermia on ICP in patients with ALF undergoing liver transplantation (OLT). During OLT for ALF, some patients develop acute increases in ICP. We tested the hypothesis that increases in ICP during OLT for ALF can be prevented by moderate hyporhermia. Sixteen patients wirh ALF undergoing OLT were studied. Depending upon the measured ICP prior to OLT, the patients were divided into three groups. Group $I(n=6)$ did not require treatment for increased ICP (ICP $<15 \mathrm{mmHg})$. Group II $(\mathrm{n}=5)$ had episodes of increased ICP that were controlled by conventional treatment. Both these groups of patients were maintained normothermic during OLT. Group III $(n=5)$ had uncontrolled increased ICP prior to OLT for which they had been cooled and remained cooled during OLT. There was a significant increase in ICP during the dissection and reperfusion phases of the OLT in the patients in Groups I and II. Patients in Group III had no significant increase in ICP during the OLT. The rise in ICP in Groups I and II was associated with an increase in CBF which was not observed in Group III. In patients from Group I and II, the increase in ICP was corrected during the anhepatic phase of the operation. There was no difference in the requirement of transfusions or incidence of post-operative infections between the groups. In conclusion, the results of this study suggest that hypothermia prevents the increase in ICP that is encountered in patients with ALF undergoing OLT, particularly during the dissection and the reperfusion phases of the operation. These protective effects of hypothermia may be mediated by decreased requirement for substrates and oxygen, which can therefore be better satisfied during the derangements of cerebral metabolism which results from the products of the 'necrotic' liver.

In Chapter 13 we conclude with an evaluation of the clinical effects and the pathophysiologic basis of moderate hypothermia in patients with ALF who develop intracranial hypertension that is unresponsive to standard medical therapy. We studied fourteen patients with ALF who were awaiting OLT and had increased ICP that was unresponsive to 2 treatments with mannitol and ultrafiltration. Core temperature was reduced to $32-33^{\circ} \mathrm{C}$ using cooling blankets. Thirteen patients were successfully bridged to OLT with a median of 32 (range 10-118) hours of cooling. They underwent OLT' with no significant complications related to cooling either prior to or after OLT and made complete neurological recovery. ICP prior to cooling was 36.5 (2.7) $\mathrm{mmHg}$ and this was reduced to $16.3(0.7) \mathrm{mmHg}$ at 4 hours, which was sustained at 24 hours (16.8 (1.5) $\mathrm{mmHg}$ ). Despite cooling, 1 patient died from cerebral herniation. Mean arterial pressure and cerebral perfusion pressure increased significantly and the requirement for inotropic agents was reduced significantly. The effect of hypothermia on ICP are likely to be mediated through multiple mechanisms such as reduction in arterial ammonia, its brain uptake and metabolism, and its effects on $\mathrm{CBF}$ and brain cytokine production. In conclusion, the results of this study show that mild-moderate hypothermia can be used safely to bridge patients with ALF and uncontrolled intracranial hypertension to liver transplantation. 


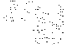

$\therefore$ 


\section{PERSPECTIVES FOR THE FUTURE}

\section{Observation:}

After an overnight fast the portal drained viscera are the major ammonia producing organs, which is mostly related to glutamine breakdown.

\section{Implication:}

Currently all standard clinical approaches aim to influence ammonia production of colonic bacteria that split urea. Our data provide insight into why these therapies, e.g. lactulose and non-absorbable antibiotics have litcle therapeutic effecr. Diminishing intestinal ammonia production that results from glutamine breakdown may be difficult since glutamine is an essential fuel source for the intestine. However, greater insight into the factors regulating intestinal glutaminase activity may provide novel therapeutic targets.

\section{Observation:}

Post-bleeding hyperammonemia in patients with cirrhosis of the liver is predominantly caused by enhanced renal ammonia production and not by enhanced intestinal ammonia production. Renal ammonia release into the circulation is acutely diminished by TIPSS insertion and an acute change in volume status increases urinary ammonia excretion with a simultaneous decrease in plasma ammonia levels.

\section{Implication:}

These findings change existing hypotheses. The kidney becomes a new therapeutic target for ammonia lowering therapies. From whole body nitrogen homeostasis it would be interesting to enhance renal glutamine breakdown into ammonia if the major part of this ammonia would be excreted into the urine. This would be an effective way to release circulating nitrogen via a non-toxic carrier (glutamine), since the notmal route of urea synthesis is impaired during liver failure. In this respect, the temporary buffer of ammonia metabolism to glutamine by muscle may be of value. "This observation is clinically relevant, since in the presence of a deficient ammonia detoxification by the cirrhotic liver, as generally assumed and shown in this dissertation, ammonia can only be detoxified by excretion into the urine. New therapies should therefore aim to increase ammonia excretion via the urine during hyperammonemia, ideally resulting in a negative renal v-a ammonia difference, showing net ammonia excretion across the kidney. For clinical practice the data indicate the important role of adequate volume status in patients with cirrhosis of the liver, give a clinical tool (volume expansion) and new insight (prevent dehydration, misuse of diuretics) to prevent and treat hyperammonemia induced hepatic encephalopathy in patients with cirrhosis of the liver. 


\section{Observation:}

Skeletal muscle from the leg removed more ammonia from the circulation than the cirrhotic liver in the basal state and after upper GI bleeding. Moreover, the upper GI bleeding studies showed that the liver was unable to increase ammonia detoxification.

\section{Implication:}

Muscle is the major ammonia removing organ during liver failure. Future research should determine if this serves as a temporary ammonia buffer, since more glutamine was released from muscle than ammonia taken up. Release of glutamine into the systemic circulation can lead to ammonia production elsewhere, more specifically in the PDV and kidney. However, as stated above, breakdown of glutamine by the kidney to ammonia and net excretion of this ammonia into the urine would imply that the muscle-kidney axis is responsible for net detoxification of ammonia. Furthermore, we found that glutamine release was partially accounted for by net protein catabolism. It therefore appears to have therapeutic benefit to prevent net protein catabolism and muscle wasting in cirrhotic patients by ensuring adequate intake. Future research should address the role of muscle mass in enhancing the ammonia buffering capacity of muscle and develop other approaches to increase muscle ammonia detoxification.

\section{Observation:}

Patients with cirrhosis of the liver exhibit net release of urea from the spleen and from the superior mesenteric vein draining viscera after an overnight fast.

\section{Implication:}

Peripheral urea production could be an important route of detoxifying ammonia in cirrhotic patients with a TIPSS. Further research is necessary to determine the quantitative rellevance of this observation.

\section{Observation:}

Simulation of an upper GI bleed induced minor deteriorations in neuropsychological function that was reflected in changes in regional cerebral perfusion, cerebral metabolism and water homeostasis. We presented the first evidence of ammonia-glutaminebrain swelling hypothesis in patients with cirrhosis.

\section{Implication:}

- We introduced a model of HE that is safe, well tolerated, reproducible and clinically relevant.

- It may therefore be a potent new research tool to study the pathophysiology of $\mathrm{HE}$ in cirrhosis. Recently, we have used this model to explore the role of inflammation in modulating the neuropsychological effects of hyperammonemia.

- Different components of the test-battery were affected differently suggesting that 
pathways subserving memory and learning were more significantly affecred by hyperammonemia than pathways involved in visuomotor coordination and vigilance. This gives direction to future studies.

- Our data suggest that the mechanism of ammonia induced alterations in mental state may be through its effects on cerebral perfusion. This opens new areas of research to understand the interactions between ammonia, glutamine and endothelial function.

- The first proof of existence of the ammonia-glutamine-brain swelling hypothesis reinforces that ammonia remains the central target for therapy of $\mathrm{HE}$.

\section{Observation:}

An upper GI bleed resulted in diminished whole body protein synthesis using the isoleucine tracer but not with the phenylalanine tracer. Infusing isoleucine improved liver and muscle protein synthesis during an upper GI bleed.

\section{Implication:}

Infusion of isoleucine in patients with cirrhosis of the liver during an upper GI bleed enhances the synthesis of proteins. This may have clinical implications since these patients are mostly undernourished and clinical deplete. Furthermore, during this acute illness there is an enhanced metabolic need to enable an adequate acute phase response and to produce clotting-factors. This should be tested in patients with an actual bleed. We are therefore planning to perform an intervention study in which patients will be randomised to receive continuous infusion of isoleucine or saline as placebo. Endpoints will be rebleeding, infection and mortality. Substitute endpoints potentially consist of the synthesis rate of clotting factors with a short half-life (e.g. factor 7).

\section{Observation:}

Our observation suggests that TIPSS-induced increased CBF is mediated by increased nitric oxide production, which is likely to be due to increased shear stress resulting from TIPSS induced increase in cardiac output. TIPSS insertion did not change arterial ammonia concentration or brain extraction, but increased ammonia uptake by the brain via the increase in $\mathrm{CBF}$, which may also increase $\mathrm{CBF}$.

\section{Implication:}

The results of this study provide the basis for developing the hypothesis that a "secondhit' may be important in the pathogenesis of $\mathrm{HE}$ and provides a more generally applicable framework for the operative mechanism. Our results have implications for prevention of the immediate cerebral complications of TIPSS insertion, particularly in patients with cirrhosis that have underlying sepsis and uncontrolled variceal bleeding and are susceptible to developing brain edema after TTPSS. 


\section{Observation:}

Patients with ALF that require treatment for uncontrolled increased ICP have significantly higher $\mathrm{CBF}$, elevated markers of inflammation and arterial ammonia concentrations than patients with lower ICP levels. Furthermore, patients that had initially lower ICP levels but developed surges of increased ICP showed increased CBF, increases in markers of inflammation but without a change in arterial ammonia concentration. In the patients that remained stable, no change in inflammatory markers was observed. Delivery of ammonia to the brain was a better predictor of uncontrolled ICP than arterial ammonia concentrations.

\section{Implications:}

Inflammation may be an important mediator of increased $\mathrm{CBF}$ and resulting ICP. The data support a multiple-hit hypothesis for the development of intracranial hypertension in patients with ALF. Accordingly, brain ammonia uptake is possibly the initial stimulus that leads to cerebral edema but the bursts of increased ICP is the result of cerebral hyperaemia which may be consequent on the action of putative vasodilators which are the result of the systemic inflammatory response and/or due to ammoniainduced glutamine accumulation. Consequently, the results provide a framework for the rational management of increased ICP in ALF and the basis for use of antibiotics, anti-inflammatory agents and treatments such as hypothermia.

\section{Observation:}

Institution of moderate hypothermia in patients with ALF that have uncontrolled ICP, reduces ICP, CBE, brain ammonia extraction, cardiac index and noradrenaline requirements, and increases cerebral perfusion pressure. Hypothermia reduced the arterial concentration of pro-inflammatory cytokines, its production by the brain, and it reduced systemic markers of oxidative stress and NO production. Furthermore, hypothermia restored the defective CBF autoregulation and the absence of reactivity to carbondioxide that is normally present in these patients.

\section{Implication:}

- The results of this study have direct implication for the management of patients with acute liver failure. This form of treatment is easy to apply, cheap and relatively safe. We demonstrated that its application was associated with sufficient prolongation in the life of the patients allowing them to be successfully transplanted. Our data provide the rationale for an appropriately powered large clinical trial to determine the place of hypothermia in ALF. We understand that our results have allowed such a trial to be initiated by the US Liver Failure group supported by a generous grant from the NIH.

- The results of our study support the concept of a multifactorial pathogenetic mechanism in the development of intracranial hypertension in ALF. 
From the pathophysiological perspective, the experiments with hypothermia provide support for the hypothesis that further reduction in the metabolic demands allows recovery of the organism.

\section{Observation:}

Institution of moderate hypothermia in patients with ALF that undergo OLT prevents increases in ICP during the dissection and reperfusion phase of the operation. Moderate hypothermia lowered systemic markers of inflammation and prevented there increase. In the normothermic ALF patients undergoing OLT, significant increases in ICP were observed during the dissection and the reperfusion phases that were abolished during the anhepatic phase. The reduction in ICP in the normothermic groups during the anhepatic phase was associated with a normalisation of CBF.

\section{Implication:}

- Moderate hypothermia proved to be a safe and successful method to prevent the rise in ICP during the dissection and the reperfusion phases of the operation and has direct relevance to patient care.

- The results of this study support the concept that liver derived factors are important in the pathogenesis of increased ICP and one of the mechanisms by which hypothermia acts is through a reduction in the production of these substances. Our data provide the framework to explore the nature of these putative toxins and targeted therapy. 


\section{ACKNOWLEDGEMENTS}

This thesis encompasses work spanning 7 years starting as a concept written at the back of a tissue paper in the pub in Newcastle during our first attendance at an Encephalopathy conference. The work has involved several departments in at least 3 major institutions and has been funded through numerous sources. The work started as a collaboration between the Royal Infirmary of Edinburgh and the Academic Hospital Maastricht. The final phases of the collaboration, particularly the aspects defining the role of inflammation in liver failure was developed with the members of the Liver Failure group at the Institute of Hepatology, London.

We would like to thank the organisations involved in funding this work, namely, NWO, Stichting 3 Lichten, the Wellcome Trust, the Research and Development Committee, Royal Infirmary, Edinburgh and the Sir Stanley Davidson Research Fund, University of Edinburgh.

Next, we would like to thank the members of the Corona that were wiling to judge our thesis: Prof. dr. MJHM van Dieijen-Visser, Prof. dr. RW Stockbrügger, Prof. dr. CDA Stehouwer and Prof. dr. MJHM Jacobs, all AZM, Maastricht; the Dutch experts Prof. dr. TM van Gulik (University Amsterdam) and Prof. dr. MJH Slooff (University Groningen); and the international experts Prof. dr. AT Blei (University Chicago, USA), Prof. dr. RF Butterworth (University Montreal, Canada) and Prof. dr. P Ott (University Arhus, Denmark).

We acknowledge that we could only perform the work described with the enormous help of a whole different range of contributors and by standing on the shoulders of Giants. Our biggest thanks goes to the patients who took part in the studies described in this thesis. The nurses of the Department of Medicine, Royal Infirmary of Edinburgh, in particular Sister Castle, Sister Davies and Sister Macbeth deserve special mention. The technicians Sue and Derrick who helped us with all kinds of technical difficulties, analysis and sample preparations. The Radiographers and nurses in Radiology and, the nursing staff of the Liver wards, the Scottish Liver Transplantation Unit and Intensive Care Unit contributed immensely for successful completion of the experiments. Paula Hynds of the department of Dietetics was always willing to take the nutritional assessment, even outside working hours. Our colleagues Hock-Fong Lui and Sherzad Balata for helping in executing the MR and SPECT studies and their contribution in interpretating the data. The members of the MRC brain Unit, especially Prof. Joanne Wardlow for the MR studies and the members of the Department of Psychiatry of the Royal Edinburgh Hospital, especially Prof Klaus Ebmeier, Nadine Dougall and Dr. Mike Glabus for the SPECT study. Stewart McLennan for his help in the acute TIPSS study. Prof Ronan O'Carroll and Maggie Couston for helping with the 
design of the neuropsychologic test battery. Prof. Ken Fearon for the use of the ventilated hood. Prof. OJ Garden and Dr. KK Madavan for their hellp and support to perform the liver transplantation study. We gratefully thank Jean Scheyen, Frank van de Vegt, Annita Rousseau, Gabrie ten Have and Hans van Eijk, for their analyticall help, the shipments of the tracers and Jean for sometimes flying to Edinburgh to facilitate the transport of the samples.

We would also thank our clinical and research colleagues of the Liver Unit, RIE: Kenneth, John, Alistair, Andy, Adrian, Toby, Carol, Ruth, George, Kostas, Matthias, Hazem, Ahmed, Philip, Geoffrey, Neil, Paddy, Lenny, Khalid, Hasnain and Boris for tolerating us, our discussions and the free rounds in Doctors on Friday. Moreover we would like to thank our clinical and research colleagues in London and Maastricht/Heerlen for providing us with the "extra space" that was necessary to perform/complete the work.

\section{The Key Players}

Professor Peter Hayes provided us with the freedom to explore our thoughts without restraint and also unwavering uninhibited, enthusiastic support in terms of access to patients, laboratory space and a fantastic infrastructure to be able to perform the work. Professor Peter Soeters provided us with the freedom to explore the Isoleucinehypothesis, the training to be able to think through the results critically and the support of Steven's stay in Edinburgh.

Dr Mick Deutz, our co-promoter was the person who has provided the analytical basis to be able to perform the studies. His contribution was central in the formulation of the study design, biochemical analyses that the thesis is based upon and also in the interpretation of the data. It has become clear that the data gathered by us during the studies performed has provided the basis for new hypotheses which are now being explored.

Dr Kees Dejong, Steven's co-promotor, introduced him in the research field of ammonia and amino acids, more specifically the metabolic consequences of an upper GI bleed and supported him to explore the Edinburgh-link to translate the experimental Maastricht observations into the patients setting.

Dr Doris Redhead, made the studies, which have been described in the thesis, easy to perform. To the uninitiated, it is impossible to describe the skill and enthusiasm that is necessary to perform the impossible experiments which we describe. No matter what time of day, she was always willing to supply us with the samples requested. We also would acknowledge her for passing some of those skills on.

Dr Alistair Lee, provided unrestricted access to his patients in the Intensive Care Unit. The most difficult experiments were performed after the days work was done. Nothing 
was too difficult or too late at night. Also his insights into the real observations from those that were of less importance provided the framework for a great part of the acure liver failure work.

\section{The people in our lives}

Steven

Major life events laten je realiseren wat belangrijk is in het leven. Goede vrienden (Martin, de dikke stella's, Philip, Roy, Mathijs), een hechte familieband (Gaby, Martin, Arjan, Agnes, Leon, Ellen, Theo, Lyda, mijn ouders Ans en Henk) en een pracht urow die me het mooiste op aarde beeft geschonken (Florine en Melle). Marja, goede wijn behoeft geen krans. Dank voor je steun en liefde.

\section{Rajiv}

I acknowledge very gratefully the support of my parents Kamal and Indra, who have endured without question my long period of absence with unflinching support, love, encouragement and inspiration. I acknowledge my late-grandfather for his unquestioning support and enthusiasm and, my late grandmother for all encompassing love. Also, I acknowledge Bhavnesh and Kamlesh for their support.

Rosy has been at the cold face of living with a clinical-academic. I am grateful (and thank my lucky stars) for the love and support Rosy has provided me through the years helping develop my academic activity by taking on more and more responsibility. About my lovely Aarti, what can I say; she makes it all worth it!

We hope that the series of studies outlined in the book provides encouragement to clinician scientist to indicate that it is possible to undertake high quality translational science in parients. It must however, also be remembered that a large and appropriate infrastructure is needed to be able to undertake these experiments safely. The funding organisations are waking up to increasing funding for translational science and this thesis is a testament that good collaboration and infrastructure is the key for translating fundamental developments into the clinical environment.

Rajiv and Steven 


\section{CURRICULUM VITAE}

Steven Olde Damink was born on September 16th, 1968 in Oldenzaal, The Netherlands. After graduating from the Thycollege (VWO) in Oldenzaal in 1986, he went to the Maastricht University, Maastricht to study Health Sciences (1986) and Medical School (1988). In 1992 he received his doctorate in Movement Sciences and Medicine. During his internships he worked as a research assistant at the department of Surgery, Academic Hospital Maastricht (head Prof, dr. G. Kootstra). After his graduation from Medical School in 1995 he continued his research at this department, which he combined with his surgical training from 1996 (AGIKO). In 1997 he received an NWO fellowship (NWO-AGIKO, nr 920-03-036), which allowed him to work as a Clinical Research Fellow at the Liver Unit, Department of Medicine of the Royal Infirmary of Edinburgh, Scotland, UK (Head Prof.dr. P. . Hayes) and perform the work that is described in this thesis. In 1998 he received funding from the Wellcome Trust (195MED R34543) to continue the collaborative work started. In 1999 he returned to the Netherlands to continue the clinical part of his AGIKO surgical training at the Academic Hospital Maastricht (Sept 1999- Jan 2001 and Sept 2004-present: Head Prof. dr. G. Kootstra and later Prof. dr. M.J.H.M. Jacobs) and the Atrium Medical Centre, Heerlen (Jan 2002- Aug 2004: Head Dr PR. Brink and later Dr. C.J, van der Linden). In 2005 he was elected as Rising Star of Gastroenterology by the Association of National European and Mediterranean Societies of Gastroenterology and was awarded with the Hendrik Casimir-Karl Ziegler fellowship of the Royal Dutch Academy of Science (KNAW), which will allow him to work at the Liver Transplant Unit of Prof. Dr. C.E. Broelsch (Essen, Germany) after the completion of his surgical training (Sept 2005). He is married to Marja Olde Damink; they have two children, Florine (4) and Melle (2). 
Rajiv Jalan was born on December 14th, 1962 in Calcurta, India. He attended secondary school at the La Martiniere for boys, Calcutta, India, after which he studied Medical School at the Nil Ratan Sircar Medical College Hospital, Calcutta, India, were he received his MBBS in 1988. He worked as a clinical fellow in Gastroenterology at the Kothari Centre of Gastroenterology, Calcutta Medical Research Institute, Calcutta (1988-1989, Head: Dr. K.N.Jalan); as registrar in General Medicine and Gastroenterology, Walton Hospital, Liverpool, United Kingdom (1989-1992, head: Dr R.J. Walker and Prof. J.M. Rhodes); as registrar in General Medicine, Gastroenterology and Hepatology, Royal Infirmary of Edinburgh, Scotland, UK (1992-1993, head: Dr. N.D.C. Finlayson). In 1991 he received his MRCP (Royal College of Physicians, London). In 1993 he started to perform his research that led to his MD (1997, 'Transjugular intrahepatic portosystemic stent-shunt, University of Edinburgh) as a Clinical Research Fellow at the Department of Medicine, Royal Infirmary of Edinburgh, and later as a MRC Research Fellow at the Department of Gastroenterology and MR Unit, Royal Postgraduate Medical School, Hammersmith Hospital, London (1994, Head: Prof. H.J.F: Hodgson). In 1995 he returned to Edinburgh to finish his clinical training as Senior Registrar, Department of Medicine (head Prof. P.C. Hayes). He received his CCST in General Medicine and Gastroenterology in 1998. In 1997 he started the work that is described in the present thesis. Since 1999 he works as a senior Lecturer at the Institute of Hepatology, Royal Free and University College Medical School (Head Prof. R. Williams). In 2003 he became a Fellow of the Royal College of Physicians of Edinburgh (FRCPE) and in 2004 of the Royal College of London (FRCP). He is married to Rosy Jalan, they have a daughter Aarti (5). 


\section{LIST OF PUBLICATIONS}

\section{Steven Olde Damink}

Full papers

1. Olde Damink SWM, Dejong CHC, Deutz NEP, Soeters PB. Effects of simulated upper gastrointestinal hemorrhage on ammonia and related amino acids in blood and brain of chronic portacaval-shunted rats. Metabolic Bran Disease 1997; 12:121-35.

2. Olde Damink SWM, Dejong CHC, Deutz NEP, Soeters PB. Decreased plasma and tissue tissue tissue isoleucine levels after simulated gastrointestinal bleeding by blood gavages in chronic portacaval shunted rats. Gut 1997; 40: 418-24.

3. Dejong CHC, Olde Damink SWM, Deutz NEP, van Berlo CLH, Soeters PB. Uremie na bloedingen hoog in de tractus digestivus. Nederlands Tijdschrift voor Geneeskunde 1998; 142: $2558-62$.

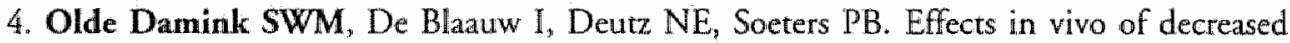
plasma and intracellular muscle glutamine concentration on whole-body and hindquarter protein kinetics in rats. Clinical Science 1999; 96:639-46.

5. Olde Damink SWM, Dejong CHC, Deutz NEP, van Berlo CLH, Soeters PB. Upper gastrointestinal bleeding: an ammoniagenic and catabolic event due to the rotal absence of isoleucine in the haemoglobin molecule. Medical Hyporheses 1999; 52:515-19.

6. Jalan R, Olde Damink SWM, Deutz NEP, Lee A, Hayes PC. Moderate hypothermia for uncontrolled intractanial hypertension in acute liver failure. Lancet 1999; 354:1164-8.

7. Jalan R, Newby DE, Olde Damink SWM, Redhead DN, Hayes PC, Lee A. Acute changes in cerebral blood flow and metabolism during portosystemic shunting. Liver Transplantation 2001; 7:274-8.

8. Jalan R, Olde Damink SWM, Deutz NEP, Hayes PC, Lee A. Restoration of cerebral blood flow autoregulation in acute liver failure by moderate hypothermia. Hepatology $2001 ; 34$ : 50.4 .

9. Olde Damink SWM, Jallan R, Deutz NEP, Redhead DN, Hayes PC, Soeters PB. Interorgan ammonia and amino acid metabolism in metabolically stable patients with cirthosis and a TIPSS. Hepatology 2002; 36: 1163-71.

10. Jalan R, Olde Damink SWM, Lui HF, Glabus M, Deutz NEP, Ebmeier K. Oral amino acid load mimicking hemoglobin results in reduced regional cerebral perfusion and deterioration in memory test in patients with cirrhosis of the liver. Metabolic Brain Disease 2003; 18: $37-49$.

11. Olde Damink SWM, Jalan R, Deutz NEP, Redhead DN, Dejong CHC, Hynd P, Jalan RA, Hayes PC, Soeters PB. Hyperammonemia following a simulated or actual upper gas- 
trointestinal bleed in patients with cirrhosis of the liver is predominantly caused by enhanced renal ammoniagenesis. Hepatology 2003; 37: 1277-85.

12. Jalan R, Olde Damink SWM, Deutz NEP, Davies NA, Garden OJ, Madhavan KK, Hayes PC, Lee A. Moderate hypothermia prevents cerebral hyperemia and increase in intracranial pressure in patients undergoing liver transplantation for acute liver failure. Transplantation 2003; $75: 2034-9$.

13. Balata S, Olde Damink SWM, Ferguson K, Marshall I, Hayes PC, Deutz NEP, Williams R, Wardlaw J, Jalan R. Induced hyperammonemia alters neuropsychology, brain MR spectroscopy and magnecization transfer in cirrhosis. Hepatology 2003; 37: $931-9$.

14. Shawcross DL, Balara S, Olde Damink SWM, Hayes PC, Wardlaw J, Marshall I, Deurz NEP, Williams $\mathbb{R}$, Jalan $R$. Low myo-inositol and high glutamine levels in the brain are associated with neuropsychological deterioration following induced hyperammonemia. Am J Physiol 2004; 287: G503-9.

15. Jalan R, Olde Damink SWM, Deurz NEP, Hayes PC, Lee A. Pathogenesis of Intracranial Hypertension in Acute Liver Failure Inflammation, Ammonia, Cerebral Blood Flow. J Hepatol; 41: 613-20.

16. Jalan R, Olde Damink SWM, Deutz NEP, Hayes PC, Lee A. Hypothermia for uncontrolled intracranial hypertension in acute liver failure. Gastroenterology 2004: 127: 133846.

17. Olde Damink SWM, Dejong CHC, Deurz NEP, Redhead DN, Hayes PC, Soeters PB, Jalan R. The kidney plays a major role in ammonia homeostasis after portasystemic shunting in patients with cirrhosis. (submitted)

18. OIde Damink SWM, Jalan R, Deutz NEP, Dejong CHC, Redhead DN, Hynd P, Hayes PC, Soeters PB. Isoleucine infusion during an upper gastrointestinall bleed improves liver and muscle protein synthesis in cirrhotics. (submitted)

19. Olde Damink SWM, Jalan R, Deutz NEP, Hayes PC, Soeters PB. An upper GI bleed impairs protein synchesis without branched chain amino acid antagonism in patients with cirrhosis (submitted) 


\section{Rajir fallan}

Full papers

1. Lowe NM, Green A, Rhodes JM, Lombard M, Jalan $\mathbf{R}$, Jackson MJ Studies of human zinc kinetics using the stable isotope $70 \mathrm{Zn}$. Clinical Science $1993 ; 84: 113-7$.

2. Jalan $\mathbf{R}$, Grose RD, Dillon JF, Simpson KJ, Hayes PC Gastric mucosal bleeding in cirrhosis. Gastrointestinal Endoscopy 1994; 40; 599-602.

3. Jalan R, Simpson KJ, Redhead DN, Chalmers N, Hayes PC. Transjugular intrahepatic portosystemic stent shunt (TIPSS): long term follow-up. QJ Med 1994; 87: 565-73.

4. Jalan R, Peviris JN, Jalan AR, Finlayson NDC, Hayes PC. A pilot study of ICG clearance in the prediction of graft function following orthotopic liver transplantation. Transplantation 1994; 58: 196-200.

5. Jalan R, Dillon JF, Harrison DJ, Elton R, Hayes PC Laparoscopy and hiscology in the diagnosis of liver disease. QJ Med 1995; 88: 559-64.

6. Ferguson J, Jalan R, Redhead DN, Hayes P, Allan PA The role of Duplex Doppler in monitoring shunt function following transjugular intrahepatic stent shunt. British I Radiology $1995 ; 68: 587-9$.

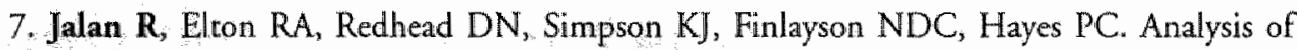
prognostic variables in the prediction of shunt failure, variceal rebleeding, early mortality and encephalopathy following the Transjugular intrahepatic portosystemic stent-shunt (TIPS) J Hepatology 1995; 23: 123-8.

8. Jalan R, John TG, Redhead DN, Finlayson NDC, Garden OJ, Hayes PC. A comparative study of the transjugular intrahepatic portosystemic stent-shunt (TIPSS) and oesophageal transection in urcontirolled variceal haemorrhage. An J Gastroenterol 1995; 90: 1932-7.

9. Jalan $\mathbf{R}_{1}$ Gooday $\mathrm{R}, \mathrm{OCarrol} \mathrm{R}, \mathrm{Redhead} \mathrm{DN}$ Simpson KJ, Hayes PC. A prospective study of changes in neuropsychological and liver function following the transjugular intrahepatic portosystemic stent shunt. J Hepatology 1995; 23: 123-8.

10. Jalan R, Sargentoni J, Bryant DJ, Coutus GA, Bell JD, Cox I], Rolles K, Burroughs AK, Taylor-Robinson SD. Assessment of in viwo hepatic phosphorus magnetic resonance spectroscopy as a prognostic marker in patients with primary biliary cirrhosis. Gut 1996; 39 : 141-6.

11. Jalan R, Harrison DJ, Redhead DN, Hayes PC. Transjugular intraheparic portosystemic stent-shunt (TIPSS) ocdusion and the role of biliary-venous fistula. J Hepatology 1996; $24: 169-76$.

12. Jalan $\mathbf{R}$, Redhead DN, Hayes PC. Haematological alterations following the transjugular intrahepatic portosystemic stent-shunt (TIPSS). Eur J Gastroenterol \& Hepatol 1996; 8: $381-5$. 
13. Forrest EH, Jalan R, Redhead DN, Hayes PC. Differing actions of the acute administration of propranolol and isosorbide-5-mononitrare on the portal circulation. Alimentary Pharmacology and Therapeutics 1996; 10:795-800.

14. Jalan R, Thomas HW, Henderson N, ORourke K, Dillon JF, Williams BC, Hayes PC. Mechanism of changes in renal function following TIPSS. European J Gastroenterology and Hepatology 1996; 8: 1111-6.

15. John TG, Jalan R, Stanley AJ, Redhead DN, Sanfey H, Hayes PC, Garden OJ. Transjugular intrahepatic portosystemic stent-shunt (TIPSS) insertion as a prelude to orthotopic liver transplantation in patients with severe portal hypertension. European I Gastroenterology and Heparology 1996; 8: 1145-9.

16. Stanley AJ, Jalan R, Redhead DN, Finlayson NDC, Hayes PC. Transjugular intrahepatic portosystemic stent-shunr: Results in 130 parients. Gut 1996; 39:479-85.

17. Bzeizi KI, Jalan R, Mcgregor I, Lee A, Hayes PC. Role of neutrophil activation in reperfusion injury. Transplantation 1996; 62: 916-20.

18. Bzeizi KI, Jalan R, Drummond I, Hayes PC. The role of cyclic GMP in reperfusion injury after OLT. Transplantation 1997; 63: 403-6.

19. Jalan R, Forrest EH, Redhead DN, Dillon JF, Hayes PC Reduction in renal blood flow following acute increase in the portal pressure: Evidence for the existence of a hepatorenal reflex in man? Gut 1997; 40: 664-70.

20. Stanley AJ, Jalan R, Ireland IM, Redhead DN, Hayes PC A comparison of gastric and oesophageal varices treated with TIPSS. Alimentary Pharmacology and Therapeutics 1997; 11: 171-6.

21. Jalan R, Forrest EH, Redhead DN, Dillon JF, Bzezie KI, Finlayson NDC, MacGilchrist AJ, Hayes PC. TIPSS vs variceal band ligation in the secondary prevention of variceal haemorrhage in cirrhosis: Resulss of a randomised controlled study. Hepatology 1997; 26: 1115-22.

22. Newby DE, Jalan R, Masumori S, Hayes PC, Boon NA, Webb DJ. Impaired central but stimulated humoral regulation of vascular tone in patients with cirrhosis and ascites. Cardiowascular Research 1998; 38:221-8.

23. Haydon G H, Jalan R, Ala-Korpela M, Hiltunen $Y$, Hanley J, Jarvis L M, Ludlam C A. Prediction of cirrhosis in patients with chronic heparitis $\mathrm{C}$ infection by artificial neural network analysis of virus and clinical factors. Journal of Viral Hepatitis 1998; 5: 255-64.

24. Dabos K J, Stanley A J, Redhead D N, Jalan R, Hayes P C. Efficacy of balloon angioplasty, restenting, and parallel shunt insertion for shunt insufficiency and transjugular intrahepatic portosystemic stent shunt (TIPSS). Min Invas Ther \& Allied Technol 1998; 37: 287-93.

25. Plevris J N, Jalan R, Bzeizi KI, Dollinger M M, Lee A, Garden O J, Hayes P C. Indocyanine green clearance reflects reperfusion injury following liver transplantation and is an early predictor of graft function. J Hepatology 1999; 30: 142-8. 
26. Jalan R, Olde Damink SWM, Lee A, Hayes PC. Moderate hypothermia for uncontrolled intracranial hypertension in acute liver failure. Lancet 1999; 354: 1164-8.

27. Helmy A, Jalan R, Newby DE, Hayes PC, Webb DJ Role of angiotensin II in the regulation of basal and sympathetically-stimulated vascular tone in patients with early and advanced cirhosis. Gastroenterology 2000; 1.18: 565-72.

28. Jalan $\mathbf{R}$, Hayes PC Renal and neurohumoral response to saline loading in pre-ascitic cirrhosis. Gut 2000;46:527-33.

29. Jalan R, N.Turjansk:, SD Taylor-Robinson, MJ Koepp, MP Richardson, J Wilson, J Schweiso, DJ Bryant, JD Bell Df Brooks Pathogenesis of CHE: A positron emission tomography (PET) with 11C-Flumazenil and 1H MRS. Gut 2000; 46: 546-52.

30. Shah SH, Jalan R, Lui HF, Helmy A, Redhead DN, Penny K. Hayes PC. Transjugular intrahepatic portosystemic stent-shunt insufficiency and the role of diabetes mellirus. Eur I Gastroenterol Hepatol 2001; 13: 257-61.

31. Helmy A, Jalan R, Newby DE; Johnston NR, Hayes PC, Webb DJ. Altered peripheral wascular responses to exogenous and endogenous endorhelin-1 in patients with well-compensated cirrhosis. Hepatology 2001; 33: 826-31.

32. Jalan $R$, Olde Damink SWM, Deutz NEP, Hayes PC, Lee A. Restoration of cerebral blood flow autoregulation and reactivity to carbon dioxide in acute liver failure by moderate hypothermia. Hepatology 2001; 34: 50-4.

33. Jalan R, Newby DE, Olde Damink SWM, Redhead DN, Hayes PC, Lee A. Acute changes in cerebral blood flow and metabolism during portasystemic shunting. Liver Transpl 2001; $7: 274-8$.

34. Lui HF, Stanley AJ, Forrest EH, Jalan R, Hislop WS, Mills PR, Finlayson ND, Macgilchrist AJ, Hayes PC. Primary prophylaxis of variceal hemorrhage: a randomized controlled trial comparing band ligation, propranolol, and isosorbide mononitrate. Gastroenterology $2002,123,735-44$.

35. Jalan R, Bzeizi KI, Tripathi D, Lui HF, Redhead DN, Hayes PC. Impact of transjugular intrahepatic portosystemic stent-shunt for secondary prophylaxis of oesophageal variceal haemorrhage: a single-centre study over an 11 -year period. Eur J Gastroenterol Hepatol $2002 ; 14: 615-26$.

36. Olde Damink SWM, Jalan R, Redhead DN, Hayes PC, Deutz NEP Soeters PB. Interorgan ammonia and amino acid metabolism in metabolically stable patients with cirrhosis and a TIPSS. Hepatology 2002, 36:1163-71.

37. Balata S, Olde Damink SWM, Ferguson K, Marshall I, Hayes PC, Deutz NE, Williams $R$, Wardlaw I, Jallan R. Induced hyperammonemia alters neuropsychology, brain MR spectroscopy and magnetization transfer in cirrhosis. Hepatology 2003; 37: 931-9.

38. Helmy A, Newby DE, Jalan R, Hayes PC, Webb DJ. Enhanced vasodilatation to endothelin antagonism in patient with compensated cirrhosis and the role of nitric oxide. Gut $2003 ; 52: 410-5$. 


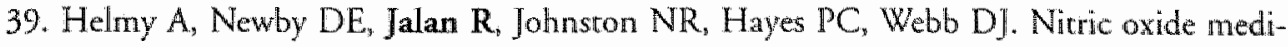
ates the reduced vasoconstrictor response to angiotensin II in patients with preascitic cirrhosis. J Hepatology 2003; 38: 44-50.

40. Jalan R, Kapoor D. Enhanced renal ammonia excretion following volume expansion in parients with well compensated cirrhosis of the liwer. Gur 2003; 52: $1041-5$.

41. Jalan R, Olde Damink SWM, Deutz NEP, Davies NA, Garden OJ, Madhavan KK, Hayes PC, Lee A. Moderate hypothermia prevents cerebral hyperemia and increase in intracranial pressure in patients undergoing liver transplantation for acure liver failure. Transplantation $2003 ; 75: 2034-9$.

42. Jalan $\mathbf{R}$, Olde Damink SWM, Lui HF, Glabus M, Deutz NEP, Hayes PC, Ebmeier K. Oral amino acid load mimicking haemoglobin results in reduced regional cerebral perfusion and deterioration in memory tests in patients with cirrhosis of the liver Metab Brain Dis. 2003; $18: 37-49$.

43. Jalan R, Sen S, Steiner C, Kapoor D, Alisa A, Williams R, Extracorporeal liver support with molecular adsorbents recirculating system in patients with severe acute alcoholic heparitis. J Hepatology 2003; 38, 24-31.

44. Kapoor D, Redhead DN, Hayes PC, Webb DJ, Jalan R. Systemic and regional changes in plasma endothelin following transient increase in portal pressure. Liver Transpl. 2003; 9: 32-9.

45. Mookerjee RP, Sen S, Davies NA, Hodges SJ, Williams R, Jalan R. Tumour necrosis factor $\mathbf{a}$ is an important mediator of portal and systemic haemodynamic derangements in alcoholic heparicis. Gur 2003; 52:1182-7.

46. Olde Damink SWM, Jalan R, Deutz NEP, Redhead DN, Dejong CHC, Hynd P, Jalan RA, Hayes PC, Soeters PB. The kidney plays a major role in the hyperammonemia seen after simulated or actual GI bleeding in patients with cirrhosis. Hepatology 2003; 37: $1277-85$.

47. Tilg $H$, Jalan R, Kaser A, Davies NA, Offner FA, Hodges SJ, Ludwiczek O, Shawcross D* Zoller H, Alisa A, Mookerjee RP, Graziadei I, Datz C, Trauner M, Schuppan D, Obrist P, Vogel W, Williams R. Anti-rumor necrosis factor-alpha monoclonal antibody therapy in severe alcoholic hepatitis. J Hepatology 2003; 38: 419-5.

48. Tripathi D, Lui HF, Heimy A, Dabos K, Forrest E, Stanley AJ, Jalan R, Redhead DN, Hayes PC. Randomised controlled trial of long term portographic follow up wersus wariceal band ligation following transjugular intrahepatic portosystemic stent shunt for preventing oesophageal variceal rebleeding. Gut 2004; 53: 431-7.

49. Shawcross DL, Davies NA, Mookerjee RP, Hayes PC, Williams R, Lee A, Jalan R. Worsening of cerebral hyperemia by the administration of terlipressin in acute liver failure with severe encephalopathy. Hepatology 2004; 39: 471-5.

50. Shawcross DL, Davies NA, Williams $R$, Jalan $R$. Systemic inflammatory response exacer- 
bates the neuropsychological effects of induced hyperammonemia in cirrhosis. J Hepatology 2004; 40: 247-54.

51. Sen S, Ytrebo LM, Rose C, Fuskevaag OM, Davies NA, Nedredal GI, Williams R, Revhaug A, Jalan R. Albumin dialysis: a new therapeutic strategy for intoxication from protein-bound drugs. Intensive Care Med 2004; 30: 496-501.

52. Shawcross DL, Balata S, Olde Damink SWM, Hayes PC, Wardlaw J, Marshall I, Deurz NEP, Williams R, Jalan R. Low myo-inositol and high glutamine levels in the brain are associated with neuropsychological deterioration following induced hyperammonemia. Am J Physiol 2004; 287: G503-9.

53. Jalan R, Kapoor D. Reversal of diuretic-induced hepatic encephalopathy with infusion of albumin but not colloid. Clin Sci (Lond) 2004 ;106: 467-74.

54. Jalan R, Olde Damink SWM, Deutz NEP, Hayes PC, Lee A. Pathogenesis of Intracranial Hypertension in Acute Liver Failure: Inflammation, Ammonia, Cerebral Blood Flow. J Hepatol; 41: 613-20.

55. Jalan R, Olde Damink SWM, Deutz NEP, Hayes PC, Lee A. Hypothermia for uncontrolled intracranial hypertension in acute liver failure. Gastroenterology 2004; 127: 133846.

56. Sen S, Davies NA, Mookerjee R, Hodges S, Cheshire L, Williams R, Jalan R. Pathophysiological effecrs of albumin dialysis in acute-on-chronic liver failure: A randomized controlled study, Liver Transplantation 2004; 10: 1109-19.

57. Ytrebo L, Sen S, Rose C, Nedredal GI, Jalan R, Revhaug A. Contractile response of femoral arteries in pigs. Scandinavian Journal of Gastroenterology 2004; 39: 1000-4. 